$$
\text { JAN POSPISIL }
$$

\title{
Die Entwicklung von Sicherheit
}

Entwicklungspolitische Programme der USA und Deutschlands im

Grenzbereich zur Sicherheitspolitik 
Jan Pospisil

Die Entwicklung von Sicherheit 
Jan Pospisil (Dr. phil.) ist wissenschaftlicher Mitarbeiter am Österreichischen Institut für Internationale Politik (oiip) und Lehrbeauftragter am Institut für Politikwissenschaft der Universität Wien. 
Jan Pospisil

\section{Die Entwicklung von Sicherheit}

Entwicklungspolitische Programme

der USA und Deutschlands im Grenzbereich

zur Sicherheitspolitik

[transcript] 


\section{(9) $(1) \Theta$}

Dieses Werk ist lizenziert unter der Creative Commons AttributionNonCommercial-NoDerivs 4.0 Lizenz (BY-NC-ND). Diese Lizenz erlaubt die private Nutzung, gestattet aber keine Bearbeitung und keine kommerzielle Nutzung. Weitere Informationen finden Sie unter https://creativecommons.org/licenses/by-nc-nd/4.o/deed.de/. Um Genehmigungen für Adaptionen, Übersetzungen, Derivate oder Wiederverwendung zu kommerziellen Zwecken einzuholen, wenden Sie sich bitte an rights@transcript-verlag.de

\section{(C) 2009 transcript Verlag, Bielefeld}

Die Verwertung der Texte und Bilder ist ohne Zustimmung des Verlages urheberrechtswidrig und strafbar. Das gilt auch für Vervielfältigungen, Übersetzungen, Mikroverfilmungen und für die Verarbeitung mit elektronischen Systemen.

\section{Bibliografische Information der Deutschen Nationalbibliothek}

Die Deutsche Nationalbibliothek verzeichnet diese Publikation in der Deutschen Nationalbibliografie; detaillierte bibliografische Daten sind im Internet über http://dnb.d-nb.de abrufbar.

Umschlagkonzept: Kordula Röckenhaus, Bielefeld

Lektorat \& Satz: Jan Pospisil

Druck: Majuskel Medienproduktion $\mathrm{GmbH}$, Wetzlar

Print-ISBN 978-3-8376-1077-2

PDF-ISBN 978-3-8394-1077-6

Gedruckt auf alterungsbeständigem Papier mit chlorfrei gebleichtem Zellstoff.

Besuchen Sie uns im Internet: http://www.transcript-verlag.de

Bitte fordern Sie unser Gesamtverzeichnis und andere Broschüren an unter: info@transcript-verlag.de 


\section{Inhalt}

$\begin{array}{ll}\text { Abkürzungen und Akronyme } & 7\end{array}$

$\begin{array}{ll}\text { Vorwort } & 13\end{array}$

$\begin{array}{ll}\text { Einleitung } & 17\end{array}$

$\begin{array}{ll}\text { Untersuchungsfeld und Problemstellung } & 27\end{array}$

Der Dispositivcharakter von Entwicklung $\quad 35$

Entwicklungskritik $\quad 36$

Entwicklung als Ausdruck gouvernementaler Rationalität 43

Zur analytischen Strategie einer Dispositivanalyse 69

Das neue Sicherheitsproblem von Entwicklung 89

Entwicklung und Sicherheit im Blick der Theoriegeschichte $\quad 90$

Post-politische Konfliktanalyse und die Etablierung neuer

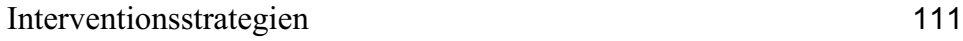

Konzepte und Gestaltung sicherheitspolitischer Entwicklungszusammenarbeit - USA und Deutschland im Vergleich 163

Der institutionelle Apparat des Dispositivs 167

USA: Akteursgruppierung zum Zwecke polyarchischer Stabilität 189

Deutschland: Aufbau demokratischer Sicherheitsstaatlichkeit 233

Zwei Spielarten der Entwicklung von demokratischem Frieden 276 
Sri Lanka - Friedensentwicklung im »Protracted Conflict $«$

Hilfe zum Frieden, Hilfe zum Krieg? Der sri-lankische Konflikt im Kontext internationaler Interventionen

Democracy Promotion und Peacebuilding revisited - Die

entwicklungspolitischen Praxen der USA und Deutschlands

320

Sicherheit und Entwicklung als »White Man's Burden«? Schlüsse und Thesen

Literatur

375

Tabellen und Grafiken

437 


\section{Abkürzungen und Akronyme}

\begin{tabular}{|c|c|c|}
\hline ADB & - & Asian Development Bank \\
\hline AKP-Staaten & - & $\begin{array}{l}\text { Gruppe von Staaten des afrikanischen, kari- } \\
\text { bischen und pazifischen Raumes, in Ver- } \\
\text { tragsbeziehungen mit der EU }\end{array}$ \\
\hline APRC & - & $\begin{array}{l}\text { All Party Representative Committee } \\
\text { (Sri Lanka) }\end{array}$ \\
\hline ATP & - & $\begin{array}{l}\text { Anti-Terrorismus-Programm der deutschen } \\
\text { Bundesregierung }\end{array}$ \\
\hline BCIS & - & $\begin{array}{l}\text { Bandaranaike Centre for International Stu- } \\
\text { dies (Colombo, Sri Lanka) }\end{array}$ \\
\hline $\mathrm{BMZ}$ & - & $\begin{array}{l}\text { Bundesministerium für wirtschaftliche } \mathrm{Zu}- \\
\text { sammenarbeit und Entwicklung } \\
\text { (Deutschland) }\end{array}$ \\
\hline CEPAL & - & $\begin{array}{l}\text { Comisión Económica para América Latina } \\
\text { (Teilorganisation der UNO) }\end{array}$ \\
\hline CFA & - & Ceasefire Agreement (Sri Lanka) \\
\hline CFSP & - & Common Foreign \& Security Policy (EU) \\
\hline CHA & - & $\begin{array}{l}\text { Consortium of Humanitarian Agencies } \\
\text { (Sri Lanka) }\end{array}$ \\
\hline CIMIC & - & Civil Military Cooperation \\
\hline CMM & - & $\begin{array}{l}\text { Office for Conflict Management and Mitiga- } \\
\text { tion (USAID) }\end{array}$ \\
\hline $\mathrm{CNC}$ & - & Ceylon National Congress \\
\hline CPA & - & Centre for Policy Alternatives (Sri Lanka) \\
\hline $\mathrm{CSO}$ & - & Civil Society Organization \\
\hline
\end{tabular}




\begin{tabular}{|c|c|c|}
\hline $\mathrm{D} \& \mathrm{G}$ & - & $\begin{array}{l}\text { Democracy and Governance (Arbeitsbereich } \\
\text { von USAID) }\end{array}$ \\
\hline DAC & - & Development Assistant Committee (OECD) \\
\hline DCHA & - & $\begin{array}{l}\text { Bureau for Democracy, Conflict, and Huma- } \\
\text { nitarian Assistance (USAID) }\end{array}$ \\
\hline DDR & - & $\begin{array}{l}\text { Disarmament, Demobilization and Reintegra- } \\
\text { tion }\end{array}$ \\
\hline DEA & - & Drug Enforcement Administration (USA) \\
\hline DFA & - & Director of Foreign Assistance (USA) \\
\hline DFID & - & $\begin{array}{l}\text { UK Department for International Develop- } \\
\text { ment }\end{array}$ \\
\hline DoD & - & Department of Defence (USA) \\
\hline EADI & - & $\begin{array}{l}\text { European Association of Development Re- } \\
\text { search and Training Institutes }\end{array}$ \\
\hline $\mathrm{EC}$ & - & European Commission \\
\hline EDA & - & $\begin{array}{l}\text { Eidgenössisches Departement für auswärtige } \\
\text { Angelegenheiten }\end{array}$ \\
\hline EPDP & - & $\begin{array}{l}\text { Eelam People's Democratic Party } \\
\text { (Sri Lanka) }\end{array}$ \\
\hline ESDP & - & EU's Security and Defence Policy \\
\hline ESF & - & Economic Support Fund (USA) \\
\hline ESS & - & Europäische Sicherheitsstrategie \\
\hline EWS & - & Early Warning System \\
\hline $\mathrm{EZ} / \mathrm{EZA}$ & - & Entwicklungszusammenarbeit \\
\hline FARC-EP & - & $\begin{array}{l}\text { Fuerzas Armadas Revolucionarias de Co- } \\
\text { lombia - Ejército del Pueblo (Kolumbien) }\end{array}$ \\
\hline FCE & - & Foundation for Co-Existence (Sri Lanka) \\
\hline FLICT & - & $\begin{array}{l}\text { Facilitating Local Initiatives for Conflict } \\
\text { Transformation (GTZ-Projekt, Sri Lanka) }\end{array}$ \\
\hline FriEnt & - & $\begin{array}{l}\text { Arbeitsgemeinschaft Gruppe Friedensent- } \\
\text { wicklung (Deutschland) }\end{array}$ \\
\hline FTO & - & Foreign Terrorist Organizations (USA) \\
\hline $\mathrm{FZ}$ & - & Finanzielle Zusammenarbeit \\
\hline GoSL & - & Government of Sri Lanka \\
\hline GTZ & - & $\begin{array}{l}\text { Deutsche Gesellschaft für Technische Zu- } \\
\text { sammenarbeit }\end{array}$ \\
\hline
\end{tabular}




\begin{tabular}{|c|c|c|}
\hline $\mathrm{HSN}$ & - & Human Security Network \\
\hline IBRD & - & $\begin{array}{l}\text { International Bank for Reconstruction and } \\
\text { Development (eine der fünf Banken der } \\
\text { Weltbank-Gruppe) }\end{array}$ \\
\hline ICG & - & International Crisis Group \\
\hline IDP & - & Internally Displaced Person \\
\hline IKRK & - & Internationales Komitee vom Roten Kreuz \\
\hline INGOs & - & $\begin{array}{l}\text { International Non-Governmental Organizati- } \\
\text { ons }\end{array}$ \\
\hline InWEnt & - & $\begin{array}{l}\text { Internationale Weiterbildung und Entwick- } \\
\text { lung GmbH (Deutschland) }\end{array}$ \\
\hline IPKF & - & $\begin{array}{l}\text { Indian Peace Keeping Force } \\
\text { (Sri Lanka, 1987-1990) }\end{array}$ \\
\hline JHU & - & Jathika Hela Urumaya (Sri Lanka) \\
\hline JVP & - & Janatha Vimtkthi Peramuna (Sri Lanka) \\
\hline KfW & - & $\begin{array}{l}\text { Kreditanstalt für Wiederaufbau } \\
\text { (Deutschland) }\end{array}$ \\
\hline LTTE & - & Liberation Tigers of Tamil Eelam \\
\hline $\mathrm{MCA}$ & - & Millennium Challenge Account (USA) \\
\hline $\mathrm{MCC}$ & - & $\begin{array}{l}\text { Millennium Challenge Corporation (USA, } \\
\text { Umsetzungsorganisation des MCA) }\end{array}$ \\
\hline MDGs & - & Millennium Development Goals \\
\hline NED & - & National Endowment for Democracy (USA) \\
\hline NGO & - & Nichtregierungsorganisation \\
\hline NPA & - & New People's Army (Philippinen) \\
\hline NPC & - & National Peace Council (Sri Lanka) \\
\hline NSS & - & National Security Strategy (USA) \\
\hline ODA & - & $\begin{array}{l}\text { Official Development Assistance (definiert } \\
\text { nach Kriterien des DAC) }\end{array}$ \\
\hline OECD & - & $\begin{array}{l}\text { Organisation for Economic Co-operation and } \\
\text { Development }\end{array}$ \\
\hline OTI & - & Office of Transition Initiatives (USAID) \\
\hline PA4 & - & Politische Abteilung IV (EDA, Schweiz) \\
\hline PACT & - & $\begin{array}{l}\text { Poverty Alleviation and Conflict Transfor- } \\
\text { mation (GTZ-Programmschwerpunkt, Sri } \\
\text { Lanka) }\end{array}$ \\
\hline
\end{tabular}




\begin{tabular}{|c|c|c|}
\hline PCIA & - & Peace and Conflict Impact Assessment \\
\hline PIMU & - & $\begin{array}{l}\text { Poverty Impact Monitoring Unit (ehemaliges } \\
\text { GTZ-Projekt, Sri Lanka) }\end{array}$ \\
\hline PIP & - & $\begin{array}{l}\text { Performance Improvement Project } \\
\text { (GTZ-Projekt, Sri Lanka) }\end{array}$ \\
\hline PRT & - & $\begin{array}{l}\text { Provincial Reconstruction Team } \\
\text { (Afghanistan) }\end{array}$ \\
\hline PSM & - & Peace Secretariat for Muslims (Sri Lanka) \\
\hline PTA & - & Prevention of Terrorism Act (Sri Lanka) \\
\hline P-TOMS & - & $\begin{array}{l}\text { Post-Tsunami Operations Management } \\
\text { Structure (Sri Lanka) }\end{array}$ \\
\hline RNCST & - & $\begin{array}{l}\text { Resource Network for Conflict Studies and } \\
\text { Transformation (Projekt der Berghof Stiftung } \\
\text { in Sri Lanka) }\end{array}$ \\
\hline RRF & - & Rapid Reaction Force \\
\hline SALW & - & Small Arms and Light Weapons \\
\hline SAPI & - & South Asia Peace Institute (Sri Lanka) \\
\hline SCA & - & Strategic Conflict Assessment \\
\hline SCOPP & - & $\begin{array}{l}\text { Secretariat for Coordinating the Peace } \\
\text { Process (Sri Lanka) }\end{array}$ \\
\hline SIDA & - & $\begin{array}{l}\text { Swedish International Development Coop- } \\
\text { eration Agency }\end{array}$ \\
\hline SIPRI & - & $\begin{array}{l}\text { Stockholm International Peace Research In- } \\
\text { stitute }\end{array}$ \\
\hline SLFP & - & Sri Lanka Freedom Party \\
\hline SLMC & - & Sri Lanka Muslim Congress \\
\hline SSA & - & Social Scientists' Association (Sri Lanka) \\
\hline SSR & - & Security Sector Reform \\
\hline TMVP & - & $\begin{array}{l}\text { Tamil Makkal Viduthalai Pulikal } \\
\text { (Karuna-Fraktion, Sri Lanka) }\end{array}$ \\
\hline TNA & - & Tamil National Alliance (Sri Lanka) \\
\hline ToT & - & Training of Trainers \\
\hline TRO & - & $\begin{array}{l}\text { Tamil Rehabilitation Organisation } \\
\text { (Sri Lanka) }\end{array}$ \\
\hline TULF & - & Tamil United Liberation Front (Sri Lanka) \\
\hline $\mathrm{TZ}$ & - & Technische Zusammenarbeit \\
\hline
\end{tabular}




\begin{tabular}{|c|c|c|}
\hline UNDP & - & United Nations Development Programme \\
\hline UNDSS & - & $\begin{array}{l}\text { United Nations Department of Safety and } \\
\text { Security }\end{array}$ \\
\hline UNHCHR & - & $\begin{array}{l}\text { United Nations High Commissioner for } \mathrm{Hu}- \\
\text { man Rights }\end{array}$ \\
\hline UNP & - & United National Party (Sri Lanka) \\
\hline USAID & - & $\begin{array}{l}\text { United States Agency for International De- } \\
\text { velopment }\end{array}$ \\
\hline USIA & - & United States Information Agency \\
\hline USIP & - & United States Institute for Peace \\
\hline $\operatorname{UTHR}(J)$ & - & $\begin{array}{l}\text { University Teachers for Human Rights (Jaff- } \\
\text { na), Sri Lanka }\end{array}$ \\
\hline ZFD & - & Ziviler Friedensdienst (Deutschland) \\
\hline
\end{tabular}





\section{Vorwort}

Die Machtstrukturen einer Gesellschaft zeigen sich besonders deutlich an jenem Punkt, an dem sie sich durch die Ingangsetzung verschiedener Technologien in die Lage versetzen, diejenigen hinter die anvisierten Projekte zu spannen, von denen gemeinhin alternative Ansätze erwartet werden würden. Zwei typische thematische Felder, die Spielraum für derartige Praktiken bieten, sind Entwicklung und Frieden, insbesondere in Gestalt einer zivilen Intervention in den Peripherien, also in ihrer nach außen gerichteten Wirksamkeit.

Ich vermute, dass in diesem Feld vielleicht noch mehr über unsere Gesellschaft und ihr Selbstverständnis zu erfahren ist als bei der Untersuchung domestischer Problematiken. Schließlich wirkt, auch wenn ich damit das Risiko eingehe, zynisch zu klingen, die zivile Intervention im politischen Sinne vielleicht noch radikaler als die militärische. Militärische Interventionen lassen klare Grenzen bestehen, ermöglichen klare Identifikationen, produzieren ein handfestes Innen und Außen, Feinde und Freunde. Davon sind auch bemüht neutralistische »Humanitäre Interventionen « in keiner Weise ausgenommen. Zivile Interventionenn dagegen arbeiten genau in die Grenzbereiche dieser Identifikationen hinein, und führen dadurch, zumindest mittel- und langfristig, ebenso zu einschneidenden Konsequenzen.

Diese Konsequenzen haben nicht zwangsläufig mit den Intentionen zu tun, mit denen sie ursprünglich gesetzt wurden. James Ferguson (1994: 19f.) hat die Funktionalität von Entwicklungsprojekten gerade auch in ihrem Scheitern mit Foucaults Idee des trotz ihrem Versagen feststellbaren »Erfolgs« von Gefängnissen (vgl. Foucault 1994) verglichen. Obwohl die Institution Gefängnis bei den eigentlich intendierten Zielsetzungen Prävention oder Korrektion wenig ausrichten konnte, war sie dennoch bei der Produktion eines zur Aufrechterhaltung disziplinier- 
ter Herrschaft überaus hilfreichen Mechanismus erfolgreich: der Delinquenz. Einen ähnlichen Prozess ortet Ferguson bei Entwicklungspolitik.

Es ist gerade dieser Prozess, der für mich die Faszination des Themas der Überschneidung von Entwicklungs- und Sicherheitspolitik ausmacht. Wahrscheinlich kann es am besten in der Frage nach dem »Warum« zusammengefasst werden,dem »Warum«, das hinter der »responsibility to protect «, dem »enlightened self-interest « oder angeblich naheliegenden geopolitischen Erwägungen liegt, alles Erklärungsmuster, die mehr verschleiern, als sie zu erhellen in der Lage sind. Zivile Intervention, speziell in der in jüngster Zeit in Mode gekommenen aktiven Überschneidung mit Sicherheitspolitik im engeren Sinne, ist, so meine Annahme, eines der ergiebigsten Felder, um sich mit diesem Problem auseinander zu setzen.

Die Fragestellung einer Studie so zu legen bietet neben dem individuellen Erkenntnisgewinn zudem selbst die Möglichkeit zu einer politischen Intervention, wenngleich sie auch minimal bemessen sein mag. ${ }^{1}$ Sie zielt auf die produktive Störung eines speziell im entwicklungspolitischen Bereich festgefahrenen Forschungsbetriebs, der sich nach dem Ende der großen Debatten der 1970er bis auf einige Außenseiter/innen vollständig auf policy-nahe Felder zurückgezogen hat. Michel Foucault hat diese für mich wesentliche Intention in etwas bombastischen (und für meinen Zweck in jedem Fall hoch gegriffenen) Worten als »Humor des Verrats« zusammengefasst:

»Oder etwas Gewaltiges erzählen, wie man die Bude in die Luft jagen könnte, aber ohne gewaltsamen Sturmangriff, sondern indem man im Gegenteil eine vertraute Haltung einnimmt, indem man sich den Anschein gibt, das Haus unbesorgt zu bewohnen, das man untergräbt, und die Sprache zu verwenden, die man dort üblicherweise spricht. Humor des Verrats« (Foucault 2002a: 520).

Ein solcher »Humor des Verrats« darf nicht mit Arroganz verwechselt werden. Schließlich beruht er entscheidend darauf, dass das Gegenüber, das Forschungsobjekt und die beforschten Subjekte, in ihren Handlungen und Intentionen ernst genommen werden. Zugleich setzt er aber voraus, die übliche Falle der Technokratie - die Suche nach Verbesserungen, im entwicklungspolitischen Jargon die berühmten »lessons learned « - zu vermeiden. Und zwar zugunsten von Annahmen und Erkenntnissen, die im Rahmen der institutionellen Beschränkungen nicht zu formulieren wären - eben »die Bude in die Luft jagen«.

1 »Wissenschaft als kulturelle Tätigkeit ist zugleich Interaktion und Intervention. Wenn Wissenschaft als kulturelle Praxis verstanden wird, dann gibt es kein bloßes Entdecken«, meint Mona Singer (2005: 20). 
An dieser Stelle kommt zumeist ein klassischer, oftmals erhobener Vorwurf. Wie leicht sei es, etwas grundsätzlich zu verdammen, viel schwerer - und natürlich auch wichtiger - sei es hingegen, Kritik produktiv zu formulieren. Im besten Fall wäre eine solche Kritik gleich mit unmittelbaren Policy-Implikationen versehen. Das Zauberwort lautet Anwendbarkeit.

Ich empfinde diesen Vorhalt als richtig und falsch zugleich. Richtig ist er, weil sich viele der so genannten Fundamentalkritiken in einfach gestrickten Mustern von Stehsätzen erschöpfen und eine Konfrontation mit der Empirie vermeiden. Seine Grenze erreicht der Vorhalt aber spätestens dann, wenn die Konfrontation mit der Empirie nicht vermieden, sondern gesucht wird. In diesem Fall kommt sofort der Praxisdruck zum Tragen, der versucht, jede/n, der/die sich mit der Materie beschäftigt, in die Pflicht zu nehmen, immer auf der Suche nach dem unmittelbaren Verwertungszusammenhang. Und mit diesem Mechanismus zielen die Praktiker/innen nicht mehr auf die Schwäche, die sich aus der Diskrepanz zwischen leichter Kritik und unverhältnismäßig schwierigerer Formulierung von Konsequenzen aus dieser ergibt. Vielmehr zeigt sich dann die generelle Unwilligkeit der Auseinandersetzung mit den Störgeräuschen, die die als gegenüber solchen Störungen immun konstruierten bequemen Nischen beinträchtigen, vielleicht sogar gefährden könnten.

Ich hoffe, es gelingt mir, sichtbar zu machen, dass der vertiefte und konkrete Umgang mit Empirie für mich der Schlüssel kritischer Wissenschaft ist. Ein solches Verständnis ist untrennbar verknüpft mit analytischer Bescheidenheit, die aus der Einsicht in die Beschränktheit der eigenen Sicht resultiert. Mir ist natürlich klar, dass mit wenig intellektuellem Aufwand aus jedem beliebigen Detail eine große Geschichte konstruiert werden kann, die dann mühelos für sich in Anspruch nimmt, auf empirischen Realitäten zu fundieren. Mit einem Verständnis von Kritik und damit auch von Wissenschaft, das nicht nur die Infragestellung der zu erforschenden Realität, sondern zuallererst die Infragestellung des erkennenden Subjekts selbst umfasst, hat dies jedoch wenig zu tun.

Natürlich wäre diese Arbeit und der ihr vorangegangene langwierige Arbeitsprozess nicht möglich und in jedem Fall bei weitem nicht so produktiv gewesen, wie ich ihn empfunden habe, ohne eine Fülle von Menschen und Institutionen, die mich dabei unterstützt haben. Einige von ihnen möchte ich an dieser Stelle erwähnen, um mich in aller Form bei ihnen zu bedanken. Allen voran steht Wolfgang Dietrich, der mich über eine Gesamtzeit von fast acht Jahren in den fortgeschrittenen Phasen meiner akademischen Ausbildung begleitet hat. Sein Einfluss ist ohne 
Zweifel spürbar, und ohne seine Unterstützung und sein Beispiel hätte diese Arbeit so nicht stattgefunden.

Gelesen und kommentiert wurde die Arbeit von Stefan Khittel, Thomas König, Eva Kreisky, Katharina Salas Torrero und Walter Trunk. Ihnen gebührt das Verdienst, den Text sowohl wissenschaftlich als auch sprachlich auf ein Niveau gebracht zu haben, zu dem ich allein nicht fähig gewesen wäre. Trotzdem tragen sie weder für eventuelle sprachliche Behäbigkeiten noch für die Fehler und Nachlässigkeiten des Textes Verantwortung. Diese sind allein mir zuzuschreiben.

Mona Singer, Baruch Wolski, Stefan Hinsch, Jelena Tošić, Helmut Lukas, Gudrun Kramer und Wilfried Graf waren Diskussionspartner/innen, die in unterschiedlicher Weise dazu beigetragen haben, meine Ideen auszurichten und zu schärfen. Sumane Liyanage, Nick Lewer, Koenraad Denayer und Norbert Ropers haben entscheidend dazu beigetragen, dass mein viermonatiger Aufenthalt in Sri Lanka zu einer für mich fruchtbaren Zeit werden konnte, die zur Entwicklung meiner Thesen wesentlich beigetragen hat.

Institutionell sei dem Jubiläumsfonds der Österreichischen Nationalbank gedankt, der jene Forschungsprojekte finanziert hat, die mir im Rahmen meiner Mitarbeit die mehr als dreijährige vollständige Konzentration auf die untersuchte Materie ermöglichten. Vor allem möchte ich aber das Österreichische Institut für Internationale Politik (oiip), besonders dessen Direktor Otmar Höll und Heinz Gärtner, sowie die Kommission für Sozialanthropologie an der Österreichischen Akademie der Wissenschaften hervorheben, die mich während des Arbeitsprozesses in verschiedenster Weise unterstützt haben.

Letztendlich möchte ich mich ausdrücklich auch bei all jenen bedanken, die sich mir zu Gesprächen und Interviews zur Verfügung stellten. Sie werden in überwiegender Zahl von meinen hier präsentierten Ausführungen alles andere als begeistert sein. Ich hoffe dennoch, dass sie im Sinne des immer positiven Einflusses auch externer fundamentaler Kritik - in der einen oder anderen Form von meiner Arbeit profitieren können. 


\title{
Einleitung
}

\begin{abstract}
$»$ Kritik heißt nicht, dass man lediglich sagt, die
Dinge seien nicht gut so, wie sie sind. Kritik heißt herausfinden, auf welchen Erkenntnissen,

Gewohnheiten und erworbenen, aber nicht reflektierten Denkweisen die akzeptierte Praxis beruht. $[\ldots]$

Kritik heißt, Dinge, die allzu leicht von der Hand gehen, ein wenig schwerer zu machen.«
\end{abstract}

(Michel Foucault)

»Not only is underdevelopment thus classified as dangerous, violence-laden, endemic conflict amongst impoverished populations appears to deepen that danger. The micro-practices of underdevelopment are both a source of conflict and a result of conflict: the locus of a vicious cycle of violence and human rights abuse. By the 1990s such sentiments provided the rationale for the widespread incorporation of a security problematic committed to conflict prevention and conflict resolution into development aid policy. This represented the securitisation of the politics of development« (Dillon 2004: 79f.).

Diese Bemerkungen von Michael Dillon legen nahe, dass es während der letzten Jahre in der entwicklungspolitischen Theorie und Praxis zu einer Veränderung gekommen ist, die er als »Securitisation of Development « charakterisiert. Wir können eine solche Veränderung - um den Prozess vor einer genaueren Diskussion und Bewertung zunächst möglichst wertungsfrei zu benennen - in der entwicklungspolitischen Debatte in Europa seit Mitte der 1990er Jahre feststellen. In der Folge der zu diesem Zeitpunkt noch frischen Erfahrungen aus den Krisen in Jugoslawien und Ruanda sind plötzlich Aussagen zu lesen, die Kriegsbeendi- 
gung und Friedenskonsolidierung zur $»$ neue[n] Aufgabe von Entwicklungspolitik« (Matthies 1995b: 20) erklären.

Natürlich ist Entwicklungshilfe ${ }^{1}$ immer schon unter sicherheitspolitischen Aspekten verstanden worden, wenn auch auf beiden Seiten des Atlantiks in unterschiedlicher Weise. Während die USA seit ihrem ersten explizit entwicklungspolitischen Programm - dem Marshall-Plan eine klare Kohärenz im sicherheitspolitischen Bereich erkennen ließen (bis heute ist die National Security Strategy das bestimmende Leitdokument für die Tätigkeit von USAID), wurde dies bei den ehemaligen großen Kolonialmächten in Europa nie derart explizit formuliert. Dass sich hier trotzdem unter argumentativen Mustern wie dem »wohl verstandenen Eigeninteresse«, das für die offizielle Entwicklungshilfe bis heute tonangebend ist, naturgemäß sicherheitspolitische Interessen finden und seit dem Beginn dieser Form internationaler Intervention auch befunden haben, liegt auf der Hand.

Dennoch werde ich in dieser Arbeit nachzuweisen versuchen, dass die Veränderung der 1990er Jahre einen Bruch mit bisherigen entwick-

1 Angesichts der im deutschen Sprachraum umfassenden Begriffsverwirrung zwischen Entwicklungshilfe, Entwicklungspolitik und Entwicklungszusammenarbeit möchte ich gleich die erste Erwähnung eines der genannten Begriffe für eine ausführliche Klarstellung nutzen. Zunächst zeigt allein die Verwendung des Begriffes »Entwicklungshilfe«, dass ich nicht gewillt bin, den in den letzten Jahren aufkommenden Usus seiner moralisch argumentierten ersatzlosen Streichung (die mit einer für gewöhnlich wahllosen Ersetzung durch einen der beiden anderen einhergeht) mitzuvollziehen, und zwar aus zwei Gründen: Einerseits sehe ich wie Gerald Hödl keinen Sinn darin, einen »die Realität höchst unzureichend beschreibende[n] Euphemismus durch einen anderen« abzulösen (vgl. Hödl 2004: 7, Fn 2). Andererseits bin ich der Überzeugung, dass der Begriff aus definitorischen Gründen nicht durch einen anderen ersetzt werden kann, da es einer solchen Dreiteilung von Entwicklungs-Aktivismus bedarf. Ich möchte versuchen, die von mir angewandten Bedeutungen anhand der klareren englischen Begriffe nachzuzeichnen: Entwicklungspolitik entspricht dem englischen Begriff »development policy«. Damit ist das Politikfeld gemeint, in dem sich Entwicklungshilfe und Entwicklungszusammenarbeit vollziehen; Entwicklungshilfe entspricht dem Begriff der »development assistance « und umfasst mehr oder minder (die in dieser Definition liegenden Probleme werden noch zu diskutieren sein) alle Leistungen, die unter die ODA-Kriterien des DAC fallen; Entwicklungszusammenarbeit schließlich umfasst die konkreten Interventionen, die im Englischen durch den Begriff »development cooperation« wiedergegeben wird. Er kann entlang der hier nützlichen offiziellen deutschen Terminologie in die Bereiche der Technischen und der Finanziellen Zusammenarbeit (TZ/FZ) weiter differenziert werden. Spreche ich hingegen vom Begriff Entwicklung allgemein, ist damit jenes gedankliche Fundament gemeint, auf dem die erwähnte Begriffsdreiteilung gebaut ist und das ich später als Dispositiv klassifizieren werde. 
lungspolitischen Ansätzen mit sich gebracht hat, und dass beide Seiten des Atlantiks davon betroffen waren, wenngleich in unterschiedlicher Form. An dieser Stelle soll es allerdings genügen festzuhalten, dass in der kontinuierlich laufenden Ausrichtungsdebatte der Entwicklungspolitik während der letzten Jahre Dinge gesagt, analysiert und vorgeschlagen wurden, die einen grundlegend neuen Gehalt aufweisen und selbst ein Jahrzehnt zuvor so nicht hätten gesagt werden können.

Historisch schließt diese Veränderung an die Diskussion um den Governance-Begriff an, der in den späten 1980ern die entwicklungspolitischen Debatten dominierte. Es ist zunächst vor allem die UNO, die die neue Fokussierung auf bewaffnete Konflikte mit einigen Schlüsseldokumenten einleitet: zunächst im Jahr 1992 mit dem Report »An Agenda for Peace. Preventive diplomacy, peacemaking and peace-keeping « (Boutros-Ghali 1992), und später mit dem programmatischen Human Development Report 1994 (UNDP 1994), der den Begriff »Human Security« in großem Stil in die Debatte einführt.

Mit einigen Jahren Verzögerung beginnt ein solches Verständnis bei den großen bilateralen Gebern Fuß zu fassen. In Europa ist die Diskussion Mitte der 1990er Jahre wie erwähnt vor allem von den auch als Versagen von Entwicklungspolitik verstandenen Katastrophen in Ruanda und Jugoslawien geprägt und primär defensiv ausgerichtet. Ruanda wird in diesem Zusammenhang als Paradefall für eine Praxis identifiziert, die nicht nur die lokalen und regionalen Gegebenheiten mangelhaft reflektiert, sondern durch die gesetzten Interventionsschritte vorhandene konfliktträchtige Situationen sogar noch verschärft. Dies hätte, so die allgemeine Einschätzung, zu einem »Schock» für das Selbstverständnis der Entwicklungshilfe geführt (vgl. Mehler/Ribaux 2000: 20; Smith 2004b: 115; Klingebiel 2001: 2; Ropers 2002: 14 oder Brock et al. 2003: 20 f. für die deutsche Diskussion).

Speziell die skandinavischen Länder und die Niederlande erweisen sich in der Folge als Vorreiter einer Debatte (vgl. Mehler/Ribaux 2000: 41), die im Jahr 1997 mit der Ausarbeitung der bis heute (nach einer Überarbeitung in 2001) als Schlüsseldokument geltenden Guidelines des Development Assistance Committee (DAC) der OECD, »Helping Prevent Violent Conflict« (DAC 2001), einen ersten Höhepunkt findet. Im Juli 2005 sorgt das DAC (2005b) mit der Einführung des DAC-Codes 152, der auf Maßnahmen mit der Zielsetzung »Conflict prevention and resolution, peace and security« zugeschnitten ist, für eine formelle organisatorische Einbindung der Thematik in den entwicklungspolitischen Kanon. 
Die Wahlsiege der sozialdemokratischen Parteien in Großbritannien (1997) und Deutschland (1998), die in beiden Ländern zur Bildung von Mitte-Links-Regierungen führen, tragen ein Übriges zur Forcierung des neuen entwicklungspolitischen Themas bei. Eine der ersten Handlungen der Regierung Blair ist die Gründung des Department for International Development (DFID) zur Zentralisierung und Bündelung der staatlichen britischen Aktivitäten auf dem Entwicklungssektor. DFID ersetzt die Overseas Development Administration, die, nachdem sie in den 1970ern für einige Jahre den Status eines Ministeriums innehat, seit 1979 als Abteilung des Foreign and Commonwealth Office die Verwaltung der entwicklungspolitischen Agenden wahrgenommen hat.

Obwohl sich DFID thematisch primär in der Armutsbekämpfung auf globaler Ebene und seit 2000 in den thematischen Komplexen der Millennium Development Goals (MDGs) engagiert und diese Ausrichtung bis heute beibehält, nimmt Großbritannien gleichwohl eine der weltweit führenden Positionen bei der Verflechtung entwicklungs- und sicherheitspolitischer Aufgabenfelder ein. Mit dem Begründungszusammenhang, dass Entwicklung wesentliche Voraussetzung für nachhaltigen Frieden sei und zugleich unter den Bedingungen eines bewaffneten Konflikts nicht stattfinden könne, wird begonnen, unmittelbar in sicherheitspolitische Kernbereiche zu intervenieren. Besonderes Augenmerk wird auf die Arbeit am Sicherheitssektor (die so genannte "Sicherheitssektorreform«, kurz SSR) der Zielländer gelegt. Dazu werden nach ersten Schritten Ende der 1990er Jahre nach der Jahrtausendwende in Zusammenarbeit mit Foreign Office und dem Ministry of Defence gemeinsame Programmlinien erarbeitet, die in der entwicklungspolitischen Szene bis heute als richtungsweisend gelten.

Eine ähnliche Entwicklung lässt sich in Deutschland nach der Regierungsübernahme durch ein sozialdemokratisch-grünes Kabinett unter Gerhard Schröder beobachten. Zwar existiert das Bundesministerium für wirtschaftliche Zusammenarbeit (BMZ) ${ }^{2}$ schon seit 1961, doch nach der Übernahme des Ministeriums durch die Sozialdemokratin Heidemarie Wieczorek-Zeul zeichnet sich eine deutliche Veränderung der Schwerpunktlegungen ab. Wieczorek-Zeul stellt ihre Amtsperiode unter das Leitmotiv der »Globalen Strukturpolitik«, woraus sich auch eine prononciert sicherheitspolitische Ausrichtung ableitet. Ausdrücklich sind damit nicht nur die mittelbaren Wirkungen »traditioneller« ökonomisch und sozial orientierter Intervention gemeint, sondern die Ausdehnung

2 Derzeit lautet der vollständige Name »Bundesministerium für wirtschaftliche Zusammenarbeit und Entwicklung«. Der Zusatz »und Entwicklung« wird, wie noch auszuführen sein wird, erst im Jahr 1993 hinzugefügt. 
auf Bereiche, die bis dahin dem Gebiet der klassischen Sicherheitspolitik vorbehalten waren, also dem Auswärtigen Amt oder dem Verteidigungsministerium. »Eine >Schmalspurlösungく ist [...] offensichtlich kaum angebracht «, kommentiert eine der zentralen Auftragsstudien jener Tage, denn »Organisationen, die sich mit dem Thema nur am Rande beschäftigen, werden kaum über die Kompetenzen verfügen, die zur Verfügung stehenden Instrumente rechtzeitig und zielgruppengerecht einzusetzen« (Mehler/Ribaux 2000: 91).

Bereits Ende der 1990er Jahre wird zu diesem Zweck mit dem »Zivilen Friedensdienst « ein erstes spezifisches Instrument geschaffen, das Interventionen im Konfliktkontext mit Mitteln ermöglichen soll, die nicht dem konventionellen Repertoire entwicklungspolitischer Intervention entsprechen (vgl. Evers 2000; BMZ 2004c). Bemerkenswert ist, dass diese Initiative nicht vom Ministerium oder anderen staatlichen Stellen ausgeht, sondern von privaten Akteuren wie NGOs und Friedensaktivist/innen, die es als großen Erfolg feiern, das BMZ zum Aufgreifen des Vorschlages bewegt zu haben.

Auf Ebene der Europäischen Union - die konkrete Organisation der Entwicklungshilfe obliegt hier der EU-Kommission - ist ein ähnlicher Prozess feststellbar, wenn auch mit relevanter zeitlicher Verzögerung. Erste Ansätze finden sich zwar schon Mitte der 1990er Jahre im Zuge der AKP-Verhandlungen und in der Diskussion um zivile Interventionen in Afrika (vgl. EC 1996). Der thematische Durchbruch ist allerdings erst im Jahr 2001 mit der Kommunikation der EU-Kommission zum Thema der Konfliktprävention festzumachen (EC 2001b). ${ }^{3}$ Darin heißt es etwa: $»$ Development policy and other co-operation programmes provide, without doubt, the most powerful instruments at the Community's disposal for treating the root causes of conflict« (ebd.: 9).

Hand in Hand mit dieser Kommunikation werden auf institutioneller Seite praktische Maßnahmen gesetzt, die sich primär auf die Kooperation mit im Bereich der Konfliktprävention tätigen Forschungseinrichtungen konzentrieren. So wird das Conflict Prevention Network (CPN), unterhalten von verschiedenen ODA-Akteuren und zu diesem Zeitpunkt an der deutschen »Stiftung Entwicklung und Frieden« in Bonn beheimatet, mit der Ausarbeitung eines »Practical Guide« beauftragt, der dem Personal sowohl in den Planungsbereichen als auch im Feld konkrete Handlungsanleitungen vermitteln soll (Lund/Mehler 2001).

In der 2003 veröffentlichten Europäischen Sicherheitsstrategie (ESS) wird die Entwicklungspolitik schließlich ausdrücklich als Be-

3 Es ist in diesem Zusammenhang wesentlich zu erwähnen, dass diese Kommunikation im April 2001, also vor 9/11, veröffentlicht wird. 
standteil der CFSP der EU bezeichnet (European Union 2003: 13). Zwar ist eine solche Zuordnung in der Tat bemerkenswert, es gilt aber zu bedenken, dass diese Rollenzuweisung ursächlich aus Gründen der PolitikKohärenz erfolgt (gerade auch der CFSP und ihrer Politikbereiche mit der ESDP). Die mitunter geäußerte Interpretation, die ESS würde in Richtung einer maßgeblich entwicklungspolitisch beeinflussten Wende in der Sicherheitspolitik gehen, scheint übertrieben. Dennoch zeigt die ESS, dass wir auch auf der Ebene des »kollektiven Bilateralismus« (vgl. Nuscheler 1996: 458) mit einer signifikanten Veränderung konfrontiert sind.

In den Vereinigten Staaten gestaltet sich dieser Prozess anders, schon allein weil angesichts der Ausgangsbedingungen US-amerikanischer Entwicklungshilfe eine »Re-Politisierung« nur schwerlich machbar ist. Entwicklungshilfe wird schließlich von Beginn an zentral an politischen Zielbestimmungen festgemacht: »The United States Agency for International Development was created in 1961 with two purposes in mind: to respond to the threat of communism and to help poorer nations develop and progress « (USAID 1994: 1). Diese politische Stoßrichtung wurde mit einer Vehemenz rund um den Globus umgesetzt, die jede europäische Bemühung um sektorale Kooperation im außen- und sicherheitspolitischen Bereich als ein fast kleinliches Geplänkel erscheinen lassen.

Dennoch meine ich, zumindest innerhalb der konzeptionellen Rahmenbedingungen der US-amerikanischen Entwicklungshilfe Veränderungen nachweisen zu können, die denjenigen in der europäischen Debatte (und auch in den ähnlich verlaufenden Debatten in den großen multinationalen Entwicklungsinstitutionen wie UNO und Weltbank) vergleichbar sind. Am besten lässt sich das anhand der strategischen Planungsdokumente zeigen, die die Basis für die jährlichen Congressional Budget Justifications bilden, denen wiederum die Funktion konkreter Operativpläne zukommt. Diese strategischen Planungsdokumente sind jeweils für etwa vier bis sechs Jahre angelegt, wobei sie in diesem Zeitraum Revisionen unterzogen werden können.

Der Blick in die Dokumente zeigt, dass die erste Hälfte der 1990er vom damals neu aufgekommenen Begriff des »Sustainable Development « geprägt ist (vgl. USAID 1994). Eine spannende Veränderung ergibt sich mit der im Jahr 2000 durchgeführten Revision des strategischen Planes von 1997, die diesem bei gleichbleibenden Zielsetzungen im Sinne der Nachhaltigkeit verschiedene »Cross-Cutting themes« hinzufügt. Eines dieser Themen ist »Crisis Management«, wo unter Anwendung des Mitte der 1990er aufkommenden Modebegriffes einer »complex cri- 
sis« ${ }^{4}$ Katastrophenmanagement mit der spezifischen Arbeit an internen und externen Konflikten verknüpft wird (vgl. USAID 2000a: 22).

Eine qualitative Veränderung ist jedoch erst mit dem 2003 veröffentlichten strategischen Plan für die Jahre 2004-2009 (U.S. Department of State\&USAID 2003) festzustellen, der unzweifelhaft stark von $9 / 11$ und dem in der Folge vom Zaun gebrochenen »War on Terrorism« geprägt ist. Dies demonstriert nicht nur das vorangestellte Motto »Security, Democracy, Prosperity«, das das letzte ausgegebene Motto von 1994, »Sustainable Development«, ablöst. Zum ersten Mal wird die strategische Planung gemeinsam mit dem State Department durchgeführt. Schon bei der Identifikation der Bedrohungsbilder zeigt sich eine deutliche strategische Neuorientierung gegenüber den vorherigen Planungen: Nicht mehr Armut und Demokratiedefizit sind das zentrale Problem, sondern Failed States und Terrorismus.

Als die zwei zentralen Arbeitskomplexe finden sich nun »Peace and Security « und »Sustainable Development « nebeneinander, wobei es auch innerhalb des traditionellen Entwicklungskomplexes zu einer Verschiebung kommt. Die im Jahr 2000 noch dominierende ökonomische Komponente wird vom Demokratie- und Menschenrechtsthema von der Spitze verdrängt und zudem um ein Sicherheitssuffix ergänzt. Aus dem breit angelegten ökonomischen Wachstum der 1990er wird jetzt »Economic Prosperity and Security« (ebd.: 21).

Dieses neue Programm zeigt zweierlei: zunächst, dass es sich bei der Verschiebung der Bedrohungsbilder nicht nur um eine semantische Operation handelt, sondern dass hier sehr wohl grundlegende Änderungen anvisiert sind. Zugleich können solche Änderungen nicht einfach als eine simple Rückführung der Entwicklungspolitik in die außenpolitische Domäne gedeutet werden, auch wenn diese Deutung zwanzig oder vielleicht sogar zehn Jahre zuvor naheliegend gewesen wäre. Vielmehr

4 Der Begriff (gleichbedeutend mit »complex emergencies «) stammt aus der Diskussion um die Katastrophen in verschiedenen afrikanischen Ländern und in Jugoslawien in der ersten Hälfte der 1990er Jahre, wo kriegerische Entwicklungen mit großen Flüchtlingsbewegungen einhergehen. In der Debatte im entwicklungspolitischen und humanitären Bereich wird in weiterer Folge darunter der traditionelle Begriff des »disaster management« subsumiert. Mark Duffield schlägt vor, diese neue Begrifflichkeit als Ausdruck des liberalen Friedens unter neuen Rahmenbedingungen zu begreifen: »The concept of a complex political emergency carries with it the ideological means of justifying, mobilising and coordinating the state and non-state actors of liberal peace. It would make more sense, however, to rephrase the encounter so that liberal peace is seen to confront not complex political emergencies but emerging political complexes on its borders« (Duffield 2001: 163). 
scheint auch in den USA der Entwicklungspolitik eine Funktion in einem sich neu konstituierenden sicherheitspolitischen Komplex zuzufallen.

Was wir anhand dieser kurzen Schlaglichter auf Vorgänge bei verschiedenen bi- wie multilateralen Gebern sehen können, ist ein trotz unterschiedlicher Gegebenheiten durchgängiger und zeitlich relativ parallel verlaufender Veränderungsprozess, der auf eine tendenzielle Änderung von Annahmen, Zielsetzungen und Prioritäten der internationalen Entwicklungspolitik hinausläuft. In Schlagworte gefasst könnten wir sagen, dass nach »Modernisierung «, dem entscheidenden entwicklungspolitischen Begriff bis Mitte der 1980er, und »Nachhaltigkeit«, der das Feld spätestens seit dem Brundtland-Report 1987 dominiert, nun »Sicherheit« zum führenden Motto aufzusteigen scheint.

So klar und einfach ein derartiges Schema auch erscheint, so vorsichtig und skeptisch müssen wir ihm allerdings entgegentreten. Denn die Annahme weist ohne Zweifel Schwächen und Unschärfen auf, die einer genaueren Betrachtung bedürfen, bevor eine Analyse von Ursachen und Konsequenzen in Angriff genommen werden kann. Die Tatsache, dass ein Großteil der ODA-Gelder nach wie vor in »traditionelle« Entwicklungsprogramme fließt, ist hier ebenso einzubeziehen wie die programmatische Dominanz der MDGs nicht nur im Kontext der UNO und ihrer Suborganisationen, sondern auch bei vielen bilateralen Gebern.

Der genaue historische Verlauf und seine Rahmenbedingungen bleiben also zu diskutieren und zu bewerten, ebenso wie die Auswirkungen in konkreten Interventionen. Dringlich notwendig ist in jedem Fall eine Klärung jener beiden Begriffe, die sich schon im Titel der Arbeit finden und die den thematischen Komplex konstituieren, um den es im Folgenden gehen soll: die Begriffe »Entwicklung« und »Sicherheit«.

Wir finden bei beiden Begriffen eine große Vielfalt an Definitionsansätzen, was angesichts ihrer amöbenhaften Struktur keineswegs überraschend ist. Genauso wenig überraschend, dafür aber umso aussagekräftiger ist die Tatsache, dass beide Begriffe in der Mehrzahl der Beiträge, die sich mit ihnen befassen, erst gar nicht definiert werden. Diskutiert werden zumeist Konzepte, Wege und Strategien, wie »Entwicklung « und »Sicherheit« zu erreichen seien. Die Frage, ob sie überhaupt erstrebenswerte Zielsetzungen markieren, oder was es eigentlich bedeutet, wenn sie letzten Endes gar erreicht oder zumindest temporär gewährleistet sein sollten, wird in diesem Zusammenhang für gewöhnlich nicht gestellt. Mit einer solchen Operation werden die Begriffe - wie ich meine gezielt - in die Rolle anthropologischer Grundkonstanten gerückt, 
die sich jeder Hinterfragbarkeit widersetzen (hinsichtlich des Sicherheitsbegriffs vgl. Dillon 1996: 13). ${ }^{5}$

Natürlich weisen beide Begriffe in ihrer Entstehung bedeutende Unterschiede auf. Wenngleich unbestreitbar ist, dass beide ihre heute dominierende Form mit der Entfaltung der Moderne angenommen haben, gibt es dennoch beträchtliche Differenzen in den jeweiligen Etymologien. Während »Entwicklung« an sich eine moderne Idee darstellt, deren Begriffsgeschichte gerade einmal in das 18. Jahrhundert zurückreicht (vgl. Esteva 1992a: 8f.), geht die Geschichte von »Sicherheit« (die im Deutschen jene zwei Komponenten umfasst, die das Englische mit »security« und »safety« getrennt konzeptualisiert) bedeutend weiter zurück und ist eng mit der Idee menschlicher Gemeinschafts- und Institutionsbildung verknüpft ${ }^{6}$, wobei insbesondere letzterer Prozess eine Verbindung mit dem Wahrheitsbegriff nach sich zieht (vgl. Dietrich 2006). Trotzdem erhält der Begriff seine heute bestimmende politische Prägung erst mit der Moderne und mit ihr verbundener Prozesse wie der Entstehung von Staatlichkeit, dem Auftauchen der Idee des Liberalismus und der mit diesem zusammenhängenden Erfindung des Phänomens der Bevölkerung (vgl. Foucault 1999: 289, Dean 1999: 116ff.). Er erhält darin eine politische Dimension, die heute trotz der unterschiedlichen Sicherheitskonzepte, die diskutiert werden (von State Security bis Human Security), eine tendenziell einheitliche Stoßrichtung im Sinne der Abwehr drohender Gefahren aufweist. Das Konzept der Abwehr beginnt sich bald geographisch in zunehmendem Maße auszuweiten, so dass es nur folgerichtig ist, wenn die innere Sicherheit Deutschlands heutzutage auch am Hindukusch verteidigt wird, wie es der ehemalige deutsche Verteidigungsminister Struck einmal formuliert hat.

Ich möchte daher vorschlagen, beide Begriffe konzeptionell gleich $\mathrm{zu}$ interpretieren, indem ich ein methodisches Konzept anwende, das sie

5 Michael Dillon sieht die Ursache dafür in einer bewussten metaphysischen Setzung des Begriffs. »[...] metaphysics first allows security to impress itself upon political thought as a self-evident condition for the very existence of life - both individual and social. One of those impulses which it is said appears like an inner command to be instinctive (in the form, for example, of the instinct for survival), or axiomatic (in the form of the principle of self-preservation, the right to life, or the right to self-defence), security thereby became the value which modern understandings of the political and modern practices of politics have come to put beyond question, precisely because they derived its very requirement from the requirements of metaphysical truth itself« (Dillon 1996: 13).

6 Der Begriff Sicherheit lässt sich etymologisch auf den lateinischen Begriff der »cura« zurückführen. »Cura« verknüpft in seinen Bedeutungen die persönliche Fürsorge und Sorgfalt über das Konzept der Obhut mit institutionellen Ideen wie Verwaltung, Leitung oder Aufsicht. 
gewissermaßen von außen betrachtet, zugleich aber auch zu starke interpretative Zuschreibungen vermeidet: das Konzept des Dispositivs.

»Das, was ich mit diesem Begriff zu bestimmen versuche, ist erstens eine entschieden heterogene Gesamtheit, bestehend aus Diskursen, Institutionen, architektonischen Einrichtungen, reglementierenden Entscheidungen, Gesetzen, administrativen Maßnahmen, wissenschaftlichen Aussagen, philosophischen, moralischen und philanthropischen Lehrsätzen, kurz, Gesagtes ebenso wie Ungesagtes, das sind die Elemente des Dispositivs. Das Dispositiv selbst ist das Netz, das man zwischen diesen Elementen herstellen kann« (Foucault 2003b: 392).

Bei einer Interpretation von Komplexen wie Sicherheit und Entwicklung als Dispositiv geht es also um mehr als eine schlichte konzeptionelle Verbindung von politischen, strategischen und ideologischen Leitlinien mit verschiedenen Ebenen der Praxis (wie sie etwa in der Trinität Entwicklungspolitik, Entwicklungshilfe, Entwicklungszusammenarbeit zum Ausdruck kommt). Ein Dispositiv entwickelt darüber hinaus einen eigenen spezifischen Charakter - als auf einen bestimmten strategischen Zweck ausgerichteter Modus, in dem sich gesellschaftliche Machtverhältnisse materialisieren.

Eine sich nun abzeichnende Verbindung zwischen Entwicklungsdispositiv und Sicherheitsdispositiv, nicht nur im Sinne einer allgemeinen (auf ideologischer Ebene determinierten) Gleichläufigkeit, sondern im Sinne einer konkreten Vermengung ihrer Diskurse und Praktiken, wirft Fragen auf, die in verschiedener Hinsicht interessant und damit auch »untersuchenswert« sind. Von einer sehr allgemeinen Ebene, wo über die konkrete Problemstellung der zivilen Intervention der Zentren in die Peripherien ${ }^{7}$ die Frage der in die postkoloniale Epoche tradierten kolonialen Sichtweisen berührt ist ${ }^{8}$, über die Auswirkungen auf die betroffenen Politikbereiche bis hin zu den Effekten jener Interventionen - auf

7 Ich beziehe mich in meiner Verwendung der Kategorien »Zentrum« und »Peripherie« (ebenso mit der Kategorie »Semi-Peripherie«) auf Begriffe der Weltsystemanalyse nach Immanuel Wallerstein $(1986,2004)$. Die Kategorien werden von ihm als Strukturelemente des Weltsystems definiert (vgl. Wallerstein 1986: 520).

8 Wir können dies etwa eindrucksvoll an der von Michael Ignatieff in den USA losgetretenen und derzeit vor allem in Großbritannien prosperierenden Diskussion um eine positive Wiederbesetzung des Imperialismusbegriffes (»Good Imperialism«) beobachten, eine Diskussion, die direkt an Rudyard Kiplings »White Man's Burden« vom Ende des 19. Jahrhunderts anschließt. 
die von der Intervention Betroffenen wie auch auf die Intervenierenden selbst - ergeben sich zahlreiche relevante Fragenkomplexe.

Es liegt auf der Hand, dass all diese Fragen im Rahmen einer Studie nicht in der notwendigen Tiefe behandelt werden können. Die Arbeit an umfassenden Antworten ist aber auch nicht das Ziel meines Unterfangens. Vielmehr geht es mir darum, einen klar eingegrenzten Bereich der empirischen Realität, der sich in dem definierten thematischen Feld vollzieht, herauszugreifen und zu untersuchen. Zu diesem Zweck habe ich versucht, den von mir behandelten Ausschnitt so zu legen, dass er Praktiken umfasst, in denen sich die Thematik in einem verhältnismäßig hohen Grad an Zuspitzung präsentiert, in der Hoffnung, damit zumindest einen kleinen Beitrag zu den aufgeworfenen Fragen auf allgemeinerer Ebene treffen zu können. Ich werde mich daher nicht nur auf eine Fallstudie beschränken, sondern auch den allgemeinen Fokus der Arbeit eingrenzen, und zwar auf den Komplex der Entwicklungszusammenarbeit.

Natürlich wäre auch ein anderer Fokus möglich, wie eine Ausweitung der Materie auf den allgemeiner definierten Bereich der Entwicklungspolitik oder die Behandlung des Problems von Seiten der Sicherheitspolitik. ${ }^{9}$ Allerdings erscheint mir die Frage der zivilen Intervention gerade in Hinblick auf die allgemeinen Problemstellungen besonders aussagekräftig, bringt sie doch die Rationalitäten des Dispositivs vielleicht sogar drastischer auf den Punkt als die Fragen globaler Ordnungsstrukturen oder militärischer Interventionen.

\section{Untersuchungsfeld und Problemstellung}

Nach der Darlegung des allgemeinen thematischen Feldes und der damit einhergehenden Positionierung der Studie sollen in der Folge das Untersuchungsdesign und die konkreten Problemstellungen geklärt werden. Bevor aber die genaue Definition der Materie und die Formulierung der forschungsleitenden Fragestellungen in Angriff genommen werden können, muss ich zwei weitere Einschränkungen vornehmen.

Erstens stellt die Untersuchung nicht die Frage, ob »Entwicklung« oder ihre Konzepte grundsätzlich richtige oder wünschenswerte Annahmen sind. Wie bereits angedeutet stellt sich eine solche Frage insofern nicht, da eine darauf folgende Antwort immer auf Wahrheiten basieren

9 Speziell der Bereich der »Humanitären Intervention« scheint bei dieser Fragestellung vielversprechend. Er bildet gewissermaßen das sicherheitspolitische Gegenstück zur hier angesprochenen Neuausrichtung der Entwicklungszusammenarbeit. 
würde, die historisch und kontextual kontingent sind. Vielmehr ist es die Herausforderung der konkreten Darstellung und Analyse der gegebenen Realitäten, der ich mich mit dieser Untersuchung stellen will.

»For a social scientist, there is always another question: what do these ideas do, what real social effects do they have? At this point the analysis more closely resembles vivisection than critique. For the question is not show closely do these ideas approximate the truth, $\measuredangle$ but $\gg$ what effects do these ideas (which may or may not happen to be true) bring about? How are they connected with and implicated in larger social processes? «" (Ferguson 1994: xv).

Im Mittelpunkt stehen demzufolge die Gestalt des Dispositivs und dessen Wirkungen, in keinem Fall dagegen moralische Kategorien, unter die auch Zuschreibungen wie »richtig« oder »falsch« fallen würden. Dies heißt jedoch nicht, dass konkrete Bewertungen von Maßnahmen und der Strukturen, in denen sie gesetzt werden, vermieden werden sollen - ganz im Gegenteil. Wie James Fergusons bahnbrechendes Werk über die »Anti-Politics Machine« der internationalen Entwicklungspolitik in Lesotho zeigt, liegt genau in der Gegenüberstellung des von ihm so bezeichneten »Settings« und der mit Hilfe eines handlungsleitenden Dispositivs konstruierten Realität einer der wesentlichen Stärken, sich der Problemstellung über Fallstudien zu nähern.

Im Gegensatz zu Ferguson allerdings, und dies ist die zweite Bemerkung, die an dieser Stelle zu machen ist, will ich die Problemstellung eben nicht über die Situation in den Zielregionen der Intervention aufrollen, sondern über die intervenierenden Institutionen. Das ist einerseits der Forschungsstrategie geschuldet, da durch einen interinstitutionellen Vergleich, der nicht nur auf einen spezifischen Länderkontext konzentriert bleibt, bessere Aussagen zu den in Frage stehenden Dispositiven zu erwarten sind.

Andererseits ist es aber auch eine grundsätzliche und forschungsethisch begründete Entscheidung, die mit der Frage von Repräsentation zusammenhängt. Interventionen aus dem Blickpunkt der von der Intervention Betroffenen zu analysieren soll auch in der Hauptsache diesen Betroffenen überlassen bleiben. ${ }^{10}$ Die Abarbeitung des gesamten Prozesses aus der Perspektive eines einzigen Fallbeispiels würde, selbst bei besten Intentionen, nahezu unweigerlich zu einer Form von Identifikation führen, die in beständiger Gefahr stünde, in die althergebrachten paternalistischen Abgründe westlicher Wissenschaft abzurutschen. Gerade

$10 \mathrm{Zu}$ dieser Problematik vgl. Spivak 1988, auch wenn ich ihre äußerst pessimistischen Schlussfolgerungen nicht teilen will. 
wenn es um Fragen epistemologisch ausgerichteter Kritik geht, halte ich eine solche Perspektivität für wenig hilfreich.

Anders als bei Ferguson, der sich das gesamte Ensemble entwicklungspolitischer Maßnahmen in einem Land, Lesotho, als Untersuchungsmaterie zurechtlegt, müssen daher in meinem Fall die definitorischen Eingrenzungen der Untersuchung also in erster Linie institutionell und materiell erfolgen. Dazu ist die bisher argumentierte Beschränkung auf den Bereich der konkreten zivilen Intervention im Rahmen der Entwicklungszusammenarbeit zwar notwendig, aber keinesfalls hinreichend. Deswegen will ich weitere Einschränkungen vornehmen, um die Materie auf ein handhabbares Maß zuzuspitzen.

In dieser Hinsicht wird sich die Untersuchung auf Maßnahmen konzentrieren, die auf bilateraler Ebene gesetzt werden und nach den ODAKriterien des DAC als Entwicklungshilfe zu klassifizieren sind. Diese Einschränkung ist primär deswegen sinnvoll als davon ausgegangen werden kann, dass die politischen Rationalitäten, nach denen die konkrete Ausgestaltung des Dispositivs erfolgt, hier verhältnismäßig deutlicher sichtbar werden als im multilateralen Bereich. ${ }^{11}$ Die Beschränkung auf ODA - und die damit einhergehende weitgehende Ausklammerung rein privater Entwicklungshilfe ${ }^{12}$ - ist dagegen primär einer praktischen Vereinfachung der Untersuchungsmaterie geschuldet, da die vergleichsweise klar gesetzten Rahmenbedingungen von ODA, die mit einer zumeist guten und überschaubaren Dokumentation einhergehen, eine vergleichende Geber-Analyse begünstigen.

Dies ist vor allem deswegen von Bedeutung, weil eine meiner Ausgangshypothesen in der Annahme liegt, dass trotz mitunter gleich oder sehr ähnlich verlaufender Argumentationsmuster tiefgreifende Unterschiede zwischen den Akteuren existieren, deren Herausarbeitung für ein Verständnis der Gestalt der untersuchten Dispositive unumgänglich ist. Ein Vergleich zwischen den USA und Deutschland verspricht besondere Spannung, nicht nur aufgrund der Differenzen in der gegenwärtigen sicherheitspolitischen Orientierung. Auch große Unterschiede in verschiedenen benachbarten Themenbereichen, von der akademischen Tradition bis zur grundsätzlichen Herangehensweise an Politik sollten

11 Das bedeutet aber keineswegs, dass eine Untersuchung der multilateralen Ebene in Bezug auf diese Fragestellung nicht sinnvoll wäre. So könnte etwa eine Analyse der sich wandelnden Interpretationsmuster des so genannten »Politikverbotes« der Weltbank interessante Ergebnisse hervorbringen.

12 Co-finanzierte Projekte zwischen privaten und öffentlichen Gebern sind sehr wohl Teil der vorliegenden Untersuchung. 
Erkenntnisse ermöglichen, die diese speziell für den europäischen Bereich problematische ${ }^{13}$ Auswahl rechtfertigen.

Eine weitere Eingrenzung muss auf inhaltlicher Ebene getroffen werden, und zwar in Hinblick auf die in die Untersuchung einzubeziehenden Maßnahmen. Da die Studie, wie eingangs gesagt, von einer substanziellen Veränderung der entwicklungspolitischen Praxis in den letzten beiden Jahrzehnten ausgeht, sollen vorwiegend jene Maßnahmen in den Fokus gerückt werden, die diese Veränderung hin zu einer verstärkt sicherheitspolitischen Ausrichtung besonders sichtbar machen. Es geht folglich um jenen Bereich, den das DAC in seinen für den gesamten Sektor grundlegenden Guidelines unter der Kategorie "working on conflict« zusammenfasst (DAC 2001: 23). Das umschließt alle gezielten entwicklungspolitischen Interventionen, die in Konfliktregionen mit dem Bewusstsein gesetzt werden, den Konflikt zu beeinflussen (im Gegensatz zu dem vom DAC als überkommen definierten Zugang eines »working around conflict« und eines »working in conflict «, bei dem zwar Maßnahmen im Konfliktkontext gesetzt werden, allerdings mit der vom DAC als unrealistisch bewerteten Intention, den Konflikt möglichst nicht zu beeinflussen). ${ }^{14}$

Die Konzentration auf den Bereich der Entwicklungszusammenarbeit ist eine logische Folge dieser Einschränkungen. Andere entwicklungspolitische Handlungen wie die Frage der Konditionalität von Entwicklungshilfe, werden, obwohl sie weitreichende sicherheitspolitische Implikationen haben können, nur insoweit in die Untersuchung miteinbezogen, als sie helfen, das zu entwerfende Bild der unterschiedlichen Rationalitäten, das für die Geber kennzeichnend ist, zu vervollständigen.

Zusammenfassend lassen sich also drei zentrale Ausgangshypothesen festhalten, die in der Studie, wenn auch angesichts der getroffenen Einschränkungen in einem zugegebener Maßen kleinen Ausschnitt, untersucht werden sollen:

Erstens geht die Untersuchung davon aus, dass seit spätestens Mitte der 1990er Jahre ein Veränderungsprozess im Bereich der Entwick-

13 Deutschland kann keineswegs als repräsentativ für die Praktiken in der Europäischen Union verstanden werden. Ein Einbeziehen aller relevanten bilateralen europäischen Akteure und der EU-Kommission hätte die Untersuchung allerdings überfordert. Für eine Darstellung der unterschiedlichen Zugänge von verschiedenen europäischen Schlüsselakteuren vgl. Khittel/Pospisil (2006).

14 Um welche Maßnahmen es sich dabei im Einzelnen handeln kann, ist in den DAC-Guidelines detailliert aufgeführt. Allerdings gibt es signifikante geberspezifische Besonderheiten, weswegen ich die spezifische Diskussion erst etwas später im empirischen Teil vornehmen will. 
lungs- wie auch der Sicherheitspolitik stattgefunden hat und stattfindet, der sich in Richtung eines Zusammenwachsens der beiden Politikbereiche entwickelt. Mit spezieller Konzentration auf das Instrument der Entwicklungszusammenarbeit soll die Frage gestellt werden, wo die Ursachen dieser Veränderung liegen, wie sie verstanden werden kann und wie sie zu bewerten ist.

Zweitens wird davon ausgegangen, dass diese Veränderung bei den verschiedenen entwicklungspolitischen Gebern unterschiedliche neue Formen von Rationalität hervorgebracht hat, die sich in Strategien, Konzepten und spezifischen Interventionsschritten wiederfinden lassen. In diesem Zusammenhang stellt sich nicht nur die Frage, wie diese Rationalitäten konzeptionell gefasst werden können, sondern auch, welche Realität, also welches spezifische Wissen sie sich in konkreten Interventionsfeldern zurechtlegen und wie sie von diesem ausgehend wirken.

Die dritte Grundannahme ist schließlich, dass es nicht nur in der geschichtlichen Entwicklung dieser Veränderung, sondern auch in ihrer praktischen Ausgestaltung gewichtige Unterschiede zwischen den Gebern gibt. Vor dem Hintergrund der derzeit laufenden großen sicherheitspolitischen Debatten soll dazu der Vergleich der USA mit Deutschland in den Mittelpunkt der Studie gestellt werden. Die Frage nach diesen Unterschieden, auch im Verhältnis zum Grad der Veränderung an sich, also ob es die Heterogenität des Prozesses überhaupt erlaubt, von einem Gesamtprozess einer solchen Veränderung auszugehen, soll anhand der Konzepte und der Praxis der beiden Geber diskutiert werden.

Die letzte grundsätzliche Entscheidung, die zu treffen bleibt, ist die vielleicht wichtigste für das Gelingen des gesamten Unterfangens: die Auswahl des Fallbeispiels, anhand dessen diese Fragen konkret untersucht werden sollen. Ich habe mich dazu entschieden, Sri Lanka als Fallstudie zu behandeln, was sich nicht zuletzt aus Gründen der besonderen Relevanz der dort gesetzten Praktiken erklärt. Nicht nur gibt es eine signifikante US-Präsenz, vor allem - aber nicht nur - im ODA-Bereich, Sri Lanka ist auch für die Neuausrichtung der deutschen Entwicklungspolitik im Sinne sicherheitspolitischer Agenden von wesentlicher Bedeutung. Das GTZ-Länderprogramm wurde schon vor längerer Zeit schwerpunktmäßig auf das Thema Konflikt ausgerichtet, und mit der Berghof-Stiftung wurde mit großem finanziellen Einsatz eine Interventionsstrategie erprobt, der zukünftig Modellcharakter zukommen könnte. Nicht zuletzt ist auch ausschlaggebend, dass die internationale Peace- 
building-Community Sri Lanka zu einem ihrer zentralen Einsatzgebiete erklärt hat. ${ }^{15}$

In der Auseinandersetzung mit den drei zentralen Hypothesen der Arbeit will ich in mehreren Schritten vorgehen, die nach einer theoretischmethodischen Auseinandersetzung den angesprochenen Veränderungsprozess auf (theorie-)geschichtlicher, institutioneller und praktischer Ebene nachzeichnen. Im Abschnitt »Der Dispositivcharakter von Entwicklung« wird zunächst eine theoretische Verortung vorgenommen. Eine Schlüsselrolle wird dabei wie erwähnt den von Michel Foucault entwickelten Konzepten von Dispositiv und Regierungsrationalität zukommen.

Der Abschnitt »Das neue Sicherheitsproblem von Entwicklung« beschäftigt sich mit den eingangs angeführten »Veränderungen« auf der Ebene der Entwicklungs- aber auch der Sicherheitspolitik, denen ich mich primär über die die Politikfelder begleitende Forschung annähern will. Es werden die Grundlinien des Prozesses auf Seiten der Entwicklungstheorie und der Konfliktanalyse nachgezeichnet, mögliche Ursachen diskutiert und schließlich einer Bewertung unterzogen.

Nach der Auseinandersetzung mit den theoretischen, methodischen und historischen Grundlagen folgt in nächsten beiden Kapiteln der empirische Hauptteil der Untersuchung. Im Abschnitt »Konzepte und Gestaltung sicherheitspolitischer Entwicklungszusammenarbeit « wird der Blick auf die institutionelle Ebene gelegt. Die konzeptionellen und strategischen Grundlagen der Geber werden verglichen, ebenso wie die organisatorischen Veränderungen der jüngsten Zeit, die sich mit dem »Sicherheits-Shift« ergeben haben. Dabei wird versucht, eine erste Standortbestimmung der unterschiedlichen Rationalitäten vorzunehmen, die dann in die Analyse der Fallstudie einfließen.

Daran anschließend wird die Intervention der beiden untersuchten Geber in Sri Lanka behandelt. Ausgehend von einer Diskussion der als Grundlage verwendeten Konfliktanalysen werden dazu die Konzepte, Programme und wesentlichen Projekte der beiden Geber dargelegt. Dabei geht es wie gesagt nicht um eine Art »Wirkungsanalyse advanced«, sondern um eine praktische Prüfung der zuvor entwickelten Thesen über die unterschiedlichen Rationalitäten der Geber.

15 Im Zuge der Arbeit an der vorliegenden Studie wurden drei weitere Feldforschungen - in Kolumbien, Mindanao/Süd-Philippinen und Norduganda - absolviert, deren Resultate in der einen oder anderen Form bereits vorliegen (vgl. Pospisil 2005, 2006b; Khittel/Pospisil 2006). In der vorliegenden Arbeit dienen sie als wesentliche Hintergrundinformationen. 
Abschließend werden nicht nur die empirischen Teile zusammengetragen und auf dieser Basis die drei zentralen Problemstellungen diskutiert, sondern auch versucht, die auf diese Art gewonnenen Erkenntnisse in einen weiteren historischen Kontext einzubetten. 



\section{Der Dispositivcharakter von Entwicklung}

$»$ Earlier, science had linked itself with enlightenment and millennial claims, before going on to associate itself with racism, sexism, imperialism and colonialism, and then settling down with development, an idea in which most

of these earlier inheritances are encoded.«

(Claude Alvares)

Wenn ich zuvor von Entwicklung und Sicherheit als Dispositiven gesprochen habe, setzt das ein weites Spektrum an Annahmen voraus. Der folgende Abschnitt ist der Klärung dieser Annahmen gewidmet und stellt sich darüberhinaus das ambitionierte Ziel, diese Annahmen in einer für ein empirisches Forschungsprojekt handhabbaren Form operationalisierbar zu machen.

In einem ersten Schritt will ich zunächst den gewählten Ansatz in einem breiteren Kontext verorten, den ich - trotz dem Bewusstsein, damit überaus verschiedene und vielfältige Ansätze zusammenzufassen - mit »Entwicklungskritik« umschreibe. Diese Zusammenfassung rechtfertigt sich aus dem all diesen Zugängen gemeinsamen Zugang, nicht auf eine Verbesserung des Projekts »Entwicklung« hin getrimmte Forschung zu betreiben, sondern »Entwicklung« als sozioökonomischen, politischen und gesellschaftlichen Prozess kritisch und radikal in Frage zu stellen.

Danach will ich den für den theoretischen und methodischen Zugang der Arbeit zentralen Begriff des »Dispositiv« herausarbeiten und charakterisieren. Wesentliche Orientierung hierbei bilden die Arbeiten von Michel Foucault, dessen Machtanalyse die Basis des Dispositivkonzeptes bilden. Wesentlicher Punkt ist darin auch die methodisch relevante Abgrenzung des Verständnisses von Entwicklung als Dispositiv zu den im 
entwicklungskritischen Spektrum ebenfalls verbreiteten Interpretationsansätzen von Entwicklung als Ideologie einerseits oder Diskurs andererseits.

Schließlich geht es um den Prozess der Operationalisierung, wobei ich mir das von Åkerstrøm Andersen (2003) vorgeschlagene Modell einer »analytischen Strategie« zueigen mache. Eine solche analytische Strategie stellt sich in bewussten Gegensatz zu Modellen strikter methodischer Operationalisierung, wie sie in verschiedenen Spielarten der empirisch ausgerichteten Diskursanalyse mittlerweile üblich ist. Das Denken und empirische Herangehen in Form einer Strategie soll, gerade angesichts der mannigfaltigen und heterogenen Momente, die ein Dispositiv auszeichnen, eine analytische Flexibilität gewährleisten, die diesen relativ starr konzipierten Ansätzen zumeist abgeht. Nur dadurch wird es möglich, sich der komplexen Figur eines Dispositivs in einem Forschungsprozess zu nähern.

\section{Entwicklungskritik}

Die kritische Auseinandersetzung mit dem Entwicklungsbegriff und insbesondere dem Komplex Entwicklungspolitik - Entwicklungshilfe Entwicklungszusammenarbeit ist keineswegs neu. Dennoch wurde eine solche Kritik bis Mitte der 1980er Jahre vor allem im konstruktiven Sinne formuliert, also mit Blick auf Verbesserungen der entwicklungspolitischen Praxis. Angesichts einer relativ starken Repräsentanz der politischen Linken in der Entwicklungspolitik, insbesondere im Bereich der Entwicklungszusammenarbeit, war zu dieser Zeit nicht nur der Solidaritätsgedanke, sondern auch der Glaube an die positive soziale Kraft von Modernisierung (sei es gesellschaftlich, sei es technisch) dominant. Es stellte sich in Hinblick auf die Entwicklungspolitik und die bis dahin schon drei Jahrzehnte währende Geschichte ihres konkreten Scheiterns also nicht die Frage nach einem »Ob«. Gefragt wurde ausschließlich nach einem »Wie«, das sich im Sinne eines »besser Machens« entlang der zwei hauptsächlichen Aufforderungen ausdifferenzieren ließ, mehr konkrete Entwicklungshilfe zu leisten und sie genauer an die Erfordernisse, also an die in den Zielregionen identifizierten Bedürfnisse anzupassen: dem Alternative Development (vgl. Nederveen Pieterse 1998).

In diesem Strom der Kritik kommt es in der Mitte der »verlorenen Dekade « zu einem Bruch. Erstmals wird der bislang geltende Rahmen, entwicklungspolitische Praxis mit dem Ziel ihrer Verbesserung zu kritisieren, durchbrochen. Plötzlich wird Entwicklungspolitik als gesamtes Projekt in Frage gestellt. Die sich in diesem Zusammenhang herauskris- 
tallisierende Strömung des Post-Development ${ }^{1}$ wird von Intellektuellen und ehemals professionellen Entwicklungshelfer/innen erarbeitet, die vorwiegend aus dem lateinamerikanischen und dem südasiatischen Kontext stammen. ${ }^{2}$

Dieser Entstehungsprozess ist nur zum Teil aus den konkreten entwicklungspolitischen Misserfolgen erklärbar. Die zeitgleich wachsende Rezeption postkolonialer Ansätze spielt in diesem Zusammenhang, speziell im südasiatischen Raum, wahrscheinlich eine ebenso wichtige Rolle wie das allmählich sichtbar werdende konkrete Scheitern von revolutionären Projekten in Lateinamerika, und zwar, wie im Fall der Sandinistas, gerade nach ihrem vermeintlichen Sieg. Diese Bedingungen ebnen den Weg für eine angepasste Rezeption von Zivilisationskritikern wie Ivan Illich ${ }^{3}$, aus der heraus eine Fundamentalkritik des Entwicklungsprojektes möglich wird.

Erste Schlüsselbeiträge, etwa von Gustavo Esteva oder Arturo Escobar, erscheinen in der zweiten Hälfte der 1980er in einem der führenden Journals für kritische und politisch orientierte Wissenschaft, Alternatives. Ende der 1980er entsteht, interessanterweise in den USA, die Idee $\mathrm{zu}$ einem Sammelband, der unter dem Titel »The Development Dictionary« (Sachs 1992) alle zu diesem Zeitpunkt wesentlichen Autor/innen der Strömung versammeln wird.

Darin finden sich auch, in der Einleitung von Wolfgang Sachs griffig zusammengefasst, die Grundannahmen des Post-Development. Zunächst wird in einem stark von Michel Foucault und Edward Said (vgl. Escobar 1995: 6) geprägten Gedankenschritt davon ausgegangen, dass Entwick-

1 Angesichts der verschiedenen und zum Teil sehr heterogenen Sichtweisen, die Post-Development umfasst, wäre es unrichtig, von einem einheitlichen Ansatz oder einer Theorieschule zu sprechen.

2 Trotz dieser Dominanz von Lateinamerika und Südasien ist die gängige Grundannahme (und auch das bestimmende Selbstbild), dass Post-Development vor allem von Theoretiker/innen aus dem Süden getragen werde, falsch. Erstens muss bedacht werden, dass eine beachtliche Zahl von Beiträgen aus den weltsystemischen Zentren stammt, während es beispielsweise aus Afrika und weiten Teilen Asiens überhaupt keine Beiträge gibt. Zweitens haben die meisten namhaften Post-Development-Denker/innen des Südens die westlichen Bildungs- und Entwicklungseinrichtungen durchlaufen.

3 Ivan Illich, 1926 in Wien geboren, machte sich in den 1960ern vor allem als Befreiungstheologe einen Namen. So veröffentlichte er unter anderem gemeinsam mit Paulo Freire und gründete (schon 1960) das Centro Intercultural de Documentación in Cuernavaca/Mexiko. 1969 brach er schließlich nach harten Auseinandersetzungen mit dem Vatikan und betätigte sich primär als zivilisationskritischer Philosoph. Dabei zog er Entwicklungspolitik schon bald in seine Grundsatzkritik mit ein (vgl. Illich 2005 [Original von 1971]). 
lung und Unterentwicklung nicht gegebene Grundkonstanten des kapitalistischen Weltsystems (sei es positiv oder negativ konnotiert) sind, sondern erfundene diskursive Kategorien. Zugeschrieben wird diese Erfindung der Truman-Administration. Harry S. Truman habe in seiner Inaugurationsrede am 20. Januar 1949 erstmals von den munderdeveloped areas« der südlichen Hemisphäre gesprochen (Sachs 1992: 2; Escobar 1995: 3f.). ${ }^{4}$

Mit dieser Rede habe Truman Annahmen getroffen, die fundamental in Zweifel zu ziehen seien und zudem verheerende Folgen gehabt hätten: (1) die USA wären an der Spitze einer gesellschaftlichen Evolutionsskala eingeordnet worden, die vom Grad der Industrialisierung bestimmt sei; (2) Truman wäre es bei seiner entwicklungspolitischen Initiative darum gegangen, die Spitzenposition der USA zu verteidigen und den wachsenden Einfluss der Sowjetunion zurückzudrängen; (3) die darauf einsetzende Entwicklung habe die Welt zwar verändert, aber keinesfalls so, wie es propagiert worden wäre. Dies habe nun (4) zur Folge, dass "suspicion grows that development was a misconceived enterprise from the beginning " (Sachs 1992: 3).

Während die Punkte 1-3 heute selbst von USAID in der einen oder anderen Form bestätigt werden würden, markiert Punkt 4 doch einen einschneidenden Bruch, der nicht so einfach aus Interessenslagen oder den Konsequenzen bisheriger Misserfolge hergeleitet werden kann. Die Ursachen dürften eher durch die neuen Möglichkeiten im philosophischen Instrumentarium seit den 1970er Jahren ${ }^{5}$ und nicht nur durch Fehlschläge, sondern auch durch sichtbar werdende konkrete Widerstandsprozesse zu erklären sein. Wenngleich die meisten der tragenden

4 Diese Analyse ist nur teilweise gerechtfertigt. Erstes explizit entwicklungspolitisches Programm der USA war der Marshall-Plan, der im Juli 1947 beschlossen wurde. Die dazu geführte politische Debatte - gerade in der Auseinandersetzung mit dem konkurrierenden Morgenthau-Plan zeigt schon deutlich jenes Denken (»Rückentwicklung zum Agrarland « vs. »Entwicklung« als Re-Industrialisierung), das Truman dann auf eine globale Ebene heben sollte. Auch die historische Kontinuität, die das europäische Entwicklungsdenken mit der Kolonialperiode verbindet, ist in einer solchen Argumentation unterbewertet.

5 Vor allem sind hier die schon erwähnten Michel Foucault und Edward Said gemeint, die stark rezipiert wurden, und ohne deren Arbeiten die Verbindung des Post-Development mit den Ideen von Ivan Illich wahrscheinlich nicht möglich gewesen wäre. Insgesamt sind aber die zunehmende Popularität postmodernen und postkolonialen Denkens und die damit einhergehende Herausforderung an den Marxismus in der politischen Linken, der die meisten der Post-Development-Autor/innen, wenn auch in verschiedener Form, zu dieser Zeit zuzurechnen waren, ein nicht $\mathrm{zu}$ vernachlässigender Faktor. 
Personen diese Erfahrungen teilen, werden ihre daraus folgenden wissenschaftlichen und politischen Konsequenzen allerdings unterschiedliche sein.

Das »Development Dictionary« bleibt denn auch das einzige wirklich gemeinsame Projekt der Strömung, ein späterer, 1997 erscheinender sehr erfolgreicher Sammelband mit dem Titel »The Post-Development Reader« (Rahnema/Bawtree 2005) beruht nahezu ausschließlich auf der kompakten Re-Publikation von vorhandenem Material. Unterdessen hat eine weitgehende Ausdifferenzierung unter den Protagonist/innen eingesetzt, die sich an analytischen Aussagen wie auch an der Umgangsweise damit, also an der daraus folgenden Praxis, festmachen lässt.

Gustavo Esteva etwa konzentriert sich, offenkundig in Auseinandersetzung mit dem Bielefelder Ansatz ${ }^{6}$, auf die Entwicklung einer politischen Praxis im Sinne der Subsistenzperspektive (vgl. dazu etwa Esteva 1992b oder Esteva/Prakash 1998). Durch seine mitunter ungenaue Rezeption von theoretisch-philosophischen Bezugspunkten und die unkritische Verklärung von kleinen Ausschnitten mittelamerikanischer Lebensrealität, die er zu den tatsächlichen Bedürfnissen der Menschen im Süden verallgemeinert, hat er zum Teil vehemente Kritik auf sich gezogen (vgl. etwa Kiely 1999; Storey 2000). ${ }^{7}$

Angesichts der Tatsache, dass Esteva mit seinen Verallgemeinerungen einen Prozess wiederholt, den er anderen, speziell denjenigen, die aus ihrer entwicklungspolitischen Praxis heraus ein tendenziell einheitliches Bedürfnis nach »Entwicklung« in den »unterentwickelten« Regionen unterstellen, vorhält, ist diese Kritik sicherlich berechtigt. Andererseits wird sie oftmals dazu benutzt, Entwicklungskritik an sich pauschal anzugreifen und Grundannahmen der Moderne, die von ihr in Zweifel gezogen werden, zu rehabilitieren. Demgegenüber sollte ein wesentlicher Einwurf von Arturo Escobar bedacht werden:

»The process of gauging experiences such as these from Western perspectives is not easy. Two extremes must be avoided: to embrace them uncritically as alternatives; or to dismiss them as romantic expositions by activists or intellec-

6 Einen guten Einblick in das Denken dieses Ansatzes, der vor allem von Maria Mies, Claudia von Werlhof und Veronika Bennholdt-Thomsen geprägt wurde (und damit seiner Herkunft nach in den feministischen Debatten in der Bundesrepublik Deutschland der 1980er festgemacht werden kann), bietet Maria Mies (2003).

7 Diese Kritik bezieht sich zumeist auch auf andere bekannte Entwicklungskritiker/innen wie den Iraner Majid Rahnema oder die indische Ökofeministin Vandana Shiva, die mit Esteva die Wortgewalt der Kritik und die Suche nach alternativen Lebensprojekten teilen. Speziell Shiva gleitet dabei merklich in Esoterik ab. 
tuals who see in the realities they observe only what they want to see, refusing to acknowledge the crude realities of the world, such as capitalist hegemony and the like. Academics in the West and elsewhere are too apt to fall into the second trap, and progressive activists are more likely to fall into the former. Instead of true or false representations of reality, these accounts of cultural difference should be taken as instances of discourse and counterdiscourse. They reflect struggles centred on the politics of difference, which often [...] include an explicit critique of development« (Escobar 1995: 170).

Escobar selbst hat sich den Versuchungen der Suche nach derartigen umfassenden Alternativen weitgehend enthalten. Ihn beschäftigt mehr die Anti-Globalisierungs-Bewegung, in der er einen epistemologischen Bruch hin zu einer »plural landscape of knowledge forms « zu erkennen meint. Er sieht in dieser Bewegung das Potenzial, moderne Denkraster, die auf gegenwärtige Probleme keine Lösung bereithalten würden, aufzubrechen (Escobar 2004: 212). ${ }^{8}$

Neben der um das »Development Dictionary« versammelten Gruppe von Autor/innen entstanden noch weitere entwicklungskritische Ansätze, die unter dem Begriff Post-Development subsumiert werden können. Fünf Jahre bevor die Erstausgabe des entwicklungskritischen Wörterbuches erscheint, dissertiert in Harvard der junge Anthropologe James Ferguson mit seiner im Titel etwas sperrig klingenden These »Discourse, Knowledge, and Structural Production in the >Development Industry: An Anthropological Study of a Rural Development Project in Lesotho«. Es sollte eine der erfolgreichsten Dissertationen der Sozialanthropologie werden. Das Buch zur »Anti-Politics Machine« der internationalen Entwicklungshilfe in Lesotho (Ferguson 1994 [Dissertation in 1985]) ist mittlerweile in der siebenten Auflage erschienen.

Auch Ferguson versteht Entwicklung primär als Diskurs und sieht strukturelle Ähnlichkeiten zum Zivilisationsbegriff in der Spätphase des Kolonialismus. Davon ausgehend unternimmt er eine eingehende und detaillierte Fallstudie, die ihn in Bezug auf Entwicklungspolitik zu überaus skeptischen Schlussfolgerungen kommen lässt (ebd.: 280). ${ }^{9}$ Es ist überraschend wie rätselhaft, dass Ferguson keinen Eingang ins »Development Dictionary« gefunden hat - nicht einmal als Literaturverweis.

Etwas zeitversetzt entsteht auch an englischen Universitäten ein Zweig der Post-Development-Strömung, vor allem an den Universitäten

8 Er bezieht sich dabei insbesondere auf Arbeiten von Boaventura de Sousa Santos und Arjun Appadurai.

9 Er bezieht seine Kritik hauptsächlich auf die Frage nach der Position des Akteurs und findet sich damit in einem Naheverhältnis zur Kritik kolonial geprägter Repräsentationsmuster von Gayatri Spivak (1988). 
von Manchester, wesentlich getragen von Uma Kothari (vgl. Kothari/Minogue 2002), und Lancaster. Dort sind mit dem ehemaligen Oxfam-Mitarbeiter Mark Duffield, der spätestens seit seinem »Global Governance and the New Wars« (Duffield 2001) als einer der prononciertesten Entwicklungskritiker gilt, und Michael Dillon, dessen Arbeit hauptsächlich versucht, die Ansätze Michel Foucaults für die Disziplin der Internationalen Beziehungen nutzbar zu machen, zwei zentrale Exponenten dieser Strömung tätig, die im Laufe dieser Arbeit noch prominent zu Wort kommen werden. ${ }^{10}$

Bei den in Lancaster vertretenen Ansätzen finden sich Parallelen zu den Arbeiten von Arturo Escobar, wenngleich sich auch die Fragestellungen grundsätzlich unterscheiden. Während sich Escobar vor allem auf Entwicklung als Gesamtprojekt konzentriert und Gegenbewegungen auslotet, konzentrieren sich Duffield, Dillon und Kolleg/innen vom Bereich der Internationalen Beziehungen kommend auf den Überschneidungsbereich von Entwicklungspolitik und dem Sicherheitskomplex. Alternativen haben sie, zumindest derzeit, keine anzubieten, und sie enthalten sich in ihren Arbeiten auch der Suche danach. Ihre Intention liegt mehr in der allgemeinen Einordnung der aktuellen Entwicklungen in historische Konfigurationen (im Mittelpunkt steht dabei der Begriff der »global liberal governance«).

Selbst angesichts der inhaltlichen, räumlichen und auch zeitlichen Distanz zwischen den verschiedenen Akteuren ist es immer noch erstaunlich, wie wenig Austausch es unter ihnen tatsächlich gibt. Wechselseitige Rezeption zwischen den verschiedenen Ansätzen der Strömung ist selten, in weiten Teilen der Publikationen sogar faktisch inexistent. Arturo Escobar ist hier die einzige relevante Ausnahme. Sein »Encountering Development « (1995) findet sich als Standardliteratur in den meisten Beiträgen zum Thema.

Aram Ziai (2004) ortet in einer vergleichenden Analyse prominenter Autor/innen die Entwicklung von zwei Tendenzen des Post-Development, die er als »neo-populistische« und »skeptizistische« Entwicklungskritik bezeichnet. ${ }^{11}$ Diese Trennlinie ist aufgrund der zahlreichen

10 Mark Duffield hat mittlerweile an die University of Bristol gewechselt, wo er eine Professur für Development Politics innehat.

11 Er rechnet dabei Majid Rahnema und Gustavo Esteva den »NeoPopulisten« zu, Arturo Escobar und James Ferguson den »Skeptizisten«. Einen der entscheidenden Unterschiede zwischen den beiden Tendenzen macht er an der Genauigkeit ihrer Foucault-Rezeption fest. Die »NeoPopulisten« würden sich dabei Foucaults Arbeiten gegenüber ähnlich verhalten wie Marxisten-Leninisten gegenüber dem Werk von Marx (vgl. Ziai 2003a: 411). Diesen Vergleich halte ich allerdings angesichts der Tatsa- 
Grautöne, die sie überspielt, zu scharf gezogen. Wenngleich sich vielleicht Gustavo Esteva ${ }^{12}$ oder Vandana Shiva ${ }^{13}$ angesichts ihres politischen Engagements, das sich generell in Richtung einfach gestrickter Lösungsvorschläge für komplexe Problemlagen bewegt, in die Kategorie »Neo-Populisten« einordnen lassen, und James Ferguson auf der anderen Seite in der Tat keine alternativen Ideen anbietet, sondern sich auf "Skeptizismus« konzentriert, ist die Lage bei nahezu allen Protagonist/innen zwischen diesen beiden Polen weniger eindeutig. So fällt weder der von Ziai ins Lager der »Neo-Populisten« geschobene Majid Rahnema durch besondere politische Ambitionen auf, noch der »Skeptizist« Arturo Escobar durch entsprechende Enthaltsamkeit.

Wodurch sich die Protagonist/innen unterscheiden, ist der Grad ihres gesellschaftlichen Aktivismus, der sich auf der Bereitschaft gründet, aus Analysen eindeutige praktische Konsequenzen zu ziehen. Was sich in diesem Zusammenhang meines Erachtens feststellen lässt (und hier spiegelt sich Ziais These sehr wohl wider), ist eine signifikante Reziprozität zwischen einer Bereitschaft zum Anbieten gesellschaftlicher Alternativen und der Qualität und Tiefe der wissenschaftlichen Beiträge.

Das ändert allerdings nichts daran, dass etwa das Werk Gustavo Estevas für die Entstehung einer entwicklungskritischen Forschungsperspektive von nicht zu unterschätzender Relevanz ist. Gerade die Ablehnung seiner wesentlichen Schlussfolgerungen bei gleichzeitiger Konfrontation mit seinen in ihren wesentlichen Aussagen stimmigen Kritiken an »Entwicklung« ermöglichen es, zu einem Ansatzpunkt zu gelangen, der diese grundsätzlichen Sichtweisen mit einer induktiven Herangehensweise zu kombinieren sucht. »Entwicklung« ist, und das ist die Grundthese dieser Arbeit, als Diskurs wie als Dispositiv, letztlich nur aus ihren konkreten Wirkungen heraus zu verstehen.

che, dass es nie die Absicht von Foucault war, ein »Werk« zu hinterlassen und er die Verwendung seiner Thesen explizit freistellt, für unangebracht.

12 Esteva ist mittlerweile Berater der zapatistischen Bewegung in Mexiko, die sich von ihrem Beginn an durch einen speziell auf die europäische Solidaritätsbewegung anziehend wirkenden Populismus ausgezeichnet hat.

13 Shiva versteht sich selbst als Aktivistin, westliche Wissenschaft lehnt sie mittlerweile (sie hat eine klassische wissenschaftliche Karriere durchlaufen) $-a b$. 


\section{Entwicklung als Ausdruck gouvernementaler Rationalität}

Die Idee, Entwicklung sollte weder als intendierte Strategie noch als simpler Pragmatismus konzeptualisiert werden, habe ich eingangs im Vorschlag zum Ausdruck gebracht, Entwicklung, ebenso wie Sicherheit, als Dispositiv zu definieren. Eine solche Definition beinhaltet in erster Linie eine zentrale Aussage: Entwicklung und Sicherheit erfüllen eine bestimmte strategische Funktion in einer gegebenen Machtkonstellation - allerdings im Sinne einer Strategie, die sich vollzieht und herausbildet, eben nicht geplant oder auch nur planbar. Damit will ich nicht sagen, dass es keine politischen Strategien gibt, die in den Feldern der Entwicklung oder der Sicherheit intentional geplant und umgesetzt werden. Allerdings meine ich sehr wohl, dass wir die Wirkungen der Dispositive nie verstehen können, wenn wir auf dieser Ebene stehen bleiben. Denn »was gemacht wird, erklärt sich durch das, was in jedem Moment der Geschichte das Machen war. Fälschlicherweise stellen wir uns vor, dass das Machen, die Praktik, sich vom Gemachten aus erklärt« (Veyne 1992: 37).

Im Folgenden möchte ich zunächst versuchen, vor dem Hintergrund einer Annäherung an den Diskurs-Begriff das Konzept des Dispositivs zu klären. Erst dadurch wird es meiner Meinung nach möglich, sich Gedanken über seine konkrete Ausgestaltung zu machen, was bedeutet, sich die Frage nach den Rationalitäten zu stellen, in denen und durch die ein Dispositiv seine Wirkungen entfalten kann. Ich werde dazu Foucaults Idee aufgreifen, diesen Prozess als dominant gouvernementale Rationalität zu interpretieren, die auf internationaler Ebene wirkt.

Neben der Eigenschaft, auf besondere und meines Erachtens gut nachvollziehbare Weise Prozesse beschreibbar zu machen, hat das Konzept einen wesentlichen Vorteil: durch die Ausrichtung auf das Machen und die dieses Machen bestimmende Rationalität ermöglicht es feine Vergleichbarkeiten, die durch die reine Konzentration auf die Interventionspraxis und ihre Wirkungen nicht möglich wären. So können Unterscheidungen getroffen werden, die bei einem auf eine allgemeinere Ebene fokussierten Blick vermutlich verborgen bleiben würden: Einerseits in Hinblick auf die Akteure - im gegebenen Fall die USA und Deutschland als in der Ausgangsthese einander gegenübergestellte Geber - andererseits aber auch in der Frage nach einer genauen historischen Einordnung jenes Problems, das der Ausgangspunkt der Untersuchung ist: die Verbindung der Dispositive von Entwicklung und Sicherheit. 
Was meint nun jener an Foucault orientierte Dispositiv-Begriff? In Bezug auf »Sicherheit« lässt sich zunächst feststellen, dass sich ein so verstandenes Sicherheitsdispositiv substanziell von jenem Verständnis unterscheidet, das in der klassischen sicherheitspolitischen Analyse beheimatet ist. Diese sicherheitspolitische Definition im engen Sinne umfasst mehr oder minder ein Regime konkreter Maßnahmen in einem spezifischen Setting mit der Zielsetzung einer in diesem Kontext definierten Sicherheit, ist also primär auf der sicherheitspolitischen Umsetzungsebene angesiedelt. Ein an der Foucaultschen Dispositiv-Definition orientierter Begriff hingegen kann schon deswegen keine praktisch orientierte sicherheitspolitische Funktionalität entfalten, weil er sich der Logik des gesamten Gegenstandes verschließt. Er erkennt das oberste Ziel jeder sicherheitspolitischen Problemstellung, die Herstellung ebenjener Sicherheit, schlichtweg nicht an.

Freilich stellt er ebenso wenig wie der traditionelle Sicherheitsbegriff die Frage nach der Notwendigkeit und Sinnhaftigkeit des Konzeptes an sich. Doch während der traditionelle Zugang diese Frage vermeidet, um die Unanfechtbarkeit der begriffsbegründeten Logik zu garantieren, anerkennt die Untersuchung eines Dispositivs im Foucaultschen Sinne, dass eine Frage nach Notwendigkeit und Sinnhaftigkeit eines Konzeptes immer kontextgebunden ist, abhängig von einem spezifischen gesellschaftlichen Wahrheitsregime, auf dem es fußt und zu dem es beiträgt. Oder wie es Michael Dillon ausdrückt: »In general, then, security is a regime of truth expressed as a discourse of danger through which government takes place in the name of fears that are nonetheless functional to the re-production of the political order« (Dillon 2004: 88).

Es geht also nicht um Bewertung, sondern um Darstellung, um die Klärung der Anordnung der verschiedenen heterogenen Elemente, die den Diskurs konstituieren, oder in einer Formulierung von Gilles Deleuze, um die Beschreibung einer Vielheit, in der »im Werden befindliche Prozesse wirken, die sich von denen unterscheiden, die in einem anderen Dispositiv wirken« (Deleuze 1991: 157).

Ein solcher Dispositiv-Begriff benötigt zu seiner genaueren Charakterisierung Abgrenzungen von zwei verwandten Konzepten: dem des Diskurses und dem der Ideologie. Es empfiehlt sich, diese Abgrenzungen anhand des Entwicklungsbegriffes zu diskutieren, da für ihn sowohl die Anwendung von Diskursanalyse wie auch Ideologiekritik bereits von einigen Arbeiten der bereits erwähnten Post-Development-Ansätze vorgeschlagen wurde.

Demnach scheint es vielversprechend, Entwicklung als Diskurs zu begreifen. Arturo Escobar ist uneingeschränkt zuzustimmen, wenn er meint: »Thinking of development in terms of discourse makes it possible 
to maintain the focus on domination $[. .$.$] and at the same time to explore$ more fruitfully the conditions of possibility and the most pervasive effects of development« (Escobar 1995: 5f.). Dennoch besteht die Gefahr, bei einer methodischen Fokussierung auf den Diskurs in eine zu weit gehende Abstraktion abzugleiten, die die konkreten Verästelungen und die faktischen Auswirkungen vernachlässigt. Natürlich ist es möglich, den Diskursbegriff so zu definieren, dass er nicht nur Geschriebenes und Gesprochenes, sondern auch Getanes mit einschließt. Trotzdem verführt ein solches Herangehen besonders in empirisch relevanten Bereichen wie der Entwicklungs- und der Sicherheitspolitik dazu, die Implikationen konkreter Interventionen in den Hintergrund rücken zu lassen.

Da das Konzept des Dispositivs ohnehin die Analyse der für den Dispositiv bestimmenden diskursiven Formation umfasst, sie allerdings nicht nur in jenes »Netz« einbettet, das auch nichtdiskursive Praktiken enthält (die natürlich ohne die Analyse des mit ihnen verknüpften Diskurses nicht interpretierbar wären), sondern auch genau nach der Natur ebendieses »Netzes« und der Anordnung seiner verschiedenen Bestandteile fragt, bietet es einen gewissen methodischen Schutz gegen Einseitigkeiten und Simplifizierungen. Auch ist es durch seine Verortung auf der Ebene der Strategie für die Analyse von Problemstellungen aus dem Bereich der internationalen Politik besonders gut geeignet. So versteht Foucault Dispositiv als »eine Art [...] Gebilde, das zu einem historisch gegebenen Zeitpunkt vor allem die Funktion hat, einer dringenden Anforderung nachzukommen. Das Dispositiv hat also eine dominante strategische Funktion« (Foucault 2003b: 392f.).

Auf der anderen Seite ist das Dispositiv wiederum von der Ideologie abzugrenzen, wenngleich es bei einer solchen Abgrenzung nicht um Absolutheit gehen kann. Ohne Zweifel muss ein Dispositiv ideologische Elemente enthalten, sonst könnte es sich nicht als Dispositiv konstituieren. Allerdings liegen Dispositiv und Ideologie auf unterschiedlichen Ebenen. So will ich den Vorschlag, Entwicklung und Sicherheit als Dispositive zu konzeptualisieren, in bewusster Abgrenzung zu Ansätzen aus der kritischen Entwicklungsforschung, die mit einer ideologiekritischen Methodik operieren (vgl. Ziai 2004a, 2004b), verstanden wissen. Zwar versprechen diese Ansätze ohne Zweifel spannende Ergebnisse, nachdem Dispositive wie gesagt notwendiger Weise ideologische Elemente beinhalten. Jedoch ist die Gefahr des Abgleitens in eine die empirischen Realitäten vernachlässigende Abstraktion, die mehr verschleiert als erhellt, vielleicht noch größer als bei der reinen Fokussierung auf das Diskurskonzept. Entwicklung aber als gesamtes Projekt über den Ideologiebegriff zu interpretieren (vgl. Ziai 2004a), scheint mir in jedem Fall verfehlt. 
Dies hat vor allem mit dem engen Wechselverhältnis zu tun, in dem Projekte wie Entwicklung und Sicherheit mit dem gesellschaftlichen Prozess der Macht stehen, beziehungsweise mit den spezifischen Funktionen, die sie darin einnehmen. Dazu ist zunächst eine Diskussion des Machtbegriffes notwendig, die umso wichtiger erscheint, als sich sein Inhalt in dem Sinne, wie ich ihn verstehen will, von seiner kolloquialen Bedeutung erheblich unterscheidet.

»Die Macht ist nicht etwas, was man erwirbt, wegnimmt, teilt, was man bewahrt oder verliert; die Macht ist etwas, was sich von unzähligen Punkten aus und im Spiel ungleicher und beweglicher Beziehungen vollzieht«, meint Michel Foucault (1999a: 115). An anderer Stelle in »Der Wille zum Wissen« schreibt Foucault von der Macht als dem Namen, »den man einer komplexen strategischen Situation in einer Gesellschaft gibt« (ebd.: 114). Es liegt auf der Hand, dass eine solche Machtkonzeption vehemente Kritik auf sich gezogen hat, was, wenn wir Didier Eribon glauben wollen, durchaus beabsichtigt war: »Im Gefolge von Überwachen und Strafen will Foucault damit die Machttheorien marxistischer Prägung schleifen, die zu dem Zeitpunkt, als er diese Bücher zu schreiben unternimmt, noch zählebig weiterexistieren und erst ins Schlingern geraten, als sie erscheinen« (Eribon 1999: 389).

Vertreter der Idee einer machtfreien Wahrheit, allen voran Jürgen Habermas, haben Foucault wegen seiner aus diesem Machtbegriff folgenden Konsequenzen - etwa der Unmöglichkeit eines kontextungebundenen Wahrheitsbegriffes - heftig kritisiert. Andere griffen und greifen das Konzept von der Perspektive der Suche nach emanzipativem Widerstand an. ${ }^{14}$ Außer von verschiedenen klassisch marxistischen Strömungen wird der Vorwurf, Foucault heble mit diesem Konzept jede normative Grundlage emanzipatorischer oder revolutionärer Bewegungen aus, vorwiegend von den auf die Tradition der kritischen Theorie rekurrierenden Strömungen im Feminismus erhoben. ${ }^{15}$

14 Ausgangspunkt der Debatte ist Foucaults Definition des Widerstandes als der Macht inhärentes Phänomen in »Der Wille zum Wissen«. „Wo es Macht gibt, gibt es Widerstand. Und doch oder vielmehr gerade deswegen liegt der Widerstand niemals außerhalb der Macht« (Foucault 1999a: 116). Foucault hat diese erstmals 1976 veröffentlichte These trotz der vehementen Kritiken niemals verworfen, allerdings präzisiert. Judith Butler interpretiert Foucaults Spätwerk vor dem Hintergrund des Foucaultschen Bedürfnisses, Macht stärker über Widerstandsprozesse verstehbar zu machen. (vgl. Butler 2001: 95).

15 Diese Kritik wurde und wird prononciert vor allem von Nancy Fraser geführt, die Foucault in mehreren Artikeln und Vorträgen seit Anfang der 1980er Jahre (der bekannteste vielleicht »Foucault: $A>$ Young Conservativer? « von 1985) sinngemäß vorwirft, dass kein emanzipatorischer Wider- 
In der Tat verunmöglicht eine derartige Denkweise die Konstruktion eines essentialistisch konzipierten emanzipatorischen (oder gar revolutionären) Subjekts. Es verunmöglicht auch die normative Herstellung potenzieller gesellschaftlicher Alternativen, also im eigentlichen Sinne jedes leninistisch geprägte Revolutionskonzept. ${ }^{16}$ Aber mitnichten ver-

stand möglich sei, wenn es kein Außerhalb der Macht gebe. Ohne diese wesentliche Debatte hier vertiefen zu wollen (dies wäre ohne eine ausführlichere Auseinandersetzung mit Frasers Ausgangspunkten nicht möglich), möchte ich festhalten, dass eine solche Kritik auf einer eingeschränkten Lesart von Foucault beruht. Später wird Foucault präzisieren: »Tatsächlich denke ich, dass der Widerstand ein Element dieses strategischen Verhältnisses ist, worin die Macht besteht. Der Widerstand stützt sich stets, in Wirklichkeit, auf die Situation, die er bekämpft« (Foucault 2005c: 917). Im gleichen Interview präzisiert er seinen Macht- und Widerstandsbegriff in definitorischer Hinsicht: $»[\ldots]$ wenn es keinen Widerstand gäbe, gäbe es keine Machtbeziehungen. Weil alles einfach eine Frage des Gehorchens wäre. Von dem Augenblick an, da das Individuum in seiner Situation nicht das tun kann, was es will, muss es Machtbeziehungen gebrauchen. « Hinsichtlich der Perspektiven des Widerstandes meint er: „Wir können uns nicht aus dieser Situation herausversetzen, und wir sind nirgendwo frei von jeder Machtbeziehung. Aber wir können stets die Situation umgestalten. Ich habe also nicht sagen wollen, dass wir stets in der Falle sitzen, sondern im Gegenteil, dass wir stets frei sind. Und dass es schließlich, kurz gesagt, stets die Möglichkeit gibt, die Dinge umzugestalten« (ebd.: 916). Eine weitere speziell an Foucaults Macht- und Widerstandskonzept gerichtete Kritik kommt von Gayatri Spivak, die auf die »institutional responsibility « des Kritikers verweist und Foucault vor diesem Hintergrund eurozentristische Tendenzen vorwirft (Spivak 1988: 280). Foucault hätte sich demnach, gerade als französischer Intellektueller, mit der Frage des Kolonialismus auseinandersetzen müssen, vor allem, da er andere Formen der Macht und des Widerstandes prominent thematisiert. Die sich daran entspinnende Debatte will ich hier ebenfalls nicht aufgreifen, ich möchte allerdings festhalten, dass dieser Vorhalt mit Foucaults (objektiv) wichtiger Rolle bei der Entstehung der gesamten Postcolonial Studies kontrastiert.

16 Explizit dafür wurde er beispielsweise von Edward Said kritisiert. Said (1983: 244ff.) setzt seine Kritik an zwei Vorhaltungen an: »For one [...] Foucault's eagerness not to fall into the Marxist economism causes him to obliterate the role of classes, the role of economies, the role of insurgency and rebellion in the societies he discusses." Der zweite, »more important criticism« richtet sich gegen die aus dem Foucaultschen Konzept folgende Unmöglichkeit, Widerstand auf ein produktives Ziel zu richten, da jede Utopie selbst Produkt bestehender Machtverhältnisse sein müsse. »In human history there is always something beyond the reach of dominating systems, no matter how deeply they saturate society, and this is obviously what makes change possible, limits power in Foucault's sense, and hobbles the theory of that power.« Den ersten der beiden Vorhalte hat Foucault zweifellos durch begriffliche Klärungen seines Macht- und Widerstandsbegriffes entkräftet. Die Auseinandersetzung mit der zweiten Frage 
unmöglicht es bewussten Widerstand, der auch auf eine Umgestaltung der Verhältnisse zielt. Ein solcher Widerstand müsste allerdings, will er erfolgreich sein, seine Ausrichtung genau bedenken und beständig reflektieren. So sei es gefährlich, Widerstand in einer Form begreifen zu wollen, einem Staat spezifische Interessenslagen oder, allgemein gesprochen, einer Gemeinschaft spezifische Ansprüche entgegenzusetzen. Es ginge vielmehr um die Infragestellung einer vorhandenen Form von Rationalität: »Die Befreiung kann nicht durch einen Angriff auf den einen oder den anderen dieser Effekte erreicht werden, sondern durch einen Angriff auf die eigentlichen Wurzeln der politischen Rationalität« (Foucault 2005a: 198).

Das Erkennen und Beschreiben solcher Rationalitäten ist denn auch die primäre Problemstellung der Diskussion von Foucaults Zugang zu den Fragen von Macht und Widerstand, und dieses Verständnis soll in der Folge als die Grundlage der Interpretation des Entwicklungs- und Sicherheitsdispositivs dienen. ${ }^{17}$ Wir müssen daher das Problem des $\mathrm{Zu}$ sammenhangs dieses Konzepts von Macht mit den bereits angesprochenen Modellen von Diskurs und Dispositiv untersuchen, jedoch ohne Foucault ein monolithisches theoretisches Gebilde zu unterstellen, das jeder dieser Kategorien ihren genau fixierten Platz zuweisen würde. Dies ist nicht der Fall, es würde vermutlich sogar der Foucaultschen Idee solcher Konzepte entgegenstehen, die im Prozess ihrer Anwendung häufig einen rhizomatischen Ausdehnungsprozess erfahren und sich so ohnehin jeder Form der definitorischen Ab- und Eingrenzung erfolgreich widersetzen.

Diesem Zugang entspricht auch die wahrscheinlich kürzeste Definition, die Foucault dem Begriff »Macht« im Zuge seiner Arbeiten gegeben hat. Macht ist demnach ein Begriff, »der viele einzelne, definierbare und definierte Mechanismen abdeckt, die in der Lage scheinen, Verhalten oder Diskurse zu induzieren« (Foucault 1992: 32). Wir erkennen an dieser Definition dreierlei: erstens die erwähnte heterogene Form der Abgrenzungen, die zusammengestückelte Form der Macht aus jenen »viele[n] einzelne[n], definierbare[n] und definierte[n] Mechanismen«,

ist komplexer. Denn einerseits ist Said sicher zuzustimmen, dass es keine totalen Wirkungen von Macht gibt (was auch Foucault so nie behauptet hat), andererseits ist diese Feststellung auch eine Idealisierung realer Prozesse, der ich in dieser Form nicht zustimmen würde.

17 Die Konzentration auf Entwicklungs- und Sicherheitsdispositiv ist auch der Grund, warum ich wesentliche Aspekte von Foucaults Machtkonzept, die sich primär mit Körperlichkeit auseinandersetzen (vgl. Foucault 1997a, 1997b) und später speziell im feministischen Poststrukturalismus eine wesentliche Rolle spielen werden, an dieser Stelle nicht in die Diskussion einführe. 
die mit einer bewussten Vagheit (»in der Lage scheinen«) der Zuschreibung einhergeht. Wir erkennen auch den spezifischen Charakter von Macht als soziales Beziehungsgeflecht, das direkt auf den Menschen wirkt und in der Lage ist, soziale Praktiken - - Verhalten oder Diskurse $\ll$ - zu bestimmen. Aus genau diesem Charakteristikum können wir den dritten wesentlichen Aspekt ableiten: den offenkundigen Zusammenhang von Macht und Wissen, den Foucault als »Macht-Wissen-Komplex« zusammenfasst. Er beschreibt diesen Zusammenhang als den »Eindruck, dass eine durchgehende Verknüpfung der Macht mit dem Wissen und des Wissens mit der Macht besteht« (Foucault 2005b: 929). ${ }^{18}$

Macht produziert also Wissen, ebenso wie Wissen Macht produziert. Die daran anknüpfende Frage ist nun, wie das vor sich geht. Wenn wir davon ausgehen, dass Macht nicht nur in der Lage ist, durch Mechanismen »Verhalten oder Diskurse zu induzieren«, sondern sich eben erst in diesen »Verhalten oder Diskursen« ausdrücken lässt, erkennen wir, dass die entscheidende machtvolle Wirkung im Macht-Wissen-Komplex eben von diesen "Verhalten oder Diskursen« ausgehen muss. Vereinfacht könnten wir sagen, dass der Diskurs jene Ebene ist, die die Verbindung zwischen Macht und Wissen herstellt.

Folgen wir dieser Idee, müssen wir zwei Dinge akzeptieren: erstens, dass es gesellschaftliche Steuerungsmechanismen geben muss, die die Produktion des Diskurses kontrollieren, zweitens aber auch, dass dies nie vollständig möglich sein wird. Auch ein regulierter Diskurs entzieht sich in seinen konkreten Ausprägungen letztendlich seiner totalen Kontrolle, er behält etwas Gefährliches, eine »schwere und bedrohliche Materialität« (vgl. Foucault 2000: 10f.). Er ist nie gleichförmig, aber dennoch zusammenhängend, um einen »epistemologischen Strang« gedreht, was aber nicht bedeutet, »dass alle das Gleiche denken. Er [der epistemologische Strang] ist vielmehr die Bedingung, damit anderes gedacht wird und sich diese Differenz politisch durchhält« (Foucault 1999b: 248). Eine diskursive Formation ist mithin als der Fall zu verstehen, »wo

18 Wie so oft bringt auch hier ein Interview wesentliche Erklärungen zu Ansätzen und Zugängen von Michel Foucault. An dieser Stelle ist vor allem die klar gezogene Abgrenzung gegenüber dem Humanismus interessant, die die Kontroverse mit Habermas noch nachvollziehbarer macht: »Der moderne Humanismus täuscht sich also, wenn er diese Teilung zwischen Wissen und Macht ansetzt. Sie sind integriert, und es geht nicht darum, von einem Augenblick zu träumen, an dem das Wissen nicht länger von der Macht abhängen würde, was nichts anderes bedeutet, als dass man denselben Humanismus in Gestalt einer Utopie weiterbetreibt. Es ist nicht möglich, dass die Macht ohne Wissen ausgeübt wird, es ist nicht möglich, dass das Wissen keine Macht erzeugt« (Foucault 2005b: 930). 
man in einer bestimmten Zahl von Aussagen ein ähnliches System der Streuung beschreiben könnte« (Foucault 1981: 58), wobei sich die Fragestellung in der Untersuchung einer solchen diskursiven Formation auf die Bedingungen dieser Verteilung, auf die Formationsregeln, richten sollte.

Gerade bei der Untersuchung von großen Komplexen wie Entwicklung oder Sicherheit ist es an dieser Stelle notwendig zu betonen, dass wir es keinesfalls mit einer rein sprachlichen Ebene zu tun haben. Diskurse sind in erster Linie gelebte soziale Praxis, wie uns Stuart Hall (1994: 150) in Erinnerung ruft: »Diskurs bezieht sich auf die Produktion von Wissen durch Sprache. Er selbst aber wird durch eine Praxis produziert: >Diskursive Praxis` - die Praxis der Bedeutungsproduktion. Da alle sozialen Praktiken bedeutungsvoll sind, haben sie auch einen diskursiven Aspekt. So treten Diskurse in alle sozialen Praktiken ein und beeinflussen sie.« Diskurs und Verhalten sind also eng miteinander verwobene Kategorien, die eigentlich nur methodisch und nicht in Hinblick auf ihren Charakter unterscheidbar sind.

Die Kontrolle des Diskurses erfolgt über vielfältige Mechanismen, die als Begrenzungen (Prozeduren der Ausschließung) und interne Prozeduren verstanden werden können, die in der Produktion des Diskurses Zufälligkeiten und Ereignisse zu bändigen haben. Einer der wichtigsten Mechanismen, der Foucault in verschiedenen Zusammenhängen intensiv beschäftigt, ist der »Wille zur Wahrheit« (Foucault 2000: 16). In ihm findet der Macht-Wissen-Komplex auf Ebene der Diskursproduktion seinen wohl vehementesten Ausdruck.

Thomas Lemke weist uns darauf hin, dass Foucault keinem radikalen Relativismus in Bezug auf den Wahrheitsbegriff das Wort reden wollte (Lemke 1999). Vielmehr wollte er betonen, dass Wahrheit ein historisch kontingentes Phänomen ist, das sich in beständiger gesellschaftlicher Produktion befindet. Wahrheit ist ein Regime oder auch ein Spiel, das nach spezifischen Regeln funktioniert und seine eigene Geschichte hat - besser gesagt, zwei Geschichten: eine, die sich nach den internen Regulationsprinzipien vollzieht, und eine externe Geschichte, die nach genau jenen Spielregeln fragt, unter denen sie produziert wird (vgl. Foucault 2003e: 12f.). Dies ist das Wesen jedes emanzipatorischen Unterfangens: »Es kommt nicht darauf an, die Wahrheit von jedem Machtsystem zu befreien - was ein Trugbild wäre, da die Wahrheit selbst Macht ist -, sondern die Macht der Wahrheit von den Formen einer (sozialen, ökonomischen, kulturellen) Hegemonie zu befreien, innerhalb derer sie derzeit funktioniert« (Foucault 2003a: 152).

Wahrheit ist also einer jener Mechanismen, entlang dessen sich Diskurse formieren und Macht konkret fassbar wird. Die Suche nach ihr ist 
ein wesentlicher Hinweis auf die Spuren der Formationsregeln des Diskurses. Dennoch braucht es eine weitere konzeptionelle Hilfestellung, einerseits um das Feld der Untersuchung strukturieren zu können, andererseits aber auch, um strategische Elemente - oder besser gesagt, die verschiedenen Prozesse auf strategischer Ebene - überhaupt fassen zu können. Genau an diesem Punkt bringt Foucault die Figur des Dispositivs ins Spiel.

Das Dispositiv hat verschiedene Elemente und Eigenheiten. In seiner definitorischen Abgrenzung habe ich bislang seine Netzförmigkeit hervorgehoben, jene »heterogene Gesamtheit«, die es beschreibt - und die Unterscheidbarkeit zum Diskurs vor allem daran festgemacht, dass es auch nicht-diskursive Elemente mit einschließt. Auf der Grundlage der vorangegangenen Überlegungen zum Machtbegriff ist es nun möglich, weitere besondere Merkmale des Dispositivs in die Diskussion einzuführen. Nach Foucaults ausführlichster Charakterisierung ${ }^{19}$ (Foucault 2003b: 392f.) sind das neben seiner gegenständlichen Eingrenzung vor allem zwei.

Zunächst stellt sich die Frage nach den Beziehungen, in denen die verschiedenen Elemente der vom Dispositiv umfassten Gesamtheit zueinander stehen. Diese Verbindung markiert den qualitativen Unterschied zum Diskurs, nachdem sie dem Diskurs erst seine Art des Sprechens ermöglicht.

»So kann irgendein Diskurs mal als Programm einer Institution, mal im Gegenteil als ein Element erscheinen, das es erlaubt, eine Praktik zu rechtfertigen oder zu verschleiern, die selbst stumm bleibt, oder er kann als Sekundärinterpretation dieser Praktik funktionieren und ihr Zugang zu einem neuen Rationalitätsfeld verschaffen. Kurz, zwischen diesen diskursiven und nichtdiskursiven Elementen gibt es gleichsam ein Spiel, gibt es Positionswechsel und Veränderungen in den Funktionen, die ebenfalls sehr unterschiedlich sein können« (ebd.).

Es ist von zentraler Bedeutung, dass dieses Spiel zwischen diskursiven und nicht-diskursiven Elementen ${ }^{20}$ nicht beliebig oder zufällig abläuft.

19 Diese Charakterisierung entstammt auch in diesem Fall einem Interview, mit »champ freudien« im Juli 1977.

20 Die Unterscheidung zwischen den Attributen »diskursiv« und »nichtdiskursiv« ist für Foucault in diesem Zusammenhang »nicht so wichtig«. Entscheidend ist für ihn die Herausstreichung des spezifischen Charakters von Institution, der zwar ohne Zweifel diskursiv gefasst werden kann (und sich ohne einen entsprechenden Diskurs nicht herausbilden könnte), sich aber doch durch schwer im Konzept des Diskurses zu fassende materielle 
Vielmehr erfüllt es einen bestimmten Zweck, oder, wie es Foucault ausdrückt, eine strategische Funktion: „Drittens verstehe ich unter Dispositiv eine Art - sagen wir - Gebilde, das zu einem historisch gegebenen Zeitpunkt vor allem die Funktion hat, einer dringenden Anforderung nachzukommen. Das Dispositiv hat also eine dominante strategische Funktion« (Foucault 2003b: 392f.). Ein Dispositiv kann immer nur um ein prävalentes strategisches Ziel wachsen, in dem es seine Funktionalität entfalten kann, wobei es darin an die epistemischen Grenzen gebunden $_{\text {ist. }}{ }^{21}$

Arturo Escobar sieht im Fall des Entwicklungsdispositivs dieses strategische Ziel in der Aufrechterhaltung der systemischen Asymmetrie oder, in politischem Vokabular ausgedrückt, in der Produktion und Reproduktion der Dominanz der Zentren gegenüber den peripheren Regionen. Dazu wird nicht nur spezifisches Wissen hervorgebracht, sondern es werden auch konkrete Praktiken der Intervention gesetzt, die das Dispositiv ausmachen: »From this critical perspective, development can be described as an apparatus (dispositif) that links forms of knowledge about the Third World with the deployment of forms of power and intervention, resulting in the mapping and production of Third World societies« (Escobar 1992a: 23).

Für die Problemstellung der vorliegenden Untersuchung ist das Verständnis der Art und Weise, wie diese strategische Funktionalität ausgeübt wird, entscheidend. Anders können die Eigenheiten von Entwicklung und Sicherheit, und der besondere Charakter, der dem Prozess ihrer

Praktiken auszeichnet. Foucault insistiert in dem Zusammenhang darauf, dass »mein Problem ja kein sprachliches ist« (Foucault 2003b: 396).

21 Dieser Zusammenhang ist freilich komplexer: "Was das Dispositiv betrifft, stehe ich vor einem Problem, für das ich noch keine richtige Lösung gefunden habe. Ich habe gesagt, dass das Dispositiv von einer wesentlichen strategischen Beschaffenheit wäre, was unterstellt, dass es sich dabei um eine bestimmte Manipulation von Kräfteverhältnissen handelt, um einen rationalen und abgestimmten Eingriff in diese Kräfteverhältnisse, um sie in irgendeine Richtung zu entwickeln, um sie zu blockieren oder um sie zu stabilisieren, sie zu verwenden. Das Dispositiv ist also immer in ein Machtspiel eingeschrieben, doch immer auch an eine oder an mehrere Wissensgrenzen gebunden, die daraus hervorgehen, es aber genauso auch bedingen. Das eben ist das Dispositiv: Strategien von Kräfteverhältnissen, die Arten von Wissen unterstützen und von diesen unterstützt werden. In Les Mots et les Choses hatte ich eine Geschichte der Episteme schreiben wollen und bin in einer Sackgasse stecken geblieben. Was ich jetzt machen möchte, ist versuchen zu zeigen, dass das, was ich Dispositiv nenne, ein viel allgemeinerer Fall der Episteme ist. Oder vielmehr, dass die Episteme ein spezifisch diskursives Dispositiv ist, im Unterschied zu dem Dispositiv, das selbst diskursiv und nicht diskursiv ist und dessen Elemente viel heterogener sind« (Foucault 2003b: 394f.) 
allmählichen Verschränkung innewohnt, schwerlich diskutiert werden. Auch die große Resistenz, die für beide Konzepte kennzeichnend ist, die spezifische Produktion von Wissen, die sie ankurbeln, weisen auf diese Funktionalitäten hin, sind aber ohne sie auch nur eingeschränkt zu verstehen.

Ein Dispositiv erfüllt seine Wirkung auf zwei unterschiedliche Arten: Einerseits auf der offensichtlichen Ebene, auf der Ebene, die gesagt wird, und die aus dem Gesagten unmittelbar abgeleitet werden kann; das Gefängnis in seiner Orientierung auf präventive Abschreckung und korrektive Reintegration, Entwicklung aus dem Ziel der Modernisierung und systemischer Integration, Sicherheit in der Durchsetzung konkreter Regime staatlicher, sozialer oder persönlicher Abwehr- und Schutzmaßnahmen. Ich will diese Wirkungen als »Wirkungen erster Ordnung« bezeichnen, da sie sich unmittelbar aus den im Rahmen des Dispositivs generierten Diskursen ableiten lassen.

Auf die zweite Art der Wirkung bin ich ebenfalls bereits eingegangen, als ich eingangs James Fergusons (1994: 19f.) Vorschlag erwähnte, Foucaults Analyse der Wirksamkeit des Gefängnisdispositivs in seinem vermeintlichen Scheitern auf Entwicklung anzuwenden. Foucault hatte in »Überwachen und Strafen« (1994) festgestellt, dass das Gefängnis trotz des Scheiterns bei der Durchsetzung seiner propagierten Zielsetzungen, Abschreckung und Integration, einen ursprünglich unintendierten Erfolg mit sich brachte: die Produktion der Delinquenz, ein Milieu, das sehr bald produktiv aufgegriffen und zum neuen Raum für Projekte der Kontrolle und der Normalisierung wurde.

Ähnliche Wirkungen können wir beim Entwicklungsdispositiv feststellen, das seinen Erfolg auch nicht in Hinblick auf seine eigentlich propagierten Zielsetzungen erreicht hat - Entwicklung in der vorgesehenen Form hat in den innerhalb des Dispositivs als unterentwickelt konstruierten Regionen nicht stattgefunden, zumindest nicht nach all den Kriterien, die sich die beteiligten Agenturen selbst als Maßstab gesetzt haben. Einen möglichen Erfolg können wir allerdings in der Verankerung von Strukturen der Unterentwicklung wahrnehmen, die zur weltsystemischen Asymmetrie beitragen, ebenso wie in der Herstellung spezifischer Formen von Wahrnehmung - eben in der Durchsetzung des Prinzips der Unterentwicklung.

Foucault hat diese Wirkungen des Dispositivs als seine strategische Erfüllung bezeichnet. ${ }^{22}$ Ich möchte vorschlagen, diese Wirkung als

22 An dieser Stelle haben wir es leider mit unglücklichen Übersetzungsvarianten zu tun. Die zwei vorhandenen deutschen Übersetzungen bezeichnen die Stelle entweder als »strategische Ausfüllung« (Foucault 2003b: 394) oder als »strategische Wiederauffüllung« in jener Übersetzung, die 
»Wirkung zweiter Ordnung« zu bezeichnen - um klar zu machen, dass sich Dispositive durch eine doppelte Wirkungsebene auszeichnen, die in ihrer Gesamtheit aus den Diskursen, die sie generieren und die sie tragen, nicht vollständig ableitbar sind. Die Stärke der Konstruktion eines Dispositivs, und damit der strategische Hauptstoßpunkt innerhalb des Kräfteverhältnisses, in dem er wirkt, ist also seine Fähigkeit, die widerstrebenden Strömungen, die sich in einem solchen Machtverhältnis notwendiger Weise entwickeln, in sein heterogenes Netz zu integrieren.

Spätestens an diesem Punkt ist ein Wort der Vorsicht angebracht: Dieses idealtypische Bild suggeriert eine Totalität, die in der konkreten sozialen Realität nie funktioniert, ja nie funktionieren kann. Die Ränder eines Dispositivs sind schwammig, und es gelingt ihm keinesfalls, sein Netz um alle widerstrebenden Strömungen, alle Zufälle, jede Ereignishaftigkeit zu spannen. Ein Dispositiv ist ein spezifischer Modus von Macht, und als solcher immer für Widerstand anfällig. Es ist konkret angreifbar, wenn auch flexibel und schwer fassbar.

Die entscheidende Frage, die nun im Raum steht, ist die nach den Handelnden. Entwicklung wird schließlich gemacht, und es benötigt nicht viel Nachforschung um festzustellen, dass es kein zentrales Planungsbüro gibt, welches die global in diesem Dispositiv laufenden Prozesse steuert oder auch nur koordiniert - auch wenn sich das so manche in dem Bereich Tätigen vielleicht sogar wünschen würden. Die Realität entspricht in ihrem Chaos Stück für Stück dem Bild, das Foucault vom Dispositiv gezeichnet hat, als einem Wirrwarr von Praktiken, Institutionen, Diskursen, die aber dennoch eine strategische Funktion erfüllen. Wie aber ist das nun möglich, wie kann sich diese strategische Funktion realisieren?

Auf diese Frage geben weder marxistische Basis-Überbau-Modelle noch Annahmen über die grundlegende Verhaltensweisen der Gattung Mensch, wie sie in realistischen Ansätzen wiederzufinden sind, eine befriedigende Antwort, was in diesem Zusammenhang allerdings nichts über ihre Stimmigkeiten erklärt. Die Aussagen, die sie treffen können, sind zu generell, um jene feinen Unterschiede herausarbeiten zu können, in denen sich Macht in unterschiedlichen Kontexten vollzieht. Der entscheidende Begriff, den Foucault hier in die Diskussion einführt, ist der Begriff der Regierung, allerdings nicht im Sinne einer institutionalisier-

im 1978 veröffentlichten Merve-Band »Dispositive der Macht« verwendet wurde. Der von Foucault gebrauchte Originalbegriff ist »remplissement «, was etwa soviel heißt wie Erfüllung. Gemeint ist offenkundig, dass ein Dispositiv erst mit diesem Mechanismus seine strategische Erfüllung findet, also als Gesamtes zur Wirkung gelangt und damit als Dispositiv charakterisierbar wird. 
ten Staatsführung. Vielmehr geht es um einen Begriff, der in der Lage ist, Führungstechniken zu beschreiben, der Führung des Selbst und der des Anderen (vgl. Lemke 2004: 63): »Unter Regierung verstehe ich die Gesamtheit der Institutionen und Praktiken, mittels denen man die Menschen lenkt, von der Verwaltung bis zur Erziehung. Diese Gesamtheit von Prozeduren, Techniken, Methoden, welche die Lenkung der Menschen untereinander gewährleisten [...]« (Foucault 2005d: 116).

Es ist möglich, in den »Prozeduren, Techniken, Methoden«, die Regierung gewährleisten, Regelmäßigkeiten festzustellen, die weiter reichen als das intentionale Handeln der einzelnen Subjekte, andererseits aber auch durch allgemeine und in diesem Zusammenhang ungenaue Begriffe wie Ideologie nicht verdeutlicht werden können. Foucault sieht hier ein Prinzip zwischengelagert, das auf einer anderen Ebene angesiedelt ist: »Die Regierung der Menschen durch die Menschen [...] erfordert eine bestimmte Form der Rationalität, und nicht eine instrumentelle Gewalt« (Foucault 2005a: 197). Das Prinzip der Regierung ist also eine bestimmte Form der Rationalität, die nicht mit instrumenteller Gewalt also dem Element des Zwanges - verwechselt werden darf.

Solche Rationalitäten von Regierung bauen auf dem Macht-WissenKomplex auf und würden ohne ihn nicht funktionieren. Wie Michael Dillon hervorhebt, liegt darin eine der wesentlichen Leistungen von Macht-Wissen in seiner konkreten sozialen Wirksamkeit: »This is its capacity to enable government at a distance. For it enables subjects to do their own self-governing in ways that, while empowering a degree of autonomy, nonetheless integrate them into a web of power/knowledge and reporting that systematically holds them to (self-)account. By these means, power is able to operate without a requirement for the direct intervention of force« (Dillon 1995: 325). Ohne die Schaffung des notwendigen Wissens und seiner Verankerung - die nach meiner These von Dispositiven vollzogen wird, wobei Regierung selbst als Dispositiv interpretiert werden kann - ist zivile Herrschaft nicht realisierbar. Dies bestätigt auch Morgan Brigg mit Blick auf das Entwicklungsdispositiv: »More analytically tenable and critically efficacious is the recognition that, in its very aims, development is bound with the contemporary modality of power that operates by bringing forth and promoting the forces and energies of human subjects« (Brigg 2002: 426).

Foucault hat sich im Laufe seiner Forschungen eingehend mit der Problematik der Rationalitäten des Regierens auseinandergesetzt. Er wurde darauf gestoßen, als er gesellschaftliche Mechanismen wie Psychiatrie, Gefängnis oder Sexualität in ihrer historischen Entwicklung auf ihre Wirkungsweisen untersuchte, um sich schließlich in der zweiten Hälfte der 1970er in drei Vorlesungen am Collège de France ausführlich 
der Frage von Regierung und ihren Rationalitäten zu widmen. ${ }^{23}$ Schon zuvor hatte er in »Überwachen und Strafen« die Idee der Disziplinargesellschaft entwickelt und sich mit der Frage der Souveränität auseinandergesetzt. Später wird er diese beiden Modelle als Rationalitäten ordnen, und in der Auseinandersetzung mit dem Liberalismus (seiner einzigen ausführlichen wissenschaftlichen Befragung zeitgenössischer politischer Prozesse) eine dritte hinzufügen: die Gouvernementalität. ${ }^{24}$

Führen diese Überlegungen zu Foucaults Lebzeiten in der Rezeption seines Werkes noch ein Schattendasein, so gewinnen sie Mitte der 1990er - einhergehend mit dem Aufkommen von »Neo-Liberalismus« als politischem Schlagwort und einer damit notwendig gewordenen wissenschaftlichen Auseinandersetzung mit diesem Phänomen - schlagartig an Bedeutung. Zahlreiche Sammelbände ${ }^{25}$ und Einführungswerke (Dean 1999) werden veröffentlicht, auch mit dem Ziel, governmentality studies speziell in der US-amerikanischen Forschungslandschaft als interdisziplinären Forschungszweig zu etablieren.

»There are several reasons why governmentality has arisen to such prominence. First, it offers a line of investigation that has appealed to researchers frustrated with the enduring positivism of the social sciences, but not content either with the textualism of certain poststructuralisms. Governmentality certainly has significant affinities with poststructuralist approaches: a view of power as fragmented; an insistence on the constitutive nature of language; and

23 Alle drei betreffenden Vorlesungen wurden in schriftlicher Form veröffentlicht und liegen mittlerweile auch in deutscher Übersetzung vor. Es sind dies die Vorlesungen aus dem Jahr 1976, »In Verteidigung der Gesellschaft« (Foucault 1999b), dem Jahr 1978, »Sicherheit, Territorium, Bevölkerung« (Foucault 2004a), und dem Jahr 1979, »Die Geburt der Biopolitik« (Foucault 2004b).

24 Aus dem Entstehungszusammenhang des Konzeptes wird deutlich, dass die Mode gewordene Interpretation, es handle sich bei gouvernementalité um eine Verbindung von gouvernement und mentalité (vgl. etwa Reichert 2004), also eine Denkweise, falsch sein muss, ebenso wie die leider oft eingebürgerte deutsche Übersetzung »Regierungsmentalität«. Es geht hier nicht um Denkweisen (also Episteme), sondern um Rationalitäten, wir würden uns also - abgesehen von der linguistischen Fehldeutung, auf die Michel Sennelart (Foucault 2004a: 564) hinweist - auf einer falschen Ebene bewegen.

25 Für den englischen Sprachraum sind neben dem grundlegenden Band von Graham Burchell (Burchell et al. 1991) vor allem die Bände von Perry und Maurer (2003), Larner und Walters (2004) und Jonathan Xavier Inda (2005) zu erwähnen. Auch im deutschsprachigen Raum wurden mittlerweile einzelne Bände veröffentlicht. Zu nennen sind etwa der von Ramón Reichert (2004) herausgegebene Band »Governmentality Studies«, der durch seine Qualität besticht, sowie der Überblicksband von Pieper und Gutíerrez Rodríguez (2003). 
a view of agency in terms of contingent rather than fixed subjectivities. But unlike much work in poststructuralism, governmentality research has been more historical and more avowedly empirical in its orientation. This has much to do with an aspect of governmentality [...] - a concern with power in its multifarious practical, technical manifestations« (Larner/Walters 2004: 3).

Die Vorteile, die Larner und Walters auflisten, machen das Konzept nicht abgetrennt, sondern verbunden mit anderen Formen von Regierungsrationalität - zu einem gewinnbringenden Ansatz für die kritische Entwicklungsforschung. Sie ermöglichen eine Feinheit der Analyse, die in bisherigen Zugängen, auch jenen, die direkt auf Foucault rekurrieren, allzu oft in Pauschalitäten verloren gegangen sind. Auf der anderen Seite liegen in dem Zugang auch Gefahren. In einem starken Hang zum Empirismus vernachlässigen zahlreiche Studien, die sich im Kontext der governmentality studies platzieren, den komplexen Zusammenhang, in den die Gouvernementalität als eine Form der Rationalität des Regierens eingebettet ist. Die Folge ist eine Isolierung des Konzeptes und seine Umwandlung in eine schlichte soziologische Methode, die Kontrollmechanismen von Staatlichkeit abbildet, allerdings zumeist mit der aus der Vernachlässigung einer komplexen Machtanalyse resultierenden Tendenz zur Totalisierung solcher Mechanismen. Speziell Thomas Osborne (2004) hat sich massiv gegen die Einrichtung solcher governmentality studies als Zweig der Soziologie gewandt und als Gegenentwurf »Studies of Governmentality« vorgeschlagen.

»In diesem Sinne ist es den Studies of Governmentality um die Historizität der Ereignisse innerhalb einer Geschichte der Regierungstechnologien zu tun. Und damit sollten sie auch nicht als Teil einer übergeordneten Methodologie (welcher Art auch immer) verstanden werden, sondern als singuläre, diskontinuierliche Forschungsarbeiten: begrenzt, empirisch, hoffentlich maßvoll, aber auch - sofern sie ihre Arbeit gut machen - unzeitgemäß, also in einer kontingenten und fragenden Weise auf die Gegenwart bezogen « (Osborne 2004: 39). ${ }^{26}$

Das Verhältnis zwischen den unterschiedlichen Rationalitäten ist in Foucaults Konzept nicht als allmählicher Übergang zu verstehen, wie es

26 Wahrscheinlich wäre es überhaupt klüger, solche Forschungen nicht auf die Suche nach Gouvernementalität, sondern nach Rationalitäten auszurichten - allein, um nicht schon im Ansatz Ergebnisse im Voraus zu determinieren. Auch wenn angesichts der globalen Dominanz des Liberalismus in seinen unterschiedlichen Spielarten Gouvernementalität zur hervorstechenden Rationalität des Regierens - was in unserem Fall heißt: der Intervention - geworden ist, bedeutet das keineswegs, dass damit andere Rationalitäten ausgelöscht worden wären. 
etwa die marxistischen Stufen der gesellschaftlichen Entwicklung nahe legen. Hier finden sich eben Reste vorkapitalistischer Produktionsweisen auch in kapitalistisch dominierten Settings, mitunter finden wir auch eigentlich unproduktiv gewordene - (heutzutage nicht mehr regional abgrenzbare) Zonen des Sklavenhaltertums. Foucaults Konzept der Rationalitäten ist nicht auf teleologische Entwicklung ausgelegt, dementsprechend kennt es auch keine Abfolge. Souveräne Rationalitäten finden sich neben der Disziplin, und ohne die Rationalität der Disziplin könnte Gouvernementalität vermutlich gar nicht greifen - Foucault hat diesen Zusammenhang einmal als Triangel bezeichnet (vgl. Dillon 1995).

Näher liegt vielmehr die Annahme, dass gewisse soziale Situationen - also Machtbeziehungen - strategische Problemlagen hervorbringen und daher in Folge zur Bildung von Dispositiven führen, die zur Lösung dieser Probleme dienen sollen. Und diese Dispositive bilden eine ihnen angemessene Konfiguration jener Rationalitäten heraus, die von der epistemischen Ebene geprägt sind, gleichzeitig aber wieder auf sie rückwirken. Michael Dillon (2004: 77) hat daher vorgeschlagen, Gouvernementalität als »a born of a technological ontology that proceeds through reflexive epistemologies« zu begreifen.

Ich will die zum Verständnis des Gedankenganges eigentlich notwendige historische Herleitung an dieser Stelle nicht vertiefen und auf die entsprechende Literatur verweisen (insb. Foucault 2004a, 2004b oder auch Dean 1999). Es soll genügen, einige Bemerkungen zum speziellen Charakter der gouvernementalen Rationalität festzuhalten, die zur Untermauerung meiner Grundthese dienen, dass der spezielle Charakter des Entwicklungsdispositivs, der sich in der Vermengung mit dem Dispositiv der Sicherheit ausdrückt, primär von dieser Rationalität geprägt ist, obwohl weiterhin wesentliche Elemente der Disziplin und der Souveränität tragend wirken.

Die zentrale Entstehungsbedingung gouvernementaler Rationalität hängt eng mit dem politischen Projekt des Liberalismus zusammen und wird erst durch diesen möglich. Die Ursache dafür hängt vor allem mit dessen Ausgangspunkt als Kritikbewegung zusammen, die in einer starken inneren Dynamik mündet: »As a practice animated by an ethos of critique, liberalism displays a remarkable degree of political invention and self-renewal. Thus the liberal regime of government not only prepares the way for positive forms of knowledge of economy and society but establishes them as absolutely necessary« (Dean 1999: 51). Die Konsequenz ist ein Wandel der konkreten Methoden und Charakteristika von Herrschaft, die sich vom Rechtsprinzip der Staatsräson und der mit der Idee der Disziplin verknüpften Einrichtung der Policey als allumfas- 
sender Kontrollinstanz erheblich unterscheiden (Lemke et al. 2000: 13) und daher eine eigene Form der Rationalität, die Gouvernementalität, hervorbringen. $^{27}$

Verknüpft ist diese Rationalität mit einer neuen, dynamischen Qualität von Regierung, die ein wesentliches Spezifikum bildet. Diese Dynamik ergibt sich aus dem liberalen Selbstverständnis der beständigen Reform, wie Mark Duffield (2002: 80) hervorhebt: »The failure of modernity is synonymous with a temporary loosening of civilisation's grip and reversing of the ameliorative effects of social organisation. [...] If the failure of modernity is a recurring motif, it is because liberal society defends itself as being in a process of never-ending reform in response to its critics and detractors. « Überhaupt bildet das Scheitern der Utopie des Marktes eine notwendige Bedingung für den Liberalismus als politisches Projekt (vgl. Polanyi 2001: 227) und wird damit zur Basis einer neuen Rationalität des Regierens.

Mitchell Dean (1999: 20f.) charakterisiert die sich daraus ergebenden speziellen Charakteristika von Gouvernementalität anhand von vier Punkten. Erstens hängt für ihn die Entstehung gouvernementaler Rationalität mit dem Aufkommen bestimmter neuer Wahrnehmungen gesellschaftlicher Phänomene zusammen. Eines der wesentlichsten ist neben der Herausbildung der wissenschaftlichen Idee einer politischen Ökonomie die Erfindung der Bevölkerung (vgl. Foucault 1999b: 291). Das Konzept Bevölkerung liefert gegenüber älteren Mustern wie Untertanen oder der von Souveränität geprägten Konstruktion des Volkes vollkommen neue Möglichkeiten der Einflussnahme auf Menschen. Diese hängen eng mit einer sozialen Anwendung des Risikobegriffs zusammen und, in der Folge der praktischen Umsetzung der Risikoanalyse, mit der Idee von Statistik und Wahrscheinlichkeit, die auch wesentlich auf das Sicherheitsdispositiv rückwirkt. ${ }^{28}$

27 Es ist bei diesen Konzepten allerdings zu bedenken, dass die Kategorienbildung von Foucault in den betreffenden Vorlesungen bei Begriffen wie Liberalismus, Gouvernementalität oder Regierung lose ist. So spricht er vom Liberalismus als »Typ von Rationalität in der Regierungskunst« (Foucault 2004a: 40). Wäre ich gezwungen, klare Begriffsabgrenzungen vorzunehmen, würde ich den Liberalismus eher der ideologischen Ebene zuordnen, was Foucault im Übrigen auch nicht ausschließt. Der entsprechende Typ von Rationalität wäre in diesem Bild dann die Gouvernementalität.

28 Foucault zeichnet in der Entstehung der Vakzination und der Impfkampagnen im 18. Jahrhundert diese Denkentwicklung nach (Foucault 2004a: 90ff.). Vakzination als medizinische Errungenschaft war genötigt, grundsätzlich mit den medizinischen Rationalitäten des 17. Jahrhunderts zu brechen, da es in diesem Kontext unmöglich gewesen wäre, Krankheitserreger einfach aufgrund statistischer Wahrscheinlichkeiten zu impfen. Es wä- 
Foucault führt für diesen Prozess einen neuen Begriff in die Diskussion ein: Biomacht, und im Weiteren, um den intentionalen Charakter zu verdeutlichen, Biopolitik, wobei Biopolitik im eigentlichen Sinn kein politisches, sondern ein tendenziell regulatives Unternehmen auf der Ebene des Lebens darstellt (vgl. Dean 1999: 99). ${ }^{29}$

»Diesseits der großen absoluten, dramatischen und dunklen Macht der Souveränität, die darin bestand, sterben zu machen, tritt jetzt mit dieser Technologie der Bio-Macht, dieser Macht über >die` Bevölkerung als solche, über den Menschen als Lebewesen, eine dauerhafte und gelehrte Macht hervor: die Macht >leben zu machen`. Die Souveränität machte sterben und ließ leben. Nun tritt eine Macht in Erscheinung, die ich als Regulierungsmacht bezeichnen würde und die im Gegenteil darin besteht, leben zu machen und sterben zu lassen« (Foucault 1999: 291).

Der Bedeutungswandel, den das Konzept der Nation im Zusammenhang mit liberaler Regierung durchläuft, steht mit dieser Biopolitik in unmittelbarem Zusammenhang. Nation wird dabei, im partiellen Bruch von Souveränität, die sich hiermit (mit zum Teil verheerenden Folgen) in ein Stadium der neuen Verhandelbarkeit begibt, de-territorialisiert und entlang sozial konstruierter Kriterien neu formiert (vgl. Burchell 2004: 53). Damit wird, gerade auch auf internationaler Ebene, über die Nation ein neuer Konnex zu Sicherheit geschaffen. Der Drang nach entwicklungs-

re auch möglich, die von Polanyi (2001) so eindrucksvoll geschilderte Überwindung der Speenhamland-Gesetzgebung im England des späten 18. Jahrhunderts mit diesem Prozess zu erklären. Wahrscheinlich ist Speenhamland und die sich in ihm vollziehende Entstehung des Marktdispositivs eines der eindrucksvollsten Beispiele, um das Zusammenwirken zwischen Dispositiven, Rationalitäten und Epistemen anschaulich nachzuvollziehen.

29 Für Foucault laufen diese Prozesse ineinander: "Zum ersten Mal in der Geschichte reflektiert sich das Biologische im Politischen. Die Tatsache des Lebens ist nicht mehr der unzugängliche Unterbau, der nur von Zeit zu Zeit, im Zufall und in der Schicksalhaftigkeit des Todes ans Licht kommt. Sie wird zum Teil von der Kontrolle des Wissens und vom Eingriff der Macht erfasst. Diese hat es nun nicht mehr bloß mit Rechtssubjekten zu tun, die im äußersten Fall durch den Tod unterworfen werden, sondern mit Lebewesen, deren Erfassung sich auf dem Niveau des Lebens halten muss. Anstelle der Drohung mit dem Mord ist es nun die Verantwortung für das Leben, die der Macht Zugang zum Körper verschafft. Kann man als >BioGeschichte jene Pressionen bezeichnen, unter denen sich die Bewegungen des Lebens und die Prozesse der Geschichte überlagern, so müsste man von >Bio-Politik sprechen, um den Eintritt des Lebens und seiner Mechanismen in den Bereich der bewussten Kalküle und die Verwandlung des Macht-Wissens in einen Transformationsagenten des menschlichen Lebens zu bezeichnen« (Foucault 1999: 170). 
politischem Nation-Building findet vor diesem Hintergrund einen Erklärungsansatz: "Nation building, in any case, illustrates the sproductive face of biopower and security « (Hardt/Negri 2004: 23; vgl. dazu auch Reid 2005).

Dies ist allerdings nur eine der Wirkungen, die das Entwicklungsdispositiv auf Ebene der Biopolitik entfalten kann. Mehr oder minder ist sein gesamtes Programm auf vielfältige Weise darin eingeschrieben, wie Mark Duffield argumentiert:

»While development programmes contain individualising disciplinary elements, typically in the form of projects, they also seek to strengthen the resilience of collectivities and populations. Towards this end, development draws widely on regulatory mechanisms, risk management techniques and compensatory programmes that act at the aggregate level of economic and social life. In particular, development is a biopolitical security mechanism associated with populations understood as essentially self-reproducing in relation to their basic social and welfare needs« (Duffield 2005b: 6).

Als zweites Merkmal gouvernementalen Regierens hebt Mitchell Dean das Verhältnis hervor, in dem es zu den anderen Formen des Regierens steht, vor allem der Souveränität und der Disziplin. Dean erkennt in der Gouvernementalität eine Neukodierung von Macht, die sich substanziell von souveränen und disziplinären Machtformen unterscheidet. Ein wesentliches Element in diesem Zusammenhang ist das Bedürfnis nach der Einschränkung von Regierung - weniger Regieren als »Selbstbegrenzung der gouvernementalen Vernunft« (Foucault 2004a: 40). Dies führt nicht nur zu einer neuen Bedeutungszuschreibung für den rechtlichen Rahmen dieser Selbstbegrenzung. Karl Polanyi hat nachgewiesen, dass gerade auf ökonomischer Ebene ein liberaler Markt nie nach dem Laissez-faire-Prinzip funktioniert hat: »There was nothing natural about laissez-faire; free markets could never have come into being merely by allowing things to take their course« (Polanyi 2001: 145). Vielmehr wären konkrete Interventionen notwendig gewesen, die oftmals in juristische Formen gegossen wurden.

Diese Selbstbegrenzung hat auch direkte Auswirkungen auf internationale Interventionen, und das Selbstverständnis derjenigen, die sie durchführen. Die Staatsräson, eine klassische Idee der souveränen Rationalität, hätte, so Foucault, den Gedanken des Imperialismus durch den Wettbewerb zwischen konkurrierenden Staaten verdrängt. Die Gouvernementalität würde dies wiederum um- und zurückwerfen, und zwar aufgrund ihrer selbst auferlegten Begrenzung des Regierens: »Die liberale Vernunft geht einher mit der Aktivierung des imperialen Prinzips, 
nicht in Form des Kaiserreichs, sondern in Form des Imperialismus, und zwar in Verbindung mit dem Prinzip des freien Wettbewerbs zwischen den Individuen und den Unternehmen « (Foucault 2004a: 42, Fn).

Das dritte Merkmal, das Mitchell Dean herausstreicht, ist für unser Thema von besonderer Bedeutung. Es geht um eine neue Wirkung von Sicherheit auf die neu erfundene Figur der Bevölkerung. Gouvernementalität, so Dean, »seeks to enframe the population within what might be called apparatuses of security« (Dean 1999: 20). Thomas Lemke weist darauf hin, dass die Einführung des Sicherheitsdispositivs eine Grundbedingung liberalen Regierens darstellt (vgl. Lemke 1997: 184ff.). Liberalismus zielt auf Freiheit ab, in einem Freiheitsbegriff, der grundsätzlich individualistisch geprägt ist. Damit fallen zwei Machtmechanismen weg: einerseits das Element der direkten Herrschaft des Absolutismus, andererseits aber auch der Rekurs auf kollektivistische Konstruktionen. Damit bedarf es eines neuen Modus, um Gefahren für die Bevölkerung, die von Individuen ausgehen können, zu kodifizieren und in der Folge abzuwehren. Dieser Modus ist Sicherheit. »Freiheit und Sicherheit [bilden] die beiden Pole der liberalen Gouvernementalität« (Lemke 1997: 186). ${ }^{30}$

Freilich ist die erwähnte Einführung des Sicherheitsdispositivs an sich keine wirkliche Innovation. Ohne Zweifel haben auch andere Rationalitäten des Regierens auf Dispositive der Sicherheit zurückgegriffen. Doch das Sicherheitsdispositiv des gouvernementalen Regierens unterscheidet sich fundamental von vorherigen Sicherheitsbegriffen, so dass es nicht korrekt wäre, von einer einfachen Veränderung auszugehen. Tatsächlich ist dieser Prozess besser als Neueinführung zu beschreiben. Entscheidendes Charakteristikum ist die umfassende Bedeutung von Sicherheit im gouvernementalen Kontext, die sich auf den sozialen Bereich ausdehnt: »In suggesting that the society we now live in has become, preeminently, a >society of security<, part of what Foucault no doubt has in mind that our government involves a distinctive circuit of interdependence between political security and social security. It is

30 Mitchell Dean analysiert dies so: »Thus the liberal problematic of security differs from the police conception of security in a first way. While the police conception makes the security of the state dependent upon the detailed regulation of >men and things $<$, the liberal problematic advises that security can be best attained by creating the conditions under which individuals can exercise various liberties « (Dean 1999: 116f.). Was wir hier auf jeden Fall immer bedenken müssen, ist, dass das neue Sicherheitsdispositiv die Freiheit zu seinem Funktionieren voraussetzt: »This means that to govern properly, to achieve security, it is necessary to respect the liberty of the governed so that the natural processes of the economy and the population might function effectively« (ebd.). 
misleading to envisage the dimension of the social as the state's antagonist or its prey« (Gordon 1991: 35).

Auf internationaler Ebene sticht in dieser Beziehung sofort das $\mathrm{Hu}-$ man-Security-Konzept ins Auge. Kontrastierend ist jedoch, dass das Konzept erst gegen Mitte der 1990er Jahre populär wird. Seinen Ausgangspunkt findet es mit dem Human Development Report von 1994 (vgl. UNDP 1994). ${ }^{31}$ Warum so spät, wenn doch der Entwicklungsdispositiv eine deutlich längere Geschichte aufweist? Eine gut nachvollziehbare These ist, diese eigentlich überraschende Verzögerung mit der speziellen Bedeutung zu erklären, die dem Prinzip der Souveränität auf internationaler Ebene, und im Speziellen im Verhältnis der zentralen Staaten zur Peripherie und Semi-Peripherie des Weltsystems zukommt.

Die Rationalitäten, die auf dieser internationalen Ebene historisch wirksam werden, sind zwar grundsätzlich dieselben, die auch innerhalb der zentralen Regionen selbst zu finden sind. Doch es gibt keine Übereinstimmung, was die jeweils konkreten Konfigurationen anbelangt, die sich aus dem Zusammenspiel der Rationalitäten in den unterschiedlichen Dispositiven der Machtverhältnisse ergeben. Wenn also in Europa das Rationalitätsprinzip der Souveränität im Inneren bestimmend wirkt, bedeutet dies keineswegs, dass dies auch für die Außenbeziehungen Europas gelten muss - so war das Prinzip der Souveränität, das in Europa während langen Phasen seiner Geschichte als Kolonialmacht dominierte, in den Kolonien selbst keineswegs bestimmend. Ebenso können wir feststellen, dass auf internationaler Ebene das Prinzip der Souveränität in der Epoche der Dekolonisierung und Gründung der Nationalstaaten in den (Semi-)Peripherien vorherrschte, während sich in den Zentren die liberale Gouvernementalität als bestimmende Rationalität des Regierens etablierte. $^{32}$

31 Ein wesentlicher Anstoß zu der Diskussion kam von der Auseinandersetzung um die Dritte Generation der Menschenrechte, die zunächst von verschiedenen gewichtigen Staaten des Zentrums abgelehnt wurde. Die Entstehung des Human Security-Konzepts kann gewissermaßen als individualisierte Antwort auf diese Bemühungen verstanden werden.

32 Michael Dillon sieht im internationalen System beide Prinzipien am Werk: »Rather, I am proposing that the state and the system of states has, to an important degree, and from very early modern times, been a function of knowledgeable governmentality as much of sovereign territoriality; and that, moreover, governmentalization of the state and of the system of states - the codes, protocols, and regimes of knowledge of (inter)national relations - has been the principal device by which the technologization of the political (and the democratic) has been disseminated globally in the modern age « (Dillon 1995: 343). Ich werde auf diese hier nur kurz angerissenen Fragen weiter hinten noch zu sprechen kommen. 
Offenbar befindet sich dieses Prinzip der Souveränität auf internationaler Ebene in einem Stadium, in dem es neu verhandelt werden kann. Es ist in der Tat anzunehmen, dass diese neue Verhandelbarkeit mit dem Ende des Kalten Krieges in engem Zusammenhang steht, aber vielleicht in einer etwas komplexeren Weise als in den Versuchen, die darauf folgenden politischen Prozesse der internationalen Politik mit einem ökonomistisch oder kulturalistisch gefüllten Globalisierungsbegriff zu begreifen, für gewöhnlich angenommen wird. Mir scheint der wesentliche neue Prozess, der damit in Gang gesetzt wird, nicht allein in einer quantitativen oder qualitativen Verstärkung internationaler Asymmetrien zu liegen, die sich durch den »Sieg« des Westens ergeben hätten - und nichts anderes ist in den meisten Fällen gemeint, wenn Begriffe wie »Globalisierung« und »Neoliberalismus« mit politischer Betonung in Debatten eingeführt werden, oftmals verbunden mit der expliziten Betonung des Souveränitätsprinzips, das durch diese Entwicklungen unterlaufen werde.

Diese Behauptung ist kurzsichtig. Was wir beobachten können ist keinesfalls ein Unterlaufen oder Aufweichen dieses Souveränitätsprinzips. Vielmehr scheint es hier um eine Neukonfiguration der Rationalitäten des internationalen Systems zu gehen, in der die Verhandelbarkeit und Relativität der Souveränität ein Charakteristikum sind - aber keineswegs deren Ursache. Es ist keinesfalls als reine Provokation misszuverstehen, wenn Francis Fukuyama 1989 das Ende der Geschichte mit der Bemerkung einläuten kann, die Idee des westlichen Liberalismus habe endgültig triumphiert: »The triumph of the West, of the Western idea, is evident first of all in the total exhaustion of viable systematic alternatives to Western liberalism« (Fukuyama 1989). Fukuyama ist letztendlich nur einer derjenigen Apologeten, die den Drang zur globalen Normalisierung im Sinne des Liberalismus behaupten wollen.

Die Kurzsichtigkeit von Fukuyamas Argument liegt also keinesfalls in der ihm oft vorgeworfenen Präpotenz - dieser Vorhalt mag stimmen oder nicht, er ist letztendlich unerheblich. Seine Kurzsichtigkeit liegt eher darin, nicht nur ignoriert zu haben, dass sich die Idee des globalen Liberalismus und die Idee einer globalen Normalisierung, die er unter dem Begriff »post-historical« zusammenfasst und die ihm - was gegen den Ignoranz-Vorhalt spricht - alles andere als zusagt, ausschließen. Es kann also eine post-historische Epoche des Liberalismus gar nicht geben. Es ist die Vorstellung einer Möglichkeit, dass die Welt gleichzeitig geteilt sein könnte »between a part that was historical and a part that was 
post-historical« (ebd.), die die eigentliche Paradoxie seiner These ausmacht. ${ }^{33}$

Die in dieser Behauptung liegende Fiktionalität ist also nicht so sehr der Annahme einer Existenz von Räumen geschuldet, in denen eine liberale Ordnung hegemonial ist und die mithin als "post-historisch« gelten könnten. Sie liegt in der Annahme einer wie auch immer gearteten Koexistenz. Die Intention, und erst mit ihr bekommt Fukuyamas Argument Gewicht, besteht darin, dass eben jene nicht normalisierten Räume zu Ausnahmen werden, zu Ausnahmen einer Norm, die nach den Rationalitäten der Zentren zurechtgezimmert wird.

Giorgio Agamben hat in seiner reflektierten Weiterführung der Theorien Carl Schmitts auf die Bedeutung der Konstruktion von Ausnahmefällen für das gegenwärtige Paradigma des Regierens, speziell auf internationaler Ebene, hingewiesen. Denn Ausnahmefälle begründen einen Ausnahmezustand: »Angesichts der unaufhaltsamen Steigerung dessen, was als >weltweiter Bürgerkrieg` bestimmt worden ist, erweist sich der Ausnahmezustand in der Politik der Gegenwart immer mehr als das herrschende Paradigma des Regierens« (Agamben 2004: 9).

Der Ausnahmezustand definiert ein spezifisches Feld politischen Handelns, das erst erlaubt, das lange dominante Souveränitätsprinzip neu zu verhandeln. Indem es sich gleichzeitig innerhalb wie außerhalb der Rechtsordnung bewegt, ermöglicht es neue Grenzziehungen. »Der Ausnahmezustand ist kein Sonderrecht (wie das Kriegsrecht), sondern er bestimmt, indem er die Rechtsordnung suspendiert, deren Schwelle oder Grenzbegriff« (ebd.: 11). Ein solcher Ausnahmezustand nimmt auf internationaler Ebene einen spezifischen Charakter an, von dem das Unterlaufen des im Falle konkreter Interventionsdrohungen von der UNO garantierten Souveränitätsprinzips nur eine von zahlreichen Erscheinungen ist.

»The zone of exception within a planetary order exists within the politicojuridical lacunae created by defending the territorial integrity of the underdeveloped or authoritarian state while making sovereignty over the life within it negotiable and contingent. Such a conditional sovereignty can be used to either promote life through technologies of sustainable development and human security, or it can allow death when these security technologies break down and mass society comes under threat from unsecured global circulation« (Duffield 2006).

33 Fukuyamas These ist eine Fortschreibung jenes imperialistischen Denkens, das in vergleichbarer Weise eine prähistorische Epoche konstruierte. Eric Wolf hat sich in »Europe and the People Without History« (1997) detailliert mit dieser Frage auseinandergesetzt. 
Erst auf dieser Grundlage wird zivile Intervention in der Form, wie wir sie heute erleben, überhaupt möglich. Sie definiert zugleich den grundlegend neuen Charakter, der die Verbindung zwischen Entwicklungsdispositiv und Sicherheitsdispositiv derzeit annimmt. Dies ist ein ausgesprochen produktiver Prozess: »The problematic of security is shifting from that of securing the economic and social processes external to the state, and the forms of snatural liberty< on which they depend, to one of constructing centres of agency and activity, of making them durable, and of implanting continuous relations of authority« (Dean 1999: 173). Entwicklungspolitik ist eine der Ebenen, auf der sich diese Herausbildung derzeit hauptsächlich abspielt.

Mark Duffield (2005a) weist in diesem Zusammenhang darauf hin, dass sich Entwicklung in ihrer Einwirkung grundsätzlich von Mechanismen in den Zentren selbst unterscheidet: »Unlike the modes of intervention associated with mass society, development represents a biopolitics of population understood to be self-reliant in terms of its basic economic and welfare needs. The security function of such a biopolitics is that of bettering self-reliance in the interests of defending international society: it is the art of getting savages to fight barbarians.«

Der an dieser Stelle von Duffield angeführte Diskurs um Zivilisation, Wildheit und Barbarei ist eine aussagekräftige Metapher. Er kann gewissermaßen als eine der Nahtstellen in der Verbindung der Dispositive von Entwicklung und Sicherheit verstanden werden. Während sich die Figur des Wilden durch seine nicht-territoriale Verankerung und seine ökonomische Primitivität - er hat es über den Tauschhandel nicht hinausgebracht - gekennzeichnet ist und damit seine Erlösung durch Zivilisation möglich wird, er aber auch ohne sie denkbar ist, ist der Barbar nichts anderes als eine simple zivilisatorische Antithese: »Ein Barbar ist ohne einen zivilisatorischen Bezugspunkt, zu dem er in ein Verhältnis des Außerhalb tritt und gegen den er ankämpfen wird, nicht denkbar. Ein zivilisatorischer Bezugspunkt - den der Barbar verachtet und den er aufsucht -, zu dem er in ein Verhältnis von Feindschaft und fortgesetztem Krieg tritt. Es gibt keinen Barbar ohne eine Zivilisation, die er zu zerstören oder sich einzuverleiben trachtet« (Foucault 1999b: 231f.).

Die Neukonfiguration des Entwicklungsdispositivs spielt gezielt in diesem Trialismus. Während lange Zeit die Befreiung des Wilden von seiner Wildheit durch die Segnungen der Zivilisation das Grundmuster der Erzählung gebildet hatte, kommt jetzt die Figur des Barbaren zu neuer Prominenz. Entwicklung soll ihre Wirkung dahingehend entfalten, das Barbarische zu unterlaufen und zu verdrängen, die Voraussetzungen für sein Aussterben zugunsten der Zivilisation zu schaffen - und sei es, in dem die Wilden dafür instrumentalisiert werden. 
Der vierte und letzte Punkt, den Mitchell Dean beleuchtet, betrifft die Gouvernementalisierung des Staates. Die sich darin entwickelnde Staatsräson, die der gouvernementalen Vernunft entspringt, bricht mit den Vorstellungen von Monarchien, Reichen oder älteren Konzepten von Staatlichkeit. Dieser spezielle Charakter wird oftmals verkannt, da sich Untersuchungen im Kontext von Staatlichkeit zumeist auf seinen institutionellen Aspekt konzentrieren - ebenso wie auch Ansätze des aktiven State-Building. Insofern bezeichnet Foucault die Frage des Staates als überbewertet, was auch in seiner eigenen Logik liege: »Alles in allem ist der Staat vielleicht nur eine bunt zusammengewürfelte Wirklichkeit, eine mythifizierte Abstraktion, deren Bedeutung viel beschränkter ist als man glaubt. [...] Das, was es für unsere Modernität [...] an Wichtigem gibt, ist also nicht die Verstaatlichung der Gesellschaft, sondern das, was ich eher die >Gouvernementalisierung « des Staates nennen würde« (Foucault 2004a: 163; vgl. dazu auch Migdal 2001: 18f.).

Der Staat ist also eine Art Zielbestimmung, »dasjenige, was am Ende der Rationalisierung der Regierungskunst stehen soll« (ebd.: 416). Natürlich baut die Idee von Staat auf vorhandenen Formen des Regierens auf - institutionell, strukturell, wie auch in der Ausprägung der steuernden Rationalitäten. Der Staat als Zielbestimmung ist keine Reißbrettkreation. Dennoch ist er ohne gouvernementale Rationalität in seinen zeitgenössischen Formen nicht denkbar.

»Ich wollte Ihnen auch zeigen, dass diese gouvernementale ratio, diese gouvernementale Vernunft etwas erscheinen ließ, das zugleich ihr Ursprung als auch ihr Ziel war, ihre Grundlage und ihr Zweck, und dieses Etwas, das zugleich Ursprung und Ziel der gouvernementalen Vernunft ist, ist der Staat. Der Staat, der, wenn Sie so wollen, in gewisser Weise ein Verständnisprinzip und strategisches Schema ist. Um einen anachronistischen Begriff im Verhältnis zu der Epoche zu verwenden, von der ich spreche, könnte man sagen, eine regulative Idee. Der Staat ist die regulative Idee der gouvernementalen Vernunft« (Foucault 2004a: 415).

Die Besonderheit, die den gouvernemental geprägten liberalen Staat von vorherigen Staatsideen unterscheidet, ist vor allem das gesellschaftliche Modell, in dem er operiert. Denn liberale Staatlichkeit funktioniert nur mit einer zivilen Gesellschaft - aber ebenso funktioniert zivile Gesellschaft nur mit liberaler Staatlichkeit. Die Idee einer Zivilgesellschaft war von ihrem Ursprung her eng mit der Idee des Liberalismus und dem Konzept des Individualismus verbunden (vgl. Kaldor 2002). 
»The scheme of civil society situates the problem of exercising political power in relation to a natural domain in which power, in the form of spontaneously developed relations of authority and subordination, already exists in an internal, dynamic relation to the play of egoistic and non-egoistic interests. Thus government can be thought of as a function of already existing social and economic relations in the form of relations of authority and subordination « (Burchell 1991: 140).

Die Vorstellung von Zivilgesellschaft als anti-hegemonialer Kraft, wie sie oft in ihrer aktivistischen Interpretation (vgl. Kaldor 2002: 6f.) zu hören ist, entbehrt daher ihrer Grundlage. Zivilgesellschaft ist integraler Bestandteil der liberalen Staatsidee, in der der Staat eben nicht souveränes Institut ist, sondern Resultat des Wirkens von heterogenen, jedoch von der gouvernementalen Vernunft getragenen Kräften. Sie muss gewissermaßen - zumindest in überwiegenden Teilen - zur staatlichen Hegemonie beitragen, was sie im Übrigen auch durch scharfe Kritik tun kann, soweit sie sich innerhalb der gewöhnlich weit gefassten Toleranzgrenze der Prinzipien gouvernementalen Regierens befindet. ${ }^{34}$ Bewegt sie sich außerhalb dieser Toleranzgrenze, ist sie per definitionem keine zivile Gesellschaft mehr - schon allein, weil sie sich innerhalb kürzester Zeit mit den repressiven Instrumenten von Herrschaft konfrontiert sehen wird.

Diese vier Charakteristika liberaler Gouvernementalität - Erfindung von neuen gesellschaftlichen Größen wie Bevölkerung, die zum Ziel von Regierungshandeln werden, die ineinander greifenden und komplexen Beziehungen zu anderen Rationalitäten von Regierung, das umfassende Sicherheitsdispositiv und die Gouvernementalisierung des Staates - prägen, wie ich in Ansätzen bereits zu zeigen versucht habe, das Entwicklungsdispositiv.

34 Dieser Rahmen ist als internationales Projekt zu verstehen, der die konkrete Toleranz eines spezifischen Staates - im Sinne »demokratischer Opposition « - sehr wohl überschreiten kann. Dennoch greifen dann oft internationale Mechanismen, sei es auf politischer, sei es auf medialer Ebene (als ein Paradefall sei Aung San Suu Kyi erwähnt, die für die Überschreitung des Toleranzrahmens des Burmesischen Staates sogar den Friedensnobelpreis erhalten hat). 


\section{Zur analytischen Strategie einer Dispositivanalyse}

Bevor ich mich den empirischen Hauptteilen der Untersuchung zuwende, ist eine Auseinandersetzung mit dem »Wie« der Analyse angezeigt. Denn nicht nur sind - mit wenigen Ausnahmen wie etwa einem Vorschlag von Siegfried Jäger (2001), auf den ich später noch zu sprechen komme - kaum konkrete methodische Vorschläge für die Analyse von Dispositiven vorgelegt worden. Auch jene Arbeiten, die sich auf empirischer Ebene mit konkreten Dispositiven auseinandersetzen - für den Entwicklungsdispositiv sind die Arbeiten von Ferguson (1994) und Escobar (1995) schon zur Sprache gekommen -, zeichnen sich durch äußerst unterschiedliche Zugänge aus. Verallgemeinerbare methodische Implikationen sind aus ihnen nicht abzuleiten.

Hannelore Bublitz sieht dies nicht durch subjektive Nachlässigkeiten bedingt, sondern als Konsequenz der foucaultschen Herangehensweise: »Die spezifischen Problemstellungen hinsichtlich der Operationalisierung ergeben sich aus der Eigentümlichkeit des foucaultschen Denkgebäudes, das weder Theorie - im Sinne eines generalisierbaren Systems, das auf verschiedene Bereiche >angewendet « wird - noch Methode - im Sinne eines Verfahrens, das quasi >von außen Gegenstand lenkt - sein will« (Bublitz et al. 1999: 15f.). Die große analytische Heterogenität von Arbeiten, die sich auf jenes »foucaultsche Denkgebäude« berufen, unterstützt ohne Zweifel diese Annahme. Zugleich tritt in diesen Arbeiten oftmals eine spezifische Tendenz zu Tage, die sich aus der von Bublitz skizzierten Problemstellung ergibt: die Frage der Operationalisierung wird umschifft und einfach durch die subjektive Kreativität der Forschenden ersetzt.

Nun meine ich nicht, dass diese Kreativität an sich das Problem darstellen würde - ganz im Gegenteil. Letztendlich ist sie einer der wesentlichen Faktoren, auf denen die Güte qualitativer Forschung gebaut ist. Allerdings sollte sie nicht als Ersatz für die Anwendung reflektierter Prozesse der Datengewinnung und -analyse herhalten, und zwar aus zwei Gründen: Erstens ist eine festgelegte Systematik bei richtiger Anwendung nicht nur kein Gegner einer kreativen Entfaltung im Forschungsprozess, sondern einer ihrer wesentlichen Verbündeten. Sie hilft bei der unvermeidbaren Eingrenzung der zu treffenden Aussagen, trägt also dazu bei, im Hinblick auf das jeweilige Forschungsinteresse bessere Fragen an einen konsequenter eingegrenzten Materialkorpus zu richten. Zweitens ermöglicht die Darlegung einer solchen Systematik einen doppelten Effekt in Bezug auf die Kommunizierbarkeit einer Studie. Zu- 
nächst erlaubt sie eine Nachvollziehbarkeit der Ergebnisse auch dann, wenn ihre epistemologischen Grundlagen umstritten bleiben.

Zugleich ist die Nachvollziehbarkeit nicht nur in Bezug auf die Ergebnisse von Bedeutung. Sie hilft auch, den Forschungsprozess selbst transparent zu halten, was wiederum aktiv zu der beständigen und meines Erachtens nach entscheidenden Debatte über die Frage der methodischen Gestaltung konstruktivistisch orientierter sozialwissenschaftlicher Forschung beiträgt. Damit soll nun keinesfalls das wohl bekannte Bild einer im Sinne teleologischen Fortschritts voranschreitenden Wissensakkumulation bemüht werden - im Gegenteil. Eine lebendige Debatte ist vielmehr die beste Voraussetzung, die Lebendigkeit analytischer Kreativität vor dem Mief der Kanonisierung zu bewahren.

Wie oben kurz erwähnt, hat Siegfried Jäger (2001) einen methodischen Ansatz zur Analyse von Dispositiven vorgelegt. ${ }^{35}$ Ausgehend von den foucaultschen Überlegungen schlägt er vor, Dispositiv als Zusammenspiel von diskursiven Praktiken mit »nichtdiskursive[n] Praktiken und sogenannte[n] Sichtbarkeiten/Vergegenständlichungen « zu begreifen (ebd.: 87). In der methodischen Annäherung an diese Aufgabenstellung gewinnt er den »Eindruck, dass die Schwierigkeiten bei der Bestimmung des Dispositivs mit der mangelnden Bestimmung der Vermittlung von Diskurs (Sagbarem/Gesagten), nicht-diskursiven Praxen (Tätigkeiten) und Sichtbarkeiten (Produkten/Gegenständen) zu tun haben« (ebd.: 95).

Auf den ersten Blick wirkt dieser Eindruck korrekt und gut nachvollziehbar. Näher betrachtet offenbart sich darin allerdings eine Problematik, die sich durch den gesamten methodischen Ansatz von Jäger zieht: die Annahme, ein Dispositiv sei die Summe der drei Teile Diskurs, Praxis, Sichtbarkeit. In der Annäherung an mögliche methodische Vorgangsweisen präzisiert Jäger diese Interpretation: »Ein Dispositiv ist der prozessierende Zusammenhang von Wissen, die in Sprechen/Denken - Tun - Vergegenständlichung eingeschlossen sind. Die Grundfigur des Dispositivs kann man sich als ein Dreieck oder besser: als einen rotierenden und historisch prozessierenden Kreis mit drei zentralen Durchlauf-Punkten bzw. Durchgangsstationen vorstellen« (ebd.: 106).

Sicher ist hier Jägers Versuch erkennbar, sein Dispositiv-Konzept mit einem dynamischen Element auszustatten. Trotzdem vernachlässigt er jene Komponente, die den eigentlich besonderen Charakter eines Dispositivs ausmacht: die Komponente der Strategie. Foucault spricht von

35 Eine englische Version seines Artikels findet sich unter dem Titel »Discourse and knowledge: theoretical and methodological aspects of a critical discourse and dispositive analysis« in Wodak und Meyer (2001: 32$62)$. 
einer »dominante[n] strategische[n] Funktion«, die Dispositive auszeichnet, sie hätten »vor allem« die Funktion, dringenden historisch gegebenen Anforderungen nachzukommen (Foucault 2003b: 393). Eine solche strategische Komponente ist für das Konzept des Dispositivs also konstituierend. Es muss sich daher auch im analytischen Zugang wiederfinden, der der Untersuchung von Dispositiven zu Grunde gelegt werden soll.

Der Vorschlag Jägers läuft darauf hinaus, mit einer Kombination verschiedener standardisierter Verfahren jenes Wissen zu rekonstruieren, das sich in »diskursiven Praxen«, »nichtdiskursiven Praxen« und »Sichtbarkeiten/Vergegenständlichungen« materialisiert (Jäger 2001: 108). Zwar sieht er sich explizit zu nicht mehr in der Lage als Anregungen für solche komplexe Analyseprozesse zu geben. Dennoch gestaltet er diese Anregungen eindeutig: »Herzstück [der Analyse] hat dabei die Diskursanalyse zu sein«, die er primär auf Texte bezogen und mit bekannten standardisierten Verfahren abgewickelt sehen will (ebd.: 111).

Dieser Ansatz scheint mir speziell aus zwei Gründen wenig zielführend: Zunächst unterscheidet sich eine Diskursanalyse nach Foucault grundlegend von einem an Texte gebundenen Zugang, wie Glyn Williams (1999: 76) hervorhebt: »What Foucault did do was to show that discourse analysis was much more than linguistic analysis pure and simple in the sense of trying to indicate which sentences might be grammatically possible. Rather, his focus was upon the systems of rules which make the appearance of certain statements rather than others possible in particular settings, at particular historical conjunctures« (vgl. dazu auch Keller 2004: 44). Eine Trennung zwischen textualem Diskurs und nicht-textualer Praxis, wie sie Jäger trifft, geht also am Konzept des Diskurses, auf das er sich selber bezieht, vorbei.

Zugleich suggeriert Jäger mit seinen Überlegungen (trotz verschiedener Einschränkungen), dass die Entwicklung standardisierter Verfahren zur Analyse von Dispositiven prinzipiell möglich wäre. Eine solche Möglichkeit kann allerdings nur dann behauptet werden, wenn vom strategischen Gehalt des Dispositivs abstrahiert wird. In der Diskussion seiner Analyse der »Formation der Strategien« bei diskursiven Formationen bekennt Foucault (1981: 94ff.) etwa, große Schwierigkeiten insofern gehabt zu haben, als die Schwerpunkte jener spezifischen Formationen immer andere gewesen seien. Es ist anzunehmen, dass auf Dispositive Ähnliches zutrifft - ihre Gestalt ist also zu verschieden, als dass es möglich wäre, sich ihnen mit standardisierten Verfahren zu nähern. Dies trotzdem zu versuchen bedeutet letztlich nichts anderes als ihren strategischen Gehalt zu ignorieren. 
Niels Åkerstrøm Andersen (2003) interpretiert diese Frage als ein grundsätzliches Problem, das aus dem Widerspruch zwischen einer ontologisch orientierten überdeterminierten und einer epistemologisch ausgerichteten Herangehensweise resultiert. ${ }^{36}$ Während die Anwendung von Methoden auf die Frage hinauslaufe, welche Regeln und Prozeduren notwendig wären, um wissenschaftliches Wissen zu produzieren, stelle sich bei einer epistemologisch orientierten Forschung die Frage, wie Wissen gewonnen werden könne, das sich kritisch vom »existing system of meaning« unterscheide (ebd.: XIII; vgl. dazu auch Miller/Fox 2004: 51). Er schlägt daher vor, sich dem Forschungsgegenstand nicht über Methoden zu nähern, da sie einen objektivierbaren Standpunkt des Forschenden suggerieren, sondern über analytische Strategien:

»Analytical strategy does not consist of methodological rules but rather of a strategy that addresses how the epistemologist will construct the observations of others $[\ldots]$ to be the object of his own observations in order to describe the space from which he describes. From an epistemological point of view the perspective constructs both the observer and the observed. Hence analytical strategy as a way to stress the deliberate choice and its implications, and to highlight that this choice could be made differently with different implications in respect of the emerging object« (ebd.).

Åkerstrøm Andersen versucht den Brückenschlag von diesem erkenntnistheoretischen Ausgangspunkt zur Forschungspraxis im Rahmen der von ihm behandelten Ansätze ${ }^{37}$ über die Identifikation eines Gegensatzpaares, das einer Art analytischem Hauptwiderspruch des jeweiligen Ansatzes gleichkommt. Daraus leitet er Grundfragen ab, die wiederum in spezifisch gegliederte Fragenkomplexe unterteilt werden können. Zugleich ermöglicht diese Zuspitzung auch eine griffige Unterscheidung der unterschiedlichen gewählten Strategien der jeweiligen Autoren. Bei Foucault unterscheidet Åkerstrøm Andersen, ähnlich wie beispielsweise Reiner Keller (2004: 42ff.), zwischen einer auf Regelmäßigkeit/Streuung des Diskurses abzielenden Archäologie, einer nach Kontinuitäten/Diskontinuitäten fragenden Genealogie, und einer auf Subjektivierung/Subjektivität zielenden Analyse der Techniken des Selbst ${ }^{38}$, wobei diese Analysestrategien nicht als voneinander abgeschlossen und einan-

$36 »$ Whereas ontologically over-determined thinking ontologises the object, epistemology de-ontologises its object « (Åkerstrøm Andersen 2003: XII).

37 Neben jenen von Michel Foucault behandelt Åkerstrøm Andersen die analytischen Strategien von Reinhart Koselleck, Ernesto Laclau und Niklas Luhmann.

38 Die Analyse der Techniken des Selbst hebt Keller (2004) nicht als eigenen analytischen Zugang bei Foucault hervor. 
der gegenüberstehend, sondern als komplementäre Nuancen zu verstehen seien. Ihre Stärke liege vor allem darin, dass »these analytical strategies are always defined in relation to a specific research question « (Åkerstrøm Andersen 2003: 2).

Die Dispositivanalyse, die Åkerstrøm Andersen als vierten Zugang unterscheidet, kann demnach nur als eine komplementäre Strategie konzeptualisiert werden, die archäologische, genealogische und selbstanalytische Elemente verbindet und auf eine Problemstellung richtet, die er im Wechselspiel zwischen strategischer Logik und Apparat, der sie hervorbringt und zugleich von ihnen geformt wird, vermutet (ebd.: 29). Zwar erscheint mir diese Zuspitzung, gerade in Hinblick auf die Komplexität und Heterogenität, die Dispositive auszeichnet, als unglücklich (es gäbe ebenso gute Gründe, das Wechselspiel der sich vollziehenden Macht und einer zielgerichteten strategischen Logik als kritischen Punkt zu destillieren). Dies ist allerdings insofern nebensächlich, als unbestritten bleibt, dass die Analyse von Dispositiven primär auf die ihnen inhärenten strategischen Komponenten zu zielen hat. Sie zu erarbeiten bleibt - so oder so - eine Aufgabe der Erarbeitung von Orientierungspunkten, was Deleuze (1992) als Kartographie umschrieben hat.

Die Wahl des Bildes der Kartographie ist dabei doppelt günstig: nicht nur führt es das (immer in der einen oder anderen Form scheiternde) Unterfangen vor Augen, gegebene empirische Komplexitäten vereinfacht darzustellen. Sie betont auch, dass es bei dieser Darstellung tatsächlich nur um Darstellung geht, und nicht um Interpretation im Sinne einer Suche nach »Eigentlichem «, »Dahinterliegendem«, um irgendeine Art von Essenz, die das Gesagte über das sprechende Subjekt erklären könnte. Vielmehr geht es bei dieser Darstellung darum, jene spezifischen Regelsysteme zu erkunden, deren Modalitäten das Sprechen und Handeln von Subjekten einrahmen, und die sie positionieren. ${ }^{39}$

Spätestens an diesem Punkt muss der besondere Charakter der beiden Dispositive, auf die sich die Untersuchung konzentriert, in die Überlegungen miteinbezogen werden. Ich möchte dies im Rahmen der fünf klassischen Phasen des Forschungsprozesses demonstrieren, wie sie etwa Peter Atteslander (2003: 21ff.) vorschlägt, auch wenn ich die Schwerpunkte der Phasen im Vergleich zu seiner Interpretation zum Teil etwas verschiebe. Auch will ich sie in Form von Problemstellungen begreifen - primär, weil sie meinen Erfahrungen nach nicht in getrennten Phasen durchlaufen werden können, sondern sich in mitunter enger

$39 »$ Discursive formations consist of specific configurations of enonciative modalities, which are seen as types of discoursive activity, each of which has its own subject positions« (Williams 1999: 87). 
Wechselwirkung zueinander bewegen: den Problemstellungen der Frage, der Eingrenzung, der Annäherung, der Analyse und der Darstellung.

(1) Frage. Eine der zentralen Fragestellungen, um die die vorliegende Untersuchung kreist, ist jene nach den sich in den Dispositiven Entwicklung und Sicherheit, im Speziellen im Prozess ihrer Vermengung vollziehenden Rationalitäten des Regierens. Diese Fragestellung setzt einen besonderen Charakter der beiden zu behandelnden Dispositive voraus, der eigentlich - orientieren wir uns am schematischen Ablauf der fünf angesprochenen Phasen im Forschungsprozess - Aussagen trifft, die erst bei einer genaueren Untersuchung der Formationsregeln dieser Dispositive, insbesondere auf Ebene der Strategie, getroffen werden können. Es ist keinesfalls gegeben, dass sich die strategische Komponente eines Dispositivs nach Rationalitäten des Regierens vollziehen muss. Mehr noch: Dieser Charakterzug als Dispositiv des Regierens ist für Entwicklung und Sicherheit auch nur ein Merkmal unter vielen, und es ist keineswegs ein durchgängiges Prinzip (wenngleich auf strategischer Ebene ein dominantes Element).

Im praktischen Ablauf der Untersuchung stand die Entwicklung dieser Fragestellung in einer späten Phase des Forschungsablaufes, keinesfalls bildete sie ihren Beginn. Zu diesem Zeitpunkt waren bereits zwei der vier Feldforschungen durchlaufen, ein Großteil des Materials nicht nur zusammengetragen, sondern bereits ausgewertet. Erst diese umfangreiche Arbeit und die damit einhergehende Konfrontation mit einer Vielzahl unterschiedlicher Abläufe und Praktiken ermöglichte es mir, zu jener Frage vorzudringen, die mir in Bezug auf das gesamte Projekt als interessanteste erschien: wie diese Vielheit zusammengehalten und im Sinne eines Zwecks in Stellung gebracht werden kann.

Die Fragestellung ist also im Kontext meiner Arbeit Resultat wie Voraussetzung der Untersuchung gleichermaßen; Resultat, da ihre Entwicklung ohne die Arbeit an der Thematik überhaupt nicht möglich gewesen wäre, Voraussetzung, da ohne sie die Analyse der Dispositive in ihrem strategischen Charakter abgerissen und hohl bleiben müsste.

(2) Eingrenzung. Das Problem der Eingrenzung besteht immer im Verhältnis der ausgewählten Blickpunkte, die im Rahmen einer Untersuchung behandelt werden, zum Gesamtkontext, in dem die Untersuchung steht. In der Dispositivanalyse zeigt sich dies insbesondere an zwei Eigenschaften, die Dispositiven eigen sind: Zunächst sind Dispositive, allein schon durch die Tatsache, dass sie sich anhand von spezifischen Streuungsmustern von Aussagen und Praxen (re-)konstruieren, auf definitorischer Ebene nicht klar abzugrenzen. Zugleich bilden sie ein eigen- 
tümliches Geflecht, das Definitionen und Eingrenzungen immer zu einer Frage des Blickwinkels werden lässt. So sind beispielsweise Entwicklung und Sicherheit (auch) als Dispositive des Regierens zu verstehen ebenso ist es legitim, das Prinzip der Regierung selbst als Dispositiv zu konzeptualisieren. Es wäre sogar eine gangbare These, den Prozess der Vermengung von Entwicklung und Sicherheit als strategische Implikationen des Dispositivs Regieren in seinen internationalen Wirkungen zu interpretieren.

Neben dem Problem der definitorischen Begrenzung steht auch die komplexe Wirkungskette, die Dispositive entfalten, einer präzisen Eingrenzung entgegen. Es ist de facto unmöglich, die gesamte Palette an Diskursen, Apparaten, Logiken, Selbsttechniken und Praxen einzufangen, die von Dispositiven generiert werden und die sie zugleich charakterisieren. Daher ist es nur möglich, Ausschnitte, Sketches zu beleuchten, deren systematische Darstellung Eindrücke vermitteln kann.

Wenn ich zu Beginn der Arbeit materielle, institutionelle und regionale Eingrenzungen getroffen habe, die sich aus der Zielsetzung der Untersuchung herleiten, will ich damit also nicht den Eindruck erwecken, eine Teilmenge definiert zu haben, aus deren Analyse sich Aussagen auf ein Gesamtes - wie auch immer das nun definiert sei - rückschließen lassen würden. Vielmehr bedeutet diese Eingrenzung nicht nur eine Eingrenzung des Materialkorpus, sondern auch die Begrenzung des Blickes, der auf die Dispositive gelegt wird. Sie ist also letztendlich die Entscheidung über einen bestimmten Ausschnitt, über den Aussagen getroffen werden sollen.

(3) Annäherung. Entgegen einer systematisch strengen Annäherung, die sich etwa aus dem oben vorgestellten Konzept Siegfried Jägers ergeben und auf einer strikten Trennung (und vorangegangenen Definition) von unter anderem textualen, praktischen, institutionellen Elementen basieren würde, empfiehlt sich auch beim Prozess der Materialgewinnung ein integrierter Zugang. Natürlich hat dies klare, auf definitorischer Ebene liegende Gründe: So sind die verschiedenen Elemente eines Dispositivs $\mathrm{zu}$ tief ineinander verstrickt, um eindeutige Abgrenzungen treffen $\mathrm{zu}$ können, womit auch eine säuberliche Aufteilung der Materialien, wie sie für eine durchgängige Systematisierung der Datengewinnung notwendig wäre, gar nicht möglich ist.

Dies ist aber nur ein Teil der Begründung, und nach meinen Erfahrungen im Zuge dieser Arbeit der kleinere. Der hauptsächlich relevante Grund, der zu einem (allerdings durch gewisse Rahmengebungen zu begrenzenden) Pragmatismus nahezu zwingt, liegt in dem, was ich das Prinzip der Unvorhersagbarkeit der Dispositivanalyse nennen würde. 
1966 sagt Foucault, befragt nach dem Problem der Auswahl im »Forschungsfeld« Archäologie: »Es darf keine privilegierte Auswahl geben. Man muss alles lesen, alle Institutionen und Praktiken kennen« (Foucault 2001: 644). Wir können davon ausgehen, dass dieser Grundsatz auch auf die Untersuchung von Dispositiven anzuwenden ist. Allerdings gibt es dabei einen entscheidenden Nachteil: angesichts der unerschöpflichen Menge an Material ist er schlichtweg nicht zu verwirklichen. ${ }^{40}$ Eine strikte Trennung zwischen einer Phase der Datengewinnung und einer darauf folgenden Phase der Analyse ist demgemäß unmöglich. Vielmehr weist sich der Weg zu den wesentlichen Problemstellungen erst im Rahmen des parallelen Durchlaufens beider Phasen, und es ist am Beginn der Untersuchung nicht möglich abzuschätzen, welche Richtung dieser Weg letztendlich nehmen wird. Foucaults Forderung nach allumfassender Kenntnis interpretiere ich in diesem Zusammenhang als die Offenheit, sich auf einen solchen Forschungsprozess einzulassen, mit all den Beschränkungen und auch Mühen, die damit einhergehen.

Die Rahmengebungen, von denen ich vorher gesprochen habe, sind also primär Orientierungshilfen in einem schwer fassbaren Feld - haben also nichts mit seiner Begrenzung oder der Identifikation von Teilfeldern zu tun. Einige dieser Rahmengebungen, jene, die standardisierten und erprobten Verfahren sozialwissenschaftlicher Datengewinnung entsprechen, standen bereits vor Beginn der Arbeit fest und wurden auch nicht mehr verändert: zunächst, als eine Art Grundprinzip, die parallele und sich wechselseitig beeinflussende Untersuchung von die strategischen und politischen Leitkonzepte der Geber betreffenden top-downund den auf Ebene der Implementation bei lokalen Projektarbeiter/innen und lokalen Projektpartnern liegenden bottom-up-Strömungen. ${ }^{41} \mathrm{Die}$ Feldforschungen in den Zielregionen erfolgten also gegenüber der Analyse der zentralen Agenturen weder nachgeordnet noch vorgelagert, sondern parallel. ${ }^{42}$

40 Der Grundsatz des »Alles kennen« ist natürlich auch bei Archäologien im Sinne Foucaults schon allein aus praktischen Gründen (der notwendigen selektiven Beschränktheit des Materials in den Archiven) nicht realisierbar. Offenkundig geht es Foucault hier einerseits um ein idealtypisches Postulat, andererseits aber auch um die Betonung, dass kein Material derart privilegiert behandelt werden kann, dass Rückschlüsse auf andere, weniger privilegierte Materialien gezogen werden.

41 Top-down und bottom-up sind hier nicht als klar abgrenzbare Kategorien zu verstehen, die objektiv gegeben wären. Sie sind lediglich Tendenzen, mit denen zu arbeiten sich grundsätzlich als praktikabel erwiesen hat.

42 Eine solche Vorgangsweise korrespondiert auch mit einem Zugang, den Foucault der Archäologie zugrunde legt und als »Forschungsstil« bezeichnet. Ein solcher Stil sei für ihn deswegen interessant, »weil dabei das 
In Bezug auf die Erhebungstechniken standen vor Untersuchungsbeginn drei Vorgangsweisen fest: erstens eine Vollerhebung aller strategisch und programmatisch relevanten Texte der zu untersuchenden Geber, zweitens die Beschränkung der sozial interaktiven Datengewinnung auf semistrukturierte Schlüsselinterviews mit Expert/innen ${ }^{43}$ und drittens die Durchführung von Feldforschungen, zunächst primär aus dem Grund, um die konkret relevanten Projekte der Geberagenturen in ihrem unmittelbaren Umfeld zu untersuchen.

Angesichts der Tatsache, dass sich die leitenden Forschungsfragen zum Teil erst im Zuge der Untersuchung ergaben, haben sich diese Orientierungen als beschränkt erwiesen. Zunächst wurde sichtbar, dass gerade die diversen Schnittstellen oder, besser formuliert, Übergänge zwischen dem, was ich zuvor als top-down- und bottom-up-Strömung definiert habe, zu den in Hinblick auf die Herausarbeitung der Regulative der Dispositive vielleicht interessantesten Bereichen gehören. Auf Ebene des Theorie-Praxis-Verhältnisses war dies zu erwarten und auch gut herauszuarbeiten. Allerdings liegt der besondere Spannungsbereich, speziell im Hinblick auf die in den Dispositiven wirkenden Rationalitäten, auf subjektiver Ebene, weil hier die Selbsttechniken, die eine wesentliche Komponente der Wirksamkeit von Dispositiven ausmachen, besonders deutlich zum Tragen kommen.

Eine zweite, allerdings besser behebbare Beschränkung meiner ursprünglichen Annahmen lag in der Unterschätzung der historischen Verknüpfungen. Ohne die Verarbeitung speziell der kolonialen Geschichte der Zielregionen muss das Verständnis der heute wirksamen Rationalitäten abgerissen wirken.

Drittens - und das ist der Faktor, der einer Anwendung standardisierter methodischer Verfahren wahrscheinlich am gewichtigsten entge-

Problem vermieden werden kann, ob die Theorie der Praxis vorausgegangen ist oder umgekehrt. Ich behandle Praktiken, Institutionen und Theorien auf derselben Ebene nach ihren jeweiligen Isomorphien und suche das gemeinsame Wissen, das sie möglich gemacht hat, die Schicht des konstitutiven historischen Wissens« (Foucault 2001: 645).

43 Die Auswahl der Expert/innen konzentrierte sich auf drei Gruppen: Erstens Entscheidungsträger/innen auf politischer (in den jeweiligen Ministerien) wie technischer Ebene (etwa Büroleiter/innen der Agenturen oder der Counterparts), in den Geberländern wie in den Zielregionen; zweitens Personal, das mit der Durchführung der entwicklungspolitischen Maßnahmen, in Konzeption wie Umsetzung betraut ist, sei es auf Ebene der ODAAgenturen, sei es auf Ebene der Projektpartner. Die dritte Gruppe schließlich bildeten Expert/innen aus dem wissenschaftlichen Feld, in den Geberländern (primär Entwicklungsforscher/innen, um die Ansätze der Geber zu durchleuchten) und in den Zielregionen, um den Prozess der Intervention in einen breiten Kontext einbetten zu können. 
gensteht - ist die überragende Bedeutung hervorzuheben, die den in wissenschaftlichen Darlegungen schwer fassbaren Gesamteindrücken zukommt, insbesondere im Rahmen der Feldforschungen in den Zielregionen entwicklungs- und sicherheitspolitischer Interventionen. Ich möchte an dieser Stelle nicht den Mythos »Feld» bemühen, wie er in manchen Ausprägungen der Sozialanthropologie zu finden ist. Ohne Zweifel ist unbestreitbar, dass allein aus einem »being there« kein Automatismus einer besonderen Kompetenz folgt. Dennoch ist ebenso unbestreitbar, dass die mit dem Aufenthalt und Reisen im Feld verbundenen Begegnungen und Eindrücke vielleicht sogar mehr Einfluss auf den Charakter einer Arbeit haben als die Absolvierung des eigentlichen Feldforschungsprogramms.

Zusammenfassend lässt sich also sagen, dass das Problem der Annäherung nicht optimal gelöst werden konnte. Dennoch glaube ich nicht, dass die sich daraus ergebenden Einschränkungen ein schwerwiegendes Problem darstellen. Schließlich geht es in meinem Verständnis qualitativer sozialwissenschaftlicher Forschung nicht um objektivierte Ergebnisse, sondern um die systematische Erarbeitung kritischer Perspektivität.

(4) Analyse. Die Frage nach dem »Wie« der Untersuchung stellt sich auf zweierlei Art. Einerseits gilt es natürlich, ein Gerüst zu konstruieren, das die systematische Bearbeitung der Materialien erlaubt. Andererseits müssen aber auch die Erwartungen an dieses Gerüst abgeklärt werden, es muss die grundsätzliche Frage gestellt werden, was es leisten kann und was nicht. Auf diesen zweiten Punkt möchte ich zunächst kurz eingehen.

Aus dem bisher Gesagten lassen sich jene zwei Rahmenbedingungen, die mir als die wesentlichsten erscheinen, bereits herauslesen. Beide betreffen die Form, mit der uns die Untersuchungsmaterie konfrontiert: erstens ihr Charakter der Zusammensetzung als Häufigkeit in der Streuung von Aussagen im Raum, und zweitens die vielfältige Beschaffenheit (als Diskurse, Praxen, Techniken, Baulichkeiten, Institutionen, Apparate) jener Aussagen selbst. Beide Rahmenbedingungen widersprechen den grundlegenden Anforderungen standardisierter analytischer Verfahren, die nicht nur eine zumindest tendenziell eindeutige Definierbarkeit der Untersuchungsmaterie benötigen, sondern auch eine weitgehende Homogenität des Materials. 
Während sich das erste Problem noch mit der inneren Dynamik eines Forschungsprozesses lösen lassen würde ${ }^{44}$, schließt das zweite Problem jede Art der Anwendung von Verfahren zur standardisierten Datenverarbeitung und -vereinfachung aus. ${ }^{45}$ Daraus ergibt sich, dass die Anforderungen an ein analytisches Gerüst prinzipiell beschränkt sein müssen, und auch nur beschränkte Wirksamkeit entfalten können. Mit dem Begriff von Deleuze kann der Prozess der Abbildung dispositiver Regelsysteme wie erwähnt als Kartographie gefasst werden, wobei diese Karte keine Kopie sein dürfe: „Die Karte ist das Gegenteil einer Kopie, weil sie ganz und gar auf ein Experimentieren als Eingriff in die Wirklichkeit orientiert ist. Die Karte reproduziert kein in sich geschlossenes Unbewusstes, sie konstruiert es« (Deleuze/Guattari 1997: 23f.).

So verstanden, verschließt sich der Raum seiner Erfassung. Bei der Erstellung eines analytischen Gerüstes kann es also um nicht mehr gehen als um grobe Orientierungshilfen im komplexen Raum, vergleichbar einem Kompass und verschiedener dafür geeigneter Anwendungserfahrungen. So gerüstet mag ein Weg durch den Raum in Blick auf jene Zielorientierung gelingen, die vorgegeben ist, aber weder wird dieser Weg der einzig mögliche sein, noch wird er uns einen umfassenden Blick auf den Raum gewährleisten können. Wir bewegen uns also gewissermaßen in einem Extrem sozialwissenschaftlicher Forschung, denn die größte Schwäche der analytischen Strategie ist ihre starke Abhängigkeit vom forschenden Subjekt. Genau darin liegt vielleicht aber auch ihre größte Stärke.

Wie lässt sich nun ein derartiges Gerüst gestalten? Ähnlich wie Åkerstrøm Andersen möchte ich vorschlagen, die Analyse von Dispositiven als komplementären Prozess unterschiedlicher analytischer Stränge zu fassen. Allerdings verstehe ich diese Stränge - im Unterschied zu Åkerstrøm Andersen - als Elemente einer analytischen Strategie, und interpretiere sie zudem formal nicht gleichartig. Ich möchte vorschlagen, zwischen vier derartigen Elementen zu unterscheiden: die archäologische Strategie, der genealogische Blick, die Frage nach den Techniken und das strategische Prinzip.

44 Die von mir anfangs eingeführten Definitionen der Untersuchungsmaterie sind ein Beispiel für einen diesem Problem entsprechenden pragmatischen Lösungsversuch.

45 Hier handelt es sich augenscheinlich nur um eine Begründung der »technischen« Unmöglichkeit. Die methodologischen Aspekte, die eine Anwendung jeder Art von Vereinfachungsverfahren bei der Analyse von Dispositiven (oder auch Diskursen) verbietet, ergibt sich aus ihrer rhizomatischen Form. »[E]in Rhizom lässt sich keinem strukturalen oder generativen Modell zuordnen. Jede Vorstellung einer genetischen Achse oder Tiefenstruktur ist ihm fremd« (Deleuze/Guattari 1997: 23). 
(a) Die archäologische Strategie beruht auf der Idee, jene vier Fragenkomplexe, die Foucault (1981: 48ff.) für die Untersuchung der Formationsregeln ${ }^{46}$ von diskursiven Formationen vorschlägt, auf Dispositive umzulegen. Die Möglichkeit für eine derartige Umlegung sehe ich insofern gegeben, als es gute Gründe gibt, jene Hypothesen, die Foucault zur Gestalt diskursiver Formationen annimmt (ebd.: 49ff.) ebenso auf Dispositive anzuwenden. Denn letztendlich ist auch das Dispositiv als »eine Gesamtheit« zu verstehen, die von »in ihrer Form verschiedenen, in der Zeit verstreuten Aussagen« gebildet wird, »wenn sie sich auf ein und dasselbe Objekt beziehen« (ebd.: 49). ${ }^{47}$

Ich bezeichne die Archäologie insofern als Strategie, als sie jene Fragelinien vereint, die sich durch den gesamten Ansatz der Dispositivanalyse durchziehen: die Fragen nach dem Gegenstand, nach den Modalitäten, nach den Begriffen und nach den Strategien. $\mathrm{Zu}$ den ersten drei Fragelinien hat Åkerstrøm Andersen (2003: 14f.) konkrete Vorgangsweisen ausgearbeitet, die Foucaults Überlegungen spezifizieren. So richtet sich das Problem der Formation der Gegenstände auf die Häufigkeiten und Verteilungen von Aussagen, die die Abgrenzung des Objekts erst möglich machen, sowie nach den grundsätzlichen Regeln ihres Auftretens und ihren Beziehungen und Hierarchien untereinander. Die Formation der Modalitäten von Äußerungen (Åkerstrøm Andersen bezeichnet diese Ebene als Formation des Subjekts) richtet sich nach der Subjektposition, und darin - insbesondere für unseren Fall relevant - nach den institutionellen Plätzen, von denen aus gesprochen wird. Die Frage nach der Formation der Begriffe untersucht den Zusammenhang zwischen Aussagen und Konzepten und den Regelsystemen, im Rahmen derer sich dieser Zusammenhang gestaltet.

Die vierte Fragelinie, die der Strategien, hat nun bei der Untersuchung von Dispositiven, die sich ja entscheidend entlang ihres strategischen Charakters definieren, besondere Bedeutung. Es empfiehlt sich daher, Strategie nicht gleich den drei bislang erwähnten Fragelinien zu verstehen, sondern als Prinzip zu definieren, das entlang seiner prakti-

$46 »$ Man wird Formationsregeln die Bedingungen nennen, denen die Elemente dieser Verteilung unterworfen sind (Gegenstände, Äußerungsmodalität, Begriffe, thematische Wahl)« (Foucault 1981: 58).

47 Diese sehr allgemein gehaltene Hypothese zu den diskursiven Formationen, die Foucault im expliziten Zusammenhang mit der Archäologie trifft, ist auch der Hauptgrund dafür, warum ich nicht der Meinung bin, dass bei Foucaults Arbeitsweisen im Laufe der Zeit von unterschiedlichen analytischen Strategien ausgegangen werden kann, die distinkt zu unterscheiden wären. Eine Interpretation der Entwicklung der Foucaultschen Arbeitsweisen im Sinne zunehmender Differenzierung und Akzentuierung erscheint mir stimmiger. 
schen Konsequenzen als viertes Element neben Archäologie, Genealogie und der Technikenfrage in allen analytischen Prozessen zu beachten ist.

(b) Ich bezeichne Genealogie ${ }^{48}$ insofern als Blick, da sie sich weniger durch speziell zu stellende Fragestellungen auszeichnet denn durch eine spezifische historische Herangehensweise: „Genealogy is a history of the present designed to outline the historical conflicts and strategies of control by which knowledge and discourses are constituted and operate, and to use these descriptions as a counter-memory« (Åkerstrøm Andersen 2003: 19).

Es handelt sich hier um eine besondere Form der Geschichtsschreibung, in doppeltem Sinne: gebrochen und destruktiv. Genealogie ist gebrochene Geschichte, weil sie sich nicht in der Suche nach mythenstiftenden Kontinuitäten ergeht, sondern die Frage nach den Brüchen, den Diskontinuitäten $^{49}$, den Besonderheiten in den Mittelpunkt stellt. Dies bedeutet konsequenter Weise natürlich auch das Ende der Möglichkeit von globaler oder nationaler Geschichtsschreibung, womit sich der Übergang zum destruktiven, verräterischen, oder aber auch emanzipatorischen Charakter nahezu von selbst ergibt. Denn der Zweck des genealogischen Blickes im Rahmen einer analytischen Strategie erfüllt sich im Drang nach einer dreifachen Zerstörung (vgl. ebd.): der Zerstörung der Realität als jener Art und Weise, wie sich die Gegenwart aus ihrer Geschichte heraus konstruiert; der Zerstörung der Identität als Zerstörung der historischen Motiv- und Mythenbildung; und der Zerstörung von Wahrheit in Opposition zum historischen Prozess der Begründung von Wissen.

Es wird vor diesem Hintergrund deutlicher, warum ich Genealogie nicht als eigene analytische Strategie, sondern als Blick verstehen will. Es geht um eine grundsätzliche Haltung des erkennenden Subjekts zum Gegenstand seiner oder ihrer Forschung. Diese Haltung, gekennzeichnet durch den Mut zum Bruch und dem Willen zur Destruktion, ist allerdings, wie oben erwähnt, kein Selbstzweck. Es ist eine notwendige Operation, um Raum für jene Formen von Wissen freizulegen, die durch das Projekt Geschichtsschreibung verdeckt sind.

(c) Die Frage nach den Techniken hervorzuheben erfüllt primär die Aufgabe der Bewusstmachung. Denn natürlich ist die Frage nach den Praktiken in den Fragelinien enthalten, die die archäologische Analyse auszeichnen. Allerdings ist die Frage nach den Techniken mehr als eine simple Kombination zwischen Theorie und Praxis, Konzept und Maß-

48 Die Verwendung des Begriffes »Genealogie« erfolgt hier strikt in dem von Foucault verwendeten Sinn (vgl. Foucault 2002c).

49 »Die Diskontinuität war jenes Stigma der zeitlichen Verzettelung, die der Historiker aus der Geschichte verbannen musste« (Foucault 1981: 17). 
nahme. Vielmehr geht es um jene sich im Rahmen der strategischen Wirkungen eines Dispositivs vollziehenden Prozesse, die die Entstehung jener Theorien und Praxen, Konzepte und Maßnahmen überhaupt erst ermöglichen, indem sie die auf subjektiver Ebene liegenden Bedingungen dafür bereitstellen.

Foucault hat diese Prozesse an ihrem zugespitzten Punkt - den Techniken des Selbst - untersucht, genauso lässt sich die Frage aber auch nach den Techniken des Anderen stellen. ${ }^{50}$ Wie werden Menschen dazu gebracht, gemäß den strategischen Bedürfnissen eines Dispositivs zu handeln, oder aber, wie generiert sich das strategische Bedürfnis eines Dispositivs aus den Handlungen (im formalen Sinne: Äußerungen), die in seinem Rahmen gesetzt werden? Diese Frage etabliert das Feld der Techniken.

(d) Das strategische Prinzip ist konstitutiv für die Existenz von Dispositiven, denn es ist entscheidend für den Prozess der Selektion. Welche Aussagen - Texte, Reden, Maßnahmen, Praxen, Institutionen, Baulichkeiten - getroffen werden können, entscheidet sich nach dem im Dispositiv liegenden strategischen Gehalt. Damit ist die Strategie Ausdruck der zeitlichen und räumlichen Verortung jener Aussagen: »[A Statement] is always strategic in the sense that the statement as reactualisation emerges as one choice among other possible actualisations. Consequently, it cannot easily be displaced in time and space« (Åkerstrøm Andersen 2003: 12). Aus der Streuung der Aussagen die Kriterien jener Auswahlprozesse offen zu legen ist somit die erste Aufgabenstellung, die sich aus dem strategischen Prinzip der Dispositivanalyse ableiten lässt.

Die Selektion der Aussagen und die damit einhergehende strategische Ausgestaltung eines Dispositivs sind nun aber ohne Zweifel Prozesse, die unmittelbar im Rahmen eines gesellschaftlichen Machtgefüges wirken. Sie sind also Modalitäten von Macht, womit es unumgänglich ist, die Analyse von Dispositiven mit einer Machtanalyse zu verknüpfen. Allerdings ist das in zweierlei Hinsicht kein leichtes Unterfangen: Einerseits ist der Zusammenhang von Dispositiven als einen strategischen Zweck erfüllende Konstruktionen und Macht schwierig zu konzeptionalisieren. Eine solche Konzeptionalisierung ist in erster Linie eine Frage

50 Die in der gegenwärtigen Foucault-Rezeption gängige Praxis, die Analyse der Selbsttechniken als besonderes Element herauszuarbeiten, teile ich nur insoweit, als damit keine Abstrahierung von den Techniken des Anderen erfolgt. Dass sich Foucault in seinen späten Arbeiten vor allem mit der Ebene des Selbst auseinandersetzt, interpretiere ich weniger als methodischen Vorschlag denn als Frage des persönlichen Interesses und einer damit einhergehenden Akzentuierung. 
der eigenen Orientierung und daraus folgenden Positionierung, denn es stellt das erkennende Subjekt vor die grundsätzliche Frage, ob die »geschichtlichen Kämpfe« oder die »Machtbeziehungen und Machtdispositive« in den Mittelpunkt der Analyse gerückt werden sollen. »Dabei begegnen wir nicht denselben Bedeutungselementen, nicht denselben Verkettungen und auch nicht denselben Arten von Intelligibilität, obwohl sie auf dasselbe geschichtliche Gewebe verweisen und beide Analysen sich aufeinander beziehen müssen« (Foucault 2005b: 293).

Es ist offenkundig, dass sich vorliegende Arbeit im Sinne dieser Unterteilung auf die Untersuchung der Machtdispositive konzentriert und die geschichtlichen Kämpfe als notwendigen Kontext mitanalysiert. Es geht also nicht darum, die Geschichte von Kämpfen zu schreiben, die gegen jene Machtdispositive geführt worden sind oder noch geführt werden, wenngleich sehr wohl sichtbar werden soll, dass diese Machtdispositive (und ohne Zweifel sind Sicherheit und Entwicklung zwei Schlüsselbeispiele dafür) nicht ohne Kämpfe und Widerstandsprozesse $\mathrm{zu}$ verstehen sind, auf die sie eben strategische Antworten zu geben hätten. Es war demnach auch naheliegend, dass ich als Fallbeispiel für die Untersuchung eine Region gewählt habe, die durch solche Kämpfe und Widerstandsprozesse charakterisiert ist. ${ }^{51}$

Wo aber liegen nun mögliche Ansatzpunkte für eine Analyse? Ich habe zuvor zwischen zwei Wirkungsweisen von Dispositiven unterschieden, jenen erster Ordnung, die unmittelbar der jeweiligen strategischen Intentionalität entsprechen, und jenen zweiter Ordnung, die sich entlang der strategischen Interessen entfalten, obwohl sie sich den expliziten Zielsetzungen der Dispositive verweigern oder sich ihnen gar widersetzen. Diese beiden Arten von Wirkung bilden jene Wirkungskette, die ich als den konkreten Zusammenhang zwischen Machtverhältnis und Dispositiv verstehen würde. Auf sie muss daher das Augenmerk gerichtet werden.

Zugleich bleibt bei einer Klärung dieses Zusammenhangs das Problem, dass die Analyse von Macht selbst eine komplexe Herausforderung darstellt. Ausgehen können wir dabei von der Grundannahme, dass sich

51 Ich möchte diese Formulierungen definitiv nicht als Wertungen verstanden wissen, etwa im Sinne, dass sich solche lokale Kämpfe subjektiv oder selbst objektiv gegen eine herrschende Weltordnung richten würden. Schon gar nicht will ich das Bild eines globalen Kampfes gegen das System bemühen, was im Zusammenhang mit den von mir untersuchten Beispielen ohnehin vollkommen absurd wirken würde. Dennoch sind diese Kampfprozesse Fehlfunktionen in der globalen Machtstruktur, die Foucault (2005: 293) als »Herrschaft« definiert. Folgerichtig benötigt es strategische Antworten, ein Bedürfnis, in dessen Rahmen Dispositive ihre Funktion einnehmen. 
Macht in Dispositiven materialisiert, was folgerichtig bedeutet, dass durch die Analyse der Dispositive Elemente der darin wirkenden Machtbeziehungen offen gelegt werden können. Dieser Prozess bleibt dennoch problematisch, denn woher soll klar sein, wonach eigentlich gesucht wird? Oder umgekehrt formuliert, ist nicht eine solche Vorgangsweise dazu verdammt, an den wesentlichen Punkten vorbeizugehen, wenn diese nicht vorher klar gelegt sind?

Diese Fragen sind in der Tat schwierig, denn sie zwingen letztendlich dazu, das zu tun, was - wie ich gerade erwähnt habe - in einer Dispositivanalyse eigentlich nie getan werden sollte: Verhältnisse, Dinge anzunehmen, erfahrungswissenschaftlich und aus den ersten Ergebnissen des Forschungsprozesses, ohne sie streng aus dem gewonnenen empirischen Material abzuleiten. Ohne grundsätzliche Annahmen über internationale Konstellationen heranzuziehen, wie ich es mitunter mit Rückgriffen auf das Instrumentarium der Weltsystemansätze tue, lässt sich die Gestalt global wirksamer Dispositive, die im Rahmen dessen wirken, was Foucault als Herrschaft definiert hat, schwerlich untersuchen.

Welche Komponenten sind für eine solche Analyse der Machtbeziehungen, die vor dem Hintergrund dieser allgemeinen Annahmen im Rahmen der Dispositivanalyse erfolgen soll, nun wesentlich? In einer 1982 gehaltenen Vorlesung, die die Frage des Zusammenhangs zwischen Subjekt und Macht diskutiert, benennt Michel Foucault (2005b: 289f.) fünf konkrete Punkte, die er als notwendiger Weise zu klären ansieht: Neben dem »System der Differenzierungen«, das im Wesentlichen auf die Frage der Verortung von Aussagen zurückgeht, die ich oben diskutiert habe, stehen hier »[d]ie Art der Ziele«, »[d]ie instrumentellen Modalitäten«, »[d]ie Formen der Institutionalisierung« und »[d]er Grad der Rationalisierung«. Wir haben es also im Wesentlichen mit zwei zusätzlichen Feldern zu tun, die in die Analyse mit einbezogen werden müssen: die Frage der Ziele, Leitbilder, Rationalitäten, und jene Prozesse, die Åkerstrøm Andersen unter dem Begriff »Apparatisierung« als den zentralen Problemkontext von Dispositiven zusammengefasst hat und der ein wesentliches Element des strategischen Prinzips ausmacht.

In Dispositiven des Regierens, und als solche habe ich Entwicklung und Sicherheit interpretiert, konkretisiert sich die Suche nach den Zielsetzungen in jenen Rationalitäten, die das in den Dispositiven wirkende Prinzip des Regierens bestimmen - womit deutlich wird, dass ich diese Frage zur eigentlichen Hauptfragestellung der Untersuchung gemacht habe. Angesichts der Tatsache, dass die theoretischen Hintergründe bereits diskutiert wurden, bleibt das konkrete Problem, wie diese sich in den Rationalitäten des Regierens findenden Zielsetzungen nun aus den 
archäologischen Arbeiten im Dispositiv herausgearbeitet werden können.

Der Schlüssel zur Lösung dieser Aufgabenstellung liegt in der konsequenten Umsetzung der Verbindung von archäologischer Strategie und strategischem Prinzip, die sich in zwei konkreten Anforderungen fassen lässt: Das Material in Hinblick auf die Frage der Rationalitäten lesen oder verarbeiten und vergleichen, sowie die Ordnung des Materials an den daraus ableitbaren Zielsetzungen systematisieren. Das mag zwar letztlich nach nicht mehr klingen als einem relativ beliebigen Postulat, ich glaube aber dennoch, dass diesen beiden Anforderungen ein wesentlicher Anteil am Gelingen meines Unterfangens zukommt. Letzten Endes wird das Ergebnis zeigen, ob dieser Grad an Konkretisierung genügt hat oder ob vielleicht doch die Erarbeitung stärker formalisierter Systematiken der Analyse notwendig ist.

Schließlich fehlt noch der schon zuvor erwähnte Vorgang der Apparatisierung/Institutionalisierung, um das strategische Prinzip der Analyse abrunden zu können. Es ist damit nicht nur die Frage nach konkreten Instrumenten gemeint, auch nicht nur die Frage nach dem Prozess der institutionellen Integration von durch das Dispositiv bestimmten Praktiken, beziehungsweise der Schaffung solcher Institutionen durch eben diese Dispositive. ${ }^{52}$ Solche Fragestellungen würden sich aus der konsequenten Anwendung der archäologischen Analysestrategie ergeben.

Wenn hier also ein spezieller Verweis, eine Bewusstmachung der Einbeziehung der Apparatisierung/Institutionalisierung als Teil des strategischen Charakters der Dispositive erfolgt, hat das den Grund, auf genau diese strategische Funktionalität von Institutionalisierungsprozessen und Apparatisierungen hinzuweisen. Es reicht nicht, zu erkennen, dass sich von Dispositiven getragene Praktiken institutionalisieren - es muss vielmehr das Verhältnis untersucht werden, in dem solche Institutionalisierungsprozesse mit den strategischen Interessen, also den sich in den Rationalitäten des Regierens findenden Zielsetzungen stehen, welche Funktionen sie erfüllen, aber auch, wie sie diesen Zielsetzungen mitunter entgegenlaufen.

Die Verortung der Aussagen, der Machtkontext und die Apparatisierung/Institutionalisierung bilden die drei wesentlichen Komponenten des strategischen Prinzips bei der Analyse. Speziell hier ist es allerdings unabdingbar, den genealogischen Blick streng zu beherzigen, denn gerade bei der Suche nach Strategien ergibt sich eine speziell für das polito-

52 Es ist naheliegend, dass auch Institutionalisierung an sich als Dispositiv verstanden werden kann, auf dessen Praktiken im Rahmen der Dispositive von Entwicklung und Sicherheit immer wieder zurückgegriffen wird. 
logische Auge als klassisch zu bezeichnende Falle: die teleologische Versuchung. Der strategische Charakter eines Dispositivs ist nur dann sinnvoll zu bearbeiten, wenn er im Forschungsprozess aus dem Konkreten entwickelt wird.

Nur allzu oft suchen Studien, gerade im Bereich der Governmentality Studies, mit foucaultschen Begriffen gerüstet nach Bestätigungen von Unterstellungen, die bereits am Beginn feststehen und an denen nicht mehr gerüttelt wird. Solche Unterstellungen sind gefährlich, denn sie beeinflussen gerade in derart komplexen Konstruktionen wie Dispositiven nicht nur den Prozess der Analyse - sie determinieren faktisch den gesamten Forschungsprozess, der so in die Rolle des Bestätigens vorher angenommenen Wissens gerät. Und Forschende tendieren zumeist dazu, zu finden, was sie suchen. Wenn ich daher zuvor bereits Hypothesen über den Charakter der hier zur Disposition stehenden Dispositive angenommen und der weiteren Arbeit zugrunde gelegt habe ist zu beachten, dass diese nicht am Anfang der Arbeit als solches standen, sondern erst im Laufe der Arbeit entwickelt worden sind. Sie sind damit genauso Resultat wie Ausgangspunkt vorliegender Untersuchung.

(5) Darstellung. Die Darstellung ist insofern verhältnismäßig einfach zu lösen, als es hier im Prinzip nur um einen reproduzierenden Prozess geht: »Sie [die Archäologie] ist nicht mehr und nicht weniger als eine erneute Schreibung: das heißt in der aufrecht erhaltenen Form der Äußerlichkeit eine regulierte Transformation dessen, was bereits geschrieben worden ist« (Foucault 1981: 200). Es geht also um nicht mehr, aber auch nicht weniger als um einen gemäß den vier Elementen der analytischen Strategie regulierten Prozess der erneuten Schreibung.

Wie im Folgenden sichtbar wird, habe ich in der Umsetzung eine klassische Form gewählt: die Aufsplittung nach Akteuren und Schauplätzen. Dies ist nicht nur eine Lösung, die der besseren Übersichtlichkeit entgegenkommt. Sie bezweckt zudem die Veranschaulichung der extremen Heterogenität der Kontexte, die Schauplätze von Entwicklungspolitik und -praxis sind. Dennoch darf eines nicht vergessen werden: es geht bei der Darlegung eines »Länderbeispiels« nicht um Untersuchungen über ein Land und eine Wirkungsanalyse der dort erfolgten und erfolgenden sicherheits- und entwicklungspolitischen Interventionen (auch wenn sich Elemente davon ohne Zweifel wiederfinden lassen). Es geht um die Darstellung der Dispositive im komplexen Kontext ihrer praktischen Ausprägungen, und darin primär um die Rationalitäten, die den Handlungen der untersuchten entwicklungspolitischen Akteure zugrunde liegen. 
Die Geschichten, die im empirischen Teil erzählt werden, handeln also weniger von Sri Lanka als von den Wahrnehmungen und Praxen der dort Intervenierenden und den strategischen Wirkungen, die die Dispositive von Entwicklung und Sicherheit im konkreten Prozess ihrer Vermengung lokal entfalten. Aber selbst diese Geschichten werden alles andere als ein einheitliches Bild präsentieren können. 



\section{Das neue Sicherheitsproblem von Entwicklung}

\footnotetext{
»The constant articulation of danger through foreign policy is thus not a threat to a state's identity or existence: it is its condition of possibility. While the objects of concern change over time, the techniques and exclusions by which those objects are constituted as dangers

persist. «

(David Campbell)
}

In der Einleitung wurde die Annäherung zwischen Entwicklungs- und Sicherheitspolitik vor allem mit Blick auf die Ereignisse in den 1990er Jahren mit dem vagen Begriff der »Veränderung« bezeichnet, um dann später im Anschluss an vorwiegend theoretische Arbeiten von Michael Dillon und Mark Duffield eine tendenzielle Vermengung von Sicherheits- und Entwicklungsdispositiv zu behaupten. Eine solche hätte naturgemäß weitreichende Auswirkungen und ginge in ihrer Substanz weit über eine schlichte $»$ Veränderung « hinaus, schon allein, weil damit auch für die gesamte Konfiguration von internationaler Herrschaft schwerwiegende Konsequenzen gegeben wären.

Die Diskrepanz zwischen diesen beiden Zugängen, dem Zugang über konkrete Interventionen einerseits und das theoretische Verständnis der dahinter liegenden Prozesse andererseits, ist das Spannungsfeld, in dem sich die vorliegende Arbeit bewegt. Ich will dabei der empirischen Annäherung an das Problem die eindeutige Priorität zuweisen. Daher soll der folgende Teil erste Klärungen hinsichtlich der konkreten Entstehung der jetzigen Situation hervorarbeiten, insbesondere in Bezug auf die Theoriegeschichte, deren praktische Auswirkungen im Feld der 
Entwicklungspolitik immer schon stark spürbar wurden. Wenn seit Mitte der 1990er Jahre vom Sicherheitskomplex als der schon angesprochenen neuen Aufgabenstellung von Entwicklungspolitik die Rede sein kann, müssen im Vorlauf zwei Bedingungen erfüllt worden sein: erstens impliziert diese Aussage, dass eine sicherheitspolitische Ausrichtung zuvor, zumindest auf Ebene der Implementierung, keine bewusst angestrebte Zielbestimmung gewesen ist, und zweitens müssen offenbar verschiedene Prozesse dazu geführt haben, dass dieses Manko jetzt als Problem identifiziert werden kann.

Das wirft eine Reihe von Fragen auf: Wo liegt das potenziell Neue dieser Entwicklung? Woran schließt es an? Warum ist es entstanden, wie kann es bewertet und eingeordnet werden? Zunächst will ich dazu die Anknüpfungspunkte herausarbeiten, die in der Geschichte der Entwicklungspolitik, speziell im Feld der mit ihr verbundenen Theorieentwicklung, zu finden sind. Danach werde ich mich der Suche nach den Ursachen in einem stärker sicherheitspolitischen Bereich widmen, der Konfliktanalyse. Dabei will ich die im entwicklungs- und sicherheitspolitischen Diskurs vernehmbaren Anfangsgründe, die über die Behauptung eines neuartigen Bedrohungsszenarios laufen, mit Erkenntnissen konfrontieren, die sich aus einer historischen Betrachtung des wissenschaftlichen Diskurses zum Thema »bewaffneter Konflikt« ergeben.

\section{Entwicklung und Sicherheit im Blick der Theoriegeschichte}

Es mutet seltsam an, wenn Andreas Boeckh 1993 ausgerechnet im »Handbuch der Dritten Welt« (Nohlen/Nuscheler 1993) vorschlägt, »Rückschau« auf die Entwicklungstheorien zu halten. Denn obwohl er von einem »Scheitern der Entwicklungstheorien an der Komplexität ihres Gegenstandes« (Boeckh 1993: 125) spricht, ist für ihn trotzdem eines klar: Entwicklung an sich braucht es natürlich schon. Jene neue Bescheidenheit, die er einfordert und die $\gg$ zum dauerhaften Bestandteil der Wissenschaftskultur« im Bereich der Entwicklungsforschung werden solle (ebd.: 126), bezieht sich also nicht auf die metatheoretische Ebene.

Obwohl dies keinesfalls überraschend ist, ist es dennoch - oder vielleicht sogar umso mehr - wert, diskutiert zu werden. Wie bereits erwähnt, wäre es angesichts der (gemessen an seinen explizit formulierten Zielbestimmungen und Maßstäben) chronischen Erfolglosigkeit der bis zu diesem Zeitpunkt vier Dekaden entwicklungspolitischer Praxis eigentlich naheliegend, das Projekt Entwicklung ad acta zu legen. Dennoch hat nur eine kleine Minderheit diesen Weg eingeschlagen. Offen- 
kundig zeichnet sich das Entwicklungsdispositiv, im Rahmen dessen sich der Diskurs bewegt, durch eine hohe Integrationsfähigkeit aus. Speziell die Wirkungsmechanismen zweiter Ordnung, an denen sich diese Integrationsfähigkeit angesichts des sich parallel vollziehenden Scheiterns an den postulierten Zielbestimmungen festmachen lässt, scheint beeindruckendes Potenzial entfalten zu können.

Wie ist es möglich, von einem Scheitern der Entwicklungstheorien zu sprechen, und gleichzeitig der Metatheorie von Entwicklung volle Gültigkeit zuzusprechen? Eine umfassende Antwort auf diese Frage ist schwer möglich, würde sie doch die umfassende Untersuchung des Entwicklungsdispositivs, nicht nur in seiner historischen und thematischen Entfaltung, sondern auch in seinen zahlreichen Überschneidungsflächen mit anderen Machtdispositiven voraussetzen. Ich will im Folgenden vielmehr argumentieren, dass die Überschneidung mit dem Komplex Sicherheit ein potenzieller Weg ist, im Rahmen dessen das Entwicklungsdispositiv eine drohende substanzielle Krise zu überwinden sucht.

Dazu ist es zunächst empfehlenswert, sich den historischen Rahmenbedingungen zuzuwenden, die es überhaupt ermöglichen, von einem Ende der Entwicklungstheorien zu sprechen. Ich möchte dazu den Untersuchungszeitraum eng halten, da es im Rahmen dieser Untersuchung zu viel wäre, die Wurzeln der Entwicklungstheorie im Kolonialismus, in den grundlegenden Werken der Moderne, oder gar vermittelt durch den Fortschrittsbegriff in noch früheren Epochen herauszuarbeiten. ${ }^{1}$ Der Schwerpunkt liegt also auf der Periode nach Ende des Zweiten Weltkrieges, als mit dem Marshall-Plan das klassische Zeitalter der auf Modernisierung gerichteten Entwicklung eingeläutet wird.

Der Beginn der nachkolonialen Neukonfiguration des interventionistischen Projekts Entwicklung in den 1950ern und 1960ern ist von klaren Grundannahmen geprägt. Wirtschaftswachstum sei der primäre Weg des Fortschritts und würde ein Aufholen und letztendliches Angleichen an das Wohlstandsniveau der Metropolen gewährleisten. Auch wenn es kaum mehr glaubhaft erscheint: Jenes Aufholen ist in der Tat nichts weniger als das Ausgangspostulat in der Frühzeit der Entwicklungspolitik. Kurz gefasst: Es geht zunächst um Modernisierung um jeden Preis, wobei diesen Preis vor allem jene zu zahlen haben, die aus ihren von Subsistenz oder alternativen Marktsystemen geprägten Lebensformen in eine in den allermeisten Fällen abgerissene, unvollständige Modernisie-

1 Hier kann auf eine in großer Zahl vorliegende Literatur verwiesen werden. Neben den bereits angeführten Entwicklungskritiker/innen, die sich nahezu alle mit dieser Fragestellung auseinandersetzen, seien Ulrich Menzel (1993: 70ff.) oder Aram Ziai (2004a: 85ff.) erwähnt. 
rung gepresst werden, die zumeist in einer perspektivlosen Semiproletarisierung endet.

Die Modernisierungstheorie, auch wenn sie sich pointiert erst später in den großen ideologischen Auseinandersetzungen der 1970er formieren sollte, benennt, verkörpert vor allem in den 1944-1947 aus der Taufe gehobenen Bretton-Woods-Institutionen, die hauptsächlichen Hindernisse gegenüber einem solchen Aufholprozess in endogenen Faktoren. Die Erfindung der Armut (vgl. Escobar 1995: 23ff.) - wir könnten in diesem Zusammenhang für den spezifischen historischen Kontext von der Herausbildung eines komplex wirkenden Armutsdispositivs ${ }^{2}$ sprechen bildet die Grundlage für die Entwertung der nun als im negativen Sinne traditionell (also rückständig) definierten gesellschaftlichen Strukturen.

In dieser Wahrnehmung findet sich einerseits eine frappierende Kontinuität zum Denken des Spätkolonialismus, im Sinne der Bürde des weißen Mannes und der ihm unterstellten Problemerkennungs- und Problemlösungskompetenz. Andererseits ist es jedoch auch ein qualitativer Bruch mit der kolonialen Tradition. Statt der Tendenz zur Optimierung der kolonialen Ökonomie auf die unmittelbaren Bedürfnisse des Zentrums - eine im Prinzip ganz und gar antiliberale Idee, die sich auch um die Frage der Armut nur eingeschränkt Gedanken macht und sich eher auf die optimale Nutzung der natürlichen und menschlichen Ressourcen ausrichtet - steht von nun an die Idee der Weltmarktintegration im Zentrum der Aufmerksamkeit. ${ }^{3}$ Diese Weltmarktintegration wird zum wesentlichen Vehikel, um die langfristige Reproduktion der systemischen Asymmetrie zwischen Zentren, semi-peripheren und peripheren Regionen zu garantieren.

Es macht an dieser Stelle wenig Sinn, über die Intentionen der an diesem Prozess Beteiligten zu spekulieren. Manche mögen diese Strategie, die die koloniale Traditionen des Rassismus mehr oder minder ungebrochen fortschrieb, mit einer gewissen Bösartigkeit verfolgt haben,

2 »One of the many changes that occurred in the early post-World War II period was the >discovery< of mass poverty in Asia, Africa, and Latin America. Relatively inconspicuous and seemingly local, this discovery was to provide the anchor for an important restructuring of global culture and political economy. The discourse of war was displaced onto the social domain and to new geographical terrain: the Third World. Left behind was the struggle against fascism. In the rapid globalization of U.S. domination as a world power, the >war on poverty in the Third World began to occupy a prominent place « (Escobar 1995: 21).

3 Dieser Neuorientierung auf ökonomischer Ebene stehen bemerkenswerte Kontinuitäten gegenüber, speziell im Rahmen von Institutionenentwicklung und Capacity-Building, die schon zentraler Bestandteil der Idee von »indirect rule« waren (vgl. Cooke 2003: 47ff.). 
manche mögen vom gern ins Treffen geführten »wohlverstandenen Eigeninteresse « geleitet worden sein, und manche mögen auch wirklich an die Versprechungen der Modernisierung der Peripherie geglaubt haben. Das ist letztendlich unerheblich. So oder so bleibt der traditionelle Ablauf bestehen, nach dem die Zentren zunächst die Bedürfnisse der Peripherien bestimmen, um dann die passenden Interventionsmechanismen in Gang zu setzen.

Es dauert mehr als zwei Jahrzehnte, bis mit dem von der Weltbank in Auftrag gegebenen Pearson-Report 1969 dieser große und simpel gestrickte Anfangs-Optimismus im entwicklungspolitischen Diskurs offiziell ad acta gelegt wird. ${ }^{4}$ Die damit einkehrende Nüchternheit bezieht sich allerdings vornehmlich auf den Zeithorizont und den notwendig aufzubringenden Einsatz ${ }^{5}$ im Projekt Entwicklung - an der grundsätzlichen Zielsetzung der Angleichung von Lebensbedingungen, definiert über ökonomische Eckdaten wie Bruttosozialprodukt und Wirtschaftswachstum, rüttelt der Bericht keineswegs.

Allerdings reicht diese sanft angesetzte Kritik bereits aus, eine lebhafte Infragestellung der von den Bretton-Woods-Institutionen vorgegebenen Politik auszulösen, die zuvor von verschiedenen Teilen der Peripherie ausgegangen war. Diese Kritik setzt hauptsächlich an zwei Punkten an: Einerseits werden der Betonung der endogenen Faktoren - also den die Modernisierung bremsenden Wirkungen der so genannt traditionellen Lebensweisen - die entwicklungshemmende Wirkung der globalen Asymmetrie gegenübergestellt. Andererseits richtet sich die Kritik auch gegen das von Ulrich Menzel (1993: 133) treffend mit »Wachstum zuerst, Umverteilung und Demokratisierung später« zusammengefasste Leitmotto der ersten beiden Entwicklungsdekaden. Eingefordert wird nun statt der bislang gegebenen ausschließlichen Konzentration auf Wachstumsstrategien im Sinne von Marktintegration die gleichzeitige

4 Uwe Andersen (2004: 89) weist zu Recht auf die interessante Tatsache hin, dass die entwicklungspolitische Diskussion in ihren Periodisierungen anhand der verschiedenen UN-nahen Entwicklungskommissionen nachgelesen werden kann. Dies trifft im Fall der Pearson-Kommission ebenso zu wie für die Kommissionen unter der Leitung von Willy Brandt und Gro Harlem Brundtland, die noch zur Sprache kommen werden.

5 Der große Einsatz, den die westliche Welt zu leisten hätte, um Entwicklung in der unterentwickelten Welt erfolgreich anzustoßen, ist sicherlich der in seiner langfristigen praktischen Wirkung wesentlichste Teil des Pearson-Reports. So entspringt diesem Bericht die auch heute noch allgegenwärtige Forderung an die Industriestaaten, 0,7 \% des BIP für entwicklungspolitische Leistungen zur Verfügung zu stellen. Ursprünglich war die Zeitfrist der Realisierung bis 1975 gesetzt, wobei sich die $0,7 \%$ auf ODAMittel bezogen, bei gleichzeitiger Gewährleistung einer Gesamtleistung (inklusive privater Mittel) von $1 \%$ des BIP. 
Setzung von Maßnahmen auf den Ebenen von politischer Partizipation und Umverteilung. ${ }^{6}$

Im Verlauf dieses Prozesses entwickelt sich die Dependenztheorie zum prominentesten Gegenspieler der Modernisierungsansätze. Ursprünglich ausgehend von der bereits seit 1948 bestehenden Comisión Económica para América Latina, CEPAL, und ihrem Leiter Raúl Prebisch wird von ihr nicht nur die Ursache von Unterentwicklung gemäß einem strukturalistischen Ansatz aus systemischen Faktoren abgeleitet, sondern auch der Zusammenhang zwischen ökonomischem Wachstum, Demokratie und Wohlfahrtsstaat herausgestrichen. Wie daraus schon anklingt, nimmt auch in diesem Konzept der Staat eine wesentliche Rolle ein, als so genannter »Entwicklungsstaat« (Becker 2004: 148) vielleicht sogar noch eine bedeutendere als in den verschiedenen klassischen Modernisierungsansätzen.

Während der 1970er sind die unterschiedlichen Dependenztheorien die hauptsächlichen entwicklungspolitischen Herausforderer der Modernisierung (vgl. Hödl 2004: 53). Dabei werden unterschiedliche Ansätze vertreten, die von einem reformorientierten Ansatz wie dem der CEPAL bis hin zu marxistischen Ansätzen reichen, die sich direkt an das Leninsche Imperialismuskonzept anlehnen. Auch die Lösungsmodelle sind unterschiedlich - von mehr oder minder entwicklungskeynesianischen Konzepten bis hin zu dissoziativen Ansätzen, die auf der Idee einer SüdSüd-Kooperation aufbauen, und kommunistisch-revolutionären Ideen sind unterschiedliche Grade an Radikalität vertreten. ${ }^{7}$

6 Jenes Denken im Sinne von »Wachstum zuerst, Umverteilung und Demokratisierung später« ist keinesfalls im Sinne eines Vergessens oder Vernachlässigens zu verstehen, speziell in Bezug auf die Partizipation. Vielmehr wird angenommen, dass ein zu hohes $\mathrm{Ma}$ an Partizipation den notwendigen Modernisierungsprozess negativ beeinträchtigen oder gar gefährden könne. Das vielleicht prägnanteste, mit Sicherheit aber bekannteste Beispiel dieses Denkens ist Samuel Huntingtons »Political Order in Changing Societies«, erschienen im Jahr 1968. Huntington betont darin die Notwendigkeit politischer Stabilität für einen erfolgreichen Entwicklungsprozess, wobei die vorherrschende Instabilität an der Peripherie vor allem auf den schnell verlaufenden Modernisierungsprozess zurückzuführen sei: »Political instability was rife in twentieth-century Asia, Africa, and Latin America in large part because the rate of modernization was so much faster there than it had been in the earlier modernizing countries" (Huntington 1996: 46). Den Schlüssel zur langfristigen Stabilisierung sieht Huntington im Aufbau eines stabilen Parteiensystems, das Alternativen zu den durch die Modernisierung zerstörten traditionellen Autoritäten anbieten müsse (ebd.: 460f.).

7 Die klassischen Ansätze, hauptsächlich in den Bretton-Woods-Institutionen verortet, erarbeiten als Antwort auf die Herausforderung durch die 
Das theoriegeschichtlich Interessante an der Herausforderung des klassischen entwicklungspolitischen Mainstreams durch die Dependenztheorie ist nicht deren weitgehendes Scheitern in diesem Versuch. Aus historischer Perspektive ist es letztendlich wesentlich zu sehen, dass die Diskussion primär um die Frage des Entwicklungsweges geführt wird, während das Ziel von Entwicklung an sich weitestgehend unumstritten bleibt. Natürlich sind sich die tragenden Kräfte keineswegs einig, ob ein wohlfahrtsstaatlich oder ein neoklassisch liberal orientiertes staatliches Modell anzustreben wäre, oder wie weit und zu welchen Bedingungen eine Weltmarktintegration zu erfolgen hätte. Aber diese Auseinandersetzungen bleiben sekundär vor dem Hintergrund, dass die grundlegende Vision, ökonomische und soziale Modernisierung sowie die Einrichtung eines modernen Staatswesens durchgängig, also von Modernisierungswie Dependenztheorie, von neoklassischen Vertreter/innen einer bedingungslosen Weltmarktintegration bis hin zu radikalen Vertreter/innen einer totalen Dissoziation, geteilt wird.

Ein effektiver Wandel in der entwicklungspolitischen Konzeption ist später, konkret in den 1980er Jahren, anzusetzen und er überwindet den Grabenkampf zwischen Modernisierern und Dependistas auch insofern, als sich die offiziellen bi- wie multilaterale Entwicklungsagenturen gegenüber ihren Kritiker/innen öffnen und sie in ihrem überwiegenden Teil über neu eingebrachte Themenstellungen zurück ins Boot holen (vgl. Nederveen Pieterse 1998: 349f.). Eingeläutet wird dieser Bruch durch den 1980 veröffentlichten Brandt-Report, wiederum der Bericht einer von der IBRD eingesetzten Entwicklungskommission. Unter dem Titel »Das Überleben sichern« stellt die so genannte »Nord-SüdKommission« erstmals in dieser Deutlichkeit das geltende Motto des entwicklungspolitischen Mainstreams in Frage: »es gilt, von der ständigen Verwechslung zwischen Wachstum und Entwicklung loszukommen [...]« (Brandt 1980: 33).

Schon einige Jahre zuvor war der Grundbedürfnis-Ansatz, forciert durch den damaligen Weltbank-Präsident Robert McNamara, zum State of the Art erhoben worden, was letztendlich die politische und konzeptionelle Unterminierung der Wachstums-These einläutete. Es sollte allerdings noch einige Jahre der »verlorenen Dekade« der 1980er benötigen, bis es einer weiteren Kommission, diesmal der 1983 von der UNO eingesetzten »Weltkommission für Umwelt und Entwicklung« unter Leitung von Gro Harlem Brundtland, vorbehalten bleibt, im Jahr 1987 die

Dependenztheorie einen in der entwicklungspolitischen Praxis bis heute populären Zugang: den Grundbedürfnis-Ansatz (vgl. Hödl 2004: 56). 
neue, in der Tat bahnbrechende entwicklungspolitische Idee mit einen Begriff auf den Punkt zu bringen: Nachhaltigkeit.

Der zunächst hauptsächlich für den Umweltbereich vorgesehene Begriff, gemeint vorwiegend im Sinne einer Nutzungsweise von natürlichen Ressourcen, die ihre Reproduzierbarkeit und damit langfristige Nutzbarkeit garantiert, wird Nachhaltigkeit zu dem entwicklungspolitischen Schlüsselbegriff der 1990er Jahre, definiert als »development that meets the needs of the present without compromising the ability of future generations to meet their own needs« (Weltkommission für Umwelt und Entwicklung 1987, zitiert nach Duffield 2005a). Diese Definition ist entscheidend, markiert sie doch den wesentlichen Bruch mit dem bis zu diesem Punkt vorherrschenden Entwicklungsdenken: »it marked an end to earlier modernist assumptions that the underdeveloped world would, after passing through various stages, come to resemble the developed. Sustainable development breaks this aspirational goal and substitutes a relation of containment. Southern populations are now expected to live within the limits of their own powers of self-reliance« (Duffield 2005a).

Es sind zwei zentrale Implikationen, die dieser Wechsel mit sich bringt: zunächst das Ende der großen Theorie-Debatten, die bis in die 1980er nicht nur im entwicklungspolitischen Bereich dominieren (Menzel 1992: 129ff.). Dieser Prozess ist allerdings keineswegs so naheliegend und trivial wie es zunächst scheinen mag. Sicherlich hat die Auflösung des Warschauer Pakt-Systems seinen Teil zum Zusammenbruch ideologiegeladener Theoriegebäude beigetragen. Natürlich finden sich auch in Ulrich Menzels $(1992,1993)$ Argument der großen Differenziertheit der »Dritten Welt«, die ihn veranlasst, von ihrem »Ende« zu sprechen, stimmige Elemente. Es ist jedoch spannend, wie es durch den Begriff der Nachhaltigkeit und die durch ihn durchführbare gezielte Anbindung der Ökologiebewegung und des NGO-Sektors möglich wird, einen Großteil der konkurrierenden Ansätze in das Entwicklungsprojekt der staatlichen Eliten des Nordens zu reintegrieren. ${ }^{8}$

Der Prozess ist umso erstaunlicher, wenn wir die zweite Implikation betrachten, die Nachhaltigkeit mit sich bringt: die endgültige Abkehr von der ursprünglichen Zielbestimmung von Entwicklungspolitik. Nach-

8 Dieser Prozess spiegelt sich im entwicklungspolitischen Diskurs in einem neuen Technizismus wider, der allerdings gegenüber den ebenfalls technokratisch ausgerichteten Weltbank-Ansätzen der 1950er bis 1970er Jahre viel größere Flexibilität aufweist: »Die vielzitierte $>$ Krise der Entwicklungstheorie ist allerdings eher als Chance zu bewerten, wenn sie die Lagermentalität aufhebt und zum Verzicht auf allgemeine, mit Ausschließlichkeitsanspruch vertretene Theorieansätze führt, die sich mehr auf ideologische Überzeugung als auf empirische Bewährung stützen« (Andersen 2004: 94). 
haltigkeit bedeutet letzten Endes nichts anderes als eine Verschiebung der Modernisierungsversprechungen auf unbestimmte Zeit, also im Grunde genommen das Eingeständnis des Scheiterns der bisherigen Konzeption von Entwicklungspolitik (vgl. Duffield 2005a). Statt der versprochenen Angleichung des Lebensstandards sind es nun vor allem neue Verantwortlichkeiten, die den peripheren Regionen zugewiesen werden - eben nachhaltig zu agieren. Dies bedeutet strategisch im systemischen Kontext vor allem eines: das Ende der bisherigen Kooptierungsstrategien gegenüber den Peripherien ${ }^{9}$ und ihre Ersetzung durch Containment (vgl. Duffield 2006). ${ }^{10}$

Durch die Abschwächung seiner postulierten Zielbestimmungen ist das Entwicklungsdispositiv in der Lage, sein Integrationspotenzial wesentlich zu erweitern. Damit kann es die gefährlich weit geöffnete Schere der Heterogenität, die in eine für seine Wirksamkeit prekäre Bipolarität zwischen staatlichen und multilateralen Ansätzen sowie einem privaten, Grassroots-orientierten alternativen Entwicklungsprojekt zu kippen droht, wieder schließen. Das in diesem Zusammenhang entscheidende Manöver liegt auf diskursiver Ebene. Entwicklungspolitische Praxis wird im Sinne der Nachhaltigkeit nicht mehr länger zielspezifisch, sondern problemspezifisch argumentiert, womit all die verschiedenen Vorbehalte und Kritiken am klassischen Wachstumskonzept, die antagonistischen Charakter hätten annehmen können, plötzlich als Zielbestimmungen und Querschnittsthematiken der Entwicklungsagenturen auftauchen. Als wesentlicher Agent dieser Unternehmung werden genau jene engagiert, die die Kritik ursprünglich vorgebracht hatten: die "Zivilgesellschaft«, nun in Form professionalisierter, international wie national agierender NGOs.

Das alles bedeutet keineswegs, dass die ökonomistischen Ansätze aus dem Weltbank-Umfeld ausgespielt hätten. Speziell die neoklassische Theorie der komparativen Kostenvorteile behält ihren prominenten Platz. Aber selbst in der Weltbank werden neue Begrifflichkeiten geprägt, die die Bildung ganzer neuer entwicklungspolitischer Sektoren nach sich ziehen. Hervorzuheben sind hier vor allem die Problemstel-

9 Gegenüber der Semi-Peripherie ist die Situation ambivalenter. Je nach ökonomischer und geostrategischer Interessenslage der Zentren findet sich hier auch eine Fortsetzung der Kooptierung, die sich durch die spezifische Bedeutung der Semi-Peripherien als regionale Knotenpunkte ergibt.

10 Gerald Hödl (2004: 270) spricht im Gegensatz dazu davon, dass die Kooptierungs- durch Dominanzstrategien abgelöst worden wären. Dies ist im Einzelfall sicher nicht unrichtig, dennoch meine ich, dass die Behauptung einer solchen allgemeinen Tendenz den Charakter der seit den 1990er Jahren laufenden Prozesse auf internationaler Ebene verkennt. 
lungen Good Governance (vgl. Fuster 1998) und, etwas später, der Umgang mit bewaffneten Konflikten.

Die Integrationsfähigkeit des Dispositivs beweist sich speziell an drei anderen Themenfeldern, die zuvor zentrale Kritikbereiche an der bisherigen Entwicklungspraxis markieren: Umwelt, Frauen und Frieden. Durch ihren Einbezug nicht nur in die Diskussion, sondern in die definierten Zielbestimmungen der meisten Entwicklungsagenturen während der 1990er Jahre (einhergehend mit ihrer »Mainstreamisierung« oder Erklärung zur »Querschnittsmaterie«) wird ein doppelter Effekt erzielt. Einerseits kann durch die Integration von für gewöhnlich als »links« besetzten Thematiken in den Mainstream-Diskurs eine neue Übereinstimmung der ODA-Agenturen mit den größtenteils im NGO-Sektor verorteten Entwicklungshelfer/innen aus dem Spektrum der alternativen sozialen Bewegungen hergestellt werden. Die verstärkte Einbindung des NGO-Sektors auch in die offizielle bilaterale wie multilaterale Entwicklungshilfe ab den späten 1980ern erklärt sich folgerichtig aus diesem Prozess.

Andererseits bedeuten diese Themenfelder in weiterer Konsequenz auch, dass in der Analyse die Ursachen der Probleme an die Peripherien zurückgeschoben werden (vgl. Hödl 2004: 58ff.). Denn klarerweise geht es bei entwicklungspolitischen Umwelt-, Frauen- oder Friedensproblematiken wiederum um Probleme an der Peripherie, die durch das Knowhow der Zentren einer Lösung zugeführt werden sollen. Damit wird nicht nur die Wirkung von Ansätzen, die das systemische Ungleichgewicht als primäre Problemursache ins Zentrum rücken wollen, effektiv untergraben. Umweltschutz wird zur Frage von Luftverpestung in veralteten peripheren Schwerindustriestandorten, Feminismus zur Frage von als reaktionär und patriarchal identifizierten Praktiken traditioneller Gesellschaften, und Friedenspolitik zur Frage von zunehmend als irrational analysierten Gewaltkonflikten an der Peripherie. Damit ist es auch bei sich selbst als kritisch definierenden Entwicklungshelfer/innen mit der Infragestellung globaler systemischer, in einen historischen Kontext gesetzten Ursachen, also substanzieller Systemkritik, weitestgehend vorbei.

Nachdem im Gefolge des Brundtland-Reports Umwelt das erste jener Themenfelder ist, das als Musterbeispiel des NachhaltigkeitsDiskurses zelebriert wird, avanciert Gender Mitte der 1990er Jahre zu einem der zentralen Themenkomplexe. Werden Frauen im Prozess der entwicklungspolitischen Modernisierung zumeist in die Rolle jener unsichtbaren Arbeitskraft gedrängt, die jenseits der Wahrnehmung der Entwicklungsökonomen durch primär subsistente Tätigkeiten die verheerenden Wirkungen der Semi-Proletarisierung abzufedern haben (vgl. 
von Werlhof 1991), geraten sie jetzt als zu emanzipierende Subjekte ins Visier der entwicklungspolitischen Intervention. Gender, vorwiegend problematisiert entweder im Sinne eines Gleichberechtigungs- oder, entlang dem argumentativen Muster von Frauen als besonderer Kategorie der Opfer von Unterentwicklung, als Opferdiskurs, wird in den meisten Entwicklungsagenturen als so genannte Querschnittsmaterie eingezogen. ${ }^{11}$

Zudem wird Gender, wie in vielen Institutionen Mitte der 1990er, zu einem zu mainstreamisierenden Problemkomplex erklärt. Nach Definition der GTZ bedeutet dieser Neologismus zweierlei: »(1) the integration of a gender perspective into the analyses and formulation of all policies, programmes and projects, and (2) initiatives to enable women as well as men to formulate and express their views and to participate in decision-making processes « (Reimann 2001b: 7). Wiederum zeitigt dieser Prozess einen doppelten Effekt: Nicht nur gelingt es, mit der permanenten Betonung der Gender-Mainstreamisierung ${ }^{12}$ eine vertiefte Diskussion über den an sich patriarchalen Charakter des Entwicklungsprojektes abzuschneiden. Ebenso wird Gender im entwicklungspolitischen Kontext als Thema im Sinne eines einheitlichen modernen emanzipatorischen Projektes operationalisiert, was eine kritische Hinterfragung eines solchen Projektes wiederum untergräbt.

Zugleich ist damit intendiert, die Idee multipler Feminismen, die sich einem eurozentristischen Bias entziehen, hintanzuhalten. ${ }^{13}$ Andererseits folgte daraus notwendigerweise auch jener Prozess, den Gayatri Spivak (1988) auf einer allgemeineren Ebene bereits prägnant nachgezeichnet hat: Die ungefragte Repräsentation der subalternen Frauen durch die gender-gemainstreamten Entwicklungsagenturen führt zu einer Diskursordnung, die nach klaren und von den Zentren ausgegebenen Kriterien regelt, wer sprechen kann und wer rezipiert wird. In den meisten Fällen übernehmen diese Aufgaben ohnehin gleich die Agenturen selbst.

11 Die etwas spröde »Begriffswelt der GTZ« (GTZ 2004a) definiert Querschnittsproblematik wie folgt: »Als Querschnittsthemen bezeichnet man solche Aspekte, die bereits während des Prüfungsverfahrens von Programmen oder Projekten berücksichtigt werden müssen [...].« Darunter findet sich auch die »Gleichberechtigung der Geschlechter«.

12 Irmi Maral-Hanak (2004: 190f.) spricht in diesem Zusammenhang pointiert von »Übermainstreamisierung« und »Femokraten«, in Anspielung an den technokratischen Charakter des Mainstreamisierungs-Projekts.

13 Maral-Hanak (2004: 192f.) hält trotzdem weiterhin an dieser Forderung fest, auch wenn die Hoffnung auf die Wirkungen ihres Plädoyers allem Anschein nach gering ist. 
Das Thema Frieden wiederum wird von Anfang an nicht nur in Verbindung mit dem Thema Sicherheit gebracht (vgl. dazu als einen der ersten entsprechenden Texte Boutros-Ghali 1992), sondern auch gleich in den Kontext von Nachhaltigkeit gesetzt. Ein Schlüsselkonzept, das diese Verbindung erleichtern soll, wird mit »Human Security« gefunden, das nach seiner quasi-formellen Einführung im Human Development Report von 1994 (vgl. UNDP 1994) unter dem Begriff »Sustainable Human Development « die dazu notwendigen verbindenden Linien formuliert. In der zweiten Hälfte der 1990er Jahre gewinnt der Begriff an Prominenz, im Jahr 2001 schreiben die DAC-Guidelines »Helping Prevent Violent Conflict « die Verbindung auch formell fest (vgl. DAC 2001: 19). ${ }^{14}$

Solche sicherheitspolitischen Zielsetzungen sind der Entwicklungspolitik grundsätzlich nicht neu. Schon die berühmte Inaugural-Ansprache von Harry Truman ${ }^{15}$ enthält, wenngleich in relativ verklausuliertem Ton, Aussagen über sicherheitspolitische Motivationen des Unternehmens Entwicklung: »Our aim should be to help the free peoples of the world « - eine klare Anspielung im Ton des Kalten Krieges. Ebenso wie die nahezu mythische Kraft, die Truman der Demokratie zuschreibt: »Democracy alone can supply the vitalizing force to stir the peoples of the world into triumphant action, not only against their human oppressors, but also against their ancient enemies - hunger, misery, and despair. ${ }^{16}$

Generell können wir davon ausgehen, dass Antikommunismus neben der Transformation der kolonialen Beziehungen in die nachkoloniale Ära die zentrale Triebkraft hinter den ersten entwicklungspolitischen Bemühungen ist. Gerald Hödl (2004: 198) stellt dies etwa für die Anfänge der österreichischen Entwicklungszusammenarbeit fest, aus der

14 Interessanterweise findet sich in der Erstausgabe der DAC-Guidelines von 1997 eine solche Festschreibung nicht. Die Rolle von Human Security als Bindeglied zwischen friedens- und sozialpolitischen Zielsetzungen und dem Sicherheitskomplex war zu diesem Zeitpunkt offenbar noch nicht so prominent.

15 Harry Trumans Rede vom 20. Januar 1949 ist einsehbar über die Website der Truman Library, www.trumanlibrary.org (Zugegriffen am 08-042006).

16 Richard Saull verallgemeinert diese Ansätze in seiner Analyse der Rolle der Peripherien im Kalten Krieg: »Whereas the expansion of the Soviet social system came through war and/or revolution, the expansion of the US-led system of international capitalism did not necessitate war or direct coercive occupation. American capitalist power could be secured within the existing international political-legal framework; that is, states could be >sovereign<, as the states of Western Europe and other parts of the world were, but still subject to US political-economic influence« (Saull 2005: 270). 
Geschichte der deutschen Entwicklungspolitik, lässt sich ähnliches ableiten. Spätestens mit der kubanischen Revolution verstärkt sich jedoch auch die Angst vor einer Eskalation des Nord-Süd-Konfliktes: »Die Reaktion des Westens auf diese Ereignisse [die kubanische Revolution] bestand in verstärkten Entwicklungshilfe-Anstrengungen (besonders markant die Alliance for Progress der USA, aber auch die Gründung regionaler Entwicklungsbanken und die Schaffung der UNIDO) [...] (Hödl 2004: 24). Damit bestätigt sich die These, dass die Rolle der systemischen Peripherien während des Kalten Krieges keinesfalls passiv verstanden werden darf, denn $»$ the South was a factor in the domestic and international politics of each superpower to a much greater degree than most mainstream theories of the Cold War recognise« (Saull 2005: 255). Im Sinne der konstruktiven Bearbeitung dieser Problemstellung im Interesse der Zentren ist auch Willy Brandts bekannter Slogan zu verstehen, wonach »Entwicklungspolitik von heute« die »Friedenspolitik von morgen« sei (zitiert nach Nuscheler 2000: 17).

Die politischen Strukturen der peripheren Regionen rücken erst in den 1980er Jahren verstärkt in den entwicklungspolitischen Fokus interessanterweise, wie Franz Nuscheler (1996: 337) bemerkt, zunächst von »links«, also von Seite derjenigen, die den Aktivitäten der BrettonWoods-Institutionen kritisch gegenüberstehen. Von der CEPAL-Idee des Entwicklungsstaates ausgehend entwickelt sich eine lebhafte Diskussion um die Rolle des peripheren Staates als ökonomischer Lenkungsmechanismus. Die Weltbank nähert sich dem Thema Staat weniger über seine potenziellen Möglichkeiten, sondern vielmehr über seine Unzulänglichkeiten. Der Weltentwicklungsbericht 1983 (World Bank 1983) listet erstmals staatliche Versäumnisse in der Umsetzung effizienter ökonomischer Politiken auf (vgl. auch Nuscheler 1996: 343). Eingefordert wird ein »Reorienting Government«, gemeint als »developing strong and efficient public institutions« (World Bank 1983: 115).

Einen verstärkt politischen Aspekt, der sich zunehmend in Richtung des Sicherheitskomplexes bewegt, bekommt die Diskussion mit dem Aufkommen des Begriffes »Good Governance«. Erstmals findet sich der Terminus im Westafrika-Bericht der Weltbank von 1989, »From Crisis to Sustainable Growth« (World Bank 1989). Er wird hier jedoch, auch bedingt durch das Politikverbot der Weltbank, primär in einem technokratischen Sinn interpretiert und mit ökonomischen Problemstellungen verknüpft: »A root cause of weak economic performance in the past has been the failure of public institutions. Private sector initiative and market mechanisms are important, but they must go hand-in-hand with good governance - a public service that is efficient, a judicial system that is 
reliable, and an administration that is accountable to its public« (ebd.: xii).

Interessant ist, dass diese Zeilen aus dem Vorwort des damaligen Weltbank-Direktors Barber Conable stammen. Im eigentlichen Text wird der Begriff nur an einer Stelle - im Zusammenhang mit der Bedeutung einer freien und unabhängigen Presse - erwähnt (ebd.: 61). Offenkundig ist die Einführung des Begriffes also keine konzertierte und geplante Aktion, sondern eher ein Selbstläufer (vgl. auch Fuster 1998: 8ff.). ${ }^{17}$ In jedem Fall entwickelt sich ausgehend von dieser Studie eine richtiggehende Governance-Debatte in der entwicklungspolitischen Szene, die zunächst primär Afrika-zentriert geführt wird. Good Governance beginnt in der Folge die entwicklungspolitische Diskussion der ersten Hälfte der 1990er Jahre zu dominieren, wobei die Interpretationen durch Verknüpfungen mit Themenkomplexen wie Demokratisierung und Menschenrechte immer stärker politisiert werden.

Offenkundig gibt es einen dringenden Bedarf nach einer verstärkten Einbindung politischer Aspekte in die entwicklungspolitische Praxis, wobei als einer der häufigsten Gründe dafür angegeben wird, dass die durch den Kalten Krieg bedingte geopolitisch motivierte Rücksichtnahme, durch die speziell der multilaterale Sektor gebunden ist, nun fallengelassen oder doch zumindest abgeschwächt werden kann (vgl. Fuster 1998: 23). Die große Popularität des von Robert Keohane maßgeblich mitentwickelten Neoinstitutionalismus im Bereich der Analyse, aber auch der strategischen Ausrichtung der Bretton-Woods-Institutionen tut ihr Übriges zur verstärkten Fokussierung auf staatliche Institutionen von Seiten des entwicklungspolitischen Mainstreams.

Die bilateralen Agenturen entwickeln Good Governance in der Folge immer stärker in eine politische Richtung und verknüpfen es mit der Nachhaltigkeitsproblematik. Ausdruck davon sind die Guidelines zu »Participatory Development and Good Governance«, die 1995 veröffentlicht werden: »It has become increasingly apparent that there is a vital connection between open, democratic and accountable systems of governance and respect for human rights, and the ability to achieve sustained economic and social development« (DAC 1995: 5). Nachhaltigkeit wird somit, obwohl ursprünglich primär auf ökologischer und ökonomischer Ebene angestrebt, zu einem genuin politischen Problem. Dementsprechend wird auch die Definition merklich ausgedehnt. Spricht die Weltbank ursprünglich von drei zentralen Bereichen - effizienter

17 Thomas Fuster verweist in diesem Zusammenhang auf das bemerkenswerte Faktum, dass die Weltbank mit diesem Bericht erstmals ihr statutarisch festgelegtes auf die ökonomische Ebene beschränktes Mandat durchbricht (vgl. Fuster 1998: 10). 
öffentlicher Sektor, funktionierendes Justizsystem und eine gegenüber der Öffentlichkeit verantwortliche Administration - kommen nun weitere, explizit politische Komponenten hinzu. Herausgestrichen wird die enge Verbindung zwischen Good Governance, »participatory development«, Menschenrechten und Demokratisierung: »They [the agendas] include elements which are basic values in their own right, such as human rights and the principles of participation, and others such as accountability, transparency and high standards of public sector management, which are also means to developmental ends. Some of the objectives, such as the rule of law, must be viewed as both ends in themselves and means to viable development « (ebd.: 6).

Die von der Weltbank festgelegten Zielsetzungen werden vom DAC so als Teil einer breiteren Agenda interpretiert, die, besonders mit der Einbeziehung demokratischen Prinzipien, Elemente hervorhebt, welche die Weltbank in der Form nie gefordert hätte. Zwar werden einerseits die technischen Anforderungen von Good Governance herausgestrichen (»rule of law«, »public sector management«, »controlling corruption«, »reducing excessive military expenditure «), aber diese auf Policy-Ebene verorteten Bereiche werden unter das generelle Motto partizipativer Entwicklung und zweier eindeutig politischer Komponenten gestellt: Demokratisierung und Menschenrechte (ebd.). Dies unterstreicht einmal mehr das offenbar vorhandene Bedürfnis nach Anfangsgründen, die eine engere Anbindung von Entwicklung und politischer Intervention möglich machen können.

Der Grundgedanke der Demokratisierung, der bei Good Governance mitschwingt, liegt in der Logik von Nachhaltigkeit. Denn mit ihrer Erfindung ist praktisch das Ende der Entwicklungsdiktaturen eingeläutet. Interessanterweise läuft dieser Prozess auch zeitlich parallel mit vergleichbaren Verschiebungen in anderen Politikfeldern. William Robinson (1996) zeigt, dass die US-Administration ab Mitte der 1980er - also faktisch zeitgleich mit dem Aufkommen des Governance-Gedankens bei der Weltbank - beginnt, ihre außenpolitische Orientierung in Richtung von »democracy promotion« zu verändern. ${ }^{18}$ Robinson führt das darauf zurück, dass demokratische Systeme spezifischen Zuschnitts (er spricht von Polyarchien und bezeichnet solche Systeme als »low intensity democracies «) zur Exekution von Herrschaft in der aktuellen Phase besser geeignet seien als diktatorische Regime. Die Frage nach den Ursachen dieses Wechsels beantwortet Robinson in einem Rekurs auf Gramscis

18 Robinson weist darauf hin, dass zeitgleich mit dem außenpolitischen Richtungswechsel auch ein Aufschwung der Demokratietheorie im wissenschaftlichen Spektrum zu verzeichnen ist (vgl. Robinson 1996: 41ff.). 
Hegemonieansatz damit, dass es einfacher sei, durch aktive Zustimmung der Betroffenen zu herrschen als gegen sie. Demokratie niederer Intensität sei in diesem Modell der Schlüssel zur Produktion der globalen Hegemonie des kapitalistischen Weltsystems.

Robinsons Darstellung lässt allerdings offen, warum sich dieser Bruch ausgerechnet Mitte der 1980er abzeichnet, zu einem Zeitpunkt, als in vielen Regionen der Welt linksgerichtete Guerilla-Bewegungen gegen US-gestützte Diktaturen kämpfen, der Kalte Krieg nach wie vor das geopolitische Denken dominiert und mit Ronald Reagan der USAdministration ein alles andere als experimentierfreudiger Republikaner vorsitzt. Just zu dieser Zeit entwirft das National Endowment for Democracy, NED, erste Programme zur Demokratieentwicklung, die schnell in einem ersten Highlight münden: Ferdinand Marcos wird 1986 fallengelassen und die Philippinen werden zum ersten Vorzeigeland der neuen Demokratisierungswelle (vgl. Robinson 1996: 117ff.).

Das neue Interesse an politischen Strukturen und der Forcierung von Demokratie kann also nur zum Teil mit dem Ende des Kalten Krieges in Verbindung gebracht werden. Zumindest ebenso relevant ist der $\mathrm{Zu}$ sammenhang mit dem sich durchsetzenden Denken der Nachhaltigkeit. In dem Moment, wo die Idee der Möglichkeit des Aufholens aufgegeben wird, braucht es auch nicht mehr diejenigen, die dieses Aufholen kompromisslos umzusetzen suchen. Die Zeit der Modernisierungsdiktaturen ist vorbei, sie haben in der Welt der Nachhaltigkeit keinen Platz mehr. Die jetzt notwendige Verwaltungs- und Befriedungspolitik lässt sich in der Tat besser in formal demokratischen Systemen verwirklichen schon allein weil diese das Risiko minimieren, dass sich potenziell starke Administrationen aktiv gegen eine solche Containment-Taktik wenden. ${ }^{19}$

Einige Jahre verzögert findet auch von Seiten der traditionellen Sicherheitspolitik eine Annäherung an entwicklungspolitische Agenden statt, hier aber deutlich von der neuen globalen Konstellation nach Ende

19 Natürlich gibt es auch Gegenbeispiele zu dieser Entwicklung. China ist unter einem ökonomisch modernisierenden Zwangsregime $\mathrm{zu}$ einem ernsthaften Herausforderer der weltsystemischen Zentren geworden und auf dem Weg, mittelfristig das derzeitige Systemgefüge grundlegend in Frage zu stellen. Allerdings ist China keine klassische Entwicklungsdiktatur von Gnaden der Zentralmächte, was auch für andere Gegenbeispiele (Burma, Nordkorea, oder einige der afrikanischen Staaten) nicht behauptet werden kann. Dennoch lassen sich, etwa im Arabischen Raum, in Zentralasien und einigen afrikanischen Ländern, nach wie vor Regimetypen finden, die nicht nur von den systemischen Zentren weitestgehend gestützt werden, sondern auch als Entwicklungsdiktaturen zu bezeichnen sind. Der generelle politische Trend ist jedoch eindeutig gegenläufig. 
des Kalten Krieges geprägt. Auslöser sind dabei zwei zusammenhängende Faktoren, einer auf konkret politischer, einer auf konzeptioneller Ebene: die Auseinandersetzung um die Frage der humanitären Intervention und die Diskussion um die Erweiterung des traditionellen Sicherheitsbegriffes.

Die enge Verbindung der beiden Problemstellungen ergibt sich aus der Tatsache, dass der staatszentrierte Sicherheitsbegriff, der die traditionelle Sicherheitspolitik geprägt hatte, gerade in Interventionen, die primär von humanitären Erwägungen getragen sind (vgl. Stokke 1997: 204), nicht mehr anwendbar ist. Die Bedrohungsbilder haben sich gewandelt, wie Boutros Boutros-Ghali (1992) im historisch ersten UNOSchlüsseldokument zu dem Problemkomplex (»An Agenda for Peace«) herausstreicht: die »deepest causes of conflict« wären nun »economic despair, social injustice and political oppression« (ebd.: Abs. 15). Die Frage ist also nicht mehr die der Sicherung eines Landes gegen äußere Bedrohung oder innere Unruhen, wie sie die 1980er Jahre dominieren. Dagegen rücken gesellschaftspolitische und sozioökonomische Bedingungen in den Mittelpunkt der Aufmerksamkeit, was nicht nur das Nachhaltigkeitskonzept demonstriert - es geht nicht mehr um eine Lösung der Probleme im Sinne eines wirtschaftlichen Aufholens, sondern um deren Befriedung. Auch die Frage staatlicher Souveränität wird neu bewertet, und dies pikanter Weise gerade von der UNO, der traditionellen Hüterin dieser Souveränität: »The time of absolute and exclusive sovereignty, however, has passed; its theory was never matched by reality« (ebd.: Abs. 17).

Als Lösungsansatz schlägt Boutros-Ghali (nach dem Scheitern der üblichen diplomatischen Schritte zur Konfliktprävention) ein DreiPhasen-Konzept von Peacemaking, Peace-Keeping und Peacebuilding vor und errichtet damit das Grundgerüst des humanitären Interventionismus. »It is the essence of the concept of collective security as contained in the Charter that if peaceful means fail, the measures provided in Chapter VII should be used, on the decision of the Security Council, to maintain or restore international peace and security in the face of a >threat to the peace, breach of the peace, or act of aggression « (ebd.: Abs. 42). Ist der Krieg siegreich geführt, bekommt die zivile Intervention ihre Funktion im Aufbau einer tragfähigen Friedensordnung: »In the aftermath of international war, post-conflict Peacebuilding may take the form of concrete cooperative projects which link two or more countries in a mutually beneficial undertaking that can not only contribute to economic and social development but also enhance the confidence that is so fundamental to peace« (ebd.: Abs. 56). 
Was in diesen Stellen noch relativ sanft anklingt, markiert den Beginn zahlreicher, von »humanitären Erwägungen« getragenen bewaffneten Interventionen der Zentren in peripheren Krisengebieten, mit einer bestenfalls ambivalenten Bilanz (vgl. Paris 2004). »Nur in wenigen Fällen sind externe Interventionen tatsächlich praktikabel«, befinden etwa Tobias Debiel und Franz Nuscheler (1996: 43) als Schlussfolgerung eines Sammelbandes zur Thematik, geprägt von den zu diesem Zeitpunkt gerade aktuellen Desastern in Somalia (1993-1995) und Ruanda (1994).

Ausgehend von diesen Erfahrungen werden die Begriffe Konfliktprävention (und zwar eben nicht im Sinne einer rein präventiven Diplomatie) und Peacebuilding zum Schlagwort der zweiten Hälfte der 1990er. Die Europäische Kommission diskutiert den Problemkomplex wie erwähnt erstmals öffentlich 1996 mit der Kommunikation »The EU and the issue of conflicts in Africa« (EC 1996). Darin wird explizit die Verbindung der Konfliktpräventionsidee mit Good Governance hergeleitet. Eine wesentliche Aufgabenstellung wäre demnach: »Support for democratisation is essential for establishing a stable and legitimate political framework. [...] Long-term consolidation of democratic systems should be sought through programs on participatory development and good governance $[\ldots]$ « (ebd.). In der ultimativ festgelegten Zielbestimmung der »strukturellen Stabilität« findet auch das Konzept der Nachhaltigkeit einen prominenten Platz: »As it goes without saying that economic development and prosperity are major factors for ensuring nonviolent change, sustainable development is a central objective for the attainment of structural stability« (ebd.).

An diesen Problem- und Zieldefinitionen wird sichtbar, dass ein auf staatliche Souveränität eingeschränkter Sicherheitsbegriff nicht mehr praktikabel sein kann. In sicherheitspolitischen Kreisen wird daher die Idee einer »comprehensive security " geboren, die nicht nur die traditionelle »state security« im militärischen Sinne, sondern auch verschiedene soziale, ökonomische, politische und Umwelt-Faktoren inkludieren soll. Freilich ist die Idee eines solchen erweiterten Sicherheitsbegriffs schon älter. Erstmals wird der Begriff von Olof Palme im Rahmen der Independent Commission on Disarmament and Security im Jahr 1982 erwähnt. Durchzusetzen beginnt er sich in den 1990er Jahren, speziell seit sich das Human-Security-Konzept etabliert.

Human Security wird von Beginn an - die erste ausführliche Auseinandersetzung mit dem Begriff erfolgte im Human Development Report 1994 unter dem Titel »New Dimensions of Human Security « (UNDP 1994) - mit Nachhaltigkeit verknüpft, gefasst im Slogan »Sustainable Human Development«, für das Human Security eine Voraussetzung sei. In der Begriffsdefinition wird vom UNDP, in gewisser Komplementari- 
tät zum »State Security«-Begriff, die sicherheitspolitische Bedeutung von Themenkomplexen wie soziale Sicherheit, Umwelt, Gesundheit und Bildung hervorgehoben (vgl. ebd.: 22). Damit wird die Sicherheit von Menschen in explizitem Kontrast zum in der radikalen Form überkommenen sicherheitspolitischen Denken mit der ausschließlichen Fokussierung auf Staaten in den Mittelpunkt gerückt.

Generell sind die begriffsgeschichtlichen Parallelen und strukturellen Verwandtschaften von »sustainable development« und »comprehensive security« augenfällig. Beide Konzepte entstehen nahezu zeitgleich in von führenden europäischen Sozialdemokraten geleiteten UNO-nahen Kommissionen, durchleben über einige Jahre ein Schattendasein, um sich dann speziell ab Mitte der 1990er Jahre als neuer Standard zu etablieren. Auch ist die Problemstellung ausgesprochen ähnlich: Bedeutet Nachhaltigkeit das faktische Eingeständnis des Scheiterns der Entwicklungspolitik an ihren ursprünglich gesetzten Maßstäben, ist der erweiterte Sicherheitsbegriff die Konsequenz aus dem unzureichenden Charakter der »state security« in Zeiten verändert wahrgenommener Bedrohungsbilder, insbesondere einer vermehrt als Gefahr gesehenen Peripherie. Auch hier zeigt sich, dass dem gescheiterten Versuch der lückenlosen Durchsetzung globaler Standards wie einer etablierten Staatlichkeit nun die Eindämmung von Bedrohungen folgt - unter anderem durch den direkten Eingriff vor Ort.

In der zweiten Hälfte der 1990er Jahre formt sich Konfliktprävention neben Grundversorgung, Gesundheit, Gender und nachhaltiger Ressourcennutzung endgültig als einer der zentralen entwicklungspolitischen Themenkomplexe heraus. 1997 veröffentlicht das Development Assistance Committee (DAC) der OECD die Guidelines »Helping Prevent Violent Conflict« (vgl. DAC 2001), die als erster Referenzpunkt sicherheitspolitischer Entwicklungszusammenarbeit gelten können. In ihrer überarbeiteten Fassung von 2001 sind diese Guidelines nach wie vor der primäre Bezugspunkt zur Rolle der Entwicklungspolitik in der Aufgabenstellung Konfliktprävention. Wiederum findet sich die Konstruktion des Zusammenhanges zwischen erweiterter Sicherheit und nachhaltiger Entwicklung als Ausgangsbedingung: »Security, including >human security<, is a critical foundation for sustainable development. This implies protection from systemic human rights abuses, physical threats, violence and extreme economic, social and environmental risks, and territorial and sovereignty threats « (ebd.: 19). ${ }^{20}$

20 Wir finden ähnlich konstruierte Zusammenhänge auch in zeitgleich erscheinenden Schlüsseldokumenten der UNO. Kofi Annan benennt seinen Afrika-Report im Jahr 1998 in symptomatischer Weise »The Causes of Conflict and the Promotion of Durable Peace and Sustainable Develop- 
Die wesentliche Funktion, die der entwicklungspolitischen Intervention in dieser eigentlich genuin sicherheitspolitischen Aufgabenstellung zukommt, wird über die notwendige Langzeitwirkung argumentiert: »Promoting peace is a dynamic process that requires long-term commitment. Sustainable peace is not something that can be produced rapidly or with a technical >quick fix «. It is a process rather than a clear state that can be achieved once and for all. Long-term vision, therefore, should be maintained, even in short-term complex crises« (ebd.: 26).

Diesem Zusammenhang kann sich auch die multilaterale Ebene nicht entziehen. Die Weltbank legt den Schwerpunkt des Weltentwicklungsberichtes 1997 auf den Staat (»The State in a Changing World«, World Bank 1997). Angesichts des nach wie vor bestehenden grundsätzlichen Politikverbotes der Weltbank handelt es sich um einen bemerkenswerten Schritt, der zeigt, wie fließend die ursprünglich stark getrennten thematischen Komplexe im Kontext der Nachhaltigkeit ineinander übergehen können. Die Forderung nach einer stärkeren Rolle des Staates im Entwicklungsprozess, in den 1970ern hauptsächlich von marxistisch orientierten Entwicklungsforscher/innen zu hören, findet sich nun als einer der zentralen Ansatzpunkte der Weltbank: »Efforts to restart development in countries with ineffective states must start with institutional arrangements that foster responsiveness, accountability, and the rule of law« (ebd.: 157).

Wenig später erscheint das mittlerweile als entwicklungspolitisches Standardwerk zu bezeichnende »Do No Harm« von Mary Anderson (1999), das sich als einer der ersten Beiträge mit den potenziellen und realen Schwierigkeiten und Risiken entwicklungspolitischer Intervention in bewaffneten Konflikte auseinandersetzt und die Erfahrungen des ersten Schwunges ziviler Interventionen im Konfliktkontext, speziell von angloamerikanischen Gebern, auswertet. Das Konzept ist bemerkenswert, da es am entwicklungspolitischen Selbstverständnis kratzt. Anderson verweist nicht nur auf einfaches Scheitern an gesetzten Zielsetzungen in der Interventionspraxis. Sie arbeitet heraus, dass eine zivile Intervention auch zu schwerwiegenden Folgen in Konfliktszenarien führen kann und Entwicklungspolitik in einem solchen Umfeld jedenfalls selbst als Teil des Konfliktes gesehen werden muss: »When international assistance is given in the context of a violent conflict, it becomes a part of

ment in Africa« (Annan 1998) und behauptet, dass die Erarbeitung eines stabilen Friedens vorwiegend von den zwei Faktoren Good Governance und nachhaltige Entwicklung abhängig sei. Im Jahr 2001 schließlich schreibt Annan im Report »Prevention of Armed Conflict «: »Conflict prevention and sustainable development are mutually reinforcing« (Annan 2001: Abs. 11). 
that context and thus also of the conflict« (Anderson 1999: 1). Diese Feststellung ist in der Tat bemerkenswert, kontrastiert sie in ihrer Substanz doch mit den bis zu diesem Zeitpunkt üblichen oberflächlich gehaltenen »lessons learned«-Abschnitten der Evaluationsberichte, die gewöhnlich auf ein schlichtes »mehr Dinge besser machen« hinauslaufen. $^{21}$

Zwei Dinge stechen bei Andersons Kritik heraus und sind vermutlich auch der Grund dafür, dass ihr Text bei nahezu allen strategischen Papieren, die zu dem Thema - egal von welcher Institution - erscheinen, zitiert wird. Einerseits wird die zivile entwicklungspolitische Intervention22 eben als Teil des Konfliktes konzeptualisiert, was für einige eingefleischte Entwicklungshelfer/innen an einen Skandal grenzen muss. Diese auf der Analyse konkreter Konstellationen fußende Feststellung ist der konkrete Nachvollzug der normativen Anbindung von Sicherheits- und Entwicklungspolitik. Entwicklungspolitik kann sich dem Konflikt nicht entziehen, also ist eine gleich von Anfang an kohärent geführte Strategie mit Sicherheitspolitik, gerade auch mit ihrem militärischen Arm, angemessen. Auch wenn Anderson diese letzten Folgerungen nicht explizit zieht - sie werden sich in weiterer Folge ergeben.

Andererseits ist die bereits angesprochene Konsequenz, die Anderson einfordert, bemerkenswert. Es ist folgerichtig, sie mit dem Konzept der Nachhaltigkeit in Verbindung zu setzen. Eine Politik der Eindämmung erfordert genaueres Handeln als eine Politik der Kooptierung, speziell im Kontext bewaffneter Konflikte. Es ist nicht mehr möglich, sich in Quantitäten zurückzuziehen und versäumte Resultate sowie gescheiterte Messlatten als »verlorene Dekade« zu verkaufen. Nachhaltigkeit bedeutet präziseres Handeln, muss es bedeuten, da sich das Risikoniveau bei der Umstellung auf globales Containment unweigerlich anhebt. Trotzdem muss Entwicklungspolitik bereit sein, mit dem erhöhten Einsatz zu spielen: "we believe the good we are doing outweighs the harm« (ebd.: 38).

Die Nachwehen von 9/11 führen zu einem weiteren Auftrieb für den neuen Themenkomplex, diesmal im Zusammenhang mit der Terroris-

21 Nach Mary Anderson könnte die entwicklungspolitische Intervention laufende bewaffnete Konflikte auf zwei Arten verschärfen: »It can feed intergroup tensions and weaken intergroup connections. When aid has either of these impacts, it inadvertently exacerbates conflict. Conversely, aid can help war to end by lessening intergroup tensions and strengthening intergroup connections « (Anderson 1999: 69).

22 In ihrer Arbeit inkludiert Anderson auch den humanitären Sektor. Mit der Analyse von Katastrophen-Strategien von EZ und Humanitärer Hilfe hat sie sich zusammen mit Peter Woodrow bereits 1989 in »Rising from the Ashes« (Anderson/Woodrow 1998) auseinandergesetzt. 
musbekämpfung (vgl. etwa Klingebiel 2001). Allerdings handelt es sich bei den danach von nahezu allen größeren ODA-Agenturen weltweit eingeführten Anti-Terrorismus-Komponenten in den entwicklungspolitischen Programmen (etwa dem ATP-Programm der Deutschen Bundesregierung, das auf entwicklungspolitischer Ebene von der GTZ umgesetzt wurde) letztendlich um Konsequenzen eines schon lang vorher gestarteten Prozesses. 9/11 hatte also in dem Zusammenhang eher die Funktion eines späten Katalysators mit allerdings signifikanter Wirksamkeit. Diese Wirksamkeit bezieht sich vor allem auf die konsequente Weiterführung der sicherheitspolitischen Neuausrichtungen, die vor 9/11 mit den Konzepten von »humanitärer Intervention« und einem erweitertem Sicherheitsbegriff ihren Ausgang genommen hatte. Sowohl die EU als auch die USA entwerfen neue Sicherheitsstrategien, die diese Konzepte bündeln sollen.

Die Europäische Sicherheitsstrategie, ESS (vgl. EU 2003), schafft die Voraussetzung für eine enge Anbindung der Entwicklungspolitik an eine integrierte sicherheitspolitische Konzeption. Dazu wird das neue politische Schlagwort der Kohärenz bemüht und auf die neuen Bedrohungsbilder angewandt: »More Coherent. The point of the Common Foreign and Security Policy and European Security and Defence Policy is that we are stronger when we act together. [...] Diplomatic efforts, development, trade and environmental policies should follow the same agenda. In a crisis there is no substitute for unity of command « (EU 2003: 13). Angesichts als neuartig verstandenen Bedrohungsfeldern wie »New Wars" oder »Failed States«, die neue Konzepte von Sicherheit erforderten, wäre also die Kohärenz verschiedener damit befasster Policy-Bereiche (von Militär- über Außen-, Handels- bis hin zur Entwicklungspolitik) unter dem gemeinsamen Verständnis eines erweiterten Sicherheitsbegriffes oberstes Gebot (vgl. Reiter 2003: 319). Die ESS bringt diese Erfordernis markig mit dem militärischen Slogan »unity of command « auf den Punkt.

Auch die USA definieren die Zielbestimmungen ihrer nationalen Sicherheitspolitik auf internationaler Ebene wie aus einem Definitionskatalog von Good Governance: »the rule of law; limits on the absolute power of the state; free speech; freedom of worship; equal justice; respect for women; religious and ethnic tolerance; and respect for private property« (The White House 2002: 2). Ebenso bekommt die zivile Intervention als Teilbereich der »Foreign Aid« einen integralen Status im Kampf um die Erreichung dieser Ziele: $»[\mathrm{U}]$ se our foreign aid to promote freedom and support those who struggle non-violently for it, ensuring that nations moving toward democracy are rewarded for the steps they take«, denn es wäre unabdingbar »[to] make freedom and the de- 
velopment of democratic institutions key themes in our bilateral relations« (ebd.: 4).

Spätestens damit läuft der Mitte der 1980er Jahre eingeleitete Wandel zur globalen Forcierung von demokratischen Systemen auch in den offiziellen Zielbestimmungen auf festen Bahnen. Die Epoche der »comprehensive security« hat in der Entwicklungspolitik Einzug gehalten, ebenso wie sich die Idee der Nachhaltigkeit in der Sicherheitspolitik verankert hat.

\section{Post-politische Konfliktanalyse und die Etablierung neuer Interventionsstrategien}

Die konkrete Konfrontation von Entwicklung mit dem Phänomen bewaffneter Konflikt und die Kontextualisierung solcher bewaffneter Konflikte, ihrer Ursachen und ihrer Folgen im Feld der Nachhaltigkeit ist eine wesentliche Voraussetzung für die Konstituierung einer sicherheitspolitischen Entwicklungszusammenarbeit. Eine weitere notwendige Voraussetzung liegt, wie schon angeklungen ist, in einer neuartigen Erzählung von Bedrohungsbildern, vor allem dem im Zusammenhang mit Entwicklungspolitik besonders schlagenden Bild der gefährlichen Peripherie.

Die Konzeptualisierung einer solchen Gefahr ist zunächst eine Angelegenheit, die in den genuin sicherheitspolitischen Bereich fällt. Nach Ende des Kalten Krieges wandert die Aufgabe der Analyse und theoretischen Ausarbeitung jedoch merklich von klassischen geostrategischen Ansätzen der Internationalen Beziehungen hin zur Konfliktforschung. Neue Ansätze der Konfliktanalyse sprießen in der Folge aus dem Boden, die alle, wenn auch in unterschiedlicher Weise, ein Bild von bewaffnetem Konflikt generieren, das sich fundamental von demjenigen unterscheidet, das zur Zeit des Kalten Krieges dominierte. Der folgende Abschnitt wird sich mit diesen neuen Ansätzen auseinandersetzen und auch die verschiedenen Interventionskonzepte diskutieren, die sie bislang hervorgebracht haben.

Fassen wir die Aussagen aus der entwicklungspolitischen Szene zusammen, sind hauptsächlich vier Faktoren dafür verantwortlich, dass Konfliktprävention und Friedensentwicklung zu einem »neuen Aufgabenbereich« der Entwicklungspolitik (Ropers 2002: 2) werden konnten. Alle diese vier Faktoren hängen mit unmittelbaren oder mittelbaren Nachwirkungen des Endes des Kalten Krieges zusammen: die Tragödie von Ruanda 1994, das nach Ende des Kalten Krieges eingetretene Machtvakuum, die zunehmende Politisierung ziviler Interventionen und 
schließlich die Notwendigkeit für den entwicklungspolitischen Sektor, sich in der veränderten Gesamtkonstellation neu zu positionieren.

Der Anlass, und damit auch der erste Faktor, der für die neue Wahrnehmung einer »konzeptionelle Lücke« zwischen Entwicklungs- und Sicherheitspolitik (Matthies 2002: 123) verantwortlich zu machen ist, ist ein konkretes Ereignis, das wir im Begründungszusammenhang der neuen Bedeutung des Konfliktpräventionsbereiches nahezu immer an erster Stelle finden: die blutigen Ereignisse in Ruanda im Jahr 1994, die, so Thania Paffenholz (2001: 23), »zu einem Schlüsselerlebnis innerhalb der Entwicklungszusammenarbeit« werden.

Ruanda wird nicht nur in Deutschland (Brock et al. 2003: 20f.), sondern praktisch in der gesamten Geberlandschaft, neben den zwei weiteren zeitlich nahezu parallel stattfindenden humanitären Interventionskriegen in Somalia und Jugoslawien (Klingebiel 2001: 2), zum Wendepunkt (vgl. Ropers 2002: 14). Speziell im angloamerikanischen Raum dürfte Somalia angesichts der traumatischen Erfahrungen der gescheiterten Intervention vielleicht sogar ein noch größerer Stellenwert zukommen. Entscheidend ist jedoch, dass damit Mary Andersons Feststellung von 1999, dass sich Entwicklungspolitik der Problematik bewaffneter Konflikte nicht entziehen könne, konkretes Anschauungsmaterial erhält. Es wird aus dieser Situation heraus eine Notwendigkeit formuliert, politischer zu denken und die eigenen Interventionen in einem stärker politisch gefärbten Kontext zu verstehen.

Es ist eine Konsequenz dieser neuen Wahrnehmung, dass sicherheitspolitische Analysen in entwicklungspolitischen Bereichen an Relevanz gewinnen müssen. So kann als zweiter Faktor die Argumentation eines neu entstandenen Machtvakuums und seiner Konsequenzen herausgestrichen werden. Demnach hätte das Ende des Kalten Krieges in vielen peripheren Regionen, die vorher geopolitischen Einflusszonen zugeordnet und damit sicherheitspolitisch von Großmächten gestützt worden waren, ein politisches (vor allem sicherheitspolitisch verstandenes) Vakuum entstehen lassen (vgl. Anderson 1999: 9). Dieses Vakuum hätte nun in vielen Fällen den Weg für jenes Phänomen geebnet, das gegenwärtig prominent unter dem Begriff der »neuen Kriege« firmiert (vgl. Kaldor 2001; Münkler 2002).

Zugleich öffnet es den Blick auf die abgerissenen staatlichen Integrationsprozesse an der Peripherie, die vorher zumeist in einer Art künstlichen Aufrechterhaltung durch tatkräftige Unterstützung einer Hegemonialmacht überdeckt werden. Dieser neue Blick wird derzeit unter den Termini »weak/failed/fragile states« diskutiert (vgl. Milliken 2003) und sowohl aus der Perspektive eines aktiven State-Building (vgl. etwa DAC 2003: 16; Fukuyama 2004) als auch Nation-Building (vgl. Hippler 
2004) zum zumindest potenziellen entwicklungspolitischen Arbeitsfeld erklärt. $^{23}$

Der dritte Faktor hängt eng mit dem zweiten zusammen, insofern er sich auch auf das neu identifizierte Vakuum bezieht. Dieses hätte nämlich überhaupt erst die Möglichkeit zu einer verstärkten Politisierung ziviler Interventionen eröffnet. Zivile, speziell entwicklungspolitische Interventionen hätten zuvor, angesichts der durch den Kalten Krieg gegebenen geopolitischen Beschränkungen, primär technischen Charakter haben und sich so gezielten Interventionen in sicherheitspolitische Problemstellungen weitestgehend enthalten müssen. ${ }^{24}$ Abgesehen von den neuen Bedrohungsbildern findet sich hier auch die wesentliche Grundbedingung für die Diskussion der methodischen Erweiterung von Entwicklungspolitik auf Policy-Bereiche, die gemeinhin unter Sicherheitsund Verteidigungspolitik subsumiert werden. Der Begriff, der diese Vermengung vielleicht am ausdrucksvollsten zusammenfasst, ist jener der "globalen Strukturpolitik«, der in Deutschland (Fahrenhorst/Musto 2002: 177) und der Europäischen Union (EC 2001b: 10) gegenwärtig die Debatte bestimmt. ${ }^{25}$

Durch den neuen Spielraum können endlich auch jene »komparativen Vorteile« der Entwicklungspolitik eingesetzt werden, die sie gegenüber anderen Interventionsformen einzubringen hätte (vgl. Faust/ Messner 2004b: 409ff.): die bestehenden Erfahrung im Governance-Sektor, das hohe $\mathrm{Ma} ß$ an lokalem und regionalem Hintergrundwissen, und außerdem die Eigenschaft, in Konfliktsituationen die Rolle als »natürlicher Broker« (ebd.: 411) der unterschiedlichen Interessen, vor allem derer auf lokaler Ebene, aufzutreten.

Bei all diesen Argumenten ist durchzuhören, dass es der Entwicklungspolitik offenbar - und damit sind wir beim vierten Faktor - auch um eine eigene Neuposition in einem veränderten innenpolitischen Umfeld geht. Schließlich resultiert das Ende des Kalten Krieges zunächst einmal in einer signifikanten Neukalkulation von Budgetmitteln, speziell im außenpolitischen Bereich. Entwicklungspolitik (wie im Übrigen auch der Bereich der Sicherheits- und Verteidigungspolitik) ist nach dem Wegfall der Systemkonkurrenz und damit der ihr zufallenden Zuckerbrotfunktion im Gesamtkontext des Kalten Krieges mehr oder minder

23 Ich werde auf beide Themenkomplexe (Neue Kriege und schwache Staaten) weiter unten noch ausführlicher zu sprechen kommen.

24 Dies gilt primär für den europäischen Kontext, die Interventionen von USAID hatten demgegenüber zumeist klar formulierte politische Implikationen.

25 Oftmals findet sich auch der Begriff der »strukturellen Stabilität« (vgl. Ropers 2002: 46), der denselben inhaltlichen Komplex umreißt. 
dazu gezwungen, neue Arbeitsfelder zu lukrieren - gerade da die jetzt bestimmende Leitlinie der Nachhaltigkeit zu einer (zumindest in Relation gesehenen) Reduzierung der Mittel geradezu auffordert.

Komplementär dazu beginnt auch die markant anwachsende Gilde der Peace-Practitioner die Ansätze der großen, praxisorientiert ausgerichteten Namen der internationalen Friedensstudien-Lehrgänge wie Dieter Senghaas, Johan Galtung und John Paul Lederach ins Feld zu tragen, begleitet von rasant an Größe und Einfluss gewinnenden und auf den Konfliktbereich spezialisierten internationalen NGOs wie International Alert. Die neuen, spezifisch ausgerichteten Programme der ODA bieten dafür Möglichkeiten an Finanzierung, was allerdings auch dazu führt, dass von diesem Sektor ein zunehmender Druck in Richtung einer Finanzierung von spezifischen Peacebuilding-Aktivitäten durch den ODA-Sektor ausgeübt wird.

Mit der Definition der neuen Bedrohungsbilder aus dem sicherheitspolitischen Bereich (bei denen die budgetäre Situation sicher ebenfalls mit hineinspielt) ergeben sich dazu beachtliche Möglichkeiten. Auch wenn das damit einhergehende Verständnis von Kohärenz, das im Zweifelsfall zu einer Unterordnung entwicklungspolitischer Vorgangsweisen in einen militärisch dominierten Gesamtplan bedeuten würde, zu Widersprüchen in der entwicklungspolitischen Szene führt (vgl. Klingebiel/Roehder 2004; Knuchel 2004), scheinen sich die »nüchternen Eigeninteressen« (Matthies 2002: 124) auf Dauer durchzusetzen.

Zur selben Zeit findet auf wissenschaftlicher Ebene ein entscheidender Wandel in der für den thematischen Bereich relevanten Forschung statt. Dieser Wandel betrifft die Analyse von Konflikten, also genuine Aufgabenstellungen von der Sicherheits- und Verteidigungspolitik nahe stehenden Disziplinen wie den Internationalen Beziehungen. Verschiedene Strömungen, die in weiterer Folge zum Mainstream der Konfliktforschung aufsteigen, verdrängen die lange Jahre dominierende Lehrmeinung, wonach die meisten der internen Konflikte an der Peripherie als Widerspiegelung des Ost-West-Konfliktes begriffen werden sollten. ${ }^{26}$

26 Dass diese Einschätzung auch in den Hochphasen des Kalten Krieges alles andere als stimmig war, hat wie bereits erwähnt Richard Saull herausgearbeitet. Beachtenswert ist in dem hier vorliegenden Zusammenhang die Rolle der Sowjetunion als gegen revolutionäre Bewegungen an der Peripherie gerichtete Kraft: »Thus, in a number of crises that became >fronts in the Cold War, especially those outside the Eurasian landmass, the USSR was not directly involved and in some respects was hesitant if not opposed to supporting local revolutionary struggles« (Saull 2005: 259). 
Das klägliche Scheitern aller gängigen Theoriegebäude der Internationalen Beziehungen an jeglicher Vorhersage oder Antizipation eines Endes des Kalten Krieges bringt jene aus dieser Disziplin stammenden Ansätze der Konfliktanalyse, die sich vor allem auf den geopolitischen Zusammenhang berufen, in Verruf. In der Absage an das »Cold War paradigm« (vgl. Huntington 1996b: 56) wird damit ein Raum für neue oder wieder neu in die Diskussion gebrachte Ansätze geöffnet. Gleichzeitig existieren in diesem Raum nach dem tendenziellen Wegfall der klassischen geopolitischen Erklärungsmuster zunächst nur vage analytische Vorgaben. Diese wissenschaftliche Ausgangssituation fällt mit einem verstärkten Bedürfnis der politischen Entscheidungsträger zusammen, neue Ansätze zu benennen, die nicht nur den Weiterbestand bewaffneter Konflikte erklären können, sondern auch dazu dienen sollen, neue Bedrohungsbilder und damit eine Situation globaler Unsicherheit zu konstruieren. $^{27}$

Aus der Notwendigkeit, weiterhin griffige Feindbilder vermitteln zu können, werden die analytischen Vorgaben von der politischen Ebene schon bald auf relativ klar umrissene Bedrohungsszenarien zugespitzt. So skizziert etwa der Sicherheitsberater im ersten Kabinett Clinton, Anthony Lake, im September $1994^{28}$ : »The enemies of the tolerant society are not some nameless, faceless force; they are extreme nationalists and tribalists, terrorists, organized criminals, coup plotters, rogue states, and all those who would return newly freed societies to the intolerant ways of the past.« Ähnliche Bemerkungen sind im sicherheitspolitischen Bereich Mitte der 1990er Jahre an der Tagesordnung, wenngleich sich nicht viele durch eine derart kompakte Aufzählung der Bedrohungsszenarien auszeichnen.

Solche politischen Aussagen sind auch der Anstoß für einen Prozess, den ich als »Scramble for Paradigms« in der Konfliktanalyse bezeichnen und im Folgenden charakterisieren möchte. Dieser Wettlauf führt in den daran beteiligten wissenschaftlichen Disziplinen zu einer neuartigen Form des Branding von Begrifflichkeiten, die sich tendenziell nach den politisch vorgegebenen Bedrohungs- und Feindzuschreibungen ausrichten. Dieses Branding unterscheidet sich massiv von den politisch geprägten Debatten der Konfliktstudien bis zu den 1980er Jahren: politische und weltanschauliche Momente spielen keine entscheidende Rolle mehr, vielmehr geht es - vor dem Hintergrund der wechselseitigen Ak-

27 Mark Duffield (2005b: 7) sieht diese Neuinterpretation mit Anfang der 1990er Jahre als internationalen Konsens: »A new international consensus on the changed nature of war emerged in the early 1990s."

28 Lake macht diese Bemerkungen in einer Rede vor dem Council on Foreign Relations am 14. September 1994, in Washington D.C. 
zeptanz und Anerkennung - um die Prägung von prägnanten Konzepten, die sich vor allem durch eine spezifische Brückenfunktion in der Vermittlung zwischen akademischer, politischer, Policy- und Implementationsebene auszeichnen sollen. ${ }^{29}$ Abbildung 1 stellt jene Brands, die sich in diesem Kontext bislang behaupten konnten, in einer systematischen Einteilung entlang der Ebenen von Symptombenennung, Analyse, Diagnose und Therapie dar.

Abbildung 1: »Branding« der post-politischen Konfliktanalyse

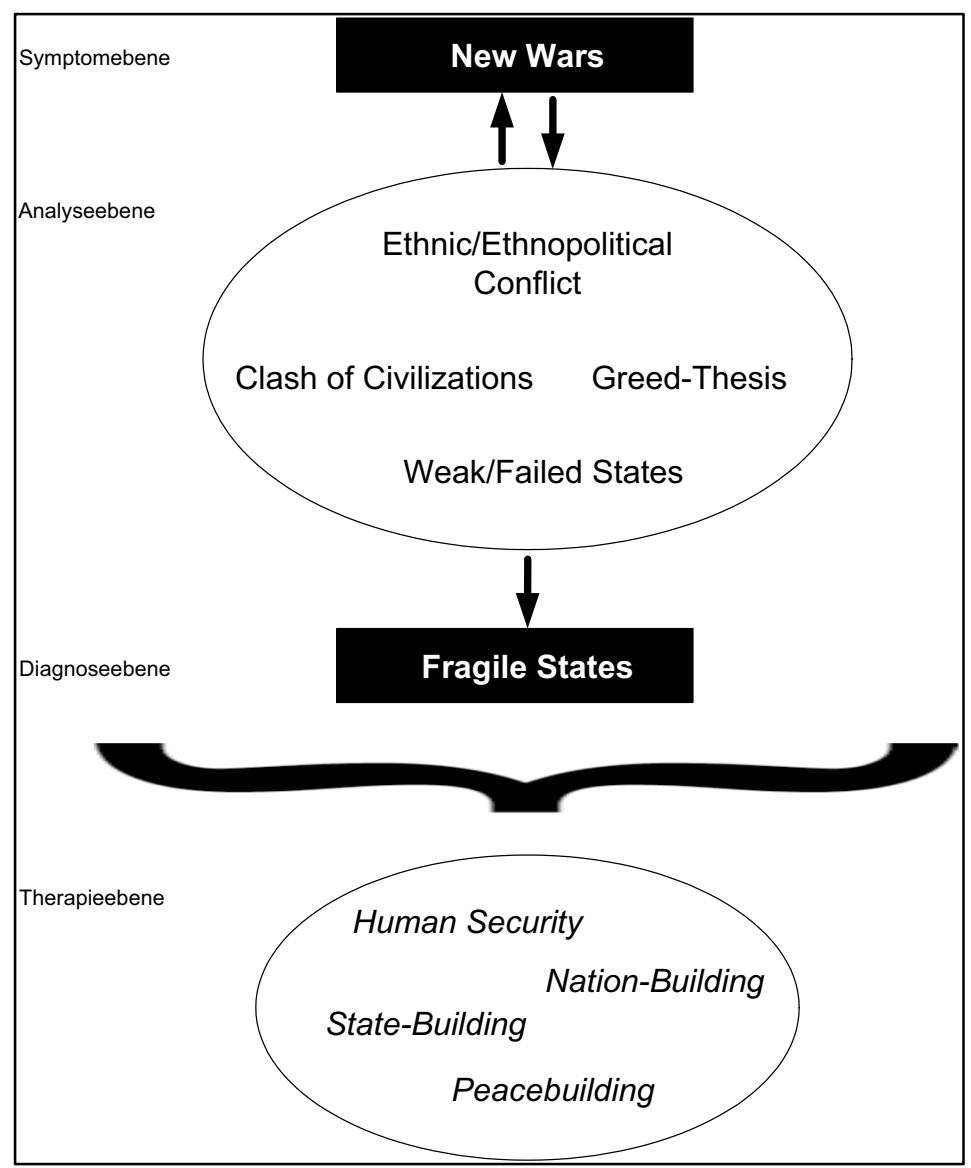

29 Eine vertiefte Analyse der Ursachen dieses Prozesses muss an dieser Stelle leider unterbleiben. Vermutlich ist die Steuerung policy-orientierter Forschung durch spezifische Grants ebenso ein relevanter Faktor wie der Bedeutungsdrang von beteiligten Wissenschaftler/innen. 
Die Abfolge der Schlagwörter in Abbildung 1 verläuft nicht chronologisch, sondern versucht, eine Systematik in Hinblick auf die Zielrichtung der dahinter liegenden Konzepte einzuführen. So ist die Samuel Huntingtons »Clash of Civilizations«-These historisch um einiges älter als der von Mary Kaldor geprägte Begriff der »New Wars«, dennoch ist Kaldors Begriff gewissermaßen vorgeordnet, versucht er doch, nicht eine Analyse anzubieten, sondern ein als neu verstandenes Phänomen zu beschreiben: »My central argument is that, during the 1980s and 1990s, a new type of organized violence has developed, especially in Africa and Eastern Europe, which is one aspect of the current globalized era. I describe this type of violence as >new war« (Kaldor 2001: 1).

Unzweifelhaft hätte Kaldor diesen Begriff ohne die bereits vorher existierenden neuen Analyseraster gar nicht entwickeln und in die Diskussion einbringen können. Seine schnelle Karriere - und vor allem auch die große Bandbreite an Beschreibungen und Analysen, mit der er gefüllt werden sollte - zeigt seine flexible Handhabbarkeit. Das ist der entscheidende Grund dafür, warum ich den Begriff auf der Symptomebene verorte. Seine erhebliche argumentative Kraft liegt darin, die Behauptung des »Neuen« in der Gestalt bewaffneter Konflikte zu untermauern, aber nicht, Erklärungs- oder Interventionsansätze zu liefern.

Die Erklärungsansätze verortet die Grafik auf der Analyseebene, wo jene vier Zugänge zusammengefasst sind, die in den 1990er Jahren zu großer Prominenz und weiter Rezeption gelangt sind und seither das Feld der Konflikt-, aber auch der Friedensforschung dominieren: die »Clash of Civilizations«-These von Samuel Huntington, die »Greed«These von Paul Collier, sowie die von einer Reihe von Autor/innen entwickelten Konzepte eines »ethnisch«, und/oder durch schwache oder gescheiterte Staatlichkeit bedingten Konflikts. ${ }^{30}$

Alle vier Ansätze spielen sowohl im Prozess der sicherheitspolitischen Neuausrichtung als auch in der entwicklungspolitischen Konfliktanalyse, wenn auch in unterschiedlicher Weise, eine wesentliche Rolle, weswegen es notwendig ist, sie genauer zu behandeln. Sie alle, insbesondere jedoch der Ansatz der schwachen/gescheiterten Staatlichkeit, bilden die unterschiedlichen theoriegeschichtlichen Vorläufer und Komponenten eines Begriffs, der sich anschickt, vor allem auf Ebene des politischen Diskurses zum Leitmotiv sicherheitspolitisch motivierter Interventionen zu werden: dem Begriff der »Fragile States«. Diesen will ich aufgrund seiner relativ geringen analytischen Kapazität, die mit einer

30 Ein »und/oder« ist an dieser Stelle insofern möglich, als sich diese Analyseansätze gegenseitig nicht ausschließen, sondern - mitunter sogar explizit - Elemente der anderen Ansätze mit einbeziehen. 
umso höheren Policy-Relevanz einhergeht, auf der Diagnoseebene verorten. Dort steht er insofern alleine, als sich die aus Sicht einer staatsorientierten Politikwissenschaft prinzipiell mögliche Vorgangsweise, dass jeder bewaffnete Konflikt notwendigerweise die vorhandenen staatlichen Strukturen, zumindest in der unmittelbar vom Konflikt betroffenen Region, schwächt, in zunehmender Weise in den relevanten PolicyDokumenten wiederfindet. Jede sicherheits- und entwicklungspolitische Intervention im Rahmen bewaffneter Konflikte wird damit quasi per Definition auch eine Tätigkeit unter der Bedingung fragiler Staatlichkeit.

Schließlich finden sich auf der Therapieebene jene Interventionsansätze, die in Form programmatischer Konzepte den gegenwärtigen Trend im bi- wie multilateralen entwicklungspolitischen Bereich dominieren. Während sich »State-Building« und, wenngleich in einem etwas anderen Kontext stehend, »Nation-Building« direkt auf die Problemdiagnose der schwachen Staatlichkeit beziehen, also eine strukturelle Intervention darstellen, ist die Ausrichtung der Idee von »Peacebuilding« eher auf der Akteursebene angesiedelt. »Human Security« wiederum versucht sich mittels einer qualitativen Erweiterung der Zielgruppen von Interventionen in einer tendenziellen Verschiebung der Akteursebene. Auch diese vier Ansätze werden im Verlauf dieses Abschnitts kurz vorgestellt, sie werden dann aber vor allem im Zuge der empirischen Untersuchungen genauer behandelt.

Das in seinen Aussagen am allgemeinsten gehaltene Konzept der neuen Konfliktanalysen in den 1990ern, das speziell im europäischen politikwissenschaftlichen Bereich schnell zu großer Popularität gelangt, ist jenes der »Neuen Kriege«. Es wird federführend von Mary Kaldor in ihrem 1999 erstmals erschienenen »New \& Old Wars - Organized Violence in a Global Era« (Kaldor 2001) entwickelt. ${ }^{31}$ Kaldor geht davon aus, dass es einen Bruch in der internationalen Kriegsführung gegeben habe, der zeitlich in den 1980er und 1990er Jahren, also mit Ende des Kalten Krieges, festgemacht werden kann (ebd.: 1). Die Bezeichnung der »Neuen Kriege« will sie als Abgrenzung von traditionellen Kriegsformen (im Besonderen dem zwischenstaatlichen Krieg) verstanden wissen, wobei sie damit speziell jene Formen meint, die bisher unter dem Begriff »low-intensity conflict« firmiert haben (ebd.: 2).

31 Mary Kaldor ist Direktorin des Centre for the Study of Global Governance an der London School of Economics. Wenngleich es übertrieben erscheint, ihre Rolle in der europäischen Politikwissenschaft mit derjenigen Samuel Huntingtons in den USA zu vergleichen, wäre die schnelle Verankerung des von ihr entworfenen Schemas ohne ihre schon davor bestehende relative Prominenz nicht erklärbar. 
Worum geht es nun bei diesen »Neuen Kriegen«? Nach Kaldor handelt es sich bei solchen Kriegen zwar sehr wohl um politische Prozesse, zugleich sei aber andererseits festzustellen, dass »[...] the new wars involve a blurring of the distinctions between war [...], organized crime [...] and large-scale violations of human rights [...] (ebd.: 2). Diese von ihr als neu behauptete Vermischung habe viel mit dem Prozess der Globalisierung zu tun, der neue Arten von Identitätspolitiken möglich, mitunter sogar notwendig machen würde.32 Herfried Münkler, der Kaldors Ansatz weiterzudenken sucht, definiert die Unterscheidung vorwiegend akteursbezogen: »Der klassische Staatenkrieg, der die Szenarien des Kalten Krieges noch weithin geprägt hat, scheint zu einem historischen Auslaufmodell geworden zu sein; die Staaten haben als die faktischen Monopolisten des Krieges abgedankt, und an ihre Stelle treten immer häufiger parastaatliche, teilweise sogar private Akteure [...] (Münkler 2002: 7).

Die »Neuen Kriege« seien faktisch ein politischer Ausdruck von Machtkämpfen, die sich auf einem neuen Terrain, der Vermischung eines wiederentdeckten Traditionalismus unter der Bedingung einer globalisierten Welt, abspielen würden:

»The political goals of the new wars are about the claim to power on the basis of seemingly traditional identities - nation, tribe, religion. Yet the upsurge in the politics of particularistic identities cannot be understood in traditional terms. It has to be explained in the context of a growing cultural dissonance between those who participate in transnational networks which communicate through e-mail, faxes, telephone and air travel, and those who are excluded from global processes and are tied to localities even though their lives may be profoundly shaped by those same processes« (ebd.: 69f.).

Schwerwiegende Folgen dieser Entwicklung seien etwa die Tendenz zur Privatisierung militärischer Strukturen (ebd.: 91ff.), oder der gezielte Einsatz von bevölkerungspolitischen Maßnahmen (wie etwa Vertreibungen oder Massakern) als Mechanismen politischer Kontrolle. Der folgenschwere Bruch, den die »Neuen Kriege« heraufbeschworen hätten, sei jedoch ihre Tendenz, sich jeglicher rechtlicher Kontrolle - und damit auch jeglicher Legitimität - zu entziehen (ebd.: 115). Dementsprechend liegt Kaldors zentraler Ansatz in der Argumentation für eine kosmopoli-

32 Es ist wichtig anzumerken, dass Mary Kaldor keinerlei Makrodaten zur Unterstützung ihrer allgemeinen Thesen präsentiert. Sie erzählt ihre Geschichte der »New Wars" vorwiegend am Beispiel Jugoslawien und garniert diese Erzählung mit weitgehend unzusammenhängenden Beobachtungen aus anderen Weltregionen. 
tische »Global Governance« (ebd.: 147ff.), wobei einer der zentralen Aspekte ein »cosmopolitan law enforcement« (ebd.: 124ff.), etwa im Rahmen der UNO, sein sollte. Einer globalisierten, also transnational verfassten Zivilgesellschaft schreibt Kaldor später eine Schlüsselrolle in der Umsetzung eines solchen Ansatzes zu (vgl. Kaldor 2003). Dabei setzt sie diese Idee explizit jedem partikularistischen Ansatz entgegen. ${ }^{33}$

So erfolgreich das Konzept auch im Sinne der Prägung eines sozialwissenschaftlichen Schlüsselbegriffes ist - jener Vorgang, den ich als Branding bezeichne - so schnell werden seine Unschärfen sichtbar, was bald Kritik hervorruft. Entlang dieser Unschärfen wird mitunter sogar der Sinn des Konzeptes an sich in Frage gestellt. Sabine Kurtenbach und Peter Lock (2004: 12) meinen etwa: »Die Unterscheidung zwischen alten und neuen Kriegen ist nicht nur ambivalent, sie ist auch ziemlich inhaltsleer, und ihre Fruchtbarkeit für die Schärfung unseres Blicks auf die Zeitgeschichte und die Strukturierung unserer Wahrnehmung erschöpft sich schnell.« Die Schwächen dieser Unterscheidung sind in der Tat sowohl auf empirischer als auch auf theoretischer Ebene evident.

Abbildung 2 verdeutlicht anhand der Daten der Hamburger Arbeitsgemeinschaft für Kriegsursachenforschung (AKUF) ${ }^{34}$ die historische Entwicklung, die die Kriegsformen weltweit seit 1945 genommen haben. ${ }^{35}$ Wir können dabei feststellen, dass sich die gegenwärtige Situation sehr gut mit den 1970er und 1980er Jahren - den Hochphasen des Kalten Krieges - vergleichen lässt. Der Ausschlag der Anti-Regime- (Typ A) und Sezessionskriege (Typ B) zu Beginn der 1990er Jahre ist nahezu vollständig auf die Kämpfe in der Folge des Zerfalls der Sowjetunion zurückzuführen, repräsentiert also eher ein punktuelles Ereignis als einen generellen Trend. ${ }^{36}$

33 Ausdrücklich hebt Mary Kaldor Samuel Huntington und Robert Kaplan als Vertreter jenes abzulehnenden Partikularismus hervor (Kaldor 2001: 142ff.), da sie deterministische Theorien vertreten würden.

34 Die Arbeitsgemeinschaft Kriegsursachenforschung (AKUF) ist an der Abteilung für Sozialwissenschaften der Universität Hamburg angesiedelt (http://www.akuf.de).

35 Die AKUF unterscheidet zwischen fünf unterschiedlichen Kriegstypen: Typ A sind Anti-Regime Kriege; Typ B Autonomie- und Sezessionskriege, Typ C zwischenstaatliche Kriege, Typ D Dekolonisierungskriege, und Typ E sonstige innerstaatliche Kriege (Typ E spielt nach 1945 keine Rolle und ist deshalb in der Grafik nicht wiederzufinden). Für eine genauere Definition siehe http://www.sozialwiss.uni-hamburg.de/publish/Ipw/Akuf/ kriege_aktuell.htm\#Def. (Zugegriffen am 15-04-2006).

$36 \mathrm{Zu}$ einem ähnlichen Schluss kommt auch der Human Security Report 2005, der die Situation seit Ende des Kalten Krieges als tendenziell friedlicher einschätzt, auch was die Ebene exzessiver Gewaltanwendung - beispielsweise Massaker - betrifft (Human Security Centre 2005: 1f., 23ff.). 
Abbildung 2: Kriegsformen im historischen Verlauf nach AKUF-

Kriegstypen

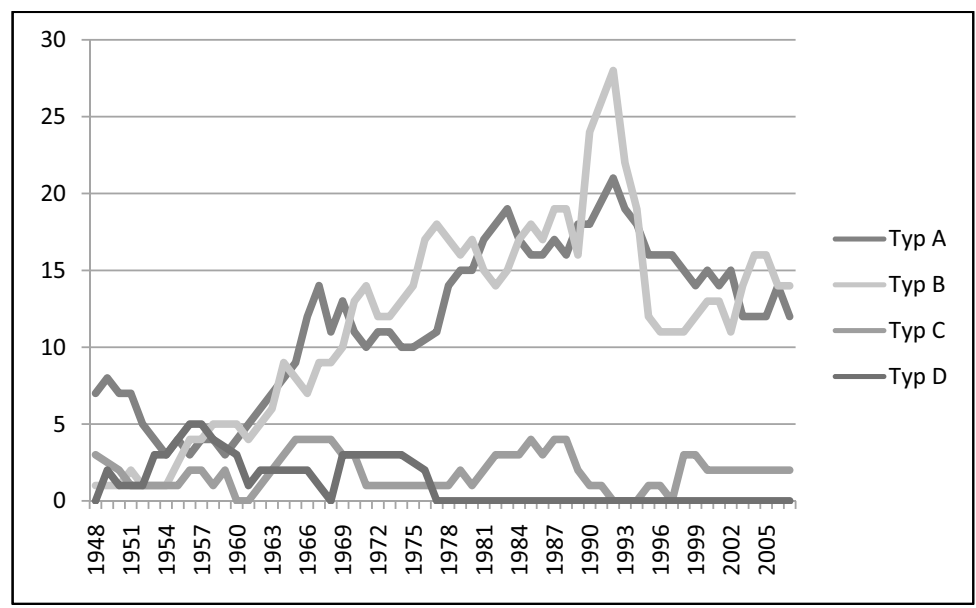

Quelle: AKUF (www.akuf.de)

Auch hat sich die marginale Rolle der zwischenstaatlichen Kriege (Typ C) seit Ende des Zweiten Weltkrieges nicht verändert, sondern zeigt vielmehr eine beeindruckende Konstanz. Dies bestätigt etwa der israelische Militärhistoriker Martin van Creveld. Nach seiner Rechnung wären etwa drei Viertel aller kriegerischen Auseinandersetzungen seit 1945 im Bereich niedriger Intensität geführt worden (van Creveld 1998: 45), wobei sich der historische Beginn dieser Form des Krieges mit dem bewaffneten Widerstand gegen den Nazi-Faschismus festgemacht ließe (ebd.: 96).

Kaldors Argument, dass sich die Form des Krieges in den letzten 20 Jahren signifikant verschoben hätte, ist gemäß diesen Daten also schwerlich haltbar. Ebenso problematisch wird es, wenn wir ihre strukturellen Behauptungen einer genaueren Prüfung unterziehen. Allem voran ist dies die Behauptung einer Vermischung zwischen Krieg, organisiertem Verbrechen und systematischer Menschenrechtsverletzungen, deren Zahl und Schwere als Konsequenz dieser Kriege nach Ende des Kalten Krieges eine neue Dimension angenommen habe.

Die erste der beiden Annahmen, die Neuheit einer Verbindung zwischen Krieg und organisiertem Verbrechen, bezieht sich vorwiegend auf die Idee einer »Kommerzialisierung der Gewalt» (Kurtenbach/Lock 2001: 21) oder mit einem Begriff von Georg Elwert, die Entstehung so genannter »Gewaltmärkte« (Elwert in Kievelitz/Poeschke 2003: 96ff.; Ehrke 2002: 143). Wenn wir uns vor diesem Hintergrund Charles Tillys 
Theorie der Entwicklung der europäischen Nationalstaaten als ursprünglich nicht intendiertes Resultat kriegerischer Prozesse in Erinnerung rufen, sehen wir allerdings, dass die Verbindung von Krieg und organisiertem Verbrechen so neu nicht sein kann: Ist einer der wesentlichen Motivationsgründe hinter Kriegen, gerade in der prä-nationalstaatlichen Epoche in Europa, ökonomischer Profit, so sind selbst mittelbare Praktiken des Krieges - seine Kosten und die daher notwendige Einführung von Steuerpflicht als einer faktischen Geiselhaft der in einem nun plötzlich eingegrenzten Territorium lebenden Menschen - nichts anderes als organisiertes Verbrechen. Auch der Umschlag hehrer Motive in eine Verselbstständigung der Gewalt ist spätestens seit den Erfahrungen im Dreißigjährigen Krieg alles andere als verschüttetes Spezialwissen der Geschichtswissenschaften.

Auch das zweite von Kaldor angenommene Charakteristikum "Neuer Kriege« ist keine Neuheit: der Krieg als systematische Verletzung menschlicher Rechte. Wir können uns dieser Frage von zwei Ebenen annähern, der empirischen und der philosophischen. Nachdem sich die empirische Ebene nicht grundsätzlich von bereits Gesagtem unterscheidet $^{37}$, ist es für unseren Zweck - der Herausarbeitung des besonderen Charakters der neuen Konfliktanalysen - interessanter, kurz die Frage nach der Stimmigkeit des philosophischen Gehalts dieser Annahme anzudiskutieren.

Wenn Mary Kaldor eine Neuartigkeit systematischer Rechtsverletzungen im Kriegsgeschehen der letzten grob 20 Jahre behauptet, behauptet sie damit auch, dass es eine derartige systematische Rechtsverletzung davor nicht gegeben habe, zumindest nicht in der heute feststellbaren Form. Dies wiederum impliziert die Möglichkeit, Krieg ohne derartige Rechtsverletzungen führen zu können, also die grundsätzliche Konzeptualisierung von Krieg als letztendlich rechtlichem Zustand.

Vorsichtig formuliert erscheint mir eine solche Idee zumindest fragwürdig zu sein. Es ist in diesem Kontext interessant, sich die Ausarbeitungen von Giorgio Agamben $(2002,2004)$ zum Problem des Ausnahmezustandes in Erinnerung zu rufen, einer Krieg oder bewaffnetem Konflikt vorgelagerten Situation. Agamben versteht - kurz gefasst - den Ausnahmezustand als einen nicht rechtlichen Zustand, der allerdings ohne eine Rechtsordnung, zu der er eben eine Ausnahme bilden würde, nicht existieren könne. Er ist also definiert durch ein Ganzes, dem er zugleich nicht angehört.

37 Wir könnten an dieser Stelle wiederum den Vergleich des Dreißigjährigen Krieges anführen, der sich in der Systematik seiner Rechtsverletzungen, gerade im individuellen Bereich, qualitativ ebenso wenig vom aktuellen Kriegsgeschehen unterscheidet wie beispielsweise der Zweite Weltkrieg. 
Wir können diese Annahme auf die Ebene des Krieges umlegen. Krieg ist so ein außerhalb des Rechtes stehender Prozess. Er kann konsequenter Weise in der Form des Rechtes auch nicht gefasst werden, wie das vollständige Scheitern aller Bemühungen, ein Kriegsrecht auf völkerrechtlicher Ebene wirksam zu verankern, demonstriert. In diese Richtung weist auch van Crevelds Definition von Krieg als einem Zustand totaler Freiheit: »Gewiss ist auch der Krieg in gewisser Hinsicht eine künstliche Tätigkeit, doch im Gegensatz zu den anderen räumt er völlige Freiheit ein, paradoxerweise auch die Freiheit vom Tod« (van Creveld 1998: 319).

Allerdings müssen wir eine notwendige Erweiterung einbeziehen. Diese Erweiterung betrifft den gesamthistorischen Kontext. Denn es ist sehr wohl möglich, Krieg vollständig im rechtsfreien Raum zu denken. Schließlich ist er ein Phänomen, das auch in nicht an Rechtskörper gebundenen Gesellschaften vorgefunden werden kann. Aber in einem solchen Kontext ist es folgerichtig auch nicht möglich, Krieg als Verletzung von Rechten zu verstehen.

So oder so, die von Kaldor zugrunde gelegte Annahme, es gebe einen an einen Rechtskörper gebundenen Krieg, der mithin auch die individuellen Rechte davon Betroffener nicht verletzen würde, ist kaum haltbar. Ebenso wenig sind die Erscheinungsformen neu, die sie unter dieser Kategorie subsumieren will. ${ }^{38}$ Es bleibt mithin die Frage offen, ob sich nun effektiv etwas verändert hat, und in jedem Fall, was Forscher/innen zu einer Annahme bewegt wie: »Inzwischen kann der Befund als gesichert gelten, dass sich die Formen kriegerischer Gewalt tatsächlich strukturell verändern« (Kurtenbach/Lock 2004: 21). Auch alle im Folgenden angeführten Ansätze - die »Clash of Civilizations«Theorie, die Greed-These, die These ethnischer oder ethnisierter Konflikte und der auf Staatlichkeit rekurrierende Erklärungsansatz - behaupten in der einen oder anderen Form eine solche strukturelle Veränderung.

Wenn wir nun mit stichhaltigem empirischem Rückhalt davon ausgehen können, dass sich der Gegenstand der Theorien - der bewaffneten Konflikt - nicht substanziell verändert hat, kommen nur zwei Möglichkeiten

38 »The literature of the $>$ new wars provides a great service in explaining patterns of contemporary conflict, and especially in drawing attention to the social and economic aspects of conflict and the relationship between security and development. However, much of this is not new: all of the factors that characterize new wars have been present, to varying degrees, throughout the last 100 years « (Newman 2004: 179; vgl. auch Brzoska 2004). 
in Frage: entweder hat sich der Wahrnehmungshorizont der Forscher/innen in der Weise verschoben, dass neue Aspekte in die Untersuchung miteinbezogen werden, die ein verändertes Licht auf bekannte Phänomene werfen, oder es haben sich die allgemeinen Bedingungen so verändert, dass sie einen Blick auf etwas bislang Vernachlässigtes freigeben. Oftmals treten diese beiden Prozesse parallel auf und bedingen einander. Es liegt nahe, auch im Fall der neuen Konflikttheorien ein derartiges Zusammentreffen anzunehmen.

Zunächst zu den Bedingungen: Tatsächlich berufen sich zahlreiche Arbeiten, die im Rahmen dieser neuen Strömungen der Konfliktanalyse entstehen, auf die Frage von Staatlichkeit, beziehungsweise ihrer Abwesenheit in vielen der untersuchten Konfliktkonfigurationen, und verknüpfen diesen so behaupteten Staatszerfall mit nach Ende des Kalten Krieges radikal veränderten Bedingungen des internationalen Systems. So schlägt Herfried Münkler vor, »Neue Kriege« als Staatszerfallskriege zu interpretieren (Münkler 2002: 20), und Mary Kaldor (2001: 78) rekurriert auf den Zerfall moderner Staatsstrukturen als eine Ursache der neuen Formen von bewaffnetem Konflikt ${ }^{39}: »$ The new identity politics arises out of the disintegration or erosion of modern state structures, especially centralized, authoritarian states. The collapse of communist states after 1989, the loss of legitimacy of post-colonial states in Africa or South Asia, or even the decline of welfare states in more advanced industrial countries provide the environment in which the new forms of identity politics are nurtured.«

Der Staat wird bei Kaldor zum einzigen wirksamen Schutzmechanismus vor bewaffneten Konflikten, deren spezifische Erklärung dann zumeist auf Besonderheiten zurückgeführt wird, die tendenziell in den Bereich der Irrationalität verschoben werden. ${ }^{40}$ Herfried Münkler, der

39 Wir finden zahlreiche ähnliche Belege für diese These (vgl. etwa Ehrke 2002: 138f.; Böge/Debiel 2003: 324). Kalevi Holsti (1996) behauptet zwar ebenfalls einen Zusammenhang zwischen Konflikten niedriger Intensität und Staatszerfall, kann in die Aufzählung allerdings insofern nicht einbezogen werden, als er (a) den Zeitrahmen anders legt, indem er den Beginn des Prozesses mit 1945 festsetzt (»The problem is that the Clausewitzian image of war, as well as its theoretical accoutrements, has become increasingly divorced from the characteristics and sources of most armed conflicts since 1945«, ebd.: 14), und (b) die speziellen Bedingungen der Staatlichkeit an der weltsystemischen Peripherie hervorhebt.

40 Es sei an dieser Stelle der Vollständigkeit halber erwähnt, dass Thomas Hobbes, dessen Annahme im Zuge solcher Argumentation oftmals schnell bei der Hand ist, ein denkbar schlechter Verbündeter ist, ist doch für ihn Krieg keine irrationale, sondern eine durch und durch rationale Angelegenheit: »And consequently it is a precept, or general rule of reason, that every man, ought to endeavour peace, as far as he has hope of obtaining it; 
selber mehr einer dem »Greed«-Ansatz von Paul Collier vergleichbaren ökonomistischen Rationalität zuneigt, erkennt darin Zweckorientierung: »Dass die ethnischen und religiösen Erklärungen der neuen Kriege so attraktiv sind, dürfte vor allem daran liegen, dass die Kriege damit, zumindest implizit, für irrational erklärt werden können: Wenn sie von antiquierten, unaufgeklärten Einstellungen und Motiven angetrieben werden, so liegt es nahe, ihnen mit dem Instrumentarium der Aufklärung zu Leibe zu rücken« (Münkler 2002: 160f.). Einen ähnlichen Schluss zieht Mark Duffield (2002: 88): »[...] the new wars have provided an opportunity to rediscover and reposition development as a second chance to make modernity work.«

Das Argument einer Zweckorientierung im Dienste der Moderne ist in der Tat äußerst stichhaltig, in jedem Fall stichhaltiger als das Argument der neuen Bedingungen von Staatlichkeit nach Ende des Kalten Krieges. Wie ich in der kurzen Diskussion der Frage der Nationalstaatsbildungsprozesse an der weltsystemischen Peripherie zu zeigen versucht habe, ist es nun keineswegs so, dass einstmals funktionierende Staatswesen seit Ende des Kalten Krieges reihenweise zusammenbrechen würden. Faktisch haben sie in der hier projizierten Form niemals funktioniert.

Vor diesem Hintergrund erscheint es also vielversprechender, von einer Wahrnehmungsverschiebung als der ursächlichen Triebfeder hinter den neuen Ansätzen der Konflikttheorie auszugehen. Denn obwohl alle vier Analyseansätze, die ich im Folgenden behandeln werde, zueinander nicht unbedingt kongruent sind und mitunter sogar heftig konkurrieren, eint sie ein gemeinsames Interesse, das fast programmatische Züge annimmt: die Entpolitisierung des Problemfeldes bewaffneter Konflikt.

Der wesentliche Schritt, der diesen neuen Ansätzen vorausgeht, ist somit nicht auf empirische Veränderungen zurückzuführen, sondern er interpretiert den Charakter des Gegenstandes radikal neu: Konflikt wird nicht länger aus politischen Kategorien heraus wahrgenommen. Einen politischen Charakter bekommt die Analyse ausschließlich dann, wenn es darum geht, Konflikt durch (zumeist internationale) politische Interventionen $\mathrm{zu}$ beeinflussen und damit einhergehende Entscheidungen über Legitimität und Illegitimität der beteiligten Akteure zu treffen. Auf diese Weise ist der endgültige Bruch mit den Analyserastern des Kalten Krieges vollzogen, die primär auf politische Problemlagen als Ausgangspunkt der Konflikte Bezug nahmen. ${ }^{41}$ Derartiges wird bei »Neuen

and when he cannot obtain it, that he may seek, and use, all helps, and advantages of war« (Hobbes 1996: 87).

41 Damit wurde im Übrigen auch die Artikulation innerhalb der Konflikte selbst verändert. Diese richtet sich mit dem Blickpunkt auf die globale Öf- 
Kriegen« nicht mehr angenommen. Vielmehr sind nun andere Faktoren entscheidend - zivilisatorische, ethnische, religiöse oder ökonomische. Dazu ist es vollkommen unwesentlich, inwieweit sich bewaffnete Konflikte mit politischen Programmatiken artikulieren oder nicht. Ebenso scheint es von untergeordneter Bedeutung, ob nun »rationale« (sprich: ökonomische) oder »irrationale« Faktoren für ihr Entstehen und ihre Verläufe hauptverantwortlich gemacht werden.

Der entscheidende Punkt ist, dass Konflikte nicht von einem politischen Standpunkt betrachtet und damit automatisch und a priori mit der Zuschreibung von Illegitimität versehen werden. ${ }^{42}$ Erst das ermöglicht das neue Spiel der Legitimierung von Akteuren in diesen Konflikten entlang der Regeln, die von den Zentren vorgegeben werden. Die neuen Konfliktanalysen, die ich als post-politisch bezeichnen will, befähigen die flexible Parteinahme bar jedes politischen Bezuges bei der gleichzeitigen Verankerung universell gedachter Lösungsansätze.

Die post-politischen Konfliktanalysen füllen jenen Raum, der durch das Ende des Kalten Krieges, oder vielleicht präziser, durch das Scheitern der meisten gängigen Ansätze am Ende des Kalten Krieges geschaffen wurde. Daher können sie auch bei jener sicherheitspolitischen Neuausrichtung mitbestimmend werden, die aus den neuen Gegebenheiten folgen muss. Es ist interessant zu beobachten, wie ähnlich sich im Zuge dieses Prozesses Sicherheitspolitik und Entwicklungspolitik verhalten. Die Idee der Nachhaltigkeit, verstanden als sicherheitspolitisches Containment mit Mitteln der Entwicklungspolitik, findet in der Konstruktion neuer Bedrohungsbilder, die von einem nun als irrational oder zumindest illegitimen Bild von bewaffnetem Konflikt an der Peripherie geprägt werden, ihre Entsprechung - konstruiert als eine entwicklungspolitische Herausforderung an die Sicherheitspolitik.

fentlichkeit immer auch an Wahrnehmungen von außen aus. Konfliktanalyse ist somit keineswegs einfach eine lineare Bestandsaufnahme von Bestehendem. Im asymmetrischen Verhältnis des Weltsystems prägt sie selbst die Erscheinungs- und Verlaufsformen der Konflikte mittels ihres Einflusses auf die politische Wahrnehmung und Interventionstätigkeit der Zentren mit.

42 Mark Duffield schlägt gegenüber post-politischen Begriffen wie jenem der »Neuen Kriege« den Begriff des post-nationalstaatlichen (und damit auch postmodernen) Konfliktes vor, um einerseits die Grenzen der traditionellen Konfliktanalyse zu brechen, andererseits die Irrationalitätszuschreibungen der neuen analytischen Stränge zu vermeiden: »Post-nation-state conflict suggests the appearance of nonstate or qualified state political projects that no longer find it necessary to project power through juridical or bureaucratic forms of control« (Duffield 2000: 74f.). 
Der erste neuartige und griffige Slogan, der in diesem Prozess hervorgebracht werden sollte, ist jener vom »Clash of Civilizations«. In einem allein in seinem Ablauf bemerkenswerten Versuch, einen neuen paradigmatischen Strang der Konfliktanalyse, aber auch der Internationalen Beziehungen allgemein zu etablieren, veröffentlicht Samuel Huntington 1993 in einem mittlerweile berühmten Artikel in Foreign Affairs (Huntington 1996a; als erste Replik auf die Kritiken Huntington 1996b) die These, dass sich die zukünftigen Bruch- und Konfliktlinien an den Grenzen von »Civilizations « ${ }^{43}$ entzünden würden: »Nation states will remain the most powerful actors in world affairs, but the principal conflicts of global politics will occur between nations and groups of different civilizations. The clash of civilizations will dominate global politics. The fault lines between civilizations will be the battle lines of the future« (Huntington 1996a: 1).

\section{Abbildung 3: Die »Civilizations« nach Huntington}

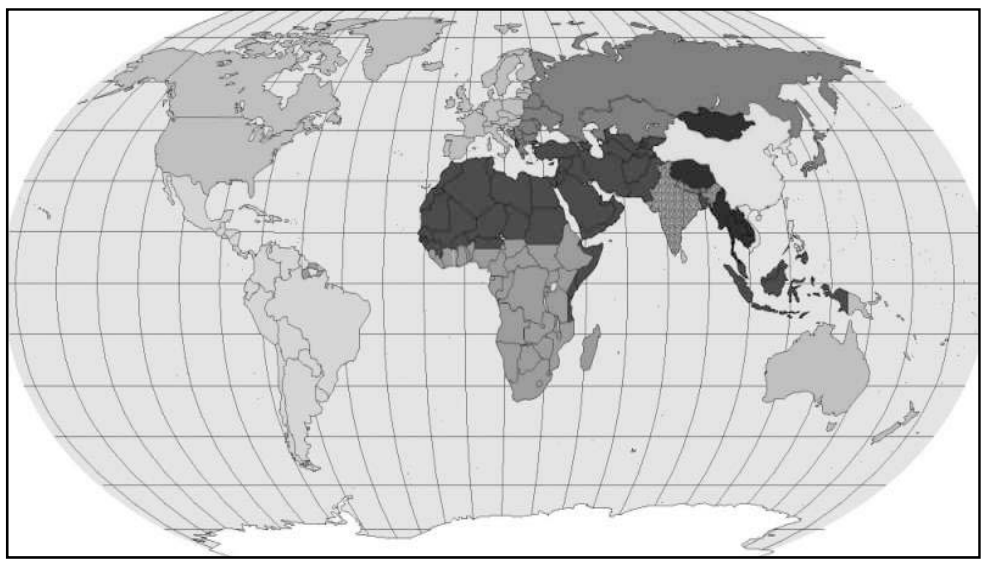

Quelle: Huntington 2003: 26-27, Map 1.3

In der Folge wird Huntington bis zu neun verschiedene derartige »Civilizations « unterscheiden (siehe Abbildung 3): eine westliche, lateinamerikanische, afrikanische, islamische, chinesische, hinduistische, orthodoxe, buddhistische und, zumindest seit der Veröffentlichung des Buches,

43 Ich will den Begriff »Civilization« nicht übersetzen, sondern im englischen Original belassen. Weder die oftmals verwendete Übersetzung mit dem Begriff »Kultur« (Huntingtons später erschienenes Buch wurde mit »Kampf der Kulturen« übersetzt), noch die Beibehaltung des ZivilisationsBegriffes im Deutschen geben das wieder, was Huntington in seiner Definition in dem Begriff auf den Punkt bringen will. 
eine japanische. ${ }^{44}$ Seine These ist dabei nicht als eine Konsequenz aus einer Fehleranalyse der Phase des Kalten Krieges zu verstehen. Vielmehr geht es ihm um die Verankerung eines neuen klaren Paradigmas, das die Zeit des Kalten Krieges mit ihrer an West- oder Ostbindung festgemachten Einteilung abzulösen hätte. Nun ginge es um eine neue Phase der Geschichte: »Conflict between civilizations will be the latest phase in the evolution of conflict in the modern world « (Huntington 1996a: 1).

Was sind nun diese »Civilizations«? Auf jeden Fall primär kulturell bestimmte Einheiten (»cultural entity«, vgl. ebd.: 2), die aber doch einen weitergehenden Charakter haben als der deutsche Kultur-Begriff suggerieren würde. Wesentliche Faktoren sind in jedem Fall Sprache (Huntington 2003: 59ff.) und Religion (ebd.: 64ff.). Zugleich hat das Konzept eine nahezu universelle historische Geltung. Huntington versteht »Civilizations « als einer Art Grundkategorie der historischen Entwicklung seit der prähistorischen Epoche. Entscheidend in der gegenwärtigen Phase sei aber nun, dass die Ablehnung der Bestrebungen der westlichen Modernisierung speziell in der Phase nach Ende des Kalten Krieges zu einer Rekulturalisierung von Identitätspolitik geführt hätte: »During the Cold War a country could be nonaligned, as many were, or it could, as some did, change its alignment from one side to another. [...] In the new world, however, cultural identity is the central factor shaping a country's associations and antagonisms « (ebd.: 125). ${ }^{45}$

Aus unterschiedlichen Gründen müssten diese »Civilizations« nun aufeinanderprallen (vgl. Huntington 1996: 4ff.): zunächst aufgrund des grundsätzlichen Charakters ihrer Unterschiedlichkeit und der nach Ende des Kalten Krieges in neuer Form bestehenden Möglichkeit, auf sie Bezug zu nehmen ${ }^{46}$, dann aber auch angesichts verschiedener Aspekte, die gemeinhin unter dem Begriff der Globalisierung zusammengefasst werden. Einerseits würde die Welt ein »smaller place« werden, andererseits hätte aber auch die Modernisierung zu einschneidenden Veränderungen

44 Tatsächlich verwundert die Beliebigkeit, mit der Huntington die »Civilizations «, die doch »not only real«, sondern auch »basic« wären (Huntington 1996a: 4), einteilt. Finden sich anfangs noch sieben derartige Entitäten, sind es in späteren Phasen acht und letztendlich neun, wobei speziell der ostasiatische Raum immer weiter unterteilt wird.

45 Jugoslawien ist in diesem Muster der Modellfall jener Entwicklung, wo ein Staat, der sich im Widerspruch zu den zwischen den »Civilizations« liegenden Bruchlinien entwickelt hatte, auseinander brechen muss, um neuen, den Bruchlinien entsprechenden Nationalstaaten Platz zu machen (vgl. Huntington 2003: 259ff.).

$46 »[\ldots]$ cultural characteristics and differences are less mutable and hence less easily compromised and resolved than political and economic ones« (Huntington 1996a: 5). 
auf subjektiver Ebene geführt, »separating people from longstanding local identities« (ebd.).

Die erstaunlichste Einschätzung von Huntington ist jedoch jene zu den Wirkungen der westlichen Zivilisierungsbemühungen. Zunächst würden sie durch ihre Konfrontation mit Bestrebungen anderer »Civilizations «, die Welt in anderer Weise zu gestalten, entscheidend zum Aufeinanderprall beitragen. ${ }^{47}$ Allerdings wären auch die dahinter liegenden Grundannahmen eines Universalismus fundamental zu hinterfragen:

» The concept of a universal civilization is a distinctive product of Western civilization. In the nineteenth century the idea of sthe white man's burden helped justify the extension of Western political and economic domination over non-Western societies. At the end of the twentieth century the concept of a universal civilization helps justify Western cultural dominance of other societies and the need for those societies to ape Western practices and institutions« (Huntington 2003: 66).

Eine solche Eurozentrismus-Kritik ist bemerkenswert, auch wenn nicht vergessen werden sollte, dass Huntington mehrmals klar zu erkennen gibt, selbst die westliche »Civilization« für die beste der gegebenen Möglichkeiten zu halten. Dennoch untergräbt eine solche Einschätzung die verschiedenen Zivilisierungsbemühungen, im Speziellen diejenigen, die die Entwicklungspolitik gerade versucht, als ihr Arbeitsfeld zu vereinnahmen.

Huntingtons Vorschläge für eine Lösung der sich an den Grenzen der Zivilisationen entspannenden Konflikte, so genannter »fault line wars«, sind in der Tat andere. Einerseits sei ein Maß an Erschöpfung der kämpfenden Kräfte vonnöten, andererseits wäre aber auch die Mediation einer dritten Partei, die keine Eigeninteressen verfolgen würde, oftmals von Nutzen (vgl. ebd.: 291ff.). Ein direkter Eingriff in lokale oder regionale Strukturen findet hier ebenso wenig Platz wie Versöhnungsprozesse oder lokale Sicherheitsarrangements. Frieden wird von oben gemacht: »Fault line wars bubble up from below, fault line peaces trickle down from above« (ebd.: 298).

Dieser an das klassische Repertoire der Diplomatie gemahnende Ansatz spielt in der Praxis nach wie vor eine wesentliche Rolle. Er kann demnach nicht als wirkliche Innovation gelten. Die hervorstechenden Wirkungen von Huntingtons Vorschlag, speziell auf entwicklungs- und sicherheitspolitischer Ebene, liegen woanders. Denn tatsächlich hat

$47 \gg$ A West at the peak of its power confronts non-Wests that increasingly have the desire, the will and the resources to shape the world in nonWestern ways« (Huntington 1996a: 5). 
Huntington mit Wirkung seines klingenden Namens und der breiten Rezeption seiner effektiv veröffentlichten und provokativ auf maximale Verbreitung angelegten These einen bemerkenswerten politischen Effekt erzielt. In der teils vehementen Ablehnung seiner relativistischen Position bezeugen die meisten seiner Kritiker/innen nicht nur ihre enge Verbundenheit mit universellen Wertgebäuden, sondern auch mit der konkreten Herrschaftsform der repräsentativen Demokratie.

Damit gelingt eine wesentliche Verknüpfung, die in Hinblick auf die Aufrechterhaltung einer hegemonialen Situation auf globaler Ebene signifikante Wirksamkeit entfaltet: die Verbindung der Ablehnung grob ethnizistischer (und im Extremfall nur oberflächlich verschleierter rassistischer und orientalistischer) Weltbilder mit der Propagierung von westlichen Wert- und politischen Strukturmustern. ${ }^{48}$ Huntington selbst wird damit zu nicht mehr als einem Advocatus Diaboli, der das Dispositiv der globalen Ordnung mit der Provokation einer Wirkung zweiter Ordnung auf eine neue Ebene hebt.

Huntingtons Ansatz ist letztendlich eine radikale Interpretation eines relativ alten Zuganges in der Analyse von Krieg und bewaffneten Konflikten: der These vom »ethnischen Konflikt«, deren Wurzeln in der kolonialistischen Wahrnehmung kolonialisierter Gebiete und deren Bevölkerungen liegen (vgl. Mamdani 1996: 61). Ab den 1960er Jahren wird der Ansatz in den Sozialwissenschaften verstärkt bearbeitet (vgl. Horowitz 2000: 6ff.). Mitunter wird Huntingtons These sogar als Teil dieses Analyserasters wahrgenommen (vgl. etwa Bowen 1996: Fn 1), was mir angesichts des Gehalts der Ansätze als eine unscharfe Wahrnehmung erscheint: während sein Begriff der »civilizations« einen großen Maßstab anlegt und auch die eigene »Civilization« miteinbezieht, zielt der Begriff der Ethnie, wie Mahmood Mamdani (1996) zeigt, nach außen: Ethnische Kategorien sind in ihrer historischen Entwicklung Unterscheidungsmerkmale für all jene, die es nicht auf das zivilisierte Niveau der Bürgerschaft gebracht haben. Dennoch ist letztendlich nicht dieser Unterschied dafür verantwortlich, dass ich die beiden Ansätze getrennt behandle. Dies liegt vielmehr daran, dass es beiden Konzepten gelungen ist, recht erfolgreiche Marken - Brands - zu etablieren, die nicht nur im

48 In der Tat ist Huntingtons Argumentation als orientalistisch im Sinne Saids zu klassifizieren. Seine Aussagen laufen im Zusammenspiel mit seinen persönlichen Positionierungen darauf hinaus, dass ein Großteil der gesellschaftlichen Strukturen der Welt nicht demokratiefähig ist. Das ist ein wesentlicher Unterschied zu einer postkolonialen Positionierung, die sich fragt, ob die repräsentative Demokratie als politisches Instrument von Herrschaft im globalen Rahmen angemessen ist. 
wissenschaftlichen, sondern auch im Policy-Bereich derzeit nicht wegzudenken sind.

Das Schlüsselwerk zur Etablierung des Begriffes »ethnischer Konflikt« entsteht im Vergleich mit den anderen drei zentralen Ansätzen verhältnismäßig früh: 1985 veröffentlicht der amerikanische Politologe Donald L. Horowitz, der sich schon seit Ende der 1960er Jahre, vor allem anhand afrikanischer Beispiele, Sri Lanka, und der »Rassenunruhen« in den Vereinigten Staaten mit der Frage von Ethnizität und Konflikt auseinandergesetzt hat, sein »Ethnic Groups in Conflict«, das mittlerweile zum Standardwerk des Ansatzes geworden ist. Dafür mitentscheidend ist sicher der Zeitpunkt seiner Veröffentlichung: Mit dem Ende des Kalten Krieges und dem darauf folgenden Ausbruch der Verteilungskämpfe in Ex-Jugoslawien und der ehemaligen Sowjetunion nimmt die Bedeutung des Konzeptes Anfang der 1990er, also nur wenige Jahre nach der Herausgabe des Werkes, schlagartig zu. So schreibt Horowitz im Vorwort zur zweiten Ausgabe im Jahr 2000: »Since this book was first published, ethnic conflict has established itself ever more securely as a subject worthy of serious study. There can be no doubt that events have driven scholarship. The most significant events followed the demise of the Soviet Union and of the former Yugoslavia« (Horowitz 2000: xi).

Tatsächlich haben diese beiden »signifikanten Ereignisse« zu zahlreichen Stellungnahmen geführt. Besonders effektvolle journalistische Beschreibungen wie »Balkan Ghosts« von Robert Kaplan ${ }^{49}$ (1994), dessen Effekt allerdings wesentlich dadurch gesteigert wird, dass sich Bill Clinton in seiner Funktion als US-Präsident öffentlich mit dem Buch in der Hand zeigt, populärwissenschaftliche Arbeiten wie von Michael Ignatieff (1993, 1997) und nicht zuletzt mediale Berichterstattung (vgl. Seaton 1999) führen zu einem Popularitätsgewinn und einer Steigerung des Einflusses in der akademischen Szene. ${ }^{50}$ Forschungsschwerpunkte

49 Robert Kaplan ist derzeit Senior Fellow an der New America Foundation und Essayist in verschiedenen einflussreichen US-Zeitungen und Magazinen. Ursprünglich Soldat bei der israelischen Armee und später Journalist, bewegt er sich seit Jahren an der Grenzlinie zwischen provokativem Reisejournalismus und Populärwissenschaft, speziell seit 9/11 mit großem Erfolg.

50 So schreibt etwa Robert Wimmer (1997: 631) unter weitgehender Vernachlässigung von zu diesem Zeitpunkt schon zugänglichen empirischen Fakten des »Minorities at Risk«-Projekts (s.u.): »Since the end of the Cold War, ethnonationalist conflicts have outweighed all other forms of political confrontation.« 
und -projekte, spezielle Professuren und Institute sowie Journals ${ }^{51}$ werden eingerichtet, die die Bedeutung des Ansatzes weiter steigern. »Today, the study of ethnic conflict is a major growth industry « (Gilley 2004: 1155).

Zugleich führen die neuen politischen Rahmenbedingungen nach Ende des Kalten Krieges, vor allem die neue Rolle von peripherer Staatlichkeit, auch zu inhaltlichen Uminterpretierungen, die in Richtung einer Universalisierung des Ansatzes streben: »ethnic conflict possesses elements of universality and uniformity that were not present at earlier times« (Horowitz 2000: 5). In diesem Zusammenhang entwickelt sich eine immer größere Nähe zur Nationalismusforschung, die insbesondere der britische Nationalismustheoretiker Anthony D. Smith, ein Schüler von Ernest Gellner, verkörpert. Sein »The Ethnic Origins of Nations« (Smith 1987) schafft dafür die inhaltlichen Voraussetzungen. Smith (1998: 222ff.) liefert auch den besten Überblick über die AnalyseAnsätze von Nationen und Ethnien, wobei er zwischen primordialen, perennialen, symbolisch-instrumentalistischen, modernen beziehungsweise modern-konstruktivistischen und postmodernen Zugängen unterscheidet.

Die auf Policy-Ebene derzeit erfolgreichste Ausprägung des Ansatzes findet sich im Begriff des »ethnopolitischen Konfliktes«. Dazu werden auf Basis der Annahme, dass »Ethnie« ein für politische Zwecke mobilisierbares Muster kollektiver Identitätsbildung darstellt, konstruktivistische und instrumentalistische Zugänge verbunden. Zentral ist es etwa für die Arbeit der deutschen Berghof-Stiftung, die das von ihr herausgegebene und speziell in Europa sehr einflussreiche Konflikttransformations-Handbuch unter das Motto »Transforming Ethnopolitical Conflict« (vgl. Austin et al. 2004) gestellt hat.

Der Ursprung einer solchen Interpretation der Verbindung von Ethnizität und Konflikt ist allerdings älter. Schon Anfang der 1980er Jahre setzt sich Donald Rothschild in seinem 1981 erschienenen Werk »Ethnopolitics: A Conceptual Framework« ausführlich mit dem Begriff auseinander und begründet dessen wissenschaftliche Grundlagen. Rothschilds Konzept wird ein Jahrzehnt später von Ted Robert Gurr dem »Minorities at Risk«-Projekt an der Universität Maryland zugrunde gelegt und weiterentwickelt. Dieses Projekt untersucht die Entwicklung von als »ethnopolitisch« klassifizierten Konflikten im globalen Rahmen

$51 \mathrm{Zu}$ diesen Journals zählen etwa Nations and Nationalism, Ethnicities, Nationalism and Ethnic Politics und Ethnic Conflict Research Digest (vgl. Gilley 2004: Fn 1). 
und gilt bis heute als die Schlüsselarbeit zu diesem Ansatz. ${ }^{52}$ Speziell für die Berghof-Stiftung, aber auch für die meisten anderen, die sich auf das Konzept »ethnopolitischer Gruppen« beziehen, ist Gurr und das von ihm geleitete Projekt nach wie vor der zentrale Referenzpunkt. ${ }^{53}$

Der analytische Ansatz des Projektes wird zusammen mit den ersten empirischen Ergebnissen im Jahr 2003 veröffentlicht (vgl. Gurr 2000a). Als Herausgeber fungiert dabei bemerkenswerter Weise das USIP (United States Institute for Peace), einer der zentralen US-amerikanischen Think Tanks im Peacebuilding-Bereich, was die praktische Relevanz des Ansatzes verdeutlicht. Ted Robert Gurr hat in den 1980er Jahren den Ethnizitäts-Begriff vor allem konstruktivistisch bearbeitet, tendiert im Zuge der Arbeit an dem Projekt allerdings in Richtung einer instrumentalistischen Interpretation, die auch essentialistische Züge aufweist: »Ethnic identities are not >primordial but nonetheless based on common values, beliefs and experiences. They are not sinstrumental but usually capable of being invoked by leaders and used to sustain social movements that are likely to be more resilient and persistent than movements based solely on material or political interest « (ebd.: 5).

Offenkundig geht Gurr also davon aus, dass »ethnischen« Kategorien eine im Vergleich zu anderen Kategorien signifikant höhere Persistenz innewohnt. Damit unterscheidet er sich aber nur mehr graduell von jenen primordialen Zugängen, die er kritisiert, ebenso wie er die instrumentelle Funktion der Kategorien hervorhebt, um sich gleichzeitig von instrumentalistischen Ansätzen zu distanzieren. Zugleich demonstriert diese Definition auch den entscheidenden Unterschied zwischen den Kategorien »ethnisch « und »ethnopolitisch ${ }^{54}$ in der Konfliktanalyse: So ist es tatsächlich erst »die Politisierung ethnischer Merkmale«, der eine »Schlüsselrolle im Konfliktprozess« zukomme, und nicht ethnisch konstruierte Kriterien an sich (Ropers 1995: 1). Die in Bezug auf bewaffnet ausgetragene Konflikte relevantesten ethnopolitischen Gruppen - und

52 Die Arbeit des noch immer laufenden Forschungsprojektes ist unter der Website http://www.cidcm.umd.edu/mar/ einsehbar (Zugegriffen am 2705-2007).

53 Auch Immanuel Wallerstein verwendet das Konzept der »Ethnopolitik«, insbesondere in seinen Analysen zur Frage von »Rassen « und Status- oder Identitätsgruppen (vgl. Wallerstein 1990a). So spricht er in Bezug auf afrikanische Staaten von »eine[r] ganze[n] Reihe ethno-politischer Rivalitäten « (Wallerstein 1990b: 231). Im Unterschied zu Gurr unterlässt er jedoch eine systematische Ausarbeitung und Propagierung des Ansatzes.

54 Die kürzeste Definition, die Gurr für das Konzept liefert, lautet: »Ethnopolitical groups are identity groups whose ethnicity has political consequences, resulting either in differential treatment of group members or in political action on behalf of group interests« (Gurr 2000a: 5). 
hier kommt der Eindruck der Auflösung Jugoslawiens und der Sowjetunion ebenso wie die enge Anbindung an die Nationalismus-Forschung voll zur Geltung - sind ethnonationalistische: »Nationalism is a distinctively modern doctrine that links ethnic identity to a hegemonic political program« (Gurr 2000a: 6). ${ }^{55}$

In der Verbindung von ethnopolitischen Gruppen mit Nationalismus greift Gurr, ebenso wie zahlreiche andere, speziell aus dem praktizistischen Bereich kommende Analysen und Darstellungen, auf Konzepte des seit den 1950ern in den USA tätigen »Ethno-Psychoanalytikers« Vamik Volkan zurück. Volkans Ansatz beruht auf der Anwendung psychoanalytischer Methoden auf groß dimensionierte Kollektive. Speziell die Tatsache, dass diese Kollektive als gegebene Größen angenommen werden, führt allerdings dazu, dass sich Volkans Analysen tendenziell in die Richtung einer verblüffend einfältigen Psychologisierung der Konfliktparteien bewegen, von der aus Rückschlüsse bis zur subjektiven Ebene gezogen werden. Zugleich versucht Volkan, die Handlungsweisen so genannter »ethno-terroristischer« Führungsfiguren aus deren persönlichen Schicksalen zu erklären (Volkan 1997: 160).

Der zentrale Begriff bei der Analyse ethnisch-national ausgerichteter Kollektive ist jener des »Chosen Trauma« (ebd.: 36ff.). Ebenso wie einzelne Subjekte von spezifischen Ereignissen traumatisiert werden könnten, könnten, so Volkan, auch Kollektive von Geschehnissen traumatisch in Mitleidenschaft gezogen werden, in Form einer sich bewusst wie unbewusst vollziehenden Überlieferungsarbeit: »[...] I maintain that the word chosen fittingly reflects a large group's unconsciously defining its identity by the transgenerational transmission of injured selves infused with the memory of the ancestors' trauma« (ebd.: 48). Solchen »chosen traumas « stehen in Volkans Ansatz so genannte »chosen glories« gegenüber, tradierte Erfolgsgeschichten, die durch ihren immanent heroischen Charakter zu wirksamen Bezugspunkten bei der Herausbildung von kollektiven Identitäten werden können (vgl. dazu auch Ropers 1995: 14).

Aufbauend auf diesen Ansätzen zeichnet Gurr ein düsteres Bild der Gegenwart und Zukunft ethnopolitischer Konflikte, das überdeutlich in Richtung der Notwendigkeit internationaler Intervention argumentiert, für die er auch selbst Leitlinien entwickelt (Gurr 1994):

55 Auch zum Konzept des »Ethnonationalismus« gibt es seit den frühen 1990er Jahren eine breite Forschung, die vor allem auf Walker Connor und sein 1994 erschienenes Standardwerk »Ethnonationalism: The Quest for Understanding« zurückgeht. 
»Protracted conflicts over the rights and demands of ethnic and religious groups have caused more misery and loss of human life than has any other type of local, regional, and international conflict since the end of World War II. [...] Ethnopolitical conflicts are here to stay. [...] The Holocaust should have enlightened us about what ethnic and religious hatred can do when used by unscrupulous leaders armed with exclusionary ideologies« (Harff/Gurr 2004: 1).

Interessanterweise zeigen die empirischen Ergebnisse des »Minorities at Risk«-Projekts in eine andere Richtung. Selbst entlang der dem Projekt zugrunde gelegten Definitionskriterien ist die Zahl der »ethnopolitischen Konflikte« nach einem Höhepunkt Anfang der 1990er Jahre, der durch die Kriege in Ex-Jugoslawien und der ehemaligen Sowjetunion bedingt war, stark zurückgegangen. Mittlerweile liegt das von Harff und Gurr (2004: 2) errechnete Niveau wieder auf dem von Mitte der 1960er Jahre (vgl. auch Gurr 2000a: 55). Trotz der ins Auge springenden Brutalität von Konflikten wie Kosovo, Osttimor oder Ruanda sei dieser Trend, so Gurr (2000b: 52) nicht zu leugnen: »a sharp decline in new ethnic wars, the settlement of many old ones, and proactive efforts by states and international organizations to recognize group rights and channel ethnic disputes into conventional politics. « Solche Ergebnisse halten Gurr jedoch offenkundig nicht vom Drang nach interventionistischer Praxis ab, auch wenn sich diese damit im tendenziellen Widerspruch zu seinen eigenen Forschungsresultaten bewegt.

Entscheidendes Problem des Konzepts ist allerdings nicht die Prävalenz oder Nicht-Prävalenz so genannter ethnischer oder ethnopolitischer Konflikte. Die Schwierigkeiten, die die Arbeit mit derartigen Kategorien aufwirft, liegen tiefer und haben verschiedenartige Kritiken auf sich gezogen. So kritisiert etwa Bruce Gilley (2004), dass »ethnischer Konflikt« an sich keine aussagekräftige und für eine Analyse brauchbare Kategorie darstellt. John Mueller (2000) wiederum kritisiert den Ansatz, da er solcherart interpretierte Konflikte eher als kriminelle Akte verstehen will, John R. Bowen (1996: 3) lehnt das Konzept aus prinzipiellen Erwägungen $\mathrm{ab}$, da es präjudizierend wirke: »The very phrase >ethnic conflict misguides us. It has become a shorthand way to speak about any and all violent confrontations between groups of people living in the same country.«

Bowens Aussage kann nicht nur für ethnische, sondern auch für ethnopolitische Konflikte Gültigkeit beanspruchen. Dies lässt sich anhand von Gurrs Analyse exemplifizieren. Denn, wie erwähnt, schwenkt er selbst im Zuge seiner Arbeit mit dem Konzept von seinem konstruktivistischen Zugang weg auf eine essentialistische Herangehensweise ein, 
gegen die der Begriff »ethnopolitisch « ursprünglich gerichtet war: »Ethnic identities may be sconstructed in the sense that they are malleable in scope and content, but that does not detract from their social reality or their persistence« (Gurr 2000a: 8). Folgen wir Ansätzen aus den Postcolonial Studies, dürfte die Ursache für diesen Schwenk im Begriff der »Ethnizität« selbst zu finden sein.

So weisen etwa die Thesen von Mahmood Mamdani (2001: xiii) und Qadri Ismail (2005) darauf hin, dass nicht historische Faktoren für die Herausbildung ethnisch definierter Gruppen verantwortlich gemacht werden können, sondern dass vielmehr eine bewusste Konstruktion von Geschichte aus dem Blickpunkt der Gegenwart erfolgt. Somit wird es allerdings schwierig, von ethnischen Kollektiven als politischen Akteuren zu sprechen. Mamdani will den Prozess einer ethnisch argumentierten Politik denn auch als originär politischen - und damit nicht ethnopolitischen - verstanden wissen: »From being only a cultural community, the ethnic group was turned into a political community, too« (Mamdani 2001: 24). Zugleich verweist Mamdani darauf, dass die Konstruktion dieser »cultural communities« als ethnische Gruppen eine durch den Kolonialismus geprägte Operation ist. Schließlich hatte insbesondere der Spätkolonialismus darauf beruht, die Ethnizität als Gegenstück zur Kategorie der »Rasse« einzuführen, wobei Ethnizität als Unterscheidungskriterium unter den Eingeborenen fungierte, die wiederum rassistisch von den weißen Bürgern, den Kolonialherren, unterschieden wurden (ebd.: 24ff.).

Qadri Ismail konzentriert sich in seiner Auseinandersetzung mit der analytischen Konstruktion des sri-lankischen Konfliktes - dem oftmals ja die Rolle des Musterbeispiels für einen »ethnopolitischen Konflikt« zukommt - auf die Rolle der Geschichtsschreibung. In der Dekonstruktion der Erzählungen eines tamilischen und eines singhalesischen Historikers eröffnet er eine radikale Schlussfolgerung: Nicht Geschichte determiniert Gegenwart, sondern Gegenwart konstruiert sich Geschichte. Wohlgemerkt, sie determiniert sie nicht, weil sie sie nicht etwa umschreibt, vielmehr produziert sie sie.

Eine derartige Schlussfolgerung ist, wie bereits angeführt, für die Arbeit mit Kategorien wie »ethnisch" oder »ethnopolitisch«, selbst wenn sie im konstruktivistischen Sinne verstanden werden, mit weitreichenden Konsequenzen verknüpft. Schließlich kann Ethnizität gar nicht auf irgendwelchen verallgemeinerbaren kollektiven, objektivierbaren, historisch bedingten Merkmalen aufbauen - maximal versucht sie, die Existenz von solchen zu erzählen und zu behaupten. Die Notwendigkeit, politische Gruppen, die sich anhand letztendlich historisch-konjunkturell bedingter Identitätsmerkmale konstruieren, mit dem Präfix »ethno« aus- 
zustatten ist folglich eine zweifelhafte Operation. Konflikte wie Ruanda, Ex-Jugoslawien oder Sri Lanka als »ethnopolitische Konflikte« zu klassifizieren erinnert an die Idee, die russische Revolution von 1917 als Klassenkampf zu interpretieren, was zweifellos einsichtig und über signifikante historische Perioden populär, nichtsdestotrotz analytisch wenig hilfreich war und ist.

Letztlich sucht auch ein Konzept, das von »ethnopolitischen Gruppen« spricht, nach kollektiven Akteuren anhand historisch und kulturell bedingter ethnischer Kriterien, die nun eben politisch mobilisiert und zur Kollektivkonstruktion herangezogen werden. Es steht für gewöhnlich zu erwarten, dass eine solche analytische Suche erfolgreich verläuft, schließlich kursieren zahlreiche derartige Erzählungen im Rahmen jener politischen Gruppen, die darauf ihre Mobilisierungsbasis errichten wollen. Ob sie angesichts der Erkenntnisse postkolonialer Forschungsansätze, wie ich sie nur bruchstückhaft mit Mamdani und Ismail angerissen habe, auch aussagekräftig sind, muss allerdings stark bezweifelt werden. ${ }^{56}$

Dennoch bietet das Konzept - und hier sind angesichts des breiten Erfolges keine Zweifel angebracht - ein sehr gut handhabbares Label für den Policy-Bereich. Wie bereits angemerkt argumentiert Ted Robert Gurr massiv in Richtung der Notwendigkeit internationaler Interventionen in »ethnopolitische Konflikte«:

»What's needed is coordinated effort by international actors and major powers to facilitate negotiated settlements, guarantee local and regional security, promote democratic power sharing, and assist economic development. [...] Building civil peace in divided societies depends not just on peace settlements and institutional engineering but on consistent, long-term engagement to ensure that agreements are implemented and that institutions work (Gurr 2000a: 288).

Einer der Akteure, die diesen Aufruf konkret aufgreifen, ist die deutsche Berghof-Stiftung, die ihre Intervention in Sri Lanka, die primär mit bilateralen Geldern Deutschlands und der Schweiz finanziert wird, um diesen Ansatz formiert. Die Stiftung bezieht sich dabei auch auf die Analy-

56 Sverker Finnström (2003: 54) dokumentiert beispielsweise anhand der nordugandischen Acholi die Flexibilität der Eigenwahrnehmungen im Rahmen des ethnisch konstruierten Kollektivs und damit die Brüchigkeit der historischen Erzählung der Acholi, die, so Heike Behrend (1999: 14), erst durch den Kolonialismus initiiert wurde. Entscheidend bei ihrer kolonialen und nachkolonialen Konstruktion war die Anthropologie, der es nach Finnström (2003: 84) zukam, »charters for Acholi nationhood« zu verfassen. 
sen von Dieter Senghaas ${ }^{57}$, der bewaffnete ethnopolitische wie ethnonationalistische Konflikte als klassische Beispiele für unzivilisiertes Verhalten versteht und daher »therapeutische Konfliktinterventionen« im Sinne der Zivilisierung des Konfliktes für notwendig erachtet (vgl. Senghaas 1992: 116ff.). In ihren Konzepten wie in ihrer Praxis baut Berghof mit dem Verweis auf das ethnopolitische Konzept auf dem Prinzip ethnisierter Politik auf, insbesondere in ihren Lösungsansätzen: »The legitimacy of ethnic demands in multi-ethnic political systems has to be acknowledged (Fischer/Ropers 2004: 14).

In der praktischen Umsetzung bedeutet dies also, mittels der aktiven Beteiligung an der diskursiven Konstruktion und, wie noch zu zeigen sein wird, konkreten Etablierung von ethnischen Gruppen »ethnopolitisch « verstandene bewaffnete Konflikte »zivilisieren« zu wollen: spätestens mit dieser Paradoxie schließt sich der analytische Teufelskreis des Konzepts.

Ein dritter einflussreicher Strang der neuen Ansätze in der Konfliktanalyse, der die herkömmlichen Denkweisen über bewaffnete Konflikte "provokativ" herausfordert (Fischer/Ropers 2004: 21), entstammt unmittelbar dem entwicklungspolitischen Spektrum. Unter der Leitung von Paul Collier ${ }^{58}$ richtet die Weltbank im Jahr 1999 eine Projektgruppe zum Thema »Economics of Civil War, Crime, and Violence« ein. Ausgangspunkt ist die Annahme, dass Konflikt nicht allein als ein Problem für Entwicklung, sondern im eigentlichen Sinne als ein prototypischer Entwicklungsdefekt verstanden werden müsse: „Civil war thus reflects not just a problem for development, but a failure of development« (Collier et al. 2003: ix). Um diese Annahme zu konzeptualisieren, unternimmt Collier eine Abkehr von den bisher primär politischen Zugängen zur Konfliktanalyse: »An economist views conflict rather differently. Economists who have studied rebellions tend to think of them not as the ultimate protest movements, but as the ultimate manifestation of organized crime« (Collier 2000a: 3).

2003 wird der erste ausführliche Report des Projekts unter dem Titel »Breaking the Conflict Trap. Civil War and Development Policy« (Collier et al. 2003) veröffentlicht. Diese Veröffentlichung wirbelt im entwicklungspolitischen, aber auch im sicherheits- und außenpolitischen Bereich viel Staub auf, nicht nur, weil die Weltbank die Veröffentli-

57 Dieter Senghaas fungierte nach der Gründung der Stiftung 1971 bis zum Ende des Jahres 2000, also nahezu 30 Jahre, als Vorsitzender des Stiftungsrates von Berghof.

58 Paul Collier ist Professor für Ökonomie am St. Anthony’s College in Oxford, UK. 
chung mit großem Aufwand und einem hohen Maß an Professionalität im Sinne einer möglichst effektiven Breitenwirkung gestaltet, sondern auch das Team der Herausgeber/innen einen dem Stil Huntingtons vergleichbaren pointierten Weg der eigenen Positionierung sucht, der die Absicht der Durchsetzung und Verankerung eines neuen konfliktanalytischen Paradigmas vermuten lässt. In einem aktuellen Sammelband des Weltbank-Teams (Collier/Sambanis 2005) wird der Ansatz denn auch bereits marketingtauglich abgekürzt als »CH-Model« bezeichnet.

Was nun behaupten Paul Collier und sein Team, und was unterscheidet ihre Annahme von anderen ökonomisch orientierten Ansätzen wie den »War Systems« (vgl. Richani 2002, 2003) und den Gewaltökonomien (vgl. Kurtenbach/Lock 2004; Spelten 2004), die keine vergleichbare Popularität erlangen konnten? Es ist zunächst der analytische Ausgangspunkt, bewaffneten Konflikt als Entwicklungsdefekt und Rebellion als Form der organisierten Kriminalität zu begreifen. Dies hat eine entscheidende Konsequenz für den Forschungsansatz. Collier plädiert entschieden dafür, die Motivationen von Rebellion grundlegend in Frage zu stellen: »Rebel organizations have to develop a discourse of grievance in order to function. Grievance is to a rebel organization what image is to a business. In each case the organization will devote advertising resources to promote it« (Collier 2000a: 3). Stattdessen nehmen Collier und sein Team die These der Ursache von Bürgerkriegen in der Nutzenmaximierung der Rebellenorganisationen an, was eine neue $\mathrm{Zu}$ spitzung der Fragestellung ermöglicht: "greed or grievance? « (ebd.: 5; Collier/Hoeffler 2001; vgl. auch Ehrke 2004). ${ }^{59}$

59 Es sei der Vollständigkeit halber erwähnt, dass sich Collier und sein Team in der Analyse dieser Fragestellung vollständig auf quantitatives Datenmaterial verlassen: »Modern economics has two powerful tools: statistics and theory« (Collier 2000a: 5). Collier selbst sieht seine Schlüsse primär auf dem statistischen Beweis gegründet, da »[p]eople who are not economists are seldom convinced simply by economic theory « (ebd.). Doch gerade die Qualität und Reliabilität des verwendeten empirischen Materials ist in der Folge stark in Zweifel gezogen worden (vgl. Duffield 2001: 132f.). So ist schon die Ausgangsbasis, auf der das Modell aufbaut, und die vorab definiert, wann und wo Bürgerkrieg vorliegt, zweifelhaft. Es wird dabei auf das Kriegsdatenprojekt der Ann Arbor Universität rekurriert, nach dem in Uganda der Bürgerkrieg mit April 1988 beendet worden wäre, just zu dem Zeitpunkt, als das Holy Spirit Movement seine bewaffnete Offensive gegen die junge Regierung des National Resistance Movement begann. Auch der Bürgerkrieg in Somalia wird mit Dezember 1992 für beendet erklärt, während beispielsweise der Irak von Jänner 1985 bis Dezember 1992 als im Bürgerkrieg befindlich definiert wird (vgl. Collier/Sambanis 2005: 4ff.). Allein diese Beispiele zeigen, dass teilweise mit schlichtweg absurdem Ausgangsmaterial operiert wird. Duffield (2001: 133) führt dazu weiter aus: »In order to defend this [Collier's] position, a number of economic 
Bei den Resultaten der Untersuchungen kommt es nun zu einer interessanten Teilung. Einerseits wird die Ursachenfrage eindeutig mit dem Greed-Faktor erklärt: »We conclude that opportunities are more important in explaining conflict than are motives « (Collier/Hoeffler 2001: 2). Diese Schlussfolgerung ist auch das klare Paradigma, das transportiert werden soll. Die Analyse politischer Missstände oder Motivlagen wäre nichts anderes als eine Sackgasse, die den Blick auf die entscheidenden Faktoren der ökonomischen Nutzenmaximierung verstellen würde.

Andererseits sind die Ergebnisse der Studien aber alles andere als eindeutig. Denn in Hinblick auf die Frage, was ein Land anfällig für bewaffnete Konflikte macht, können keine signifikanten Schlussfolgerungen gezogen werden: »The analysis of motives for rebellion has not led us to any definitive conclusions « (Collier et al. 2003: 66). Eines der wenigen greifbaren Resultate, dem Signifikanz zugeschrieben wird, ist die empirische Widerlegung einer zentralen Annahme von Huntington und der meisten ethnischen Konflikttheorien, die in der »New Wars«-Theorie eine wesentliche Rolle spielen: »Substantial ethnic and religious diversity significantly reduces the risk of civil war « (ebd.: 57). Und selbst die (als fehlend angenommene) politische Motivlage der Rebellion ist nach den empirischen Resultaten alles andere als klar: »even though rebel leaders are indeed violence entrepreneurs heading private military organizations that run protection rackets, they usually have some political agenda« (ebd.: 89).

Wenn daher dennoch nicht nur Resultate behauptet, sondern aus diesen Resultaten auch Handlungsrichtlinien abgeleitet werden, bewegen sich Collier und sein Team auf wissenschaftlich unsicherem Terrain. Das eigentliche Problem des Ansatzes liegt allerdings ein Stück tiefer. Ausgangspunkt bei dieser Überlegung ist wiederum das zentrale Argument der fehlgeleiteten ökonomischen Entwicklung, die die Bedingungen für Rebellion geschaffen habe: »the key root cause of conflict is the failure of economic development « (ebd.: 53), oder auch der Umkehrschluss, dass »successful developers« im Sinne der Weltbank-Daten eine

and grievance proxies are established from a large database of different countries (former Zaïre, Liberia, Sudan, Chad, for example) that one would not automatically equate with reliable, extensive and comparative statistical information. [...] In many respects, such proxies are a good example of the manner in which underdevelopment has been reproblematised as dangerous. [...] Quantitative analysis of these proxies is cited to demonstrate that economic agendas rather than grievances are the overwhelming cause of most conflict." Kurz gefasst bewegen sich Collier und sein Team gefährlich nahe an einer Self-Fulfilling Prophecy. 
signifikant niedrigere Anfälligkeit für bewaffnete Konflikte aufweisen würden (ebd.: 107).

Damit müssten in der Bekämpfung potenzieller Konfliktursachen aber auch in der Lösung laufender Konflikte - Prioritäten gesetzt werden, die von einer genuin politischen Konfliktlösungsagenda abweichen: »If disputes are generated by opportunities rather than by objective grievances, it is at least doubtful whether the subjective grievance that may motivate the rebellion can be accommodated by redressing grievances« (Collier/Sambanis 2002: 11). Demgegenüber wären vor allem zwei Ansatzpunkte vielversprechend ${ }^{60}$ - nicht überraschend im Wesentlichen ökonomische: »Thus stimulating development in the slow-growing, lowincome countries is one of the two critical interventions to reduce the global incidence of conflict. The other critical intervention is to weaken the conflict trap, thereby increasing the chances of sustained peace in postconflict situations « (Collier et al. 2003: 118).

Doch auch gute Entwickler müssten beständige Reformbereitschaft an den Tag legen um das Konfliktrisiko zu minimieren, ebenso wie auf politisch-struktureller Ebene im Sinne der Good Governance-Agenda. Speziell Korruption, ein zu starker und unkontrollierter Militärsektor oder Sezessionsbestrebungen seien gängige Problemstellungen (ebd.: $121 \mathrm{ff}$.). Doch selbst in diesem Kontext dominiert letztendlich der ökonomische Ansatz, schneller zu wachsen und die Profite dieses Wachstums zum Ausgleichen sozialer Disparitäten zu verwenden. Wir finden also klassische Weltbank-Rezepturen zur Lösung des Problems bewaffneter Konflikte. Dennoch handelt es sich nicht um eine geradlinige Fortschreibung bestehender Ansätze, dafür hätte es das Collier-Projekt nicht benötigt. Die Absicht der Arbeiten scheint vielmehr in einer Adaption der traditionellen Ansätze der Weltbank zu liegen.

Dabei stört es nicht, dass nicht nur - wie bereits erwähnt - das von Collier und seinem Team eingesetzte Datenmaterial fragwürdig und die

60 Der ebenfalls von der Perspektive der Kosten - und zwar auf Seite der Intervenierenden - getragenen Idee des $»$ Let them fight it out among themselves « (Collier et al. 2003: 33) setzen Collier und sein Team zwei Argumente für eine Intervention entgegen. Einerseits würde jeder Konflikt verschiedene Arten von Kosten verursachen, die zumeist einem »development in reverse « (ebd.: 13) gleichkommen würden, andererseits müsse jeder Konflikt schon allein aufgrund der Gefahren von Spillover-Effekten eingedämmt werden (ebd.: 33ff.). Auch globale Effekte wie Krankheit, Drogenschmuggel, Flüchtlingswellen und Internationaler Terrorismus würden mit der Gefahr zusammenhängen. So lautet das Fazit, dass die Entscheidung über Bürgerkriege nicht den Aktiven allein überlassen sein dürfe, da sie nur einen Bruchteil der dadurch entstehenden Kosten zu tragen hätten (ebd.: 49). 
darauf basierenden Schlussfolgerungen zweifelhaft sind, sondern auch die Grundannahmen der Untersuchung. Zum einen widerspricht die Annahme von Konflikt als Entwicklungsdefekt diametral jenen historischen Einsichten der Staatstheorie, die Kriege, Konflikte und Verbrechen als eine notwendige Begleiterscheinung im Entstehungs- und Entwicklungsprozess von Staaten sehen. ${ }^{61}$ Überhaupt wird der historische Kontext - also auch die Frage des Kolonialismus und des postkolonialen Kontextes, in dem sich der Großteil der analysierten Konflikte bewegt großzügig ausgeblendet, was die ökonomistisch geprägten Schlussfolgerungen der Untersuchungen überhaupt erst ermöglicht.

Zum anderen vernachlässigen Collier und sein Team wesentliche Aspekte auf der Seite der Rebellengruppen, just jene, die der »Greed «These inkompatibel gegenüberstehen. Ein dafür hervorstechendes Beispiel ist jenes der kolumbianischen FARC. Auf den ersten Blick bildet die FARC - wie überhaupt die gesamte kolumbianische Situation - ein Paradebeispiel für die These von Collier. Im offiziellen Diskurs als "Narcoguerrilla" gebrandmarkt, geht ein Großteil der internationalen Öffentlichkeit mittlerweile davon aus, dass die Praxis der FARC tatsächlich als von "greed « gesteuert verstanden werden muss.

Francisco Gutiérrez Sanín (2003) erhebt dagegen erhebliche Einwände, die er vor allem aus einer Analyse der individuellen Motivation von Kadern und Soldat/innen der Organisation argumentiert. Denn im Gegensatz zu den rechtsgerichteten paramilitärischen Gruppen werden die Militanten von der FARC nicht entlohnt, können selten, wenn überhaupt, ihre Familien sehen, und haben strikte innerorganisatorische Regeln zu befolgen (die etwa das Verbot von Privateigentum vorsehen): »Thus, even admitting the possibility that a handful of individuals actually gets rich with the war, which until now has not been proved, the vast majority of the organisation has no possibility whatsoever of doing so, and knows it« (ebd.: 12). Stattdessen begeben sich die Mitglieder der Organisation bewusst und freiwillig in den Prozess einer von ihnen verlangten »life-long militancy« mit strengem moralischem Regulativ, wobei verhältnismäßig hohe Todesraten im Gefecht einer hohen Kampfmoral und einer nur marginalen Rate an Desertion gegenüberstehen. »All this is uncomfortable, to say the least, for any homo economicus story « (ebd.: 14).

Obwohl die FARC ein prägnantes und ohne Zweifel schwer zu vergleichendes Einzelbeispiel ist, deuten diese Beobachtungen dennoch auf ernsthafte Schwächen der Collierschen These von Rebellion als Form

61 Vgl. dazu aus historischer Perspektive etwa die Arbeiten von Charles Tilly (1985, 1992) und Martin van Creveld (1999). 
von Kriminalität hin. In jedem Fall ist der Ansatz des WeltbankProjektes, der von einem Prinzip der individuellen Nutzenmaximierung ausgeht und zugleich im Zuge der Untersuchung eben diese individuelle Ebene vollständig ignoriert, in Hinblick auf die damit getroffenen Aussagen mangelhaft: »The idea that collective action problems are, or can be, solved through ethnic or class solidarity, like in the mafia, is untenable« (ebd.: 15).

Allerdings schafft sich die Weltbank durch eine solche Konzeptualisierung von Konflikt als einem der neuen Themen der Nachhaltigkeit ein Mittel, um ihre ökonomischen Modernisierungsbemühungen, die im post-modernen Mainstream der Nachhaltigkeit vom Zeitgeist überholt $\mathrm{zu}$ werden bedroht sind, mit politischen Ansatzpunkten zu verknüpfen. Der Sicherheitskomplex bekommt damit die Rolle des Vehikels, das die Anpassung traditionell entwicklungspolitischer Praxis an neue Zielsetzungen vornimmt.

Der vierte analytische Ansatz, der im Zuge der 1990er Jahre zu Prominenz gelangen sollte, bezieht sich schließlich auf politisch-institutionelle Strukturen und legt damit auch die Schienen zu Konzeptionen und Begrifflichkeiten, mit der sich zivile wie auch militärische Interventionen rechtfertigen lassen: der Ansatz von schwacher Staatlichkeit, in Begriffen wie »weak« und »failed states« griffig zusammengefasst.

In die Rolle, die Entwicklung dieses Konzeptes durch einen provokanten inhaltlichen Einwurf loszutreten, schlüpft wiederum Robert Kaplan, dessen Beschreibungen eines durch »ancient hatreds« geprägten Balkan schon die Repopularisierung des Konzepts der »ethnischen Konflikte« mit bedingt hatte. 1994 veröffentlicht er seine Schlüsse aus Erfahrungen von mehreren Reisen nach Westafrika ${ }^{62}$ im Artikel »The Coming Anarchy« in The Atlantic Monthly. ${ }^{63}$ Besonderes Aufsehen erregt er nicht nur mit seiner eindringlichen Schilderung der zu diesem Zeitpunkt chaotischen Situation, sondern auch mit seiner Ursachenanalyse, wo er einen Zusammenhang mit der Problematik post-kolonialer Staatlichkeit erkennen will: »The state, recall, is a purely Western notion, one that until the twentieth century applied to countries covering only 3 percent of the earth's land area« (Kaplan 2001: 39).

Explizit bezieht sich Kaplan auf Samuel Huntington, den er zwar ob seiner Tendenz zur Pauschalisierung kritisiert (ebd.: 27), sonst aber in

62 Im Besonderen bezieht sich Kaplan auf die Situation in Côte d'Ivoire Anfang der 1990er Jahre, speziell in deren größter Stadt, Abidjan.

632001 veröffentlicht Kaplan ein gleichnamiges Buch, das auch verschiedene weitere Artikel zum Themenbereich gescheiterter Staatlichkeit, internationaler Sicherheit und US-Intervention enthält (Kaplan 2001). 
den wesentlichen Grundelementen seines Ansatzes stützt. Zugleich jedoch nimmt er auch Ideen von Paul Collier und Mary Kaldor vorweg, indem er etwa die Nichtunterscheidbarkeit zwischen Krieg und Kriminalität zu einem der wesentlichen Charakteristika künftiger bewaffneter Konflikte erklärt (ebd.: 49). Somit gelingt es Kaplan mit seinem Artikel, zum richtigen Zeitpunkt die zentralen zeitgemäßen Ansätze mit prägnanten und für die politische Praxis tauglichen Schlussfolgerungen zu verbinden, was ihm breite Rezeption im politischen wie im wissenschaftlichen Bereich garantiert.

Auf wissenschaftlicher Ebene ist die Idee der Konzeptualisierung von Staatsversagen als sicherheitspolitischem Problem noch ein Stück älter. ${ }^{64}$ Erstmals wird das Thema von Steven Ratner und Gerald Helman 1992 in dem Artikel »Anarchy Rules: Saving Failed States« in Foreign Policy aufgebracht. Ratner und Helman, beide aus einflussreichen Positionen der US-Administration ${ }^{65}$, geben darin jene Leitlinien vor, die Kaplan mit seinen journalistischen Wahrnehmungen verknüpft und die in weiterer Folge zur Orientierungslinie der »State Failure«-Forschung werden sollten. Ausgehend von der Beobachtung einer neuen Welle von Staatsversagen $^{66}$ wird gleich $\mathrm{zu}$ Beginn die Motivation der Analyse markant hervorgehoben: »As those states descend into violence and an-

64 Zur historischen Entwicklung der »Failed States«-Theorie vgl. Bilgin/Morton (2002), wenngleich ich in einigen wesentlichen Punkten nicht mit ihnen übereinstimme. Einerseits ist der Zusammenhang zwischen den von der US-Administration definierten »Rogue States" und »Failed States" alles andere als derart linear wie von Bilgin/Morton behauptet (»When the >weak state that causes a threat to international security happens to be a >foe<, it is invariably constructed as a >rogue state< and containment becomes the recommended policy course«, ebd.: 66). Andererseits halte ich die Einordnung von Joel Migdal in das Schema der Analyse von Staatsstärke in dieser Theorierichtung für unzutreffend. Bilgin/Morton unterstellen Migdal (1988) die Verwendung eines westlichen Idealtypus von Staatlichkeit, dessen Schwäche er analysieren würde. Dagegen meinen sie: $»[\ldots]$ by emphasising the straddling between state-civil society relations it is possible to appreciate the role played by more informal networks within the sshadow state<, through which alternative modes of political authority can be exercised « (Bilgin/Morton 2002: 74). Das ist nun aber in der Tat genau das, was Joel Migdal mit seinem »State-in-SocietyApproach « versucht.

65 Gerald B. Helman ist pensionierter Diplomat und unter anderem ehemaliger US-Botschafter am UNO-Sitz in Genf, Steven R. Ratner kommt aus dem State Department.

$66 »$ From Haiti in the Western Hemisphere to the remnants of Yugoslavia in Europe, from Somalia, Sudan, and Liberia in Africa to Cambodia in Southeast Asia, a disturbing new phenomenon is emerging: the failed nation-state, utterly incapable of sustaining itself as a member of the international community« (Helman/Ratner 1992: 3). 
archy - imperiling their own citizens and threatening their neighbors through refugee flows, political instability, and random warfare - it is becoming clear that something must be done« (Helman/Ratner 1992: 3).

Obwohl Ratner und Helman ähnlich Robert Kaplan die von ihnen identifizierte Phase des Staatszerfalls primär auf die »vast proliferation of nation-states « nach Ende des Zweiten Weltkrieges zurückführen (ebd.), plädieren sie entschieden für den Einsatz staatserhaltender Maßnahmen, vor allem von Seiten der Vereinten Nationen: »The international community should now be prepared to consider a novel, expansive and desperately needed - effort by the U.N. to undertake nation-saving responsibilities« (ebd.: 9). Dazu wird jene Neuverhandlung von Souveränität vorgeschlagen, die wir bereits vom Thema der Nachhaltigkeit und den Interventionen zu ihren Gunsten auf entwicklungspolitischer Ebene kennen: »Those barriers [to the international involvement in internal matters] stem from the talisman of ssovereignty<. That illdefined and amorphous notion of international law has been used to denote everything from a state's political independence [...] to the more extreme view that all the internal affairs of a state are beyond the scrutiny of the international community« (ebd.).

Eine jener Institutionen, die das Thema früh in ihrem wissenschaftlichen Portfolio aufgreifen und weiter bearbeiten, ist das US Army War College. Seit 1996 erscheinen Artikel zur Problematik von Staatsversagen und seinen sicherheitspolitischen Implikationen. Ab Februar 1998 wird in Zusammenarbeit mit der Purdue University eine WorkshopReihe organisiert, die die erste Sammlung zu wissenschaftlichen Arbeiten in dem neuen Themenfeld bündelt. Schon zuvor war an der Purdue University unter Leitung von Daniel C. Esty ein policy-nahes, von der US-Administration in Auftrag gegebenes Forschungsprojekt zum Thema Staatsversagen eingerichtet worden. ${ }^{67}$

Auch in Harvard wird 1998 unter der Leitung von Robert I. Rotberg ein Forschungsprojekt zu »Failed States« eingerichtet. Im expliziten Gegensatz zu Esty und seinen Kolleg/innen an der Purdue University, die einen sehr engen Begriff von Staatsversagen zugrunde legen und versuchen, hauptsächlich quantitative Indikatoren dafür abzuleiten, fassen Rotberg und sein Team die Problematik breiter (vgl. Rotberg 2004: 22f.). Rotberg findet den Zugang zur Stärke des Staates über seine Fä-

67 Der vollständige Titel dieses Projektes lautet »The State Failure Project: Early Warning Research for U.S. Foreign Policy Planning «. Das Projekt wurde von der Central Intelligence Agency kommissioniert (vgl. Esty et al. 1998). Die Projektrohdaten und der Ergebnisbericht der Projektphase III können unter http://www.cidcm.umd.edu/inscr/stfail/ eingesehen werden. 
higkeit zur Verteilung politischer Güter: »Nation-states exist to provide a decentralized method of delivering political (public) goods to persons living within designated parameters (borders)« (Rotberg 2003: 2).

Dieser qualitative Zugang ermöglicht Rotberg und Kolleg/innen die Einführung verschiedener Kategorien von Staatsstärke. Den »Failed States« werden die »Endangered« oder »Failing States« hinzugefügt, die sich im Prozess des Scheiterns befinden würden, sowie auch die Extremversion von Staatsversagen: der »Collapsed State«. »A collapsed state is an extreme version of a failed state. It has a total vacuum of authority« (Rotberg 2002: 90).

Grundsätzlich bauen sie dabei auf der Idee von starken und schwachen Staaten $^{68}$ auf, die Joel Migdal schon 1988 entwickelt hat. Im Gegensatz zu Migdals später als »State-in-Society-Approach« (Migdal 2001) klassifiziertem Zugang beziehen sich Rotberg und sein Team jedoch auf den Staat als alternativlose Form von politischer Autorität. Für sie ist der Kollaps eines Staates nicht das Zeichen dafür, dass er sich nicht gegen andere, konkurrierende Formen politischer Autorität durchsetzen konnte, wie es für Migdal der Fall ist, sondern ein autoritatives Vakuum, ein, wie sie es ausdrücken, »black hole into which a failed polity has fallen« (Rotberg 2003: 9). Dagegen geht es um den Staat als den Garant in der Abwehr von sich zunehmend globalisierenden Bedrohungsbildern: »Now, however, as much as their citizens suffer, the failings of states also pose enormous dangers beyond their own borders. Preventing nation-states from failing and resuscitating those that have failed and will fail, have thus become the critical, all-consuming, strategic and moral imperatives of our terrorized time« (ebd.: 24).

Damit bauen Rotberg et al., ebenso wie das von ihnen so kritisierte Projekt um Daniel C. Esty, auf einem normativen Zugang auf, dem es weniger um ein vom Ansatz her offenes Verständnis von Prozessen als um die Fassung politischer Antworten auf sicherheitspolitisch definierte Problemstellungen geht, deren Dreh- und Angelpunkt die dominante sicherheitspolitische Form, der Staat, bleiben muss. Der historische Anknüpfungspunkt der Idee von »schwachen Staaten« liegt damit nicht bei Joel Migdal, sondern bei den Arbeiten des britischen Kriegsforschers Lawrence Freedman, der im Jahr 1993 unter dem Titel »Weak States and the West: Warfare has a future im Economist einen viel beachteten Artikel zum Thema vorgelegt hat (Freedman 1993). Dabei skizziert

68 Ein starker Staat ist gemäß dieser Definition einer, der alle notwendigen politischen Güter zur Verfügung stellen kann: »Strong states unquestionably control their territories and deliver a full range and a high quality of political goods to their citizens « (Rotberg 2003: 4). Schwache Staaten sind demnach das Gegenteil - sie können dies nicht garantieren (ebd.: 5). 
Freedman zwar Bedrohungsbilder und Szenarien schwacher Staatlichkeit $^{69}$, vernachlässigt jedoch im Gegensatz zu Migdal eine entscheidende Frage: $»[\ldots]$ in analysis such as this, no reference is made to the processes through which these states have become >weak gained >strength . In other words, the question >who's failed the >failed state $<?<$ is almost never asked « (Bilgin/Morton 2002: 66).

Zeitlich parallel entwirft Dieter Senghaas $(1994,1995,1997)$ ein von einer vergleichbaren Problemstellung ausgehendes Modell, das speziell im deutschsprachigen Raum wesentliche Bedeutung erlangen sollte: das zivilisatorische Hexagon. Obwohl vielleicht noch normativer als die ohnehin schon sehr normativ ausgerichteten angloamerikanischen Ansätze entwickelt sich das zivilisatorische Hexagon schnell zu einer - wenn auch nach einer Zielbestimmung und nicht nach einem Problemkomplex benannten - Variante der »Failed States«-Theorie.

\section{Abbildung 4: Das zivilisatorische Hexagon von Senghaas}

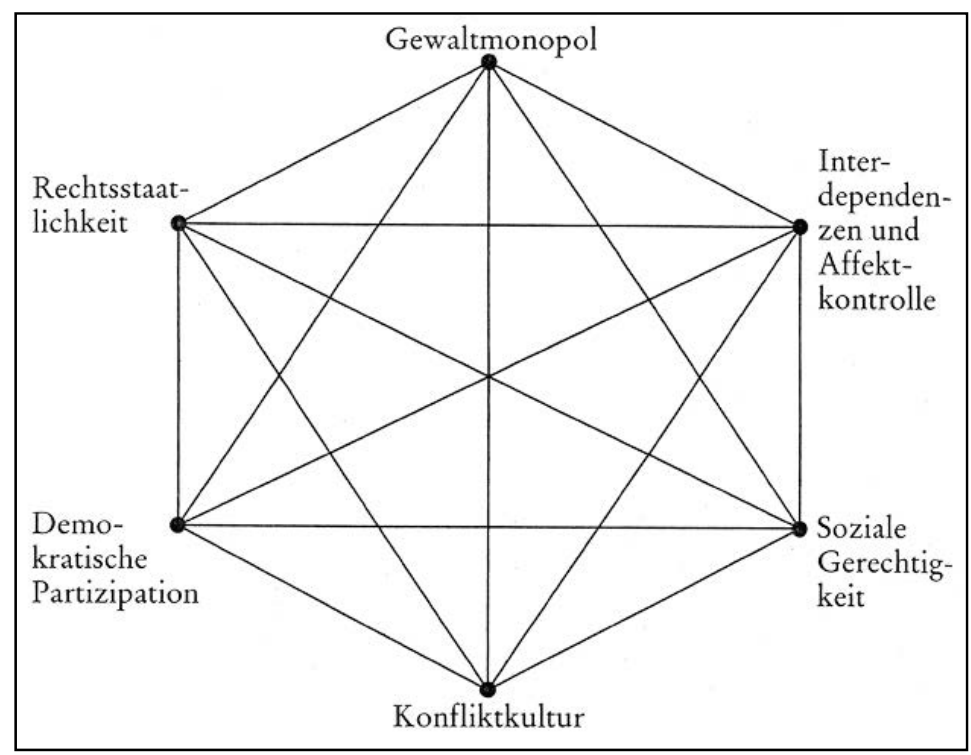

Quelle: Senghaas 1994: 26

$69 »$ The prototypical conflict now is a function of a weak state. States are weak because of the fragile nature of the civil society upon which they have been built, their underdeveloped institutional structures, which are often unable to contain and channel political tensions, and their problems of poverty and economic adjustment« (Freedman 1993). 
Von den sechs im zivilisatorischen Hexagon gebündelten Elementen (siehe Abbildung 4) sind drei unmittelbar mit einem erfolgreichen Durchstaatlichungsprozess verknüpft: Gewaltmonopol, Rechtsstaatlichkeit und demokratische Partizipation (zumindest in dem von Senghaas zugrunde gelegten Sinne) ${ }^{70}$, also ähnliche Zielgrößen, wie wir sie auch bei Freedman oder Rotberg finden können. Senghaas vollzieht mit seinem Modell einen doppelten Brückenschlag: Einerseits versöhnt seine Theorie die vormals zumeist in diametral entgegengesetzten politischen Lagern stehenden wissenschaftlichen Stränge der Friedensforschung und der internationalen Sicherheitspolitik, andererseits schafft er einen unmittelbaren Anknüpfungspunkt für Entwicklungspolitik in einer originär sicherheitspolitischen Problemstellung (vgl. als Beispiel etwa Nuscheler 1996: 198), der speziell im deutschsprachigen Raum immanent praktische Bedeutung bekommen wird.

Den naheliegenden und bereits oftmals erhobenen Vorwurf, sein Modell habe einen »eurozentristische[n] Bias« (Matthies 1995: 29) ${ }^{71}$, wischt Senghaas mit erstaunlich simplen Argumenten vom Tisch. Zwar sei der Vorwurf grundsätzlich nicht von der Hand zu weisen, meint er, aber er würde sich schlicht überleben: »Die universelle Geltung stellt sich vielmehr dadurch ein, dass es auch in allen übrigen Teilen der Welt einen Übergang in die Moderne, d.h. in sozial mobile und darauf aufbauend politisierte Gesellschaften gibt« (Senghaas 1995: 206). Die europäischen Erfahrungen, denen Senghaas globale Beispielwirkung zuweist (vgl. Senghaas 1982; Menzel/Senghaas 1986; und vor allem Senghaas 1992), liefern denn auch den primär gültigen Weg, mit einer solchen Situation umzugehen. Eurozentrismus ist für Senghaas somit auch kein Problem, sondern wünschenswert (vgl. Faschingeder 2001: 99ff.).

Prinzipielle Zweifel an der Steuerbarkeit politischer Entwicklungen, also die Infragestellung der Möglichkeit einer solchen universellen Durchsetzung, verwirft er, ohne argumentativ darauf einzugehen: »[...] das prinzipielle Argument, die westeuropäische Erfahrung sei nicht wiederholbar, wird gerade auch hinsichtlich gesellschaftlicher und politischer Veränderungsprozesse [...] widerlegt« (Senghaas 1997: 574). Zyniker/innen, die sich ausführlicher mit europäischer Geschichte speziell im Zusammenhang mit der Nationalstaatsbildung auseinandergesetzt haben, mögen Senghaas wahrscheinlich zustimmen, soweit es den not-

70 Für eine genauere Beschreibung der sechs im zivilisatorischen Hexagon zusammengefassten Elemente und ihrer Interdependenzen vgl. Senghaas (1995: 198ff.).

71 Es ist anzumerken, dass der von mir an dieser Stelle als Beispiel zitierte Volker Matthies dem Schema von Senghaas trotzdem grundsätzlich positiv gegenübersteht. 
wendigen kriegerischen Vorlauf betrifft. Dieser ist nun auch in globalem Rahmen festzustellen. Das von Senghaas intendierte Argument, die westeuropäische Erfahrung sei in peripheren Regionen durch staatliche Kopfgeburten als Ausdruck eines Zivilisierungsprozesses zu wiederholen, ist allerdings von einer bemerkenswert ahistorischen Qualität. Das ändert jedoch nichts am faktischen Erfolg des Konzeptes, speziell auf Policy-Ebene.

Aus allen vier genannten Analyseansätzen lassen sich verschiedene Handlungsoptionen für die Policy-Ebene ableiten: während aus Huntingtons Konzept primär Strategien der Nichtintervention im Sinne eines geostrategischen Containment abzuleiten sind, gehen die drei anderen Konzepte klar in Richtung einer interventionistischen Politik, wobei institutionellen Strukturen wesentlicher Raum bei der Transformation bewaffnet ausgetragener Konflikte zugebilligt wird. Es ist daher nicht verwunderlich, dass ein solches um die Frage der Staatlichkeit gruppiertes Konzept starke Politik- und Policy-Relevanz bekommen wird, insbesondere auf Ebene der Problemdiagnose.

Im Rahmen der strategischen Planung der Akteure des weltsystemischen Zentrums wird Staatszerfall, auch auf entwicklungspolitischer Ebene, seit der zweiten Hälfte der 1990er Jahre problematisiert. Schon die ersten DAC-Guidelines zur Thematik der Konfliktprävention im Jahr 1997 beziehen sich auf »Failed States« (DAC 2001: 113), wenngleich die Problematik erst in der Neuausgabe von 2001 eine signifikante Betonung erfährt. Hier wird der Staat zum Schlüssel friedlicher Entwicklung: »Donors need to maximise opportunities to help strengthen state capacity to respond appropriately to conflict« (DAC 2001: 62). 2003 richtet das DAC eine eigene Arbeitsgruppe für so genannte »difficult partnerships« ein, die 2005 in eine »Fragile States Group« übergeführt wird.

In den USA wird Staatszerfall weniger als Ansatzebene denn als Bedrohungsszenario wahrgenommen, und zwar als eines der zentralen. Gleich auf der ersten Seite der Nationalen Sicherheitsstrategie von 2002 heißt es: »America is now threatened less by conquering states than we are by failing ones« (The White House 2002: 1). Dies findet seine Umsetzung im Strategischen Plan von State Department und USAID aus dem Jahr 2003: »Failed and failing states can provide fertile ground for terrorist organizations to thrive. The heightened threat of terrorism in states with despotism, weak institutions, and neglected social, economic, and political capacity, requires greater emphasis on moving states toward more accountable, legitimate, and democratic governance« (Department of State/USAID 2003: 10). 
Ähnliches finden wir, wenn auch in einer etwas anders gefärbten Sprache, bei der Europäischen Union. Geht schon die Europäische Sicherheitsstrategie vom Staatsversagen als einem der zentralen Bedrohungsbilder aus (vgl. EU 2003: 5), finden wir auf entwicklungspolitischer Ebene vergleichbare Anschlusspunkte: »Tackling poverty and inequality should therefore be an essential component of any credible and effective security strategy, which may not be limited to addressing the symptoms and must pay attention to the real factors that undermine global, regional and in-country security, and lead to the emergence of >failed states« (EC 2004a: 113).

Mittlerweile scheint sich der Term »Fragile States« im PolicyBereich als der zentrale diagnostische Begriff bei der Ursachenbestimmung von bewaffneten Konflikten an der Peripherie durchzusetzen. Wir finden ihn etwa beim aktuellen White Paper von USAID in den Zielbestimmungen (»strengthening fragile states«, USAID 2004c: 5) und in einem eigens ausgearbeiteten Strategiepapier (der so genannten $»$ Fragile States Strategy«, USAID 2005d), bei der EU-Kommission (vgl. EC 2004a: 114), oder auch in der Sektorstrategie des deutschen BMZ (2005b: 5, 9) und bei DFID im Arbeitspapier »Why we need to work more effectively in fragile states " (DFID 2005b). Auch das DAC hat sich mit Policy Guidelines (DAC 2006b) und Prinzipien des Engagements in fragilen Staaten (DAC 2007) auf den Begriff festgelegt. »Fragile States« werden damit zum übergreifenden Konzept, das die Problematisierung von peripherer Staatlichkeit zusammenfassen soll, egal ob nur »weak« oder schon »failed«. Entscheidend ist der stringent gebündelte Problemkontext, der zugleich mit einer diplomatischen Abschwächung in der Formulierung einhergeht - lässt doch Fragilität einiges mehr an Bedeutungsspielraum offen als Schwäche oder gar Verfehlung. ${ }^{72} »$ Fragile States« werden so gewissermaßen zum Leitbegriff des neuen Programms,

72 Zur Verdeutlichung der Schwierigkeiten, die eine Klassifizierung eines Staates als »weak« oder »failed« nach sich ziehen kann, genügt ein Blick ins US-amerikanische Magazin Foreign Policy. Nachdem Pakistan im »Annual Failed States Index« auf Rang 9 eingestuft wurde (die Liste wird vom Sudan angeführt, Somalia, gewöhnlich als Paradebeispiel gescheiterter Staatlichkeit zitiert, steht interessanterweise nur auf Rang 6, vgl. Foreign Policy, May/June 2006, S. 50ff.), findet sich in der folgenden Ausgabe eine empörte Stellungnahme der pakistanischen Botschaft in den USA, die nicht nur mit angeblichen ökonomischen Fortschritten, sondern auch mit Pakistans politischer Rolle argumentiert (vgl. Foreign Policy, July/August 2006, S. 6f.): »Instead of relying on alarmist media reports and commentary, the ranking should have been based on Pakistan's cooperation as a frontline state in the war on terror.« 
das sich das Joint Venture von Entwicklungs- und Sicherheitspolitik in der Phase der Nachhaltigkeit gegeben hat.

Entscheidend ist dabei die negative Annäherung an das Problemfeld - eine Sicht, die schon »Good Governance« ausgezeichnet hatte. Es geht nicht mehr entscheidend darum, die Peripherie den Zentren gleich zu bilden, eine nachholende Entwicklung auch auf politischer Ebene in Gang zu setzen. Es geht vielmehr darum, Bedrohungsszenarien konzeptionell zu fassen und damit die Dispositive von Entwicklung und Sicherheit neu aufzuladen. Damit wird die durch die drei angeführten Strömungen der Konfliktanalyse angestrebte Entpolitisierung von Konflikt in einem Maße repolitisiert, die dem Projekt der Nachhaltigkeit strategisch entspricht.

Dennoch stellt sich die Frage, warum gerade die Problematisierung peripheren Staatsversagens in jene Katalysator-Rolle bei der Verbindung zwischen Entwicklung und Sicherheit schlüpfen konnte, was sie also gegenüber ökonomischen, kulturalistischen oder mit dem Faktor der Irrationalität argumentierenden Erklärungsmustern auszeichnet. Dies mag einerseits mit den positiven Zuschreibungen zu tun haben, die dem peripheren Staat von entwicklungspolitischer Seite lange entgegengebracht wurden, wie Jennifer Milliken und Keith Krause vermuten: »One (somewhat ironic) way to think about the contemporary anguish over state collapse is to note that what has collapsed is more the vision (or dream) of the progressive, developmental state that sustained generations of academics, activists and policy-makers, than any real existing state« (Milliken/Krause 2003: 10).

In jedem Fall jedoch ermöglicht der Zugang über die Entwicklung von Staaten, entwicklungspolitisches State-Building (vgl. Fukuyama 2004; Ottaway 2003; Debiel et al. 2005; Nöst 2006), wahrscheinlich die effektivste Möglichkeit der Intervention im Sinne einer repolitisierten Nachhaltigkeit. »The historical role of political development theorists, as avatars of global capitalism, was crucial in this sense in subsequently engendering a >doctrine for political development $\iota$, based on containing demands for mass participation as a prelude to the dissemination of liberal democracy throughout the post-colonial world « (Bilgin/Morton 2002: 60). Wenn wir diesen Ansatz weiterdenken, können wir nachhaltige Sicherheit als das eigentliche strategische Programm der gouvernementalen Rationalität entwicklungspolitischer Intervention identifizieren. Dem mit »Fragile States« umrissenen Problemkomplex kommt darin die Rolle einer zentralen taktischen Marschroute zu. Er ermöglicht die Operationalisierung des neu entstehenden Dispositiv-Komplexes von 
Entwicklung und Sicherheit in einer konkreten Programmatik sozialer Kontrolle.

In der Folge können wir auf zwei entscheidenden Ebenen eine qualitative Neubestimmung feststellen, die nicht nur auf eine Veränderung in der Konfiguration, sondern auf eine Ausdehnung der Dispositive von Entwicklung und Sicherheit hinauslaufen: auf der Ebene der Souveränität und der Ebene der Bevölkerung. Eine entscheidende Rolle als Bindeglieder in diesem an der Peripherie seit der Erfindung dieser Konzepte von externer Intervention geprägten Verhältnis kommt in der Programmatik des State-Building der Zivilgesellschaft zu. »Hence, once again, it is possible to trace the $>$ Huntingtonian formula from the modernisation and development theories of the 1950s and 1960s to the democratisation theories of the 1980s and 1990s, albeit with a shifting emphasis from the state as the centre of social control to advocating and supporting the construction of conformist civil societies as supposedly autonomous spaces of individual freedom and association« (Bilgin/Morton 2002: 65).

So rückt die Zivilgesellschaft - in ihrer Grundidee untrennbar mit modernem Staatswesen verbunden und ohne ein solches nicht vorstellbar - in die Rolle eines effizienten Hebels gouvernementaler Kontrolle. Wenngleich in der Umsetzung geberspezifisch signifikante Unterschiede festzustellen sind, wird Zivilgesellschaft durchwegs als ein Schlüssel bei der demokratischen Durchstaatlichung der Peripherien verstanden. Dabei wird Zivilgesellschaft entlang jener Linien identifiziert, die sich die Geberagenturen jeweils normativ zurechtgelegt haben, und wird damit $\mathrm{zu}$ einem der primären Einstiegspunkte des mit einem State-BuildingProzess einhergehenden Social Engineering.

Damit zusammenhängend wird, speziell bedingt durch die Misserfolge der USA in der Befriedung Afghanistans und des Irak im Anschluss an ihre bewaffneten Invasionen, Nation-Building als neuer Interventionsweg diskutiert (Fukuyama 2006; Dobbins et al. 2003; Hippler 2004a; Ottaway 2002), getragen von der Erkenntnis dass die Konzentration auf den institutionellen Aufbau und die Etablierung einer demokratischen zivilgesellschaftlichen Elite offenbar nicht ausreicht, um bewaffnete Konflikte zu beenden.

Dieses Konzept ist keineswegs neu. Der Beginn der Idee von durch externe Akteure induziertem Nation-Building kann vor etwas mehr als 100 Jahren im späten Kolonialismus angesetzt werden. Insbesondere das britische Kolonialsystem der »indirect rule« (zur Entstehung des Konzeptes und seiner Umsetzung vgl. Pakenham 1992) ist als erster Versuch zu verstehen, im Sinne besserer Verwaltbarkeit durch konkrete institutionelle Maßnahmen auch kollektives Bewusstsein zu erzeugen, das mit 
einer (zuvor von den zu Kolonisierenden zumeist ja nicht so verstandenen) räumlichen Dimension - dem kolonialen Verwaltungsgebilde verknüpft wurde. Die Brüchigkeit dieses Konzeptes liegt vor allem in jener Komponente, die Mahmood Mamdani (1996) als dezentralisierten Despotismus bezeichnet, also der Tatsache, dass die Einwohner/innen eines Gebietes sich trotz der Bemühungen keinesfalls als gleichberechtigt fühlen können, sondern in komplexen Systemen der internen und externen Herrschaft eingebunden sind.

Eine weitere Region, die durch Maßnahmen der britischen (und in einem bemerkenswerten Fall auch der amerikanischen) Kolonialmacht verschiedene Formen extern beeinflusster Nationsbildungen durchlief, war Südostasien. In seinem klassischen Werk zur Entstehung von Nationalismus zeigt Benedict Anderson (1998a, vgl. auch 1998b) am Beispiel Indonesien auf, welchen Einfluss Maßnahmen im Bereich der Verwaltungs-, Bildungs- und Kulturpolitik auf die Heranbildung eines nationalen Bewusstseins in kolonisierten Regionen haben.

Mit der Herstellung des philippinischen Nationalstaates, und hier insbesondere mit der konfliktiven Einbindung der Südinsel Mindanao, betreten die USA - hier zum ersten wie letzten Mal als Kolonialmacht die Bühne des aktiven Nation-Building (vgl. Abinales 1998, 2000, Kramer 2006: 285ff.). Bald danach folgt mit der Unterstützung der Sezession Panamas von Kolumbien und der danach folgenden Institutionalisierung der Eigenstaatlichkeit der nächste Schritt, an den sich Nicaragua, Kuba und die Dominikanische Republik in der ersten Hälfte des 20. Jahrhunderts anschließen sollten (vgl. Pei et al. 2006). Weitere Beispiele, die in der Literatur als weitgehend erfolgreich wahrgenommen werden, sind die nach dem Zweiten Weltkrieg erfolgenden Interventionen in Japan und verschiedenen europäischen Ländern, insbesondere Deutschland, die in ihrer Ausrichtung auf eine politische Demokratisierung auch die Neudefinition der nationalen Identität zum Ziel haben (vgl. Dobbins 2003).

Nachdem die 1950er und frühen 1960er Jahre, unter tatkräftiger Mitwirkung der Ford Foundation als potentem privaten Partner (vgl. Sutton 2006), noch einige als erfolgreich wahrgenommene Beispiele von induziertem Nation-Building aufzuweisen haben (genannt wird hier vornehmlich das Beispiel Südkorea im Anschluss an den Korea-Krieg, vgl. Ekbladh 2006: 22ff.), muss das Konzept mit dem desaströs endenden »Crucible of Vietnam« (ebd.: 27) einen Rückschlag hinnehmen, mit dem es für nahezu zwei Jahrzehnte auf beiden Seiten des Atlantik in einer tiefen Schublade verschwinden sollte.

Mit dem Ende des Kalten Krieges zeichnet sich jedoch ein erfolgreiches Comeback ab. Im Zuge des massiven Anstiegs von militärischen 
wie zivilen Interventionen, die über das State-Building-Konzept ohnehin den Zugriff auf die politische und institutionelle Verfasstheit von Staaten zum Ziel haben, wird das Konzept der Nation ebenfalls wieder in zunehmend positiver Weise aufgefasst. Von den USA wird beispielsweise das Eingreifen in Haiti gerade auf Ebene des zivilen Einschreitens als großer Erfolg wahrgenommen, wobei die Komponente NationBuilding als zentral interpretiert wird.

Wo liegt nun der Unterschied zwischen den Ansätzen von State- und Nation-Building? Grundsätzlich ist angesichts der dünnen praktischen Unterfütterung in den derzeitigen Ausformungen beider Konzepte - die theoretische Formation, oder zumindest die Formation von »StateBuilding « und »Nation-Building« als konzeptionellem Markenzeichen, ist in jedem Fall viel weiter fortgeschritten als jede Form der Implementierung $^{73}$ - nur von einem tendenziellen Unterschied auszugehen, wo eine laufende Intervention je nach Situation mehr in die eine oder andere Richtung argumentiert wird. Generell kann davon ausgegangen werden, dass sich der Unterschied daran festmachen lässt, dass sich StateBuilding-Interventionen eher auf formale und institutionelle Aufgabenstellungen konzentrieren, während Nation-Building eher auf Prozesse der Standardisierung und, vor allem, der Partizipation abzielt, die allerdings nicht nur als politische Teilhabe, sondern im Sinne der bewussten Annahme einer kollektiven nationalen Identität verstanden wird. ${ }^{74}$

Insbesondere die USA haben diese Verschiebung mit ihrem »Democracy Promotion«-Konzept, auf das ich im nächsten Teil noch ausführlich zu sprechen kommen werde, prononciert durchgeführt. So ist der These von Francis Fukuyama (2006: 3) nicht zuzustimmen, wonach die Nation-Building-Interventionen der USA eigentlich als StateBuilding-Interventionen mit begleitenden ökonomischen Maßnahmen zu verstehen seien, wohingegen sich europäische Akteure der gesamten Dimension von Nation besser bewusst seien. Die empirischen Erfahrungen deuten eher auf das Gegenteil hin: während die USA eine tendenziell akteursnahe Herangehensweise bevorzugen, verfolgen europäische Geber - und hier insbesondere Deutschland - stark schematisierte Wege

73 Francis Fukuyama (2006) sieht die Interventionen im Irak und in Afghanistan als Aufgaben des induzierten Nation-Building. Allein schon die bisherige Bilanz dieser beiden Beispiele zeigt die Brüchigkeit des Konzepts.

74 Vergleiche dazu das von Stein Rokkan (2000: 167) entwickelte VierPhasen-Schema der Nationalstaatsintegration. Er unterscheidet dabei zwischen Penetration (Phase I), Standardisierung (II), Partizipation (III) und Umverteilung (IV). Nation-Building-Interventionen sind entlang dieses Schemas tendenziell in den späteren Phasen angesiedelt, während aktives State-Building primär auf die Phasen I und II abzielt. 
der Implementierung, die für institutionelles State-Building gut, für das, was Fukuyama unter Nation-Building verstehen will, jedoch denkbar schlecht geeignet sind.

Ohnehin ist es aber so, dass, obwohl Nation-Building auch bei europäischen Gebern explizit zur sicherheits- und entwicklungspolitischen Aufgabenstellung erhoben und mit konkreten Programmen unterfüttert wird (als ein Beispiel siehe Hopp/Kloke-Lesch 2004), daraus kaum praktische Maßnahmen folgen. Das Projekt Nation-Building bleibt vorerst theoretisch, wenngleich erste Pilotprojekte auf entwicklungspolitischer Ebene durchgeführt werden, auch in für die EZ mitunter recht exotischen Bereichen: so startete etwa die Schweizer DEZA im Rahmen des internationalen »Jahres des Sports« ein Programm zum entwicklungspolitischen Nation-Building durch Sportprojekte.

Eine sich gleichzeitig entwickelnde taktische Spielart, die sich ebenfalls im Grenzbereich entwicklungs- und sicherheitspolitischer Intervention ansiedelt, weist strukturelle Ähnlichkeiten mit den Programmen von Nation- und State-Building auf: Human Security.

Die Ähnlichkeiten beziehen sich nicht nur auf das sich folgerichtig ergebende Ineinandergreifen der taktischen Ansatzpunkte in der Praxis von State-Building und Human Security, wie sie etwa Bøås und Jennings (2005: 389ff.) vorschlagen. Denn bereits in ihrem Artikel klingt an, welche Möglichkeiten die Verbindung eines auf Kollektivstrukturen zielenden Konzepts wie State-Building mit einem auf Individuen orientierten Ansatz wie Human Security eröffnet: »[...] it is equally important to note that a benefit of using human security as the basis for conceptualising state failure is that it implicates states that are not typically considered as failing, particularly those states with strong - perhaps overly strong - institutions and rules« (ebd.: 391f.).

So verstanden liefert Human Security nicht nur den konkreten Anknüpfungspunkt für die direkte Überleitung der »Rogue States«-Idee der Clinton-Administration an die aus dem wissenschaftlichen Kontext kommende Debatte über Staatsversagen. Sie bietet darüber hinaus eine Potenzierung der Möglichkeiten, die State-Building in seiner qualitativen Neudefinition von Souveränität - im Sinne weitestgehender Flexibilität - mit sich bringt, hauptsächlich durch die Konstruktion eines Begründungszusammenhanges für den umfassenden Zugriff auf periphere Bevölkerung.

Der offizielle Stand der Diskussion um Human Security ist derzeit noch - relativ unbestimmt. Selbst unter jenen Staaten, die im Human Security Network (HSN) engagiert sind (von den EU-Mitgliedern sind das Österreich, Griechenland, Irland, die Niederlande, und Slowenien, 
dazu kommen Norwegen und die Schweiz als europäische Nicht-EUStaaten), herrscht über die Füllung des Begriffes keine Einigkeit. Die Kompromiss-Formel, die das HSN derzeit anbietet, erinnert stark an den Good Governance-Begriff: »Human security is advanced in every country by protecting and promoting human rights, the rule of law, democratic governance and democratic structures, a culture of peace and the peaceful resolution of conflicts« (»Principles emerged from the first Ministerial Meeting of the Human Security Network in Lysøen, Norway«, May 20, 1999).

Jedoch hat sich der Ruf nach einer direkten Ausrichtung der Interventionspraxis an Human Security in den letzten Jahren merklich verstärkt. Gerade diese Forderung hat aussagekräftige Begründungsmuster hervorgebracht. Marlies Gladius und Mary Kaldor etwa sehen in der internationalen Anwendung von Kriterien der Human Security eine Art von nach außen gerichteter Anwendung der Prinzipien der Europäischen Integration: »In a sense, the human security approach is an extension of the internal methods of integration. The European Union is a political experiment that cannot be confined by territory « (Glasius/Kaldor 2005: 79f.). Sie liefern damit eine Steilvorlage für die außenpolitische Praxis der Europäischen Union. ${ }^{75}$

Und für diese präsentiert der deutsche General a.D. und Politikwissenschaftler Klaus Reinhardt ${ }^{76}$ im September 2005 bei der EADIKonferenz zum Thema »Insecurity and Development « das Konzept einer »Human Security Response Force« (Reinhardt 2005: 8ff.), das nach seiner Aussage mit den Führungsebenen der CFSP der Europäischen Union, insbesondere mit Javier Solana, akkordiert ist. In einer derartigen »integrierte[n] zivil-militärischen Organisation« (ebd.: 9) sollen 5.000 Leute in permanentem Bereitschaftsstatus stehen, um bei auftretenden Krisen schnell und nachhaltig intervenieren zu können. Während der militärische Anteil aus den bestehenden Kräften der Rapid Reaction Force der EU gebildet werden soll, ist die zivile Komponente der humanitären Hilfe und der Entwicklungszusammenarbeit vorbehalten.

Reinhardts Ideen untermauern Mark Duffields Einschätzung, die Verschmelzung von Entwicklung und Sicherheit unter der Agenda von Human Security hätte im entwicklungspolitischen Feld zu »Wachstum

75 Wir können davon ausgehen, dass ihre als klassisches Think-Tank-Paper zu klassifizierende »Human Security Strategy for the European Union« (Glasius/Kaldor 2005) vorher von der politischen Ebene bestellt und in Abstimmung mit ihr erarbeitet worden ist.

76 Klaus Reinhardt war Kommandant der Alliierten Landstreitkräfte Europa Mitte (LANDCENT) der NATO, von 1999 bis 2000 leitete er die KFORMission in Kosovo. 
und Wandel« geführt (Duffield 2004: 81). Wohl nie zuvor in der europäischen Geschichte hat es Konzepte für eine derartige Verschmelzung entwicklungspolitischer und militärischer Ansätze gegeben. Folgen wir der Argumentation von Mark Duffield, ist eine solche Entwicklung in der Struktur des Human Security-Begriffes angelegt: »Menschliche Sicherheit erweitert die Macht der internationalen Gemeinschaft deutlich, das Leben nicht-westlicher Völker zu >billigen«" (Duffield 2004: 76). Dieser Zugriff auf nicht-westliches Leben ist demnach das besondere Spezifikum der Nachhaltigkeit, die damit biopolitischen Charakter annimmt: »Sustainable development defines the type of >development is securitised in human security. In promoting diversity and choice, sustainable development is a biopolitics of life« (Duffield 2005b: 6).

Ein vierter Begriff, der bei der Entwicklung und Vermarktung neuer Interventionsansätze stark an Relevanz gewinnt, ist »Peacebuilding«. Die Bedeutung des Konzeptes ist vielschichtig, im weitesten Sinne könnten wir denn auch alle bislang genannten Strategien als Peacebuilding klassifizieren. Erstmals explizit auf großer Bühne erwähnt wurde der Begriff »Peacebuilding« im Jahr 1992 in dem bereits erwähnten "Agenda for Peace«-Report des damaligen UN-Generalsekretärs Boutros-Ghali (1992). Dort wird der Begriff vor allem im Unterschied zu präventiver Diplomatie, sowie den primär militärisch besetzten Konzepten des »Peacemaking« und »Peace-Keeping" als weitere Interventionsmöglichkeit eingeführt (vgl. auch Schneckener 2005a: 18f.). Im Zuge des bislang wohl systematischsten Versuches einer Evaluation des Feldes, der Utstein-Peacebuilding-Studie77, die die Aktivitäten der vier ursprünglichen Utstein-Partnerländer, Norwegen, Deutschland, Großbritannien und der Niederlande vergleichend untersuchte, wurden vier Bereiche identifiziert, die als Peacebuilding-Aktivitäten zu qualifizieren seien

77 Die Utstein-Gruppe wurde von den für Entwicklungspolitik zuständigen Minister/innen Norwegens, Großbritanniens, Deutschlands und der Niederlande im Jahr 1999 bei einem Treffen im norwegischen Kloster Utstein nahe Stavanger etabliert. Sie war von Beginn an jenen Prinzipien verpflichtet, die später im Zuge der Pariser Deklaration, der »Paris Declaration on Aid Effectiveness« im Jahr 2005, zu Prominenz gelangen würden: Partizipation, Kohärenz, Koordination, Partnerschaft, Schuldennachlass, »Untying« der Hilfsgelder, sowie eine Stärkung des multilateralen Systems (vgl. dazu auch die Utstein-Prinzipien, einsehbar unter http:// www.u4.no/projects/utstein/utsteinprinciples.cfm, zugegriffen am 31-052007). Nach dem Peacebuilding-Fokus liegt der derzeitige Arbeitsschwerpunkt der Gruppe in der Problemstellung Korruptionsbekämpfung. Im Jahr 2005 haben sich in diesem Rahmen auch Schweden und Kanada der Utstein-Initiative angeschlossen. 
(vgl. Smith 2004a: 20): Herstellung unmittelbarer Sicherheit, Etablierung der für einen Frieden notwendigen sozioökonomischer Voraussetzungen sowie des entsprechenden politisch-institutionellen Rahmens und der Bereich der Reconciliation.

Entscheidend bei einer solchen Definition ist aber wahrscheinlich weniger der Versuch materieller Abgrenzungen und der Bestimmung von Arbeitsbereichen als die Konsequenzen auf personeller Ebene, die dieses Konzept sowohl für Entwicklungs- als auch für internationale Sicherheitspolitik mit sich bringt. Denn tatsächlich waren und sind die konkreten Aufgabenstellungen, die mit Peacebuilding neu ins Implementations-Portfolio aufgenommen werden, nicht mit dem bisherigen Personalstamm an Entwicklungsexpert/innen, Diplomat/innen oder Militärattachés zu lösen. So tut sich damit eine Lücke auf, die, trotz vehementer Bemühungen in diese Richtung, durch interne Ausbildungsmaßnahmen nicht zu schließen ist. $^{78}$

In diese Bresche springt nun eine neue Gruppe von Expert/innen, die ich entlang der von ihnen häufig gebrauchten Eigenbeschreibung als Peacebuilding-Practitioner bezeichnen will. Ihr Engagement ist allerdings keineswegs passiv. Durch einflussreiche Beratungstätigkeit (als Beispiel siehe etwa Ropers 2002) und die Vermarktung griffig klingender, einfach gehaltener Konzepte wie Mary Andersons (1999) »do no harm« oder die von mehreren Autoren erarbeiteten und in unzähligen Manuals verbreiteten Skizzen von »Conflict Sensitivity« und »Peace and Conflict Impact Assessment (PCIA)« (vgl. Paffenholz/Reychler 2007: 71ff.) können sie den gegebenen konzeptionellen Bedarf schnell befriedigen und sich somit, zumindest in der Form einzelner Personen, auch als Faktor in der bi- wie multilateralen Policy-Formulierung etablieren.

Es sind mehr oder minder fünf Richtungen, aus denen sich die Peacebuilding-Practitioner beiderseits des Atlantiks rekrutieren ${ }^{79}$ : eine Gruppe sind jene, die aus bereits lange Zeit aktiven PeacebuildingNGOs entstammen, wie etwa aus International Alert, das schon 1984/85 in der Auseinandersetzung einer internationalen professionalisierten $\mathrm{Zi}$ vilgesellschaft mit dem zu diesem Zeitpunkt frisch ausgebrochenen Krieg in Sri Lanka entsteht. Dies ist allerdings, angesichts der notwendigen langen persönlichen Geschichte in dem Bereich, ein eng begrenzter

78 Das Problem der Heranbildung von spezifisch für PeacebuildingInterventionen qualifizierten Personals wurde mir gegenüber in Interviews von mehreren bilateralen Agenturen hervorgehoben, unter anderem mehrfach von Mitarbeiter/innen der GTZ.

79 Diese Einteilung basiert nicht auf einer systematischen Untersuchung, sondern auf Erfahrungswerten aus Interviews mit Peacebuilding-Practitionern in Sri Lanka, Uganda, Kolumbien und auf den Philippinen. 
Personenkreis, der zumeist auch schon weit fortgeschrittene Karrierewege im akademischen oder Policy-Bereich aufzuweisen hat.

Eine zweite Richtung setzt sich aus Spezialist/innen für verschiedene Teilbereiche zusammen, die das neue Arbeitsfeld mittlerweile hervorgebracht hat. Sie haben zumeist spezifische Kurse für das jeweilige Thema durchlaufen, die von den renommierten Namen der Peacebuilding-Szene geleitet worden sind: so gibt es mittlerweile »do no harm«Expert/innen ebenso wie solche für Konfliktsensitivität, PCA/PCIA, Mediation oder methodenorientiertes Training ${ }^{80}$. Jene Practitioner, die für die Gestaltung der Mehrzahl der Peacebuilding-Maßnahmen im Implementierungsstadium den größten praktischen Einfluss ausüben, lassen sich zumeist mit drei Namen verbinden, die zugleich - bei aller bestehenden Unschärfe - mit den drei einflussreichsten Schulen der praxisorientierten Friedensforschung identifiziert werden können: Dieter Senghaas, Johan Galtung und John Paul Lederach. ${ }^{81}$

Dieter Senghaas, auf dessen Konzept ich zuvor bereits ausführlich eingegangen bin, ist nicht nur direkt und über sein unmittelbares Vehikel, die Berghof-Stiftung, speziell im deutschsprachigen Bereich des Peacebuilding omnipräsent, sondern auch durch seine langjährige Lehrtätigkeit an verschiedenen deutschen Universitäten (vor allem Frankfurt und Bremen). Zudem war und ist er an weiteren im außenpolitischen Bereich einflussreichen deutschen Stiftungen wie der Hessischen Stiftung für Friedens- und Konfliktforschung und der Berliner Stiftung Wissenschaft und Politik aktiv und hat so zahlreiche PeacebuildingPractitioner herangebildet. Allerdings ist sein Einfluss außerhalb des deutschen Sprachraums eng begrenzt, was auch daran liegt, dass kaum eine seiner Arbeiten auf Englisch vorliegt (eine Ausnahme ist sein Beitrag im Berghof-Handbuch, vgl. Austin et al. 2004). Zudem ist der Großteil seiner Schützlinge, und das mag mit der Staatszentriertheit seines Ansatzes zu tun haben, nicht im aktivistischen Bereich zu finden, sondern entweder im akademischen Feld oder im staatlichen oder staatsnahen Instituten und Agenturen engagiert.

80 Letztere werden vorwiegend für das immer beliebter werdende »Training of Trainers $($ ToT) « angeworben.

81 Freilich ist der Einfluss verschiedener universitärer Peace-Studies-Institute nicht zu unterschätzen, wobei hier Bradford, UK, als das wohl einflussreichste derartige Institut hervorzuheben ist. Dennoch spielen diese Institute in der Schulung und Orientierung der Practitioner im Vergleich zu den drei genannten Namen eine untergeordnete Rolle, was wahrscheinlich durch die größere Komplexität ihrer Zugänge und das Versäumnis (oder die Verweigerung), marktgerechtes Branding von eingängigen Konzepten zu betreiben, bedingt ist. 
Weder Johan Galtung noch John Paul Lederach haben im Zuge ihrer Praxis ein mit dem Senghaasschen Ansatz vergleichbares zentrales umfassendes Theoriemodell entwickelt, weswegen es bei beiden eigentlich schwierig ist, von »Schulen« im engeren Sinn zu sprechen. Beider Einfluss begründet sich primär über Lehr- und Trainingstätigkeit und unermüdlichen Aktivismus, sowie speziell bei Galtung über das Bemühen, durch institutionelle Maßnahmen aktiv eine solche eigene Schule zu begründen. Aufbauend auf seiner langjährigen Praxis im Bereich der Friedensforschung, in deren Rahmen einige seiner Konzepte wie seine Gewalttypologie (vgl. Galtung 1996) nicht mehr wegzudenken sind, gründet Galtung 1993 den Verein »Transcend«, über den die Dissemination seiner Lehren fortan primär läuft (siehe dazu Galtung 2000). Der Verein liefert gut vermarktetes und mit Zertifikaten bestätigtes Training, das seinen relativen Erfolg vor allem dem stark steigenden Bedarf verdankt. Viele der in Trainings, Mediation oder weiteren Capacity-BuildingMaßnahmen im Peacebuilding-Bereich Beschäftigten haben denn auch in der einen oder anderen Form mit einem der verschiedenen Ausläufer von Galtungs Lehrtätigkeit Kontakt gehabt.

Speziell in den USA ist der Einfluss von John Paul Lederach derzeit wahrscheinlich noch größer. Lederach entstammt selbst dem christlich motivierten Friedensaktivismus, den er über akademische Arbeit rationalisiert und erneut disseminiert. Ähnlich wie Galtung liefert auch er kein Gesamtprogramm im Sinne eines Senghaasschen »Frieden machen«, sondern einzelne, gut handhabbare Konzepte - etwa die »Peace constituencies« oder die neue Begrifflichkeit der »Conflict Transformation«, den er der zuvor gängigen »Conflict Resolution« entgegenstellt (vgl. Lederach 1997, 2003). Verbunden werden diese Konzepte mit einem stark moralisch aufgeladenen christlichen Gemeinschaftsdenken, das mit praktischer Hartnäckigkeit gekoppelt wird (vgl. Lederach 1997: xvi). Sehr gut lässt sich daran das selbst gegebene Mandat der PeacebuildingPractitioner ablesen. Als entscheidende Aufgabe von Konflikttransformation definiert Lederach (2003: 30): »How do we end something not desired and build something we do desire? « Das »wir« in diesem Satz bleibt unbestimmt, und es ist für Lederach vollkommen selbstverständlich, dass alle, die sich betroffen fühlen wollen, in diesem »wir« inkludiert sind. Dies ist letztendlich allerdings nichts anderes als eine Selbstermächtigung zur Intervention, die - auch wenn sie definitionsgemäß »nonviolently«, also frei von direkter Gewalt erfolgt - Lederachs Ansatz einen anmaßenden Charakter verleiht.

Es ist gerade die mit eingängigen Schlüsselbegriffen verknüpfte Vagheit der Arbeiten von Galtung und Lederach, die ihnen das ermöglicht, was als ihre wahrscheinlich nachhaltigste Leistung im Bereich des 
Peacebuilding angesehen werden kann: die Transformation eines akademischen Feldes - der Friedens- und Konfliktforschung - in die Form eines Handwerks, das als mit einem gewissen Maß an Kursbesuchen und zertifizierten Trainings erlernbar verkauft wird. In Lehr- und Sammelbänden, die auf die Anwendbarkeit in Trainings und Projektlukrierung ausgelegt sind, wird ein eigener Wissenskorpus produziert und reproduziert, der simplifizierte Erkenntnisse von Friedens- und Konfliktforschung mit einer weitgehend redundanten Theoretisierung fragwürdiger Praxis- und Felderfahrungen kombiniert. Thania Paffenholz und Luc Reychler $(2001,2007)$ haben sich etwa auf solche Arbeiten spezialisiert. In ihrem jüngsten Werk, das der Etablierung eines so genannten »Aid for Peace«-Ansatzes gewidmet ist, gelingt es Paffenholz und Reychler (2007), den gesamten Ablauf eines Peacebuilding-Projektes, von den Anfangsgründen über Konfliktanalysen, Setup, Implementierung, Assessments und Evaluation, auf 150 Seiten darzulegen: Peacebuilding im »Quick User Guide «.

Neben den post-politischen Ansätzen der Konfliktanalyse, die von Beobachtungen wie den »Neuen Kriegen« kommend Konfliktursachen in »ethnischen« oder »ethnopolitischen« Konstellationen, einem »Clash of Civilizations«, ökonomisch-kriminellen Motiven oder in schwacher oder scheiternder Staatlichkeit erkennen will, sind diese vier angeführten Ansätze der Intervention wesentlicher Bestandteil des Prozesses der Verschmelzung der Dispositive von Entwicklung und Sicherheit. Sie erzählen gewissermaßen diesen Prozess, da sie neuartige Problemstellungen definieren und neuartige Wege der therapeutischen Intervention vorschlagen - so zumindest eine legitime Annahme, die sich aus dieser Darstellung ergibt. Der empirische Teil wird zu fragen haben, inwieweit es sich dabei wirklich um neuartige Dinge handelt, und in welchem Maße dabei altbekannte entwicklungspolitische Muster mitspielen, die nun einfach in einer neuen Aufmachung präsentiert werden. 



\title{
Konzepte und Gestaltung
}

\section{sicherheitspolitischer}

\section{Entwicklungszusammenarbeit - \\ USA und Deutschland im Vergleich}

\begin{abstract}
»If >development < interventions look very similar
from one country to the next, one reason is that they are designed and implemented by a relatively small, interlocked network of experts.«

(James Ferguson)
\end{abstract}

Der folgende Abschnitt dient dem Vergleich der Konzepte, Ansätze und Praktiken der beiden entwicklungspolitischen Akteure USA und Deutschland im Umgang mit dem Phänomen »Bewaffneter Konflikt«. Zur Eingrenzung der Untersuchung werde ich die Konzentration auf jene Bereiche legen, die als ODA anrechenbar sind - rein private Initiativen, die keinerlei Zusatzfinanzierung aus dem öffentlichen Bereich aufweisen, werden also im Folgenden nicht miteinbezogen.

Zunächst ist es sinnvoll, anhand einiger Statistiken die Bedeutung der beiden Akteure im internationalen Rahmen einzuordnen. Abbildung 5 versucht eine solche Quantifizierung mit der Darstellung der historischen Entwicklung der ODA-Beiträge ausgewählter entwicklungspolitischer Akteure. Dazu wurde das jährliche vom DAC angerechnete ODAVolumen ins Verhältnis zu den gesamten globalen ODA-Aufwendungen gesetzt, um so eine in historischer Relation stehende Aufstellung der entwicklungspolitischen Relevanz der einzelnen Geber zu erhalten soweit eine solche aus finanziellen Aufwendungen herauslesbar ist. 
Abbildung 5: ODA der größten bilateralen Geber in Anteilen an Gesamt-ODA

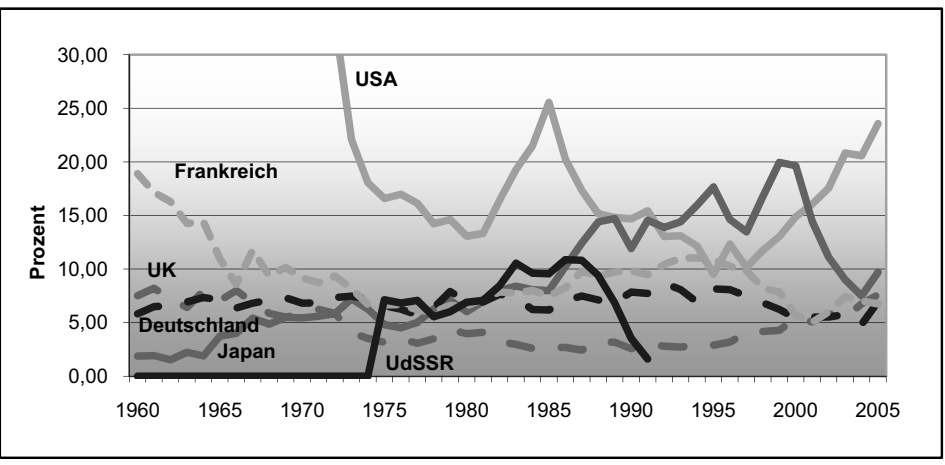

Der Verlauf der US-Beiträge weist in seiner sehr unruhigen Entwicklung auf eine bewegte entwicklungspolitische Geschichte hin, die sich, und hier sprechen die Zahlen eine eindeutige Sprache, immer mit einem im Vergleich zu anderen Gebern hohen finanziellen Einsatz vollzogen hat (natürlich nur, soweit absolute Zahlen angenommen werden: Wie noch $\mathrm{zu}$ zeigen sein wird, sind die USA relativ gesehen, gemessen am Anteil der ODA-Mittel am BIP, einer der zurückhaltendsten Geber). Nachdem die USA die Entwicklungshilfe bis in die späten 1960er Jahre mit Gesamtanteilen von weit über zwei Drittel faktisch im Alleingang bestritten haben, folgt in den 1970er Jahren eine Krisensituation, auf die später noch ausführlicher einzugehen sein wird. Die danach deutlich sichtbaren zwei signifikanten Anstiege sind mit den republikanischen Präsidentschaften Reagan und Bush (Sohn) verbunden. Dafür sind einerseits auBenpolitische Strategieentscheidungen verantwortlich - Democracy Promotion als antikommunistische Strategie bei Reagan, sowie die stark ansteigenden entwicklungspolitischen Anteile im War on Terror bei Bush -, andererseits aber auch handfeste innenpolitische Gründe: Beide Präsidenten sehen sich im Kongress bei entwicklungspolitischen Schritten keiner Ablehnungsfront gegenüber wie etwa die Administrationen Carter oder Clinton. Aus diesem Prozess eine generell besonders ausgeprägte entwicklungspolitische Affinität republikanischer Administrationen herauszulesen wäre folglich übertrieben, in jedem Fall ist allerdings das Gegenteil - eine prinzipielle Skepsis republikanischer Administrationen diesem außenpolitischen Instrument gegenüber - auszuschließen.

Auch bei der Interpretation der Anteile der anderen aufgelisteten Geber muss angesichts der Relationalität der Werte Vorsicht gelten. So ist der vermeintlich starke Abfall Japans, des weltweit größten Gebers 
der 1990er Jahre, nach der Jahrtausendwende nicht notwendigerweise mit einer politischen Umorientierung verbunden. Zwar waren die japanischen ODA-Aufwendungen kurzfristig auch in absoluten Zahlen rückläufig, befinden sich derzeit aber wieder auf dem hohen Niveau von 1998. Allerdings war Japan nicht bereit, den finanziellen Boost der Entwicklungshilfe nach der Jahrtausendwende mitzutragen, der vor allem von den seither außerordentlich ansteigenden Beiträgen der USA getragen wird. Deren ODA-Aufwendungen haben sich im Zeitraum 1998 bis 2005 verfünffacht.

Davon ausgehend ist auch der Schluss richtig, dass jene Geber, die wie etwa Deutschland auf einen relativ steten Verlauf ihrer relativen Aufwendungen verweisen können, in absoluten Zahlen einen starken Anstieg vollzogen haben. Deutschlands ODA-Gelder befinden sich heute auf dem doppelten Niveau von 1998, wenngleich sich dieser Anstieg auch aus der Entwicklung der deutschen Wirtschaftsleistung erklärt: im Verhältnis zum BIP beträgt die Zunahme gerade 0,1 Prozentpunkte, von 0,26 \% des BIP im Jahr 1998 zu 0,36 \% im Jahr 2005.

Interessant sind besonders die Aufwendungen der Geber in den für die Studie relevanten Bereichen. Sie sind - mit allen damit verbundenen Ungenauigkeiten, da sich die ODA-Anrechnungsgewohnheiten der DAC-Mitglieder zum Teil erheblich voneinander unterscheiden - am besten anhand der Ausgaben im Rahmen des DAC-Codes 150 (»Government and Civil Society«) und des neuen, erst im März 2005 offiziell eingeführten Subcodes 152 (»Conflict, Peace and Security«) festzumachen. Nachdem die Anrechnung in diesem neuen Subcode noch ziemlich lückenhaft ist, so hat etwa Großbritannien keine Aufwendungen für Code 152, was vermutlich nicht sachlich, sondern abrechnungstechnisch begründet ist, soll primär der übergeordnete Code 150 als Orientierungspunkt dienen.

Wir sehen an Tabelle 1, dass die USA auch hier der mit Abstand größte Geber sind. Über die Hälfte aller unter Code 150 (oder auch Code 152) fallenden Aufwendungen werden von den USA bestritten. Nach Großbritannien ist Deutschland bei Code 150 der drittgrößte Geber. Seine Aufwendungen von nicht ganz 500 Mio. US\$ entsprechen etwas über $5 \%$ des gesamten Volumens der im Rahmen dieses Codes angerechneten Gelder.

Einen guten Überblick über die Bedeutung des gesamten Bereiches gibt der Abgleich der für Code 150/152 aufgewendeten Gelder mit dem ODA-Gesamtvolumen des jeweiligen Akteurs, wobei der Gesamtschnitt, also der Anteil des Volumens aller Geber im Rahmen der jeweiligen Codes am gesamten globalen ODA-Aufkommen, als Orientierungswert dienen kann. 11,23 \%, also etwas über ein Zehntel aller ODA-Ausgaben 
weltweit, sind demnach dem Governance-Thema gewidmet, wobei nicht ganz $2 \%$ spezifisch auf den Subcode 152 entfallen $^{1}$.

Tabelle 1: Aufwendungen ausgewählter Geber für DAC-Codes 150/152

\begin{tabular}{|c|c|c|c|c|c|c|c|}
\hline & $\begin{array}{c}\text { Code } 150 \\
\text { (Mio US\$) }\end{array}$ & $\begin{array}{c}\text { Code } 152 \\
\text { (Mio US\$) }\end{array}$ & $\begin{array}{c}\text { Anteil } \\
\text { Code } \\
150\end{array}$ & $\begin{array}{c}\text { Anteil } \\
\text { Code } \\
152\end{array}$ & & $\begin{array}{c}\text { Code } 150 \\
\text { Gesamt- } \\
\text { ODA }\end{array}$ & $\begin{array}{c}\text { Code } 152 \\
\text { Gesamt- } \\
\text { ODA }\end{array}$ \\
\hline Alle Geber & 9222,21 & 1462,4 & & & Alle Geber & $11,23 \%$ & $1,78 \%$ \\
\hline USA & 4732,44 & 827,49 & $51,32 \%$ & $56,58 \%$ & Australien & $21,40 \%$ & $0,66 \%$ \\
\hline UK & 1090,31 & 0 & $11,82 \%$ & $0,00 \%$ & USA & $18,72 \%$ & $3,27 \%$ \\
\hline Deutschland & 491,13 & 116,79 & $5,33 \%$ & $7,99 \%$ & Schweden & $16,23 \%$ & $2,79 \%$ \\
\hline Kanada & 440,71 & 60,66 & $4,78 \%$ & $4,15 \%$ & Norwegen & $16,14 \%$ & $3,71 \%$ \\
\hline Schweden & 366,07 & 62,85 & $3,97 \%$ & $4,30 \%$ & Kanada & $15,56 \%$ & $2,14 \%$ \\
\hline Norwegen & 328,03 & 75,34 & $3,56 \%$ & $5,15 \%$ & UK & $13,36 \%$ & $0,00 \%$ \\
\hline Australien & 310,04 & 9,51 & $3,36 \%$ & $0,65 \%$ & Niederlande & $8,40 \%$ & $2,14 \%$ \\
\hline Niederlande & 309,44 & 78,67 & $3,36 \%$ & $5,38 \%$ & Deutschland & $6,60 \%$ & $1,57 \%$ \\
\hline Japan & 102,25 & 7,66 & $1,11 \%$ & $0,52 \%$ & Japan & $0,98 \%$ & $0,07 \%$ \\
\hline
\end{tabular}

Die USA sind im Vergleich der großen Geber an zweiter Stelle, mit einem Aufkommen von nicht ganz 19\%. Das bedeutet, dass nicht ganz ein Fünftel aller US-amerikanischen ODA-Gelder in den GovernanceBereich fließen, wobei 3,27 \% ausdrücklich für den Bereich Frieden und Sicherheit gewidmet sind. Deutschland liegt in diesem Vergleich, obwohl im Gesamtvolumen der Aufwendungen für Code 150 an dritter Stelle, mit einem Anteil von 6,6\% (respektive 1,57\%) relativ weit zurück. Eine gänzlich andere entwicklungspolitische Orientierung lässt sich anhand dieser Zahlen bei Japan feststellen. Der weltweit zweitgrößte Geber widmet nicht einmal $1 \%$ seiner Aufwendungen den unter Code 150 zusammengefassten Aufgaben und spielt so, selbst in absoluten Zahlen gemessen, eine vergleichsweise kleine Rolle.

Bevor ich mich nun mit den historischen Bedingungen, dem institutionellen Umgang und den aktuellen Strategien und Konzepten der beiden Geber im Zusammenhang mit dem Phänomen bewaffneter Konflikt aus-

1 Auch hier wäre es allerdings falsch, aus diesem Prozentsatz Rückschlüsse auf die tatsächliche Bedeutung des Bereiches im Vergleich zu anderen entwicklungspolitischen Maßnahmen zu ziehen. Unter den damit Befassten ist nahezu einhellig die Einschätzung zu finden, dass Interventionen im Governance- und Peacebuilding-Bereich relativ billig seien, insbesondere im Vergleich zu Infrastruktur-lastigen Maßnahmen. 
einandersetze und sie dann vergleichend gegenüberstelle, möchte ich zunächst noch den Apparat des Dispositivs Entwicklung genauer beleuchten. Durch eine solche Darstellung der einzelnen Akteursknoten, die auf den verschiedenen Ebenen des Dispositivs zu finden sind, sowie der Verhältnisse und Abläufe zwischen ihnen sollten nicht nur die Praktiken der beiden schwerpunktmäßig untersuchten Geber deutlicher werden. Auch der Zusammenhang mit den historischen Voraussetzungen auf Seiten der beiden Dispositive Entwicklung und Sicherheit sollte damit vom Gesichtspunkt des Apparates, in dem sich diese Prozesse vollziehen, nachvollziehbar werden. Erst mit einem solchen Apparat bekommt der Macht-Wissen-Komplex, der ja den Kern einer Dispositivkonstruktion bildet und den ich bislang vor allem unter dem Aspekt des Wissens behandelt habe, seine gegenständliche Form.

\section{Der institutionelle Apparat des Dispositivs}

Spätestens die Auseinandersetzung mit dem Dispositivcharakter von Entwicklung hat die Frage aufgeworfen, wer denn nun eigentlich die Akteure sind, die den Dispositiv Entwicklung gestalten, und wie sie untereinander zusammenhängen. Bislang habe ich mich vorwiegend auf Einschätzungen und Statements bezogen, die aus dem politischen und wissenschaftlichen Bereich kommen, und nur vereinzelt Stellungnahmen aufgenommen, die unmittelbar den Entwicklungsagenturen der zu untersuchenden Länder entstammen - diese werden im folgenden empirischen Vergleich der Rationalitäten US-amerikanischer und deutscher entwicklungspolitischer Praxis im Zusammenhang mit dem Phänomen des bewaffneten Konfliktes ausführlich zur Sprache kommen.

Dennoch stellt sich zunächst die Frage nach der Anbindung der Policy-Ebene an die bislang hauptsächlich behandelte Ebene der Politik, sowie an jene multilateralen Akteure, die sich im permanenten Spannungsfeld zwischen Politik und Policy bewegen, insbesondere der UNO. Auch habe ich behauptet, dass sich die wissenschaftliche Ebene, etwa gerade im Prozess der Paradigmenbildung im Rahmen der Konfliktforschung, in engen Wechselwirkungen sowohl zur politischen als auch zur PolicyEbene der Dispositive von Entwicklung und Sicherheit bewegt. Wie sich diese Verhältnisse gestalten, habe ich weitgehend offen gelassen.

Bevor ich mich nun der Aufarbeitung der verschiedenen Materialien widme, die eine Darstellung der Rationalitäten ermöglichen sollen, ist es notwendig zu klären, mit welchen Akteuren wir es auf welchen Ebenen zu tun haben, und in welchen Wechselwirkungen sie zueinander stehen. Es geht also darum, jenen Prozess, den ich mit Åkerstrøm Andersen als 
»Apparatisierung" bezeichnet und als, wenn auch widersprüchlichen, Ausdruck der strategischen Qualität eines Dispositivs angenommen habe, konkret darzustellen.

Diese Darlegung jetzt vorzunehmen bedeutet jedoch, verschiedene Widersprüchlichkeiten und Simplifizierungen in Kauf zu nehmen. Zunächst ist es im Sinne einer einigermaßen geordneten Darlegung unvermeidbar, dass ich im Folgenden Dinge behaupten werde, die erst im weiteren Verlauf mit praktischen Beispielen unterfüttert werden. Ich präsentiere hier also gewissermaßen ein Resultat der Untersuchung vor ihrem eigentlichen Beginn. Zugleich abstrahiere ich in einer allgemeinen systematischen Darlegung des Apparats Entwicklung - und auf diesen werde ich mich konzentrieren ${ }^{2}$ - von den Unterschieden zwischen den einzelnen Gebern, wenn auch in der Hoffnung, dass mir dieses allgemeine Schema die Darstellung ebendieser Unterschiedlichkeiten in der Praxis der entwicklungspolitischen Prozesse erleichtert. Schließlich sind Simplifizierungen, wie bei jeder Modellbildung, unvermeidbar und jeder Blick auf ein Detail des Schemas, das ich einführen will, würde viel komplexere Wechselwirkungen offenbaren, als ich sie hier darlegen kann. Dennoch soll dies nicht als Rechtfertigung dafür dienen, überhaupt von Versuchen derartiger Aufschlüsselungen Abstand zu nehmen.

In der Tat stellt sich der institutionelle Apparat des Dispositivs Entwicklung nicht als geordnetes Schema mit klar verteilten Aufgaben und Verantwortungsbereichen dar. Viel eher lässt er sich als komplexes Dickicht charakterisieren, das verschiedenste Akteure und Akteursgruppen aus unterschiedlichen Feldern (vor allem Politik, Policy und Wissenschaft) mit unterschiedlichen Interessen auf unterschiedlichen Ebenen auf verschiedenste Weise in Beziehung setzt.

Abbildung 6 versucht, dieses Beziehungsnetz grafisch zu veranschaulichen. Der eigentliche Schwerpunktbereich der Untersuchung, der Bereich der bilateralen Entwicklungspolitik, findet sich dabei im Zentrum der Skizze, während sich links und rechts hauptsächliche Einflussfelder - die multilaterale und die wissenschaftliche Ebene - wiederfinden. Pfeile symbolisieren Einwirkungen, deren Stärke durch die Form der Verbindungslinien dargestellt wird. Eine ungebrochene Linie steht für relativ starke, eine gestrichelte für mittelstarke und eine gepunktete für schwache Wirkungen. Ich werde diese Wechselwirkungen im Folgenden entlang der mitunter mit etwas kryptischen Kürzeln bezeichneten Akteursgruppen genauer aufschlüsseln.

2 Auf eine vergleichbare Darstellung für den Dispositiv Sicherheit verzichte ich, da ich mich in der Arbeit wie erwähnt auf den entwicklungspolitischen Bereich konzentrieren will. 
Abbildung 6: Der institutionelle Apparat des Dispositivs Entwicklung

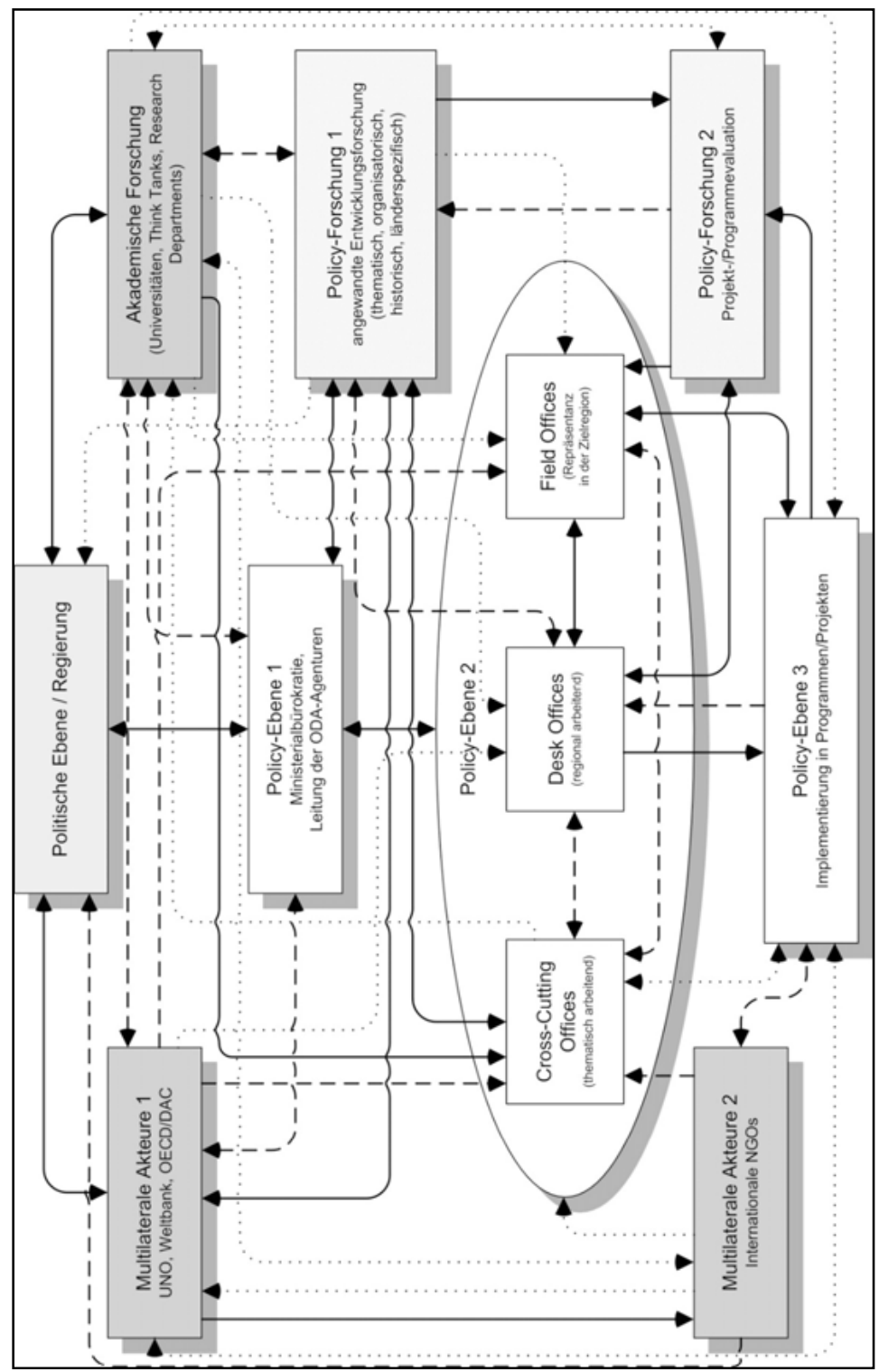




\section{Politische Ebene}

Als politische Ebene verstehe ich jenen quantitativ gesehen äußerst kleinen Teil des entwicklungspolitischen Betriebs, dem die formelle politische Gestaltungshoheit obliegt, und mit ihm auch die politische Letztverantwortung. Konkret handelt es sich dabei für gewöhnlich um eine/n Minister/in mit seinem/ihrem Stab, sowie im Falle schwerwiegenderer politischer Eingriffe um die jeweilige Regierung als Kollektivorgan. Entweder ist ein/e eigene/r Minister/in für das Ressort Entwicklungspolitik zuständig (wie etwa in Deutschland und Großbritannien, oder auch in der Europäischen Union, wo ein eigenes Kommissariat für die Agenden der Entwicklungspolitik und der Humanitären Hilfe zuständig ist ${ }^{3}$ ), oder die entwicklungspolitischen Agenden sind dem außenpolitischen Ressort unterstellt. Einen gewissen Spezialfall bilden hier die USA, wo USAID als unabhängige Agentur operiert, allerdings unter der allgemeinen außenpolitischen Leitung des/der Secretary of State.

Bei allen Gebern läuft der primäre Einfluss, den die politische Ebene auf die entwicklungspolitische Praxis ausübt, über deren unmittelbar staatlich (beispielsweise USAID) oder parastaatlich (etwa die deutsche GTZ) organisierte ODA-Agenturen. Üblicherweise verläuft dieser Prozess hierarchisch, also durch Anweisungen, die über die Policy-Ebene 1, also die oberste Stufe der entwicklungspolitischen Bürokratie, disseminiert werden. Die Formen, die diese Dissemination annehmen kann, unterscheiden sich von Geber zu Geber zum Teil erheblich, was auch der divergierenden institutionellen Verankerung (auf Ebene eines Ministeriums, einer teil-unabhängigen Agentur, oder gar beidem) der PolicyEbene 1 geschuldet ist.

Trotzdem gilt generell, dass die Vorgaben der politischen Ebene relativ allgemein gehalten sind. Unmittelbare Weisungen hinsichtlich konkreter Interventionen sind selten und zumeist anlassbezogen. ${ }^{4}$ Die Form dieser allgemeinen Vorgaben kann dabei divergieren, allerdings ist die endgültige formelle Festlegung für gewöhnlich bei allen untersuchten Gebern das Resultat informeller oder teilformalisierter Diskussionen

3 Grundsätzlich ist die Kategorisierung eines EU-Kommissars als »politische Ebene« problematisch. Im eigentlichen Sinne handelt es sich bei den Kommissaren um Teile der Spitzenbürokratie, die von der politischen Ebene der Union, also den sich im Rat artikulierenden Mitgliedsstaaten, bestellt werden. Mithin ist die oberste Ebene der EU-Entwicklungspolitik also eher auf Policy-Ebene 1 zu verorten.

4 Ein Beispiel für derartige anlassbezogene Weisungen ist etwa die Forcierung der Darfur-Intervention der deutschen EZ im Laufe des Jahres 2005 auf Anweisung der zuständigen Bundesministerin Wieczorek-Zeul. 
zwischen der gewählten politischen Ebene und der entwicklungspolitischen Führungsbürokratie.

Der vielleicht größte Einfluss, den die politische Ebene real ausübt, liegt allerdings, wie die folgenden Abschnitte bestätigen werden, nicht in der Vorgabe von Arbeitsaufträgen, wie allgemein diese auch immer gehalten sein mögen. Er liegt vielmehr in der Verankerung eines spezifischen Diskurses, dessen Eigenart es ist, nicht nur das Denken, sondern auch die praktische Umsetzung von Entwicklungszusammenarbeit maßgeblich zu beeinflussen. Dies soll nun keinesfalls bedeuten, dass die Übernahme eines Schlagwortes durch eine/n Minister/in mit sofortiger Wirkung alle Arbeitsprogramme und Projekte auf bilateraler Ebene umstößt. Aber etwa die Diskussion um fragile Staatlichkeit, die sich in politischen Strategiepapieren wie der ESS oder der NSS findet, zeigt, dass das Aufkommen derartiger Begrifflichkeiten zusammen mit den dahinter liegenden Analysemodellen signifikante und nachhaltige Auswirkungen selbst bis in die kleinsten Verästelungen des entwicklungspolitischen Handelns eines Gebers hat.

Dieser Prozess erklärt auch die enge Wechselwirkung, die ich zwischen der politischen Ebene und der akademischen Forschung annehme, was angesichts der als chronisch zu bezeichnenden Beschwerden von wissenschaftlicher Seite, gerade auf entwicklungspolitischer Ebene nicht genug in den konkreten Gestaltungsprozess miteinbezogen zu werden, seltsam anmuten mag. Die Qualität dieser Wechselwirkung findet sich praktisch nur in Ausnahmefällen, wenn auch in auf entwicklungspolitischer Ebene sehr bedeutenden ${ }^{5}$, in der unmittelbaren Einbindung von Wissenschaftler/innen in den Prozess der Politikgestaltung. ${ }^{6}$ Vielmehr

5 Einige Proponent/innen solcher bedeutender Ausnahmefälle sind bereits zur Sprache gekommen: Mary Kaldor, die als Professorin an der LSE regelmäßig eng mit der Europäischen Union zusammenarbeitet, Paul Collier, Professor am St. Anthony's College in Oxford, der von 1998 bis 2003 die Development Research Group der Weltbank geleitet hat und auch seither vorwiegend im Zusammenhang der Weltbank forscht. Auch Robert I. Rotberg, Professor an der Harvard University, arbeitet immer wieder direkt mit USAID zusammen, der Einfluss seiner Forschung zu Staatsschwäche auf die Politik der Agency ist signifikant, wie noch zu zeigen sein wird.

6 Wenngleich eine Einbindung von Wissenschaftler/innen in den Prozess der Politikgestaltung unüblich ist, hat eine personelle Fluktuation zwischen Wissenschaftler/innen und führenden Politiker/innen insbesondere in den USA doch eine lange Tradition. Henry Kissinger oder Condoleezza Rice seien als nur zwei besonders prominente Beispiele genannt, auf der anderen Seite wurde dem ehemaligen deutschen Außenminister Joschka Fischer unmittelbar nach seiner Demission eine Senior Fellow-Position an der Princeton University angeboten. 
liegt sie im gegenseitigen Antizipieren von Trends, was sich auf wissenschaftlicher Ebene im Drang nach der Kreierung analytischer Markenzeichen ausdrückt.

Dieses Antizipieren begründet sich, neben einem originär wissenschaftlichen und politisch-gestalterischen Anspruch, der ohne Zweifel vorhanden ist, auch durch Eigeninteressen der beiden Akteursgruppen. Solche von der akademischen Forschung »gelieferten« Diskurse lassen sich bei der Vermarktung politischer Programmatiken mitunter gut verwenden, bieten also eine argumentative Basis für das traditionell in der öffentlichen politischen Auseinandersetzung schwer vermittelbare Feld der Außen- und Entwicklungspolitik. Auf der anderen Seite ist der Bereich der akademischen Forschung, auf beiden Seiten des Atlantiks, stark auf die Lukrierung öffentlicher Gelder angewiesen, wobei gerade sozialwissenschaftliche Disziplinen, die nur schwerlich unmittelbar oder selbst mittelbar auf Profit ausgerichtet werden können, einen schweren Stand haben. Die Aufnahme und Verarbeitung politisch gewünschter Themenkomplexe ist eine logische Konsequenz dieser Situation.

Der Einfluss der Policy-Forschung ist demgegenüber als gering zu bewerten. Allein schon aus zeitlichen Gründen dringen spezifischere Forschungen, die sich eher an der entwicklungspolitischen Praxis und weniger an den »großen«, an einer globalen Perspektive ausgerichteten Prozessen orientieren, kaum bis zur politischen Ebene vor. Den entscheidenden Einfluss auf Policy-Ebene üben vielmehr, abgesehen von der direkten Rückkoppelung der eigenen Bürokratie, multilaterale Akteure aus. Während internationale NGOs vor allem über Lobbying und den Aufbau öffentlichen Drucks auf die politische Ebene der Geber versuchen, gestalterischen Einfluss zu nehmen, ist das Verhältnis zu den drei primären multilateralen Akteuren auf entwicklungspolitischer Ebene, der UNO, der Weltbank und der OECD, enger und wechselseitiger.

Auch bedingt durch persönliche Anbindungen kommt diesen multilateralen Akteuren die Rolle zu, den verschiedenen national laufenden Diskussionen ein internationales Forum zu bieten und im Rahmen dessen einen Prozess zu regulieren, der als Formierung eines entwicklungspolitischen Leitdiskurses bezeichnet werden kann. Wir haben bereits gesehen, wie eng sich das Aufkommen von Schlüsselbegriffen in den nationalen Agenturen an jenen Diskussionen orientiert, die im Rahmen dieser multinationalen Akteure geführt werden.

Zugleich kommt ihnen, aufgrund der ihnen eigenen großen bürokratischen Kapazität, auch im Bereich der angewandten Entwicklungsforschung wesentliche Bedeutung zu. So ist festzustellen, dass Resultate aus diesem Bereich über die multilaterale Ebene mittelbar auf die nationale politische Ebene rückwirken. Damit nehmen die multilateralen Ak- 
teure in Bezug auf die Rückkoppelung der Erkenntnisse von PolicyEbene auf die Ebene der Politik eine Schnittstellenfunktion ein, die darin besteht, Erkenntnisse aus der Praxis in gebündelter Form und mit entsprechendem politischen Gewicht zurückzuspielen. ${ }^{7}$

\section{Policy-Ebene 1}

Unter dieser Kategorie fasse ich die oberste bürokratische Leitung der nationalen entwicklungspolitischen Institutionen zusammen. Wie oben bereits erwähnt, verteilt sich diese Top-Bürokratie auf die jeweils zuständigen Ministerien (entweder eines, das unmittelbar für entwicklungspolitische Agenden zuständig ist, oder das Außenministerium) und die entwicklungspolitischen Agenturen, die für die Policy-Administration und, wie etwa GTZ, DFID oder USAID, auch für die Implementation verantwortlich sind.

Logischerweise besteht ihr engster Austausch mit der politischen Ebene einerseits und den enger an der Implementation gelegenen Teilen der Administration, der Policy-Ebene 2, andererseits. Die Aufgabe der Policy-Ebene 1 ist hier zumeist eine Art Scharnierfunktion, die sich praktisch auf organisatorische und budgetäre Maßnahmen konzentriert. Ihre wichtigste gestalterische Aufgabe im Rahmen des Dispositivs Entwicklung besteht somit in der beständigen Formung des Apparates selbst. Dies geschieht in enger Wechselwirkung mit den jeweiligen Leitmotiven des entwicklungspolitischen Diskurses, bei deren Produktion der Policy-Ebene 1 eine ebenfalls nicht zu unterschätzende Rolle zukommt.

Es sind vor allem zwei Instrumente, mit denen zu diesem Zweck gearbeitet wird. Zunächst, auf diskursiver Ebene, die Festlegung der strategischen Zielbestimmungen und ihre inhaltliche Untermauerung, die zumeist in Form von entwicklungspolitischen Gesamtprogrammen und Jahresberichten vorgenommen wird. Natürlich erfolgen diese Schritte immer in Abstimmung mit der politischen Ebene, der in grundsätzlichen Fragen die Letztentscheidung überlassen ist. Im Rahmen der vorgegebenen Leitthemen ist der Einfluss der Spitzenbürokratie auf deren spezifische entwicklungspolitische Ausformulierung jedoch überragend. Dies erklärt auch, warum dieser bei den meisten Akteuren quantitativ

7 Ein Beispiel dafür ist etwa die Kohärenz-Debatte im entwicklungspolitischen Bereich, die über das DAC der OECD den Weg in die nationalen Geberstrukturen gefunden hat, nachdem sie aus der speziell auch von der Policy-Forschung geäußerten Kritik an eben diesen Geberstrukturen gewachsen war. 
bewusst klein gehaltenen Ebene ein verhältnismäßig großer Anteil politisch-strategischer und regionaler Expert/innen angehört. ${ }^{8}$

Auch die enge Zusammenarbeit mit der Policy-Forschung (insbesondere jener Ebene, die ich unter der Kategorie Policy-Forschung 1 zusammenfasse und die sich mit angewandten entwicklungspolitischen Themenstellungen befasst, also nicht mit klassischen Evaluationen) ist aus diesem Zusammenhang zu erklären. Ziel dieser Kooperation ist die Schaffung und Schärfung von Expert/innenwissen zur Formung des entwicklungspolitischen Diskurses, der im Prozess der Abstimmung mit der politischen Ebene in Richtung der praktischen Umsetzung getrimmt wird.

Das zweite Instrument, das der Policy-Ebene 1 zur Verfügung steht, ist ein organisatorisches: die Gestaltung der Policy-Ebene 2 im Sinne der thematischen Schwerpunktlegungen, was bei Themen, die als bislang unterbelichtet wahrgenommen werden, für gewöhnlich zur Einrichtung von Cross-Cutting-Offices führt. Neben Themen wie Umwelt und Gender verfügt die Problemstellung Konflikt bei den meisten Gebern mittlerweile über die dazu notwendige inhaltliche Prominenz und dementsprechend auch über eine unmittelbare organisatorische Verankerung auf Policy-Ebene 2.

\section{Policy-Ebene 2}

Die Policy-Ebene 2 bildet gewissermaßen das Kernstück der entwicklungspolitischen Bürokratie der Geber. Sie ist für die Gestaltung der entwicklungspolitischen Praxis im Sinne der Übersetzung der allgemeinen Vorgaben, die von der Policy-Ebene 1 ausgegeben werden, in implementierbare Programmatiken verantwortlich. Grundsätzlich lässt sich

8 Dieser rückwirkende Einfluss auf die politische Ebene gehört für gewöhnlich auch zu den definierten Aufgabenstellungen der Spitzenbürokratie. So unterhält das deutsche BMZ einen eigenen Dienstsitz in Berlin, an dem sechs Referate unter anderem für die politische Beratung zuständig sind (der Hauptsitz des Ministeriums befindet sich in Bonn). DFID unterhält auf Leitungsebene gar eine eigene wissenschaftliche Einheit, die direkt dem zuständigen Minister zuarbeitet. Auch die Aufgaben des Administrators von USAID sind klar im Sinne einer Wechselwirkung mit der politischen Ebene definiert: »The Administrator (A/AID) formulates and executes U.S. foreign economic and development assistance policies and programs, subject to the foreign policy guidance of the President, the Secretary of State, and the National Security Council. Under the direct authority and foreign policy guidance of the Secretary of State, the Administrator serves as a principal advisor to the President and the Secretary of State regarding international development matters « (USAID ADS Chapter 101: Agency Programs and Functions, Revision from 02/01/2006). 
die Policy-Ebene 2 in drei Teile untergliedern, die jeweils unterschiedliche Funktionen wahrnehmen und auch auf unterschiedliche Weise in den dispositiven Apparat eingebunden sind: die mit regionalem Fokus arbeitenden Desk Offices, die in den Zielländern platzierten Field Offices, und die thematisch arbeitenden Cross-Cutting-Offices. ${ }^{9}$

Desk und Field Offices kommt dabei die zentrale Schnittstellenfunktion zur Implementationsebene, also der Policy-Ebene 3, zu. Programme werden entworfen, Projekte entweder ausgeschrieben oder direkt eigenen Einheiten zur Umsetzung aufgetragen. Länder- und Regionalprogramme werden hier ausgearbeitet, und die Information der übergeordneten Ebenen durchläuft in diesen Offices ihre zentrale Filterungs- und Aufbereitungsinstanz. Dies wird zumeist auch durch die Einschaltung externer Überprüfungsinstanzen, also klassischer Projekt- und Programmevaluierungen unterstützt, was von mir unter der Kategorie PolicyForschung 2 zusammengefasst wird. ${ }^{10}$

Desk Office, Field Office, Policy-Ebene 3 und Policy-Forschung 2 bilden so gesehen einen in sich geschlossenen Kreislauf, der als entwicklungspolitischer Implementierungszyklus bezeichnet werden könnte. Der klassische Zyklus verläuft dabei von den Desk Offices, die die Aufträge definieren, zur Policy-Ebene 3, die vor Ort implementiert, und dann unmittelbar über Berichtswesen und mittelbar über das Medium der Evaluation zurück. Den Field Offices kommt in diesem Zyklus einerseits die Rolle der wechselseitigen Vermittlung zwischen Desk Offices und Policy-Ebene 3 zu (sie liefern daher ein ebenfalls externes, aber unmittelbarer verfügbares Feedback als die Evaluationen), andererseits sind sie zumeist für die Festlegung der Programme in den Verhandlungen mit den Gegenparteien der jeweiligen Zielländer zuständig. ${ }^{11}$

9 Ich wähle hier einschlägige englische Begrifflichkeiten, um die allgemeine Vergleichbarkeit zu wahren und die begriffliche Verwirrung zwischen Referaten, Sektorvorhaben und Ähnlichem zu vermeiden.

10 Zur Unterscheidung zwischen den Kategorien Policy-Forschung 1 und Policy-Forschung 2 siehe weiter unten.

11 Dieser Implementierungszyklus bildet das historisch gewachsene Kernstück der entwicklungspolitischen Praxis, anders formuliert ist es das Wesen der Entwicklungszusammenarbeit. Verschiedene Eigentümlichkeiten dieses Zyklus, deren Vorhandensein sich einzig aus Traditionalismus erklärt, sind in jüngster Zeit auch in der entwicklungspolitischen Szene unter Beschuss geraten. Ein mittlerweile fast schon klassisches Beispiel für diese Form der Selbstkritik sind die Projekt- und Programmzyklen, die sich zumeist im Spielraum von zwei bis drei Jahren bewegen, zumeist mit der Option auf eine Verlängerung. Somit liegt die gängige Laufzeit »erfolgreicher « Projekte bei sechs Jahren, wobei der Bewegungsspielraum in der Implementation für gewöhnlich relativ gering ist. Dass diese Vorgangsweise jeder praktischen Logik entbehrt, gehört mittlerweile zum Stehsatz 
Den Cross-Cutting-Offices kommt gegenüber diesem eingespielten Zyklus letztendlich die Rolle eines, wenn auch qualifizierten, Außenseiters zu. Diese Einschätzung mag überzeichnet erscheinen, und vielleicht ist meine Darstellung der Verbindung zu den Desk Offices und der Police-Ebene 3 mit gestrichelten und gepunkteten Linienführungen zu scharf. Schließlich sind die Cross-Cutting-Offices zumeist nicht nur für spezifische Qualifizierungsmaßnahmen und die Ausarbeitung von Guidelines zuständig, sondern auch oftmals (zumindest in als konfliktgefährdet identifizierten Regionen) in die Ausarbeitung von Länderprogrammen eingebunden.

Dennoch kann das Verhältnis der klassischen Desk Officers zu den sich thematisch einbringenden Kolleg/innen oftmals als eines charakterisiert werden, das sich salopp formuliert mit dem Begriff »gesunde Skepsis« umschreiben lässt. Maßgeblich ist dafür zunächst der Zustand der permanenten inhaltlichen Überforderung, dem die Desks in ihrer Arbeit der Programm- beziehungsweise Projektgestaltung und -abwicklung tendenziell ausgesetzt sind. $\mathrm{Zu}$ viele Cross-Cutting-Themen sind mittlerweile obligatorisch in die Konzeptionen einzubauen und zugleich mit den verschiedenen allgemeinen strategischen und konkreten länderspezifischen Zielsetzungen der Agenturen für ein betreffendes Land oder eine betreffende Region in Einklang zu bringen. Diese Tatsache steht in enger Wechselwirkung mit einer ohnehin vorhandenen konservativen Tendenz der mittleren Entwicklungsbürokratie, die Änderungen im Ablauf zumeist nur zögerlich aufzunehmen bereit ist.

So ist der inhaltliche Austausch dieser Einheiten mit der wissenschaftlichen Ebene (speziell mit der Policy-Forschung 1, die in vielen Fällen die Funktion der Ausarbeitung der thematischen Problemstellun-

der meisten Praktiker/innen, speziell auf Policy-Ebene 1, wobei der Schwenk hin zur Programmorientierung oder das Umschichten der eingesetzten Mittel auf den Budgetsupport in den Zielregionen als mögliche Auswege aus der so genannten »Projektitis« hin zu langfristig orientiertem strukturellen Engagement gesehen werden (vgl. etwa Nuscheler 2006: 201ff.). Trotz all dieser Bemühungen erweist sich der Projektzyklus jedoch als ungemein zäher Mechanismus und hat bei den meisten Gebern jedem bislang unternommenen Verdrängungsversuch erfolgreich widerstanden. Dies mag daran liegen, dass er im Zuge seiner langjährigen Existenz einen beachtlichen Markt von auf diesen Zyklus orientierten Implementierungsspezialist/innen gezüchtet hat, die unter dem Label eines entwicklungspolitischen NGOs in zumeist gutem öffentlichen Ruf stehen und sich somit der Tendenz zur Trockenlegung ihres Marktes höchst effizient widersetzen können (jedenfalls gehört das Paradox der gleichzeitigen Forcierung von Budgetsupport und Programmorientierung einerseits und der Kooperation mit NGOs andererseits zu den großen blinden Flecken der gegenwärtigen entwicklungspolitischen Programmdiskussion). 
gen entlang des vorhandenen akademischen State-of-the-Art vornimmt) enger als mit den Kolleg/innen an den Desks oder vor Ort in den Regionalbüros. Diese Tendenz, wenngleich in vielen Fällen stimmig, ist allerdings keinesfalls verallgemeinerbar, wie wir in der folgenden genaueren Auseinandersetzung mit zwei dieser Geberstrukturen sehen werden.

\section{Policy-Ebene 3}

Die Policy-Ebene 3 zeichnet für die Umsetzung der Projekte im Feld ${ }^{12}$ verantwortlich. So einfach und klar diese Definition ist, umreißt sie doch ein vielfältiges und komplex vernetztes Sammelsurium an Akteuren, die nicht nur von Geber zu Geber, sondern auch von Zielregion zu Zielregion variieren. Trotz dieser zum Teil erheblichen Variationen werde ich versuchen, die im Rahmen dieser Kategorie involvierten Akteure zumindest so weit zu gruppieren, dass ein Blick auf ihren Einfluss in der Gestaltung des Dispositivs Entwicklung möglich wird.

Vier Akteursgruppen lassen sich unterscheiden, die im Rahmen der bilateralen Entwicklungszusammenarbeit in der Projekt-Implementierung engagiert sind: die jeweiligen staatlichen/parastaatlichen Agenturen der Geber selbst, kommerzielle Kontraktoren, internationale NGOs, sowie lokal/regional situierte NGOs.

Die Voraussetzung dafür, dass die Agenturen selbst unmittelbar in der Implementation tätig werden, bildet natürlich eine entsprechende organisatorische Ausrichtung, vor allem die Unterhaltung eines dafür vorgesehenen Personalstammes. DFID und GTZ sind Musterbeispiele von Agenturen, die mit eigenem Personal direkt in der Projektumsetzung engagiert sind. Die EU-Kommission verfolgt hingegen eine Art Mittelweg bei der Umsetzung: Zwar existiert, zumindest derzeit, kein eigener Pool an EU-Entwicklungshelfer/innen, die Durchführung wird bei Großprojekten aber auch selten an Dritte übergeben. Zumeist wird von den bestehenden Strukturen vor Ort internationales oder nationales Personal befristet für die Projektadministration angeworben.

USAID wählt in der Umsetzung immer einen eigenständigen Weg. Eine Zusammenarbeit mit NGOs ist dabei die Ausnahme, die unmittel-

12 Dies gilt natürlich nur insoweit, als die festgelegte Geber-Programmatik solche vorsieht. Bei entwicklungspolitischen Maßnahmen wie Budgetsupport und Schuldenstreichungen ist die Policy-Ebene 3 nicht eingebunden, da die dafür notwendigen Verhandlungen entweder von den Field Offices, also der Policy-Ebene 2, oder ohnehin direkt auf politischer Ebene geführt werden. Ein solcher vollkommener Ausschluss der Policy-Ebene 3 ist allerdings, schon allein aus den bereits angeführten historischen Gründen, unüblich. Die meisten Geber verfügen so auch über eine mehr oder weniger große Policy-Ebene 3. 
bare Implementierung wird fast ausschließlich von eigenem Personal ausgeführt. Allerdings arbeitet USAID, und auch das ist eine USamerikanische Eigenheit, in einigen Ländern ausschließlich mittels kommerzieller Kontraktoren, ist also organisatorisch (sehr wohl allerdings politisch und finanziell) nicht präsent. Ein Beispiel einer solchen Kooperation sind die Philippinen, wo die Präsenz von USAID vollständig (also auch auf Policy-Ebene 2) vom Kontraktor Louis Berger Group übernommen wird, auch in Sri Lanka wird ein Teil der Programme durch Kontraktoren umgesetzt. ${ }^{13}$

Die europäischen Agenturen wählen in der Umsetzung, sofern sie Projekte nicht durch eigenes Personal implementieren, zumeist den NGO-Sektor als strategischen Partner. Zu unterscheiden ist dabei zwischen entwicklungspolitisch ausgerichteten internationalen NGOs (dies meint auch NGOs der jeweiligen Geberländer), sowie NGOs aus den Empfängerregionen. Gesteuert wird die Tätigkeit zumeist entweder über konkrete Projektausschreibungen oder, sofern die Zielsetzungen in einem etwas weiteren programmatischen Rahmen gefasst sind, über so genannte Calls for Proposals, bei denen die konkreten Vorschläge im Rahmen eines gegebenen Programms vom NGO-Sektor selbst eingebracht werden. $^{14}$

Die komplizierten Vorgänge im Zuge der Vergabe, Abwicklung und des Abschlusses von Projekten setzen ein relativ hohes Maß an Spezialisierung des NGO-Sektors (international wie lokal) voraus. Damit war die Herausbildung eines eigenen Sektors an entwicklungspolitischen NGOs sowohl in den Geberländern wie in den Zielregionen ein historisch unvermeidlicher Prozess. Die Diskussionen zu dieser Problematik sind ebenso zahlreich wie kontrovers und sollen an dieser Stelle weder

13 Ein anderes Land, wo ein großer Teil der USAID-Aktivitäten von privaten Kontraktoren übernommen wird, ist etwa Afghanistan, wenngleich hier die zentralen Aktivitäten bei USAID selbst verbleiben.

14 Der Vollständigkeit halber muss angemerkt werden, dass NGOs keineswegs der einzige mögliche Vertragspartner solcher Aktivitäten sind. Weitere potenzielle Partner sind Wirtschaftsunternehmen (was unter dem Schlagwort der Public-Private-Partnerships zunehmend an Popularität gewinnt), sowie verschiedene Sektoren des Staates, insbesondere auf administrativer und justizieller Ebene. Dennoch bilden entwicklungspolitische NGOs nach wie vor das Gros der Partner bei Projekt-Fremdvergaben. Zur Koordination ihrer Tätigkeiten unterhalten derartige NGOs mitunter auch Regionalbüros, die jedoch vorwiegend administrativ tätig sind und damit nicht der Policy-Ebene 2 zugerechnet werden können. Dies bildet einen wichtigen Unterschied zu jenen privaten kommerziellen Kontraktoren, mit denen USAID kooperiert und deren Tätigkeiten bis hin zur Übernahme ganzer Länderrepräsentanzen gehen können. 
nachgezeichnet noch vertieft werden. ${ }^{15}$ Angesichts der sehr unterschiedlichen und vielfältigen Arten der Projektumsetzung ist eine große Differenziertheit der lokalen Kontexte und organisatorischen Strukturen unvermeidlich, was in extremen Fällen bis zu einer vollkommenen Unüberschaubarkeit reichen kann.

Der primäre Austausch der Policy-Ebene 3 findet im Rahmen des oben angesprochenen entwicklungspolitischen Implementierungszyklus statt, also mit der Policy-Ebene 2. Zu einem gewichtigen Teil ist dieser Austauschprozess über Programme, Verträge, Berichte und (unter Einschaltung der Policy-Forschung erstellte) Evaluationen formalisiert. Der nicht-formalisierte Austausch mit der Policy-Ebene 2 beschränkt sich zumeist auf die ebenfalls lokal präsenten Field Offices. Die Kommunikation mit den Desk Offices verläuft für gewöhnlich gebrochen, wobei der sichtbarere Teil durch die formal bestimmenden Auftragserteilungen von den Desks zur Policy-Ebene 3 verläuft. Die Rückkopplung geschieht in den meisten Fällen allerdings indirekt (zumeist über die Field Offices, schriftliche Berichte und Evaluationen). Direkter Kontakt zu den Cross-Cutting-Offices ist ohnehin nur in den seltensten Fällen gegeben. Deren direkter Einfluss auf die Policy-Ebene 3 beschränkt sich für gewöhnlich auf Literatur wie Manuals und Einschätzungspapiere, sowie auf Ausbildungs- und Trainingsmaßnahmen.

Die Kommunikation im Feld verläuft zumeist mit anderen international präsenten Kräften, also entweder mit Akteuren der Policy-Ebene 3 anderer Geber oder multilateralen Akteuren, vor allem präsenten internationalen NGOs und UN-Kräften. Oftmals lässt sich bei diesem Prozess beobachten, dass sich eine tendenziell abgeschlossene ExpatCommunity herausbildet.

\section{Wissenschaftliche Forschung}

Die wissenschaftliche Forschung spielt eine wesentliche Rolle bei der Gestaltung des Dispositivs Entwicklung, auch wenn sie selbst nur selten (etwa über Instrumente wie Stipendienvergaben, Kooperationen mit Universitäten in den Zielregionen oder direkte wissenschaftliche Beteiligungen in der Umsetzung von Projekten, aber auch im Rahmen der schon angesprochenen wissenschaftlichen Planstellen auf den PolicyEbenen 1 und 2) unmittelbar in entwicklungspolitische Aktivitäten eingebunden ist. Ihre Hauptrolle liegt daher außerhalb des Stranges der Po-

15 Vgl. zu dieser Diskussion so widersprüchliche Beiträge und Stellungnahmen wie etwa DAC (2001: 64, 2004: 13); Schade (2004); Stepanova (2003: 37); Nuscheler (1996: 511); Kaldor (2002); Anderson (1999: 64); Lister (2003); Glagow (1993); Esteva 1992b: 11). 
licy-Entwicklung und lässt sich in allgemeinen Worten als Erfahrungsbündelung und (strategische wie taktische) Rückkoppelung zu den im Bereich der Umsetzung tätigen Akteuren interpretieren.

Was sind nun diese Bündelungen und Rückkoppelungen, wie gestalten sie sich konkret? Den Abläufen im Prozess der Politikgestaltung und -umsetzung vergleichbar verläuft auch die wissenschaftliche Intervention mehrschichtig. Grob umrissen können dabei drei Akteursgruppierungen unterteilt werden, denen unterschiedliche Aufgaben zukommen: jene der akademischen Forschung, der Policy-Forschung 1 und der PolicyForschung 2. Diese Unterteilung ist sicher etwas künstlich, da es den entwicklungspolitischen Institutionen vergleichbare klar definierte Jobkompetenzen nicht gibt, also Think Tanks (oder zumindest einzelne Forscher/innen) auch auf allen drei Ebenen, mitunter sogar gleichzeitig, tätig sein können. Dennoch hilft die Unterscheidung, um die Diversifizierungen und vor allem die Spezialisierungen der wissenschaftlichen Rollen deutlicher nachzuzeichnen, die sich im Projekt Entwicklung historisch herausgebildet haben.

Die Policy-Forschung 2 liegt der unmittelbaren Umsetzung am nächsten. Sie ist für die Evaluierungen der verschiedenen Programme und Projekte zuständig und somit im Graubereich zwischen Wissenschaft, Consulting und bürokratischem Berichtswesen angesiedelt. Formale Möglichkeiten des eigenen steuernden Eingreifens sind letztendlich weder angestrebt noch erwünscht, es handelt sich um klar umrissene dienstliche Aufträge (die entweder extern oder auch, speziell in finanzschwachen entwicklungspolitischen Gesamtprogrammen, intern vergeben werden) mit einem Referenzrahmen, den der Auftraggeber vorgibt und der für den oder die Evaluierende/n verbindlich ist. Es geht also um eine zumindest vorgeblich unabhängige Rückkopplung des Geschehenen an Beteiligte, Verantwortliche und Geldgeber und damit in weiterer Folge um eine Überprüfung abgelieferter Berichte einerseits sowie die Grundlage für anstehende taktische (somit auf Policy-Ebene $2 \mathrm{zu}$ treffende) Entscheidungen andererseits.

Die reale Funktion der Policy-Forschung 2 erschöpft sich jedoch nicht in diesem formalen Prozess. Denn jenseits der Erarbeitung weiterer Berichte nimmt sie auch eine soziale Rolle ein, die wiederum maßgeblich auf die Etablierung eines spezifischen entwicklungspolitischen Diskurses (und damit der Gestaltung des gesamten Dispositivs) rückwirkt. Die Praxis der Evaluierungstätigkeit bedeutet neben dem Durcharbeiten der für gewöhnlich zahlreichen vorliegenden schriftlichen Berichte zu einem spezifischen Vorhaben vor allem einmal eines: Gespräche führen, zumeist mit allen unmittelbar und den wesentlichen mittelbar an dem Vorhaben beteiligten Akteuren, also der Policy-Ebene 3 und den zwei 
zentralen Akteuren auf Policy-Ebene 2, den Desk Offices (von denen zumeist der konkret angepasste Referenzrahmen vorgegeben wird) und den Field Offices.

Im Zuge dieser Diskussionen kommt es unvermeidlich zu Wirkungen (wenngleich, wie in jedem sozialen Prozess, in sehr unterschiedlicher Qualität), die von den Auftraggebern oftmals explizit gar nicht beabsichtigt sind. Diese Wirkungen resultieren in erster Linie aus der Art, wie über verschiedene konkrete Problemstellungen gesprochen wird, also in einer gewissermaßen indirekten nochmaligen Verankerung der allgemeinen Zielsetzungen des Projektes über den Referenzrahmen im Prozess der Evaluation. Jede Evaluation dient also auch, bewusst oder unbewusst, der vertieften Verankerung der programmatischen Leitlinien, insbesondere innerhalb der verschiedenen Akteure auf Policy-Ebene 3.

Durch diese Gespräche entsteht also ein nochmaliger Austausch, der das vorhandene Wissen zusammenfasst und zugespitzt definiert, es also verdichtet. Insbesondere die Diskussion der leitenden Begrifflichkeiten, und dabei vor allem die Diskussion ihrer praktischen Interpretation, ist in diesem Zusammenhang wesentlich. Denn der Trickle-Down-Effekt der Begrifflichkeiten von der politischen Ebene hin zur Policy-Ebene 3 funktioniert in erster Linie oberflächlich. Allgegenwärtige Begriffe wie »Failed States« oder »Krisenprävention« kommen zwar in jedem Fall in allen Ebenen an (allein schon bedingt durch die Notwendigkeit, diese Begriffe im Antrags- und Berichtswesen ausführlich anzuwenden), sie werden im Zuge dieses Prozesses jedoch tendenziell ihres Inhaltes entleert. Die kommunikative Intervention der Policy-Forschung 2 trägt durch ihre Diskussionen mit den beteiligten Akteuren somit auch dazu bei, Begrifflichkeiten zuzuspitzen und ihren Gebrauch zu vereinheitlichen; und sei es nur durch die Feststellung spezifischer Interpretationsschwächen, die von der Policy-Ebene 2 in der Folge (zumeist mittels Trainingsmaßnahmen) gezielt bearbeitet werden können.

Dass die Policy-Forschung 2 dabei selbst nur in den seltensten Fällen über ein klares und aus eigener empirischer und theoretischer Forschung stammendes Bild dieser Begriffe verfügen kann, da sich ihre Forschung ja hauptsächlich im Rahmen der von der Agentur ausgegebenen Referenzpunkte bewegt und globale Kontexte nur als Randerscheinung wahrnimmt, ist dabei kein Nachteil, sondern eher hilfreich: Die Intervention ist tatsächlich mehr als Mediation der unterschiedlichen vorhandenen Interpretationen zu sehen denn als Prozess des Lehrens und Lernens.

Die konkrete praxisnahe Füllung dieser Begrifflichkeiten findet vielmehr auf Ebene der Policy-Forschung 1 statt, meistens in engem Austausch mit den Policy-Ebenen 1 und 2, sowie den Forschungseinhei- 
ten von multilateralen Akteuren wie der Weltbank oder der OECD. Zwar ist dieser Prozess nicht mit der Erfindung und Definition entwicklungspolitischer Problemstellungen zu verwechseln, der tendenziell eher im Bereich des Wechselspiels zwischen politischer Ebene und akademischer Forschung verortet ist, allerdings ist seine Bedeutung auch keinesfalls zu unterschätzen.

Der verhältnismäßig größere thematische Einfluss, den die PolicyForschung 1 auf den entwicklungspolitischen Prozess ausüben kann, zeigt sich schon an der viel flexibleren Situation des Forschungsablaufes selber, angefangen von der Auftragserteilung über die Definition der Problemstellungen und Aufgaben bis hin zu den Resultaten. Zwar sind die jeweiligen entwicklungspolitischen Agenturen zumeist enge Partner und verlässliche Auftraggeber für Studien, die generell entweder thematischen, organisatorischen oder regionalspezifischen Fokus haben (der historische Bereich, den ich in der Skizze ebenfalls als Teilbereich der Policy-Forschung 1 aufgezählt habe, wird eher nachrangig behandelt, was mittlerweile als klassische Schwäche der entwicklungspolitischen Policy-Forschung gesehen werden muss). Jedoch sind sie bei weitem nicht die einzigen: NGOs, humanitäre Hilfsorganisationen oder Teile der staatlichen Bürokratie gehören ebenso zu den Kunden. Zugleich ist der Anteil der drittmittelfinanzierten Projektforschung aus diversen wissenschaftlichen Fördertöpfen nicht zu unterschätzen. Auf den Punkt gebracht bedeutet dies, dass die Policy-Forschung 1 als die eigentliche Domäne der so genannten Think Tanks zu verstehen ist. ${ }^{16}$

Die von der Policy-Forschung 1 im Zuge ihrer Arbeit produzierten Studien wirken als eine Art Operativplan zur institutionellen und praktischen Verankerung diskursiver Trends im entwicklungspolitischen Apparat. Die Policy-Forschung 1 legt den Fokus der Fragestellungen dazu eine Ebene höher als die Policy-Forschung 2. Es geht nicht um die Wirksamkeit einzelner Projekte und Programme, sondern um Wirkungen und Funktionsweisen von Entwicklungspolitik auf allgemeiner Ebene. Dabei wird einerseits nach der Identifikation erfolgreicher Schritte wie zentraler Hindernisse auf dem Weg zu den definierten entwicklungspolitischen Zielsetzungen gefragt (zumeist allerdings, ohne diese selbst zu hinterfragen), andererseits, ob nicht neue Wege im Sinne von Programmschienen, Arbeitsweisen und Partnern gefunden werden müssen, um diese zu erreichen. Es geht ihr also um eine Ausformulierung

16 Think Tanks, ursprünglich eine primär US-amerikanische Einrichtung, haben sich auch im Bereich der Entwicklungsforschung in Europa längst etabliert. Das Deutsche Institut für Entwicklungsforschung (DIE) oder das britische Overseas Development Institute (ODI) sind nur zwei Beispiele für diese Entwicklung. 
der diskursiven Vorgaben, um die wissenschaftliche Vorbereitung der organisatorischen Gestaltung des Dispositivs.

Der akademischen Forschung obliegt schließlich die Aufgabe, die großen thematischen Vorgaben der Entwicklungspolitik, ihre zentralen Problemstellungen nicht nur zu definieren und zu erforschen, sondern im eigentlichen Sinne auch, sie zu erfinden. Gerade in Hinblick auf die Verschmelzung der Komplexe Entwicklung und Sicherheit ist ihre Rolle dabei kaum zu überschätzen, ist sie es doch, die im Gegensatz zu den Policy-Ebenen und der Policy-Forschung, die in ihren jeweiligen Domänen verhaftet sind, in der Lage ist, Brücken zwischen diesen Komplexen zu schaffen, ihre Verbindung zu denken und damit diskursiv vorzubereiten. Dazu agiert sie in einer schon oben angesprochenen engen Wechselwirkung mit der politischen Ebene und übt zugleich wichtigen Einfluss auf den Bereich der Policy-Forschung, aber auch auf die PolicyEbene 1 aus. Gerade die Policy-Ebene 1 ist ja wiederum dafür zuständig, in Wechselwirkung mit der Policy-Forschung institutionelle Wege zu öffnen, um die neu erfundenen oder neu definierten Problemstellungen in bearbeitbarer Form im Apparat zu verankern.

Neben den schon erwähnten Formen, die dieser wechselseitige Einfluss annehmen kann, wie dem wechselseitigen Antizipieren von Trends oder der personellen Fluktuation zwischen den Ebenen, hat dieser Konnex auch institutionelle Verbindungen hervorgebracht. Als ein herausragendes Beispiel dafür sei die International Crisis Group (ICG) genannt. Gegründet und geleitet von ehemaligen Spitzenpolitiker/innen ${ }^{17}$ analysieren über 100 akademische Expert/innen internationale Konfliktlagen mit dem Ziel, unabhängige Lösungsansätze für die politische Ebene der weltsystemischen Zentralstaaten zu liefern. Finanziert wird die Arbeit ausschließlich durch private Spenden, die vorwiegend von US-amerikanischen Stiftungen ${ }^{18}$ bestritten werden.

Der ICG nimmt damit faktisch die Rolle einer Plattform ein, die sowohl von Staaten, multilateralen Akteuren und den verschiedenen Ebenen von Wissenschaft und Forschung genutzt wird. Zugleich ist sie auch in der praktischen Implementation von Konfliktpräventionsprogrammen aktiv, insbesondere mittels Mediation. So vereinigt die ICG gewissermaßen Komponenten der politischen Ebene, der akademischen Forschung, der Multilateralen Akteure und der Internationalen Privaten Ak-

17 Gegründet wurde die ICG vom ehemaligen EU-Außenkommissar Chris Patten. Der derzeitige Geschäftsführer ist mit Gareth Evans ein ehemaliger australischer Außenminister.

18 Diese US-amerikanischen Stiftungen sind primär philanthropische Finanzierungseinrichtungen und in diesem Sinne nicht mit den deutschen parteinahen Stiftungen zu vergleichen. 
teure, wobei ihr Schwerpunkt, inhaltlich wie personell, eindeutig in der akademischen Forschung und der Policy-Forschung 1 liegt.

\section{Multilaterale Akteure}

Ein zweiter Strang, der auf den Bereich der Gestaltung und Implementierung bilateraler Entwicklungspolitik einwirkt, ist jener im multilateralen und privaten entwicklungspolitischen Bereich. Auch diese Akteure sind sowohl in ihren Zielsetzungen als auch in ihrer institutionellen Funktion und internationalen Bedeutung vielfältig. Zwei große Gruppen lassen sich dennoch unterscheiden: einerseits jene multilateralen Organisationen, die auf Ebene der offiziellen nationalstaatlichen Politik funktionieren, also die Multilateralen Akteure, und andererseits die breit gefächerte Gruppe an internationalen Nichtregierungsorganisationen, die im entwicklungspolitischen Bereich tätig sind und die ich unter der Kategorie Internationale Private Akteure zusammenfassen will.

Die Kategorie der Multilateralen Akteure besteht hauptsächlich aus drei Organisationen, die alle in der Gestaltung der internationalen Entwicklungspolitik, auch auf bilateraler Ebene, wesentlichen Einfluss ausüben: die UNO und ihre Unterorganisationen (insbesondere das UNEntwicklungsprogramm UNDP und das in Krisenregionen zumeist präsente Hochkommissariat für Menschenrechte UNHCHR), die formal ebenfalls im UN-System integrierte Weltbank-Gruppe, sowie die OECD, vor allem das Development Assistance Committee, dem angesichts seiner Funktionen als Denkfabrik, Diskussionsforum der politischen Ebene und schließlich als eine Art Clearing House der bilateralen Entwicklungspolitik ${ }^{19}$ eine zentrale Rolle zukommt.

Schon in der Einleitung ist deutlich geworden, wie entscheidend der Einfluss dieser drei Organisationen in der Ausrichtung der entwicklungspolitischen Diskussion auf das Thema Sicherheit aus historischer Sicht gewesen ist. So hat die Weltbank mit dem von ihr erfundenen Konzept der Good Governance den grundlegenden Anstoß zur expliziten Politisierung der Entwicklungshilfe gegeben, und die UNO hat mit der Einbindung entwicklungspolitischer Konzepte in ursprünglich rein militärisch besetzte Peace-Keeping- und Peacebuilding-Strategien der An-

19 Die so genannten DAC-Codes sind der internationale Standard für die $\mathrm{Zu}-$ ordnung von Geldern zu spezifischen entwicklungspolitischen Themenbereichen. Das DAC entscheidet damit auch (natürlich als Resultat der Debatten der Mitgliedsstaaten), welche Gelder als Entwicklungshilfe deklariert werden können und welche nicht. 
näherung von Sicherheits- und Entwicklungsdispositiv einen Anfangsgrund geliefert. ${ }^{20}$

Neben dieser wichtigen Rolle in der Produktion von Schlüsseldiskursen verfügt die UNO, speziell über die schon angesprochenen Suborganisationen UNDP und UNHCHR, aber auch das UNHCR, das WFP und nicht zuletzt ihre militärischen Einsätze über eine starke Feldpräsenz. UN-Mitarbeiter/innen sind also unmittelbarer Teil der (in ihrem bilateralen Zweig) auf Policy-Ebene 3 gelagerten ImplementierungsCommunities und verfügen somit über außergewöhnlich gute Möglichkeiten der Diskurs-Dissemination. Gerade im Sicherheitsbereich übernehmen UN-Missionen zumeist mehrfache Schlüsselagenden, die von der Koordination der humanitären Hilfsprozesse über Menschenrechtsmonitoring bis hin zur Überwachung der unmittelbaren Sicherheitssituation für die präsenten internationalen $\mathrm{Kräft}^{21}$ reichen. Diese starke Verankerung an der entwicklungspolitischen Basis ist eine wesentliche Stärke der UNO, sie stellt gewissermaßen die Wurzel des starken Stammes dar, den sie im Dickicht des Dispositivs Entwicklung bilden kann ${ }^{22}$.

Ähnlich wie die UNO ist auch das DAC in der OECD als internationaler Zusammenschluss nationaler Akteure zu verstehen, sein Eigenleben ist allerdings vergleichsweise gering ausgebildet. Wie der OECD insgesamt kommt dem DAC mehr die Rolle einer Art permanenten Konferenz zu, die von den darin vertretenen Bilateralen primär zur Ausrichtung und Standardisierung der entwicklungspolitischen Anstrengungen verwendet wird. So übernimmt das DAC hauptsächlich die Funktion, bereits angestoßene und von den verschiedensten Akteuren weiterentwi-

20 Neben den drei genannten Organisationen gewinnen in zunehmendem Maße auch internationale Rechtsinstitutionen einen für die Entwicklungspolitik in Krisengebieten spürbaren Einfluss, der nicht nur auf diskursiver Ebene festzustellen ist. Die Intervention des Internationalen Strafgerichtshofes in Uganda (vgl. Pospisil 2006b) ist ein plakatives Beispiel für einen Prozess, der in Zukunft, zumindest nach dem Willen vieler einflussreicher staatlicher und nicht-staatlicher Akteure in den weltsystemischen Zentren, stark an Bedeutung gewinnen wird.

21 Diese Art der Überwachung der Sicherheitssituation, wobei Sicherheit hier unmittelbar, also im Sinne des englischen »safety« gemeint ist, obliegt dem UNDSS, dem United Nations Department of Safety and Security, das faktisch alle präsenten Hilfs- und Entwicklungskräfte in der Region über Internet und Telefon über die Lage informiert.

22 Dieses Bild würde mit gewissen Abwandlungen auch auf das Dispositiv Sicherheit zutreffen - durch die UN-Missionen ist die UNO auch im militärischen Bereich »on the ground « aktiv, wenngleich es sich bei den Soldaten nicht um UN-eigene Truppen handelt, was die Situation etwas komplexer gestaltet. 
ckelte Diskursstränge wieder einzusammeln, mit Hilfe einer starken Einbindung der Policy-Forschung 1 neu auszurichten und in dieser beständigen Ausrichtung auch ihre Implementierung voranzutreiben. Dazu hat das DAC zwei maßgebliche Instrumente eingerichtet: den PeerReview-Prozess, dem sich jeder der im DAC versammelten Geber unterwirft, und die Anrechenbarkeitskriterien, die in den DAC-Codes aufgelistet sind. Diese Codes geben dem DAC die Oberhoheit über die Qualifikation aufgewendeter Finanzmittel als Entwicklungshilfe, machen es also faktisch für das gesamte öffentliche entwicklungspolitische Feld zur international anerkannten Oberinstanz.

Die Weltbankgruppe schließlich, obwohl formal Teil des UNSystems, bildet als dritter der Multilateralen Akteure auch einen eigenen Pol. Im Gegensatz zu DAC und UNO verfolgt sie einen eigenen prononcierten Kurs, der angesichts des formell bestehenden Politikverbotes zwar nicht auf allgemein politischer, aber dafür umso stärker auf der entwicklungspolitischen Policy-Ebene festzustellen ist. Formal sind die fünf Organisationen der Gruppe im Eigentum der Mitgliedsstaaten, und durch die klaren Mehrheitsverhältnisse ${ }^{23}$ ist eine langfristig ausgerichtete kontinuierliche Arbeit garantiert.

Dieser klare entwicklungspolitische Weg der Weltbank-Gruppe, der sich mit allen Wendungen letztlich nach wie vor am ökonomistischen Bild der Modernisierungstheorien orientiert, hat innerhalb der Bank zur Herausbildung einer speziellen Art von Unternehmenskultur geführt. Dies ist einerseits am schroffen Umgang mit Kritiker/innen aus den eigenen Reihen bemerkbar ${ }^{24}$, andererseits an der scharfen Form, mit der wissenschaftliche Auseinandersetzungen nach außen geführt werden. ${ }^{25}$

23 Die Stimmverhältnisse im Gouverneursrat richten sich nach den Anteilen, die von den jeweiligen Mitgliedsstaaten an den jeweiligen Organisationen gehalten werden. So verfügen die USA durchschnittlich über ein Fünftel der Stimmrechte, Japan als zweitstärkstes Land über (je nach Organisation) $5-8 \%$.

24 So schreibt etwa William Easterly, nachdem er sich als Ökonom der Weltbank mit seiner Kritik an eigentlich anti-marktwirtschaftlichen Unternehmungen seines Arbeitgebers zu weit aus dem Fenster gelehnt hatte: »[...] many readers have asked if my statement in the original prologue that >my employer ... the World Bank ... encourages gadflies like me to exercise intellectual freedom ' was really accurate. Well almost. It should be modified slightly to $>$ the World Bank ... encourages gadflies like me to find another job« « (Easterly 2002: x).

25 Als Beispiel dafür sei die schon ausführlich behandelte Greed-GrievanceDebatte angeführt, die Paul Collier explizit gegen politikwissenschaftliche Ansätze geführt wissen will (vgl. Collier et al. 2003). Erst als die zunehmende Schärfe der Debatte beginnt, die Autorität der Weltbank zu untergraben, geht Collier in einer zweibändigen Publikation (Collier/Sambanis 
Auf der anderen Seite trägt gerade dieser Mikrokosmos dazu bei, Entwicklungspolitik nicht nur zu vollziehen, sondern auch zu gestalten. Und tatsächlich sind die wesentlichen entwicklungspolitischen Diskussionen der letzten Jahrzehnte von der Weltbank zum Teil direkt angestoßen und in jedem Fall entscheidend geprägt worden.

\section{Internationale Private Akteure}

Die Internationalen Privaten Akteure umfassen all jene Organisationen, Gruppen oder Initiativen, die in der Lage sind, aufgrund privat lukrierter Mittel Entwicklungshilfe oder, um einen relativ neuen Teil der Implementierungs-Community im Konfliktbereich mit einzubeziehen, Peacebuilding zu betreiben. Grob ist zwischen zwei Gruppen zu unterscheiden: international tätigen NGOs (von zum Teil sehr unterschiedlicher Größe) einerseits, sowie kirchlichen Gruppen andererseits. Sie unterscheiden sich von Kontraktoren und kommerziellen Anbietern entwicklungspolitischer Dienstleistungen, die auf den Policy-Ebenen 2 und 3 tätig sind, vor allem durch zwei Charakteristika: ihre (zumeist bestehende) Ausrichtung auf Gemeinnützigkeit ${ }^{26}$ und das Vorhandensein eines spezifischen entwicklungs- oder friedenspolitischen Programms.

Natürlich sind diese beiden Unterscheidungskriterien um einiges fließender, als sie zunächst scheinen. Schließlich bedeutet eine gemeinnützige Ausrichtung alles andere als einen Verzicht auf das Verdienen von Geld, denn selbstverständlich müssen die betreffenden Organisationen, Gruppierungen und Initiativen in der Lage sein, ihren Betrieb langfristig ökonomisch absichern zu können. Diese Notwendigkeit des Geldverdienens beeinflusst logischer Weise auch das entwicklungspolitische Programm, insbesondere auf der Ebene seiner Umsetzung. Nachdem sich die Einkommensquellen in den meisten Fällen auf Spendenkampagnen und Kooperationen mit bilateralen und multilateralen Gebern beschränken ${ }^{27}$, sind Tendenzen wie das schnelle Springen von Katastrophe zu Katastrophe, von bewaffnetem Konflikt zu bewaffnetem Konflikt, oder die Aufweichung von Programmatiken in Werbungen um

2005) einen Schritt zurück, und Colliers Auftraggeber wünscht sich, die selbst losgetretene Debatte zu Grabe zu tragen (»An important additional benefit of this research is that it may lay to rest the >greed versus grievance< caricature. «, ebd.: xi).

26 Eine Ausnahme bilden hier oftmals auch in der Entwicklungshilfe tätige private Missionskirchen, die zumeist aus den USA stammen. Ihre Tätigkeiten sind gewöhnlich profitorientiert, richten sich aber auch dann sehr wohl nach einer eigenen spezifischen Programmatik.

27 Eine international bedeutende Ausnahme ist Oxfam, die sich primär über ihre gleichnamige Ladenkette finanziert. 
öffentliche Aufträge unverkennbar ${ }^{28}$ und notwendiger Teil eines widersprüchlichen Geschäfts. ${ }^{29}$

Aufgrund der großen Vielfalt der Akteure kann ihr diskursiver Einfluss nur in einem sehr groben Trend wiedergegeben werden, der allerdings keinesfalls für jeden Einzelfall gültig sein muss. Generell ist bei Internationalen Privaten Akteuren jedoch aus den schon erwähnten ökonomischen Gründen die Tendenz festzustellen, überaus drastische, nicht selten stark überzeichnete Bilder gegebener Situationen zu vermitteln und Diskurse mit einer vehement moralischen Komponente zu untermauern. Die säkularen Organisationen neigen zudem dazu, einen RightsBased-Approach (basierend auf dem Menschenrechtsansatz) zu forcieren, was einerseits dadurch bedingt ist, dass sich die Arbeit oftmals anwaltschaftlich mit als benachteiligt analysierten Gruppen auseinandersetzt ${ }^{30}$, andererseits schlicht dadurch, dass dieser stark von der UNO forcierte Zugang einen auch für staatliche Akteure verbindlichen und nachvollziehbaren Referenzrahmen zur Verfügung stellt.

Merkliche Rückwirkungen auf den bilateralen Bereich gibt es in jedem Fall zwei: erstens die allgemeine Stärkung entwicklungspolitischer Öffentlichkeitsarbeit in den Geberländern. Oftmals wird dabei auch unmittelbar mit öffentlichen Gebern kooperiert. Dazu kommt als zweiter Punkt ein gewisser programmatischer Einfluss, der auch durch eine bestehende Personalfluktuation zwischen öffentlichem und privatem Bereich unterstützt wird. Historisch beginnt dieser Einfluss schon in der hitzigen Debatte um Entwicklungstheorien, wo vor allem der Dependencia-Ansatz von vielen privaten Gruppierungen, interessanter Weise auch stark von kirchlichen Gruppen, forciert wurde. Später wurde vor allem die Komponente der Anwaltschaft zentral. Die mittlerweile obligatorische Einbeziehung von Gender-Aspekt und Kinder-Problematik in der öffentlichen EZ ist zweifellos auch auf den beständigen konkreten Druck Internationaler Privater Akteure zurückzuführen, zusammen mit

28 Wiederum stellen hier viele kirchliche Organisationen eine Besonderheit dar, da sie auf regelmäßige und ereignisunabhängige Finanzquellen zurückgreifen können. Dies ermöglicht längerfristiges Engagement ohne jenes Spektakel, das speziell für im humanitären Bereich engagierte internationale NGOs ohne diesen Background unverzichtbar ist.

29 Für eine Kritik dieser Praxis im entwicklungspolitischen Bereich vgl. Zoë Marriage (2006). Für den humanitären Bereich liegt mittlerweile ein breites Spektrum an kritischen Studien vor, die von David Keens 1994 erschienenem Klassiker »The Benefits of Famine« inspiriert wurden (vgl. etwa de Waal 1997; Vaux 2001).

30 Save the Children und War Child International seien als nur zwei international bekannte Beispiele hervorgehoben. 
einem allgemeinen Wahrnehmungswandel, auf den sie auch wesentlichen Einfluss genommen haben.

Einen speziellen Platz in der Reihe der Internationalen Privaten Akteure nehmen die politischen Stiftungen ein. Sie sind in Europa ein spezifisch deutsches Instrument, wo sie hauptsächlich in Form von Parteistiftungen vorzufinden sind. Diese verfolgen trotz ihrer primär öffentlichen Finanzierung einen prononciert eigenständigen entwicklungs- und friedenspolitischen Kurs. So sind sie die derzeit einzigen Instrumente der europäischen EZ, die sich dem Aufbau, der Stärkung und der Förderung politischer Parteien in den Zielregionen widmen (vgl. Youngs 2003: 136). Gerade in politisch heiklen Bereichen erhofft sich die deutsche ODA durch eine verstärkten Kooperation mit den Stiftungen wesentliche Vorteile (vgl. Mehler/Ribaux 2000). Der international beispielgebende Charakter dieser Stiftungen wird auch dadurch deutlich, dass sie bei der Einrichtung des US-amerikanischen National Endowment for Democracy (NED) durch die Reagan-Administration Pate gestanden haben (vgl. etwa Diamond 1995), worauf ich im folgenden Abschnitt noch spezifischer eingehen werde.

Ihr Einflussbereich in den Zielregionen ist primär auf der PolicyEbene 2 anzusiedeln und bis zu einem gewissen Grad mit der repräsentativen Arbeit der Field Offices vergleichbar. Generell ist ihre Tätigkeit als Verknüpfung von politischer Arbeit und Entwicklungszusammenarbeit zu verstehen, wobei sie gerade in Krisenregionen mitunter auch die Rolle eines Fazilitators informeller Friedensgespräche einzunehmen versuchen.

\section{USA: Akteursgruppierung zum Zwecke polyarchischer Stabilität}

Im folgenden Abschnitt will ich zunächst einen gerafften Überblick über die Entstehung und die verschiedenen Phasen des Verlaufes der USEntwicklungspolitik geben, um dann jene strategischen und taktischen Elemente herauszuarbeiten, die den derzeitigen entwicklungspolitischen Umgang mit der Problemstellung bewaffneter Konflikt bestimmen. Ich werde dabei zu zeigen versuchen, dass zwar wesentliche historische Kontinuitäten vorliegen, die sich durch die 110 Jahre der Geschichte interventionistischer US-Außenpolitik ziehen, dass es aber dennoch signifikante Brüche gibt, ohne die die derzeitige Ausrichtung der USEntwicklungspolitik im Kontext bewaffneter Konflikte nicht zu verstehen ist. 
Auf diesem geschichtlichen Überblick aufbauend werde ich die derzeitige institutionelle Verankerung der sicherheitspolitischen Komplexes im entwicklungspolitischen Apparat des State Department und der Partnerorganisationen darstellen, und dann - primär anhand von Schlüsseldokumenten - die zentralen strategischen Linien nachzeichnen, mit denen dieser Apparat bewaffneten Konflikt problematisiert und Lösungen konzipiert.

\section{Historische Entwicklung und institutionelle Verankerung}

Die Geschichte der Außenpolitik der Vereinigten Staaten, die über die zumeist kriegerische Erweiterung und Fixierung des eigenen Staatsgebietes hinausgeht, ist eine vergleichsweise kurze: nur wenig mehr als hundert Jahre ist es her, seit der durch die Monroe-Doktrin geprägte Isolationismus, dessen primäres Interesse in einer Schwächung Mexikos und der Abwehr europäischer Expansionsgelüste bestand, durchbrochen wurde. Bis dahin war als einziges signifikantes, über Nord- und Zentralamerika hinausgehendes außenpolitisches Ereignis der Ankauf Alaskas von Russland im Jahr 1867 zu verzeichnen, und die USA waren, von ihren eigenen Bürgerkriegswehen geprägt, zunächst weder willig noch fähig, großflächig auf internationaler Bühne tätig zu werden. Doch in den letzten Jahren des 19. Jahrhunderts nehmen auch sie schließlich den »White Man's Burden« auf und beginnen ihre globalen Aktivitäten zur Verankerung und Verteidigung von Frieden und Freiheit.

Der Drang nach neuen Absatzmärkten im Anschluss an die abgeschlossene Binnenkolonisierung weckt Begehrlichkeiten gegenüber Spanien, wobei insbesondere die letzten spanischen Besitzungen in der Karibik, Kuba und Puerto Rico, im Zentrum des US-Interesses stehen. Im April 1898 provozieren die USA den Kriegsbeginn vor Havanna, die entscheidenden Schlachten des Krieges werden jedoch nicht in der Karibik, sondern im Pazifik ausgefochten. Die Eroberung Manilas am 13. August 1898 beendet nicht nur die spanische Kolonialherrschaft, sondern auch die philippinischen Unabhängigkeitsbestrebungen, und sie markiert einen entscheidenden Wendepunkt in der Geschichte der USAußenpolitik: den Beginn eines von bereitwilliger Übernahme internationaler Verantwortung und zivilisatorischen Vorstellungen getragenen Interventionismus, der in unterschiedlicher Ausprägungen das 20. Jahrhundert dominieren und sich bis heute fortsetzen wird.

Der maßgebliche Grundgedanke ist denkbar einfach und formuliert sich im kolonialen Kontext gegenüber den Philippinen überaus deutlich: Vollständige Unabhängigkeit benötigt gesellschaftliche Reife, eine Reife, die die neuen Kolonialherren bei den Philippinos nicht feststellen 
können. Der Unterschied zwischen Kolonisatoren und Kolonisierten wird dabei in nahtlosem Anschluss an die spanischen Kolonisatoren rassistisch kodiert (vgl. Kramer 2006). Es wäre somit unverantwortlich, die Philippinen einfach in die Unabhängigkeit zu entlassen, vielmehr gelte es, langwierige Vorbereitungen auf sozialer, politischer und institutioneller Ebene zu treffen. Fast fünfzehn Jahre wehrt sich die philippinische Unabhängigkeitsbewegung verbittert und bewaffnet gegen diesen Plan, bis sie sich schließlich dem Druck der amerikanischen Militärmacht beugen muss.

Die Philippinen werden in den folgenden hundert Jahren ein bevorzugtes Objekt der Neuorientierungen der US-Außenpolitik bleiben: so wird die Unterstützung von Ferdinand Marcos zu einem Musterbeispiel antikommunistischer Containment-Politik, die wenig später in Lateinamerika Schule machen wird. Zugleich wird allerdings das Abrücken von der Diktatur Marcos im Jahr 1986 auch das erste Beispiel und damit der historische Beginn der Phase der Democracy Promotion in der USAußenpolitik, die auch die entwicklungspolitische Komponente in einen neuen Begründungszusammenhang setzen wird.

Ein zweites Land, das wenig später von der neuen Dynamik des Interventionismus erfasst wird, ist Kolumbien. Im Zuge von Streitigkeiten mit der kolumbianischen Regierung um Landbesitzrechte in der Zone des zukünftigen Panama-Kanals entdecken die USA ihre Verantwortung für eine im Rahmen des kolumbianischen Staates als benachteiligt identifizierte Provinz im Norden Kolumbiens: Panama. Nachdem sie wenige Monate zuvor noch tatkräftig mitgeholfen hatten, panamasche Unabhängigkeitsbestrebungen zu unterdrücken, arrangieren die USA einen Putsch von Großgrundbesitzern, der mit Unterstützung der USMarine gegenüber Kolumbien abgesichert wird. Anfang November 1903 wird Panama unabhängig, und die US-Armee erbringt eine der ersten großen Leistungen der nachkolonialen Entwicklungszusammenarbeit: den Bau des Panama-Kanals. US-Präsident Theodor Roosevelt kann ruhigen Gewissens sagen, dass er nur dank der US-Unterstützung den Einwohner/innen Panamas zugute kommen werde. Diese Intervention ist nur der Anfang einer Reihe von vergleichbaren Operationen in Lateinamerika: Nicaragua, Haiti und die Dominikanische Republik werden noch in den ersten beiden Jahrzehnten des 20. Jahrhunderts Zielregionen von Interventionen, die in einem klaren politischen Kontext stehen.

Sie alle sind die Vorläufer der Erfindung von Entwicklungspolitik im engeren Sinn, die letztlich vor dem Hintergrund der durch die Truman-Doktrin im März 1947 paradigmatisch verankerten Systemkonkurrenz mit der UdSSR erfolgt. Erste Zielgebiete entwicklungspolitischer Schritte sind zwei durch den Krieg zerstörte und politisch zunächst fra- 
gile Regionen: die Frontstaaten in Europa, allen voran Deutschland, sowie Ostasien, insbesondere Japan. Im Zuge dieser Interventionen wurden nicht nur ökonomische Maßnahmen durchgeführt, wie etwa der im Juni 1947 verkündete Marshall-Plan, der auf das gesamte westliche Europa abzielt. In jenen Ländern, die bis 1945 von faschistischen Diktaturen beherrscht worden waren, werden auch genuin politische Maßnahmen gesetzt, wobei einer privaten Institution, der Ford Foundation, die federführende Rolle zukommt. Speziell die Erfahrungen mit der Installierung einer demokratisch legitimierten Regierung und der Verankerung einer neuen Verfassung in Japan (wobei dieser Prozess den Beamtenapparat aus der faschistischen Periode weitgehend unangetastet lässt) werden als nachhaltiger Erfolg interpretiert und als beispielgebend für kommende Interventionen verstanden (vgl. Orr 2004: 169ff.; Sutton 2006: 43f.).

Am 20. Jänner 1949 erklärt Harry Truman schließlich in seiner Antrittsrede vor dem Capitol in Washington Entwicklungspolitik zu einer der vier Säulen seines Programms zur Bekämpfung der kommunistischen Bedrohung. ${ }^{31}$ Dass diese »Erfindung der Unterentwicklung« (vgl. Esteva 1992: 6f.) schon von Beginn an aus einer antikommunistischen Strategie erwächst und mit der Initiative eines nordatlantischen Verteidigungsbündnisses - Punkt Drei von Trumans Programm (Punkt Eins ist die Stärkung des UN-Systems, Punkt Zwei die Weiterführung der Bemühungen zur globalen ökonomischen Stabilisierung) - verknüpft wird, zeigt ihren immanent sicherheitspolitischen Charakter. Zugleich bezieht sich Truman aber ausschließlich auf ökonomische Maßnahmen: »Our aim should be to help the free peoples of the world, through their own efforts, to produce more food, more clothing, more materials for housing, and more mechanical power to lighten their burdens."

Es geht also bei Entwicklung zunächst nicht um politische Intervention, wie sie auf den Philippinen, in Lateinamerika, in Deutschland und Japan zu diesem Zeitpunkt bereits gesetzt worden sind. Diese politischen Operationen bleiben im Geflecht zwischen verdeckt agierenden geheimdienstlichen Apparaten und privaten Stiftungen, allen voran der genannten Ford Foundation, angesiedelt. Allerdings ist Entwicklung mit ihrer Konzentration auf die Kombination makroökonomischer Anschubmaßnahmen sowie der Produktion und dem Transfer eines spezifischen Wissenskomplexes $^{32}$ als dazu komplementäre Maßnahme zu verstehen.

31 Trumans Inaugural Address ist im American Presidency Project nachzulesen, http://www.presidency.ucsb.edu.

32 »Greater production is the key to prosperity and peace. And the key to greater production is a wider and more vigorous application of modern scientific and technical knowledge«, so Truman in seiner Rede. 
Durch die Erfindung der Unterentwicklung, die historisch als Resultat der Ablehnung eines »old imperialism $\aleph^{33}$ zu sehen ist, verschiebt sich zugleich der Fokus von den kriegszerstörten Gebieten in Europa und Ostasien auf die »Dritte Welt«, also in jene Gebiete, wo die als gegeben angenommene Freiheitsliebe der Völker noch nicht in entsprechende Form gegossen werden konnte und die kommunistische Gefahr angesichts der als von Not und Armut geprägt verstandenen Lebensumstän$\mathrm{de}^{34}$ besonders groß zu sein scheint. Es geht somit um eine »expansion of the American dream to the more than half of the world stage « (Moore 1996: 129), wobei die enge Wechselwirkung dieser neuen Form der Unterstützung mit politischen Operationen nicht nur in Lateinamerika, sondern bald auch in Ost- und Südostasien spürbar wird (vgl. Ekbladh 2006).

Entwicklungshilfe bleibt trotzdem zunächst eine primär makroökonomische und technologische Operation, und die entscheidenden Schritte der nächsten Jahre finden vorwiegend auf institutioneller Ebene in den USA selbst statt: 1953 wird die Foreign Operations Administration eingerichtet, die als erste offizielle Entwicklungsagentur außerhalb des State Department angesiedelt wird. Schon ein Jahr später wird sie in der International Cooperation Administration aufgelöst (vgl. hier und im Folgenden USAID 2006a). 1954 wird die Entwicklungshilfe mit einer Überarbeitung des schon 1952 in Kraft getretenen Mutual Security Act wieder enger an ein originär sicherheitspolitisches Konzept herangeführt. Entwicklungs- und Humanitäre Hilfe bekommen eine offiziell festgeschriebene Funktion bei der Intervention in Krisenregionen. Im Mittelpunkt steht dabei der Export von Agrarprodukten, ein Programm, das die Kennedy-Administration später »Food for Peace« benennen wird und das bis heute eines der Schlüsselprogramme der Humanitären Hilfe von USAID darstellt. ${ }^{35}$

Dennoch wird Präsident Eisenhower in seiner zweiten Amtszeit mit massiver Kritik an den US-Entwicklungshilfebemühungen konfrontiert. Der Präsidentschafts-Wahlkampf 1960 ist denn auch der erste Wahlkampf, in dem Foreign Assistance eine substanzielle thematische Rolle spielt. Richard Nixon, einflussreicher Vizepräsident der EisenhowerAdministration, bekommt dabei die Schwächen des bisherigen Pro-

33 »The old imperialism - exploitation for foreign profit - has no place in our plans. What we envisage is a program of development based on the concepts of democratic fair-dealing«, wie es Truman formuliert.

34 Zur Konstruktion dieser Erzählung von Armut vgl. Escobar (1995: 23ff.).

35 Der ursprüngliche Name des von Präsident Dwight Eisenhower am 10. Juli 1954 unterzeichneten Gesetzes ist Agricultural Trade Development and Assistance Act. 
gramms zu spüren. Die Ursache dafür ist bemerkenswert: 1958 veröffentlichen der politiknahe Publizist und Spezialist für Südostasien, William J. Lederer, und der Schriftsteller Eugene Burdick den Roman »The Ugly American«, der zu einem überraschenden Bestseller wird. ${ }^{36}$

Die fiktive Handlung, die in der erfundenen südostasiatischen Nation Sarkhan spielt, demonstriert anhand verschiedener prototypisch gezeichneter US-amerikanischer Akteure das Versagen der US-Außenpolitik in Asien. Propagandistisch fügen die beiden Autoren dem Roman ein nicht-fiktionales Nachwort an, das die Notwendigkeit eines Strategiewechsels im Ringen mit dem Kommunismus in Asien herausstreicht. Dabei wird der politischen Komponente eine Schlüsselrolle zugeschrieben: $»$ We have been offering the Asian nations the wrong kind of help. We have so lost sight of our own past that we are trying to sell guns and money alone, instead of remembering that it was the quest for dignity of freedom that was responsible for our own way of life « (Lederer/Burdick 1999: 284f.). Durch seinen überraschenden Erfolg und die dadurch angezettelten Diskussionen trägt der Roman das seine zum unerwarteten Sieg Kennedys über Richard Nixon bei.

Nachdem Entwicklungshilfe erstmals zu einem Thema eines Präsidentschaftswahlkampfes werden kann ist es folgerichtig, dass der neue Präsident, John F. Kennedy, die erste große Blütezeit der USamerikanischen Entwicklungspolitik einläutet. Aufbauend auf der Einsicht, die Strategie des Antikommunismus auch auf einen starken zivilen Arm abzustützen, forciert die Kennedy-Administration die zivilen Teile der internationalen Operationen, wobei die Ausrufung der »Alliance for Progress « und die vollständige Neuorganisation der US-Entwicklungshilfe durch den Foreign Assistance Act mit der daraus resultierenden Gründung der U.S. Agency for International Development (USAID) zusammen mit der Einführung eines »Development Loan Fund « und eines »Development Grant Fund«, alles im Jahr 1961, die Eckpunkte markieren. ${ }^{37}$

In der Begründung dieser Neuordnung bemüht Kennedy - deutlich wie kein Präsident vor ihm - den engen Zusammenhang zwischen ökonomischer Entwicklung in den »unterentwickelten Nationen« und dem

36 Der Roman wird im Jahr 1963 mit Marlon Brando in der Titelrolle verfilmt.

37 Zusätzlich wird als ein weiteres Finanzierungsinstrument ein »supporting assistance«-Programm eingeführt, das für gezielte Unterstützung wichtiger Verbündeter gedacht ist. Unter dem späteren Namen »Economic Support Fund« wird es ab den 1970er Jahren in der Verknüpfung von Entwicklungs- und Sicherheitsagenden eine wesentliche Rolle bekommen. 
Sicherheitsbedürfnis der Vereinigten Staaten ${ }^{38}:$ »The economic collapse of those free but less-developed nations which now stand poised between sustained growth and economic chaos would be disastrous to our national security, harmful to our comparative prosperity and offensive to our conscience.«

Die als Abteilung des State Department neu gegründete USAID vereint verschiedene relevante Bereiche der finanziellen und technischen Zusammenarbeit auf bi- wie multilateraler Ebene. Die vier Agenturen und Programme, die zusammengeführt werden, sind die International Cooperation Agency, die bisher für das entwicklungspolitische Kerngeschäft zuständig war, der Development Loan Fund, der die Kreditvergaben regelte, die Export-Import Bank, die für Maßnahmen auf dem Währungssektor zuständig war und das zuvor beim Landwirtschaftsministerium angesiedelte humanitäre Hilfsprogramm Food for Peace.

Das erste große neue Unterfangen, das USAID jenseits der traditionell dominanten Asienhilfe in Angriff nimmt, ist der massive Ausbau der Tätigkeiten in Lateinamerika. So ist USAID für den zivilen Teil der Alliance for Progress zuständig, ein vor dem Hintergrund der neuen kubanischen Bedrohung im August 1961 in Punta del Este/Uruguay von allen lateinamerikanischen Staaten außer Kuba und der Dominikanischen Republik unterzeichnetes Programm, das versucht, die scharfen Kritiken der späten 1950er Jahre aufzunehmen und in einer groß angelegten gesamt-kontinentalen Anstrengung zu bündeln.

Unter dem Motto »progreso sí, tiranía no!« kündigt Kennedy ein umfassendes Programm zur Durchsetzung der politischen Freiheit an ${ }^{39}$, das auf der als untrennbar angenommenen Verbindung dieser Freiheit mit durch Modernisierung hervorgerufenem sozialen Wandel beruht: »This political freedom must be accompanied by social change. For unless necessary social reforms, including land and tax reform, are freely made - unless we broaden the opportunity for all of our people - unless the great mass of Americans share in increasing prosperity - then our alliance, our revolution, our dream, and our freedom will fail.« Die Allianz führt die US-Hilfe in Lateinamerika in bislang nicht gekannte Höhen, die - im Verhältnis zu anderen Regionen - später auch nicht mehr erreicht werden sollten (siehe Tabelle 2).

38 Kennedys Rede (»Special Message to the Congress on Foreign Aid«, gehalten am 22. März 1961) ist einsichtig über das American Presidency Project, http://www.presidency.ucsb.edu.

39 Das Programm findet sich in der von Kennedy am 13. März 1961 gehaltenen »Address at a White House Reception for Members of Congress and for the Diplomatic Corps of the Latin American Republics«, einsichtig über das American Presidency Project, http://www.presidency.ucsb.edu. 
Tabelle 2: Bilaterale US-Hilfe nach Regionen in Prozentanteilen

\begin{tabular}{|c|c|c|c|c|c|}
\hline Region & $\mathbf{1 9 5 5}$ & $\mathbf{1 9 6 5}$ & $\mathbf{1 9 7 5}$ & $\mathbf{1 9 8 5}$ & $\mathbf{1 9 9 5}$ \\
\hline Afrika & 1 & 5 & 6 & 11 & 12 \\
\hline Asien & 54 & 52 & 51 & 14 & 6 \\
\hline Europa / GUS & 36 & 13 & 5 & 14 & 18 \\
\hline Lateinamerika & 3 & 20 & 11 & 15 & 9 \\
\hline $\begin{array}{c}\text { Naher Osten und } \\
\text { Nordafrika }\end{array}$ & 6 & 10 & 27 & 45 & 55 \\
\hline
\end{tabular}

Quelle: The Congress of the United States 1997: 10

Diese spezifische Ausprägung der US-Politik der 1960er Jahre - eine Kombination aus makroökonomischen und technischen (auch militärisch-technischen) Maßnahmen zur Bekämpfung des Kommunismus und der Erlangung eigener Sicherheit - wird vermutlich von keiner Person derart idealtypisch verkörpert wie von Walt Whitman Rostow. Der in Yale ausgebildete und später in Cambridge und am Massachusetts Institute of Technology lehrende Ökonom ist nicht nur federführend an der Erarbeitung des Modells eines in Stufen verlaufenden ökonomischen Wachstums im Kapitalismus (dem so genannten Take-Off-Modell) und schon an der Ausarbeitung des Marshall-Plans beteiligt, sondern ab 1961 während den Administrationen Kennedy und Johnson in unterschiedlichen Funktionen auch für die sicherheitspolitische Ausrichtung der USA verantwortlich.

Im Jahr 1966 wird Rostow zum »Assistant for National Security Affairs« ernannt, einer Funktion, die dem heutigen Berater für Nationale Sicherheit vergleichbar ist. Sein 1959 als Artikel und 1960 erstmals als Buch erscheinendes Werk »The Stages of Economic Growth« (Rostow 1991), das den bezeichnenden Untertitel »A Non-Communist Manifesto« trägt, wird zum politisch-ökonomischen Schlüsselwerk jener Zeit und bereitet auf wissenschaftlicher Ebene das vor, was Kennedy zum Programm seiner Präsidentschaft auf internationaler Ebene machen wird: »We must demonstrate that the underdeveloped nations - now the main focus of Communist hopes - can move successfully through the preconditions into a well established take-off within the orbit of the democratic world, resisting the blandishments and temptations of Communism. This is, I believe, the most important single item on the Western Agenda" (ebd.: 134). 
In seinen verschiedenen Funktionen ist Rostow nicht zuletzt auch federführend für die Ausweitung des Vietnam-Krieges verantwortlich. Dieser Krieg bedeutet allerdings gleichzeitig den Höhepunkt und das ultimative Scheitern des von ihm verkörperten Ansatzes, eine Erkenntnis, die sich in den späten 1960er Jahren durchzusetzen beginnt (vgl. Ekbladh 2006: 27ff.). Diese nicht nur militärische, sondern auch politische - insbesondere entwicklungspolitische - Niederlage resultiert in einem tiefen Pessimismus, der zu einer unmittelbaren tiefen Krise der bilateralen US-Entwicklungspolitik führt und so wesentlich zur Hervorbringung des Grundbedürfnisansatzes auf internationaler Ebene beiträgt.

Die Lyndon B. Johnson nachfolgende Nixon-Administration muss sich mit den Konsequenzen dieses neuen Pessimismus sofort unmittelbar auseinandersetzen. So verankert Nixon in den so genannten »New Directions in Foreign Aid « ${ }^{40}$ den Grundbedürfnisansatz als neue paradigmatische Ausrichtung der entwicklungspolitischen Aktivitäten der USA. Dennoch werden im Oktober 1971 die Budgetvorschläge für die internationale Hilfe - erstmals in der Geschichte - vom Kongress abgelehnt. ${ }^{41}$ Die Folge dieser Ablehnung ist nicht nur eine noch weitergehende Verankerung des Grundbedürfnisansatzes, als sie in den »New Directions« ohnehin schon vorgesehen war, sondern auch eine vollkommene Neuorganisation von USAID - die gestrafften Strukturen, die eingezogen werden, sind bis zum heutigen Tag in Kraft.

Letztendlich zeigen alle diese Aktivitäten, dass das erstmals von der Kennedy-Administration eingeführte Bewusstsein der Notwendigkeit eines politischen Sieges über den Kommunismus auch nach der Niederlage in Vietnam als gültig angenommen wird: »[The] >lessons` of Vietnam led to a simple yet fundamental premise: the ultimate objective of unconventional engagements is to achieve the political, not the military, defeat of adversaries. Crucial here is the shift from military to political competition as the core of US undertakings abroad, even when the military dimensions of these undertakings appear as the most salient « (Robinson 1996: 81f.). Trotzdem bleibt bis Mitte der 1980er die US-

40 Angekündigt am 28. Mai 1969 in einer »Special Message to the Congress on Foreign Aid«, einsichtig über das American Presidency Project, http://www.presidency.ucsb.edu. Entscheidende Komponenten der Neuausrichtung sind die Ausweitung der »Technical Assistance«, die Konzentration auf die Ankurbelung der Agrarproduktion, Familienplanung sowie die Forcierung von privaten Investitionen.

41 Für diese Ablehnung sind laut David Ekbladh (2006: 31) auch die zunehmende Unzufriedenheit mit der Militärhilfe und der Glaubwürdigkeitsverlust der Entscheidungsträger in den zuständigen Institutionen verantwortlich. 
amerikanische Entwicklungspolitik auf ökonomische und technische Maßnahmen beschränkt. Zwar setzen die USA in immer stärkerem Maße auch eindeutig politische Maßnahmen im Zuge ihrer Interventionen ein, diese bleiben jedoch weiterhin eine Domäne der Geheimdienste, allen voran der CIA, und werden verdeckt realisiert.

Auch der prononcierte Menschenrechtsfokus der Administration Carter ändert an dieser Ausrichtung wenig. Zwar trägt diese neue Politik ihren Teil zur mittelfristigen Schwächung der US-gestützten lateinamerikanischen Diktaturen bei, Demokratieförderung im aktiven Sinne wird jedoch kaum betrieben. »[The Carter human rights policy] relied mainly on diplomatic measures, aid cutoffs, and some forms of economic pressure; it did not rest on the use of new types of aid « (Carothers 1999: 29).

Diese Ausrichtung ändert sich interessanterweise mit der wiederum stärker ideologischen, also antikommunistischen Orientierung von Ronald Reagan, einer Administration, die gemeinhin als der Prototyp eines geostrategisch ausgerichteten außenpolitischen Realismus gilt. Nicht wenige Elemente seines Amtsvorgängers aufgreifend kündigt Reagan im Juni 1982 - bei einer Rede im britischen Unterhaus, was doch einige Symbolwirkung in Bezug auf die internationale Ausrichtung in sich trägt - das so genannte »Project Democracy« an: »The objective I propose is quite simple to state: to foster the infrastructure of democracy, the system of a free press, unions, political parties, universities, which allows a people to choose their own way to develop their own culture, to reconcile their own differences through peaceful means. This is not cultural imperialism, it is providing the means for genuine self-determination and protection for diversity. ${ }^{42}$

Obwohl es angesichts der markigen Selbstinszenierung des ReaganUmfeldes als Hardliner des Kalten Krieges nahezu paradox erscheint, ist es tatsächlich diese Administration, die sich von der Praxis der Unterstützung von Diktaturen verabschiedet und die entscheidenden Schritte der Neuausrichtung der Foreign Assistance und damit auch der Offizialisierung von deren politischen Operationen im Rahmen der Entwicklungshilfe setzt. Es darf dabei allerdings nicht vergessen werden, dass die dahinter stehenden Schlüsselerfahrungen - ähnlich wie bei der Durchsetzung des Grundbedürfnisansatzes - im Wesentlichen Niederlagen sind. Das Scheitern der Strategie der Stützung autoritärer Regimes in Ländern wie Persien und Nicaragua demonstriert unmissverständlich die Notwendigkeit eines Kurswechsels (Robinson 1996: 79).

42 Ronald Reagan am 8. Juni 1982 in der »Address to Members of the British Parliament«, einsichtig über das American Presidency Project, http:// www.presidency.ucsb.edu. 
Als theoretischer Rückhalt fungiert dabei ein außenpolitisches Konzept, das bereits auf eine lange Tradition verweisen kann: aufbauend auf den idealistischen Ansätzen von Woodrow Wilson (kurz gefasst im Begriff Wilsonianism, vgl. Wittkopf et al. 2003: 25f.) wird die These des demokratischen oder liberalen Friedens nach einer relativ langen Zeitspanne der Dominanz des Realismus wieder handlungsrelevant. Aufbauend auf der durch quantitative Daten untermauerten Aussage, dass demokratische Staaten signifikant weniger dazu neigen würden, gegeneinander Krieg zu führen, dient der Ansatz als hervorragende Rechtfertigung eines solchen Kurswechsels (für einen Überblick über die verschiedenen Arbeiten und Zugänge im Rahmen dieses Ansatzes vgl. Paris 2004: 40ff.).

Tatsächlich dürfte der Kurswechsel weniger mit einem neu entdeckten Glauben an Ideale oder selbst mit den handfest zu verwertenden Aussagen der These vom demokratischen Frieden zu tun haben, sondern mit konkreten Problemstellungen, zu deren Bearbeitung die bisherigen Zugänge nicht mehr im gewohnten Maße funktionieren. So weisen die erwähnten Niederlagen in Persien und Nicaragua einen Weg, der dann etwa in Haiti und den Philippinen zur Abkehr der Unterstützung der diktatorischen Regime von Duvalier und Marcos führen. Speziell auf den Philippinen ist dieser neue Weg aus der Sicht der USA auch als Erfolg $\mathrm{zu}$ bewerten, gelingt es doch, mit der Etablierung einer populistischen Eliten-Demokratie die als strategische Gefahr bewertete kommunistische Guerilla NPA nachhaltig zu schwächen. Die NPA hat ihre gesamte Propaganda auf die von ihr als untrennbar angenommene Verbindung zwischen Marcos und dem so genannten US-Imperialismus gerichtet und ist nach dem Abrücken der USA vom diktatorischen Regime nicht nur mit einem massiven Glaubwürdigkeitsverlust konfrontiert, sondern auch in ihrer eigenen Strategieentwicklung desorientiert (vgl. Pospisil 2004, 2005).

Diese praktischen Erfolge korrespondieren mit einer guten Verwertbarkeit auf entwicklungspolitischer Ebene, die im weiteren Verlauf mit dem Ansatz der Nachhaltigkeit eine produktive Verbindung eingehen wird: »Liberal peace aspires to secure stability within the political complexes that it encounters on its shifting borders through the developmental principles of partnership, participation and self-management. People in the South are no longer ordered what to do - they are now expected to do it willingly themselves. Compared to imperial peace, power in this form, while just as real and disruptive, is more nuanced, opaque and complex« (Duffield 2001: 34).

Zugleich bleibt natürlich die militärische Option offen, sollte dieses Self-Management nicht funktionieren oder aber die Rahmenbedingun- 
gen zur Implementierung derartiger Mechanismen als nicht gegeben eingeschätzt werden. Die Rückkehr zum Idealismus bedeutet demgemäß auch eine Zunahme des militärischen Interventionismus, der im Kalten Krieg noch besonderen geopolitischen Konstellationen (wie Korea oder Vietnam) vorbehalten war. Eine Vervielfachung der militärischen Einsätze ab Mitte der 1980er, aber dann vor allem nach dem Zusammenbruch der Sowjetunion in den 1990er Jahren ist das unmittelbare Resultat. Dies liegt in der Tradition Woodrow Wilsons. Gemessen an der Zahl der militärischen Einsätze der USA steht er, natürlich auch bedingt durch das Eingreifen im Zuge des Ersten Weltkrieges, nach wie vor an der Spitze aller US-Präsidenten, wobei die Administrationen Reagan, Bush und Clinton die danach höchsten Werte im 20. Jahrhundert aufweisen (vgl. Wittkopf 2002: 87, Zahlen für die Administration Bush Sohn liegen hier allerdings noch nicht vor). Auch wenn es auf den ersten Blick paradox erscheinen mag: in Hinblick auf die Vereinigten Staaten resultiert die Durchsetzung der Theorie vom demokratischen Frieden in einer sofort und nachhaltig spürbaren Zunahme der Kriegstätigkeit nach außen.

Der Kurswechsel führt Mitte der 1980er Jahre auch zu einer wesentlichen institutionellen Neukonfiguration, die sich in der Neuverteilung der politischen Operationen und ihrer nun zunehmend öffentlichen Gestaltung ausdrücken. Neben der verstärkten Inanspruchnahme der bereits 1953 eingerichteten United States Information Agency (USIA) ${ }^{43}$ werden zu diesem Zweck zwei neue, formell unabhängige Institute eingerichtet, die noch auf Initiativen aus Carter-Zeiten zurückgehen: das National Endowment for Democracy (NED), gegründet 1983, und das United States Institute for Peace (USIP), gegründet 1984.

Faktisch übernehmen diese beiden Institute, die organisatorisch außerhalb des außenpolitischen Apparates angesiedelt sind und damit nicht dem State Department unterstehen - wenngleich ihre Finanzierung zu einem relevanten, beim NED sogar überwiegenden Teil durch Gelder des US-Kongresses erfolgt -, Aufgaben, die zuvor zum Arbeitsbereich der CIA gehört haben. Das NED, das, wie schon erwähnt, explizit nach dem Vorbild der deutschen parteinahen Stiftungen eingerichtet wird, ist in der neuen Aufgabenteilung vor allem für die finanzielle Unterstützung von Akteuren zuständig, denen eine Schlüsselrolle bei der Demokratieentwicklung in einem schwierigen Umfeld zugebilligt wird (vgl. Carothers 1999: 95).

43 Die USIA ist traditionell primär für die Organisation der globalen USMedienpräsenz, repräsentiert etwa durch das Radio-Flagschiff Voice of America, zuständig. 1999 wird die USIA formell aufgelöst und ihre Strukturen dem State Department eingegliedert. 
Formal agiert das NED dabei völlig transparent, und alle Zuwendungen, die ausgeschüttet werden ${ }^{44}$, sind in einer Datenbank erfasst und öffentlich zugänglich. ${ }^{45}$ Trotzdem sind die wirklichen Empfänger der Gelder in vielen Fällen nur schwer zu identifizieren, da jene Organisationen, Institutionen und Gruppierungen, denen die Zuwendung gewährt wird, oftmals Kleingruppen ohne nennenswerte lokale Verankerung sind, die sich auf Kongressen und Tagungen gegenseitig besuchen und so eine Art US-subventionierten Mikrokosmos von Para-NGOs bilden. ${ }^{46}$ Die tatsächlichen Zielsetzungen der Arbeit bleiben oftmals unklar. So entpuppt sich die formale Offenheit des NED mitunter als diskrete Methode der Verschleierung.

Neben diesen Unterstützungen engagiert sich das NED auch in der wissenschaftlichen Diskussion um Demokratieförderung, wozu es Fellowships vergibt und ein eigenes Journal, das »Journal of Democracy«, herausgibt. Obwohl dieses Journal vom äußeren Eindruck her nicht von einer der gängigen wissenschaftlichen Publikationen zu unterscheiden ist, bezeichnet es William Robinson (1996: 99) als »pseudo-academic«, da »articles are commissioned by the NED staff rather than being submitted, manuscripts are not sent out for peer review and the journal is not attached to any scholarly institution «. So interpretiert Robinson das NED auch nicht als wissenschaftlich tätige Institution, sondern als »clearing-house for the exchange of ideas and debate among intellectuals and public and private sector officials around the world.«

44 Die Zuwendungen werden zumeist jährlich gewährt und bewegen sich in einem verhältnismäßig kleinen Bereich (nur wenige Grants übersteigen die Summe von 100.000 US\$).

45 Die Grants sind über die Website des NED einzusehen, http:// www.ned.org/grants/grants.html.

46 Diese Einschätzung lässt sich anhand der Grant-Empfänger in Uganda verdeutlichen: den mit Abstand größten Grant (mit 300.000 US\$) erhielt im Jahr 2005 das International Republican Institute (IRI), eines jener vier Institute, die 1983 als Implementierungsagenturen der NED-Initiativen gegründet wurden (die anderen drei sind das National Democratic Institute for International Affairs, das American Center for International Labor Solidarity, und das Center for International Private Enterprise). Zunächst ist das nach eigener Diktion parteiunabhängige Institut, das sich jedoch unzweifelhaft konservativen Werten verpflichtet sieht, vorwiegend in Osteuropa aktiv, und hat nach dem Ende des Kalten Krieges seine nicht unumstrittenen Tätigkeiten (Unterstützung der venezolanischen Oppositionsbewegung, Training der mexikanischen Regierungspartei PAN) seine Tätigkeiten in anderen Weltregionen verstärkt. Ein anderer Empfänger von größeren Zuwendungen (47.042 US\$ in 2005) ist Lwo Development Inc., ein in Norduganda tätiges NGO, das seinen Sitz bemerkenswerter Weise in Washington hat. 
Die Ausbildung, Sammlung und Ausrichtung der akademischen Szene im Bereich der Demokratieförderung im Sinne der Zielsetzungen der US-Administration zählt jedoch eher zum Aufgabenbereich der zweiten Mitte der 1980er neu gegründeten Schlüsselinstitution: dem USIP. Das USIP vergibt großzügige Grants für wissenschaftliche Forschung, sowie Fellowships für Wissenschaftler/innen, die sich mit spezifischen, als relevant eingeschätzten Friedensprozessen auseinandersetzen. In der unmittelbaren Intervention engagiert sich das USIP weniger in der Förderung von Projekten und Tätigkeiten von Akteuren, sondern in der Organisation und Begleitung von Gesprächsrunden und Verhandlungsprozessen. ${ }^{47}$

Auch an USAID geht der neue Fokus auf Demokratieförderung und Demokratieentwicklung nicht vorbei, selbst wenn er zunächst von vielen skeptisch betrachtet wird. Larry Diamond (1992) beispielsweise sieht USAID aufgrund der Ausrichtung auf ökonomisch orientierte Entwicklungspolitik als für diese neuen Aufgaben denkbar schlecht vorbereitet und damit gegenüber Institutionen wie NED und USIP im Nachteil. ${ }^{48}$ Dennoch wird im Jahr 1984 bei USAID das »Office for Democratic Initiatives $(O D I)$ « eingerichtet, dem zunächst vor allem die Finanzierung von Wahlprozessen obliegt. Weitere inhaltliche Schwerpunkte liegen im Justizsektor und einem Bereich, der später mit Sicherheitssektorreform umschrieben werden wird. Hier liegt das Augenmerk vor allem auf der Stärkung des Dialogs ziviler Administrationen mit militärischen Führungen (vgl. Diamond 1995). Der geographische Fokus wird primär auf Lateinamerika und Asien gelegt.

Wirklichen Schwung bekommt der neue Ansatz mit dem Zusammenbruch des Warschauer Pakt-Systems, der nicht nur Osteuropa für offizielle politische Interventionen der USA öffnet, sondern auch den Handlungsspielraum in anderen Weltregionen spürbar erweitert. Dies zeigt den vorausschauenden Charakter des neuen Trends, der schon Anfang der 1980er auf eine Situation gemünzt ist, die erst knapp zehn Jahre später eintreten sollte. Die wissenschaftliche Interpretation im USamerikanischen Mainstream ist denn auch euphorisch: »In short, the U.S. decision following the end of the cold war to promote democracy was not so much a policy put into motion by U.S. policy makers as it

47 Als Beispiel sei hier auf die Interventionen des USIP im MindanaoKonflikt verwiesen (vgl. Pospisil 2005: 117f.).

48 Larry Diamonds berufliches Engagement im NED - also eine unmittelbare Konkurrenzsituation - mag ein Mitgrund für diese Einschätzung sein. $\mathrm{Zu}$ gleich steht Diamond allerdings exemplarisch für viele Skeptiker/innen, die auch innerhalb von USAID selbst zu finden sind. 
was a phenomenon brought about by this global wave of democracy« (Travis 1998: 252).

Diese Interpretation mutet insofern etwas eigenwillig an, als die USA durch ihren Policy-Shift selbst eine der bestimmenden Kräfte hinter dieser globalen Welle sind. Musterbeispiel in der Theoretisierung dieser selbsterfüllenden Prophezeiung ist Samuel Huntington, der die These einer dritten Welle der Demokratisierung entwickelt. Diese Welle hätte mit der portugiesischen Nelkenrevolution von 1974 eingesetzt (Huntington 1991: 15ff.) und nicht nur in Südeuropa, sondern weltweit Auswirkungen gezeitigt: »Overall, the movement toward democracy was a global one. In fifteen years the democratic wave moved across southern Europe, swept through Latin America, moved on to Asia, and decimated dictatorship in the Soviet bloc« (ebd.: 25). Ohne die aktive Rolle der USA - und zwar im Vorfeld dieser demokratischen Transitionen, bis hin zu der aktiven Durchsetzung solcher Prozesse wie etwa in den von Huntington angeführten Beispielen Grenada, Panama oder Haiti - wäre die Dynamik einer solchen demokratischen Welle wohl kaum erklärbar.

Abbildung 7: US-Demokratieförderung in den 1990ern nach Regionen

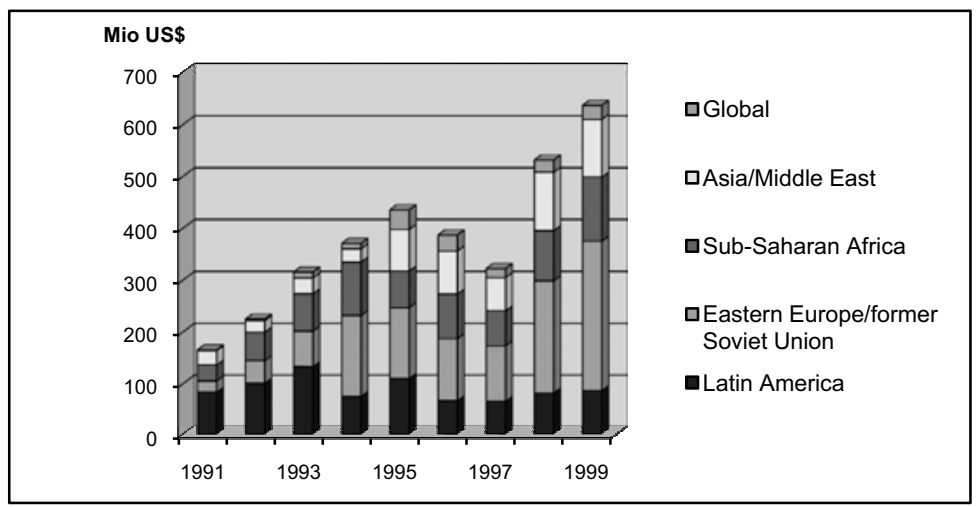

Quelle: Carothers 1999: 49ff.

Es ist Ende der 1980er Jahre vor allem Ost- und Südosteuropa, wo das Hauptgewicht der US-Demokratieförderung zum Tragen kommt. Der Schwerpunkt der Aktivitäten wird im Rahmen eines unter der Präsidentschaft von George Bush 1989 eingerichteten Programms namens »Support for Eastern European Democracy (SEED)« umgesetzt. Das Programm ist mit einem jährlichen Durchschnittsbudget von etwa 300 Mio. US\$ ausgestattet, wobei Polen quantitativ gesehen der Hauptempfänger 
ist. Der größte Anteil des Budgets für politische Maßnahmen wird jedoch in Bulgarien umgesetzt, gefolgt von Polen, Bosnien und Rumänien. $^{49}$

Gesamt gesehen trägt vor allem der massiv steigende Einsatz in Osteuropa die verhältnismäßig große Ausdehnung der Mittel in den 1990er Jahren (siehe Abbildung 7). Demgegenüber verläuft die Programmausweitung in Asien und dem südlichen Afrika verzögert ab, während das Aufkommen für Lateinamerika praktisch stagniert, wenngleich auf dem vergleichsweise hohen Niveau der späten 1980er. Der Arabische Raum (in Carothers Statistik leider nicht getrennt aufgeführt) ist von der Neuentwicklung überhaupt am wenigsten berührt (vgl. Carothers 1999: 44).

Um die Behauptung der stark ansteigenden Relevanz des Bereiches Demokratieförderung im gesamten Kanon der außenpolitischen Maßnahmen der USA zu überprüfen, ist ein Blick auf Vergleichswerte notwendig, die den quantitativ unbestreitbaren Anstieg in ein Verhältnis zum Gesamtkanon der außenpolitischen Maßnahmen setzen. Hier zeigt ein Abgleich mit dem vom DAC kommissionierten Gesamtvolumen der US-Entwicklungshilfe (siehe Abbildung 8), dass sich der Bereich der Demokratieförderung im Zuge der 1990er Jahre tatsächlich im beständigen Anstieg befindet und im Jahr 1999 schließlich fast $10 \%$ des Gesamtvolumens umfasst ${ }^{50}$ (wobei dies insofern relativiert wird, als die ODA-Aufwendungen der USA in dieser Dekade weitgehend stagnieren).

49 Insgesamt acht Länder - die Tschechische Republik, Estland, Ungarn, Lettland, Litauen, Polen, die Slowakei und Slowenien - haben im Rahmen des Programms »graduiert«, es also aus Sicht des State Department erfolgreich durchlaufen. Alle begünstigten Länder, sowie auch Bulgarien und Rumänien, die beiden nicht "graduierten « Hauptempfänger, treten in der Folge der NATO und der EU bei, was das State Department als Zeichen des überwältigenden Erfolgs des Programms interpretiert (vgl. http://usinfo.state.gov/partners/partnership/europe_eurasia.html. Zugegriffen am 03-01-2007). Die magere Ausbeute in Bosnien, das immer stärker in den Mittelpunkt der Aktivitäten rückt, steht dieser Einschätzung entgegen.

50 In diesem Zusammenhang ist allerdings darauf hinzuweisen, dass sich Carothers in seiner Rechnung nicht auf das Kriterium der ODA-Anrechenbarkeit bezieht, es kann von diesem Vergleich also nicht die Aussage getroffen werden, dass 1999 9,3 \% der ODA für Demokratieförderung ausgegeben wurden, sondern nur, dass das Volumen der Demokratieförderung 9,3\% des Volumens der gesamten ODA der USA betragen hat. Zudem bezieht sich Carothers für 1999 auf die budgetierten (und nicht die tatsächlichen) Werte (vgl. Carothers 1999: 49ff.). 
Abbildung 8: US-Demokratieförderung im Verhältnis zur Gesamt-ODA in den $1990 e^{51}$

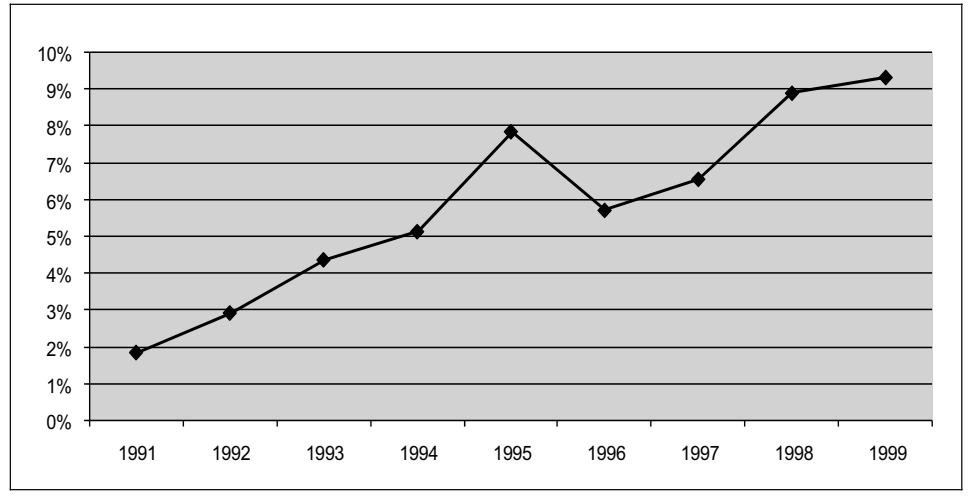

Abbildung 9: US-Demokratieförderung im Verhältnis zu den Gesamtmilitärausgaben der USA in den 1990ern ${ }^{52}$

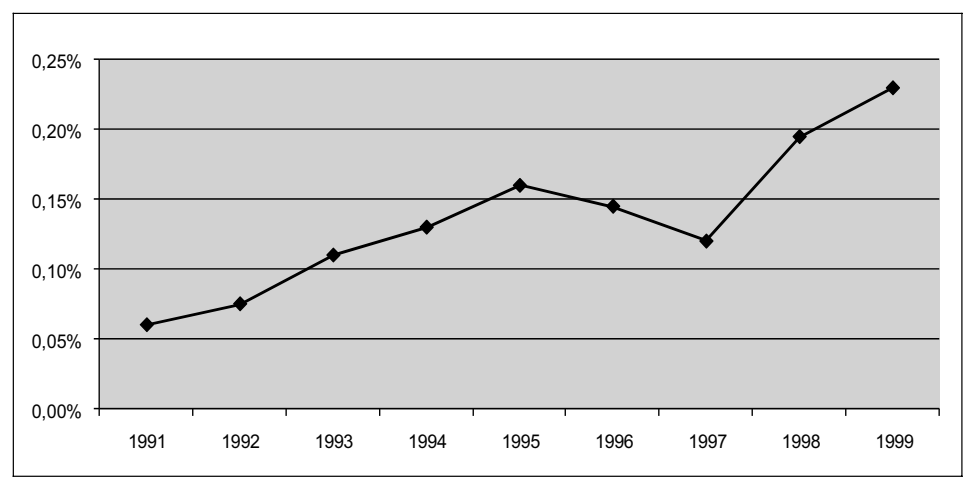

Diese relative Stärkung der zivilen Komponente der Demokratieförderung zeigt sich auch im Abgleich mit den von SIPRI errechneten Gesamtmilitärausgaben der USA in den 1990ern (siehe Abbildung 9). Zugleich eröffnet ein solcher Abgleich auch den Blick auf eine weitere Relativierung: die der tatsächlichen Bedeutung des neuen Trends. Obwohl ein signifikanter Anstieg unbestreitbar ist, belaufen sich die Aufwendungen für Demokratieförderung im Jahr 1999 gerade mal auf 0,23 \%

51 Das Volumen der Demokratieförderung ergibt sich aus den Berechnungen von Carothers (siehe Abb. 7), die Gesamt-ODA ist nach den Daten des DAC aufgelistet (vgl. http://www.oecd.org/dac/stats).

52 Das Volumen der Demokratieförderung ergibt sich aus den Berechnungen von Carothers (siehe Abb. 7), die Gesamt-Militärausgaben entsprechen den von SIPRI ausgewiesenen Werten für »military expenditure« (vgl. http://www.sipri.org). 
der US-Militärausgaben im selben Jahr. Zwar ist dieser Anteil aufgrund der im nicht in linearem Verhältnis stehenden Kosten für zivile politische und militärische Interventionen nicht absolut $\mathrm{zu}$ bewerten - es ist weitgehend unbestritten, dass ein anhand der angestrebten Wirkungen vergleichbar dimensioniertes ziviles Programm zur politischen Intervention um vieles kostengünstiger ist als jede militärische Maßnahme (dieser komparative Kostenvorteil wird in der Debatte um die weitere Forcierung solcher zivilen Maßnahmen auch immer wieder ins Treffen geführt). ${ }^{53}$ Dennoch kann nicht davon ausgegangen werden, dass diese zivilen Maßnahmen den Militärsektor im Bereich der internationalen Intervention der USA zurückdrängen würden.

Abbildung 10: Aufteilung der US-amerikanischen Demokratieförderung nach Institutionen im Jahr 1998

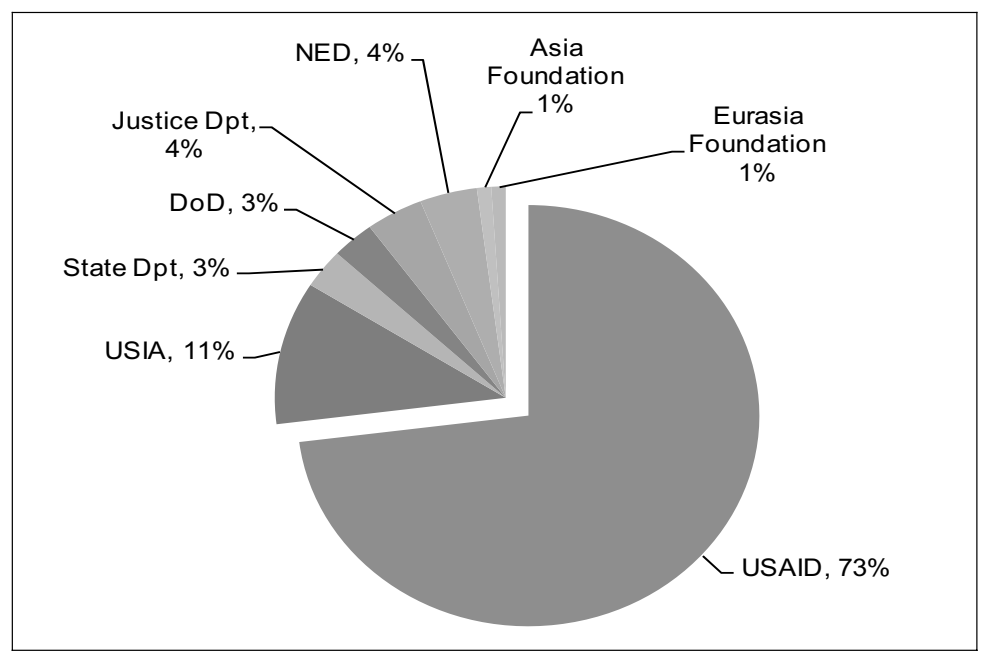

Quelle: Carothers 1999: 54

Vielmehr geht es um Komplementarität und Kooperation. Dies bedeutet einerseits, dass das US-Militär selbst beginnt, Maßnahmen im Bereich der Demokratieförderung zu setzen, die eigentlich einen originär politischen Charakter haben. Bei der Betrachtung der institutionellen Auftei-

53 Vergleichbare Einschätzungen gibt es im Übrigen auch für das Verhältnis politischer Komponenten der EZ zu klassischen Einsatzbereichen der finanziellen und technischen Zusammenarbeit, die ebenfalls viel kostenintensiver sind. Eine »objektive« Einschätzung der Relevanz der politischen Maßnahmen ist also über einen Abgleich der aufgewendeten Mittel nicht möglich. 
lung der Gesamtaufwendungen für Demokratieförderung im Jahr 1998 (siehe Abbildung 10) fällt beispielsweise auf, dass $3 \%$ vom DoD, dem US Department of Defense, getätigt werden. Auch ist in diesem Zusammenhang die schon angeführte wichtige Rolle zu erwähnen, die die Universitäten des US-Militärs (insbesondere das U.S. Army War College) bei der Theorieentwicklung und der Definition der neuen von der Peripherie ausgehenden Sicherheitsbedrohungen spielen.

Auf der anderen Seite rückt speziell die Zusammenarbeit der zivilen und militärischen Komponenten im Rahmen so genannter CIMICOperationen (»Civil Military Cooperation«) ins Zentrum der Bemühungen. Diese Aktivitäten werden ein struktureller Schwerpunkt der Clinton-Administration, die eine solche Notwendigkeit aus den Schwierigkeiten der konventionellen militärischen Interventionen in komplexen Konfliktsituationen ableitet. Insbesondere die negativen Erfahrungen im Rahmen der von der UNO sanktionierten Intervention in Somalia 1993 die Operation wurde nach dem Tod von 18 US-Soldaten im Zuge von Straßenkämpfen in Mogadischu abgebrochen - haben zu dieser Einsicht beigetragen.

Die im Herbst 1994 durchgeführte Intervention in Haiti, die angesichts der Erreichung der Kernzielsetzungen - Wiedereinsetzung des gestürzten Präsidenten Aristide und Herstellung relativer Ruhe - bei gleichzeitiger Minimierung der eigenen Verluste (nur ein getöteter USSoldat) als Erfolg gewertet wird, bestätigt in dieser Sichtweise den eingeschlagenen Weg: nach allgemeiner Einschätzung ist gerade die enge Verknüpfung des militärischen Vorgehens mit eingebetteten zivilen Maßnahmen für den Erfolg ausschlaggebend. ${ }^{54}$

In der Folge erlässt Präsident Clinton im Mai 1997 die Presidential Decision Directive 56, die angesichts der Erfahrungen in verschiedenen »complex emergencies« (zur Geschichte und Bedeutung des Begriffes vgl. Dillon/Reid 2000; vgl. auch USAID 2000a: 22) die Notwendigkeit so genannter »complex contingency operations« herausstreicht (The White House 1997). Neben verschiedenen begleiteten Maßnahmen liegt der Schlüssel solcher Operationen in ihrem integrierten Vorgehen: »integrate all components of a U.S. response (civilian, military, police, etc.) at the policy level and facilitate the creation of coordination mechanisms at the operational level« (ebd.), wobei die Implementierung im Rahmen eines politisch-militärischen Planes (»pol-mil plan«) erfolgen soll. Obwohl die Direktive nicht jene Wirkungen entfalten kann, die sich die

54 Siehe dazu den Literaturüberblick der Air University Library des USMilitärs, einsehbar unter http://www.au.af.mil/au/aul/bibs/haiti/haiti99.htm (Zugegriffen am 03-01-2007). 
Administration ursprünglich von ihr verspricht, gibt sie dennoch die Richtung für die weitere Integration entwicklungspolitischer und militärischer Aufgaben in der US-Außenpolitik des 21. Jahrhunderts vor.

Diese Erfahrungen und Einschätzungen verdichten sich schließlich 1999 im Zuge des Angriffes auf Jugoslawien, der mit der Lage im Kosovo argumentiert wird, in Form einer Doktrin. In einem Interview anlässlich des G8-Gipfels in Köln 1999 antwortet Präsident Clinton auf die Frage, ob angesichts dieses Krieges von einer Clinton-Doktrin gesprochen werden könne, mit Verweis auf das Versagen der internationalen Gemeinschaft im Zuge des ruandischen Genozids: »Well, I think there's an important principle here that I hope will be now upheld in the future and not just by the United States, not just by NATO, but also by the leading countries of the world, through the United Nations. And that is that while there may well be a great deal of ethnic and religious conflict in the world - some of it might break out into wars - that whether within or beyond the borders of a country, if the world community has the power to stop it, we ought to stop genocide and ethnic cleansing. $\ll^{55}$ Die Idee des Wilsonianism hat damit also wiederum ihre militärisch-interventionistische Festlegung gefunden.

Die inneradministrative Mittelaufteilung der US-amerikanischen Demokratieförderung (siehe Abbildung 10) zeigt aber auch, dass USAID trotz einer relativ großen Zahl von im selben Bereich aktiven Institutionen zunächst zentral bleibt (vgl. Lancaster 2000a: 33). Speziell in den Jahren 1993/94 kommt es unter dem Slogan »Strategy of Enlargement « zu einer signifikanten Ausweitung des Bereiches (siehe Abbildungen 7, 8, 9), in der der Nationale Sicherheitsberater der ClintonAdministration von 1993 bis 1997, Anthony Lake, eine entscheidende Rolle spielt (vgl. Travis 1998: 256). Einerseits obliegt ihm die Identifikation der neuen Bedrohungsbilder, andererseits zeichnet er auch für die politische Ausgestaltung des US-amerikanischen Bosnien-Engagements verantwortlich, was angesichts der bitteren Nachwehen jedoch als nicht sehr erfolgreiche Übung wahrgenommen wird. Lake scheitert auch später an der Übernahme der Leitung des CIA, für die Clinton ihn vorgesehen hatte. ${ }^{56}$ Dennoch ist er es, dem die Neuformulierung der Bedrohungsbilder in den Begriffen der post-politischen Konfliktanalyse in den USA primär zuzuschreiben ist.

Ebenfalls schon im Jahr 1993 hat eine von Clinton eingesetzte Kommission unter Leitung des Unterstaatssekretärs im State Deparment,

55 »Interview with Wolf Blitzer of Cable News Network's >Late Edition〈 in Cologne«, June 20th, 1999, einsichtig über das American Presidency Project, http://www.presidency.ucsb.edu.

56 Lake scheitert an der republikanischen Mehrheit im Kongress. 
Clifton Wharton, eine Neuausrichtung der Foreign Aid auf die neuen globalen Konstellationen in deutlichen Worten angeregt. Auch die $\mathrm{Na}$ tional Performance Review, von Clinton zur Reformierung des gesamten Apparates der föderalen Administration eingerichtet, fordert eine solche im Jahr 1993 ein: »NPR agrees with AID's administrator that the agency must make radical departures from past practices if it is to succeed in the volatile and complex international arena of the future. $\aleph^{57}$ Bei der Neubesetzung des Leiters von USAID wird diese Forderung bereits einbezogen: 1994 übernimmt mit Brian Atwood jemand diese Funktion, der angesichts seiner mehrjährigen Aktivität im privaten DemocracyPromotion-Institut »National Democratic Institute for International Affairs« auf Expertise in dem nun immer relevanter werdenden Politikfeld verweisen kann (vgl. Travis 1998: 257).

Mit dem folgenden Strategischen Plan von USAID wird die Heranbildung von Demokratien auch zu einer der vier Kernzielsetzungen im politischen Kontext der nachhaltigen Entwicklung (vgl. USAID 1994: 17ff.). Die dazu angeführten Bedrohungsbilder spiegeln die Trendwende in der Wahrnehmung der Rolle von Entwicklungspolitik bereits deutlich wider: »Narco-terrorism, ethnic warfare, uncontrolled migration, and religious intolerance threaten the very notion of a world community and international peace« (ebd.: 17). Auch auf organisatorischer Ebene findet diese neue Zieldefinition ihren Niederschlag. Noch im Jahr 1994 werden gleich zwei mit diesen Aufgaben befasste Subeinheiten innerhalb von USAID eingerichtet: das Center for Democracy and Governance, das für die Planung und Implementierung der Democracy Promotion innerhalb der Agency verantwortlich sein wird, und das Office of Transition Initiatives (OTI), das für kurzfristige Aufgabenstellungen flexibel handhabbare Förderungen zur Verfügung stellen soll.

Eine entscheidende Komponente, die neben der politischen Neuausrichtung als notwendig erachtet wird (vgl. Lancaster 2000a: 2), ist die vollständige Umorganisierung eines mittlerweile unüberschaubar komplexen Geflechts all jener Institutionen, die in der einen oder anderen Form für Foreign Aid zuständig sind. Ziel der Administration ist es, die verschiedenen Komponenten der Foreign Aid zu vereinheitlichen und näher an das Weiße Haus zu holen. Diese Bemühungen stoßen im republikanisch dominierten Senat jedoch auf Widerstand und können nicht wie vorgesehen umgesetzt werden - mit der Konsequenz, dass die Rolle

571993 NPR Agency Report über die U.S. Agency for International Development (AID), einzusehen über die virtuelle Bibliothek der NPR, http://govinfo.library.unt.edu/npr/library/review.html (Zugegriffen am 1006-2007). 
von USAID im Bereich der Democracy Promotion institutionell wichtiger wird (vgl. Travis 1998: 256).

Im Strategischen Plan von 1997, der in einer Revision bis zum Jahr 2004 gültig sein wird, systematisiert USAID ihr Arbeitsprogramm. »Democracy and Good Governance Strengthened « wird zur zweiten von sechs Kernzielsetzungen, wobei dazu vier prioritäre Subziele definiert werden: »Rule of Law«, die Förderung eines legitimen und kompetitiven politischen Prozesses, eine politisch aktive Zivilgesellschaft sowie das klassische Good-Governance-Kriterium von Transparenz und Verantwortlichkeit (vgl. USAID 1997). 1998 wird dazu ein »Framework«Papier veröffentlicht, das diese Bemühungen im Governance-Bereich nochmals unterstreicht. Darin wird die Anbindung zwischen der auf politischer Ebene populären Fokussierung auf Wahlen mit einer tendenziellen Governance-Orientierung der Policy-Ebene angestrebt, wobei der Schlüsselansatz wie schon im Strategischen Plan »Rule of Law« ist, mit dessen Hilfe dem Problem der »incomplete democratic transitions « (USAID 1998: 3) zu begegnen wäre (zur Bedeutung und den Umsetzungs-Problematiken von »Rule of Law« vgl. Carothers 2003a).

Die drei weiteren angeführten Elemente sind Wahlen und politischer Prozess, Zivilgesellschaft, sowie weitere Maßnahmen im Bereich der Governance. Ähnlich wie bei den meisten europäischen Geberstaaten wird die Zivilgesellschaftsförderung in weiterer Folge zunehmend ins Zentrum der Aufmerksamkeit rücken, was entscheidend mit der Rolle der akademischen Forschung zu tun hat: »This growth [of civil society as objective] in stature was associated with, and partially the result of, the influence of a reevaluation of civil society by academicians « (Travis 1998: 269). Von USAID wird die zunehmende Bedeutung der Zivilgesellschaft über Verbindung der Democracy Promotion mit der Zielsetzung einer ökonomischen Liberalisierung argumentiert: »An intensified focus on the role of civil society accompanied two of the principal development trends of the 1980s and 1990s: economic liberalization (reduction in the economic role of the state vis-à-vis markets) and democratization (change in the nature of state-society relationships) « (vgl. USAID 2000a: 21). Ein Trend, dem allerdings bald enge konzeptionelle Grenzen gesetzt werden.

Der Widerstand in Senat und Kongress gegen die weitere Aufwertung der Foreign Aid durch Clinton verschärft sich unterdessen. Es ist vor allem der Leiter des Senate Foreign Relations Committee, der Republikaner Jesse Helms, der mit seiner Überzeugung, jegliche Art der Foreign Aid sei Verschwendung von Steuergeldern, die innerhalb der USA besser einzusetzen wären, durch immensen persönlichen Einsatz relevanten Einfluss gewinnt. Nicht nur ist er federführend daran betei- 
ligt, die Reorganisations-Bemühungen der institutionellen Strukturen zu unterminieren, seine Initiative führt auch zu einem relevanten Rückgang der Entwicklungshilfe-Gelder in der zweiten Hälfte der 1990er Jahre, was sich auf den Bereich der Democracy Promotion auswirkt (vgl. Hook 1997, siehe auch Abbildungen 9, 10, 11).

Erst mit dem Angebot der Auflösung der USIA und der Übertragung ihrer Kompetenzen auf das State Department kann die ClintonAdministration Jesse Helms zum Einlenken bewegen. So kommt es 1998 durch den Foreign Affairs Reform and Restructuring Act doch noch zu einer Reorganisation der verschiedenen Institutionen der Foreign Aid, wobei die neue Schlüsselrolle dem State Department zukommt. Nicht nur wird die USIA tatsächlich aufgelöst und die nach Ende des Kalten Krieges verbliebenen Strukturen unmittelbar ins State Department eingegliedert, es wird auch USAID unter dessen Oberhoheit gestellt, wenngleich es formell als eigenständige Institution erhalten bleibt. Dennoch hat diese Eingliederung weitgehende Konsequenzen, deren sichtbarster Ausdruck die ab diesem Zeitpunkt einsetzende Praxis der gemeinsamen strategischen Planung zwischen State Department und USAID ist. ${ }^{58}$

Die Administration Bush forciert ab 2001 schließlich alle drei der unter Clinton angelegten Trends: sowohl die Intensivierung der Arbeit an der Problemstellung bewaffneter Konflikt, als auch eine generelle Ausweitung der Foreign Aid werden einhergehend mit einer Weiterführung institutioneller Reformprozesse vorangetrieben. So beginnt mit dem Jahr 2001, schon vor 9/11, eine hauptsächlich auf USAID ausgerichtete Konferenzreihe, die sich mit dem Thema »Conflict Prevention« auseinandersetzt (vgl. USAID/Woodrow Wilson International Center for Scholars 2001; siehe auch Davitt 2003). Dabei wird vor allem die Argumentation des Zusammenhangs zwischen außenpolitischer Konfliktprävention und nationaler Sicherheit innerinstitutionell eingeübt. Dem neu bestimmten USAID-Direktor Andrew Natsios, einem Oberstleutnant der Reserve der US-Armee und in dieser Kapazität Veteran des Golfkrieges, obliegt die Umsetzung des Programms. Einer seiner ersten Schritte besteht denn auch darin, das bereits bestehende Center for De-

58 Das von dieser Entscheidung am direktesten betroffene Instrument ist der so genannte Economic Support Fund (ESF), ein schon lange existierender Fonds zur Unterstützung von als geostrategisch wichtig angesehenen Partnerländern. Bislang wurde die Entscheidung über die Mittelvergabe vom State Department getroffen, USAID war für die Umsetzung der nichtmilitärischen Projekte des ESF zuständig. Nun wird der Prozess kollektiviert, wenngleich formell das State Department entscheidungsbefugt bleibt. 
mocracy and Governance innerhalb von USAID zu einem Office aufzuwerten.

9/11 fungiert dann aber als wesentlicher Katalysator all dieser Prozesse. Nicht nur werden die Zielbestimmungen der Foreign Assistance und insbesondere im entwicklungspolitischen Bereich neu definiert neben der Humanitären Hilfe und der Stützung geostrategischer Interessen sind es nun fragile Staaten, die Verbindung politischer mit ökonomischer Liberalisierung, gefasst im Begriff »transformational development «, sowie die von der Bush-Administration zu einem außenpolitischen Schwerpunkt ernannte Problemstellung globaler Seuchen und Krankheiten (vgl. USAID 2004b) - auch die generelle Bedeutung der Entwicklungshilfe erhält durch die rapide Zunahme der öffentlichen Unterstützung nach 9/11 einen ungeahnten Auftrieb (vgl. Lancaster/van Dusen 2005: 3).

So kommt es in den Jahren nach $2001 \mathrm{zu}$ der bereits eingangs erwähnten Vervielfachung der US-amerikanischen ODA-Gelder, was die USA wieder zum eindeutig größten bilateralen Geber werden lässt. George W. Bush spricht in diesem Zusammenhang vom »largest increase in development assistance since the Marshall Plan. $"{ }^{59}$ Trotzdem ist diese quantitativ enorme Zunahme im historischen Abgleich nicht so eindrucksvoll wie sie zunächst scheinen mag (vgl. Shapiro/Birdsall 2002). Inflationsbereinigt ist das Volumen der ODA-Gelder nach wie vor unter den Spitzenwerten der Reagan-Ära (siehe Abbildung 11), relativ, gemessen am Anteil der ODA am BIP, sind die USA mit nicht annähernden $0,2 \%$ im internationalen Vergleich im Hintertreffen. Dennoch bekommt die Vorstellung, die USA würden sich in ihren ODAAusgaben auch nur den festgelegten EU-Zielwerten von 0,56 \% annähern, angesichts der bereits mit dem derzeitigen Aufwand bestehenden Dominanz einen fast bedrohlichen Charakter.

Auffällig an diesem starken Anstieg ist auch der unverhältnismäßig hohe Anteil an ODA-Geldern, die über das Verteidigungsministerium vergeben werden. Nachdem im Jahr 2002 noch 5,6 \% der Mittel über das DoD liefen, waren es im Jahr 2005 bereits 21,7\%, also über ein Fünftel der gesamten entwicklungspolitischen Mittel der USA (vgl. DAC 2006a: 12). Dies erklärt sich vor allem aus den primär militärisch getragenen Wiederaufbauprogrammen in Irak und Afghanistan, sowie dem afghanischen Anti-Drogen-Programm und den in hohem Maße militärisch verwalteten Post-Tsunami-Wiederaufbauprozessen in Süd- und

59 George W. Bush am 31. Mai 2007, „President Bush Discusses United States International Development Agenda«, einsichtig über das American Presidency Project, http://www.presidency.ucsb.edu. 
Südostasien. ${ }^{60}$ Zugleich ergibt sich ein gewichtiger Teil des Anstieges auch aus Schuldennachlässen für den Irak (vgl. DAC 2006a: 25).

\section{Abbildung 11: ODA-Aufwendungen der USA (im Wert von 2003-US\$)}

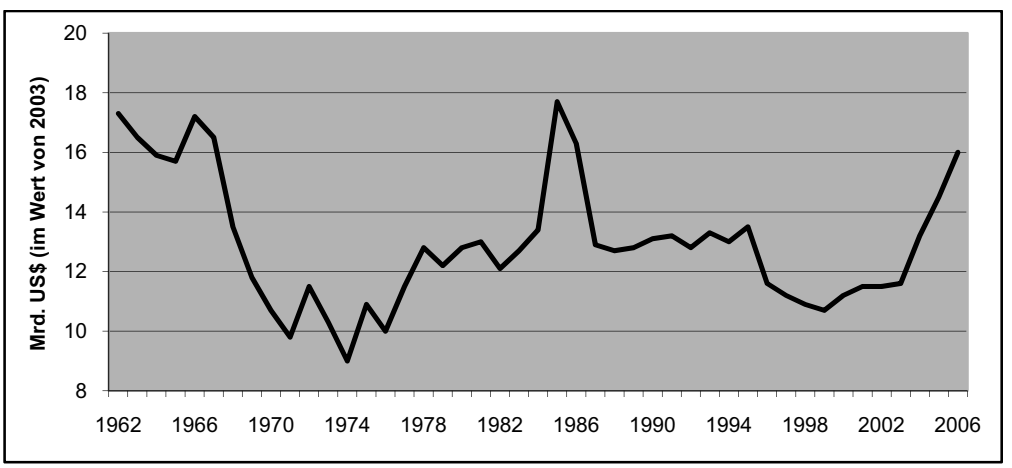

Quelle: Shapiro/Birdsall 2002: 6

Abbildung 12: ODA-Aufwendungen der USA im Verhältnis zum BIP

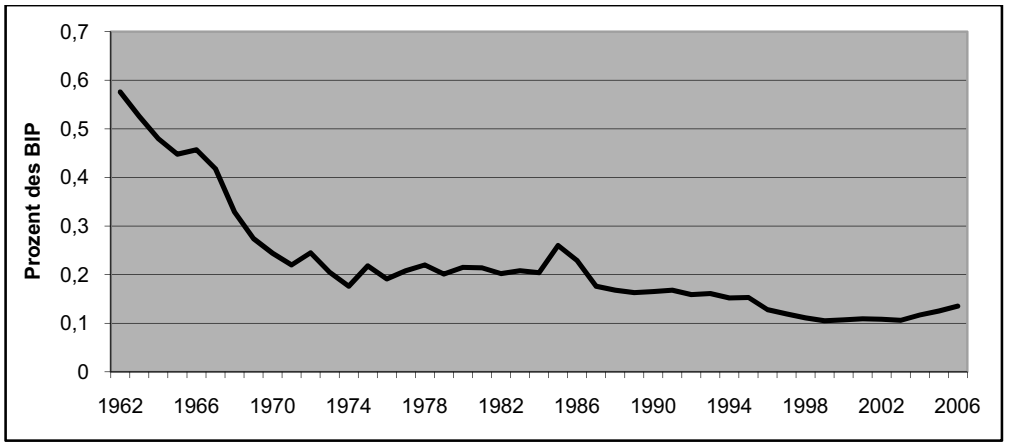

Quelle: Shapiro/Birdsall 2002: 6

Doch Entwicklungspolitik erfährt auch eine strategische Aufwertung im Verhältnis zu den anderen außenpolitischen Instrumenten. Erstmals wird in der Nationalen Sicherheitsstrategie im Jahr 2002 Entwicklung auf

60 Als Konsequenz der zunehmenden Bedeutung des DoD im entwicklungspolitischen Bereich wird mit Herbst 2005 innerhalb von USAID ein Office of Military Affairs (DCHA/OMA) eingerichtet (vgl. DAC 2006a: 47). Es ist innerhalb des Büros für Demokratie, Konflikt und Humanitäre Hilfe angesiedelt und dient primär zur Formalisierung der Kooperation mit dem DoD, wozu auch allen fünf geographischen Unified Combattant Commands USAID-Personal beigeordnet wird. 
derselben Augenhöhe wie Diplomatie und Verteidigungspolitik behandelt (vgl. The White House 2002; USAID 2004b: 3). Dies veranlasst die Peer Review des DAC dazu, die Strategie als ein "watershed document" $\mathrm{zu}$ bezeichnen, »which has set the stage for some of the most farreaching reforms in American development co-operation since 1961« (vgl. DAC 2006a: 18). In Bezug auf die drei zentralen Elemente der USAußenpolitik ist ab diesem Zeitpunkt von den 3Ds die Rede (Development, Diplomacy, Defence, vgl. DAC 2006a: 10). Dieser Trend setzt sich auch in der Nationalen Sicherheitsstrategie von 2006 fort (vgl. The White House 2006). Zugleich kritisiert das DAC, dass die USA dem Bereich traditioneller Entwicklungspolitik (Infrastruktur, Wachstum), wo doch komparative Vorteile gegenüber anderen Gebern bestünden, zu wenig Aufmerksamkeit schenken würde. Vielmehr würden die Ausgaben vor allem im Governance-Sektor, den »social services« sowie in der Nothilfe und im Wiederaufbau unverhältnismäßig ansteigen, es sei eine »escalating distribution of aid to crisis countries« feststellbar.

Institutionell erfährt der Bereich der unmittelbaren Konfliktarbeit innerhalb von USAID mit der Gründung des Office of Conflict Management and Mitigation (CMM) im Jahr 2002 eine weitere Aufwertung. ${ }^{61}$ Das CMM ist vor allem für die inhaltliche Arbeit am Komplex bewaffneter Konflikt und das so genannte Mainstreaming des Themas innerhalb der gesamten Organisation zuständig. Dazu erarbeitet es etwa Handreichungen zur Erstellung von Conflict Assessments (vgl. USAID 2005b) oder Ratgeber zum Zusammenhang von bewaffnetem Konflikt mit verschiedenen Themen- und Problemstellungen wie Land, Rohstoffe, Wasser, Jugend oder Frauen, so genannte Toolkits (vgl. USAID 2005i, 2005j, 2005k, 2005l, 2005m, 2007). Zusammen mit dem schon seit 1994 bestehenden Office of Transition Initiatives (OTI) und dem Kernprogramm Democracy and Governance wird das CMM in einer der

61 Ebenfalls im Jahr 2002 richtet die Bush-Administration ein neues Finanzierungsinstrument ein, den so genannten Millennium Challenge Account (MCA). Er dient zur verstärkten Förderung identifizierter Good Performer (nach einem umfassenden Kriterienkatalog, der neben ökonomischen Basisdaten auch Demokratie-, Governance- und Menschenrechtskriterien enthält), wobei ambitionierte Entwicklungsprojekte der jeweiligen Partnerregierungen mit vergleichsweise hohen Summen unterstützt werden. Abgewickelt wird das Programm über eine eigene Organisation, die Millennium Challenge Corporation (MCC). Wenngleich das Programm angesichts seiner Ausrichtung auf traditionellen makroökonomischen und infrastrukturorientierten Entwicklungsprojekten nicht in den Rahmen dieser Studie fällt, ist es doch bemerkenswert, dass Sri Lanka seit Beginn des Programms zu den identifizierten Empfängern, also zu den Good Performern auch im Governance- und Menschenrechtsbereich gezählt wird. Diese Einschätzung wurde im Frühjahr 2007 erneut bestätigt. 
drei administrativen Kerngruppen des Büros für Demokratie, Konflikt und Humanitäre Hilfe (DCHA) zusammengefasst (siehe Abbildung 13). Dies bestätigt die sich aus den bisher angeführten internen Prozessen ergebende Annahme, dass die spezifische Arbeit an bewaffneten Konflikten eng mit der seit Mitte der 1980er bestehenden Schiene der Democracy Promotion zusammenhängt, zeigt aber auch die enge Verbindung mit der Humanitären Hilfe, die den konkreten lokalen Gegebenheiten geschuldet ist.

Abbildung 13: Institutionelle Verankerung des Demokratie- und Konfliktbereiches in USAID

The CMM Office within the Bureau for Democracy, Conflict, And Humanitartian Assistance (DCHA)

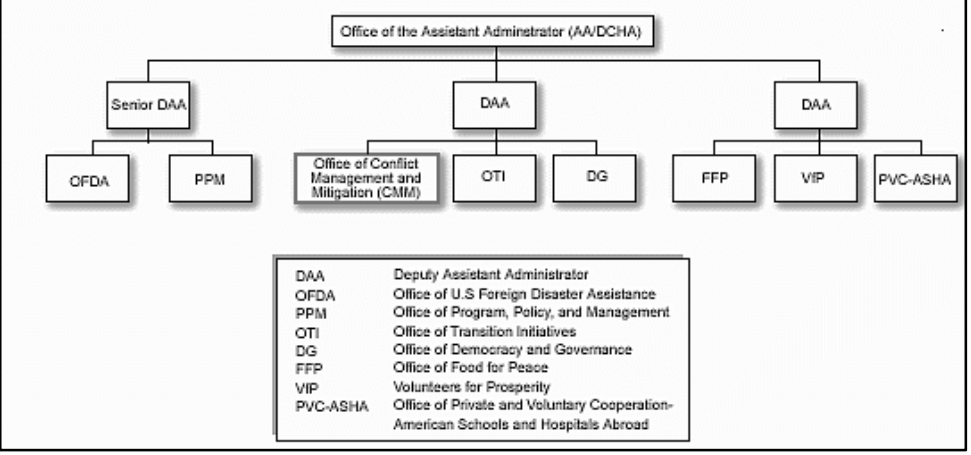

Quelle: http://www.usaid.gov/our_work/crosscutting_programs/conflict/about/org_chart.html (Zugegriffen am 20-01-2008)

Insgesamt sind in der derzeitigen Organisationsstruktur von USAID drei thematische Büros eingerichtet, die inhaltliche Schwerpunktlegungen repräsentieren. Neben DCHA sind das die Büros für »Global Health« und »Economic Growth, Agriculture, and Trade«. Interessant ist die Besetzung des Leiters des DCHA, einer Position, die auf Vorschlag des Präsidenten dem Senat zur Bewilligung vorzulegen ist. Hier vertraut die derzeitige Administration auf militärische Erfahrung: seit 2005 bekleidet mit Michael Hess ein in West Point ausgebildeter Karrieremilitär den Posten. Hess verfügt über militärische Erfahrungen in der Türkei, Bosnien und Kosovo und war zuletzt im Jahr 2003 im Zuge der IrakInvasion in militärischer Funktion für die Leitung des Office of Recon- 
struction and Humanitarian Assistance verantwortlich sowie in der Folge auch an der Installierung einer irakischen Übergangsregierung beteiligt. Er symbolisiert so die noch immer enge Anbindung dieses politisch sensiblen Bereiches ziviler Intervention mit dem militärischen Sektor.

Auch die bislang organisatorisch nicht ausreichend umgesetzte Oberhoheit des State Department über USAID, die auf Clintons Reform von 1998 zurückgeht, wird mit Beginn des Jahres 2006 in eine institutionelle Form gegossen. Mit der Einrichtung der neuen Position eines Director of Foreign Assistance (DFA) erreicht die Bush-Administration nicht nur eine taktische Befriedung von zahlreichen Kritiken, die die mangelnde Koordination der verschiedenen außenpolitischen Instrumente anprangern, sondern auch eine verstärkte direkte Kontrolle der Administration über USAID - etwas, woran die Clinton-Administration einige Jahre zuvor an republikanischem Widerstand gescheitert war. Mit dem Fiskaljahr 2008 geht die Vereinheitlichung schließlich erstmals so weit, dass nicht nur ein strategischer Plan, sondern auch eine Congressional Budget Justification von State Department und USAID gemeinsam herausgegeben wird (vgl. Department of State/USAID 2007b).

Die Position des DFA wird derzeit in Personalunion vom Direktor der USAID eingenommen, eine Entscheidung, die als Aufwertung der organisatorischen Rolle von USAID verstanden werden kann, insbesondere da die Entscheidungen über die Mittelvergabe aus dem ESF, die bislang zentral dem State Department unterlagen, tendenziell zu USAID wandern. Im Jahr 2006 beliefen sich diese Mittel auf etwa 2,6 Mrd. US\$. ${ }^{62}$ Außerhalb des Einflusses des DFA bleiben momentan zwei entwicklungspolitische Instrumente: die Umsetzung des bereits erwähnten Millennium Challenge Account und die Befugnisse des Global AIDS Coordinator. Alle anderen Kompetenzen der Foreign Assistance finden sich nun in der Kompetenz des DFA. ${ }^{63}$

Der Start für diese neue Funktion verläuft allerdings denkbar unglücklich. Randall Tobias, der im Jänner 2006 Andrew Natsios (2001-

62 Zugleich wird USAID in der relativen Verteilung der Mittel durch eine starke Ausweitung der ODA-Mittel für das DoD geschwächt - wie angeführt wurden im Jahr 2005 21,7 \% der ODA-Gelder durch das DoD vergeben, gegenüber nur $38,8 \%$ von USAID. Im Jahr 2002 war USAID noch für über die Hälfte der ODA-Gelder zuständig gewesen (vgl. DAC 2006a: 12).

63 Im State Department wird noch eine zweite Koordinations-Stelle eingerichtet, die primär für den Konfliktbereich relevant ist, hier aber vor allem die humanitäre Komponente betreut: das Office of the Coordinator for Reconstruction and Stabilization (O/CRS), das direkt der/dem Secretary of State verantwortlich ist und derzeit mit einem Karrierediplomaten, John Herbst, besetzt ist. 
2005) als Leiter von USAID ablöst, wird als erster mit der Position des DFA betraut. ${ }^{64}$ Seiner Amtszeit in State Department und USAID ist jedoch ein schnelles und unglückliches Ende beschieden. ${ }^{65}$ Mittlerweile ist Henrietta Fore als Nachfolgerin nominiert. Sie hat einen primär ökonomischen Hintergrund, dürfte jedoch angesichts ihrer langen Dienstzeiten im öffentlichen Bereich vor allem im Hinblick auf die Konsolidierung der internen Reorganisation von USAID bestimmt worden sein.

\section{Strategien und Programme}

Ist die US-Entwicklungszusammenarbeit mit bewaffnetem Konflikt konfrontiert - und sie ist es, laut eigener Rechnung, in $60 \%$ der Länder, in denen sie aktiv ist (vgl. USAID 2005a: 2) - hat sie einen mittlerweile relativ starken Korpus an Literatur, auf den sie zurückgreifen kann. Dieser Korpus ist allerdings nicht nur kompakt gehalten, sondern auch in einer klaren Prioritätenrangliste gesetzt ist (siehe Abbildung 14). An der Spitze steht eindeutig die Nationale Sicherheitsstrategie (The White House 2006), die nicht nur für USAID, sondern für alle außenpolitisch relevanten Institutionen das primäre Referenzdokument darstellt. Dieses von der politischen Ebene kommende Leitdokument hat tatsächlich eine überraschend große Umsetzungsrelevanz.

Die strategische Planung auf Policy-Ebene, die seit dem Jahr 2004 von State Department und USAID gemeinsam abgewickelt wird, enthält einen präzisen Verweis jeder festgelegten strategischen Zielsetzung auf die von der Nationalen Sicherheitsstrategie vorgegebenen Aufgabenstellungen (vgl. State Department/USAID 2007: 11). Zugleich fungieren die in dieser strategischen Planung festgelegten konkretisierten Ziele wiederum als die explizit hervorzuhebenden Bezugspunkte in der jährlichen Congressional Budget Justification - seit 2007 ebenfalls von State Department und USAID (2007b) gemeinsam vorgelegt - die als das zentrale Implementierungsdokument dient und schon insofern sehr genau eingehalten wird, als der Kongress dafür bekannt ist, Mittelvergaben insbesondere im entwicklungspolitischen Bereich punktgenau zu überprüfen

64 Aus der Privatwirtschaft kommend war Tobias politisch vor allem durch großzügige Spenden an die Republikanische Partei in Erscheinung getreten und seit dem Jahr 2003, vor seiner Ernennung zum USAID-Direktor, mit der Funktion des AIDS-Koordinators betraut.

65 Tobias tritt Ende April 2007, nach etwas über einem Jahr Amtszeit, aus persönlichen Gründen zurück, nachdem sein Name im Zuge der Ermittlungen gegen einen Callgirl-Ring genannt wird, vgl. International Herald Tribune, Online Edition, 28. April 2007. 
(vgl. DAC 2006a: 21ff.). Dadurch ist jedoch auch eine hohe PolicyRelevanz der politischen Leitdokumente gewährleistet.

\section{Abbildung 14: Schlüsseldokumente der US-Entwicklungspolitik im} Konfliktkontext

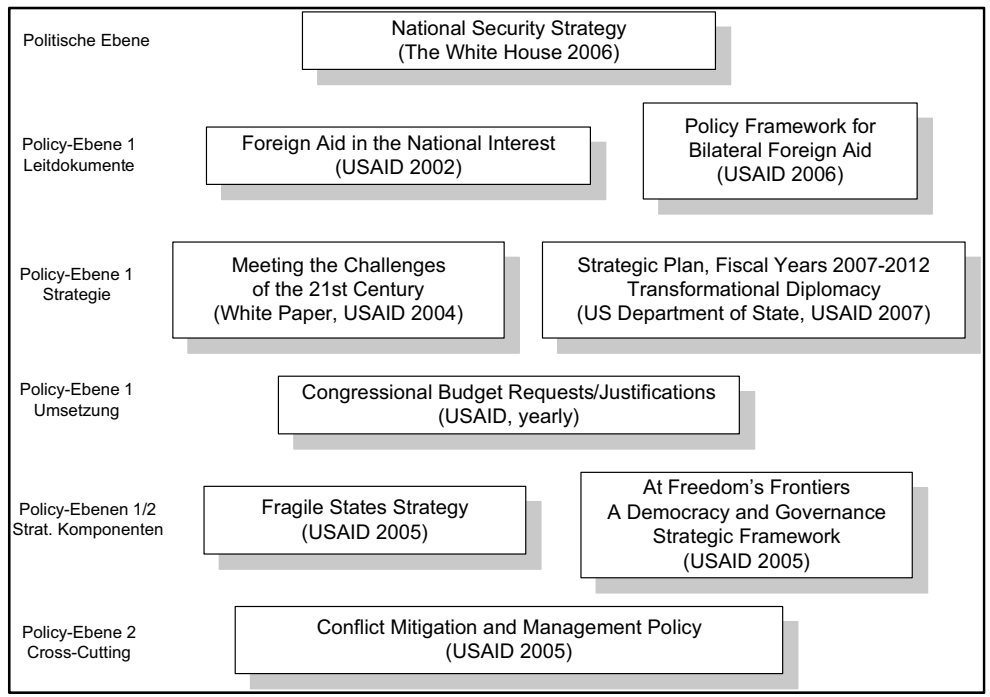

$\mathrm{Zu}$ diesem klaren dreistufigen Aufbau kommen nun Studien und Denkschriften, die für die strategische Weiterentwicklung von USAID gedacht sind und so gewissermaßen die inhaltliche Ausdifferenzierung der politischen Leitlinien vorbereiten, wie etwa der so genannte NatsiosReport »Foreign Aid in the National Interest « (USAID 2002), das White Paper zur Ausrichtung von USAID (USAID 2004b) oder das kurz und präzise gehaltene »Policy Framework« (USAID 2006b), das als Leitdokument auf Policy-Ebene 1 für die Ausarbeitung der strategischen Planung dient. Auf Policy-Ebene 2 werden primär Dokumente und Studien produziert, die entweder als strategisch angelegte Leitlinien für die Implementierung dienen, wie etwa die Fragile States Strategy (2005c) und das Strategiepapier des Democracy\&Governance-Bereiches (USAID 2005d), zum Mainstreaming der Problematik bewaffneter Konflikt innerhalb der Organisation beitragen sollen, wie die Conflict Mitigation and Management Policy (USAID 2005a), oder konkrete Programmierungshilfen für die spezifische Programmierung und Projektgestaltung darstellen. $^{66}$

66 Diese Dokumente sind in der Grafik nicht erfasst, als Beispiele seien die schon erwähnten Toolkits des CMM angeführt (vgl. USAID 2005i-m). 
Trotz diesem formell relativ hohen Niveau an Konsistenz wäre es falsch, von einer einheitlich konzipierten Vorgangsweise der USA selbst im nicht-militärischen Umgang mit bewaffneten Konflikten auszugehen. Die innere Kohärenz der verschiedenen Texte ist zwar entlang der strategischen Hauptader zwischen Nationaler Sicherheitsstrategie und Strategischem Plan relativ hoch, ab der Budget Justification, die auch die Maßnahmen in den jeweiligen Zielregionen umfasst, jedoch durch eine starke Differenzierung gekennzeichnet, die sich aus zwei Faktoren ergibt: einerseits sind die verschiedenen Leitlinien und Handreichungen von den Schwerpunktlegungen der jeweils zuständigen Offices geprägt und weisen daher, obwohl sie eigentlich für die selben Problemstellungen gestrickt sind, unterschiedliche Fokussierungen auf. Andererseits ist die Umsetzung der Strategie von Zielregion zu Zielregion unterschiedlich, was mit dem nach wie vor bedeutenden geostrategischen Element in der US-Außenpolitik zu tun hat.

So geht die derzeitige Strategie von fünf Länderkategorien aus, für die unterschiedliche Regelungen und Zielsetzungen zur Anwendung kommen (vgl. State Department/USAID 2007b: 58). Die nicht nur auffälligste, weil explizit geostrategisch geprägte, sondern auch historisch älteste Kategorie ist jene der »Sustaining Partnership Countries«. Diese Länder werden unabhängig der konkreten Situation vor Ort und der Einhaltung der verschiedenen Entwicklungsmaßstäbe so lange unterstützt, wie es politisch als notwendig erachtet wird. Derzeit fallen 43 Länder in diese Kategorie, wobei Israel, die Türkei, Saudi-Arabien oder Kuwait zu den bekanntesten Beispielen gehören. Traditionell wird die finanzielle Unterstützung hauptsächlich aus einem eigenen Topf, dem bereits erwähnten Economic Support Fund, bestritten, wobei die konkrete Mittelverwendung vom State Department entschieden wird und durch keine formellen Kriterien beschränkt ist. Geltende Policies der Entwicklungszusammenarbeit werden in diesen Ländern also nur insoweit umgesetzt, als es geostrategischen Maßgaben dienlich erscheint.

Das eigentliche Gegenstück zu dieser Kategorie sind die so genannten »Restrictive Countries«, derzeit 11 Länder, mit denen ebenfalls aus politischen Gründen grundsätzlich keine offizielle Zusammenarbeit läuft. Mehr oder minder sind damit jene Staaten gemeint, die die ehemalige Secretary of State der Clinton-Administration, Madeleine Albright, markig als »Schurkenstaaten« bezeichnet hat: neben Nordkorea und dem Iran fallen etwa Zimbabwe oder Weißrussland in diese Kategorie. Nachdem sich die offizielle US-Position gegenüber diesen Ländern auf einen Systemwechsel festgelegt hat, ist ODA im engeren Sinne nicht möglich, auch wenn natürlich verschiedene Maßnahmen gesetzt werden. Diese beschränken sich allerdings hauptsächlich auf den politischen, diploma- 
tischen oder geheimdienstlichen Bereich und weisen einen mehr oder minder subversiven Charakter auf.

Die verbleibenden drei Kategorien von Partnerländern unterteilen sich nun entlang von zwei Kriterien: einerseits der Entwicklungsstand, der anhand von verschiedenen Performance-Indikatoren zur Qualifikation für das Millennium Challenge Account-Programm festgemacht wird, und andererseits an dem Kriterium eines laufenden oder vor kurzem beendeten bewaffneten Konflikts, wobei das Performance-Kriterium, also der Anspruch auf eine Partnerschaft mit der MCC, vorrangig behandelt wird. Derzeit qualifizieren sich 24 Länder für dieses MCA-Programm (»Transforming Countries«), wobei mit Sri Lanka oder den Philippinen auch solche mit laufenden bewaffneten Konflikten miteinbezogen sind. Demgegenüber sind die »Rebuilding Countries« direkt über das Vorhandensein eines bewaffneten Konfliktes definiert ${ }^{67}$, während unter den "Developing Countries" jene nicht MCA-Berechtigten zusammengefasst sind, in denen nicht primär über die Konfliktproblematik interveniert wird, auch wenn so definierte Staaten wie Uganda, Burundi oder Pakistan anderes nahelegen würden.

Solcherart primär politisch zu erklärende Inkonsistenzen innerhalb der grundlegenden, allgemein verbindlichen Zuordnung tragen ebenso wenig zur Herausbildung eines festgeschriebenen charakteristischen Programms von US-amerikanischer Friedensentwicklung bei wie die je nach Policy-Ebene und thematischer Verortung der einzelnen befassten Offices unterschiedlichen Zielsetzungen. Trotzdem zieht sich die zentrale Rolle der Konfliktproblematik in allen relevanten Dokumenten durch. Im Strategischen Plan ist »Peace and Security« die Zielsetzung Nummer Eins, gefolgt von »Governing Justly and Democratically«. Erst danach finden sich, zusammengefasst unter den Kategorien »Investing in People« und »Economic Growth« die traditionellen Felder von Entwicklungsund Außenwirtschaftspolitik, sowie der Bereich der Humanitären Hilfe (vgl. State Department/USAID 2007a: 10).

Im auf Entwicklungszusammenarbeit konzentrierten Rahmenpapier findet sich eine andere Zusammenstellung der gleichen Problemstellungen (USAID 2006b: 2, vgl. auch USAID 2004b: 14f.). Hier wird als erste Zielsetzung das Motto des Strategischen Planes genannt und auf den Entwicklungsbereich gemünzt: »Transformational Development«, also die Kombination von institutionellen Maßnahmen, Governance und ökonomischer Entwicklung, mit dem Ziel der »Graduation«, also der

67 Unter diese Kategorie fallen neben Afghanistan und dem Irak Kolumbien, Cote d'Ivoire, die Demokratische Republik Kongo, Haiti, Liberia, Nepal, Sierra Leone, Somalia, der Sudan und der bereits als eigenständig definierte Kosovo (vgl. State Department/USAID 2007b: 758). 
Überwindung der derzeit angenommenen Notwendigkeit von externen Hilfsgeldern. An zweiter Stelle steht mit der Zielsetzung der Stärkung fragiler Staaten die Konfliktkomponente, zusammengefasst als Kombination der beiden Schlüsselbegriffe Sicherheit und Stabilität (vgl. USAID 2006b: 10), was den schon in der Fragile States Strategy (USAID 2005c) vorgegebenen Prioritätensetzungen entspricht. Als weitere zwei Schwerpunkte werden die Ermutigung zu Reformschritten und der Ausbau der Kapazität von Institutionen angeführt (ebd.: 5). Jenseits dieser zwei Zielsetzungen - "promote transformational development und »strengthen fragile states" - finden sich faktisch keine weiteren spezifisch entwicklungspolitischen Zielsetzungen, sondern geostrategische (»support strategic states«), humanitäre (»provide humanitarian relief «), sowie eine weit gefasste Restkategorie, zusammengehalten durch den schwammigen Term "global issues" (USAID 2006b: 2).

Anhand dieser Zielsetzungen ist also sichtbar, dass die originär entwicklungspolitische Intervention de facto vornehmlich entlang der Frage von bestehenden bewaffneten Konflikten, umschrieben mit dem »Fragile States«-Begriff, kategorisiert wird. Trotz der mitunter inkohärenten Vielfalt an Ansätzen und Zielbestimmungen kann somit ein relativ klarer Blick gewonnen werden, der im Zuge der Implementierung ${ }^{68}$ zu einer relativen Einfachheit tendiert: ist ein bewaffneter Konflikt vorhanden, sind die Maßnahmen der US-Entwicklungshilfe in unmittelbarer oder mittelbarer Form zu seiner Lösung oder Transformation einzusetzen. Darauf aufbauend, aber auch darüber hinausgehend kann zudem, so zumindest meine hier vorgeschlagene Lesart der verschiedenen Schlüsseldokumente, von einem relativ geschlossenen Bild ausgegangen werden, durch das die dazu bestimmte Vorgangsweise charakterisiert ist.

Über die grundsätzliche Herangehensweise bei der Entwicklung von Frieden lässt schon die Nationale Sicherheitsstrategie keine Zweifel aufkommen: »The most effective long-term measure for conflict prevention and resolution is the promotion of democracy « (The White House 2006: 15). Demokratieentwicklung ist also selbst bei laufenden bewaffneten Konflikten das bestgeeignete Instrument zur Intervention, wenngleich sie, und das macht den speziellen Charakter der entwicklungspolitischen Demokratieentwicklung unter Konfliktbedingungen aus, mit einer starken Governance-Komponente verknüpft ist - sowie auch mit der Heranbildung neuer oder Stärkung bestehender »responsive institutions « ${ }^{69}$, mit

68 Darauf weisen zumindest die Ergebnisse der Feldforschungen in allen vier Regionen deutlich hin.

69 Die Übersetzung der Zuschreibung »responsive« fällt schwer, da ein vergleichbarer Begriff im Deutschen nicht existiert. Gemeint ist eine Verbindung von Verantwortlichkeit, Ansprechbarkeit, Flexibilität und Umset- 
Hilfe derer der fragile Charakter der Staatlichkeit stabilisiert und schließlich überwunden werden soll: »Institutions matter most«, wie das White Paper von USAID unmissverständlich vorgibt (USAID 2004b: 11).

Wie die vier Prioritäten für die Arbeit in fragilen Staaten zeigen »enhance stability«, »improve security«, »encourage reform«, »develop the capacity of institutions « (vgl. USAID 2005c: 5) - ist mit diesen Ansatzlinien schon viel abgedeckt. Allerdings ergibt sich entlang der Zielsetzungen von Stabilität, Sicherheit und, vor allem, der Motivierung zu Reformen in einem definitionsgemäß hochkonfliktiven Umfeld letztlich auch für die US-Entwicklungszusammenarbeit die Notwendigkeit der Implementierung spezifischer Peacebuilding-Maßnahmen. Gehen wir nach dem Natsios-Report, sind solche Maßnahmen vor allem auf gesellschaftlicher Ebene zu setzen: »At a very general level, conflict prevention and management entail a continuum of interventions that have as their primary objective strengthening the capacity of states and societies to sustainably manage sources of tension and strain in their own countries" (USAID 2002: 22, meine Hervorhebung). Der wesentliche Begriff, der in diesem Zusammenhang zur Anwendung kommt, ist der der Partnerschaft (vgl. etwa USAID 2005a: 4, 2005d: 12).

Von diesen Interventionslinien ausgehend lassen sich also drei Ansatzpunkte herausarbeiten, die in ihrem Zusammenspiel jenes Instrumentarium bilden, mit dem die US-Entwicklungszusammenarbeit, insbesondere USAID, dem Phänomen »Bewaffneter Konflikt« begegnen will: (1) Demokratieentwicklung in Verbindung mit Good Governance, (2) sich auf Institutionen konzentrierendes State-Building, und (3) spezifische Maßnahmen, die ich mit dem etwas schwülstigen Begriff von Friedenspartnerschaften ${ }^{70}$ umschreiben möchte. Anhand einer genaueren Betrachtung dieser drei Stränge lässt sich die Rationalität der im zivilen Bereich angesiedelten US-Interventionen zur Befriedung bewaffneter Konflikte gut nachzeichnen.

zung. In diesem Sinne kann »responsive« als das Adjektiv von Good Governance verstanden werden.

70 Diese Friedenspartnerschaften sind nicht mit der um die NATO gebauten »Partnerschaft für den Frieden« zu verwechseln - das eine ist eine nach Definition zivile und gesellschaftliche, das andere eine militärische und staatliche Konstruktion. Sie befinden sich also auf anderen Ebenen des Diskurses, wenngleich sie die grundsätzliche strategische Komponente der Einbindung zunächst nicht notwendigerweise engagierter Akteure, denen gleichwohl ein objektives Interesse an diesem Engagement unterstellt wird, teilen. 
(1) Demokratieentwicklung und Good Governance. Schon im Ansatz wollen die USA keinen Zweifel über den grundlegenden Wandel in der außenpolitischen Orientierung aufkommen lassen, den sie Mitte der 1980er Jahre vollzogen haben: »We will not let the challenges of democratic transitions frighten us into clinging to the illusory stability of the authoritarian« (The White House 2006: 7). Auch wenn Demokratie ein potenziell riskantes und konfliktträchtiges Projekt ist - so werden etwa Wahlen durchaus als möglicher Trigger-Faktor für den Ausbruch bewaffneter Kämpfe verstanden (vgl. USAID 2005b: 28) -, ist es nicht nur der am ehesten erfolgreiche, sondern auch aus prinzipiellen Gründen richtige Weg der Intervention.

Um die dennoch bestehenden Risiken einer Demokratisierung zu minimieren, aber auch um Demokratie nicht nur als formal durchzusetzenden Rahmen definiert zu haben, wird die Governance-Komponente auf gleicher Augenhöhe innerhalb derselben Zielsetzung einbezogen. Dies ist keinesfalls eine Selbstverständlichkeit. Dementsprechend wird ausdrücklich festgehalten, dass Demokratie keine essenzielle Voraussetzung für Good Governance sei und auch schlechte Governance innerhalb formaldemokratischer Systeme möglich wäre, aber: »[D]emocracy and good governance together provide the strongest guarantee of security, justice, and economic development« (USAID 2005d: 3). Zugleich ist die Frage der Governance die Wasserscheide in Hinblick auf die staatliche Fragilität: »Research indicates that the instability associated with fragile states is the product of ineffective and illegitimate governance« (USAID 2005c: 3).

Es ist also keine Überraschung, wenn die vier Kerndimensionen, mit denen der Bereich abgesteckt wird, auf beides - Demokratie und Good Governance - abzielen (explizit gemacht in den Punkten "promoting more genuine and competitive elections \& political processes «, sowie $»$ more transparent and accountable governance $\ll) .{ }^{71}$ Dazu kommen noch zwei Elemente, die tendenziell den zwei weiteren Interventionslinien, State-Building und der Suche nach Partnerschaften, zuzuordnen sind: »strengthening the Rule of Law and Respect for Human Rights«, eine Zielsetzung, die den hohen Stellenwert des Rights-Based-Approach in der US-Entwicklungspolitik wiedergibt, sowie »increased development of a politically active civil society«, ein Ansatz, der - wie ich im Folgenden zeigen werde - jedoch eine sehr spezifische Bedeutung hat und

$71 \mathrm{Zu}$ finden sind diese Zielsetzungen auf der Website des Democracy\&Governance-Programms von USAID, vgl. http://www.usaid.gov/our_ work/democracy_and_governance/ (Zugegriffen am 15-06-2007). 
dem gängigen Bild der Förderung politisch engagierter NGOs nur bedingt entspricht.

Was ist nun mit dem genuinen demokratischen Prozess gemeint? Es geht dabei um drei voneinander unterscheidbare Komponenten. Zunächst steht natürlich die Einrichtung von formalen Strukturen für einen politischen Wettbewerb im Mittelpunkt: »Encourage formal means of political competition «, heißt es schlicht in der Fragile States Strategy (USAID 2005c: 7), womit nichts anderes als die Etablierung freier und fairer Wahlen gemeint ist. Diese Zielsetzung ist keineswegs überraschend. Sie ist eine der zentralen Elemente der Democracy Promotion seit den 1980er Jahren und hat alle Kritiken - mit der zuvor erwähnten Einschränkung, dass Wahlen unter gewissen Umständen als potenzieller Trigger-Faktor für den Ausbruch eines bewaffneten Konfliktes interpretiert werden - letzten Endes überdauert.

Die zweite Komponente ist für die Umsetzung der Demokratisierung von zumindest ebenso großer Bedeutung und im Vergleich mit der Garantie eines formellen demokratischen Prozesses für die Entwicklungszusammenarbeit relativ neu: die Arbeit mit und die Förderung von politischen Parteien. Dabei sieht sich USAID in einer besonderen Position und die eigene Arbeit kritisch abgegrenzt von üblichen Geber-Praktiken: »Political parties are among the core elements of democracy. [...] Instead, parties are often seen as internally authoritarian, corrupt, even venal. Donors often agree - and prefer to work with spurer actors in civil society. This would be a mistake. Donors need to help reform parties, no less than governmental institutions on NGOs« (USAID 2002: 6). Auch in persönlichen Gesprächen mit regionalen Verantwortlichen des Democracy\&Governance-Programms wird dieser Schwerpunkt - und das dementsprechende Versäumnis der meisten anderen Geber (mit Ausnahme der deutschen Parteistiftungen) - beständig hervorgehoben.

Die dritte Komponente ist schließlich der flexible Einsatz von Mitteln im Zusammenhang von so verstandenen demokratischen Revolutionen. OTI ist hierfür das Vehikel der Wahl, da unabhängig von langfristigen Planungen die für eine solche Intervention notwendigen schnellen, kurzfristig beschlossenen Schritte gesetzt werden können (USAID 2001b: 4f.). Zusammengefasst wird dies unter dem Stichwort des »democratization momentum «, das es zu unterstützen gelte (USAID 2005d: 12). Auch werden nach einer erfolgreichen demokratischen Umwälzung sofortige Mittelumwidmungen in Betracht gezogen, die das eindeutige Primat der politischen Gesellschaft untermauern: »In particular, our support often shifts to a larger degree from the private sector and civil society to the new government to help establish and strengthen institutions of democratic and accountable governance« (USAID 2005d: 12). 
Die Maßnahmen im Bereich der Good Governance beinhalten auf den ersten Blick wenig Besonderheiten: neben dem schon erwähnten Rechtsschwerpunkt gehören Korruptionsbekämpfung, die Effektivierung der Policy-Implementation und die Arbeit im Sicherheitssektor zu den Ansatzpunkten. Im Bereich der Sicherheitssektorreform (SSR) zählen vor allem die zivile Kontrolle des Militärs - angepackt interessanterweise über Capacity-Building bei zivilen Akteuren, um deren Verständnis militärischer Angelegenheiten zu erhöhen (vgl. USAID 2001b: 18) und der Aufbau einer fähigen Polizei (USAID 2005c: 7) zu den Schwerpunkten. $^{72}$

Es sind vor allem zwei Dinge, die den US-Zugang zur GovernanceProblematik in besonderer Weise kennzeichnen. Da ist zunächst, und dies steht mit der Arbeit im Sicherheitssektor in engem Zusammenhang, die fast vollständige Abwesenheit der Frage des staatlichen Gewaltmonopols, ein Faktor, der beispielsweise in der deutschen Diskussion (vgl. dazu den Ansatz von Dieter Senghaas, Abbildung 4) von überragender Bedeutung ist. In den Schlüsseldokumenten wird darauf nicht Bezug genommen, die einzige Erwähnung finden wir in der Handreichung zur Durchführung einer Konfliktanalyse, und selbst da in einer in Bezug auf das Staatsgebiet relativierenden Weise: »One critical factor is the state's ability to maintain a monopoly on the use of violence throughout the country or in certain regions « (USAID 2005b: 27, meine Hervorhebung). Offenbar wird der Durchsetzung eines solchen Monopols kein kritischer Stellenwert bei der Verankerung verantwortlicher, demokratischer Governance zugeschrieben und dem gegenwärtigen Trend zur Privatisierung selbst militärischer Sicherheitsaufgaben demgegenüber kein Bedrohungspotenzial zugeschrieben.

Die zweite Auffälligkeit ist die starke Betonung von Dezentralisierung und der Stärkung von »local government« als wesentlicher Maßnahme zur Forcierung von Good Governance: »The decentralization of authority from national to local government authorities can bring a number of significant benefits« (USAID 2005b: 27). An dieser Stelle wird der enge Zusammenhang mit dem Bereich der Institutionsentwicklung schlagend. Denn eine der zentralen Aufgaben von politischen Institutionen bei der Verhinderung bewaffneter Konflikte in dieser Lesart ist die

72 Interessant ist dabei die Verknüpfung zu den zentralen Zielsetzungen der Sicherheit und Stabilisierung, gerade im Sinne der ökonomischen Sicherheit, die durch Rule of Law garantiert werden soll: »[...] a strong and accountable security sector and an equitable and impartial rule of law can guarantee personal security and property rights, leaving little room for the opportunistic political and economic behavior discussed earlier« (USAID 2005b: 26). 
Rahmengebung für ökonomische Prozesse, die die wirtschaftlichen Anreize für eine Austragung solcher Konflikte minimieren sollen. Die Verantwortung solcher Institutionen wäre somit $»[$ to] provide economic alternatives that do not rely on the exploitation of scarce resources or [to] mediate between groups to use the same resource« (USAID 2002: 104), also im eigentlichen Sinne klassische Aufgabenstellungen von lokaler Governance. Neben dem Feld der nationalen Eliten ist es also auch das Feld der peripheren Eliten, in dem sich die Verbindung der Demokratisierung mit Aufgaben von Good Governance und Institutionenentwicklung zu beweisen hat, will sie bewaffnete Konflikte erfolgreich transformieren oder deren Ausbruch verhindern.

(2) State-Building. Die Bedeutung, die dem Ausbau der institutionellen Kapazitäten speziell bei fragilen Staaten zugemessen wird, verunmöglicht es, diese Komponente einfach als Subeinheit der Praktiken auf Governance-Ebene zu verstehen. Ich möchte in der Benennung sogar noch weiter gehen und diesen Institutionen-Schwerpunkt in Verbindung mit einigen Ansätzen, die eigentlich in den Governance-Bereich fallen und die ich daher unter Punkt (1) benannt habe, mit dem weitreichenden Begriff des State-Building bezeichnen, obwohl dieser Begriff in den Policy-Papieren von USAID kein einziges Mal verwendet wird. Er ist derzeit eindeutig auf die Policy-Forschung beschränkt, wo er wesentliche analytische Funktionen erfüllt ${ }^{73}$, speziell in der zumeist evaluierenden Untersuchung von Interventionen. Zugleich fasst er jedoch auch die gegenwärtige Praxis der Stärkung von Governance in Zusammenhang mit dem Schwerpunkt des Institution-Building am griffigsten zusammen (vgl. etwa Fukuyama 2004).

Warum ist nun gerade die Entwicklung von Institutionen, und hier vor allem staatlicher Institutionen, für die Transformation oder Verhinderung bewaffneter Konflikte so entscheidend? Die Antwort ist im Prinzip sehr einfach: weil sie die logischen Mediatoren sind, die im Kontext staatlich regulierten Zusammenlebens für die Behebung von Missständen, den Ausgleich von Ungerechtigkeiten und - ein wichtiger Punkt auch für die Kontrolle des Verhaltens der verschiedenen Akteure ver-

73 Wir finden ein ähnliches Phänomen auch beim Begriff des NationBuilding. Analytisch und in den Vorschlägen der Policy-Forschung ein ungemein beliebtes Konzept, ist es im Bereich der entwicklungspolitischen Praxis der USA explizit nicht präsent. Implizit können verschiedene vorhandene Elemente, die sich auch in der hier vorliegenden Zusammenfassung finden, jedoch sehr wohl in diese Richtung interpretiert werden, wenngleich keinesfalls so offensichtlich wie für den Ansatz des StateBuilding. 
antwortlich zeichnen sollen: »[I]f there are strong institutions in place that can address grievance or check the behavior of >conflict entrepreneurs,, then whatever incentives for violence exist in a society can find legitimate channels of expression or be successfully controlled « (USAID 2002: 22). Speziell bei der Bearbeitung fragiler Staatlichkeit ist daher der Schwerpunkt auf diese Problemstellung zu legen: »Therefore, our focus is on building - or rebuilding - institutions of democratic and accountable governance based on the rule of law, and promoting justice and human rights« (USAID 2005d: 13).

Neben dem Management und dem Ausgleich von Interessen und der konkreten Gewährleistung von Rahmenbedingungen für den demokratischen Prozess - wobei im Speziellen die Kontrollfunktionen hervorgehoben werden (vgl. USAID 2005c: 7) - gibt es noch eine zweite wesentliche Aufgabenstellung für Institutionen: die Erbringung grundlegender staatlicher Versorgungsleistungen. In diesem Bereich wird Kapazitätsentwicklung als potenziell besonders wirksame Maßnahme verstanden: »[R]eform and build the technical and administrative capacity of those parts of the civil service responsible for economic management, core services, and food security« (USAID 2005c: 7). Wir werden in Punkt (3) sehen, dass dieser Komponente eine zentrale Rolle in der USamerikanischen Herangehensweise zukommt, ist doch damit auch, zum Teil ausdrücklich, eine Abwendung von der Förderung zivilgesellschaftlicher Akteure gemeint, die bei einem Nicht-Funktionieren staatlicher Institutionen als logische Partner/innen bei diesen Aufgabenstellungen gesehen werden. Zeichnet sich ein solches Funktionieren jedoch $a b$, ist konzeptionell eine sofortige Umschichtung der Mittel von zivilgesellschaftlichen zu staatlichen Akteuren vorgesehen.

Der Zusammenhang zwischen der Vermeidung bewaffneter Konflikte und der spezifischen Art und Weise der Institutionenentwicklung wird nicht so sehr in offiziellen Leitdokumenten deutlich wie in der Umsetzung beziehungsweise in persönlichen Gesprächen mit an dieser Umsetzung Beteiligten. Ein Bericht einer USAID-internen Konferenz bringt diese Verbindung auf den Punkt:

» [Conflict p]revention is a >push-package < wherein democratic institutions and ideals are planted in a nation state. Countries, however, often do not know how to spull or respond to democratization. It is important to mentor newly democratizing states and remain with them over an extended period of time, in order to ensure the successful implementation of a healthy democracy. A vital component of the >pull package is instilling the belief that formal institutions cannot be created without developing a corresponding set of values « (USAID/Woodrow Wilson International Center for Scholars 2001: 5). 
Aber auch in den Leitdokumenten kommt diese Wertorientierung in jüngster Zeit stärker zum Ausdruck. So ist dem aktuellen Strategischen Plan von State Department und USAID nicht nur ein innerinstitutionell verbindlicher Wertekatalog vorangestellt (State Department/USAID 2007a: 8), auch die strategischen Zielsetzungen werden von einem stark wertorientierten Mission Statement ${ }^{74}$ eingeleitet (ebd.: 9). Es wird somit deutlich, dass neben den verschiedenen Elementen der Governance die Demokratisierung wesentlicher Bestandteil der Entwicklung dieser staatlichen Institutionen zu sein hat. Ich möchte sogar noch einen Schritt weiter gehen und behaupten, dass die Institutionsentwicklung ein wesentliches, wenn nicht sogar das entscheidende Vehikel zur Umsetzung der Democracy Promotion darstellt, weshalb sich der Begriff des StateBuilding in der analytischen Beschreibung dieses Prozesses geradezu aufdrängt.

(3) Friedenspartnerschaften. Die große Bedeutung, die der Frage von Partnerschaften bei der Bearbeitung bewaffneter Konflikte zukommt, ist vermutlich aus einem speziellen Verständnis des Ownership-Prinzips zu erklären, das für eine sich im Sinne der Nachhaltigkeit verhaltende Entwicklungszusammenarbeit unabdingbar ist. Die genauere Eingrenzung der erwünschten Partner deutet auf ein klar definiertes Bild von Ownership hin: »Most important are our partnerships with local leaders and organizations promoting reform and democratization« (USAID 2005d: 2). Negativ formuliert bedeutet das denn auch nichts anderes als dass bei weitem nicht jede und jeder als Partner/in in Frage kommt - offenbar sind dazu in erster Linie politische Kriterien zu erfüllen, die sich eng am eingangs geschilderten Demokratie-Konzept orientieren.

Genau genommen kommt hier eine diskursiv verhältnismäßig offensiv vorgetragene Interpretation von Ownership zum Tragen, die das Prinzip an sich zwar nicht in Frage stellt, die Kontrolle über den fraglichen Prozess jedoch keinesfalls aus der Hand geben will. ${ }^{75}$ Obwohl sich jede Geberinstitution - sei sie öffentlich oder privat, bilateral oder multi-

74 »Advance freedom for the benefit of the American people and the international community by helping to build and sustain a more democratic, secure, and prosperous world composed of well-governed states that respond to the needs of their people, reduce widespread poverty, and act responsibly within the international system《 (State Department/USAID 2007a: 9).

75 Simon Harris (2005: 9) hat die Problematik in einem Interviewzitat für seine Aid Effectiveness-Studie für Sri Lanka auf den Punkt gebracht, als er einen Geberrepräsentanten zu Wort kommen lässt, der in Hinblick auf die sri-lankische Regierung meint: »[T]he Government is firmly in the driving seat, but not necessarily in the car that we would want them to drive." 
lateral - dieses Widerspruchs bewusst ist, gibt es dennoch verschiedene Formen des Umganges. Diejenige von USAID, den Kontrollmechanismus nicht nur über die genaue Auswahl, sondern im eigentlichen Sinne über die Eingrenzung der Partner im Sinne von demokratiepolitisch argumentierten Kriterien zu begründen, ist jedoch eine US-spezifische Eigenart und führt auch zu eigentümlichen Problemstellungen. Denn so gerne USAID den gegenwärtigen Trend in der Konfliktbearbeitung, sich auf die Arbeit mit den so genannten »Spoiler«-Gruppierungen zu konzentrieren, mitvollziehen würde (vgl. etwa USAID 2005a: 6) - wirkliche Konzepte können dafür angesichts der bestehenden Einschränkungen nur schwer angeboten werden. So bleibt entweder die Hoffnung, dass sich das Engagement mit derartigen Gruppierungen ohnehin in eine Richtung bewegt, die in geeigneter Weise interpretiert werden kann wie etwa die Partnerschaft von USAID mit islamischen Gruppierungen im Süden der Philippinen (vgl. dazu Pospisil 2005) - oder die Hoffnung auf eine explizit formulierte oder implizit vorausgesetzte Arbeitsteilung mit anderen entwicklungspolitischen Akteuren - wie etwa im Umgang mit der LTTE in Sri Lanka, auf den noch näher einzugehen sein wird.

In jedem Fall erleichtert sich die Zusammenarbeit mit Partnern mit der Zunahme der ihnen zugeschriebenen politischen wie gesellschaftlichen Bedeutung. Denn bei der Frage, wer nun eigentlich für USAID die zentralen Partner sind, die für eine Kooperation idealtypisch in Frage kommen, stehen vor allem politische Eliten im Mittelpunkt. Einerseits geht es dabei um die Etablierung von strategischen Partnerschaften, die speziell bei demokratischen Eliten als wesentlicher und vor allem wirksamer als zivilgesellschaftliche Netzwerke wahrgenommen werden. Solche Netzwerke wären grundsätzlich zwar ebenfalls wünschenswert, » [a]t the end of the day, however, the partnerships with the country's democratic leaders are central« (USAID 2005d: 7). Andererseits wird einem Dialog zwischen Eliten eine wesentliche Rolle bei der Transformierung, vor allem aber bei der Verhinderung bewaffneter Konflikte zugeschrieben: »Therefore intra-elite dialogue may [sic] an important intervention in terms of conflict management « (USAID 2005b: 33 ). ${ }^{76}$

Neben diesen politischen Eliten ist es vor allem die Business Community, die als potenziell am besten geeigneter Partner identifiziert wird, auch wenn diese Möglichkeiten, so wird eingestanden, noch nicht voll

76 Diese Arbeit mit Eliten ist auch auf im Bereich der direkten Verhandlungsunterstützung feststellbar. OTI kümmert sich dabei zwar auch um lokale Friedensinitiativen und die Graswurzel-Ebene, ist aber ebenso für die Unterstützung der Verhandlungsteams auf diplomatischer Ebene, die Einrichtung von »Special Advisors« oder die Erweiterung der Verhandlungen um neue Partner zuständig (vgl. USAID 2001b: 6). 
ausgeschöpft sind: eine »underexplored but potentially important area« (USAID 2002: 107). Die Vorteile der Arbeit in diesem Feld werden wiederum hauptsächlich anhand objektiver Interessenslagen festgemacht - nicht nur hätte der private Wirtschaftssektor ein logisches Bedürfnis nach einer stabilen politischen Situation (»[t]heir interest in stability is self-evident«, vgl. USAID 2005b: 34), er hätte auch im Vergleich zu NGOs bessere Möglichkeiten, dieses Interesse gegenüber politischen Entscheidungsträgern wirksam zum Ausdruck zu bringen: »[T]hey can often bring more pressure to bear on local and national government officials to adopt constructive policies than traditional Peacebuilding NGOs« (ebd.). Auch wäre der Sektor ethnischen Spannungen nicht in der Form ausgesetzt wie die Zivilgesellschaft, die sich ja als Widerspiegelung bestehender gesellschaftlicher Tendenzen diesen nicht entziehen könnte (ebd.).

Diese Ansätze fallen zudem mit der ohnehin bestehenden Notwendigkeit zusammen, die wirtschaftliche Situation zu stabilisieren und damit ökonomische Konfliktanreize zu minimieren: »Support for economic activities that lead to job creation, improved family incomes, and better functioning markets can, in most cases, contribute to greater economic stability« (USAID 2005c: 5). Klarerweise ist schon dafür eine enge Partnerschaft mit der Business Community Voraussetzung.

Ein weiterer wesentlicher Partner sind die Medien, deren Unterstützung vor allem in ihrer alltäglichen Arbeit entscheidend ist: »Develop the professionalism of the media, particularly in investigative journalism, and expand access to information « (USAID 2005c: 7, siehe auch USAID 2005b: 32). Medien sollen also mittels Maßnahmen, die von Training über die Unterstützung bei der Produktion von Inhalten bis zum Marketing reichen (vgl. USAID 2005d: 10), befähigt werden, nicht nur die traditionelle Überwachungsfunktion, sondern vor allem eine Multiplikatorenrolle einzunehmen (vgl. USAID 2001b: 38-41).

Angesichts dieser relativ breiten Auflistung möglicher Partner und der weitreichenden Definition der von ihnen potenziell abzudeckenden Aufgabenstellungen stellt sich natürlich die Frage, welche Rolle für das Feld der Zivilgesellschaft, dem traditionellen Liebkind der europäischen Entwicklungszusammenarbeit, nach Einschätzung von USAID zu spielen übrig bleibt. Die Beantwortung dieser Frage ist in erster Linie von den Bedingungen abhängig, mit denen die US-Intervention konfrontiert ist.

So ist klar, dass im Falle einer Infragestellung der Legitimität der politischen Sphäre in der Zielregion, wie es etwa bei der Kategorie der »Restrictive Countries« definitionsgemäß der Fall ist, die Zivilgesellschaft der primäre Ansprechpartner für entwicklungspolitische Zusam- 
menarbeit ist: »Where legitimacy is an issue, options generally narrow, and programs often shift to nongovernmental and private sector actors" (USAID 2005c: 6). Hier ginge es primär darum, einen Reformprozess außerhalb der bestehenden Strukturen anzustoßen - und wie das Paradebeispiel der osteuropäischen Machtwechsel von Ende der 1980er Jahre demonstriert, ist die Zivilgesellschaft von heute in solchen Kontexten zumindest potenziell die politische Gesellschaft von morgen. Daher ist bei der Intervention in autoritären Staaten klar (natürlich nur insoweit, als sie nicht als geostrategische Partner definiert sind): »Civil society, private sector actors, and the media are our logical partners, and they usually receive the bulk of our assistance« (USAID 2005d: 12).

In einem anderen Umfeld - also etwa in jeder Art von formaldemokratischem System - wird die Rolle der Zivilgesellschaft nicht im Sinne einer Triebkraft gesellschaftlicher Transformation interpretiert, sondern im Sinne dreier relativ klar umgrenzter Aufgabenstellungen: als Multiplikator politischer Prozesse, speziell in der Kommunikation von Verhandlungsprozessen auf Graswurzelebene (vgl. etwa USAID 2001: 34), als demokratiepolitische Überwachungsinstanz, etwa im Bereich des Menschenrechtsmonitoring (vgl. USAID 2005b: 35) sowie als ServiceProvider, allerdings nur dort, wo die staatliche Versorgung nicht in ausreichendem Maße funktioniert.

Dieser Zugang basiert einerseits auf der grundsätzlichen Annahme, dass zivilgesellschaftliche Akteure in gewissen Konstellationen über besondere Fähigkeiten in ihnen vertrauten Bereichen, also über einen Wettbewerbsvorteil verfügen, der sich primär aus ihrer Verortung in einer betreffenden Lokalität ergibt. Andererseits aber zieht dieser Zugang die gerade im friedenspolitischen Bereich populäre Annahme, Zivilgesellschaft wäre mit jenen Kaldorschen (vgl. Kaldor 2001: 117ff.) »Inseln der Zivilität« gleichzusetzen, die die logischen Partner in der Friedensentwicklung wären, ausdrücklich in Zweifel: »Donors need to focus as much on engaging groups and individuals with incentives to engage in violence as on those committed to peace. [...] civil society groups have been asked to carry far too much of the burden in addressing conflict « (USAID 2002: 108). Selbst im Falle eines Engagements bleibt Vorsicht wie Umsicht geboten: »Before engaging with civil society, donors need to conduct a careful analysis of how civil society groups line up along society’s fault lines« (USAID 2005b: 35).

Es wird also deutlich, dass die Zivilgesellschaft nicht unter allen gegebenen Umständen als notwendiger zentraler Partner bei der Transformation bewaffneter Konflikte gesehen wird. Dies entspräche jedoch auch nicht dem Bild, das sich aus den verschiedenen anderen Komponenten 
des US-amerikanischen entwicklungspolitischen Peacebuilding ergibt. Denn nicht nur die Fokussierung auf ausgewählte Partnerschaften, sondern auch der grundsätzliche Ansatz der Demokratisierung mit der daraus folgenden Arbeit in der politischen Gesellschaft, insbesondere mit den politischen Parteien, verdeutlicht, dass die Wirksamkeit einer Intervention über das Potenzial jener Akteure realisiert werden soll, die zu diesem Zweck als politisch geeignet betrachtet werden.

Die richtige Platzierung solcher Akteure in der politischen Arena und die Verankerung von als universal gültig verstandenen demokratischen Spielregeln sollen so im Zusammenspiel mit der Gewährleistung eines stabilen institutionellen Apparates und ökonomischem Wachstum jene Mixtur ergeben, die die friedliche Austragung von Interessenskonflikten garantiert. Eine solche Austragung soll demnach von der Sphäre des bewaffneten Kampfes einerseits auf den demokratischen Wettbewerb von Parteien, die dem Primat des Politischen verpflichtet sind, und andererseits auf die Ebene dezentral wirkender und fähiger Institutionen, die Interessensunterschiede speziell auf lokaler Ebene auszugleichen in der Lage sind, verlegt werden. Das demokratische Spiel ist per Definitionem elitär und auf politische Führungen - sei es lokal, sei es national - beschränkt. Es entspricht tatsächlich, wie schon William I. Robinson (1996) feststellt, jener Konfiguration, die Robert Dahl (1971) als Polyarchie charakterisiert hat: ein Konzept von Demokratie, das sich auf die zwei Komponenten der Liberalisierung des politischen Systems im Sinne eines demokratischen Wettbewerbs und die Beteiligung von Repräsentant/innen möglichst aller Elemente der Gesellschaft an diesem Wettbewerb auszeichnet. ${ }^{77}$

Trotz dieser fast schon bescheiden anmutenden Zielbestimmung handelt es sich, wie sich schon aus dem historischen Ablauf ergibt, um ein stark idealistisch geprägtes Konzept, das von USAID, aber auch von der US-Außenpolitik generell verfolgt wird. Denn tatsächlich ist der Glaube an den globalen Drang zur Demokratie und die sich darin manifestierende Annahme der immanenten Friedfertigkeit demokratischer Systeme handlungsrelevant und hat die Idee geostrategischer Partnerschaften, die um jeden politischen Preis zu halten sind, in der überwiegenden Tendenz abgelöst. ${ }^{78}$ Freilich ist diese starke idealistische Ten-

77 Robert Dahl (1971: 2) räumt schon eingangs seiner Untersuchung ein, dass dieses Konzept keineswegs alle möglichen Elemente einer demokratischen Gesellschaft beinhaltet - aus diesem Grund führt er den Begriff der Polyarchie überhaupt ein.

78 Natürlich ist dies nicht durchgängig der Fall, wie beispielsweise die Kategorie der geostrategischen Partnerländer im »Foreign Policy Framework« 
denz von realistischen Elementen durchzogen, die sich in der Behauptung der Notwendigkeit von Stabilität und einer starken institutionellen Komponente manifestieren.

Der Glaube an die Selbstregulierung des Systems ist also begrenzt, wiewohl die gouvernementale Rationalität der Intervention ihr bestimmendes Merkmal bleibt. Doch zeigt der elitär geprägte Fokus auf die politische Gesellschaft merkliche Tendenzen in Richtung souveränen Regierens, während die Kontrolle und Disziplinierung vermittels speziell in den lokalen Bereich schielenden Institutionen verankert werden soll. Die Regulierung benötigt also Unterfütterung, bleibt aber dennoch das zentrale Element im Rahmen der Intervention. Der Zugriff der Intervention ist definitionsgemäß beschränkt, beschränkt auf Partner, die und das lässt sich aus den formulierten Kriterien klar ablesen - bewusst oder unbewusst diese Partnerschaft wollen und auch benötigen. Der institutionelle Rahmen dient letztendlich primär der Garantie der Bedingungen des Wettbewerbs (vgl. dazu auch Foucault 2004b: 303), wobei natürlich jenen Akteuren, die im Zuge des Prozesses zu Partnern mutieren, die Schlüsselrollen zugedacht sind.

\section{Deutschland: Aufbau demokratischer Sicherheitsstaatlichkeit}

Für die deutsche Entwicklungszusammenarbeit ist das Arbeitsfeld »Krisenprävention und Friedensentwicklung«, wie es derzeit offiziell genannt wird, vergleichsweise neu. Es bedurfte dreier schockartiger Ereignisse auf ganz unterschiedlichen Ebenen - den Zusammenbruch des Warschauer Pakt-Systems mit der daran anschließenden deutschen Wiedervereinigung, den Eindruck des ruandischen Genozids und schließlich die Abwahl von Helmut Kohl und der von ihm angeführten konservativliberalen Bundesregierung - damit diese Aufgabenstellung im Verlauf der 1990er Jahre zu einem der Schwerpunkte nicht nur in der generell außenpolitischen, sondern auch in der entwicklungspolitischen Ausrichtung der Bundesrepublik Deutschland werden konnte. Dennoch wäre es falsch anzunehmen, davor hätte die Idee, Entwicklungspolitik als Element internationaler Sicherheits- und Friedenspolitik wahrzunehmen, nicht existiert. Immer wieder in der mittlerweile 50jährigen Geschichte deutscher Entwicklungspolitik kam dieser Faktor zum Tragen, wenn-

und die konkrete Partnerschaft mit Regimes wie etwa jenem in SaudiArabien untermauern. 
gleich oftmals nur randständig und abhängig von besonderen politischen Konstellationen.

Innerhalb kurzer Zeit scheint Deutschland diesen »Entwicklungsrückstand « gegenüber den USA und anderen im Bereich der sicherheitspolitischen Entwicklungszusammenarbeit führenden Gebern wie Großbritannien aufgeholt zu haben. Eine Vielzahl von Konzeptpapieren, Studien und Handreichungen wurde in den letzten zehn Jahren produziert. Zusammen mit den Rahmendokumenten von Bundesregierung und dem zuständigen Bundesministerium für wirtschaftliche Zusammenarbeit und Entwicklung (BMZ) ermöglichen sie einen guten Einblick in die Rationalitäten jener Form des deutschen Interventionismus, der mit zivilen Mitteln - je nach Perspektive - zur Friedensentwicklung beitragen oder gar »Frieden machen« (vgl. Senghaas 1997a) will.

\section{Historische Entwicklung und institutionelle Verankerung}

Wie gesagt spielen politische Gründe am Beginn der ersten zögerlichen Versuche deutscher Entwicklungshilfe keine große Rolle. Es kann mit Recht als programmatisch gesehen werden, dass die ersten Handlungen des noch durch den verlorenen Krieg und die darauf folgende Teilung geschwächten Landes im multilateralen Rahmen liegen: 1952 beteiligt sich Deutschland am Beistandsprogramm der Vereinten Nationen, dem Vorläufer des heutigen UNDP. Erste bilaterale Schritte werden vom Wirtschaftsministerium ab Mitte der 1950er gesetzt - der erste Partner ist 1956 Indien (vgl. BMZ 2006a: 2), wobei die Bedienung handfester ökonomischer Interessen, die sich an der traditionellen Exportorientierung der wieder erstarkenden deutschen Wirtschaft festmachen lassen, im Mittelpunkt (vgl. Korff 1997: 2; Hein 2006: 29), und dezenter amerikanischer Druck, den Marshall-Plan-finanzierten Wiederaufbau auch im Sinne der Unterstützung antikommunistischer Verantwortungsübernahme auf internationaler Ebene zu verwenden, im Hintergrund stehen.

Dennoch bleiben die Interessen der exportorientierten Wirtschaft nicht der einzige Motivationsfaktor. Seit den späten 1950er Jahren wird die Entwicklungshilfe als definiertes Instrument deutscher Außenpolitik in den Geltungsbereich der Hallstein-Doktrin gesetzt. Entwicklungshilfe bekommt demnach nur, wer den deutschen Alleinvertretungsanspruch der Bundesrepublik akzeptiert. Dafür werden dann aber breit und relativ konzeptlos Hilfsgelder ausgeschüttet. Bis 1973, dem Jahr der Aufnahme beider deutschen Staaten in die UNO, bleibt die Doktrin für den entwicklungspolitischen Bereich weitgehend gültig, was zeigt, dass die Idee politischer Konditionalität von Entwicklungshilfe in Deutschland - die sich auch heute noch ungebrochener Beliebtheit erfreut - auf eine lange 
Geschichte zurückblicken kann. Bastian Hein (2006: 31) schreibt dieser politischen Konditionalität sogar eine substanzielle Rolle bei der Entstehung der deutschen Entwicklungshilfe zu: »Das Bedürfnis, einen Hebel für eine systematische und präventive Deutschlandpolitik zu erhalten, half zu Beginn der 1960er Jahre die bundesdeutsche Entwicklungspolitik zu schaffen.«

Institutionell wird Deutschland schnell zu einem der entwicklungspolitischen Vorreiter in Europa. Schon 1959 wird die »Deutsche Stiftung für internationale Entwicklung« (DSE) in West-Berlin, die heutige InWEnt ${ }^{79}$, gegründet, mit dem Ziel, die durch die bis auf wenige Ausnahmen weitgehend fehlende Kolonialgeschichte nur schwach ausgeprägten sozialen und kulturellen Beziehungen Deutschlands zu den jetzt als »Entwicklungsländer« definierten Staaten zu forcieren (vgl. Holtz 2006: 32). 1961 kommt es dann zur Einrichtung des ersten speziell für Entwicklungspolitik zuständigen Ministeriums in Europa: das Bundesministerium für wirtschaftliche Zusammenarbeit, BMZ. Es nimmt unter dem FDP-Politiker Walter Scheel seine Arbeit auf (vgl. BMZ 2006a), wenngleich noch als Rumpf. Erst drei Jahre später, 1964, werden jene Agenden, die später unter der Bezeichnung »technische Zusammenarbeit« als eine der Kernaufgaben von Entwicklungshilfe gelten werden, vom Auswärtigen Amt in das BMZ überführt.

In der entwicklungspolitischen Ausrichtung sucht sich die Bundesrepublik, jenseits der klar definierten politischen Konditionalität um die Hallstein-Doktrin, mit unproblematischen Themensetzungen in der sich aufladenden Blockkonfrontation gerade nicht zu profilieren: Armutsbekämpfung ist die oberste Prämisse (vgl. Korff 1997: 3), immer begleitet von der Durchführung von Großprojekten, die den Interessen der Schlüsselbetriebe der »Deutschland AG« entgegenkommen. Im Allgemeinen bleibt Entwicklungspolitik bis Ende der 1960er Jahre ein weitgehend irrelevantes Politikfeld, mit dem keine eigentliche Außenpolitik jenseits der Hallstein-Doktrin betrieben wird. Immer wieder ist amerikanischer Druck vonnöten, um Deutschland an seine entwicklungspolitischen Verpflichtungen als Teil der »Freien Welt« und dem sich daraus ableitenden aktiven Antikommunismus zu erinnern. Speziell die Kennedy-Administration tut sich bei den Durchhalteparolen für diesen primär ökonomischen Abwehrkampf hervor.

Der Wandel erfolgt mit der Übernahme der Regierungsgeschäfte durch die sozialliberale Koalition unter Willy Brandt. Der exponierte SPD-Linke Erhard Eppler wird Minister für wirtschaftliche Zusammenarbeit und definiert Entwicklungspolitik forthin als »internationale Sozi-

79 »Internationale Weiterbildung und Entwicklung GmbH« 
al- und Friedenspolitik«, die er mit einem soliden moralischen Fundament untermauert. Eppler macht seine Präsenz sofort spürbar, viel später wird er in einem Interview sogar meinen, dass er als Minister vielleicht sogar »zu sehr« engagiert gewesen wäre. ${ }^{80}$ Er reklamiert, in den sechs Jahren seiner Amtszeit (1968-1974) aus dem »Laden« BMZ erst ein Ministerium gemacht zu haben. Auf seine Anregung geht auch die 1974 erfolgte Gründung der Gesellschaft für Technische Zusammenarbeit GTZ als das zentrale Instrument der Projektimplementation zurück.

1971 entwickelt das BMZ die erste »Entwicklungspolitische Konzeption der Bundesrepublik Deutschland« als Strategieentwurf für die gerade angebrochene zweite Entwicklungsdekade (vgl. BMZ 2006a). Die Konzeption gruppiert sich vor allem um die Bereiche Beschäftigung, Gesundheit und Ernährungshilfe. Eppler selbst betont - im Übrigen bis zum heutigen Tag - immer wieder die friedensstiftende Komponente der Entwicklungszusammenarbeit. Unzweifelhaft sieht er sich in der Rolle eines in politische Verantwortung gelangten Aktivisten, der nun versucht, in diesem Rahmen seine Überzeugungen institutionell zu verankern und umzusetzen. Damit symbolisiert Erhard Eppler als erster einen für die weitere Entwicklung der staatlichen deutschen Entwicklungspolitik charakteristischen Prozess: den engen Austausch mit friedens- und entwicklungspolitischen Aktivist/innen, speziell aus einem Bereich der im linken politischen Spektrum verorteten sozialen Bewegungen (vgl. Herf 1986).

Es ist daher nur konsequent, dass Eppler als Minister den Rücktritt Brandts und die Macht- und Regierungsübernahme durch die pragmatisch-liberal orientierte SPD-Strömung um Helmut Schmidt nicht übersteht. Schmidt ist gegenüber Entwicklungspolitik ein deklarierter Skeptiker und tendiert dazu, Ausgaben gerade in Zeiten beginnender ökonomischer Schwierigkeiten, wie sie durch die Ölkrise von 1973 gegeben sind, als unnötige Geldverschwendung zu interpretieren (vgl. Bellers et al. 1998). Unter Epplers Nachfolger Egon Bahr wird 1974 die Auswahl jener Länder, in denen die deutsche Entwicklungshilfe präsent ist, »konzentriert«, wie es in der offiziellen Geschichtsdarstellung heißt (vgl. BMZ 2006a). Unter Vergabekriterien, die als »pragmatisch« beschrieben werden (vgl. Bellers et al. 1998: 42), jedoch »vermehrt das wirtschafts- und rohstoffpolitische Eigeninteresse der Bundesrepublik« ins Kalkül ziehen (ebd.: 40), wird Entwicklungshilfe vor allem auf die Gruppe der durch die Erdölpreiserhöhung am härtesten getroffenen Länder eingeschränkt. Ansonsten gibt Bahr, mit einer merklichen Spitze ge-

80 »30 Jahre GTZ - Ein Interview mit Erhard Eppler«, http://www.gtz.de/de/ top-themen/7222.htm (Zugegriffen am 12-07-2007). 
gen den Zugang Epplers, das Motto aus: »Umstellung von karitativem Denken auf Kooperation« (ebd.: 42).

Dieser für Konfliktprävention und Friedensentwicklung vermeintlich wenig interessante Zugang wird allerdings von einem Ereignis durchbrochen, das in Bezug auf die deutschen Bemühungen in diesem Bereich zwar keine unmittelbar spürbaren Auswirkungen zeitigt, aber dennoch als richtungsweisend angesehen werden kann. Im April 1974 wird in Portugal die parafaschistische Diktatur des Estado Novo unter Marcelo Caetano von antifaschistischen Truppenteilen gestürzt. Die so genannte Nelkenrevolution läutet eine Demokratisierung ein, die auch für die griechischen und spanischen antidiktatorischen Kräfte Vorbildwirkung haben wird. Zugleich konfrontiert sie die entwicklungspolitischen Bemühungen der demokratischen EG-Staaten mit einer Grundsatzentscheidung: soll mit Soforthilfen zugunsten des demokratischen Neubeginns interveniert werden oder nicht? Während sich die EG gegen solche Soforthilfen ausspricht und zuerst greifbare Resultate der Demokratisierung abwarten will, entscheidet sich die sozialliberale Regierung der Bundesrepublik - gegen heftigen Widerstand der CDU/CSU-Opposition - für eine solche Intervention. Damit schafft sie den Präzedenzfall für eine klar politisch motivierte Intervention in einem riskanten politischen Umfeld (vgl. Holtz 2006: 34), eine Intervention, die noch dazu angesichts der weiteren historischen Entwicklung unzweideutig als Erfolg gewertet wird.

Zusammen mit dem zunehmend spürbaren politischen Druck der Friedensbewegung ist diese Erfahrung mitverantwortlich dafür, dass die am 30. Mai 1979 verabschiedeten »Thesen zur Entwicklungspolitik« der Bundesregierung Friedenssicherung als explizites Ziel die »langfristige Friedenssicherung durch die Förderung des wirtschaftlichen und sozialen Fortschritts der Entwicklungsländer« (Bundesregierung 1979:These 2) festlegen. Spezifische Maßnahmen zur Friedenssicherung sind hier allerdings nicht vorgesehen. Entwicklungshilfe dient vielmehr als ein außenpolitisches Steuerungsinstrument, das primär auf ökonomischer Ebene wirksam werden soll, wobei insbesondere die Betonung der Blockfreiheit in den entwicklungspolitischen Grundsatzpapieren jener Zeit von einer gewissen Eigensinnigkeit der sozialliberalen Koalition gegenüber der generellen internationalen Ausrichtung des NATOBlocks zeugt. Dies ist für das ansonsten stark transatlantisch orientierte Kabinett Schmidt ein bemerkenswertes Detail.

Im Jahr 1980 erfolgt, nicht zuletzt bedingt durch Anstöße auf internationaler Ebene, eine noch stärkere Betonung der friedensfördernden Qualität deutscher Entwicklungspolitik. Zunächst veröffentlicht die auf Anregung der UNO und der Weltbank - vor allem Weltbank-Präsident 
Robert McNamara zeigt sich sehr interessiert - eingerichtete Nord-SüdKommission ihren Bericht »Das Überleben sichern«, der unter dem

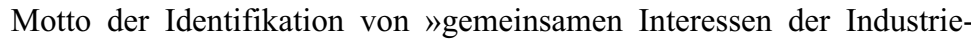
und Entwicklungsländer« steht (Brandt 1980). ${ }^{81}$ Dass der Vorsitz dieser Kommission einem Deutschen obliegt, garantiert dem Bericht verhältnismäßig große Aufmerksamkeit in der Bundesrepublik, und dass dieser Vorsitzende auch noch Willy Brandt heißt, der zentrale Exponent des mit Kanzler Schmidt konkurrierenden linken, in relevantem Maße von der Friedensbewegung beeinflussten Parteiflügels der SPD, schafft eine für die Bundesregierung heikle Situation.

So bleibt dem BMZ unter dem neuen zuständigen Minister Rainer Offergeld trotz politisch tendenziell gegenläufiger Tendenzen ${ }^{82}$ nichts anderes übrig, als die von Brandt vorgegebene Linie in die entwicklungspolitische Ausrichtung einzubauen. So schlägt sich der Bericht in den »Entwicklungspolitischen Grundlinien der Bundesregierung« von 1980, dem ersten strategischen Policy-Paper für den entwicklungspolitischen Bereich der Bundesrepublik, nieder, wenngleich seine explizite Erwähnung sorgsam in den Annex verbannt wird. Obwohl der Schwerpunkt der Grundlinien in der Verankerung des Grundbedürfnisansatzes liegt, hinterlässt der Brandt-Report auch im inhaltlichen Teil Spuren: neben der schon zuvor immer wieder betonten Menschenrechtsorientierung wird auch die Zielsetzung der Friedenssicherung durch Entwicklungspolitik explizit unterstrichen (vgl. Holtz 2006: 34). Entwicklungspolitik trage demnach mit ihrem Beitrag zur wirtschaftlichen und sozialen Entwicklung der Dritten Welt etwa zum »Abbau von Spannungen« und zur »Sicherung des Friedens« bei (BMZ 1983: 30).

81 An den unterschiedlichen Motivationen hinter dem Brandt-Bericht lässt sich ein interessanter Unterschied zwischen US-amerikanischer und deutscher außen- und damit entwicklungspolitischer Orientierung ablesen. Während Brandt als ohne Zweifel genuiner Friedensaktivist die dem Bericht zugrunde gelegten "gemeinsamen Interessen« aus moralischen und nicht zuletzt auch innenpolitischen Erwägungen hervorheben will, verkörpert McNamara den zu dieser Zeit auch bei USAID stark verankerten Ansatz der ökonomischen Entwicklung zur Eindämmung des Kommunismus an der weltsystemischen Peripherie, der mit der Durchsetzung geostrategischer Interessenslagen einhergeht. Beiden Interessen ist mit dem Bericht gleichermaßen gedient.

82 Offergeld, Jurist und enger Gefolgsmann von Kanzler Schmidt, hatte zuvor etwa den Leiter der BMZ-Grundsatzabteilung, Wilfried Böll, sowie den Leiter der Abteilung »Internationale Zusammenarbeit und Ziele der Entwicklungspolitik«, Rainer Oppelt, zwei Vertraute von Brandt und Vertreter eines moralisch konnotierten Bildes von Entwicklungshilfe, aus politischen Gründen entlassen (vgl. Bellers et al. 1998: 43). 
Der Bruch der sozialliberalen Koalition im Jahr 1983 hat für das BMZ schwerwiegende Konsequenzen. Das Ministerium wird in der innerkoalitionären Aufteilung der CSU überantwortet, was einen markanten Bruch in seiner politischen Ausrichtung zur Folge hat. Zwar hatten wie erwähnt schon die SPD-Minister nach Erhard Eppler die SPDLinken sorgsam aus den Leitungspositionen des Ministeriums entfernt, unter dem ersten CSU-Minister Jürgen Warnke kommt es jedoch zu einer regelrechten Umfärbung des Ministeriums, was diesem sogar den Spitznamen »Bayernbrigade« einbringt (vgl. Nuscheler 1996: 385). Politisch orientiert sich das BMZ fortan eng an den von Weltbank und IWF vorgegebenen Linien, wobei die in den Jahren zuvor eingezogene Konzentration auf Armutsbekämpfung durch eine marktwirtschaftliche Orientierung abgelöst wird, deren Umsetzung vor allem über Mittelstandsförderung läuft. Zugleich kommt es wieder zu einer Verschärfung der politischen Konditionalität von deutscher Entwicklungshilfe: Staaten, die außenpolitisch die UdSSR unterstützen, werden von der Vergabe ausgeschlossen. ${ }^{83}$

Diese Ausrichtung an einem wachstumsorientierten politischökonomischen Entwicklungskonzept, das später als »Washington Consensus ${ }^{84}$ in die Geschichtsbücher eingehen sollte, durchzieht die Entwicklungspolitik in Deutschland in der zweiten Hälfte der 1980er Jahre. Entlang der Debatte auf internationaler Ebene ist der einzige wirklich relevante Schnitt dieser Phase die Einführung des Nachhaltigkeitskonzeptes, das erstmals in der Fortschreibung der entwicklungspolitischen Grundlinien der Bundesregierung im März 1986 auftaucht. Zusammengefasst wird es mit dem popularisierten Motto »Hilfe zur Selbsthilfe« und dem Begriff der »Eigenanstrengung« (BMZ 1988: 40). Diese »Eigenanstrengung « ist es auch, die die Aufrechterhaltung und im weiteren

83 Trotz dieser Veränderungen wird in der Zielorientierung von Entwicklungspolitik die friedenspolitische Komponente auch unter der CSUFührung beibehalten. So heißt es im Sechsten Entwicklungspolitischen Bericht der Bundesregierung von 1985: »Die Bundesregierung versteht ihre Entwicklungspolitik als Teil ihrer weltweiten Friedenspolitik. In einer Zeit wachsender Spannungen und Krisen ist es für sie ein wichtiges entwicklungspolitisches Ziel, zur politischen Stabilität durch wirtschaftliche und soziale Entwicklung beizutragen« (BMZ 1985: 35f.).

84 Der Begriff »Washington Consensus« wurde im Jahr 1990 vom Weltbanknahen Ökonomen John Williamson geprägt. Der Konsens bezieht sich auf ein wirtschaftspolitisches Programm, als dessen Eckpfeiler die makroökonomische Stabilität bei gleichzeitigem Abbau des Protektionismus gilt. Im Rahmen der namensgebenden Konferenz einigten sich die Anwesenden auf zehn Policy-Instrumente, die dies garantieren sollten, wobei speziell der Haushaltsdisziplin eine Schlüsselrolle zukommt (vgl. dazu BMZ 2004d). 
Verlauf sogar die Zuspitzung politischer Zielsetzungen ermöglicht, politische Zielsetzungen, die sich im Unterschied zur wirtschaftspolitischen Orientierung des BMZ interessanter Weise kaum von jenen unterscheiden, die unter sozialliberaler Leitung verfolgt wurden.

So werden Menschenrechte und Demokratie nach wie vor als Kernelemente des entwicklungspolitischen Engagements verstanden (vgl. BMZ 1988: 40). Das zentrale Element in der Umsetzung ist der »offene, partnerschaftliche Politikdialog« (BMZ 1988: 38). Eigentlich gedacht, um »eine übereinstimmende Beurteilung entscheidender Entwicklungsengpässe und -hemmnisse und geeigneter Entwicklungs- und Anpassungspolitiken des Entwicklungslandes zu erreichen « (BMZ 1988: 41), wird er zunehmend offensiv interpretiert und so $» z u$ einem Instrument der Einflussnahme« auf die Politik der Partnerländer (Holtz 2006: 34). Er bereitet somit eine verstärkte Konditionalität entlang von Menschenund Bürgerrechtskriterien vor, die die erste Hälfte der 1990er Jahre kennzeichnen wird.

Mit der Übernahme des Ressorts durch Carl-Dieter Spranger im Jahr 1991 kommt es zu einer signifikanten Justierung in der Arbeit und den Schwerpunktlegungen des BMZ, was sogar Franz Nuscheler, generell ein scharfer Kritiker der Ausrichtung des BMZ jener Jahre, dazu veranlasst, von überraschenden Fortschritten in der Vergabepolitik zu sprechen (vgl. Nuscheler 1996: 388). Es sind vier miteinander verwobene Faktoren, die für diese Veränderung verantwortlich sind, und auch über Sprangers Amtszeit hinaus inhaltlich bestimmend bleiben. Zunächst sind es natürlich die schon angeführten weltpolitischen Ereignisse, die auf zwei Ebenen unmittelbare Auswirkungen auf die entwicklungspolitische Ausrichtung haben: Der Zusammenbruch des Ostblocks mit der deutschen Wiedervereinigung sowie der Ausbruch des Jugoslawien-Krieges nötigen nicht nur zu einer inhaltlichen Neuorientierung, sondern auch zu einer umfassenden Neuverteilung der Entwicklungshilfegelder. Zugleich wirken diese globalen Ereignisse auch auf einer zweiten Ebene mittelbar auf die deutsche Situation zurück. Die Veränderungen in der internationalen entwicklungspolitischen Debatte, insbesondere die immer stärker werdende Governance-Diskussion dieser Zeit, wird angesichts der traditionell starken multilateralen Auswirkung der deutschen Entwicklungspolitik rasch und umfassend übernommen.

Aber auch innerdeutsche Kräfte wirken in relevantem Maße auf die Diskussion ein. So kommt es Ende der 1980er und Anfang der 1990er Jahre zu einer richtungsweisenden Umorientierung in der deutschen Zivilgesellschaft: Die Neuen Sozialen Bewegungen, vor allem in ihren drei Hauptsträngen der Friedens-, Ökologie- und Frauenbewegung, wandeln sich in ihrer dominierenden Tendenz von definiert außerparlamentarisch 
agierenden alternativen Kräften zu einer sich zunehmend professionalisierenden Form von Zivilgesellschaft. Konsequenterweise transformiert sich auch die Palette ihres Aktivismus von zeitweise sogar gewaltsamem Protest zu Lobbyarbeit und beginnenden Formen kommerzialisierten Wirtschaftens im Rahmen von Dienstleistungen, die durch das spezifische Know-how der Aktivist/innen ermöglicht werden. Diese Entwicklung spiegelt zwar einen Trend wider, der in ganz Westeuropa zu finden ist, dennoch sind seine Auswirkungen in Deutschland, speziell im entwicklungspolitischen Sektor, unverhältnismäßig stark. Sie führen beim BMZ schon unter CSU-Ägide zur aktiven Aufnahme der drei zentralen Themenstellungen der Neuen Sozialen Bewegungen: Frauen, Ökologie und Frieden (vgl. Korff 1997: 6).

Der vierte Faktor ist schließlich eine generelle Neudefinition der deutschen Außenpolitik, die aus der sowohl global wie auch national veränderten Konstellation resultiert. So kommt es in der Folge der deutschen Wiedervereinigung zu einer Richtungsdebatte, die sich zwischen den beiden Polen einer "Normalisierung « der deutschen Außenpolitik im Sinne einer wieder gewonnenen Großmachtstellung einerseits und der Betonung der Kontinuität entlang des bisherigen, auf niedriges Profil und Multilateralismus setzenden Kurses andererseits entspinnt (vgl. Risse 2004: 24). Ein dritter Strang, der eine gewisse Mittelposition zwischen diesen beiden Polen einnimmt, fordert wiederum die Ausrichtung der deutschen Außenpolitik im Sinne der »Zivilisierung « der internationalen Beziehungen, auch im Sinne eines aktiv zivilisierend wirkenden internationalen Eingreifens (vgl. Peters 2001: 24). Das Schlagwort, das in diesem Zusammenhang speziell von einigen aus der anwendungsorientierten Friedensforschung stammenden Namen wie Ernst-Otto Czempiel und Dieter Senghaas oder dem Außenpolitikspezialisten Hanns Maull forciert wird, lautet »Zivilmacht«.

Gemeint ist damit eine außenpolitische Orientierung, die bei Hervorhebung ziviler und einer weitgehenden Enthaltsamkeit gegenüber militärischen Mitteln vor allem multilaterale und durch internationales Recht abgesicherte Wege einschlägt. Hanns Maull analysiert Deutschland und Japan in ihrer außenpolitischen Ausrichtung zu Beginn der 1990er Jahre in Foreign Affairs als Zivilmächte, womit er offenbar einen doppelten Zweck, eine Werbung für die deutsche Orientierung in den USA bei gleichzeitiger Stärkung des Konzepts in Deutschland selbst, verfolgt (vgl. Maull 1990). Maull verortet sich in der Richtungsdebatte eindeutig, und zwar im Sinne der Kontinuität. Demnach wäre die »Zivilmacht «-Strategie schon in den 1950er und 1960er Jahren in Deutschland entwickelt und seither bewusst verfolgt worden (Maull 2004: 22). Wenig später widerspricht er dieser Einschätzung allerdings selbst, 
wenn er meint, dass $»[i] n$ der deutschen Außenpolitik [...] das Zivilmachts-Konzept vor 1989 weder in der wissenschaftlichen Analyse noch in der politischen Diskussion um die Ausrichtung der deutschen Außenpolitik eine Rolle« gespielt habe (Maull 2007: 75).

Im Zusammenhang mit dieser Unschärfe stellt sich nun die in Bezug auf die Neuausrichtung deutscher Entwicklungspolitik interessante Frage, ob es bereits Anfang der 1990er unter konservativ-liberaler Ägide eine auch entwicklungspolitisch spürbare Veränderung der außenpolitischen Orientierung gegeben hat, oder ob diese erst mit dem Regierungswechsel zur rot-grünen Koalition im Herbst 1998 eintritt. Maull selbst tendiert in früheren Analysen eher zur ersten Einschätzung, wenn er meint, dass sich mit Ausnahme der Frage nach der zukünftigen Rolle der Bundeswehr »Konturen der ıneuen deutschen Außenpolitik«« bereits in der Ära Kohl verfestigt hätten (Maull 1999: 172).

Der entscheidende Schritt zur Klärung dieser Frage scheint in der Trennung zwischen einer Analyse der Praxis, einer Analyse der PolicyDiskurse und der Erarbeitung einer normativ-konzeptioneller Ausrichtung zu liegen. So ist Maull sicherlich zuzustimmen, dass es Anfang der 1990er Jahre außenpolitisch, aber auch entwicklungspolitisch zu keinen bahnbrechenden Änderungen gegenüber den vorangegangen Phasen der konservativ-liberalen Koalition gekommen ist. Dennoch kommt es - und zwar schon Anfang und Mitte der 1990er - zu einem merklichen Wandel im Diskurs, speziell auf entwicklungspolitischer Ebene. Und dieser neue Diskurs nimmt das Konzept der »Zivilmacht«, das bis zu diesem Zeitpunkt in expliziter Weise fast ausschließlich auf die Europäische Gemeinschaft angewendet wurde, in einer normativen Interpretation auf. Dabei spielt es bemerkenswerter Weise keine Rolle, dass die Promotor/innen der normativen Zivilisierung, allen voran Ernst-Otto Czempiel und Dieter Senghaas, politisch eindeutig nicht auf Regierungslinie zu verorten sind. Wie schon die verstärkte Aufnahme von zivilgesellschaftlichen Ideen in den offiziellen entwicklungspolitischen Diskurs, die ebenfalls in diesen Zeitraum fällt, beweist sich auch hier, dass die parteipolitische Orientierung für die Gestaltung außenpolitischer Strategien von untergeordneter Bedeutung ist.

Nachdem sich die Rolle der Bundeswehr in Hinblick auf ihre Ausrichtung auf Kampfeinsätze im Bündnisrahmen, wie sie letztlich von der rot-grünen Koalition im Verlauf des Kosovo-Krieges getroffen wird, erst später klärt - was angesichts des »humanitären« Charakters dieser Einsätze im Übrigen von einigen Kommentator/innen nicht als Widerspruch, sondern als logische Fortführung des Zivilmachtsorientierung verstanden wird (vgl. Maull 2004: 22 oder Schneckener 2005b: 10, der in Bezug auf die EU den fast schon absurden Begriff der »robusten $\mathrm{Zi}-$ 
vilmacht « einführt) - wird die Entwicklungspolitik zu einem jener Elemente, wo die Neuorientierung am schnellsten auch in den praktischen Auswirkungen sichtbar wird. Dies ist auch insofern nachvollziehbar, als das Konzept in seinem Gehalt stark auf entwicklungspolitische Komponenten baut: »Die Gestaltungsspielräume von Zivilmacht beruhen wesentlich darauf, inwieweit sie in der Lage ist, effektive Hilfestellungen in den Prozessen der Überwindung von Unterentwicklung zu leisten« (Maull 1997: 70).

Dies führt unter der Ägide Carl-Dieter Sprangers nicht nur zu einer Verstärkung der Konditionalität entlang der 1991 eingezogenen so genannten "Spranger-Kriterien" wie Achtung der Menschenrechte, Rechtsstaatlichkeit und der Entwicklungsorientierung staatlichen Handelns ${ }^{85}$, sondern auch zu einer verbalen Aufwertung des Entwicklungsbegriffes in seiner Eigentümlichkeit gegenüber der Wirtschaftskooperation: 1993 erhält das bisherige Bundesministerium für wirtschaftliche Zusammenarbeit den Zusatz »und Entwicklung «, womit es jenen Namen erhält, unter dem es bis heute firmiert. Nach einer substanziellen Sinnkrise, die noch bis Ende der 1980er Jahre dominierend war (vgl. BMZ 1988), befindet sich der Politikbereich wieder im Aufwärtstrend.

Schon relativ früh zeichnet sich auch der Trend zur direkten Anwendung von Entwicklungspolitik im Bereich der Konfliktprävention ab. Im Neunten Entwicklungspolitischen Bericht im Jahr 1993 wird eine Problemanalyse vorgenommen, die die verschiedenen zu diesem Zeitpunkt gängigen konfliktanalytischen Ansätze unter dem Grundsatzproblem der »Destabilisierung von Regimen« zusammenfasst: »Parallel zur gesellschaftlichen Neuordnung in Osteuropa kam es zu offenen Ausbrüchen bereits latent vorhandener nationaler, sozialer, ethnischer und religiöser Konflikte in Ländern der Dritten Welt« (BMZ 1993: 44).

Entlang dieses neuen Bedrohungsbildes engagiert sich die deutsche Politik schon sehr früh an der internationalen Debatte um die Frage eines erweiterten Sicherheitsbegriffes. Im September 1993 veranstaltet der Bundestag gemeinsam mit der Society for International Development eine zweitätige europäische Parlamentarier-Konferenz zum Begriff der Human Security, also noch vor dem Erscheinen des UNDP-Reports von 1994, der die internationale Debatte dazu anstößt. An diesem Beispiel zeigt sich die überraschend große Bereitschaft der beiden von Helmut

85 Praktisch wird diese Konditionalität allerdings nicht durchgehalten, insbesondere wenn handfeste wirtschaftliche Interessen im Spiel sind: »[D]ie Anfang der neunziger Jahre konzipierte und eingeleitete Politik der Bindung von Entwicklungshilfe an bestimmte politische Standards wird zumindest in Asien - inzwischen weitgehend überlagert vom Schielen auf die Auftragsbücher deutscher Firmen« (Maull 1999: 184). 
Kohl geführten Regierungen in den 1990er Jahren, neue außen- und sicherheitspolitische Profilierungsmöglichkeiten zu finden, die bei weitgehender Vermeidung der militärischen Komponente dennoch internationale Einflussmöglichkeit garantieren.

In der Folge wird das Thema vom BMZ aufgegriffen und auf Praxisrelevanz getrimmt. Entwicklungszusammenarbeit soll von nun an für den Wiederaufbau nach Bürgerkriegen Verantwortung übernehmen, über die Forcierung der wirtschaftlichen Entwicklung krisenpräventiv wirken, aber auch schon erste spezifische Maßnahmen wie die Demobilisierung und Reintegration von Kombattant/innen, sowie den Ausbau des Rechtswesens und der politischen Partizipation in der praktischen Umsetzung ausloten (BMZ 1995: 26). Carl-Dieter Spranger liefert dazu eindeutige Stellungnahmen, worin er beispielsweise bereits das $\gg \mathrm{Zu}-$ sammenwirken von Entwicklungs-, Außen- und Sicherheitspolitik « (Spranger 1996: 2), das unter dem Schlagwort »Kohärenz« zu einem der entwicklungspolitischen Generalthemen des folgenden Jahrzehnts werden sollte, als dafür wesentliche Voraussetzung hervorhebt. Auf der anderen Seite wird im ersten explizit auf die »Krisenvorbeugung « bezogenen Policy-Dokument des BMZ die Einrichtung neuer Instrumente und Institutionen zunächst ausgeschlossen (BMZ 1997: 9).

So ist die Entstehung des Bereichs »Krisenprävention und Friedensentwicklung«, wie der sicherheitsrelevante Sektor der deutschen Entwicklungszusammenarbeit mittlerweile offiziell genannt wird, letztlich doch unmittelbar mit dem Regierungswechsel vom 27. Oktober 1998 verknüpft. Im ersten rot-grünen Kabinett unter der Kanzlerschaft von Gerhard Schröder wird die Leitung des BMZ von der dem linken SPDFlügel zuzuordnenden Heidemarie Wieczorek-Zeul übernommen. Ihr werden die Grünpolitikerin Uschi Eid sowie der aus dem Ministerium selbst stammende Sozialdemokrat Erich Stather als Staatssekretär/innen zur Seite gestellt. In der Koalitionsvereinbarung von 1998 wird Entwicklungspolitik erstmals als "globale Strukturpolitik« definiert, wobei die Bedeutung dieses Begriffes zunächst noch unpräzise schwammig bleibt und sich von der sicherheitspolitischen Interpretation entwicklungspolitischer Maßnahmen der Kohl-Ära nicht unterscheidet.

Dennoch wird dieses Bekenntnis zu "globaler Strukturpolitik«, speziell wenn es im Sinne einer aktiven Gestaltung von Global Governance als Spielart globaler Gerechtigkeitspolitik interpretiert wird, von den meisten Kommentator/innen als Bruch mit den bisher gültigen Grundsätzen gesehen: »Entwicklungspolitik steht nach 40 Jahren theoretisch vor ihrer umfassendsten Reform « (Klemp 2001: 13). Speziell der starke friedenspolitische Sektor der Zivilgesellschaft, der im Unterschied zu Ökologie- und Frauenbewegung bis zu diesem Zeitpunkt kaum in die 
bilateralen entwicklungspolitischen Strukturen eingebunden war, wünscht sich in diesem Kontext eine Umorientierung der bisher allgemein gehaltenen Interpretation von Krisenprävention hin zur Ausarbeitung und Umsetzung spezifischer interventionistischer Maßnahmen.

$\mathrm{Ob}$ mit der neuen Bundesregierung wirklich eine Zeitenwende bei der deutschen Entwicklungspolitik eingeleitet wird, lässt sich anhand einiger quantitativer Daten klären. Die erste erstaunliche Erkenntnis dabei ist, dass die deutschen ODA-Gelder (wertbereinigt) ein Jahr nach dem Regierungswechsel leicht anzusteigen beginnen, allerdings nicht annähernd das Niveau von Anfang der 1990er Jahre erreichen (siehe Abbildung $15^{86}$ ).

Abbildung 15: Historische Entwicklung der deutschen ODA-Leistungen (in 2005-US\$)

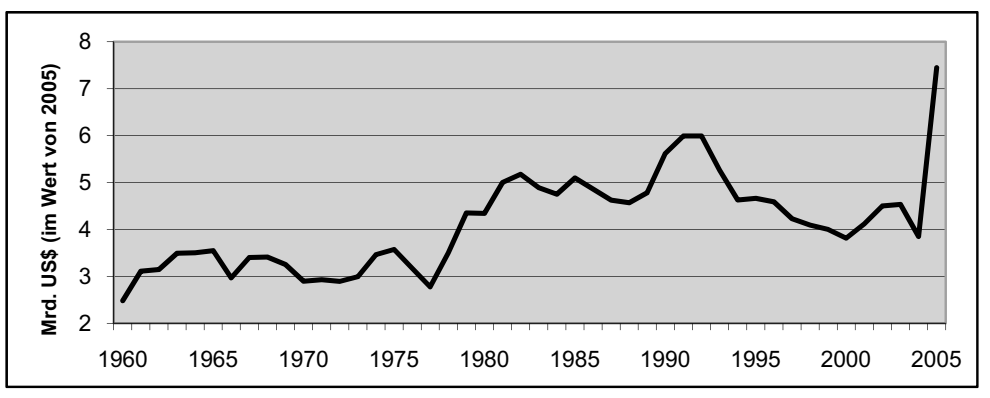

Abbildung 16: Deutsche ODA-Aufwendungen für DAC-Code 150

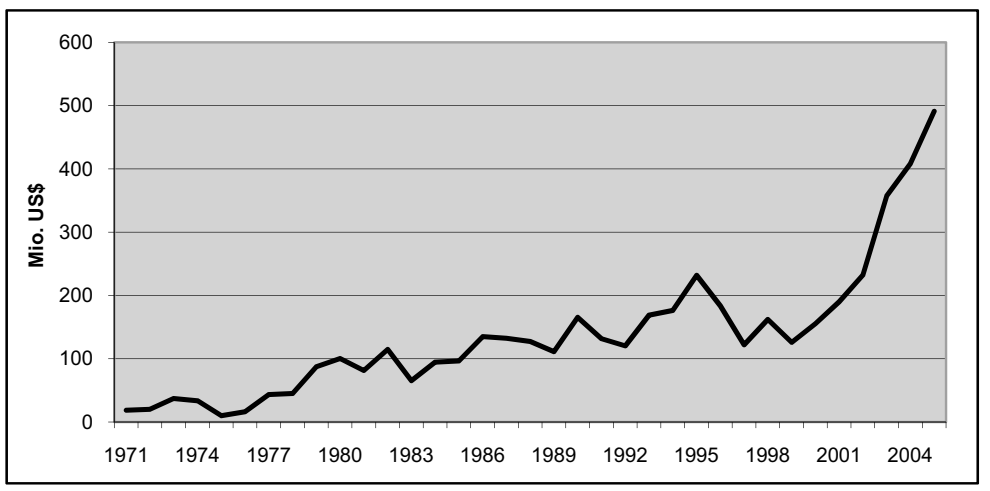

86 Der extreme Anstieg von 2004 auf 2005, nahezu eine Verdopplung des absoluten ODA-Volumens, erklärt sich faktisch zur Gänze aus Schuldenstreichungen für den Irak und Nigeria. 
Um die Bedeutung der inhaltlichen Neuorientierung in der praktischen Umsetzung zu erfassen, empfiehlt sich ein Blick auf die Entwicklung der ODA-Ausgaben, die für Tätigkeiten im Rahmen des DAC-Codes 150 (Governance und Zivilgesellschaft) gewidmet sind (siehe Abbildung 16). Tatsächlich ist hier ein massiver Anstieg zu beobachten, der mit Ende der 1990er Jahre einsetzt. Daraus ist zu schließen, dass sich die eingesetzten Interventionsmethoden nach dem Regierungswechsel tatsächlich verschieben.

Abbildung 17: Deutscher Anteil an den Gesamtaufwendungen der DACMitgliedsstaaten für Code 150

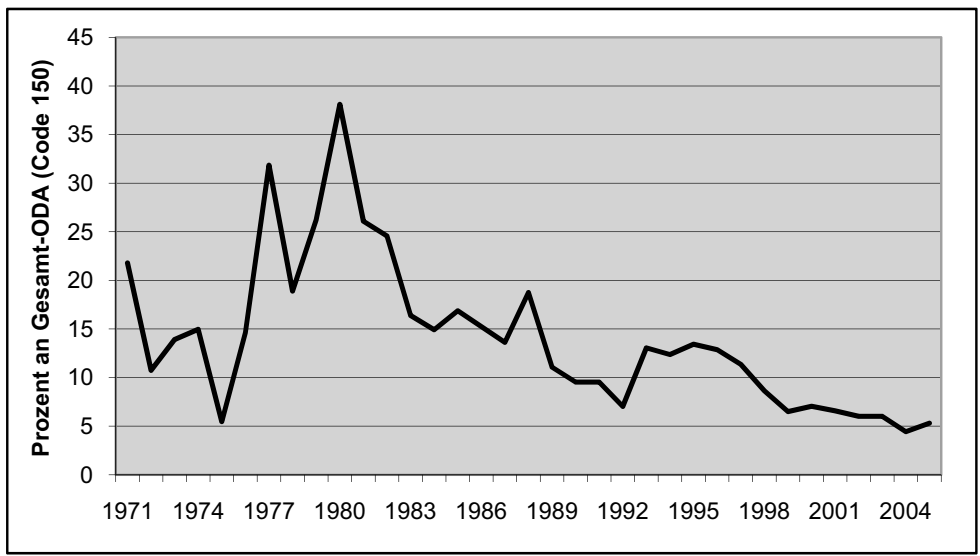

Interessant ist in diesem Zusammenhang allerdings auch, die deutschen Ausgaben im Rahmen des Codes 150 ins Verhältnis zu den internationalen Trends zu setzen (siehe Abbildung 17). Hier ist wiederum festzustellen, dass der deutsche Anteil an den Gesamtausgaben nach dem Regierungswechsel nicht etwa ansteigt, sondern kontinuierlich zurückgeht. Dies erklärt sich natürlich zum einen aus dem massiven Anstieg der Summen, die von den USA in diesem Bereich investiert werden. Zum anderen demonstriert es aber auch, dass der bemerkenswerte Anstieg in den deutschen Aufwendungen auf internationaler Ebene keine Besonderheit ist, sondern eine generelle Tendenz widerspiegelt. Die entwicklungspolitische Neuausrichtung, und hier speziell die Annahme neuer, speziell auf politische Prozesse abzielender Interventionsmethoden, ist also ein Phänomen, das zwar im deutschen Kontext eindeutig mit dem Regierungswechsel in Verbindung gesetzt werden kann und damit zum Durchbruch gelangt, sich aber aller Wahrscheinlichkeit nach nicht daraus erklärt, sondern hauptsächlich von internationalen Trends bestimmt ist. 
In jedem Fall nimmt der ohnehin durch die noch frischen Erfahrungen von Ruanda und Somalia sowie den gerade laufenden Konflikt in Rest-Jugoslawien erschütterte entwicklungspolitische Sektor in der Folge die bereits laufende internationale Diskussion zur Krisenprävention als Aufgabe der Entwicklungszusammenarbeit auf. So definiert die neue Ministerin Wieczorek-Zeul am Beginn ihrer Amtszeit drei Arbeitsschwerpunkte: Neben dem schon im Koalitionsvertrag festgeschriebenen Verständnis von Entwicklungspolitik als globaler Struktur- und als Friedenspolitik, die sich beide nicht substanziell vom Verständnis der Vorgängerregierung unterscheiden, führt sie »Krisenprävention« ins Feld, was zwar, wie sie selbst betont ${ }^{87}$, auch im Rahmen des BMZ keine bahnbrechende Neuerfindung ist, dennoch aber einer Abkehr von der bisherigen Politik gleichkommt (vgl. Wieczorek-Zeul 1999a). Als Symbol dieser Neuorientierung verweist die grüne Staatssekretärin Uschi Eid (1999) auf die Aufnahme des BMZ in den Bundessicherheitsrat: »Dies spiegelt symbolisch den neuen und erweiterten Sicherheitsbegriff der Bundesregierung wider.«

Die Forcierung neuer Methoden der entwicklungspolitischen Intervention vor dem Hintergrund eines jetzt auch formell stärker verankerten erweiterten Sicherheitsbegriffes rückt in der Folge immer mehr in den Mittelpunkt. Im Mai 1999 spricht Wieczorek-Zeul in einem Vortrag in der Bundesakademie für Sicherheitspolitik davon, dass eine der Folgen der Tragödie im Kosovo die Überwindung eines engen Sicherheitsbegriffes sein müsse. Die deutsche Entwicklungspolitik könne in der praktischen Umsetzung dieses Prozesses zwei zentrale Aufgaben erfüllen: »dismantle the structural causes of armed conflict«, sowie »establish mechanisms for peaceful resolution of conflict« (Wieczorek-Zeul 1999b: 10). Dies sei, so Wieczorek-Zeul, einerseits durch die klassische entwicklungspolitische Herausforderung der Armutsbekämpfung zu erreichen, andererseits sei aber auch ein eindeutig politischer Fokus notwendig, vor allem im Bereich der Demokratieförderung: »Therefore democracy must be assessed and strengthened in a historical, cultural and social context« (ebd.: 11).

Diese Forcierung der Demokratieförderung findet sich auf praktischer Ebene allerdings nur sehr eingeschränkt. In einem Indikatorenkatalog, der 1999 zur Stärkung der Frühwarnkapazitäten des BMZ von Angelika Spelten $(1999,2000)$ vorgelegt wird, ist - im krassen Unterschied zu vergleichbaren US-amerikanischen Dokumenten um die Jahr-

87 »Es gibt bereits verschiedene Ansätze im BMZ zur Krisenprävention. Diese wollen wir ausbauen und stärker in bestehende und zukünftige Entwicklungsvorhaben einbeziehen« (Wieczorek-Zeul 1999a). 
tausendwende - ein Indikator »Demokratie« nicht auszumachen, sondern findet in dem Multiple-Choice-Fragenkatalog, kodifiziert über die staatliche Konfliktlösungskompetenz ${ }^{88}$, nur indirekt Erwähnung. Diese Tendenz zeigt sich auch in anderen praxisrelevanten Papieren zur Jahrtausendwende. So bezieht sich das Policy-Paper »Effektive Krisenprävention«, das die SPD-nahe, von Willy Brandt gegründete Stiftung Entwicklung und Frieden vorlegt ${ }^{89}$, viel stärker auf Konzepte wie Friedensallianzen und zivilgesellschaftliche Beteiligung an Prozessen konstruktiver Konfliktaustragung, was regional möglichst flächendeckend zu erfolgen habe (vgl. Debiel et al. 1999: 11f.). Auf staatlicher Seite wird die Bedeutung von Rechtsstaatlichkeit und Good Governance betont, Demokratie im Sinne der Forcierung eines durch staatliche Rahmensetzungen zu gewährleistenden politischen Wettbewerbs jedoch nirgends erwähnt.

Auch in der ersten Querschnittsevaluierung des deutschen Krisenpräventionsbereiches, die das BMZ 1999 in Auftrag gibt, findet sich die Demokratie-Komponente nur randständig wider, was zeigt, dass sie auch in der praktischen Umsetzung im bilateralen Bereich keine wesentliche Rolle spielt. Dies begründet die Studie mit dem kurz- wie mittelfristig hohen Risiko einer konfliktverschärfenden Wirkung von Demokratieförderung (Klingebiel 1999: 29). In einem langfristigen Kontext wird der Ansatz begrüßt, jedoch primär als Aufgabenstellung für die entwicklungspolitische Praxis der parteinahen Stiftungen.

Diese parteinahen Stiftungen stellen, wie auch von anderen bilateralen Akteuren immer wieder betont wird, eine deutsche Eigenart dar: »Kein anderes Geberland verfügt über solch eigentümliche, parteinahe und größtenteils aus öffentlichen Mitteln finanzierte Subunternehmen der Politikszene« (Nuscheler 1996: 406; vgl. auch Fuster 1998: 314). Die Stiftungen versetzen die deutsche Entwicklungspolitik in die besondere Rolle, neben den USA der einzige Akteur zu sein, der - trotz der

88 »Sind die staatlichen Institutionen willens und fähig, die gesellschaftlichen Konflikte zu verhandeln und Lösungen herbeizuführen?« (Spelten 2000).

89 Das Papier wurde von vier Personen aus der »anwendungsorientierten Friedensforschung« erarbeitet, die für die Gestaltung der deutschen Strategien und Konzepte auch in den Folgejahren von großer Bedeutung sein werden: Norbert Ropers und Martina Fischer von der Berliner BerghofStiftung, sowie Tobias Debiel und Volker Matthies, die beide primär im universitären Bereich aktiv sind. Sie repräsentieren die Nachfolgegeneration der Gründerväter des deutschen Zweigs jener »anwendungsorientierten Friedensforschung «, Ernst-Otto Czempiel und Dieter Senghaas. Der stark idealistische Gehalt ihres Ansatzes wird schon im Eröffnungssatz ihres Policy-Papers deutlich: »Krisenprävention ist ein Gebot vernünftiger Politik« (vgl. Debiel et al. 1999: 2). 
offiziellen Zurückhaltung im Bereich der Demokratieförderung - auch direkte Parteienförderung in peripheren Ländern durchführt (vgl. Youngs 2003: 136). Diese funktioniert größtenteils über die (programmatische, propagandistische, strategische) Beratung von Partnerparteien. $^{90}$ Allerdings ist die Praxis der Stiftungen nicht als bilaterale Zusammenarbeit im engeren Sinne zu klassifizieren, sie entscheiden entlang klarer parteipolitischer Kriterien über ihre Interventionsformen und ihre regionalen wie lokalen Partner. Es wäre daher auch verfehlt, ihre Tätigkeit mit einem demokratiepolitischen Schwerpunkt der deutschen Entwicklungspolitik zu verwechseln.

Auf bilateraler Ebene ist es vor allem ein spezifisches Instrument, dessen Einrichtung die Trendwende in der entwicklungspolitischen Ausrichtung wie kein anderes markiert (vgl. Eid 1999): der Zivile Friedensdienst (ZFD). Wurde er von »der frühere[n] Leitung des Hauses« noch »vehement abgelehnt« (ebd.), wird er nun zum Symbol eines neu entdeckten Aktivismus der deutschen Friedenspolitik. Dieser Symbolgehalt erklärt sich aus der Geschichte der Idee des ZFD. Sie entstammt dem zivilgesellschaftlichen Sektor und entsteht in Grundzügen bereits Anfang der 1990er Jahre. Unter dem Eindruck des Jugoslawien-Krieges bündeln sich die verschiedenen an seiner Einrichtung interessierten Organisationen und Initiativen im »Forum Ziviler Friedensdienst« (vgl. zur historischen Entwicklung Uwe Trittmann in Evers 2000). Schon Mitte der 1990er Jahre beginnen intensivere Kontakte mit dem politischen Bereich, die sich nach der Ablehnung durch die Regierungsparteien vorwiegend auf die damaligen Oppositionsparteien SPD und Grüne konzentrieren. Nach dem Regierungswechsel wird der ZFD auf direkte Initiative von Heidemarie Wieczorek-Zeul ins Regierungsprogramm übernommen und in der Folge umgesetzt.

Für die überraschend große Bedeutung, die einem relativ schmal dimensionierten Element wie dem ZFD in der Selbstdarstellung der deutschen Entwicklungshilfe zukommt (vgl. etwa BMZ 2003b: 102f.), zeichnen vor allem zwei Faktoren verantwortlich. Einerseits sorgt die basisnahe Implementation des ZFD für eine gute öffentliche Nachvollziehbarkeit, die eine uneigennützige, von Eigeninteressen freie, schlichtweg gute Form von friedenspolitischer Intervention suggeriert. Diese Praxis resultiert auch aus dem Selbstverständnis, dass der »ZFD [...] kein Instrument offizieller Außen- und Verteidigungspolitik« sei (vgl. Helga Tempel in Evers 2000: 24). Vielmehr ginge es darum, als »Gast

90 Ein Beispiel aus den Philippinen ist die Förderung der vom Ex-NPAMitglied Joel Rocamora geleiteten sozialdemokratischen Parteiliste Akbayan durch die Friedrich-Ebert-Stiftung. 
aus dem Ausland « (ebd.: 29) unter Einbringung spezieller Kompetenzen unterstützend tätig zu werden. Die festgelegten Aufgabenfelder kreisen um die drei Ansätze (1) Stärkung von Friedenspotenzialen; (2) Vermittlung bei Konflikten; (3) Beiträge zur Versöhnung und Wiederaufbau (vgl. BMZ 2004c: 5). Gesteuert werden die Einsätze über das »Konsortium Ziviler Friedensdienst $~{ }^{91}$, wobei das BMZ gemeinsam mit dem Auswärtigen Amt über die Auftragsvergabe entscheidet.

Erfolgreiche Antragssteller/innen durchlaufen vor ihrem Einsatz ein nach ihren spezifischen Bedürfnissen zusammengestelltes umfangreiches Ausbildungsprogramm, bevor der zeitlich befristete Feldaufenthalt beginnt. Der Ansatz im Feld wird gemeinsam mit lokalen/regionalen Partnerorganisationen entwickelt, wobei den ZFDler/innen bei der Wahl ihrer Aufgabengebiete weitgehend freie Hand gelassen wird. So bietet das Instrument eine Vielzahl von Möglichkeiten, auf lokaler Ebene flexibel tätig zu werden, und ist in der Tat insofern bemerkenswert, als es sich einer direkten Steuerbarkeit weitestgehend entzieht.

Andererseits ist der ZFD das wahrscheinlich markanteste Symbol für die mit dem Regierungswechsel massiv einsetzende Integration weiter Teile der friedenspolitischen Aktivist/innen in die offizielle Politik. Nicht ohne Grund sieht ihn Wieczorek-Zeul »[a]ls Gemeinschaftswerk staatlicher und nichtstaatlicher Träger der Friedens- und Entwicklungsarbeit« und als »beispielhaft für das Zusammenwirken verschiedener Akteure in diesem Bereich « (Wieczorek-Zeul in Evers 2000: 10). Die Protagonist/innen selbst geben sich alle Mühe, ihn nicht als bilateralstaatliche, sondern als »zivilgesellschaftliche Aufgabe« zu verstehen, die die zusätzliche Bereicherung aufweise, dass sie »subsidiär in pluraler Trägerschaft mit staatlicher Unterstützung wahrgenommen« werde (Tempel in Evers 2000: 23).

Diese Aussagen machen das beiderseitige Interesse an der Integration der Friedensbewegung in die offizielle Politik deutlich, ein Prozess, der von der Übernahme von politischen Argumentationsmustern, über die Integration einzelner Personen, die verstärkte Finanzierung des friedenspolitischen zivilgesellschaftlichen Sektors bis hin zu einem ausgeprägten Symbolismus durchgängig sichtbar wird. So findet sich auf der zweiten Seite der für die breite Öffentlichkeit gedachten BMZInformationsbroschüre »Recht - Demokratie - Frieden« ganzseitig das Bild einer jungen, offenbar europäischen Friedensaktivistin, die allem

91 Mitglieder des Konsortiums Ziviler Friedensdienst sind die so genannten »anerkannten Entwicklungsdienste«. Es sind dies die Arbeitsgemeinschaft für Entwicklungshilfe (AGEH), Christliche Fachkräfte International (CFI), der Deutsche Entwicklungsdienst (DED), der Weltfriedensdienst (WFD), der Evangelische Entwicklungsdienst (EED), sowie EIRENE. 
Anschein nach gerade im Rahmen eines offiziellen politischen Treffens für Frieden protestiert (siehe Abbildung 18), womit sie im Übrigen auch den Widerstand gegen verschiedene sicherheitspolitische Entscheide der rot-grünen Bundesregierung symbolisiert. Das BMZ, und mit ihm die auf Krisenprävention ausgelegte Entwicklungspolitik, inszeniert sich damit als innerinstitutionelle Opposition zu außenpolitischen Entscheidungen der Bundesrepublik, die nicht zuletzt im Jahr 1999 erstmals seit dem Zweiten Weltkrieg in einem Kampfeinsatz deutscher Soldaten, noch dazu ohne entsprechendes UNO-Mandat, mündet. Während in Denkschriften und Dialogforen über die Möglichkeiten einer verstärkten Kooperation mit der Bundeswehr nachgedacht wird (vgl. Development Policy Forum 2004; Klingebiel/Roehder 2004), spielt das BMZ zugleich den Kitt, der die Reste der aktiven Friedensbewegung nicht nur mit der zivilen, sondern mit der umfassenden deutschen Sicherheitspolitik verbinden soll.

Abbildung 18: Friedensaktivistin in BMZ-Publikation

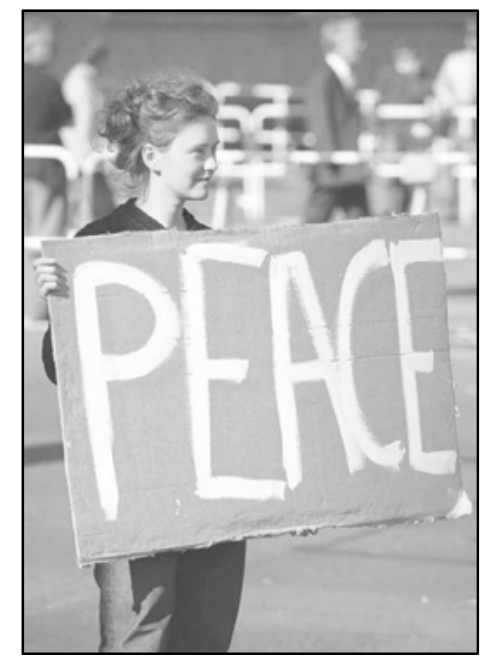

Quelle: BMZ 2003b: 2

Diese Verbindung rückt tatsächlich schnell in den Mittelpunkt. Im Jahr 2000 beschließt der Bundessicherheitsrat, ein geheim tagender Ausschuss der Bundesregierung, dessen Bedeutung nach dem Regierungswechsel nicht nur durch die Neuaufnahme des bislang nicht inkludierten BMZ signifikant aufgewertet wird, ein kurzes, aber prägnantes so genanntes »Gesamtkonzept« zum Thema der Krisenprävention und Kon- 
fliktbeilegung. Dieses Konzept, das als ressortübergreifendes Orientierungspapier gedacht ist, wird im Unterschied $\mathrm{zu}$ den meisten anderen Beschlüssen des Bundessicherheitsrates auch veröffentlicht (Bundesregierung 2000). ${ }^{92}$ So wird nicht nur ein erneutes Bekenntnis $\mathrm{zu}$ einem erweiterten Sicherheitsbegriff abgegeben, »der politische, ökonomische, ökologische und soziale Stabilität umfasst«, sondern auch explizit eine Rolle für die Entwicklungspolitik in der Implementation der Strategie formuliert. »Im Rahmen der Gesamtstrategie ist es die Aufgabe der Entwicklungspolitik, in den betroffenen Partnerländern durch Verbesserung der wirtschaftlichen, sozialen, ökologischen und politischen Verhältnisse zur Verhinderung und zum Abbau struktureller Ursachen von Konflikten sowie zur Förderung von Mechanismen gewaltfreier Konfliktbearbeitung beizutragen« (ebd.). Diese Absichtserklärung hat praktische Konsequenzen, zunächst primär auf institutioneller Ebene. Als erster Schritt wird eine Pilotstudie in Auftrag gegeben, die sich der Aufarbeitung der zu diesem Zeitpunkt neben Großbritannien vor allem von den skandinavischen Ländern und den Niederlanden dominierten internationalen Diskussion widmet (Mehler/Ribaux 2000). Danach wird im Juli 2001 zur Auslotung der konkreten Interventionsmöglichkeiten bei der GTZ ein so genanntes Sektorvorhaben ${ }^{93}$ Krisenprävention eingerichtet. Ende August 2001 (noch vor 9/11) wird unter der Ägide des BMZ von sieben entwicklungspolitischen Einrichtungen die Gruppe Friedensentwicklung, FriEnt, installiert, die die den Austausch zwischen staatlichen, kirchlichen und zivilgesellschaftlichen Akteuren auf nationaler Ebene regeln und die inhaltliche Arbeit koordiniert voranbringen soll.

Aber auch in der Programmumsetzung gibt es relevante Konsequenzen. Schon im Jahr 2000 einigt sich Deutschland mit dem Partnerland Sri Lanka, das Thema Konflikttransformation und Peacebuilding zum übergreifenden Arbeitsschwerpunkt der bilateralen ODA zu machen. Weitere Länder, zunächst Kolumbien und Guatemala, ein wenig später dann Senegal und Burundi, folgen diesem Beispiel (vgl. Kievelitz et al.

92 Der Großteil der Aufgabenstellungen des Bundessicherheitsrates betrifft Bewertungen von als heikel eingestuften Waffenexporten, aber auch geheimdienstliche Fragen.

93 Sektorvorhaben sind ein Spezifikum der Zusammenarbeit von BMZ und GTZ. Im Gegensatz zur normalen Arbeitsweise der GTZ, die sich als unabhängiges Dienstleistungsunternehmen versteht, sind Sektorvorhaben eine unmittelbare Kooperation auf Anforderung des BMZ. Sie werden zu Sektoren, denen zukünftig steigendes Potenzial zugeschrieben wird, auf direkte Anweisung des BMZ bei der GTZ zeitlich befristet eingerichtet und dienen einerseits der inhaltlichen Erkundung des Themas in Bezug auf Analyse und Möglichkeiten der Implementation, andererseits aber auch der innerinstitutionellen Verankerung dieser Thematik. 
2003: 30). Eine solche Einstufung hat für die Länderprogramme einschneidende Konsequenzen: Das Projektportfolio wird zumeist komplett umgestellt, neue Projekte angestoßen, andere, zum Teil auch als erfolgreich wahrgenommene Projekte werden wiederum eingestellt. In Kolumbien trifft diese Maßnahme nicht nur beim nationalen Counterpart, sondern auch beim GTZ-Personal vor Ort zunächst auf wenig Gegenliebe, wird nach einer harten Einarbeitungsphase dann aber durchgängig begrüßt. Unzweifelhaft hat diese praktische Radikalität in der Umstellung einzelner Programme auch Auswirkungen auf den Bereich der internen Bewusstseinsentwicklung, die im Bereich der Krisenprävention ohnehin als Manko wahrgenommen wird.

Im Aktionsprogramm 2015, dem am 4. April 2001 von der Bundesregierung veröffentlichten Umsetzungsprogramm für die MDGs, wird Konfliktbeilegung und Friedenskonsolidierung als ein »Schwerpunkt« der Entwicklungszusammenarbeit festgeschrieben, neben ihrer Kernkompetenz Armutsbekämpfung, die zu diesem Zweck allerdings ebenfalls »unerlässlich« sei (vgl. Bundesregierung 2001: 7). Mit der Betonung der »politischen Dimension« von Entwicklungspolitik (ebd.: 12) legt das Aktionsprogramm eine Verbindung der für Deutschland traditionell wichtigen multilateralen Orientierung und der neuen entwicklungspolitischen Schwerpunktlegung, die in den MDGs selbst so nicht vorgesehen ist.

Die Anschläge vom September 2001 wirken auf den Prozess wie ein Katalysator. Als Folge von 9/11 werden die institutionellen Vorarbeiten, die sich in der praktischen Umsetzung bis zu diesem Zeitpunkt auf ausgewählte Schwerpunktländer ${ }^{94}$ konzentriert hatten, in einer überregionalen Kampagne zusammengefasst. Im Rahmen des Ende 2001 beschlossenen Anti-Terrorismus-Pakets der Bundesregierung (ATP), das sich mit einem Gesamtvolumen von $3 \mathrm{Mrd}$. $€$ hauptsächlich auf innenpolitische Sicherheitsmaßnahmen konzentriert, wird ein Budget von 30,2 Mio. $€$ für entwicklungspolitische Sondermaßnahmen zur Verfügung gestellt (vgl. Paffenholz/Brede 2004: 1; zu den vorangegangenen Überlegungen BMZ 2001b). Zwar nimmt sich diese Summe im Verhältnis zu den 1,5 Mrd. $€$, die allein die Bundeswehr im Zuge des ATP zur Verfügung gestellt bekommt, verschwindend gering aus, dennoch bereitet sie in der Praxis die Basis für eine bemerkenswerte, auch im europäischen Kontext hervorstechenden Initiative: Entwicklungspolitik wird, zumindest im

94 Neben den erwähnten fünf Schwerpunktländern Sri Lanka, Kolumbien, Guatemala, Senegal und Burundi waren dies Kambodscha, Algerien, Uganda und Malawi, sowie in einem zweiten Schritt Nepal, der Tschad, die Balkanregion und der Kaukasus. 
Rahmen eines Versuchsballons, erstmals explizit als Mittel der präventiven Terrorismusbekämpfung eingesetzt.

Das Geld wird in zwei Tranchen vor allem mittels Fonds global in Krisengebieten in Spezialmaßnahmen umgesetzt, was eine Initialzündung für das Thema, insbesondere in den Länderbüros und auf Implementationsebene, zur Folge hat (Paffenholz und Brede sprechen von einem »Kick«, vgl. ebd.: 29). Insgesamt werden, wie es heißt, »weltweit 34 regionale und fünf überregionale Sondermaßnahmen zur Krisenprävention und Friedensentwicklung als Beitrag zur strukturellen Terrorismusprävention durchgeführt« (ebd.: 1). Die Palette der eingesetzten Maßnahmen ist allerdings ebenso breit gefächert wie die Wahl der Zielregionen weit gestreut. Dies lässt vermuten, dass es sich letzten Endes weniger um ein strategisch angelegtes Programm als viel mehr um eine Mischung zwischen politischem Aktionismus und einem vergleichsweise groß dimensionierten Versuchsballon für die Anwendung des Krisenpräventionsprogramms handelt.

Die generell positive Evaluierung, die den ATP-Maßnahmen zufällt, lässt die strukturellen Schwächen denn auch tendenziell unter den Tisch fallen. Dabei sticht insbesondere ins Auge, dass die von BMZ und GTZ immer wieder geäußerte Einschätzung, bei entwicklungspolitischen Maßnahmen im Kontext der Terrorismusprävention könne es sich nur um mittelbar wirksame Schritte handeln, die auf strukturelle Faktoren einwirken würden und somit längerfristig zu konzipieren seien, von politischer Seite nicht gewürdigt wird.

Vielmehr stellt das ATP dem entwicklungspolitischen Sektor kurzfristig verhältnismäßig viel Geld zur Verfügung, beschränkt sich aber letzten Endes auf eine einmalige Aktion. So werden die Länderbüros in kurzer Abfolge zweimal vor vollendete Tatsachen gestellt. Zunächst müssen sie dafür sorgen, unerwartete Sondermittel in einem äußerst knappen Zeitrahmen sinnvoll entlang der Vorgaben zu verteilen ${ }^{95}$, dann stehen sie wiederum vor dem Problem, dass vorgesehene Follow-UpMaßnahmen durch die Einmaligkeit des Geldflusses im vorgesehenen Rahmen nicht realisierbar sind. ${ }^{96}$ Letztendlich stellt die Evaluierung fest, dass die besondere Fokussierung auf Terrorismus keinen wesentlichen Gewinn für die thematische Debatte darstellt: »Weiterhin wurde klar,

95 Das Länderbüro auf den Philippinen berichtet in diesem Zusammenhang von vehementen Schwierigkeiten, im für die Mittelvergabe identifizierten Zielgebiet (Mindanao) überhaupt genügend förderungswürdige Projekte ausfindig zu machen.

96 Die Länderbüros in Kolumbien und den Philippinen gehen zu diesem Zeitpunkt etwa davon aus, dass es entsprechende Folgemittel geben würde. Diese Annahme erweist sich als falsch. 
dass der Diskurs über EZ im Kontext terrorgefährdeter Länder keine grundlegend neue Dimension aufweist, die nicht bereits durch Erkenntnisse der Debatte um Krisenprävention, Konfliktbearbeitung und Friedensentwicklung in der EZ abgedeckt wird« (ebd.: 42). Dies relativiert sich allerdings insoweit, als die angestoßene Diskussion für den Bereich Entwicklungspolitik im interministeriellen Wettstreit um außenpolitische Verantwortlichkeiten sehr wohl hilfreich ist.

Dies zeigt sich in weiterer Folge in der zunehmenden Einbindung von entwicklungspolitischen Komponenten in die militärischen Einsätze der Bundeswehr. Wie auch für Großbritannien ist dabei der AfghanistanKrieg das erste Schlüsselereignis dieser Art, wo eine zivil-militärische Kooperation im Rahmen des Provincial Reconstruction Teams (PRT) in Kunduz praktiziert wird. Unter Federführung des BMZ werden Mitarbeiter/innen von GTZ, der Kreditanstalt für Wiederaufbau (KfW) und dem Deutscher Entwicklungsdienst (DED) in das militärisch geführte PRT integriert, ebenso wie zivile Polizeiberater des Bundesministeriums des Inneren (vgl. Hett 2005: 14ff.).

Obwohl die PRTs derzeit mitunter als Vorzeigemodell für zukünftige Entwicklungen präsentiert werden, verläuft die konkrete Umsetzung im Feld schwierig. Während die Bundeswehr für die konsequente Umsetzung der so genannten CIMIC-Operation unter ihrer Führung (CivilMilitary Cooperation) plädiert und auf die Entsendung eigener ziviler Trupps drängt, lehnt dies das BMZ mit der Begründung der notwendigen konsequenten Trennung von zivilem und militärischem Beitrag ab (vgl. ebd.: 18). Die implizit dahinter stehende Befürchtung, dass Entwicklungspolitik ansonsten vollkommen vom durch das budgetär unverhältnismäßig stärker ausgestattete Militär vereinnahmt und instrumentalisiert werden könnte (vgl. Klingebiel/Roehder 2004: 10ff.), kann im allgemein als frostig und friktionsreich charakterisierten Klima zwischen den verschiedenen an der Umsetzung beteiligten Ministerien nicht ausgeräumt werden.

Trotzdem bildet die Afghanistan-Erfahrung im Jahr 2004 die Basis für einen Aktionsplan der Bundesregierung, der unter dem Titel »Zivile Krisenprävention, Konfliktlösung und Friedenskonsolidierung« versucht, ein die verschiedenen Akteure einendes übergreifendes Konzept bereitzustellen und zivile Krisenprävention als »Querschnittsaufgabe« $\mathrm{zu}$ verankern (Bundesregierung 2004: 59). Besonders angesprochen werden dabei das Auswärtige Amt, das Verteidigungsministerium und das BMZ. Mitarbeiter/innen aus den jeweiligen Ministerien werden zu Themenbeauftragten ernannt und in einem »Ressortkreis Zivile Krisenprävention« zusammengefasst, das unter Federführung des Beauftragten 
für zivile Krisenprävention im Auswärtigen Amt als Steuerungsgruppe fungieren soll (vgl. Debiel 2004: 274, 276).

Zugleich wird »Friedensentwicklung und Krisenprävention« als eine der elf Prioritäten der deutschen Entwicklungspolitik verankert. Das Sektorvorhaben Krisenprävention wird zunächst bis 2006 verlängert und danach als »Kompetenzfeld « in die reguläre Angebotspalette der GTZ integriert. Jedes der vier großen Regionalgebiete in der GTZ verfügt zudem über eine/n Themensprecher/in, der/die für die Verankerung der Querschnittsmaterie Konfliktprävention in den Länderprogrammen verantwortlich zeichnet und zugleich den Rückfluss konkreter Erfahrungen aus der Implementationspraxis in das Kompetenzfeld organisieren soll.

Auf Initiative des BMZ werden noch weitere Sektorvorhaben in das Kompetenzfeld integriert, die sich mit spezifischen Problemstellungen beschäftigen. Neben dem Sektorvorhaben Kleinwaffenkontrolle ist in diesem Zusammenhang vor allem das Vorhaben zur Reform des Sicherheitssektors (SSR) relevant, arbeitet es doch an einem der angestrebten inhaltlichen Schwerpunktsetzungen des BMZ. Dazu wird eine Person aus dem Bundesministerium für Verteidigung beauftragt, die eine konkrete Verknüpfung verteidigungs- und entwicklungspolitischer Ansätze in dem sensiblen Bereich garantieren soll. ${ }^{97}$

Die Abwahl der rot-grünen Koalition scheint nichts an dem eingeschlagenen Weg zu ändern. Nach wie vor gilt, so der Koalitionsvertrag zwischen CDU/CSU und SPD, der umfassende Sicherheitsbegriff als Grundlage des Verständnisses internationaler Sicherheitspolitik, wobei Krisenprävention auch weiterhin eine Schlüsselrolle zukommen soll: »Dabei wollen wir insbesondere unsere Instrumentarien zur Krisenprävention und -reaktion ausbauen. Diese prioritäre Querschnittsaufgabe erfordert die Zusammenführung vorhandener finanzieller und personeller Ressourcen und zusätzliche Mittel« (Koalitionsvertrag zwischen CDU, CSU und SPD vom 11.11.2005, zitiert nach Bundesregierung 2006: 7). Dass Heidemarie Wieczorek-Zeul als Ministerin im BMZ mit der Weiterführung des Amtes beauftragt wurde, garantiert nicht nur Kontinuität, sondern wahrscheinlich auch eine Stärkung des BMZ im Kabinett. Wieczorek-Zeul ist nach mittlerweile zehn Jahren im Amt nicht nur die längstdienende Leiterin des BMZ in dessen Geschichte, sondern auch Deutschlands derzeit dienstälteste Ministerin.

97 Die Verbindung zwischen entwicklungspolitischem und militärischem Sektor hat sich im Zuge der fortschreitenden Institutionalisierung des Krisenpräventions-Bereiches noch verstärkt. Mittlerweile ist ein Offizier im Generalstabsdienst zur Arbeit an den militärischen Aspekten des Aktionsplans Zivile Krisenprävention abgestellt und dem BMZ beigeordnet (vgl. Bundesregierung 2006: 69). 


\section{Strategien und Programme}

Trotz oder vielleicht auch wegen der mittlerweile relativ langen Zeit, die die Institutionen der deutschen Entwicklungshilfe bislang in konzeptionelle Arbeit investiert haben, wird das Resultat von der Gilde der den Gesamtprozess begleitenden »praxisorientierten « Friedensforscher/innen überwiegend kritisch beurteilt. »Deutschland tut sich im Bereich der Krisenprävention äußerst schwer mit einer thematischen und regionalen Prioritätensetzung. Hier und da sind inhaltliche Schwerpunkte zwar erkennbar [...]. Doch werden diese Akzente nicht schlüssig zusammengebunden« (Debiel 2004: 294).

Abbildung 19: Schlüsseldokumente der deutschen Entwicklungspolitik im Konfliktkontext

\begin{tabular}{|c|c|c|}
\hline $\begin{array}{l}\text { Politische Ebene } \\
\text { Grundsatz }\end{array}$ & $\begin{array}{c}\text { "Zivile Krisenprävention, Konfliktlösung und } \\
\text { Friedenskonsolidierung“ } \\
\text { Gesamtkonzept } \\
\text { (Die Bundesregierung 2000) }\end{array}$ & $\begin{array}{c}\text { Aktionsprogramm } 2015 \\
\text { Armut bekämpfen. Gemeinsam } \\
\text { handeln. } \\
\text { (Die Bundesregierung 2001) }\end{array}$ \\
\hline $\begin{array}{l}\text { Politische Ebene } \\
\text { Strategie }\end{array}$ & \multicolumn{2}{|c|}{$\begin{array}{c}\text { „Zivile Krisenprävention, Konfliktlösung und } \\
\text { Friedenskonsolidierung“ } \\
\text { Aktionsplan } \\
\text { (Die Bundesregierung 2004) }\end{array}$} \\
\hline $\begin{array}{l}\text { Policy-Ebene } 1 \\
\text { Strategie }\end{array}$ & \multicolumn{2}{|c|}{$\begin{array}{c}\text { Übersektorales Konzept zur Krisenprävention, Konfliktbearbeitung und } \\
\text { Friedensförderung in der deutschen Entwicklungszusammenarbeit } \\
\text { Eine Strategie zur Friedensentwicklung } \\
\text { (BMZ 2005) }\end{array}$} \\
\hline $\begin{array}{l}\text { Policy-Ebene } 1 \\
\text { Umsetzung }\end{array}$ & $\begin{array}{l}\text { Recht - Demokratie - Frieden } \\
\text { Politik für Entwicklung } \\
\text { (BMZ 2003) }\end{array}$ & $\begin{array}{c}\text { BMZ Spezial } \\
\text { Positionspapiere }\end{array}$ \\
\hline $\begin{array}{l}\text { Policy-Ebene 2 } \\
\text { Implementierung }\end{array}$ & $\begin{array}{l}\text { Leistungsangebot Friedensentwicklung } \\
\text { und Krisenprävention } \\
\text { (GTZ 2007) }\end{array}$ & $\begin{array}{l}\text { Leistungsangebot } \\
\text { Good Governance } \\
\text { (GTZ 2007) }\end{array}$ \\
\hline
\end{tabular}

Erstaunlich ist dieser Befund insofern, als die bestehenden konzeptionellen Dokumente, denen Verbindlichkeit zukommt, nicht nur relativ wenige an der Zahl, sondern auch in einer klar definierten Hierarchie angeordnet sind (siehe Abbildung 19). Den Leitdokumenten der politischen Ebene sind auf Policy-Ebene 1 ministerielle Aktionspläne nachgeordnet, die über die bestehenden Interventionsmöglichkeiten, die in den so genannten »Leistungsangeboten« der parastaatlichen Durchführungsorganisation GTZ zusammengefasst sind, in konkrete Arbeitspläne umgesetzt werden sollen. Die Krux der deutschen Situation liegt allerdings im Detail, denn nahezu keines dieser Schlüsseldokumente - und das ist ein bemerkenswerter Gegensatz zur US-amerikanischen Heran- 
gehens- und Arbeitsweise - kann sich der Formulierung eines präzisen Zielsetzungskataloges in Verbindung mit einem möglichst breiten Spektrum an Maßnahmen, die zu treffen seien, enthalten. Wie nicht anders zu erwarten sind daher weder die Zielkataloge noch die daraus abgeleiteten Maßnahmen der unterschiedlichen Konzeptpapiere zueinander kohärent und widersprechen sich zum Teil erheblich, was für die Implementation konsistenter Programme relevante Schwierigkeiten aufwirft. Für die Analyse der Rationalitäten des deutschen Herangehens wiederum ist dies kein wesentliches Problem: in all ihrer Widersprüchlichkeit und übertriebener Detailgenauigkeit ist ein durchgängiger Raster herauszulesen, der eine einigermaßen aussagekräftige Darstellung jener Grundlinien ermöglicht.

Das zentrale Dokument für den sicherheitspolitisch relevanten Bereich, für den sich in Deutschland der Begriff »Zivile Krisenprävention« eingebürgert hat, ist der im Frühjahr 2004 von der Bundesregierung veröffentlichte »Aktionsplan Zivile Krisenprävention, Konfliktlösung und Friedenskonsolidierung «, der das schon seit dem Jahr 2000 bestehende gleichnamige Gesamtkonzept - ein Prinzipiendokument mit nur zwei Seiten Umfang - konkretisiert. Diese Konkretisierung fällt in der Tat äußerst präzise aus. Insgesamt sind nicht weniger als 161 so genannte "Aktionen«, also Handlungsanweisungen, aufgelistet (Bundesregierung 2004; für eine kritische Lesung aus Sicht der dieser Entwicklung grundsätzlich sehr positiv gegenüberstehenden "praxisorientierten « Friedensforschung vgl. Debiel 2004). Mittlerweile wurde auch bereits der erste Umsetzungsbericht veröffentlicht (unter dem Titel »Sicherheit und Stabilität durch Krisenprävention gemeinsam stärken«, Bundesregierung 2006), der insofern für die konzeptionelle Ausrichtung von Bedeutung ist, als er die vom Aktionsplan vorgegebenen Schwerpunktsetzungen leicht verschiebt. Dies kann als Nachwirkung des Regierungswechsels von Herbst 2005 gedeutet werden kann.

Die durch ein derart detailliert ausdifferenziertes Grundlagendokument wie den Aktionsplan vermeintlich vorgegebene Klarheit verschwimmt bereits auf Policy-Ebene 1. Denn auch hier wurde ein Grundlagendokument erarbeitet, ein »übersektorales Konzept« zur zivilen Krisenprävention als Arbeitsfeld der deutschen Entwicklungszusammenarbeit (BMZ 2005b), das zwar die Vorgaben des Aktionsplans aufgreift, jedoch - ebenfalls in sehr konkreter Art und Weise - eigene Schwerpunktsetzungen vornimmt.

Hierarchisch unter diesem als verbindlich definierten Strategiepapier angeordnet gibt es nun auf Ministeriums-Ebene, also nach wie vor auf Policy-Ebene 1, eine relativ große Zahl an Papieren (»BMZ-Konzepte« 
oder $\left.» B M Z-S p e z i a l ~ ~^{98}\right)$, die zu einzelnen Arbeitsbereichen genauere Ansatzpunkte und Interventionsschritte definieren sollen. Im Unterschied zu vergleichbaren Dokumenten der US-amerikanischen Entwicklungszusammenarbeit handelt es sich hier jedoch keineswegs um reine Handreichungen, die dazu dienen sollen, den Background der an der Umsetzung Beteiligten zu stärken. Jedes dieser Papiere beansprucht in der einen oder anderen Weise Verbindlichkeit, während die jeweiligen Schwerpunktlegungen zugleich zu weit auseinander liegen (und sich mitunter auch auf dieser Ebene widersprechen), als dass eine kohärente Umsetzung möglich wäre. So ist die Arbeit mit diesem Korpus insbesondere für jene Institutionen, die mit der Umsetzung dieser vielfältigen Vorgaben betraut sind, eine schwer zu meisternde Aufgabe.

Dies gilt umso mehr, als diese Umsetzung formell unabhängigen Organisationen obliegt. Das Gros der Aufträge verteilt sich auf die Kreditanstalt für Wiederaufbau (KfW) für den Bereich der finanziellen $\mathrm{Zu}-$ sammenarbeit und die GTZ für den Bereich der technischen Zusammenarbeit (für einen genaueren Überblick der Implementierungsstruktur der deutschen Entwicklungshilfe vgl. Nuscheler 1996, 2006). Die Auftragsvergabe an die GTZ ist über einen so genannten Generalvertrag ${ }^{99}$ geregelt, der im Prinzip gewährleistet, dass die GTZ effektiv als die zentrale Dienstnehmerin im Bereich der Implementation technischer Programmund Projektarbeit fungieren kann. Derzeit werden rund $57 \%$ der unter dem Begriff Peacebuilding zu subsumierenden Maßnahmen von der GTZ implementiert (vgl. Kievelitz et al. 2003: 25).

Die GTZ hat über die bereits angesprochenen Sektorvorhaben (die mittlerweile im Bereich der Konfliktprävention im Rahmen eines Kom-

98 Folgende Dokumente wurden für die vorliegende Untersuchung herangezogen: BMZ-Konzept 127, »Menschen haben ein Recht auf Entwicklung. Entwicklungspolitischer Aktionsplan für Menschenrechte 2004-2007« (BMZ 2004e); BMZ-Konzept 136, »Konzept für Maßnahmen der Entwicklungsorientierten Not- und Übergangshilfe« (BMZ 2005c); BMZKonzept 149, „Entwicklungsorientierte Transformation bei fragiler Staatlichkeit und schlechter Regierungsführung « (BMZ 2007); dazu noch das BMZ-Spezial 124, »Förderung von Demokratie in der deutschen Entwicklungspolitik« (BMZ 2005a) und das ebenfalls vom BMZ herausgegebene, aber interministeriell vom Ressortkreis erarbeite »Interministerielle Rahmenkonzept zur Unterstützung von Reformen des Sicherheitssektors in Entwicklungs- und Transformationsländern« (BMZ 2006b).

99 Laut Begriffswelt der GTZ (2004a) bildet der Generalvertrag »die rechtliche Grundlage für das Auftragsverfahren und regelt die einzelnen Schritte zur Beauftragung der GTZ mit der Prüfung und Durchführung von Projekten und Programmen sowie die Vergütung der GTZ nach öffentlichem Preisrecht. Daneben legt der Generalvertrag die Unterstützungs- und Beratungsfunktion der GTZ gegenüber dem BMZ fest." 
petenzfeldes gebündelt wurden) auch ein direkt vom BMZ überantwortetes Budget, um den Sektor thematisch, methodisch und analytisch weiterzuentwickeln (vgl. Fahrenhorst/Musto 2002: 185). Zugleich verfügt sie natürlich auch ein spezifisches Programm, in dem sie ihre besonderen Kompetenzen verortet sieht. Die erste Erarbeitung einer solchen Produktpalette wurde bereits Ende der 1990er Jahre durchgeführt und 2002 in der Endfassung unter dem Titel »Friedensentwicklung, Krisenprävention und Konfliktbearbeitung« als »konzeptionelle Grundlage für diesen neuen Arbeitsbereich« vorgelegt (Ropers 2002: 3). Nach der Überführung des Sektorvorhabens Krisenprävention in ein für die konkrete GTZ-Arbeit nun mitverantwortliches Kompetenzfeld wurde, in Zusammenarbeit mit weiteren zuständigen Kompetenzfeldern vor allem aus dem Governance-Bereich, ein Leistungsangebot für den Bereich »Friedensentwicklung und Krisenprävention« als Teilgebiet des Kataloges für »Staat, Demokratie und Armutsbekämpfung« erarbeitet (GTZ 2007a). Es liegt auf der Hand, dass darin trotz der unbestreitbaren Relevanz des Aktionsplanes und des übersektoralen Konzeptes, die den verbindlichen Rahmen der Auftragsvergabe durch das BMZ bilden, wiederum GTZ-eigene Schwerpunktlegungen herauszulesen sind.

Abbildung 20: Institutionelle Verflechtung der zivilen

Friedensentwicklung in Deutschland

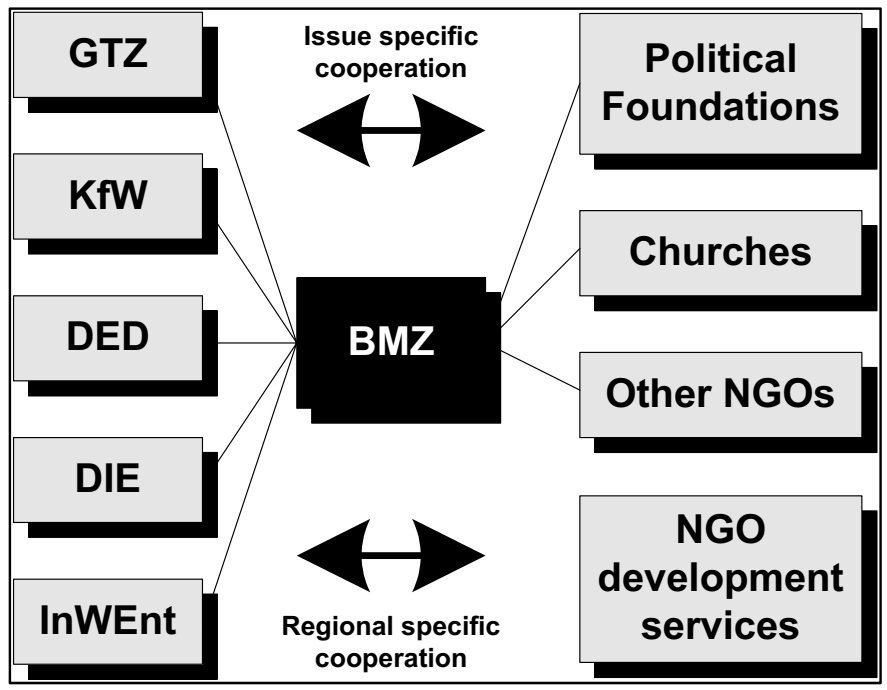

Quelle: Kievelitz et al. 2003: 47 
Abgesehen von der generellen Tendenz, mit Strategiepapieren möglichst genaue und präzise Regelungen vorzuschreiben, ist für diese Vielfalt an unterschiedlichen konzeptionellen Grundlagen die große Zahl an mitspracheberechtigten und eingebundenen Institutionen verantwortlich. Allein im zivilen entwicklungspolitischen Bereich zeigt sich ein dichtes Gemisch aus staatlichen und privaten Akteuren (siehe Abbildung 20), das in seinem staatlichen Teil über das BMZ gesteuert, in seinem privaten Teil über formelle Zusammenschlüsse, allen voran FriEnt, koordiniert wird. Eine Mischung aus ideologischen und finanziellen Motiven garantiert in diesem Spektrum eine permanent breite Palette an Vorschlägen und Forderungen an die staatlichen entwicklungspolitischen Instanzen, die diese zwar einerseits aufzunehmen gewillt - auch um sie im interministeriellen Wettstreit um Budgetgeld einsetzen zu können andererseits aber durch ihren Steuerungsdrang und den Kohärenzdruck anderer relevanter Machtgruppen im Bereich der Krisenprävention insbesondere des Außenamts und des Verteidigungsministeriums mit den gewichtigen Eigeninteressen der Bundeswehr - daran gehindert sind. Folglich ist jede inhaltliche Festschreibung das Resultat eines politisch ausgehandelten Kompromisses.

Der Begründungszusammenhang für die entwicklungspolitische Intervention in bewaffnete Konflikte lässt sich schon in den vier Leitmotiven der deutschen Entwicklungspolitik erkennen: Armut bekämpfen, Frieden sichern und Demokratie verwirklichen, Globalisierung gerecht gestalten, Umwelt schützen. ${ }^{100}$ Es sind vor allem die ersten beiden Motive, die in einen Zusammenhang konstruiert und mit einer merklichen Gerechtigkeitskomponente untermalt als hauptsächliche Argumentation des Engagements dienen. So wird im Aktionsprogramm 2015, dessen Aufgabe es ist, eine Verbindung zwischen den multilateralen Vorgaben, speziell den MDGs, und der deutschen entwicklungspolitischen Praxis herzustellen, ein direkter Zusammenhang zwischen Armut, Konflikt und Krisenprävention konstruiert: »Armut und Gewalt können sich gegenseitig verstärken. Krisenprävention und friedliche Konfliktbeilegung zu fördern, ist deshalb zur Armutsbekämpfung unerlässlich. Gleichzeitig

100 Diese vier Leitmotive gliedern sich wiederum in elf Themen, die faktisch als Zielbestimmungen der deutschen Entwicklungspolitik fungieren: Armut bekämpfen; Frieden sichern; Umwelt und Ressourcen bewahren; Entschuldung vorantreiben; Ernährung sichern; Menschenrechte wahren, Demokratie fördern; Bildung fördern; Gesundheit, AIDSBekämpfung, Bevölkerungspolitik; Globalisierung und Handel; Nachhaltige Wirtschaftsentwicklung; Zusammenarbeit mit der Privatwirtschaft. Die Zielsetzung »Frieden sichern « wird als Auftrag zur Intervention im Sinne einer zivilen Krisenprävention und Friedensentwicklung verstanden. 
leistet Armutsbekämpfung wichtige Beiträge zur Krisenprävention und -bewältigung « (Bundesregierung 2001: 37). Erstaunlich ist dies insofern, als selbst die GTZ bemerkt, dass die wissenschaftliche Grundlage für die Behauptung eines derartigen Zusammenhangs nicht gegeben ist (Paffenholz/Brede 2004: 31).

Dennoch liefert der Zusammenhang Armut - Konflikt die Grundlage für die Ausrichtung der Praxis. Wenngleich festzuhalten ist, dass sich der strategische Ansatz der deutschen Entwicklungspolitik seit Ende der 1990er Jahre merklich verändert hat und nach wie vor alles andere als fixiert ist, hat sich die Diskussion auf eine um »Human Security« kreisende Interpretation eines erweiterten Sicherheitsbegriffes zugespitzt: »Ausgangspunkt für Maßnahmen der Krisenprävention, der Konfliktbeilegung und der Konsolidierung in der Nachkonfliktphase ist ein erweiterter Sicherheitsbegriff, der politische, ökonomische, ökologische und soziale Stabilität umfasst« (Bundesregierung 2000; zur Bedeutung von Human Security vgl. auch Ropers 2002: 10; BMZ 2005b: 8). Angesichts der Tatsache, dass das jüngste in diesem Kontext veröffentlichte Dokument der Bundesregierung eine »Weiterentwicklung und Förderung des internationalen Konzepts zur Human Security« (Bundesregierung 2006: 12) verspricht, dürfte tatsächlich eine Festlegung getroffen worden sein, die in ihrer Eindeutigkeit selbst in der europäischen Geberlandschaft eine Besonderheit darstellt.

Interessant ist ein genauerer Blick auf die Definition von Human Security, die die Bundesregierung in ihrem Gesamtkonzept einführt: »Grundlage dafür sind die Achtung der Menschenrechte, soziale Gerechtigkeit, Rechtsstaatlichkeit, partizipatorische Entscheidungsfindung, Bewahrung natürlicher Ressourcen, Entwicklungschancen in allen Weltregionen und die Nutzung friedlicher Konfliktlösungsmechanismen« (Bundesregierung 2000). Auffällig ist, dass in dieser Definition praktisch alle wesentlichen thematischen Arbeitsbereiche, in denen die deutsche Außen-, speziell aber die deutsche Entwicklungspolitik aktiv ist, zusammengefasst werden. Zu deuten ist dies auf zweierlei Weisen: Einerseits könnte es als Zeichen dafür gesehen werden, dass die Steuerungskompetenz der Bundesregierung tatsächlich ihre Widerspiegelung in den konkreten Arbeitsprogrammen findet. Ebenso gut wäre es allerdings möglich, dass das Human Security-Konzept angesichts seiner großen Spannweite dafür verwendet wird, ohnehin eingeschlagene Wege in einen prägnanten konzeptionellen Begriff zusammenzufassen.

Die genauere Ausdifferenzierung dieser allgemeinen Kriterien, wie sie in den strategischen Planungsdokumenten erfolgt, legt ein Zusammenspiel dieser beiden Möglichkeiten nahe. So ist ohne Zweifel festzustellen, dass das Konzept in seinen Bestandteilen so gestreckt wurde, dass 
alle eingeschliffenen Arbeitsschwerpunkte darin Platz finden und mit der neu entdeckten Querschnittsproblematik Friedensentwicklung verknüpft werden können. Zugleich transportiert Human Security allerdings auch einen speziellen Gehalt des Zugriffs auf periphere Gebiete, der auf die gewohnten thematischen Ausrichtungen schwerwiegende Konsequenzen hat. Diese Konsequenzen äußern sich in einem vehementen Drang nach Steuerung, ein Drang, der sich um den Begriff der »Struktur« formiert.

Die Affinität zum Strukturbegriff zeigt sich schon im leitenden Motto der deutschen Entwicklungspolitik, die ja wie bereits angeführt seit dem Regierungswechsel 1998 als »Globale Strukturpolitik« verstanden wird. Dies umfasst im Verständnis des BMZ einerseits den Abbau struktureller Ursachen von bewaffneten Konflikten, also jener Teil an Maßnahmen, der die traditionellen Arbeitsgebiete der EZ ebenso anspricht wie die Arbeit an den internationalen Rahmenbedingungen, speziell im ökonomischen und dem Rechtsbereich, und andererseits den Aufbau konkreter Mechanismen zur Prävention und Transformation bewaffneter Konflikte (vgl. Fahrenhorst/Musto 2002: 179). Für diesen zweiten Bereich dient ebenfalls ein Konzept, das auf den Struktur-Begriff rekurriert: die »strukturelle Stabilität«.

Wiewohl der Begriff auch auf Ebene des DAC (2001: 31) und der EU (vgl. Klingebiel 2002: 2) zu finden ist, hat er im deutschen Kontext eine spezielle Ausprägung. So sieht etwa Norbert Ropers (2002: 46f.) diese Spielart der »strukturellen Stabilität« primär entlang der Aufgabenstellung definiert, »zivilgesellschaftliche und staatliche Strukturen $[\ldots]$ in die Lage « zu versetzen, »konstruktive und gewaltfreie Mechanismen zur Austragung grundlegender und akuter Interessensgegensätze und deren Abbau zu entwickeln.« Der »Kerngedanke« liege demnach in der »dauerhaften Überwindung fragiler und instabiler gesellschaftspolitischer und staatlicher Erscheinungsformen«, wobei dazu insbesondere die »wirksame Gestaltungsmacht des Staates« von entscheidender Bedeutung wäre.

Auch wenn sich Ropers Begriffsverständnis auf politischer Ebene nicht durchsetzen kann, da sich aus Gründen der besseren politischen Verwertbarkeit ein weiter gefasstes und tendenziell beliebiges Begriffsverständnis von »struktureller Stabilität« durchsetzt (vgl. Klingebiel 2002) und der Begriff in weiterer Folge an Bedeutung verliert, ist seine Definition dennoch interessant. Sie demonstriert eine eigenartige Vermengung zwischen einem starken, eben gestaltungsmächtigen Staat und einer Zivilgesellschaft, die sich in der Wertigkeit ihrer Relevanz mit dem Staat auf gleicher Augenhöhe befindet. Diese Vermengung ist ein Charakteristikum jener Vorgangsweise, die auf Regierungsebene als 
Schlüsselmechanismus zur Überwindung der strukturellen Ursachen bewaffneter Konflikte und der Etablierung friedlicher Konfliktlösungsmechanismen festgelegt wird (vgl. Wieczorek-Zeul 1999b).

Die sich in diesen Konzeptionen abzeichnende Tendenz zur Einflussnahme auf Gesamtprozesse wird durch traditionelle und immer wieder betonte Schwerpunkte der deutschen Entwicklungspolitik wie Multilateralismus (Bundesregierung 2000; BMZ 2003b: 105f.) und internationale Verrechtlichung, die ja beide für sich in Anspruch nehmen, eben just keine deutschen Spezifika zu sein, keineswegs konterkariert, sondern vielmehr unterstrichen. Sowohl im Menschenrechtsansatz (BMZ 2003b: 12ff.), aber vor allem im Bereich der Verrechtlichung der Konfliktaustragung (Bundesregierung 2000), einem »Markenzeichen deutscher Präventionspolitik« (Debiel 2004: 258), wird dies deutlich sichtbar. Legt die Menschenrechtsorientierung über den Slogan der »Responsibility to Protect « (Bundesregierung 2006: 98) die Schiene zur unmittelbaren Intervention im Sinne von Human Security ${ }^{101}$, eröffnet der Zugriff einer internationalen Gerichtsbarkeit auf bewaffnete Konflikte an der Peripherie ein neues Feld im Spiel der Legitimierung/Delegitimierung von Konfliktparteien. Somit obliegt eine solche Einstufung nun vermeintlich neutralen, objektiven Instanzen, deren Durchsetzungsmacht jedoch entscheidend von der Rückendeckung der Schlüsselmächte im Weltsystem abhängt.

Die Konsequenzen auf Ebene der Technischen Zusammenarbeit zeigen sich in der Operationalisierung des Bereiches von Friedensentwicklung und ziviler Krisenprävention. Entlang der üblichen Vorgangsweise bei Aufgabenstellungen, denen umfassende Relevanz zugebilligt wird, geht es zunächst um durchgängige Verankerung. Folgerichtig wird Krisenprävention als »fester Bestandteil deutscher Friedenspolitik« zur »Querschnittsaufgabe, die in der Gestaltung der einzelnen Politikbereiche verankert sein muss« (Bundesregierung 2004: 1), schlägt damit also einen Weg ein, den das Thema Gender bereits beschritten hat. Es werden bürokratische Marksteine definiert, die diese Querschnittsverankerung auch in der konkreten Umsetzung vor Ort - nach Möglichkeit also

$101 »$ Das Konzept der Human Security sowie das Prinzip der Responsibility to protect können große Bedeutung für die auf dem erweiterten Sicherheitsbegriff fußende Krisenprävention und die Anforderungen an die Akteure haben. Die Bundesregierung wird sich mit Blick auf die eigene Positionsbestimmung im Ressortkreis unter Einbeziehung von Experten aus der Zivilgesellschaft mit den Chancen und Auswirkungen des Konzepts [...] für krisenpräventives und ressortübergreifendes Handeln befassen« (Bundesregierung 2006: 98). 
bis in die Policy-Ebene 3 - gewährleisten und überprüfbar machen sollen.

$\mathrm{Zu}$ diesem Zweck führt das BMZ ein zweistufiges Verfahren ein. Als erster Schritt wird als Krisenfrühwarnung eine jährliche Bewertung aller Kooperationsländer durchgeführt. Diese Bewertung basiert zunächst auf den bereits erwähnten so genannten Spelten-Kriterien (Spelten 1999, 2000), dann auf einem darauf aufbauenden Indikatorenkatalog, der als qualitativer Fragebogen von Länderexpert/innen des Deutschen Übersee-Institutes beantwortet wird. Zusammen mit Informationen des Verteidigungsministeriums, des Auswärtigen Amtes und des Bundesnachrichtendienstes werden die Kooperationsländer dann entlang der drei Kategorien »geringer«, »wachsender« oder »akuter Präventionsbedarf« klassifiziert (vgl. BMZ 2005b: 31). Diese Einordnung dient als Grundlage für die Ausrichtung des jeweiligen Länderprogramms.

Um auch auf dieser Ebene die Konfliktsensibilität zu garantieren, wird ein Zertifizierungsinstrument entwickelt, das einen einfachen Blick auf die Verankerung von Krisenprävention als Querschnittsthema auf Implementierungsebene garantieren soll: die K-Kennung. Demnach wird jede von der deutschen EZ gesetzte Maßnahme in Hinblick auf ihre krisenpräventive Ausrichtung bewertet, wobei eine K2-Maßnahme Krisenprävention als Hauptziel, eine K1-Maßnahme eine solche als Nebenziel verfolgt und K0-Maßnahmen anderen Zielen gewidmet sind. (vgl. BMZ 2005b: 32). Diese recht rigide und umfassende Klassifizierung dient offenbar primär dem Zweck interner Disziplinierung, da entsprechende Zahlen - ebenso wie übrigens auch die Resultate der jährlichen BMZFrühwarnung - nicht veröffentlicht werden. Gerade in Hinblick auf die angestrebte Disziplinierung zeigt die Methode allerdings Wirkung: nicht nur in den Länderbüros zählt die K-Kennung zu den Arbeitsinstrumenten, es kann davon ausgegangen werden, dass ihre Rolle auch bei der Planung und Steuerung von Länderprogrammen relevant ist. Sie sind damit der wesentliche Hebel, mit dem die Umsetzung der Strategiepapiere innerinstitutionell gewährleistet wird.

Wo liegen nun die inhaltlichen Schwerpunkte, die es umzusetzen gilt? ${ }^{102}$ Die wesentliche Aufschlüsselung der Interventionsebenen findet sich in den »strategischen Ansatzpunkten« des Aktionsplans: »Als strategische Ansatzpunkte werden die Herstellung verlässlicher staatlicher Strukturen (Rechtsstaatlichkeit, Demokratie, Menschenrechte und Sicherheit), aber auch die Schaffung von Friedenspotenzialen in der Zivilgesellschaft, bei den Medien sowie in Kultur und Bildung identifiziert.

102 Diese Maßnahmen werden auch als »Maßnahmen der politischen Dimension« bezeichnet (vgl. Bundesregierung 2001: 12). 
Ein weiterer Ansatzpunkt ist die Sicherung der Lebenschancen der betroffenen Menschen durch geeignete Maßnahmen auf den Gebieten Wirtschaft, Gesellschaft und Umwelt« (Bundesregierung 2004: 2). Es geht also um die Arbeit auf drei Ebenen, die grob mit den Begriffen Staat - Gesellschaft - Ökonomie umrissen werden können. Die sich im Aktionsplan findende Bezeichnung dieser drei Ebenen ist gut gewählt, denn tatsächlich schafft sie es, die Kernelemente dieser Arbeitsbereiche auch nach Einbeziehung aller nachgeordneten Dokumente markant auf den Punkt zu bringen.

(1) »Herstellung verlässlicher staatlicher Strukturen«. Das zentrale, sich durch alle Dokumente durchziehende Element bei der Herstellung verlässlicher staatlicher Strukturen ist der Rechtsstaat (vgl. etwa 2004: 2). Dies ist angesichts der ohnehin auch im internationalen Bereich starken Rechtsorientierung wenig überraschend, hat aber dennoch einen eigentümlichen Charakter, der sich vor allem im Bedeutungsverhältnis Rechtsstaatlichkeit - Governance - Demokratie zeigt. Überraschend sind dabei die Gewichtung dieser drei Komponenten sowie die Bedeutung, die ihnen zugeschrieben wird.

Die Basis des Konzepts liegt im Zusammenspiel von Rechtsstaatlichkeit und Good Governance, die als einander bedingend verstanden werden: »Verantwortliche Regierungsführung beinhaltet neben guten politischen Rahmenbedingungen und Rechtsstaatlichkeit auch den verantwortungsvollen Umgang des Staates mit politischer Macht und öffentlichen Ressourcen« (Bundesregierung 2004: 36). Dabei wird auch der Zusammenhang zwischen Good Governance und der übergeordneten Zielsetzung der "globalen Strukturpolitik« betont, da es eine »Bedingung für Armutsbekämpfung und Friedenssicherung«, zwei zentrale Leitlinien dieser globalen Strukturpolitik, wäre (GTZ 2007b: 4). Die Achse zwischen Rechtsstaatlichkeit und Good Governance wird zugleich in Verbindung mit einer Orientierung am Ziel der nachhaltigen Entwicklung gesetzt (ebd.: 5). Folgerichtig erhält sie so eine eigene neue Definition: Entwicklungsorientierung.

Eine solche Entwicklungsorientierung ist nicht nur als Postulat zu verstehen. Genauso dient sie als Indikator der Stabilität von Staatlichkeit, was darauf schließen lässt, dass Entwicklungsorientierung als notwendiges Resultat der Kombination von funktionierender Rechtsstaatlichkeit und ohnehin an den Prinzipien nachhaltiger Entwicklung orientierter Good Governance verstanden wird. Daher lassen sich fragile Staaten auch anhand des Grades ihrer Entwicklungsorientierung identifizieren. Die Richtlinie zur »Entwicklungsorientierten Transformation bei fragiler Staatlichkeit« unterscheidet zwischen den drei Kategorien 
eines "positiven Trends entwicklungsorientierter Regierungsführung", einer "wenig entwicklungsorientierten Regierungsführung " und einer "nicht entwicklungsorientierten und sich weiter verschlechternden Regierungsführung« (BMZ 2007). Je nach Einordnung verschiebt sich der zentrale Partner der Intervention von den staatlichen Institutionen, die in ihren positiven Anstrengungen unterstützt werden sollen, zur Zivilgesellschaft, die als »driver of change« bei wenig entwicklungsorientierten Staaten identifiziert wird, und schließlich zur internationalen Gemeinschaft, da bei der letztgenannten Kategorie bilateralen Eingriffen nur minimale Erfolgschancen zugebilligt werden.

Interessant ist nun das Verhältnis zur dritten Komponente im deutschen State-Building-Konzept, der Demokratie. Formal ist Demokratie eine allgegenwärtige Zielbestimmung. So führt die zentrale für das breite Publikum gedachte BMZ-Hochglanzbroschüre nicht nur den programmatisch anmutenden Titel »Recht - Demokratie - Frieden«, sondern wartet auch mit einem Verweis auf das für die USA so wesentliche Theoriegebäude vom demokratischen Frieden auf, auch wenn das Konzept von der Theorie- auf die Erfahrungsebene herabgestuft wird: »Die Erfahrung belegt: Demokratien sind friedlicher als autoritäre Regime« (BMZ 2003b: 29). Auch verweisen Bundesregierung (etwa 2006: 38ff.) und BMZ wiederholt darauf, dass der Bereich »Demokratie, Zivilgesellschaft und öffentliche Verwaltung« in 30 Partnerländern schwerpunktmäßig bearbeitet wird.

Bei genauerer Betrachtung ergeben sich allerdings schnell überraschende Eigenheiten. Diese beginnen spätestens dann, wenn festzustellen ist, dass das »Produkt« Demokratie im Leistungsangebot der GTZ für den Bereich der politischen Maßnahmen (im Gegensatz zu immerhin sieben anderen Arbeitsfeldern allein im Governance-Bereich) noch nicht verfügbar ist, sondern sich erst »in Vorbereitung « befindet (GTZ 2007a). Dies mag auf den ersten Blick erstaunlich anmuten, ist aber letzten Endes nur konsequent. Schließlich handelt es sich bei Demokratie um ein für die deutsche Entwicklungspolitik konzeptionell in doppelter Weise bedingtes Konzept.

Einerseits wird die potenzielle Gefahr, die von Prozessen des demokratischen Wettbewerbs für die politische Stabilität ausgehen kann, in Verbindung mit der ohnehin nur unzureichenden Breitenwirkung eines solchen Ansatzes ins Treffen geführt: »Eine einseitige Konzentration der Demokratieförderung auf die Vorbereitung und Durchführung von Wahlen wird den Erfordernissen nicht gerecht und ist zuweilen kontraproduktiv. Wahlen sind zwar >sine qua non<-Bedingung für die Entwicklung von Demokratien, sind aber meist nicht ausreichend, um demokratische Formen der Machtausübung und sozialen Inklusion zu festigen« (BMZ 
2007: 30). Andererseits wird, und das mutet angesichts der überragenden Staatsorientierung der deutschen Herangehensweise fast absurd an, der Faktor kultureller Eigenheiten - die so genannte "Grundorientierung " einer Gesellschaft - hervorgehoben: »[Die deutsche Entwicklungspolitik] erkennt gleichzeitig ausdrücklich an, dass Demokratie in Abhängigkeit von historischen und kulturellen Grundorientierungen der jeweiligen Gesellschaften unterschiedlich ausgeprägt sein kann. Gefördert wird deshalb nicht eine bestimmte Form der Demokratie, sondern die Durchsetzung demokratischer und rechtsstaatlicher Prinzipien" (BMZ 2005a: 6). Diese vermeintlich in sich widersprüchliche Formulierung ist vermutlich so aufzulösen, dass mit »demokratischen Prinzipien« nichts anderes als das ohnehin zentrale Konzept entwicklungsorientierter Good Governance gemeint ist.

Abbildung 21: Schema des deutschen Konzepts zur Herstellung verlässlicher staatlicher Strukturen

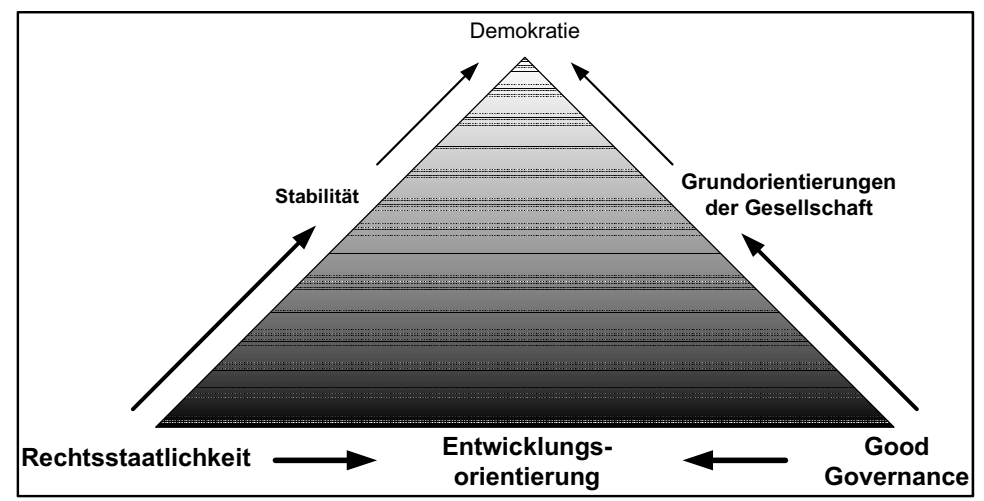

Demokratie im Sinne von parteipolitischem Wettbewerb wird so zu einer prototypischen Aktivität im Kanon der Nachhaltigkeit: ähnlich der Modernisierung wird sie zu einer vagen Zielbestimmung, die zunächst vor dem Ziel eines dichten, funktionierenden, gesellschaftlich verankerten Rechtsstaat zurückzutreten habe (siehe Abbildung 21). Die vermeintlich kultursensible Argumentation von unterschiedlichen »gesellschaftlichen Grundorientierungen« offenbart den eigentlichen Gehalt der Operation, denn die Linie der Entwicklungsorientierung ist dagegen nicht verhandelbar. Kultursensibilität besteht nur jenseits der Durchsetzung eines mit solidem Gewaltmonopol ausgerichteten Rechtsstaates, der den Kriterien von Good Governance genügt. Trotzdem wird die Ebene der politischen Gesellschaft keineswegs aufgegeben, sondern auch partnerschaftlich bearbeitet, wenngleich nicht über den offiziellen bilateralen 
Bereich. Es obliegt den deutschen Parteistiftungen, potenzielle Partner $\mathrm{zu}$ identifizieren und entsprechend $\mathrm{zu}$ fördern. Allerdings bleibt diese Komponente nicht nur in der Praxis, sondern auch im konzeptionellen Verständnis eine zweitrangige Aufgabe.

Demgegenüber sind es vor allem zwei Felder, die die Interventionen dominieren. Einerseits die Arbeit an den rechtsstaatlichen Institutionen, die natürlicherweise »primär bei der Reformbereitschaft der Partnerregierung« ansetzt (BMZ 2005a). Neben dem Capacity-Building, das besonders im Bereich der Verwaltung (vgl. GTZ 2007b: 18), speziell im kommunalen Bereich, bei Prozessen der Dezentralisierung, also der Verankerung staatlicher Dienste in den bislang zu wenig verstaatlichten Peripherien, und im Justizbereich vorangetrieben wird, ist es die Sicherheitssektorreform, die es den Verantwortlichen im Ressortkreis Krisenprävention besonders angetan hat (vgl. Bundesregierung 2006: 26). So soll SSR, auch wenn bislang nur wenige praktische Erfahrungen bestehen und zumindest eine von diesen - das SSR-Programm der BerghofStiftung in Sri Lanka, das noch zur Sprache kommen wird - desaströse Auswirkungen gezeigt hat, zu einem Schwerpunkt der deutschen Intervention ausgebaut werden. Konzeptionell ist das nur konsequent, denn es geht dabei nicht nur um die Bewahrung, die Durchsetzung und den Ausbau des staatlichen Gewaltmonopols ${ }^{103}$, sondern auch um die Verbindung dieses Gewaltmonopols mit der Komponente der Rechtsstaatlichkeit: »[Zur Sicherheitssektorreform] gehören eine effektive demokratische Kontrolle der staatlich eingesetzten Sicherungskräfte [...] beziehungsweise ihre rechtsstaatliche Einbindung in Staat und Gesellschaft« (Bundesregierung 2004: 38).

Andererseits ist es auch im Bereich des State-Building die Arbeit an der Handlungsfähigkeit nicht-staatlicher ziviler Akteure, der wesentliches Augenmerk zukommt. Die Frage der institutionellen Legitimität wird beispielsweise dual interpretiert: »Der Aufbau bzw. die Stärkung legitimer Institutionen in Staat und Zivilgesellschaft wurde zum vorrangigen Ziel der Zusammenarbeit mit fragilen Staaten erklärt (state building)« (BMZ 2007: 10). Die Bedeutung der Zivilgesellschaft für die politischen Interventionen der deutschen Entwicklungszusammenarbeit ist derart überragend, dass ihr unter dem Begriff der »Friedenspotenziale« ein eigener Arbeitsschwerpunkt zukommt. Dennoch ist es wichtig, ihre Rolle auch im Zusammenhang mit den State-Building-Bemühungen hervorzuheben, da nur so die starke Orientierung auf Durchstaatlichung,

$103 \mathrm{Zu}$ diesem Zweck wird das Mandat der zentralen deutschen TZUmsetzungsorganisation, der GTZ, zunehmend offensiv interpretiert. So ist die »Entwicklung von nationalen Sicherheitsstrategien « mittlerweile ein definierter GTZ-Aufgabenbereich (BMZ 2006b: 11). 
die gesellschaftliche Verankerung staatlicher Strukturen, in notwendigem Maße deutlich wird.

(2) »Schaffung von Friedenspotenzialen«. Zwar wird die im einleitenden Überblickssatz des Aktionsplanes verwendete Formulierung der »Schaffung von Friedensallianzen" (Bundesregierung 2004: 2) im weiteren Verlauf des Dokumentes zu einer »Förderung « relativiert, dennoch ist diese Formulierung als symptomatisch für das deutsche Selbstverständnis im Bereich der zivilgesellschaftlichen Komponente der Friedensarbeit zu sehen. Das zentrale Moment bei der Etablierung und Förderung solcher Potenziale ist das von John Paul Lederach entworfene Konzept der "peace constituencies«, das sich im Gegensatz zu anderen in Diskussion befindlichen Ansätzen vor allem dadurch auszeichnet, die verschiedensten nicht-staatlichen Akteure als zentrale aktive Komponente bei der Friedensentwicklung zu konzeptualisieren (vgl. Paffenholz 2002: 7f.): »Der Begriff Friedensallianz umfasst alle zivilgesellschaftlichen, nicht-bewaffneten, organisierten Akteure, die sich für eine friedliche Konfliktbeilegung einsetzen « (ebd.: 11; vgl. auch Ropers 2002: 60ff.).

Dieses Verständnis schränkt sich allerdings insofern ein, als der Wille allein nicht ausreicht. Die angesprochenen »Individuen und Gruppen « müssen, so die offizielle deutsche Definition im Anschluss an Lederach (Bundesregierung 2004: VIII), auch in der Lage sein, »einen gewissen Einfluss auf andere Gruppen auszuüben.« Damit lässt sich der ursprünglich recht breite, fast einem Fetisch gleichkommende Begriff der Zivilgesellschaft, dem in nahezu allen relevanten Dokumenten geradezu reflexartig Referenz erwiesen wird (vgl. etwa BMZ 2003b: 34ff.; GTZ 2007b: 11f.), elegant auf für im Zuge entwicklungspolitischer Interventionen verhältnismäßig leicht zu handhabende Akteure eingrenzen: »Für erfolgreiche Krisenprävention ist es wichtig, friedensbereite zivilgesellschaftliche Akteure zu identifizieren und zu stärken. Als Partner der Friedenspolitik der Bundesregierung kommen Nichtregierungsorganisationen und soziale Bewegungen, aber auch nicht-staatliche Akteure wie Kirchen und Verbände in Betracht« (Bundesregierung 2004: 44).

Wenn dann auch noch angeführt wird, dass es sich bei den zivilgesellschaftlichen Akteuren um eine »entwickelte Zivilgesellschaft« handeln soll, denn diese sei »von zentraler Bedeutung für die gewaltfreie Austragung von Konflikten« (ebd.: 44), wird schnell deutlich, dass sich das zugrunde gelegte Verständnis des Konzepts zwar vielleicht in der Identifikation, jedoch nicht in der Zielvision durch besonders große Heterogenität auszeichnet. Es geht um die Etablierung professionalisierter Zivilgesellschaftsorganisationen, die bei dem auf Lederach fußenden dreistufigen Track-Verständnis der Konfliktmediation (vgl. Diamond/ 
McDonald 1996, Ropers 2002: 43) die Tracks 2 und 3, also die mittlere und untere politische und gesellschaftliche Führungsebene sowie den Grassroots-Bereich bearbeiten sollen. ${ }^{104}$ Speziell der Grassroots-Bereich benötigt wesentliche Förderung, soll er diese professionalisierten Funktionen wahrnehmen können. Nachdem zusätzlich bei allen zivilgesellschaftlichen Partner/innen ein Bewusstsein der verschiedenen weiteren Querschnittsproblematiken, insbesondere der GenderThematik, zu vermitteln ist (vgl. BMZ 2005a: 13), ist es offensichtlich, dass die entscheidende Maßnahme zur Schaffung oder Förderung von Friedensallianzen neben der Vernetzung der Akteure und der Stützung von als krisenpräventiv eingeschätzten Medien die Arbeit an der zu entwickelnden Kapazität der Partner/innen ist.

Es sind vor allem zwei Methoden, die in der angestrebten dreifachen Stärkung der Kapazität der zivilgesellschaftlichen Partner - der ökonomisch-institutionellen Unterstützung, der Professionalisierung und dem politischen Capacity-Building - zur Anwendung kommen. Einerseits sind es Fonds, deren Arbeit sich aber nicht in der simplen Finanzierung von vorgeschlagenen Anträgen erschöpft, sondern die diese Finanzierung mit einer Mentor-artigen Begleitung sowie Ausbildungs- und Trainingsmaßnahmen verbinden (vgl. Ropers 2002: 68, Paffenholz 2002: 25ff., und zu den Erkenntnissen aus der Evaluation des ATP, die diesen Ansatz ausgesprochen positiv bewertet, Paffenholz/Brede 2004: 9). Der wesentliche Vorteil solcher Fonds ist, dass sie ein relativ breites Spektrum an Partnerorganisationen abdecken und zugleich durch entsprechende Schulungsmaßnahmen erfassen können. Die gesellschaftliche Breitenwirkung, und damit die Kontrolle über den aktiven zivilgesellschaftlichen Sektor und dessen Ausrichtung, sind damit gut zu gewährleisten. ${ }^{105}$

Andererseits ist es der Zivile Friedensdienst, dem durch seine explizite Ansiedlung »im politisch-gesellschaftlichen Bereich« (Bundesregierung 2001: 38) eine nicht zu unterschätzende Rolle im Aufbau einer krisenpräventiv wirkenden Zivilgesellschaft zukommt (vgl. BMZ 2003b: 102f.). Zwar mag es selbst innerhalb der eigenen Konzeption übertrieben sein, ihn als »[d]as wichtigste friedenspolitische Instrument zur För-

104 Track 1 ist in diesem Konzept der klassischen Diplomatie vorbehalten und umfasst jenen Bereich, der in der Öffentlichkeit als Friedensverhandlung wahrgenommen wird. In jüngster Zeit hat sich im professionellen Peacebuilding-Bereich auch ein vierter Track, Track 1.5, etabliert. Dabei handelt es sich um Personen der politischen Gesellschaft, denen stabiler und nachhaltiger Einfluss auf die Entscheidungsträger/innen von Track 1 zugeschrieben wird.

105 Ein für die gesamte deutsche EZ beispielgebendes Modell ist der FLICT-Fonds in Sri Lanka, auf den noch einzugehen sein wird. 
derung von Friedenspotenzialen der Zivilgesellschaft« (Bundesregierung 2004: 45) zu bezeichnen, dennoch sind seine Wirkungen ohne Zweifel relevant. Den offenkundigen Nachteilen gegenüber dem Fonds-Modell eine relativ geringe Breitenwirkung und eine verhältnismäßig schlechte Steuerungsmöglichkeit bedingt durch die weitgehende Eigenständigkeit der ZFDler/innen - steht dabei ein entscheidender Vorteil gegenüber: die permanente persönliche Präsenz, die bei den Fonds-Modellen oder auch bei der "gewöhnlichen « Projekt-Kooperation mit NGO-Partnern nicht, oder zumindest nicht permanent, gegeben ist. Damit ist ein zwar mit gewissen Nachteilen verbundener, aber dennoch wertvoller direkter Zugriffspunkt auch in peripheren Zielregionen des Partnerlandes gegeben.

Die Relevanz dieser Maßnahmen zur Förderung zivilgesellschaftlicher Friedenspotenziale wie auch die generelle Bedeutung von Zivilgesellschaft für das deutsche Gesamtkonzept als ein im Rahmen dieses Gesamtkonzeptes gleichberechtigter Punkt neben dem Aufbau von Staatlichkeit lässt sich nur verstehen, wenn das Konzept in Verbindung mit dem zuvor bereits diskutierten Demokratieverständnis gesetzt wird. Starke zivilgesellschaftliche Strukturen werden als Basis von Demokratisierungsprozessen gesehen (vgl. etwa Bundesregierung 2004: 43-44), wobei bei ihnen das Argument der kulturellen und gesellschaftlichen Bedingtheit im Unterschied zum Konzept von Demokratie als politischem Wettbewerb nicht ins Treffen geführt wird. Stattdessen wird an diesem Punkt Universalität betont: »In allen Ländern gibt es - in den unterschiedlichsten Ausprägungen - Zusammenschlüsse von Menschen, die sich nicht nur um die Verbesserung ihres eigenen Loses kümmern, sondern auch Anliegen der gesamten Gesellschaft vertreten. Sie lassen sich überwiegend den Nichtregierungsorganisationen zuordnen« (BMZ 2003b: 34).

Die Hervorhebung kultureller Faktoren ausgerechnet anhand der Frage von Demokratisierung sagt also weniger über die Kultursensibilität deutscher Entwicklungspolitik als über ihre Affinität zu einem spezifischen Demokratiekonzept aus. Es ist die Idee einer gesamtgesellschaftlichen Durchdringung professionalisierter, und damit professionalisiert demokratischer Organisationen, die sich dennoch des tendenziell schädlichen politischen Wettbewerbs enthalten und, idealtypisch gesprochen, in kollektiver Vielfältigkeit krisenpräventiv handeln, also Frieden, verstanden im Sinne eines »positiven Friedens « als entwicklungsorientierter Gesellschaftsorientierung, schaffen.

(3) »Sicherung der Lebenschancen«. Die dritte Komponente des deutschen Ansatzes umfasst schließlich jene entwicklungspolitischen Pro- 
gramme, die eher traditionellen Arbeitsweisen entsprechen, also, um den deutschen Fachbegriff zu verwenden, K0-Vorhaben, die nun allerdings auch konfliktsensibel auszurichten sind. Allerdings beschränkt sich die behauptete Wirksamkeit nicht auf eine mittelbare Instanz. Durch den krisenpräventiven Fokus hat sich zwar nicht die unmittelbare Ausrichtung, aber sehr wohl das Verständnis der in diesem Bereich gesetzten Maßnahmen geändert. So werden zumeist dieselben oder nur minimal veränderte Interventionen mit einer relevant veränderten Zielsetzung durchgeführt. ${ }^{106}$

Der erste Punkt, der im Zusammenhang mit der Frage der »Sicherung der Lebenschancen « auffällt, ist die Betonung der Gerechtigkeitsfrage, wobei vor allem die Verteilungsgerechtigkeit angesprochen wird. So wird die »ungleiche Verteilung des Wohlstandes« (Bundesregierung 2004: 50) ebenso problematisiert wie Knappheit oder unausgewogener Zugang zu natürlichen Ressourcen (ebd.: 54). Einerseits entspricht dieser Ansatz einer bis in die Amtszeit Erhard Epplers im BMZ zurückreichenden deutschen Tradition, die die Frage globaler Verteilungsgerechtigkeit vergleichsweise stark hervorhebt und speziell im Rahmen internationaler Wirtschaftspolitik beansprucht, einen Standpunkt globaler Vernunft zu vertreten. Andererseits erfährt diese Gerechtigkeitskomponente durch ihre Verknüpfung mit dem auch auf die Ebene der unmittelbaren ökonomischen Lebensbedingungen der Menschen - besser gefasst mit dem englischen Begriff der Livelihood - rekurrierenden Sicherheitskonzept von Human Security eine grundlegende Veränderung.

Die Verbindung der Problemstellungen von Gerechtigkeit, angemessenen Lebensbedingungen und Human Security liefert die konzeptionelle Basis für zwei wesentliche Operationen. Erstens gibt sie dem permanenten Drang der Entwicklungshilfeagenturen nach Zugriff auf die gesellschaftliche Mikro-Ebene eine neue Rechtfertigungsbasis. Denn selbst auf lokaler Ebene identifizierte Ungerechtigkeiten werden zu einer Bedrohung von Sicherheit und müssen folgerichtig im Namen der Krisenprävention bearbeitet werden. Die zweite, damit eng verbundene Konsequenz ist nicht nur die Befähigung, sondern die Verpflichtung der beteiligten entwicklungspolitischen Institutionen zu einer unmittelbar politischen Aktion: sie sind es nun, die über die Frage der Gerechtigkeit zu

106 Dass sich die sichtbare Praxis nicht relevant geändert hat dürfte auch daran liegen, dass der Bereich auch ein gewisses Refugium für den noch vorhandenen Rest an Wachstums- und Modernisierungsideologie bietet. Das gängige Schema der 1960er und 1970er Jahre, nach dem Entwicklung über ihre ökonomischen Wirkungen zu gesamtgesellschaftlichem Wohlstand und damit zu Frieden beiträgt, wird zwar nicht prominent behauptet, ist aber unübersehbar. 
entscheiden haben. Während die Anmaßung der Befähigung zu einer solchen Entscheidung gegenüber früheren Phasen entwicklungspolitischer Intervention nichts substanziell Neues ist, stellt die sich aus der Verbindung mit Human Security ergebende Verpflichtung zu einer daraus folgenden Intervention sehr wohl einen qualitativen Bruch dar. Was früher Dritte-Welt-Solidaritätsgruppen, also einem vergleichsweise kleinen und stark eingeschränkt handlungsmächtigen Feld vorbehalten blieb, ist jetzt essenzieller Bestandteil staatlichen entwicklungspolitischen Handelns.

In allen drei strategischen Linien der Intervention wird das Bedürfnis deutlich, auf möglichst allen identifizierten gesellschaftlichen Ebenen unmittelbar präsent zu sein. Diese angestrebte Omnipräsenz ergibt sich aus dem Zusammenspiel eines spezifischen Verständnisses von Staatlichkeit, das die notwendige Voraussetzung zur Erlangung von Sicherheit und Frieden bilden soll, und dem ohnehin auf Mikrosteuerung und das unmittelbare Leben der zu Entwickelnden fokussierenden Konzept von Human Security, das sich angesichts eines solchen Gehaltes nicht zufällig zum konzeptionellen Ankerpunkt der deutschen Entwicklungspolitik, aber auch der deutschen Außenpolitik generell entwickelt hat.

\section{Abbildung 22: Zivilisatorisches Hexagon der staatlichen deutschen}

\section{Friedensentwicklung}

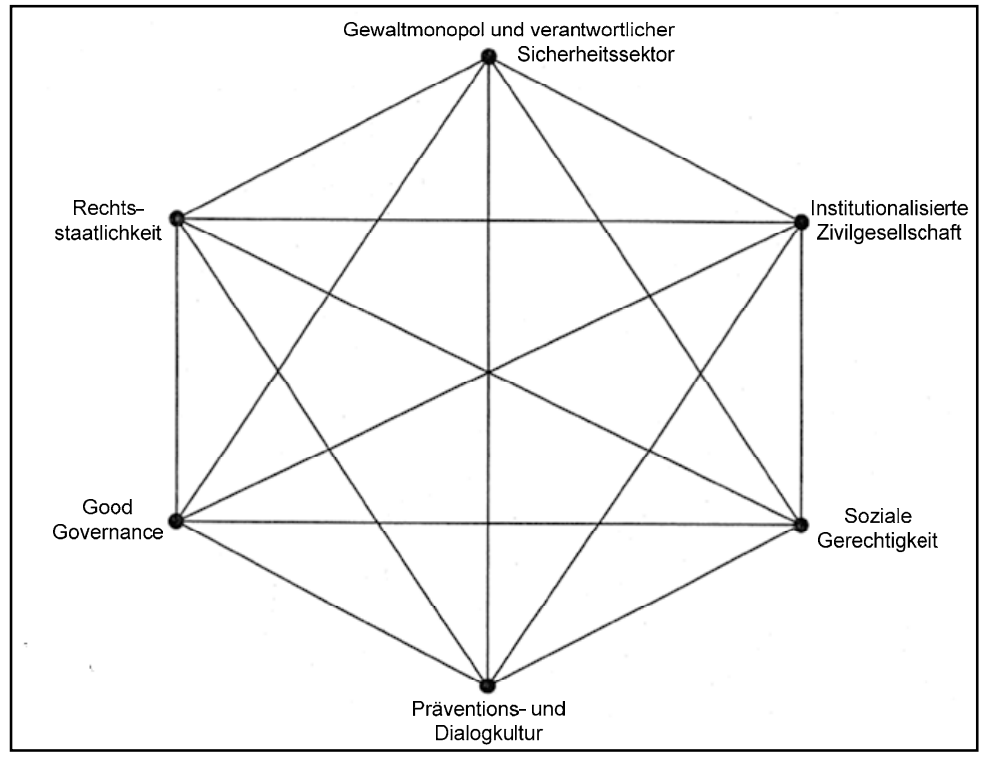


Eine sich durchziehende Besonderheit der deutschen Situation ist, wie erwähnt, der merkliche Einfluss der sozialen Bewegungen der 1980er Jahre, deren Glaube an die unumstößliche Vernünftigkeit der eigenen Ideen und ein damit einhergehendes, nach praktischen Umsetzungsmöglichkeiten suchendes Sendungsbewusstsein in den staatlichen Instanzen Einzug gehalten hat. Insbesondere die Vertreter/innen der sich selbst "pragmatisch« nennenden Friedensforschung, dem wissenschaftlichen Zweig der Friedensbewegung, spielen in der Neuausrichtung der deutschen Entwicklungspolitik, entgegen ihrer permanenten Larmoyanz, nicht genügend eingebunden zu sein, eine nicht zu unterschätzende Rolle. So ist besonders das Senghaassche Konzept des »Frieden-Machens« über den Ansatz des zivilisatorischen Hexagons (siehe Abbildung 4) in den deutschen Strategiepapieren deutlich sichtbar, wenngleich die einzelnen Komponenten leicht verschoben sind (siehe Abbildung 22).

An vier Eckpunkten zeigt sich das zivilisatorische Hexagon der staatlichen deutschen Friedensentwicklung gegenüber dem Entwurf von Senghaas weitgehend unverändert. Rechtsstaatlichkeit bleibt genauso substanziell wie das staatliche Gewaltmonopol, das um den spezifischen Schwerpunkt eines verantwortlich agierenden und zivil kontrollierten Sicherheitssektors ergänzt wird. Ebenso unverändert ist der traditionelle Schwerpunkt der sozialen Gerechtigkeit, wenngleich er in seiner Bedeutung, wie erwähnt, zu einer neuen Qualität von Immanenz gelangt ist, und das Feld der Konfliktkultur, das entlang der offiziellen Diktion allerdings mit dem etwas technisch anmutenden Doppelbegriff der »Präventions- und Dialogkultur« umschrieben wird.

An zwei Punkten kommt es hingegen zu wesentlichen Verschiebungen. So findet sich gemäß der zuvor in Abbildung 21 demonstrierten Achse der »Entwicklungsorientierung« Good Governance als zentrales Element im deutschen Hexagon wieder. Die Frage der demokratischen Partizipation, im Senghaasschen Ansatz an dem entsprechenden Punkt genannt, spielt eine demgegenüber untergeordnete und tendenziell subsumierte Rolle. Denn demokratische Partizipation hat, um die Absicherung gegenüber Risiken in Bezug auf die staatlichen Stabilität und zugleich die notwendige gesellschaftliche Angepasstheit zu gewährleisten, einen klar definierten Träger: die institutionalisierte Zivilgesellschaft. Dieser kommt zugleich aber auch jene Rolle des Verbindungsgliedes zwischen verschiedenen Instanzen und der systemischen Checks \& Balances $\mathrm{zu}$, die Senghaas mit den relativ unbestimmten Begriffen von »Interdependenzen und Affektkontrolle« umschrieben hat. Dieser Punkt ist im deutschen Ansatz in dieser institutionalisierten, damit professionalisierten wie organisierten Zivilgesellschaft operationalisiert, ebenso wie 
eine solche Zivilgesellschaft auch das Feld jener Akteure bildet, die die deutschen Vorstellungen von Demokratie transportieren.

Es geht bei der deutschen Intervention also um die Entwicklung, oder auch erst um die Errichtung eines demokratischen Sicherheitsstaates, wobei sowohl Demokratie als auch Sicherheit in einer vom kolloquialen Gebrauch abweichenden Bedeutung zu verstehen sind. So ist Sicherheit nicht nur im Sinne einer Garantie der Abwehr von physischer Bedrohung zu verstehen, sondern in seiner umfassenden, durch Human Security umrissenen Bedeutung, die auch ökonomische und soziale Fragen umfasst. Demokratie wiederum ist ausdrücklich nicht primär im Sinne eines parteipolitischen Wettbewerbs zu verstehen, sondern in der Errichtung einer Bürgergesellschaft, die vorwiegend auf die Involvierung einer institutionalisierten und auf vordefinierte Problemfelder Krisenprävention, Gender, Umwelt - fokussierenden Zivilgesellschaft aufbaut.

Ein solcher demokratischer Sicherheitsstaat umfasst damit in seiner idealtypischen Ausprägung soziale und totalitäre Komponenten. Diese zielen auf einen möglichst mikroskopischen, zugleich aber auch möglichst umfassenden Zugriff auf ein gesellschaftliches Ganzes. Es geht also nicht in erster Linie um Regulierung. Eine solche Regulierung würde einen hohen Grad an Selbststeuerung voraussetzen, und allein die wesentliche Bedeutung von Capacity-Building im deutschen Ansatz demonstriert, dass das Niveau der Selbststeuerung als nicht ausreichend bewertet wird. Dementsprechend geht es primär um Steuerung, verstanden im Sinne einer effizienten und wirksamen Mischung aus Disziplinierung, Ausrichtung und Kontrolle. Ein Ansatz, der aufgrund seiner auf allen Seiten überbordenden Dimensionierung das permanente Scheitern an einer wahrhaft gewaltigen Aufgabenstellung impliziert - damit aber natürlich auch seine permanente Notwendigkeit untermauert.

\section{Zwei Spielarten der Entwicklung von demokratischem Frieden}

Bevor ich in einer ersten Zwischenbilanz die Unterschiede zwischen den Zugängen der beiden Geber USA und Deutschland herausarbeiten will, die aus ihren strategischen und konzeptionellen Dokumenten sichtbar werden, ist es wesentlich, auf die Gemeinsamkeiten einzugehen. Obwohl die Fragestellung natürlich auf die Differenzen fokussiert, sind jene Komponenten, die beiden Gebern - und es ist anzunehmen, ebenso einem Großteil der restlichen Gebergemeinschaft, die im DAC zusammengefasst ist - gemeinsam sind, keineswegs nebensächlich. Das Ge- 
genteil ist der Fall: hätte ich die Fragestellung anders gelegt, etwa die Perspektive vom Blick auf die intervenierenden Agenturen auf diejenigen gelenkt, die von den Interventionen betroffen sind, wäre es vermutlich kaum möglich gewesen, diese Unterschiede so herauszuarbeiten. Ohne Zweifel hätten die Gemeinsamkeiten dominiert.

Kurz gefasst sind es drei Komponenten, die den strategischen Ansätzen beider Geber inhärent sind: Zunächst die gemeinsame Grundannahme, dass Entwicklung, insbesondere nachhaltige Entwicklung, eine unbedingte Notwendigkeit in der gegenwärtigen globalen Situation darstellt. Trotz aller unterschiedlichen Interpretationen im Detail, und auch trotz der vermeintlichen Logik, dass ein solches Denken für Entwicklungsagenturen wohl allein schon aus ihrem Eigeninteresse an Selbsterhaltung simple Notwendigkeit ist, scheint es mir dennoch wesentlich, diesen Punkt festzuhalten. Dies ist nicht unbedingt als Abgrenzung gegenüber entwicklungskritischen Strömungen gemeint, denn es ist einleuchtend, dass diese Strömungen keine relevanten Auswirkungen in Entwicklungsagenturen zeitigen. Allerdings ist diese Abgrenzung sehr wohl notwendig gegenüber neuen entwicklungspolitischen Strömungen, die die traditionelle Geberlandschaft in zunehmender Schärfe herausfordern. Insbesondere China trifft mit seinem Konzept einer sehr liberalen, wirtschaftsorientierten Intervention, die nicht nach Entwicklungs- oder sonstigen Kriterien schielt, sondern nach dem eigenen ökonomischen Vortei $1^{107}$, derzeit vor allem am afrikanischen Kontinent auf überaus positive Resonanz. Und ein solches Konzept von Entwicklungspolitik ist in der Tat ein Gegenentwurf zu dem, was die Gemeinsamkeit des grundlegenden Ansatzes faktisch aller im Rahmen des DAC versammelten Geber ausmacht.

Die zweite Gemeinsamkeit der beiden untersuchten Geber ist das Verständnis der neuen Dimension von Bedrohung, die von Entwicklungsländern ausgeht. Obwohl hier unterschiedliche Nuancen spürbar sind, Terrorismus und Proliferation von Massenvernichtungswaffen auf der einen Seite des Atlantiks stärker betont werden, während auf der an-

107 Diese Einschätzung ist keinesfalls moralisch zu werten, im Gegenteil: es ist das Wesen der Theorie liberaler Marktwirtschaft, dass die Orientierung am eigenen Vorteil zugleich den bestmöglichen Beitrag zum Gemeinwohl darstellt, oder wie Milton Friedman einmal sinngemäß gemeint hat: »Es gibt nur eine soziale Verantwortung von Unternehmen: Gewinne zu machen. « Umgelegt auf das chinesische Afrika-Engagement würde das heißen, dass die Verantwortung Chinas gegenüber der Entwicklung Afrikas darin besteht, von Afrika zu profitieren. Die geradezu euphorische Resonanz vieler afrikanischer Staaten gegenüber diesem Zugang kann für die traditionellen Geber als provozierende Herausforderung verstanden werden. 
deren der Flüchtlingsproblematik größeres Augenmerk zukommt, bleibt doch die zentrale Botschaft da wie dort die gleiche: Peripherien sind potenziell gefährlich, und das Potenzial ihrer Gefährlichkeit hat sich mit der neuen Unübersichtlichkeit nach Ende des Kalten Krieges überproportional gesteigert.

Die wohl überraschendste Gemeinsamkeit ist allerdings der strategische Gehalt der Antwort, die von beiden gegeben wird: denn sowohl die USA als auch Deutschland verlassen sich in ihrem jeweiligen Umgang mit dem neu entdeckten Phänomen der gefährlichen Peripherie auf strikt idealistische Konzepte: ein sich auf Demokratieförderung zuspitzendes State-Building einerseits, und ein auf idealtypische soziale, rechtliche und politische Faktoren rekurrierendes erweitertes Verständnis von Sicherheit und Stabilität andererseits.

An diesem Punkt setzen jedoch die Unterschiede zwischen den beiden Gebern ein, die ich im Folgenden anhand von fünf Punkten aufschlüsseln will, die zueinander in wechselseitigem Zusammenhang stehen.

(1) Historische Entwicklung. Wie aus dem historischen Abriss deutlich wird, verläuft die Entstehung und Veränderung des sicherheitspolitischen Feldes in den jeweiligen Apparaten äußerst unterschiedlich. Speziell die letzte Phase der entwicklungspolitischen Neuorientierung hin zur direkten Auseinandersetzung mit dem Phänomen bewaffneter Konflikte gestaltet sich nahezu konträr. Während die Veränderung in den USA von einem relativ elitären Kreis um politische Entscheidungsträger/innen und diesen nahe stehenden Wissenschaftler/innen ausging und als Konsequenz schwerwiegender außenpolitischer Niederlagen zu interpretieren ist, ist es in Deutschland eine Mischung aus einem sozialen Prozess, der durch die Integration sozialpolitischer Bewegungen und ihrer Ideen in den staatlichen Apparat gekennzeichnet ist, und dem Nachvollziehen einer internationalen Debatte, die, katalysiert durch einen Regierungswechsel, der zugleich als politischer Zeitenwechsel verstanden werden kann, für diese Neudefinition der Rolle von Entwicklungspolitik verantwortlich zu machen ist.

(2) Unterschiedliches Verständnis von Staat. Es ist folgerichtig, dass diese unterschiedlichen historischen Voraussetzungen in verschiedenen strategischen Ansatzpunkten resultieren. Vor allem am Umgang mit der Problemstellung peripherer Staatlichkeit lässt sich dies verdeutlichen. Der US-amerikanische Ansatz sieht im Staat den notwendigen Rahmen, der auf allen erforderlichen Ebenen, also national wie lokal, die institutionellen Bedingungen für politischen Wettbewerb bereitstellen soll. $\mathrm{Zu}$ - 
gleich ist dieser demokratische Wettbewerb auch von Seiten der daran Beteiligten $\mathrm{zu}$ forcieren, was idealtypisch gesehen wiederum auf die staatlichen Institutionen selbst in einer fragilen Situation verstärkend rückwirken soll.

Für den deutschen Zugang ist der Staat mehr als ein institutioneller Rahmen, wenngleich der Stärkung von Institutionen auch hier große Bedeutung zugemessen wird. Trotzdem ist der Staat nicht nur durch die Verankerung des Gewaltmonopols als einziger bewaffneter Akteur und damit als ultimativer Für- oder Absprecher politischer Legitimität verankert, sondern zugleich der konzentrierte Ausdruck eines organisch zu verstehenden Wechselspiels mit einer starken Gesellschaft, die in Form der institutionalisierten Zivilgesellschaft den zentralen mit dem Staat gemeinsamen Wert, die Entwicklungsorientierung, in allen Segmenten der Bevölkerung verankern soll.

(3) Unterschiedliches Verständnis von Zivilgesellschaft. Notwendige Konsequenz dieses grundlegend unterschiedlichen Verständnisses der Rolle des Staates ist eine verschiedenartige Interpretation der Rolle, die der Zivilgesellschaft zugedacht wird. Während für die USA, die sich an tendenziell liberalen Leitbildern von gesellschaftlicher Aktivität orientieren, die Zivilgesellschaft primär in der Rolle eines Service Providers in jenen Bereichen gesehen wird, wo sie gegenüber dem staatlichen Dienstleistungsangebot komparative Vorteile aufweist, und sich ihre demokratiepolitische Rolle - immer gesetzt den Fall, dass die US-Politik nicht auf einen Regimewechsel aus ist - auf die einer Watch-Dog reduziert, die sie zusammen mit den demokratisch orientierten Medien einzunehmen hat, geht der deutsche Ansatz bedeutend weiter. Hier wird Zivilgesellschaft zu der entscheidenden Methode, mit der stabile demokratische Verhältnisse in einer Gesellschaft zu operationalisieren sind. Während der Staat in diesem Verständnis neben der politischen Steuerung vor allem die Rolle des Service-Providers und des juristischen Schiedsrichters einnimmt, ist die Zivilgesellschaft der eigentliche Träger der Idee von Demokratie.

Dieses unterschiedliche Rollenbild resultiert auch in einer überaus divergenten Idee einer entwicklungspolitischen Schlüsselmethode, die vor allem im zivilgesellschaftlichen Sektor zum Einsatz kommt. Capacity-Building wird im Schema der US-Intervention primär als Transfer von Know-how und der Entwicklung von Managementkapazitäten verstanden, die in der Umsetzung von Dienstleistungsfunktionen, mitunter auch im Sinne politischer Dienstleistungen verstanden, relevant ist. Demgegenüber ist Capacity-Building im deutschen Ansatz eine immanent politische Operation, die nicht umsonst mehr und mehr unter dem 
treffenderen Begriff »Training « figuriert. Trainiert wird dabei zumeist nichts anderes als jene konzeptionellen Ansätze, die für die Entwicklungsagenturen selbst handlungsleitend sind. Angestrebt wird so eine Multiplikation von Friedensentwicklung, eine Durchdringung möglichst weiter Verästelungen der Gesellschaft in den Zielregionen mit Praktiker/innen, die von den von den Intervenierenden vorgegebenen Leitbildern und Handlungsleitfäden der Organisation friedlichen Zusammenlebens angeleitet sind.

(4) Konzeptioneller Unterschied: State-Building vs. Human Security. Konzeptionell zu fassen ist die Differenz entlang der beiden Konzepte von demokratischem State-Building, das für den US-Ansatz kennzeichnend ist, und Human Security, das den deutschen Zugang bestimmt. Das US-amerikanische State-Building beruht entscheidend auf der Idee der Kombination starker staatlicher Institutionen mit der Etablierung von Prozessen des politischen Wettbewerbs, und damit der wahrscheinlich effektivsten Möglichkeit der Intervention im Sinne einer repolitisierten Nachhaltigkeit. Das Konzept tendiert so zu einer liberalen Interpretation von Social Engineering, das primär auf den Mechanismus der Selbstregulierung von Akteuren vertraut, immer auf Basis der Grundannahme eines gegebenen Bedürfnisses nach Demokratie.

In der deutschen Interpretation von Human Security offenbart sich der Drang nach mikroskopischer Kontrolle von als gefährlich definierten Peripherien. Human Security impliziert so im Gegensatz zum liberalregulativ wirkenden State-Building eine tendenziell totalitäre Vorstellung gesellschaftlicher Transformation. Die Bedrohung menschlicher Sicherheit ist gleichzusetzen mit einem Versagen jener Techniken der Selbststeuerung, die ein demokratisches Zusammenleben auf substaatlicher Ebene garantieren würden. Die Etablierung möglichst präziser Systeme sozialer Kontrolle - natürlich mit der implizit oder explizit vorhandenen Möglichkeit der Überführung einer solchen Kontrolle in ein System der Selbstregulierung, wie es der Ansatz von Nachhaltigkeit verlangt - ist daher die notwendige Konsequenz.

(5) Regulation vs. Disziplin und Kontrolle. Führen wir die verschiedenen Komponenten zusammen zeigt sich, dass sich die sicherheitspolitische Entwicklungszusammenarbeit der USA tatsächlich durch ein gouvernementales Verständnis von Regierung auszeichnet. Direkter Zugriff wird zumeist auf gesellschaftliche Eliten beschränkt, der Glaube an das Ausreichen der Gewährleistung institutioneller Rahmenbedingungen offenbart Vertrauen in die Fähigkeiten zur Selbstregulierung seitens der ge- 
sellschaftlichen Triebkräfte. Es geht primär darum, diejenigen Akteure, die als relevant und politisch wünschenswert identifiziert werden, in geeigneter Weise in einer abgrenzbaren Arena des Wettbewerbs zu platzieren. Obwohl sich Elemente souveräner Rationalität finden, sind diese zumeist in begrenzter Form definiert. Natürlich braucht es stabile staatliche Institutionen, denn sie sind es, die den Rahmen der ohnehin zu erwartenden demokratischen Entfaltung der Gesellschaft zu gewährleisten haben - wiewohl sie diese Entfaltung nicht selbst hervorbringen. Zugleich sind auch sie es, denen, zusammen mit den Störfeuern antidemokratischer, also feindseliger Kräfte, die Verantwortung bei einem Scheitern dieses Konzeptes zukommt.

Demgegenüber ist das Vertrauen in eine gouvernementale Selbstregulierung der demokratischen Akteure im Rahmen des deutschen $\mathrm{Zu}$ ganges nur in äußerst eingeschränktem Maße zu finden. Im Versuch des Zugriffs auf die Gesamtgesellschaft, sei es über die Institutionen des Staates oder die Institutionen der Zivilgesellschaft, zeigt sich vielmehr die Grundtendenz der Disziplin, die, gepaart mit Kontrollmaßnahmen, deren Notwendigkeit das Konzept der Human Security mit sich bringt, die Steuerung gesellschaftlicher Transformation gewährleisten soll. 



\title{
Sri Lanka - Friedensentwicklung im „Protracted Conflict»
}

\author{
»Donor assistance was an integral part of the \\ construction of this fragmented state. Right \\ throughout the post-independence period, donor \\ support was never apolitical.«
}

(Sunil Bastian)

Eingangs habe ich meine Entscheidung, Sri Lanka als Fallstudie herauszuarbeiten und darzulegen, mit der besonderen Relevanz begründet, die der sri-lankische Konflikt für den Prozess der Verbindung von »Entwicklung« und »Sicherheit» hat. Das und in welcher Weise Sri Lanka diese Bedeutung tatsächlich zukommt, hoffe ich im folgenden Abschnitt zeigen zu können. Zuvor möchte ich aber kurz auf die Frage eingehen, warum Sri Lanka für den Bereich des entwicklungspolitischen Peacebuilding so populär werden konnte. Es sind vermutlich fünf Komponenten, die in jeweils unterschiedlicher Zusammensetzung für die meisten der heute aktiven Personen und Institutionen im Zuge ihrer Interventionsentscheidung bestimmend waren ${ }^{1}$; und für sich neu Engagierende,

1 Der Faktor Zufall ist, obwohl er in der Aufzählung nicht angeführt wird, ohne Zweifel ebenfalls relevant. So geht das österreichische Peacebuilding-Engagement in Sri Lanka - ein im Vergleich mit den großen in dem Bereich aktiven Gebern und Projekten minimal dotiertes Dialogprojekt, das vom der Galtung-Schule zuzurechnenden Wiener NGO Institute for Integrative Conflict Transformation and Peacebuilding (IICP) betreut wird - auf die guten persönlichen Kontakte eines ehemaligen srilankischen Botschafters in Wien und derzeitigen Spitzenpolitikers, Karu Jayasuriya, mit der ehemaligen österreichischen Außenministerin Benita Ferrero-Waldner zurück. Ohne die gute persönliche Beziehung dieser bei- 
neben den mittlerweile beträchtlichen Geldsummen, die für Peacebuilding ausgeschüttet werden, höchstwahrscheinlich immer noch relevant sind: ${ }^{2}$

(1) Zunächst handelt es sich bei dem in den frühen 1980er Jahren aufgenommenen Kampf der LTTE - es sei schon an dieser Stelle betont, dass dies keinesfalls den Beginn bewaffneter Konflikte im unabhängigen Sri Lanka markiert, wenngleich andere bewaffnete Kämpfe, wie der blutige Aufstand der JVP im Jahr 1971, nicht so stark im Bewusstsein der internationalen Öffentlichkeit verankert sind - um einen Konflikt, der mit dem gängigen Instrumentarium des Kalten Krieges nicht zu interpretieren ist. Diese Herausforderung führt zu einer vergleichsweise frühen Anwendung alternativer Analyse- und Interventionskonzepte, was etwa die Geschichte von International Alert demonstriert, die in ihren frühen Jahren untrennbar mit Sri Lanka verbunden ist. Eine der beiden Vorläuferorganisationen von International Alert, das Standing International Forum on Ethnic Conflict, Development, and Human Rights (SIFEC), wurde unter starker sri-lankischer Beteiligung gegründet, das erste Länderprogramm von International Alert konzentrierte sich denn auch auf Sri Lanka.

(2) Diese frühe Popularität liegt vermutlich auch darin begründet, dass die aufständische Partei, die LTTE, mit einem rationalen Analyseinstrumentarium gut zu greifen war und sich in ihrer politischen Ausrichtung von Beginn an stark danach ausrichtete. Das Unabhängigkeitsprogramm der LTTE mag für den sri-lankischen Staat ebenso wie für einige der relevanten internationalen Akteure eine nachhaltige Provokation darstellen, im Gegensatz zu vielen anderen aufständischen Bewegungen, die zu gleicher Zeit oder wenig später aktiv sind - etwa im afrikanischen Kontext - ist es nicht nur leicht zugänglich, sondern auch gut nachvollziehbar. Der Diskurs des bewaffneten Kampfes der LTTE bewegt sich von Beginn an entlang der international vorgegebenen Linien. Die Frage ist also nur, ob die LTTE auf diplomatischer Ebene als Mitspieler akzeptiert wird, das diskursive Regulativ dazu beherrscht sie nicht nur, sondern bedient es auch.

den hätte diese Intervention, die auf Wunsch Jayasuriyas zustande kam, vermutlich nicht stattgefunden.

2 Der Abschnitt zu Sri Lanka schließt zeitlich mit dem Sommer 2007 ab. Die danach einsetzenden Entwicklungen, insbesondere die bewaffnete Eskalation des Konfliktes und die damit einhergehende massive Einschränkung des Betätigungsraumes internationaler Organisationen durch die srilankische Regierung (wovon insbesondere die Berghof Foundation betroffen ist) sind somit nicht mehr enthalten. 
(3) Von seinem Ausbruch an wird dem bewaffneten Konflikt großes Potenzial für eine vergleichsweise rasche erfolgreiche Befriedung zugebilligt. Der Krieg wird faktisch ständig von Verhandlungsbemühungen begleitet und sogar zeitweise unterbrochen (Uyangoda 2005: 308ff.), die im Abstand von einigen Jahren, also absehbaren Zeiträumen, die Möglichkeit einer friedlichen Lösung als naheliegend erscheinen ließen. Speziell der jüngste Waffenstillstand, der im März 2002 geschlossen wurde und formell immer noch in Kraft ist, hat zu einer wahren Invasion von Peacebuilding-Expert/innen und einer starken Zunahme von für diese vorgesehenen bilateralen Programmschienen und Projekten geführt, die vermutlich auch nach dem Neuausbruch der Kämpfe weiterlaufen werden.

(4) Ein zurzeit besonders relevanter Faktor, der allerdings nur zeitlich begrenzt wirksam bleiben wird, hängt mit dem Tsunami zusammen, der Sri Lanka am 26. Dezember 2004 traf und zirka 30.000 Tote und maßlose Zerstörungen hinterließ. Die Katastrophe löste eine Welle internationaler Hilfe aus, die alle bislang bekannten Maßstäbe in den Schatten stellte. Der Zusammenhang der Tsunami-Hilfen mit dem srilankischen Konflikt wird in der Folge noch zur Sprache kommen, im Zusammenhang mit der Popularität von Sri Lanka als PeacebuildingDestination ergibt sich seine Relevanz aus einem verblüffend einfachen ökonomischen Faktum: Nachdem die meisten der Hilfsgelder und der davon finanzierten Wiederaufbauprojekte mittlerweile ihrem Ende entgegengehen, sind zahlreiche Akteure im Tsunami-Wiederaufbau, vor allem aus dem zivilgesellschaftlichen Bereich, zu einer programmatischen Neuorientierung gezwungen. Dies gilt sowohl für jene internationalen NGOs, die im Zuge der Tsunami-Hilfe Präsenzen in Sri Lanka aufgebaut haben und diese beibehalten möchten, als auch für die zahlreichen sri-lankischen NGOs, die nun vor der Herausforderung stehen, sich in einer nach Auslaufen der Tsunami-Nothilfe zuspitzenden Situation im Wettbewerb um internationale Finanzierungen zu behaupten. Für beide Gruppen bildet der Peacebuilding-Bereich in seinen Verzweigungen eine logische Alternative zur bisherigen Wiederaufbau-Tätigkeit, umso mehr, als jene Organisationen und Initiativen, die ihre Hilfstätigkeit im Osten/Nordosten umgesetzt hatten, auf besondere Kompetenzen in einer höchst konfliktrelevanten Region verweisen können. Durch die vorhandenen Arbeitsbeziehungen vieler der NGOs mit den internationalen Gebern geraten letztere in eine nicht zu unterschätzende Drucksituation, da sie mit Lobbying und einer Fülle von Projektvorschlägen eingedeckt werden, die jetzt um konfliktspezifische Problemstellungen kreisen. 
(5) Schließlich, und dieser Faktor ist keinesfalls zu unterschätzen ${ }^{3}$, ermöglicht Sri Lanka Peacebuilding-Intervention in einer Annehmlichkeit, die die meisten anderen Konfliktregionen der Welt missen lassen. In kaum einer anderen Region ist ein bewaffneter Konflikt so unmittelbar und gleichzeitig so risikoarm zu analysieren und zu bearbeiten wie in Sri Lanka. Die meisten Fäden laufen in der Hauptstadt Colombo zusammen, die - von einzelnen Zwischenfällen abgesehen - für die ExpatCommunity kaum nennenswerte persönliche Risiken birgt. Gebiete, in denen gekämpft wird, sind zumeist klar umgrenzt, und wo sonst können jene, die in die riskanteren Zonen vorzudringen haben, auf die Unterbringung in gut ausgebauten All-Inclusive-Beach-Ressorts zählen, eine Situation, wie sie etwa in Trincomalee an der volatilen Ostküste vorzufinden ist. Dieser Sri Lanka eigene Krieg-/Frieden-Dualismus, der eine eigenwillig zynische »On the ground«-Realität kreiert, trägt in relevantem Maße zur Bedeutung des Landes in den internationalen Bemühungen um Friedensentwicklung bei.

Diese spezifischen Bedingungen garantieren auf subjektiver Ebene eine permanente Relevanz der Problematik des bewaffneten Konfliktes bei den in Sri Lanka aktiven Gebern. Zugleich ist diese Relevanz nur vor dem Hintergrund eines historischen Prozesses zu verstehen, den ich im ersten Abschnitt dieses Teils nachzeichnen will. Denn alleine die Präsenz und Intensität der drei großen bewaffneten Konflikte, die die Insel im Verlauf der letzten vier Jahrzehnte prägten, war dafür keine ausreichende Bedingung. Vor dem Hintergrund des historischen Kontextes werde ich in einem zweiten Abschnitt die konfliktrelevanten entwicklungspolitischen Interventionen der USA und Deutschlands diskutieren und sie mit den allgemeinen strategischen Linien abgleichen, die ich zuvor herausgearbeitet habe. Wenngleich es natürlich vermessen wäre, anhand dieses einzelnen Fallbeispiels die allgemeine Frage zu beantworten, wie weit sich diese strategischen Linien in der Implementation wiederfinden, sollten sich doch interessante Aspekte finden, die zu einer Verfeinerung der Schlussfolgerungen, die sich aus dem konzeptionellen Vergleich ergeben, beitragen können.

3 Die meisten der von mir auf diese Frage angesprochenen sri-lankischen Kolleg/innen hoben diesen Punkt hervor. 


\section{Hilfe zum Frieden, Hilfe zum Krieg? Der sri-lankische Konflikt im Kontext internationaler Interventionen}

Ziel des folgenden Überblicks ist es, die entwicklungspolitischen Interventionen der USA und Deutschlands, die ich im nächsten Abschnitt analysieren will, in einen weiteren Rahmen einzubetten. $\mathrm{Zu}$ diesem Zweck will ich nicht nur einen historischen Abriss der entwicklungspolitischen Interventionen in Sri Lanka geben, sondern mich vorwiegend darauf konzentrieren, wie diese Interventionen wahrgenommen und analysiert werden, welche Bruchlinien sich im Selbstverständnis der Geber und in ihrer Außenwahrnehmung durch die wesentlichen politischen Kräfte in Sri Lanka auftun und wie sich vor diesem Hintergrund die gegenwärtige Situation gestaltet, im Rahmen derer sich jene Praktiken vollziehen, auf die ich mich im Anschluss konzentrieren will.

Ebenso wichtig wie das Herausstreichen der Zielsetzungen des folgenden Abschnitts ist eine Erwähnung der zu treffenden Einschränkungen. Angesichts der Tatsache, dass den meisten Policy-Papers, die sich mit entwicklungspolitischen Interventionen in Sri Lanka auseinandersetzen, eine kurze, zumeist oberflächliche und manchmal auch überaus uninformierte Analyse der bewaffneten Konflikte vorangestellt wird (für ein derartiges Beispiel vgl. Paffenholz 2004), ist es wichtig zu betonen, dass es im Folgenden genau darum nicht gehen wird. Weder habe ich vor, eine historische Darlegung der drei bewaffneten Konflikte zu geben, die in Sri Lanka seit 1971 stattgefunden haben, noch will ich den ohnehin in großer Zahl vorliegenden Konfliktanalysen eine weitere hinzufügen. Soweit für das Verständnis der zentralen Prozesse im entwicklungspolitischen Feld notwendig, werde ich mich auf historische Daten beziehen sowie die für die Akteure relevanten Konflikteinschätzungen diskutieren, auf eine Auseinandersetzung auf dem Gebiet der srilankischen Geschichte möchte ich mich jedoch in keinem Fall einlassen.

Eine solche Einschränkung hat nicht nur pragmatische Gründe. Geschichte, und dieser Aspekt ist auch für die internationalen entwicklungspolitischen Akteure von spürbarer Relevanz, ist in Sri Lanka selbst ein unmittelbares Schlachtfeld des Krieges, mit wahrscheinlich sogar größerer Bedeutung als manche der physischen Schlachtfelder, in denen nicht diskursiv, sondern bewaffnet gekämpft wird. »For many Sri Lankans $[\ldots]$ history just seems to be a very >present reality« (Frerks/Klem 2005: 30), und es wird von allen Seiten viel dazu getan, damit das so bleibt. So widmet der Doyen der singhalesisch dominierten srilankischen Geschichtswissenschaft, K.M. de Silva, ganze 600 der 700 Seiten seines voluminösen Standardwerks über die Geschichte der Insel 
der vorkolonialen und kolonialen Periode, nur 100 Seiten sind der Geschichte des unabhängigen Staates gewidmet (de Silva 2005). Ziel ist die Konstruktion einer Erzählung, die ethnisch begründete Ansprüche an Territorium und Macht über historische Prozesse $\mathrm{zu}$ argumentieren sucht. $^{4}$

Diese Geschichts-Obsession ist von kritischen Sozialwissenschaftler/innen speziell seit Mitte der 1990er Jahre immer wieder angegriffen worden. Der 1995 von Pradeep Jeganathan und Qadri Ismael herausgegebene Sammelband »Unmaking the Nation« umfasst verschiedene Beiträge, die mit der Idee von Nationalität und ihrem Zusammenhang mit Geschichte provokativ dekonstruktivistisch umgehen, und zwar mit allen vier zur Debatte stehenden Nationalismen: dem sri-lankischen, dem eng damit verknüpften singhalesischen, dem tamilischen und auch dem mehr religiös erzählten Versuch der Herausbildung einer kollektiven Identität der sri-lankischen Moslems (vgl. dazu Ismael 1995). David Scott (1995: 12, vgl. auch Scott 1999) fasst die grundlegende These dieser Auseinandersetzung zusammen, »that history ought to be taken as what shall be thought «, eine Notwendigkeit, die sich daraus ergebe, dass die mühsame und zugleich für die Aufrechterhaltung gegebener Machtverhältnisse

4 An dieser Stelle will ich mit kurzen Bemerkungen auf einige historische Arbeiten zur Geschichte Sri Lankas verweisen: Éric Meyer (2003) liefert eine betont sachlich und objektiv gedachte Einführung, die für einen Überblick über den geographischen, sozialen und politischen Kontext überaus brauchbar ist. Die Position eines qualifizierten Außenstehenden mag dafür alles andere als ein Nachteil sein. Der schon angesprochene K.M. de Silva hat mit »Reaping the Whirlwind « die wohl einflussreichste Geschichtsdarstellung vorgelegt, die der offiziellen Sichtweise des srilankischen Staates, und damit der oft zitierten »singhalesischen Mehrheitsposition« am nächsten kommt (de Silva 1998). Für der LTTE nahestehende, und damit stark mit de Silva kontrastierende Darstellungen der politischen Ereignisse seit 1983 sind die ausführliche und betont sachlich gehaltene Arbeit des kürzlich verstorbenen LTTE-Chefverhandlers im Friedensprozess von 2001, Anton Balasingham (2004), oder die sehr persönlich gehaltene Erzählung seiner aus Australien stammenden Frau Adele (Balasingham 2003) nahezulegen. Eine hervorragende Dekonstruktion solcher ethnisch argumentierten Geschichtsdarlegungen hat Qadri Ismail (2005) vorgelegt. Die für die internationale Gemeinschaft wohl einflussreichste, informierte und auch zu den gängigen ethnisch argumentierten Erklärungsmustern bewusst kontrastiert gehaltene Arbeit ist im von Jonathan Goodhand (2001) erarbeiteten ersten Strategic Conflict Assessment nachzulesen, das aufgrund seiner praktischen Bedeutung noch Erwähnung finden wird. Schließlich ist noch auf die aus einem Nationalismuskritischen Ansatz stammenden anthropologischen Arbeiten von Michael Roberts zu verweisen, die jedoch dem an sich problematischen Ansatz folgen, eine möglichst objektive geschichtliche Schilderung der Entwicklung der gegenwärtig dominanten Nationalismen liefern zu wollen. 
unabdingbare Produktion und Reproduktion ethnisch konstruierter kollektiver Identitäten als gezieltes Projekt (ebd.: 21) zu verstehen sei.

Was an dieser Stelle nach einem zu weiten Ausholen in Bezug auf die wissenschaftliche Auseinandersetzung mit Geschichte und Nation in Sri Lanka wirken mag, hat in der Tat eine immanent praktische Bedeutung. Es ist erwähnenswert, dass die wenigsten der in dieser kritischen Debatte Aktiven (mit der bemerkenswerten Ausnahme von Sumanasiri Liyanage) zu den bevorzugten Dienstnehmer/innen der internationalen Gebergemeinschaft gehören - was angesichts der Tatsache, dass ein Gutteil der sozialwissenschaftlichen Forschung in Sri Lanka von ODAGeldern finanziert wird, als Ausdruck einer politischen Haltung zu interpretieren ist. Diese politische Haltung bedeutet, dass ethnische Kollektive von der Gebergemeinschaft nicht nur als sri-lankische Realitäten angenommen werden, sondern dass die Existenz dieser ethnischen Kollektive sogar eine essenzielle Voraussetzung für die Interventionen der Geber darstellt, paradoxerweise gerade dann, wenn die Intervention als »konfliktsensibel« verstanden wird.

Obwohl ich nicht nur einen historischen Überblick, sondern auch die in Policy-Papers vorherrschende Unsitte eines »Konflikt-Mapping«, in dem in einem Kurzüberblick die wesentlichen Parteien eines bewaffneten Konfliktes und ihre Motivationen herausgeschält und damit in einer auch für Executives verdaubaren Form veranschaulicht werden sollen, vermeiden will, ist ein Kurzüberblick über die geographischen Gegebenheiten Sri Lankas in Anbetracht der Nachvollziehbarkeit meiner folgenden Ausführungen dennoch notwendig.

Die Darlegung spitzt sich angesichts der nationalistischen Erzählung des Konfliktes zwischen den Liberation Tigers of Tamil Eelam (LTTE) und der sri-lankischen Regierung (Government of Sri Lanka - GoSL) und der darauf aufbauenden Debatte um Minderheiten und Mehrheiten vorwiegend auf demographische Elemente zu: von den derzeit nicht ganz 20 Millionen Einwohnern werden etwa $80 \%$ als singhalesisch klassifiziert $^{5}, 8 \%$ als muslimisch, und jeweils $5 \%$ werden den beiden tamilischen Gruppen zugerechnet, den ceylonesischen Tamil/innen und den indischen Hochland-Tamil/innen, die Ende des 19. Jahrhunderts als Plantagen-Arbeiter/innen angeworben wurden. ${ }^{6}$

5 Eine solche Klassifizierung ergibt sich auch insofern, als sie bei Volkszählungen eingefordert, also gewissermaßen erzwungen wird.

6 Diese und folgende Daten nach Fischer Weltalmanach von 2007. Angesichts der Tatsache, dass die letzte die ganze Insel umfassende Volkszählung Anfang der 1980er Jahre durchgeführt wurde und die aktuellen Daten in Bezug auf die von der LTTE kontrollierten Gebiete von Schätzungen ausgehen, sind die Zahlen mit Vorsicht zu genießen. 
Abbildung 23: Politische Karte von Sri Lanka

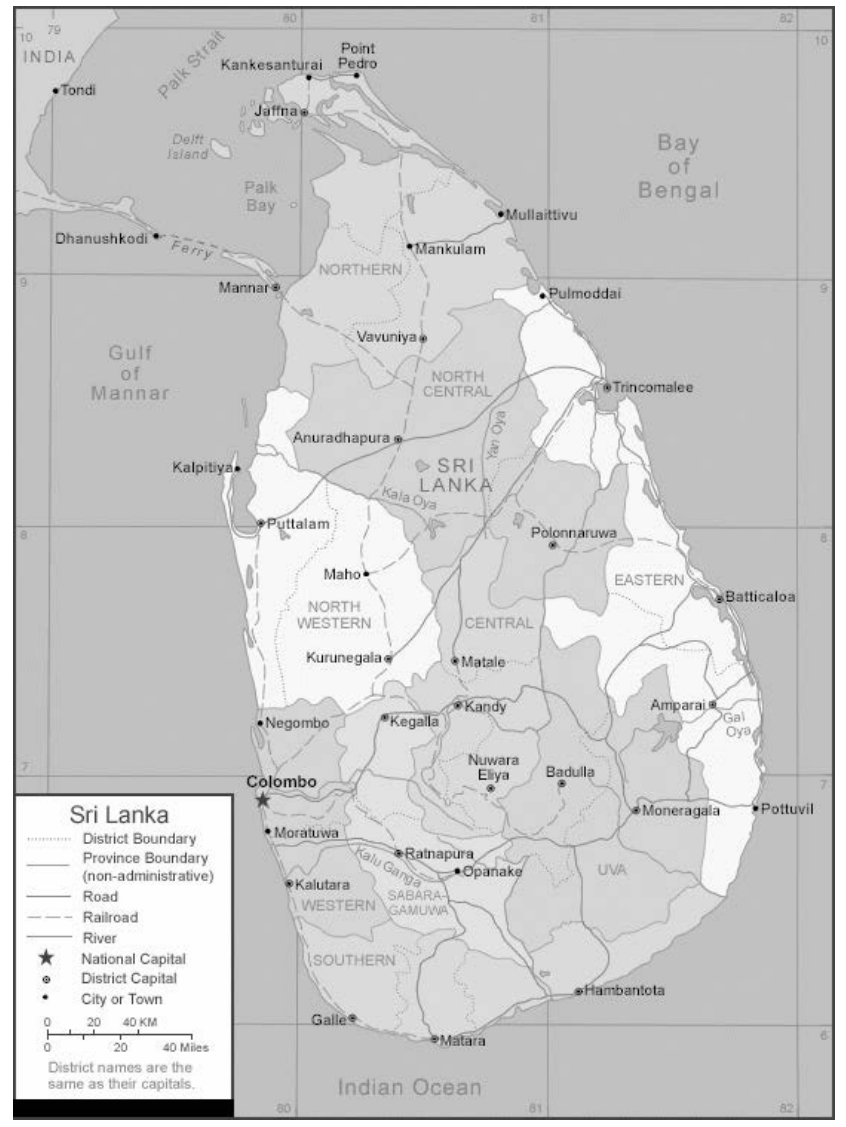

Entscheidend bei diesen Bevölkerungszahlen sind die Siedlungsgebiete der jeweiligen Gruppen, deren Zuordnung einer der zentralen Streitpunkte der historischen Dimension des Konfliktes ist. Das singhalesische Siedlungsgebiet liegt im Süden der Insel und erstreckt sich in etwa bis in die Gegend um die alte historische Hauptstadt Anuradhapura. Der Norden, mit dem Zentrum Jaffna, ist tamilisch dominiert, ebenso die Ostküste, wo aber auch ein Gutteil der moslemischen Bevölkerung ansässig ist. Die beiden mit diesem Territorium überlappenden Provinzen, die Northern und die Eastern Province, die erst seit kurzem nach einer kontroversiellen Entscheidung des Höchstgerichtes getrennt wurden, bilden, grob gesprochen, das von der LTTE als »Tamil Eelam« beanspruchte Gebiet. Die zweite tamilische Bevölkerungsgruppe, die vorwiegend aus den Nachkommen von in Indien angeworbenen Plantagenarbeiter/innen besteht und im zentralen Hochland um die Zentren Nuwara 
Eliya und Hatton verortet ist, ist im Vertretungsanspruch der LTTE nicht mit eingeschlossen. ${ }^{7}$

Das von der LTTE tatsächlich kontrollierte Gebiet beschränkt sich derzeit, nach dem Verlust von Jaffna im Dezember 1995 und den Rückschlägen an der Ostküste, wo sie im ersten Halbjahr des Jahres 2007 alle Basen an die sri-lankische Armee verliert, auf das Gebiet des so genannten »Vanni«, das nach Norden zur unter Armee-Kontrolle stehenden Jaffna-Halbinsel abgegrenzt ist und nach Süden in etwa bis zur Verbindung von der Halbinsel Mannar (die selbst momentan von der Regierung kontrolliert wird und stark umkämpft ist) über den zentralen Knotenpunkt der Demarkationslinie, Vavuniya, bis nach Pulmoddai reicht. Die Hauptstadt des LTTE-Gebietes, Kilinochchi, befindet sich im Nordwesten der Zone, während das vom Tsunami stark in Mitleidenschaft gezogene Mullaitivu nicht nur als zentrale Basis der LTTE-Marine, der Sea Tigers, dient, sondern auch den wesentlichen Hafen der Zone bildet. ${ }^{8}$ In dem von ihr kontrollierten Gebiet ist es der LTTE gelungen, einen verhältnismäßig gut funktionierenden Quasi-Staat aufzubauen, der über ein eigenes Justiz-, Polizei-, Steuer- und Meldesystem verfügt (vgl. Stokke 2006).

Alle drei großen Städte an der Ostküste repräsentieren für sich komplexe Problemstellungen. Dem malerisch an Lagunen gelegenen Trincomalee kommt dabei eine Schlüsselrolle zu, spiegelt es doch alle Facetten des sri-lankischen Konfliktes in komprimierter Form wider. Alle drei großen Bevölkerungsgruppen, Singhales/innen, Tamil/innen und Moslems, leben in etwa gleichen Anteilen innerhalb der Stadt, und Zuspitzungen auf Ebene des »großen« Konfliktes finden immer wieder

7 Obwohl historisch keine Verbindung zum tamilischen Unabhängigkeitskampf besteht, gewinnt die LTTE in den letzten Jahren im Hochland politischen Einfluss, was auch mit einer sich aus einem gegenüber Tamil/innen bestehenden Generalverdacht speisenden Diskriminierung durch den sri-lankischen Staat zu erklären ist, die sich vor allem in repressiven Maßnahmen äußert. Im Zuge der Entkolonialisierung war die Frage, ob und inwieweit den Hochland-Tamil/innen überhaupt Bürgerrechte zuzugestehen sein, eine wesentliche Debatte. Es ist in diesem Zusammenhang ein pikantes Detail, dass der vermutlich international bekannteste Sri Lanker ein noch dazu mit einer Inderin verheirateter Hochland-Tamile ist: der Cricket-Spieler Mutthia Muralitharan, der als bester Spin-Bowler in der Geschichte des Sports gilt und, trotz seiner Herkunft, im ganzen Land als Idol verehrt wird.

8 Auch an der Westküste der von der LTTE kontrollierten Zone befinden sich Hafenanlagen, die vor allem dem ausgedehnten Schmuggel mit der nur wenige Kilometer entfernten indischen Provinz Tamil Nadu dienen. Regelmäßige Seegefechte in der Palk Strait deuten darauf hin, dass hier die zentrale Nachschublinie der LTTE verläuft. 
ihre Entsprechung in gewaltsamen Auseinandersetzungen in der Stadt selbst. Zugleich ist Trincomalee auch der Brennpunkt der internationalen Interessen, was durch die hervorragende Eignung der Lagunen als Tiefseehafen begründet ist. Tatsächlich wird Trincomalee bereits seit dem 12. Jahrhundert als Hafen genutzt, und gelegentliche Verwendungen durch die US-Marine haben zu Zeiten des Kalten Krieges wiederholt indisches Misstrauen auf sich gezogen (vgl. Somasundaram 2005: 141). Zudem wird den USA auch ein Interesse an bestehenden Ö1Lagerkapazitäten unterstellt, was allerdings nicht nur beständig von diesen dementiert wird, sondern auch angesichts des schlechten Zustandes der 1945 errichteten Tanks und der Tatsache, dass den USA mit Singapur ein zentraler Hafen in einem politisch äußerst stabilen Umfeld zur Verfügung steht, einigermaßen absurd anmutet. ${ }^{9}$

Das weiter südlich gelegene Batticaloa ist wiederum vorwiegend tamilisch besiedelt und die wesentliche politische Hochburg der LTTE an der Ostküste. Nach der Abspaltung zahlreicher im Osten stationierter Kräfte unter Colonel Karuna wird die Stadt in einer informellen und fragilen Einheit von Karunas Organisation, der TMVP, und der srilankischen Armee kontrolliert. Das dritte regionale Zentrum in der Ostprovinz ist schließlich das hauptsächlich moslemische Ampara, das von der LTTE aber ebenfalls als Teil des tamilischen »Homeland « verstanden wird. Spätestens seit der Vertreibung der moslemischen Bevölkerung aus Jaffna nach dessen Eroberung durch die LTTE im Oktober 1990 ist das Verhältnis zwischen den meisten moslemischen Gruppierungen und der LTTE angespannt, immer wieder kommt es zu auch gewalttätigen Auseinandersetzungen.

Bedingt durch die vom Krieg hervorgerufenen Fluchtbewegungen und die sich durch einen permanenten Ausnahmezustand düster darstellende ökonomische Situation in Jaffna und anderen tamilischen Zentren weist die Hauptstadt Colombo ${ }^{10}$ mittlerweile einen substanziellen tamilischen Bevölkerungsanteil auf, der innerhalb des Stadtgebietes sogar an $50 \%$ heranreichen soll. Hier stellt der Bezirk Colombo 06, Wellawatte,

9 Tatsächlich zeigte Mitte der 1980er Jahre eine private US-Firma Interesse an der Sanierung der Öllagerkapazitäten und Errichtung einer Raffinerie in Trincomalee. Indien intervenierte massiv gegen ein solches Vorhaben und drängte die sri-lankische Regierung, den Auftrag an einen indischen Staatsbetrieb zu vergeben. In einem 1985 global ausgeschriebenen Tender erhielt schließlich eine Firma mit Sitz in Singapur den Zuschlag (vgl. Somasundaram 2005: 139ff.).

10 Der Regierungssitz befindet sich seit Anfang der 1980er Jahre in einem Vorort von Colombo, Sri Jayawardenapura, wo mit japanischen Geldern ein neues Parlamentsgebäude errichtet wurde. Trotz dieser formalen Einschränkung kann Colombo weiterhin als Hauptstadt bezeichnet werden. 
das tamilische Zentrum dar. Nationalistisch-singhalesische Kräfte verwenden diese Situation zur Untermauerung ihrer Argumentation, dass sie, obwohl objektiv die Mehrheit im Land, dennoch von Tamil/innen umkreist und damit eigentlich in einer Minderheitensituation wären, was Verteidigungsmaßnahmen geradezu herausfordern würde.

Der Beginn der internationalen entwicklungspolitischen Intervention ist bereits in der Periode der britischen Kolonialherrschaft auszumachen. Aus der Perspektive der Entstehung eines sri-lankischen Nationalbewusstseins entwickelt es sich schnell zu einem Nachteil, dass die damalige Kronkolonie Ceylon als Musterbeispiel einer erfolgreichen kolonialen Intervention gilt. Nach einigen landwirtschaftlichen Misserfolgen war 1860 die Einführung einer nachhaltig profitablen Teewirtschaft gelungen, die Gummiproduktion für den einsetzenden Automobil-Boom in Europa liefert ein zweites ökonomisches Standbein. Die wirtschaftliche Situation der Kronkolonie entwickelt sich in der zweiten Hälfte des 19. und am Beginn des 20. Jahrhunderts so gut, dass eine militant nach Unabhängigkeit strebende Nationalbewegung nicht aufkommen will. Neben der ökonomischen Situation sind dafür auch politische Schritte der britischen Kolonialmacht maßgeblich: schon 1833 wird mit den so genannten Colebrooke-Cameron-Reformen ein Verwaltungssystem in Kraft gesetzt, das als das liberalste aller Kolonien gilt und jede potenzielle Formierung einer antagonistisch orientierten Nationalbewegung untergräbt.

Zugleich versuchen die Reformen auch, mit einer ethnisch-kulturell begründeten administrativen Aufteilung der Insel zu brechen. Stattdessen wird die Administration einerseits vereinheitlicht und in fünf Provinzen organisiert, die einer strikt geographischen Aufteilung entsprechen sollen, andererseits wird vor diesem Hintergrund eine für diese Zeit bemerkenswert weitgehende Dezentralisierung der Machtbefugnisse verankert, die die zuvor nahezu absolute Macht des Gouverneurs beschränken und verschiedenen Gremien, die auch mit Ceylones/innen besetzt sind, Mitspracherecht einräumen. Damit wird bereits Mitte des 19. Jahrhunderts die koloniale Zivilverwaltung für Ceylones/innen geöffnet, was maßgeblich zur Durchsetzung von Englisch als der primären Sprache in der höheren Ausbildung beiträgt. 1937 wird mit der Donoughmore-Verfassung schließlich die Unabhängigkeit strategisch vorbereitet, indem ein System von Komitees eingerichtet wird, deren Mitglieder in allgemeinen Wahlen bestimmt werden.

Die durch den Zweiten Weltkrieg, in dem Ceylon als wichtige Basis der britischen Marine im Kampf gegen Japan dient (und auch von Japan 
militärisch angegriffen wird), etwas verzögerte Unabhängigkeit ${ }^{11}$ wird nach einer kurzen Zwischenphase als Dominion am 4. Februar 1948 gewährt, ohne dass darum gekämpft oder militant gestritten hätte werden müssen. Vielmehr hinterlässt Großbritannien sogar noch die erste Verfassung des unabhängigen Ceylon, die Soulbury Constitution von 1946, die bis zum Jahr 1972 in Kraft bleiben wird. Sie beruht auf dem Westminster-Modell und garantiert über nicht abänderbare Bestimmungen weitgehende Anti-Diskriminierungsbestimmungen (vgl. Marasinghe 2007: 110ff.), die oftmals ungenau als Minderheitenschutz bezeichnet werden.

Angesichts dieses gesteuerten Prozesses wird es auch nicht der Ceylon National Congress, die Nationalpartei der ceylonesischen Oberschicht, sein, der die Forderung nach Unabhängigkeit vorantreibt. Der antikoloniale Diskurs wird vielmehr von buddhistischen Kräften forciert, die seit dem späten 19. Jahrhundert eine viel zitierte »Wiedererweckung « erleben. ${ }^{12}$ Es ist ein Charakteristikum des buddhistischen Revivalismus in Sri Lanka, dass er nach einer kürzeren Phase unpolitischer Ausrichtung relativ bald beginnt, sich an der politischen Auseinandersetzung zu beteiligen. Das zentrale Thema ist der Nationalismus, der nicht ceylonesisch - also supra-ethnisch -, sondern singhalesisch verstanden wird. Und angesichts der im Süden des Landes weit verbreiteten Annahme, die Tamil/innen, verstanden als ethnische Gruppe, wären von der britischen Kolonialverwaltung systematisch bevorzugt worden ${ }^{13}$, ist es konsequent, dass der antikoloniale Nationalismus beginnt, sich rassistisch zu kodieren. Diese Erzählung wird Mitte der 1960er Jahre von der sich militant formierenden linksradikalen Bewegung um die Volksbefreiungsfront JVP aufgegriffen.

11 Ökonomisch hat Ceylon vom Zweiten Weltkrieg durch die angekurbelte Gummiproduktion nachhaltig profitiert.

12 Unzweifelhaft hängt dieser buddhistische Boom mit der Abwehr christlicher Missionsbemühungen zusammen. Inwieweit die Erweckungsbewegung selbst durch amerikanische und europäische Intellektuelle, vor allem die Theosophen, die über den US-amerikanischen Colonel Olcott zur fraglichen Zeit in den entsprechenden Debatten stark präsent sind, beeinflusst ist, ist heute eine immanent politische Auseinandersetzung, die am Selbstverständnis der buddhistisch-nationalistischen Kräfte rührt.

13 Eine bewusst neutral gehaltene Zusammenfassung liefert Robert I. Rotberg (1999: 5), der sich parallel zu seiner Failed-States-Forschung auch mit Fragen der Friedensentwicklung in Sri Lanka auseinandersetzt: »Within the largely agricultural and rural nation that emerged from British colonial rule, with thriving civil service, military, and commercial establishments, Tamils were more prominent and in greater numbers than predicted by their population percentages.« 
Die erste groß angelegte entwicklungspolitische Intervention endet also nur oberflächlich mit dem großen Erfolg der gesteuerten Unabhängigkeit einer ökonomisch erfolgreichen Musterkolonie. ${ }^{14}$ Gleichzeitig bereitet sie den Boden für jene Erzählungen, die schon wenige Jahre nach der Unabhängigkeit eine politische Spirale in Gang setzen, die in einem langjährigen blutigen Krieg münden werden. Paradoxerweise sind es gerade die wohlgemeinten politischen Maßnahmen ${ }^{15}$, die im Zusammenspiel mit der angenommenen positiven Diskriminierung ${ }^{16}$ einer so verstandenen ethnischen Gruppe ${ }^{17}$ für diesen Prozess verantwortlich sind.

Entsprechend der kolonialen Struktur bleibt auch die internationale Entwicklungshilfe im unabhängigen Ceylon zunächst auf den englischen Sprachraum beschränkt: neben Großbritannien sind es vor allem die USA und Kanada, die das Gros der internationalen Leistungen bestreiten, wobei ab Mitte der 1960er Jahre Deutschland als vierter großer Geber dazukommt. Diese vier Geber bestreiten die bilateralen ODA-

14 Gerne wird an dieser Stelle die Geschichte erzählt, wonach Lee Kuan Yew, der spätere langjährige Premierminister Singapurs, Ceylon bei einem Besuch im Jahr 1956 speziell in Hinblick auf seine gut ausgebaute Infrastruktur und seine erfolgreiche wirtschaftliche Entwicklung als Vorbild für Singapur bezeichnete.

15 Es muss erwähnt werden, dass singhalesische nationalistische Kräfte, auch entlang ihrer antikolonialen Ausrichtung, diese gut gemeinte Intention der legislativen antidiskriminatorischen Maßnahmen in Zweifel ziehen und von einer Verschwörung zwischen der britischen Kolonialverwaltung und einflussreichen Kräften aus der Vellalar-Kaste in Jaffna ausgehen, die ohne Zweifel eng mit den Kolonialbehörden kooperierten. Demgegenüber zeigt sich in den der Soulbury Verfassung vorangegangenen Debatten, dass ein nachhaltig wirksamer Schutz von Rechten der "Communities《 tatsächlich eine genuine Intention bei der Erarbeitung der Verfassung war (vgl. Marasinghe 2007: 118).

16 Der singhalesisch-nationalistische Diskurs stützt sich darauf, dass Tamil/innen, insbesondere aus Jaffna, das Gros der »einheimischen« Kräfte in der Kolonialverwaltung ausmachten. Dies war allerdings eher pragmatisch bedingt. Jaffna verfügte, vor allem bedingt durch die Errichtung eines methodistischen Colleges im Jahr 1830, über ein unvergleichlich großes Reservoir an für den Dienst in der Kolonialverwaltung Qualifizierten, die derart vehement auf den kleinen Arbeitsmarkt der britischen Bürokratie drängten, dass sie in der Folge auch in Singapur und Indien eingesetzt wurden.

17 Interessanterweise wird sich der beginnende tamilische Nationalismus erst relativ spät entlang eines ethnischen Diskurses formieren. Liyanage (2005: 141) weist darauf hin, dass das heute für die LTTE bestimmende Konzept des »Tamil Homeland « nicht im engeren Sinne ethnisch, sondern territorial, im Sinne von Siedlungsgebieten tamilischer Sprachgruppen, definiert ist. 
Leistungen im ersten Jahrzehnt nach der Unabhängigkeit faktisch im Alleingang. Während für Großbritannien die entscheidende Motivation jener Zeit die postkoloniale Transformation der noch kolonial geprägten Wirtschaftsbeziehungen ist, stehen für die USA auch geostrategische Interessen im Vordergrund. So gilt Sri Lanka nicht nur als Gegengewicht zum politisch tendenziell als unverlässlich eingestuften Indien (vgl. Bastian 2007: 33), sondern speziell in den 1960er Jahren durch seine Hafenanlagen und seine geographische Nähe zum ab 1965 zentralen US-Truppenstützpunkt im Indischen Ozean, Diego Garcia, auch als geostrategisch bedeutsam (vgl. Somasundaram 2005: 71ff.).

Die entwicklungspolitische Ausrichtung entspricht voll und ganz dem Trend jener Zeit. Vollzogen wird ein »top-down aid delivery model« (Burke/Mulakala 2005: 11), das auf den Staat als dem primären Entwicklungsmotor aufbaut und sich durch einen hohen Grad an Zentralisierung auszeichnet (vgl. Bastian 2007: 43). Zwar wird allgemeiner Budget-Support nur relativ sparsam eingesetzt, dafür wird umso mehr in staatlich gesteuerte Großprojekte investiert, die im Gesundheits-, Erziehungs- und Infrastrukturbereich angesiedelt sind. Angesichts der sich signifikant verbessernden Basisdaten werden diese Interventionen als überaus erfolgreich wahrgenommen. Der Erfolg erweist sich aber wiederum als zweischneidiges Schwert. So wird mit dem entwicklungsstaatlichen Ansatz nicht nur eine ohnehin schon überzentralisierte Struktur weiter zentralisiert. Es werden auch politische Entwicklungen negiert, die direkt in verschiedene gewaltsame Konflikte hineinführen sollten.

Zunächst gewinnt die von S.V.R.D. Bandaranaike angeführte sozialdemokratisch-nationalistisch orientierte Sri Lanka Freedom Party (SLFP) die Wahlen 1956 mit einem stark singhalesisch-nationalistisch gefärbten Programm. Nach der Regierungsübernahme im Juli 1956 beschließt die SLFP-Regierung den so genannten "Sinhala Only Act», der Singhalesisch als einzige offizielle Landessprache festlegt. Dies mündet im Jahr 1958 in schweren Unruhen, die nur mit der Verhängung des Ausnahmezustandes unter Kontrolle gebracht werden können. Parallel spitzt sich auch die ökonomische Situation zu. Die SLFP vollzieht von 1956 bis 1965 eine von Verstaatlichungen begleitete ${ }^{18}$ ausgabenfreudige Wirtschaftspolitik, die nach der Regierungsübernahme durch die konservative United National Party (UNP) im Jahr 1965 von einer fünfjährigen Liberalisierungsperiode abgelöst wird. Die Konsequenz dieser wirtschaftspolitischen Unstetigkeit ist zwar, trotz einem ungekannt hohen Niveau an Verschuldung, kein ökonomischer Kollaps, aber die Zu-

18 Nach der Nationalisierung der Ölindustrie im Jahr 1963 stellen die USA sogar kurzfristig ihre Entwicklungshilfe ein (vgl. Bastian 2007: 38). 
spitzung regionaler Disparitäten, die speziell im singhalesischen Süden in einem sich organisierenden militanten Widerstand gegen die als USfreundlich geltende UNP-Regierung resultieren.

Nachdem die SLFP unter S.V.R.D. Bandaranaikes Witwe Sirimavo ${ }^{19}$ die Wahlen von 1970 gewinnt, versucht sie, die im Süden des Landes bereits gärende Situation - die 1965 gegründete militante, auf maoistischen Wurzeln aufbauende linksradikal-nationalistisch orientierte JVP $^{20}$ hatte eine faktische Doppelherrschaft errichtet und drohte relativ unverhohlen mit einer bewaffneten Revolution - durch politische und ökonomische Maßnahmen in den Griff zu bekommen. Politisch bindet Bandaranaike die JVP und andere linksradikale Parteien in die Regierung ein, während zugleich neue Großprojekte im Infrastrukturbereich geplant werden, allen voran das Mahaweli Ganga Development Project, das gleich nach der Regierungsübernahme 1970 mit massiver Geberunterstützung in die Planungsphase geht.

Trotzdem lässt sich der bewaffnete Zusammenstoß nicht mehr vermeiden. Nach einigen Bombenexplosionen lässt Bandaranaike den JVPFührer Rohana Wijeweera verhaften, was zum Beginn des Aufstandes führt. Mit massiver Unterstützung durch indische Truppen kann die srilankische Armee die JVP zurückschlagen, das Ausmaß der Gewalt erreicht jedoch eine in Sri Lanka bis dato ungekannte Dimension: die nur zwei Wochen dauernden Kämpfe fordern 15.000 Todesopfer. Nach der

19 Bandaranaike wurde im Jahr 1959 von einem buddhistischen Mönch erschossen, der in Verbindung mit noch stärker nationalistisch orientierten Kräften in der SLFP stand.

20 Gerne wird die JVP, die nach zwei blutig niedergeschlagenen Revolten mittlerweile in ihrer dritten Reinkarnation existiert, gerade von internationalen Beobachter/innen als chauvinistisch und xenophob-rassistisch klassifiziert. Wenngleich die Ideologie der Partei während ihrer gesamten Geschichte ohne Zweifel mit rassistischen Elementen durchzogen ist, wäre eine solche Klassifizierung dennoch verkürzt. Einerseits ist ein antiimperialistisches Element vorhanden, das sich allerdings, wie bereits erwähnt, in wesentlichen Bereichen rassistisch kodiert, andererseits ist aber der Werdegang und der nachhaltige Erfolg der Partei ohne die spezifische regionale Konstellation Sri Lankas nicht zu verstehen. Die JVP ist in diesem Kontext die genuine Partei der »Southeners«, die im marginalisierten Dreieck zwischen Galle, Hambantota und Ratnapura auch ein spezifisches Lebensgefühl und nicht zuletzt ein tief verwurzeltes Misstrauen gegenüber der Hauptstadt Colombo und der dortigen als elitär wahrgenommenen Politikerschicht repräsentiert. Einige der heute prominenten Friedensaktivist/innen haben am JVP-Aufstand von 1971 teilgenommen, so der Leiter des Instituts für Politikwissenschaft an der Universität Colombo, Jayadeva Uyangoda, oder der Publizist und Zeitungsherausgeber Victor Ivan, der während des Aufstandes bei der Explosion einer von ihm hergestellten Bombe an beiden Händen schwere Verletzungen davontrug. 
Niederlage offeriert Bandaranaike zwar eine Amnestie, die JVP bleibt als Partei bis zur Regierungsübernahme durch die UNP 1977 allerdings verboten, formiert sich aber im Untergrund neu.

Für die internationalen Akteure stellt der JVP-Aufstand ihre Ausrichtung nicht grundsätzlich in Frage, vielmehr bestärkt er noch den ohnehin eingeschlagenen Weg. ${ }^{21}$ Denn schließlich kann, so die dominierende Lesart Anfang der 1970er Jahre, nur eine konsequente Modernisierung einhergehend mit infrastrukturellem Ausbau jene regionalen Disparitäten beseitigen, die als Ursache des JVP-Aufstandes wahrgenommen werden. So bleiben die ODA-Flüsse vorerst konstant, wenngleich ein merklicher Druck in Richtung einer verstärkten Liberalisierung einsetzt, die dem wirtschaftspolitischen Programm der regierenden SLFP entgegenlaufen. Die politischen Schritte, die die SLFP-geführte Regierung setzt und die nachhaltige Folgen haben werden, sind zu diesem Zeitpunkt strikt innere Angelegenheiten: 1972 wird eine neue Verfassung verabschiedet, die den Anti-Diskriminierungsabschnitt der Soulbury-Verfassung streicht. Zugleich finden nicht nur von tamilischen politischen Gruppierungen, insbesondere der Federal Party, geforderte föderale Elemente keine Berücksichtigung, es werden auch positive Diskriminierungen im Hochschulzugang für die singhalesische Bevölkerungsgruppe eingezogen (vgl. Bastian 2007: 81, der die Ursache für diese Maßnahme in der Popularität anti-singhalesischer Verschwörungstheorien verortet). Ceylon wird in Sri Lanka unbenannt, was angesichts des aus dem Singhalesischen stammenden neuen Namens von vielen Tamil/innen als weitere Provokation verstanden wird. Die Folge ist eine sich stärker nationalistisch orientierende tamilische Formierung, die als Tamil United Front (ab 1976 dann Tamil United Liberation Front, TULF) die bis zu diesem Zeitpunkt bestehenden Parteien zusammenfasst. Zugleich sammeln sich militante tamilische Gruppen unter der Sammelbezeichnung Tamil Tigers, die Velupillai Prabhakaran beginnt, in der von ihm gegründeten militärischen Organisation Liberation Tigers of Tamil Eelam, LTTE $^{22}$, zusammenzufassen.

Die internationalen Akteure konzentrieren sich zu diesem Zeitpunkt, angeführt von der die Policy prägend vorgebenden Weltbank, auf die Liberalisierungsagenda. Als 1977 wieder die UNP, diesmal angeführt

21 Die einzige relevante Ausnahme ist USAID, die ab Anfang der 1970er, also in der unmittelbaren Nachfolge des Aufstandes, ein Dezentralisierungsprogramm beginnt. Da dies ein überregional ausgerichtetes Programm ist, kann es jedoch nicht als unmittelbare Konsequenz des Aufstandes verstanden werden (vgl. Burke/Mulakala 2005: 11).

22 Zur Geschichte der LTTE vgl. Balasingham (2004), Balasingham (2003) oder Narayan Swamy (2002). 
von J.R. Jayawardene, die Regierung übernimmt und einen rigiden Liberalisierungskurs einleitet, ist die Begeisterung der internationalen Gemeinschaft groß. Die ohnehin vergleichsweise hohe internationale Hilfe für Sri Lanka - während der 1960er Jahre weist Ceylon eine der höchsten Raten von Entwicklungshilfe im Verhältnis zur Bevölkerungszahl auf (vgl. Goodhand 2001: 45) - wird, auch bedingt durch die starke Ausweitung der japanischen ODA-Gelder, auf ungeahnte Höhen geschraubt (siehe Abbildung 24). Dafür verantwortlich zeichnet eine Neuausrichtung schon laufender Großprojekte, insbesondere die Beschleunigung des Mahaweli-Projektes, sowie der Beginn eines Mammutproramms zur Integrierten Ländlichen Entwicklung (ebd., vgl. auch Bastian 2007: 122, 2006: 257).

\section{Abbildung 24: ODA in Sri Lanka (in 2005-US\$)}

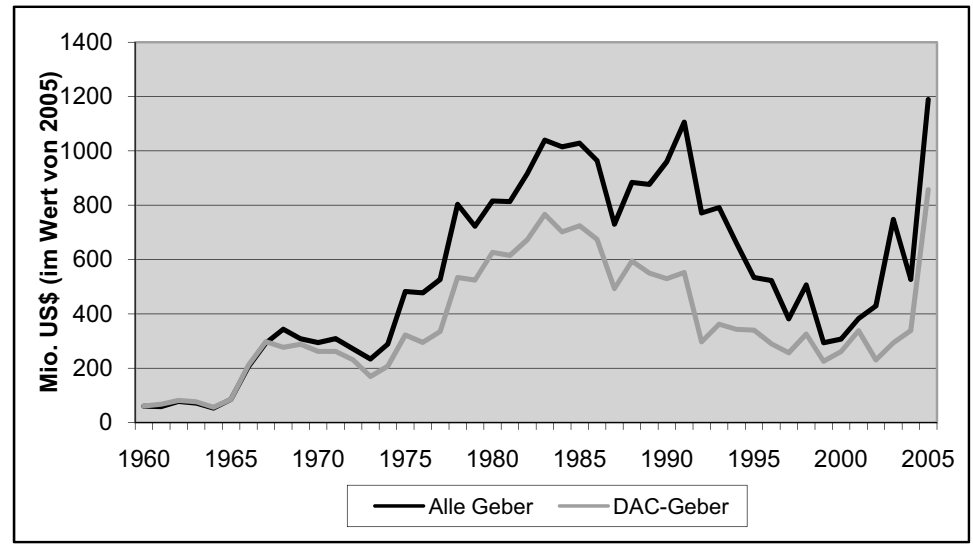

Auf politischer Ebene versucht Jayawardene, die Eskalationsschritte zurückzunehmen, die die Verfassung von 1972 mit sich gebracht hat. Die Verfassung von 1978 geht auf verschiedene tamilische Forderungen ein, die Diskriminierungen im Hochschulzugang werden beispielsweise aufgehoben. Dies verknüpft Jayawardene allerdings mit einer nachhaltigen Stärkung seiner eigenen Rolle: die Verfassung bricht endgültig mit dem Westminster-Modell und führt ein Präsidial-System ein, Jayawardene wechselt von der Funktion eines Premierministers in die des Staatspräsidenten.

Obwohl das Mahaweli-Projekt in seinen Ausläufern bis zum heutigen Tag verfolgt wird, haben sich die Einschätzungen der meisten in den 1970er Jahren großflächig beteiligten Geber geändert. Selbst die Weltbank äußert sich selbstkritisch über die vielfach missachteten regionalen Problemstellungen, die zur Verschärfung verschiedener sozialer Bruch- 
linien - und damit indirekt zur Eskalation des bewaffneten Konfliktes beigetragen haben. Neben der »Konfliktblindheit« der mit 1977 einsetzenden ODA-Leistungen (vgl. Goodhand 2001: 78) wird auch die mittelbare Wirkung der Gelder auf die Budgetsituation als Problem analysiert, da diese maßgeblich zum Aufrüstungsprogramm der JayawardeneRegierung beitragen (ebd.: 79). ${ }^{23}$

Dieses Aufrüstungsprogramm erweist sich aus Sicht der UNPgeführten Regierung als dringend notwendig, da die LTTE ab 1978 bewaffnete Angriffe auf zunächst niedrigem Eskalationsniveau beginnt. Sie untergräbt damit die Autorität der an der Regierung beteiligten TULF, die sich auf die Forderung nach Autonomie konzentriert, die sie auf politischem Weg durchsetzen will. Jayawardene, auch beeinflusst durch die Erfahrungen des JVP-Aufstandes, der als Resultat eines zu passiven Agierens des Staates interpretiert wird, entscheidet sich schnell für massives Eingreifen. 1979 wird der Prevention of Terrorism Act (PTA) beschlossen, der faktisch die Verhängung des Ausnahmezustandes bedeutet. Trotzdem oder angesichts des schrumpfenden Raumes für politische Auseinandersetzung vielleicht auch deswegen gewinnt die $\mathrm{LTTE}^{24}$ zunehmend an Stärke und beginnt, die primär politisch agierenden tamilischen Kräfte in der innertamilischen Auseinandersetzung zu marginalisieren.

Im Zuge von Lokalwahlen in der Nordprovinz im Jahr 1981 eskaliert die Gewalt erstmals spürbar, ein UNP-Kandidat wird erschossen und die Bibliothek von Jaffna, in der Wahlboxen gelagert werden, niedergerannt. Dies ändert an der Wahrnehmung der internationalen Geber und ihrer Darstellung der politischen Situation wenig: »In 1981, the simmering violence in Sri Lanka was all but invisible to most students of international politics including international donors. Indeed, the island was still portrayed as a >model Third World democracy« (Bush 1999: 22). Diese vermeintlich unpolitische Haltung führt jedoch, gerade durch das

23 Inwieweit die Liberalisierung nach 1977 selbst unmittelbar für die Zuspitzung des Konfliktes und seines gewaltsamen Ausbruchs verantwortlich ist, wie es etwa Fricke (2002) behauptet, kann an dieser Stelle nicht beantwortet werden. Angesichts der verzögerten Wirksamkeit wirtschaftspolitischer Maßnahmen, die zwar politisch, schwerlich aber auf ökonomischer Ebene auf die Ereignisse des Jahres 1978 wirken können, stehe ich der These skeptisch gegenüber.

24 In den späten 1970er und frühen 1980er Jahren ist die LTTE nur eine unter mehreren bewaffneten tamilischen Organisationen, die sich entweder parallel entwickeln oder sich, wie die, wenn auch in anderer Form, bis heute bestehenden PLOTE (People's Liberation Organization of Tamil Eelam) oder TELO (Tamil Eelam Liberation Organization), von der LTTE abspalten. 
staatsnahe Agieren der Geber, zu überaus politischen Konsequenzen: »The perceived exclusionary character of the government's development programme created incentives for the Tamil leadership not to be seen to be working with the government - otherwise there was a risk that they would lose the support of its Tamil constituency« (ebd.: 24).

Als nach einem Anschlag der LTTE auf einen Militärkonvoi im Juli 1983 Unruhen ausbrechen, die von den meisten Tamil/innen als eine eindeutig gegen sie gerichtete gewaltsame Kampagne verstanden wer$\operatorname{den}^{25}$, und in der Folge die LTTE den bewaffneten Kampf auch auf konventioneller Ebene aufnimmt, kommt es zwar nicht zu einem Bruch, aber doch zu einer merklichen Verschiebung im Verhalten der Geber. Zwar fließen die ODA-Gelder zunächst weitgehend unbeeinflusst weiter, sie werden jedoch in einem relevanten Teil von Großprojektfinanzierungen in Humanitäre Hilfe umgeleitet, vor allem von Seiten der westlichen Geber.

Der für die internationalen Akteure wirklich spürbare Schock, der die Entwicklungshilfe vorübergehend auch einbrechen lässt (siehe Abbildung 24), folgt vier Jahre später. 1987 beginnt die JVP ihren zweiten bewaffneten Aufstand, der sich über drei Jahre hinziehen und etwa 50.000 Todesopfer $^{26}$ fordern sollte. So berichtet ein zu dieser Zeit aktiver Büroleiter einer bilateralen Agentur über die Situation während der Zeit des Aufstandes, dass »for some bilaterals, the island of Sri Lanka shrunk to the island of Colombo - and for some it shrunk even further to the island of Colombo $7^{27}$ « (zitiert nach Bush 1999: 25).

Als Konsequenz beginnen Ende der 1980er Jahre einige kleinere Geber wie Kanada, die Niederlande oder die skandinavischen Staaten, nachhaltig von ihrem bisherigen Kurs abzurücken, Menschenrechtskriterien einzuführen und sogar, wie im Falle Kanadas, die Zusammenarbeit mit staatlichen Einrichtungen weitestgehend $\mathrm{zu}$ sistieren (vgl. Bastian 2007: 128ff.). Im Rahmen eines Gebertreffens in Paris im Jahr 1990 spricht die Gebergemeinschaft erstmals in offiziellem Rahmen ihre Besorgnis über die Menschenrechtssituation aus, im Zuge dessen reduzieren viele der westlichen bilateralen Geber ihr Programm. So bleiben die

25 Die - politisch umstrittene - Zahl der Toten dürfte mehrere Hundert umfassen. Zugleich verlassen 150.000 Tamil/innen in der Folge das Land, vor allem in Richtung Kanada, Australien und Großbritannien.

26 Diese Zahl ist auch insofern schockierend, wenn bedacht wird, dass der nahezu 25 Jahre dauernde Kampf zwischen der LTTE und Sri Lanka bislang zirka 68.000 Todesopfer gefordert hat.

27 Colombo 07, Cinnamon Gardens, gilt als das Luxusviertel der Hauptstadt, in dem sich zahlreiche Botschaften und Residenzen befinden. 
Weltbank, die ADB und Japan als die drei bestimmenden Akteure in der sri-lankischen Geberlandschaft übrig (vgl. ebd.: 136).

Zeitgleich kommt es 1987 im Norden des Landes zur einzigen großflächigen internationalen militärischen Intervention in Sri Lanka. Im Zuge der Belagerung von Jaffna durch eine frisch hochgerüstete srilankische Armee nimmt der Druck auf Indien, vor allem von Seiten der in Indien sehr aktiven und zu diesem Zeitpunkt auch politisch gut verankerten tamilischen Gruppen, zu, in Sri Lanka eine Ordnungsfunktion einzunehmen. Bedingt dadurch, und auch durch gegebene strategische Interessen $^{28}$, entscheidet sich die Regierung unter Rajiv Ghandi zu einem aktiven Einschreiten. ${ }^{29}$

Hatte Indien zuvor bei den gescheiterten Friedensverhandlungen im bhutanischen Thimpu ${ }^{30}$ noch als Vermittler gewirkt, erzwingt es nun ohne Einbindung der LTTE - ein Abkommen mit der sri-lankischen Regierung, den so genannten Indo-Sri Lanka Accord von $1987^{31}$ (vgl. Keethaponcalan 2005: 214). Vor dem Hintergrund eines klaren Bekenntnisses zu einem einheitlichen Sri Lanka werden Schlüsselforderungen der

28 Nicht nur gibt es seit 1983 etwa 15.000 tamilische Flüchtlinge aus Sri Lanka im indischen Gliedstaat Tamil Nadu (vgl. Samaranayake 2006: 168), auch die verstärkte Involvierung pakistanischer und israelischer Militärberater auf Seiten der sri-lankischen Armee und das ohnehin bestehende Misstrauen gegenüber den USA, denen geostrategische Interessen in Sri Lanka unterstellt werden, tragen zu der Entscheidung bei, militärische Präsenz zu markieren.

29 Diese Entscheidung sollte Rajiv Ghandi das Leben kosten. Am 21. Mai 1991 wird er bei einem Besuch im Gliedstaat Tamil Nadu von einer Selbstmordattentäterin der LTTE getötet. Dieses Attentat wird von der LTTE nahestehenden Kommentator/innen mittlerweile als schwerer Fehler bezeichnet, führt es doch zu einem Kurswechsel in der indischen Politik: Wurde der »tamilische Unabhängigkeitskampf« zuvor mitunter sogar offen auf politischer wie logistischer Ebene unterstützt, schwenkt Indien nach dem Attentat auf eine harte Anti-Terror-Linie ein, die sich an entsprechenden Vorgangsweisen der USA orientiert. Rajiv Ghandis ab 1989 verantwortliches Gegenüber auf sri-lankischer Seite überlebt nicht viel länger: Sri Lankas Präsident Ranasinghe Premadasa fällt am 1. Mai 1993 während eines Maiumzuges einem Selbstmordattentat der LTTE zum Opfer.

30 Zwar scheitern die Verhandlungen in Thimpu im Sinne einer Einigung zwischen den tamilischen separatistischen Kräften und dem GoSL, sie bringen aber eine gemeinsame Verhandlungsposition der verschiedenen tamilischen nationalistischen Organisationen hervor, die als ThimpuPrinzipien bis heute die zentralen Forderungen der LTTE, aber auch anderer tamilischer Gruppen repräsentieren: die Anerkennung des »Tamil Homeland «, die Anerkennung der tamilischen Nation und die Respektierung des tamilischen Rechts auf Selbstbestimmung (vgl. dazu Liyanage in Rupesinghe 2006a: $1 \mathrm{ff}$.).

31 Der Volltext des Accord findet sich etwa bei Balasingham (2004: 467ff.). 
LTTE und anderer radikaler tamilischer Gruppen festgeschrieben: eine Zusammenlegung der Nord- und Ostprovinz mit einer einheitlichen Administration, was über ein Referendum - über dessen Ausgang vermutlich kaum Zweifel bestanden - zu sanktionieren sei, als Reminiszenz an das »Tamil Homeland«-Konzept, eine explizite Anerkennung der ethnischen Identitäten, die, wie es heißt, gepflegt werden müssten ${ }^{32}$, sowie eine Aufhebung des Ausnahmezustandes und eine Generalamnestie für die zu entwaffnenden militanten tamilischen Gruppen.

Zur militärischen Umsetzung lässt sich Indien zur Installierung einer Schutztruppe einladen, die in Kooperation mit der sri-lankischen Armee die Überwachung des Abkommens in die Hand nehmen soll. Diese Indian Peace Keeping Force (IPKF) stößt in ihrem Vorhaben der Entwaffnung der LTTE jedoch auf vehementen Widerstand. Die auf ihrem Höhepunkt ungefähr 100.000 Mann starke Truppe sieht sich nicht nur außerstande, die Vertragsbedingungen, allen voran die Entwaffnung gegenüber der LTTE durchzusetzen, sondern lässt sich bald in einen politisch wie militärisch verlustreichen Kleinkrieg verwickeln. Insgesamt beklagt die IPKF im Zuge der dreijährigen Kämpfe 1.100 Gefallene. Parallel mit ihrem militärischen Misserfolg verstärkt sich der von singhalesisch-nationalistischen Kräften genährte Vorwurf einer imperialen Einmischung in innere Angelegenheiten, was schließlich im Frühjahr 1989 zur Aufforderung des neuen sri-lankischen Präsidenten, Premadasa, an Indien mündet, die IPKF abzuziehen. Der Abzug wird bis Anfang 1990 vollzogen, während parallel von Premadasa angestrengte Verhandlungen zwischen dem GoSL und der LTTE scheitern.

Diese schwere Niederlage des internationalen Peace-Keeping hat auf die internationale Gebergemeinschaft nachhaltige Auswirkungen. Kein anderes Land hat mit Indien vergleichbare Interessen in Sri Lanka, und wenn sogar Indien an einer militärischen Durchsetzung eines Abkommens scheitert, rückt die Perspektive eines, selbst auf Einladung geschehenden und rein überwachenden, militärischen Engagements eines anderen Drittlandes in weite Ferne. Dies wird auf die Bemühungen im Bereich der Friedensentwicklung wesentlichen Einfluss ausüben und trägt vermutlich auch dazu bei, dass zivile Mittel in Sri Lanka im internationalen Vergleich gesehen unverhältnismäßig populär sind.

Die indische Erfahrung führt noch zu einer zweiten wichtigen Einsicht, die allerdings nicht sehr verbreitet existiert und wenn, dann nur

32 Der Accord geht von vier distinkten ethnischen Identitäten aus: Singhales/innen, Tamil/innen, Muslim/innen und Burger (Nachfahren der portugiesischen und niederländischen Kolonialist/innen). Der Accord anerkennt »that each ethnic group has a distinct cultural and linguistic identity which has to be carefully nurtured« (nach Balasingham 2004: 467). 
hinter vorgehaltener Hand ausgesprochen wird $^{33}$ : die 1987 von Indien diktierten Bedingungen entsprechen, mit der einzigen Ausnahme des Fehlens eines definitiven Verweises auf eine föderale Umsetzung eines als unabdingbar wahrgenommenen Devolutionsprozesses, mehr oder minder dem, was sich die meisten der engagierten Geberagenturen und Botschaften nach wie vor als bestmögliche politische Lösung des Konfliktes vorstellen. So wird, in einer Art Pflichtoptimismus, die Ablehnung der im Vertrag eingeschriebenen Konzepte zumeist mit der Art und Weise ihrer zwangsweisen Durchsetzung begründet, die jede Genuinität des Prozesses untergraben hätte. Zugleich erklärt die indische Erfahrung aber in jedem Fall die verhältnismäßig große Passivität der internationalen Akteure im Friedensprozess von 2001, auf den noch einzugehen sein wird. $^{34}$

Zunächst kommt es allerdings ohnehin zu einer militärischen Zuspitzung. Nach der Niederschlagung des JVP-Aufstandes kann die Armee wieder konzentriert gegen die LTTE zum Einsatz gebracht werden, was die 1994 gewählte neue Präsidentin, Chandrika Kumaratunga ${ }^{35}$, nach einem weiteren gescheiterten Verhandlungsversuch auch prompt mit

33 Dass sich nur wenige internationale Akteure mit dieser Frage auseinandersetzen ist dem zum Teil geringen Wissen über die historische Entwicklung des Konfliktes zuzuschreiben, das bei der Mehrzahl der Internationalen in Colombo vorhanden ist.

34 Die Erfahrungen des Indo-Sri Lankan Accord werden selbst von professionellen Friedensentwickler/innen mitunter als Zeichen für die tendenzielle politische Unlösbarkeit des Konfliktes gesehen. So gab ein in diesem Zusammenhang nicht genannt werden wollender prominenter Vertreter der internationalen Gemeinschaft in Colombo mir gegenüber in einem Interview zu verstehen, dass er nicht an eine Verhandlungslösung glaube, sondern nur an einen erzwungenen Frieden durch eine bewaffnete Intervention internationaler Akteure. Eine solche wäre auf Dauer unvermeidlich. Dies wäre zumindest seine Hoffnung. Eine offiziell natürlich niemals einnehmbare Haltung, die eine Stimmung wiedergibt, die ohne die Erfahrung der IPKF nicht erklärbar wäre.

35 Kumaratunga folgt dem Interimspräsidenten nach der Ermordung von Premadasa, ihrem Parteifreund Wijetunga, nach. Sie entstammt dem Familienclan der Bandaranaikes, der die Geschichte der SLFP seit ihrer Gründung bestimmt hat. Ihr Vater war, wie erwähnt, schon 1959 von einem radikal-nationalistischen buddhistischen Mönch erschossen worden, ihr Mann, der populäre Schauspieler und - zunächst trotzkistisch orientierte und dann der SLFP angehörige - Politiker Vijaya Kumaratunga wird im Zuge des JVP-Aufstandes 1988 in Colombo, vermutlich von JVP-Kadern, erschossen. Ende Dezember 1999 verliert sie im Zuge eines Attentates der LTTE auf eine ihrer Wahlveranstaltungen ein Auge, was für ihre harte Linie gegenüber den tamilischen militanten Gruppen mitverantwortlich gemacht wird. Zur großen Bedeutung von Familienclans in der srilankischen Politik siehe Wickramasinghe und Wickramasinghe (2005). 
ihrer »War for Peace«-Strategie umsetzt. Entscheidend ist das Verhalten der Gebergemeinschaft, die in ihrer dominierenden Ausrichtung, noch immer primär angeleitet von der Idee infrastruktur- und freihandelsgesteuerten Wachstums, Kumaratungas militärischen Weg mitvollzieht, freilich ohne ihn politisch explizit zu begrüßen. »This reality, and the Kumaratunga strategy, has been facilitated by an international donor community that has been fixated on the economic reform agenda" (Rainford 2007: 4).

Die zum Teil auch humanitär argumentierten Aktivitäten der Geber erfüllen für die Regierung einen wesentlichen Zweck. Erstens garantieren sie die konkrete, und damit auch die symbolische Präsenz des Staates in den rückeroberten Gebieten. Speziell bei der Einnahme von Jaffna im Dezember 2005, die zu einer Fluchtbewegung von etwa 350.000 Menschen führt, die sich mit den bewaffneten tamilischen Kadern ins von der LTTE kontrollierte Vanni zurückziehen, zeigt sich die kriegswichtige Bedeutung der internationalen Hilfsbemühungen, da der Wiederaufbau von Jaffna »began to take on symbolic significance for the government« (ebd.: 6). Speziell die GTZ ist mit ihrem Jaffna Rehabilitation Project federführend an diesem Vorgehen beteiligt. Der in diesem Zusammenhang von der Regierung forcierte Triple-R-Approach (Rehabilitation, Reconstruction, Reconciliation) wird mit dem Argument unterstützt, dass Krieg in jedem Fall Entwicklung behindern würde. Insofern sei das Engagement sachlich wie moralisch gerechtfertigt (vgl. Samaranayake 2006: 171), wenn auch, maßgeblich etwa von den USA, von der Regierung eine Verhandlungslösung gefordert wird.

Angestoßen durch die internationale Debatte setzen gegen Ende des Jahrtausends schließlich nicht nur bei verschiedenen kleinen bilateralen Gebern, sondern auch bei den großen Drei der sri-lankischen Geberlandschaft - Japan, ADB und Weltbank - selbstkritische Prozesse ein. Verstärkt wird die eigene Rolle hinterfragt und, insbesondere bei Japan und der Weltbank, mit dem Jahrtausendwechsel eine Änderung der Strategie eingeläutet. Dieser Kurswechsel geht allerdings mit keiner Stärkung der Position der westlichen Geber einher. Vor allem das immer massivere Auftreten anderer asiatischer Geber, allen voran China, die sich den Diskussionen in den Geberforen weitgehend entziehen, resultiert im Gegenteil. Die zwei Jahrzehnte zuvor noch starke und mit einigem Druckpotenzial verbundene Rolle der westlichen Geber verliert nachhaltig an Bedeutung, was allerdings - wie die folgenden Diskussionen über die Einführung von Friedenskonditionalitäten zeigen werden - nicht in gegebenem Ausmaß akzeptiert wird.

Auch auf diplomatischer Ebene erfolgt nach der Schockphase als Nachwirkung des IPKF-Desasters eine internationale Initiative. Norwe- 
gen, das schon zuvor gemeinsam mit Kanada in der Forcierung eines »working on conflict «-Ansatzes im entwicklungspolitischen Bereich federführend war $^{36}$ (vgl. Goodhand 2001: 72), bietet seine diplomatischen Vermittlerdienste an, um eine neue Runde an Friedensgesprächen zu initiieren. Überraschend, bedingt durch den Druck einer durch ausbleibende Investitionen immer stärker bedrängten Business-Elite, militärische Erfolge der LTTE im Vorfeld - speziell die Rückeroberung des Elephant Pass, der die Jaffna-Halbinsel mit dem Vanni verbindet - und angesichts einer sich dramatisch verschlechternden allgemeinen Wirtschaftslage nimmt Kumaratunga das Angebot an. Der erste Verhandlungsversuch im Jahr 2000 scheitert allerdings schon an der Vereinbarung eines Waffenstillstandes.

Die meisten internationalen Geber richten sich ab diesem Zeitpunkt jedoch bereits auf einen möglichen Friedensprozess ein und ihre Arbeit auf das neue Primärziel Konflikttransformation aus. DFID gibt ein erstes Strategic Conflict Assessment (im gegenwärtigen Sprachgebrauch der internationalen Gemeinschaft in Colombo SCA1 genannt) in Auftrag, das mit seiner Analyse, den Konflikt nicht als »ethnisch« zu betrachten, sondern als Ausdruck einer Krise des sri-lankischen Staates zu sehen, Staub aufwirbelt (vgl. Goodhand 2001). Wesentlicher ist allerdings die scharfe Kritik an der bisherigen Praxis der Geber, an der Realität des Konfliktes vorbeigearbeitet und damit seine Zuspitzung bis zu einem gewissen Grad auch mitverschuldet zu haben. Nicht nur DFID leitet als Konsequenz eine Umorientierung des Länderprogramms ein, die sich an den Vorgaben des im März 2001 frisch eingerichteten britischen Gesamtkonzeptes eines Global Conflict Prevention Pool (vgl. Khittel/Pospisil 2006: 54ff.) orientiert: Die entwicklungspolitischen, diplomatischen und militärischen Kapazitäten der britischen Präsenz in Sri Lanka werden zusammengezogen und im Sinne eines einheitlichen Konfliktpräventionsprogramms gepoolt. Ebenfalls im Jahr 2001 richtet sich das deutsche Länderprogramm mit dem $P A C T$-Schwerpunkt ${ }^{37}$ auf Konflikttransformation aus, womit faktisch alle in Sri Lanka aktiven bilateralen DAC-Geber - mit Ausnahme Japans ${ }^{38}$ und bis zu einem gewis-

36 Goodhand (2001: 72) führt diese Vorreiterrolle von Norwegen und Kanada auf drei Faktoren zurück: Erstens haben beide Länder keine geostrategischen Interessen in Sri Lanka und können daher einigermaßen frei agieren, zweitens lebt in beiden Ländern eine relativ große tamilische Diaspora, und drittens entspricht die Neuausrichtung auch den generellen Leitlinien der jeweiligen bilateralen entwicklungspolitischen Programme.

37 Siehe dazu den folgenden Abschnitt.

38 Japan verstärkt seine diesbezüglichen Aktivitäten im Jahr 2002, wobei primär Maßnahmen auf diplomatischer Ebene getroffen werden. Das Entwicklungshilfe-Portfolio bleibt weitgehend traditionell orientiert. 
sen Grad der USA ${ }^{39}$ - ihr entwicklungspolitisches Programm zentral auf die Problematik des bewaffneten Konfliktes ausgerichtet haben.

Die durch die sich stark verschlechternde wirtschaftliche Situation bedingte Wahlniederlage der SLFP und die Übernahme der Regierung durch die UNP unter Premier Ranil Wickremasinghe ermöglichen schließlich einen Durchbruch bei den Verhandlungsbemühungen: Ende 2001 erklären GoSL wie LTTE einen Waffenstillstand, was unter norwegischer Vermittlung in formelle Verhandlungen übergeleitet wird und zur Unterzeichnung eines Waffenstillstandsabkommens (Ceasefire Agreement, CFA) Ende Februar 2002 führt. Das Abkommen wird allerdings gegen den expliziten Widerstand der der SLFP zugehörigen Präsidentin Kumaratunga geschlossen.

Für die LTTE, die schon ein Jahr zuvor einen Waffenstillstand erklärt hatte, folgt nach den spektakulären Erfolgen Ende der 1990er Jahre eine militärisch durchwachsene Phase, die mit einem in der Folge von 9/11 immer enger werdenden politischen Spielraum einhergeht (vgl. Narayan Swamy 2002: 363f.). Die USA hatten die LTTE schon 1997, im Zuge der Erstveröffentlichung der FTO-Liste ${ }^{40}$, als Terror-Organisation gekennzeichnet. Während auf ihrer Seite die Motivationen hinter der Verhandlungsbereitschaft daher relativ offen liegt ${ }^{41}$, ist die Situation auf Regierungsseite komplexer. Primär dürfte der schon im Verlauf des Jahres 2000 immer stärker werdende Druck der mit der UNP eng verbündeten wirtschaftlichen Eliten ${ }^{42}$ im Zusammenspiel mit einer schwindenden Popularität des Krieges im nicht stark nationalistisch geprägten Teil der singhalesischen Bevölkerung - die UNP gewinnt die Wahl mit dem expliziten Versprechen folgender Friedensbemühungen ${ }^{43}$ - für die neue

39 Wie auch der folgende Abschnitt zeigen wird, setzen die USA zwar wesentliche entwicklungspolitische Maßnahmen im Bereich der Konflikttransformation, richten jedoch ihr Länderprogramm nicht vollkommen danach aus wie ein Großteil der anderen bilateralen Geber.

40 Liste der Foreign Terrorist Organizations der USA, einsehbar unter http://www.state.gov/s/ct/rls/rpt/fto/.

41 Demgegenüber steht die Interpretation der singhalesisch-nationalistischen Kreise, dass die LTTE vor allem deswegen in Verhandlungen eintreten würde, um das strategische Kalkül nach internationaler Anerkennung mit einer Atempause für die Aufrüstung und die Eliminierung innertamilischer Konkurrenzorganisationen zu verbinden.

42 Speziell der im militärischen Sinn erfolgreiche Angriff auf den internationalen Flughafen von Colombo am 24. Juli 2001 hat folgenschwere ökonomische Auswirkungen. Der Tourismus kommt vorübergehend fast zum Erliegen, und 2001 wird zum ersten Jahr seit der Unabhängigkeit, in der die sri-lankische Wirtschaft schrumpft (vgl. Bastian 2006: 248).

43 Genau genommen läuft der Wahlkampf zwischen zwei Wahlbündnissen: die UNP ist dabei mit dem Ceylon Workers Congress, einer primär bei den 
Verhandlungsbereitschaft verantwortlich sein. Zudem sichert dieser Schritt der Wickremasinghe-Regierung den »overwhelming support provided by the international community« (Rupesinghe 2006b: xlvii), was in den sich zuspitzenden innenpolitischen Auseinandersetzungen mit der Präsidentin ohne Zweifel als hilfreich empfunden wird.

Der sich in der Folge entspinnende Friedensprozess - zwischen September 2002 und März 2003 finden insgesamt sechs Verhandlungsrunden statt - ist von Beginn an durch eine starke Internationalisierung gekennzeichnet (vgl. Bastian 2007: 152). Nicht nur richten all diejenigen, die ihr Programm schon bisher unter die primäre Zielsetzung der Konflikttransformation gestellt hatten, ihre Tätigkeit nachdrücklich auf die Unterstützung des Prozesses aus. Auch Japan vollzieht einen Wechsel in diese Richtung, was auch damit zusammenhängt, dass die japanische Entwicklungshilfe Anfang des Jahrtausends im asiatischen Raum erstmals in einzelnen exemplarischen Fällen, neben Sri Lanka beispielsweise in Aceh, in unmittelbar friedensstiftender Mission eingesetzt wird (vgl. Bastian 2006: 264). Japan ernennt im Oktober 2002 auch einen offiziellen Repräsentanten für »Peace-building, Rehabilitation and Reconstruction in Sri Lanka«, den vormaligen UN-Vizegeneralsekretär und Friedensforscher Yasushi Akashi. Auf formeller diplomatischer Ebene übernimmt Japan zudem die Funktion eines Co-Chairs des Friedensprozesses, zusammen mit den USA und der Europäischen Union. ${ }^{44}$

Die strategische Einbindung der Entwicklungspolitik in den Friedensprozess erfolgt allerdings erst zu einem Zeitpunkt, als er praktisch bereits wieder zu Ende ist. Für Juni 2003 wird eine groß angelegte Geberkonferenz in Tokio angesetzt, die die internationale Unterstützung sammeln und koordinieren soll. Schon zuvor sistiert die LTTE jedoch ihre Beteiligung. War die Stimmung an der sechsten Verhandlungsrunde in Hakone, Japan, schon schlecht, ist die Weigerung der USA, die Teil-

Tamil/innen des Hochlandes verankerten Kleinpartei, in der United National Front (UNF) zusammengefasst, während die SLFP zusammen mit einigen linken und linksradikal-nationalistischen Parteien wie der kommunistischen Partei oder der trotzkistischen LSSP als People's Alliance (PA) antritt. Die JVP tritt unabhängig an, ebenso wie die das letzte Mal eigenständig antretende TULF, die im Verlauf der nächsten Jahre mit LTTEnahen Kräften zur Tamil National Alliance (TNA) fusionieren wird.

44 Die EU zeigt sich zunächst zurückhaltend und muss, vor allem durch UNP-Minister Milinda Moragoda, massiv zur Übernahme dieser Rolle gedrängt werden. Die Ursache dafür dürfte in technischen Problemen liegen, da sich die EU im Juli $2001 \mathrm{zu}$ einer vollständigen Umgestaltung ihrer Repräsentanz in Colombo entschlossen hatte und sich daher ursprünglich personell zur Übernahme dieser Funktion nicht in der Lage sah (vgl. Noyahr 2006: 389). 
nahme der LTTE an einem Vorbereitungstreffen für die TokioKonferenz in Washington zu ermöglichen, schließlich der Auslöser der Entscheidung. Die USA hatten zuvor angekündigt, die LTTE trotz des Friedensprozesses weiterhin als terroristische Vereinigung zu behandeln, was ihre Teilnahme an dem Washingtoner Treffen faktisch verhinderte.

Formell wird die Tokio-Konferenz zunächst sowohl vom GoSL als auch von den internationalen Gebern als großer Erfolg verkauft. Hilfszusagen in Höhe von 4,5 Mrd. US\$ werden gegeben, zugleich wird die neu entdeckte politische Verantwortung der Geber durch eine Kopplung der Gelder an Fortschritte im Friedensprozess verankert. Diese FriedensKonditionalität zeigt jedoch keine andauernde Wirkung, wofür zwei Faktoren verantwortlich sind. Zunächst überschätzen die Geber ihre Bedeutung, was auf die Missachtung gegebener ökonomischer Verhältnisse in Sri Lanka in einem Rückgriff auf das Denkmuster der Hilfsabhängigkeit der 1960er und 1970er Jahre schließen lässt. »Arguably, in Tokyo, the donors overplayed their hand, and this had perverse impacts on the peace process. Both the government and donors had an inflated view of the importance of economic levers « (Goodhand/Klem 2005: 79f.). Trotz der ohne Zweifel angestiegenen Arbeit im zivilgesellschaftlichen Bereich, speziell zahlreicher Track 2-Initiativen, sehen Goodhand und Klem die Ursache auch darin, dass Geber nahezu instinktiv dazu tendieren würden, mit dem Staat zusammenzuarbeiten (ebd.), und zugleich keine verlässlichen Benchmarks eingeführt hätten (vgl. Goodhand 2006: 218).

Der zweite Faktor ist ein Naturereignis. Im Dezember 2004 trifft der Tsunami Sri Lanka, führt zu maßloser Zerstörung und fordert etwa 30.000 Todesopfer. Die sofort danach einsetzende Hilfswelle bricht auch in finanzieller Hinsicht alle bis dahin vorstellbaren Rekorde und macht jede Idee einer Konditionalität von Hilfsgeldern, seien sie nun Entwicklungs- oder Humanitäre Hilfe, zur Makulatur. ${ }^{45}$ Die Hoffnung, dass die Tragödie zu einer Wiederaufnahme der Friedensgespräche führen würde, erweist sich als Karikatur des überbordenden internationalen Enthusiasmus zu Beginn des Friedensprozesses drei Jahre zuvor, und erklärt sich aus einer Mischung von Naivität, Zweckoptimismus und einer weitgehenden Ignoranz gegenüber den längerfristigen Wirkungen eines solchen Hilfsprozesses. Die Gesamtsumme der Hilfsleistungen wird, angesichts des nicht geringen Anteils von rein privaten Leistungen, nie vollständig feststellbar sein, sie dürfte sich aber in jedem Fall in der Nä-

$45 \gg$ Following the tsunami and the massive international response, the idea of peace conditionalities became virtually redundant because of the massive inflows of unconditional aid« (Goodhand 2006: 219). 
he von 3 Mrd. US\$ bewegen. Abbildung 24 dokumentiert den massiven Anstieg allein bei den ODA-Geldern, zugleich beginnen Hundertschaften internationaler NGOs kurz- wie mittelfristig angelegte Tätigkeiten, unzählige private Kleininitiativen komplettieren das sich entfaltende Chaos.

Die Schlüsselakteure des Konfliktes versuchen nach einem ersten Schock eine möglichst weitgehende Kontrolle über die Hilfe zu monopolisieren. Dies dient allerdings nicht der besseren Koordination oder der Steigerung der Effizienz, wie das Scheitern der Einrichtung eines von GoSL und LTTE vereinbarten, gemeinsam verwalteten, auf rein technischer Ebene gehaltenen Koordinations-Mechanismus (P-TOMS) zeigt. Vielmehr geht es um politische Aspekte: »As a consequence aid became not only quickly politicised, but also a much-wanted political commodity itself« (Frerks 2006: 86). Von den meisten Akteuren, speziell aus dem privaten Hilfsbusiness, wird dies vollkommen ignoriert. Geld wird dahin geschaufelt, wo es maximale kurzfristige Wirksamkeit garantiert, welche mittel- und langfristigen Wirkungen auch immer damit einhergehen. ${ }^{46}$ Die mit dieser kurzfristigen Orientierung oftmals einhergehende schlechte und verzögerte Umsetzung der Maßnahmen trägt, gerade da sie mit den gut organisierten und generell effizienten Hilfsleistungen der militärisch bzw. militant organisierten Organisationen wie der LTTE oder der JVP verglichen werden, zu einer Demontage des Rufes vor allem der Zivilgesellschaft, speziell der INGOs, bei (vgl. Rajasingham Semanayake 2006: 10f.). ${ }^{47}$

Die bilateralen Akteure, aber auch die multilateralen Agenturen und die meisten der schon vor dem Tsunami in Sri Lanka aktiven NGOs finden einen anderen Weg des Umganges, der sich an zwei Modebegriffen des Sicherheitsdiskurses im entwicklungspolitischen Bereich orientiert: »do no harm« und Konfliktsensitivität. Dies wirft wiederum zwei Probleme auf, die sich einerseits aus der spezifischen Umsetzung dieser Begriffe, andererseits aus einer sich aus ihnen beinahe notwendigerweise resultierenden Ignoranz ergeben. Die spezifische Umsetzung im Kontext der Tsunami-Hilfe bedeutet nicht nur eine gute Nachvollziehbarkeit der

46 Eine der aussagekräftigen, weil paradoxen Praktiken ist das aggressive An- und Abwerben von Personal, das vor allem von auf den humanitären Bereich spezialisierten INGOs betrieben wird. Jene NGOs, die sich zu niedrigen Lohnniveaus verpflichtet hatten, um ihre persönliche Einsatzbereitschaft zu dokumentieren, sehen sich angesichts dieses Booms in einem kleinen, elitären Segment des sri-lankischen Arbeitsmarktes bald mit ernsthaften Problemen konfrontiert.

47 Insbesondere das IKRK sowie die verschiedenen aktiven nationalen Rotkreuz-Organisationen stehen in einem medial beständig reproduzierten Ruf des chronischen Versagens (vgl. Rajasingham Semanayake 2006: 11). 
Verwendung der Gelder und die möglichst »opfernahe« Umsetzung der Hilfsleistungen, sondern auch die Annahme, dass konfliktsensitive Umsetzung im als »ethnisch« interpretierten sri-lankischen Konflikt konsequenterweise die möglichst gleichmäßige Bedienung all dieser »Ethnien« bedeuten muss. Dieser Mechanismus, den wir bei den politischen Maßnahmen der Entwicklungszusammenarbeit, auf die im nächsten Abschnitt eingegangen wird, wiederfinden werden, hat letztendlich die fatale Konsequenz, dass ethnische Selbstzuschreibungen nicht nur reproduziert werden, sondern diese Reproduktion in ganz spezifische, organisierte Bahnen gelenkt wird. Denn natürlich ist eine derart interpretierte konfliktsensitive Hilfe nicht in der Lage, mit dem Prozess der politischen Verwertung der Hilfe zu brechen. Es geht ihr primär darum, diese Verwertung gleichmäßig unter den relevanten Akteuren und ihren Einflussbereichen $\mathrm{zu}$ verteilen, womit die eigentlich $\mathrm{zu}$ Begünstigenden wiederum gezwungen sind, sich noch stärker hinter diesen Akteuren zu scharen. Zweifellos wird dieser Prozess durch die Tsunami-Hilfe nicht ausgelöst, in jedem Fall jedoch verschärft, insbesondere an der umkämpften Ostküste, aber auch im Süden des Landes.

Zugleich wird mit der konkreten Verteilungsfrage, welche Community nun im Sinne einer konfliktsensitiven Hilfe zu begünstigen sei und welche nicht, die Problematik der budgetären und makroökonomischen Auswirkungen der Hilfswelle vollkommen ausgeblendet. Es gibt tatsächlich bislang keine wissenschaftliche Untersuchung zu dieser Problemstellung in Sri Lanka, und die Frage, inwieweit die einfließenden Hilfsgelder nicht mittelbar zur Wiederaufnahme der Kämpfe beigetragen haben, gehört zu den großen Tabuthemen der ansonsten pflichtbewusst selbstkritischen Auseinandersetzung in Geberkreisen. Wenngleich die Frage an dieser Stelle nicht beantwortet werden kann, seien nur zwei Prozesse genannt, die es zumindest wert wären, in diesem Zusammenhang genauer untersucht zu werden.

Einerseits versucht die sri-lankische Regierung von Anfang an mit dem durchaus vernünftig klingenden Argument der notwendigen Nachhaltigkeit der Maßnahmen die Hilfsleistungen auf den Infrastrukturbereich auszudehnen. Damit werden jedoch wiederum Budgetmittel freigespielt, die dem Verteidigungsetat zugeführt werden können, wie es einem bekannten Mechanismus entspricht: »It can be argued [...] that aid creates an economic buffer for the government, allowing the defence budget to grow, and to an extent postponing the costs of the conflict« (Goodhand 2001: 79; vgl. auch Ofstad 2002: 192). Andererseits führen allein die Summe der Hilfsgelder und die sich daraus ergebenden wirtschaftlichen Tätigkeiten zu einem Boom, von dem die Staatskasse zumindest mittelbar profitiert. Wenngleich es angesichts der chronisch ho- 
hen Inflation derzeit zweifelhaft scheint, ob dieser Boom in eine Neuauflage der günstigen ökonomischen Entwicklung in den Anfangsjahren des Krieges umgemünzt werden kann, konnte damit in jedem Fall die Rezession von 2001 in kürzester Zeit vergessen gemacht werden.

Zugleich tragen auch politische Ereignisse $\mathrm{zu}$ einem endgültigen Scheitern des Friedensprozesses bei. Schon im März 2004 kommt es zu einer Spaltung innerhalb der LTTE. Einer der führenden Kommandanten, Colonel Karuna, setzt sich mit seinen Truppen von der LTTE ab, unter dem Vorhalt, diese würde Jaffna-zentriert agieren und die Interessen der Tamil/innen an der Ostküste vernachlässigen. ${ }^{48}$ Faktisch läuft Karuna, der eine Partei unter dem Namen TMVP formiert, nach einer politisch indifferenten Zwischenphase ins Regierungslager über, was eine empfindliche Schwächung der LTTE, insbesondere in den zu diesem Zeitpunkt noch von ihr kontrollierten Gebieten um Batticaloa, nach sich zieht (vgl. ICG 2006: 9; Philipson/Thangarajah 2005: 39ff.). Das sieht die sri-lankische Armee wiederum als gute Möglichkeit zu einer Rückeroberung der Gebiete im Osten, was sie als ersten Schritt zu einem militärischen Sieg gegen die LTTE versteht.

Aber auch die Verhältnisse auf Seiten der sri-lankischen Regierung ändern sich im Sinne einer Weiterführung der militärischen Option. Ende 2003 setzt die Präsidentin Kumaratunga, mit gewichtiger Stützung der Armee, die Entlassung der UNP-geführten Regierung unter Ranil Wickremasinghe durch. Bei den Neuwahlen kann das SLFP-Wahlbündnis in einer taktischen Koalition mit der JVP einen relativ knappen Wahlsieg einfahren und eine Regierung unter Leitung des aufstrebenden SLFP-Politikers Mahinda Rajapakse bilden. Damit sind die Friedensbemühungen des GoSL faktisch beendet. Im November 2005 endet Kumaratungas Präsidentschaft, ihre Wiederwahl ist durch eine Verfassungsklausel ausgeschlossen. ${ }^{49}$ Um die Nachfolge Kumaratungas bewerben sich Premier Rajapakse und UNP-Führer Wickremasinghe, wobei sich Rajapakse, auch aufgrund eines überraschenden Aufrufes der LTTE zum Wahlboykott - Wickremasinghe hatte mit der überwiegenden Zahl der tamilischen Stimmen im Norden und Osten gerechnet -mit 50,29 \% der Stimmen knapp durchsetzen kann. ${ }^{50}$

48 Inoffiziell gelten schwere persönliche Differenzen zwischen Karuna und dem Leiter des LTTE-Geheimdienstes, Pottu Amman, als Auslöser der Spaltung.

49 Kumaratunga akzeptiert das zunächst nicht. Sie argumentiert, dass ihr noch ein Jahr Präsidentschaft zustehen würde, da die Wahl im Jahr 1999 ein Jahr verfrüht durchgeführt wurde. Das Höchstgericht weist dieses Ansinnen zurück.

50 Der Boykottaufruf der LTTE hat - auch wenn stark ansteigende soziale Disparitäten sicherlich ebenso für den Popularitätsverlust der UNP ver- 
Mahinda Rajapakse setzt seine Präsidentschaft von Beginn an unter das Leitbild demonstrativer Stärke. Zwar beruft er unter dem neuen Premier Wickremanayake eine Regierung ein, die mit 107 Minister/innen ${ }^{51}$ nicht nur die größte in der Geschichte Sri Lankas, sondern vermutlich auch eine der größten Regierungen weltweit ist, diese dient aber eher zur Bedienung verschiedener Klientelinteressen denn einem pluralistischen Führungsstil. Beobachter (vgl. etwa CPA 2007) gehen von einem zunehmend kleinen, diktatorisch agierenden Zirkel aus, der die wesentlichen politischen Entscheidungen trifft und sich um Rajapakse und seine beiden Brüder, Gotabaya Rajapakse, dem als Sekretär seines Bruders das Verteidigungsministerium unterstellt ist (das formelle Ministeramt liegt beim Präsidenten), sowie Basil Rajapakse gruppiert, der keine offizielle Funktion ausübt, in seiner Kapazität als einflussreicher Bruder des Präsidenten aber dennoch Staatsbesuche durchführt und das Kabinett koordiniert. Dazu kommt, dass über $60 \%$ des Budgets direkt unter Verantwortung des Präsidenten verwaltet werden, was dem Großteil des Kabinetts effektiv jede Arbeitsgrundlage entzieht.

Zusammen mit dieser Zentralisierung setzt sich mit der Übernahme der Präsidentschaft durch Mahinda Rajapakse auch eine neue Entwicklungsvision durch, die mit der von der UNP vollzogenen und eng mit einem Friedensschluss verknüpften Liberalisierungspolitik bricht, die zentral auf die Stützung durch die etablierte Gebergemeinschaft aufbaute. ${ }^{52}$ Speziell die Idee staatlich angekurbelter großer Infrastrukturprojekte wird in Rajapakses Manifest »Mahinda Chintanaya« wieder in den Mittelpunkt der Bemühungen gerückt, wobei sich die den internationalen Akteuren zugedachte Rolle signifikant verschiebt: »[Mahinda Chintanaya] questioned the existing framework of the peace process and external interference, whilst stressing autarkic economic development and the unitary nature of the Sri Lankan state« (Goodhand/Walton 2007: 6).

antwortlich sind (vgl. Bastian 2005: 38) - wesentlich zur Wahlniederlage Wickremasinghes beigetragen, was eine Wiederaufnahme der Friedensverhandlungen, die er angekündigt hatte, verunmöglichte. Diese allgemein als unlogisch wahrgenommene Vorgangsweise der LTTE hat Verschwörungstheorien aufkommen lassen. Vor allem wird über ein mögliches geheimes Abkommen zwischen Rajapakse und der LTTE spekuliert, wofür aber bislang weder die lustvoll darüber schreibende Tagespresse noch jene SLFP-Politiker, die diese Anschuldigungen zuerst erhoben, Beweise vorlegen konnten.

51 Angesichts der Menge ist die genaue Anzahl einer verhältnismäßig großen Schwankung ausgesetzt. Die genaue Zusammensetzung ändert sich allein im ersten Halbjahr 2007 drei Mal.

52 Die Entwicklungsvision der Wickremasinghe-Regierung ist im Manifest »Regaining Sri Lanka« zusammengefasst, einsehbar unter http://poverty2. forumone.com/files/Sri_Lanka_PRSP.pdf (Zugegriffen am 16-07-2007). 
Gesucht werden nun internationale Großinvestitionen, die nicht mit politischen Bedingungen, sondern mit einer mittel- und langfristigen Profitorientierung verknüpft sind: neben Pakistan, Südkorea und dem Iran wird dabei China als strategischer Partner immer wichtiger. Allein für den Ausbau des als Hafen- und Industriegebiet vorgesehen Wahlbezirkes von Rajapakse, Hambantota, stellt China 1 Mrd. US\$ zur Verfügung.

Obwohl sich die objektive ökonomische Bedeutung der im DAC organisierten Geber damit weiter relativiert, - überhaupt fällt Entwicklungshilfe gegenüber den Devisen, die durch Arbeitsmigration ${ }^{53}$ und Tourismus ins Land kommen, relativ weit ab, wobei sich aber der Tourismus in einer gewissen Wechselwirkung mit der politischen Situation befindet - werden sie auch von der Regierung Rajapakse keineswegs ignoriert. Neben den willkommenen finanziellen Hilfen ist es vor allem die internationale politische Unterstützung für den Krieg gegen die LTTE, die gerade angesichts jüngst erzielter bemerkenswerter diplomatischer Erfolge in diesem Zusammenhang - die EU hat die LTTE Ende Mai 2006 zur terroristischen Organisation erklärt - nach wie vor politische Priorität besitzt. Dennoch hat sich die Position des GoSL darauf festgelegt, den Krieg als »internal matter« verstehen zu wollen (Keethaponcalan 2005: 206), in dem allgemeine Unterstützung erwünscht ist, konkrete Einmischung jedoch abgelehnt wird.

Angesichts dieser Situation ist das Verhältnis zwischen den DACGebern und der Rajapakse-Regierung von einer fast paradox anmutenden doppelten Selbstüberschätzung geprägt. Einerseits hängen die Geberagenturen - speziell die europäischen, die USA schätzen ihre Rolle demgegenüber viel schwächer sein - nach wie vor stark am Bild eines objektiv einflussreichen Akteurs, der faktisch in der Lage ist, Verhalten durch seine Aktivitäten maßgeblich zu beeinflussen: der für 2007 verhängte Stopp neuer ODA-Zusagen durch Deutschland als Protest gegen den Kriegskurs des GoSL demonstriert diesen auf schwachen Füßen stehenden Glauben an die Macht der Konditionalität. ${ }^{54}$

Auf der anderen Seite sieht die gegenwärtige sri-lankische Regierung die Insel als strategisch derart bedeutsam, dass es für die verschie-

53 Die Migration von Arbeitskräften mit dem Hintergrund der Entlastung des sri-lankischen Arbeitsmarktes und der Gewährleistung eines Devisenstroms ins Land wird von Sri Lanka schon seit den 1970er Jahren massiv forciert (vgl. Donner/Thibault 1994: 356).

54 Der relativ hohe Organisationsgrad der internationalen bilateralen und multilateralen Agenturen in Colombo im sicherheitspolitischen Bereich mit der Donor Peace Support Group wurde ein eigenes Forum geschaffen, das diesem Thema exklusiv gewidmet ist - deutet allerdings auf die sich durchsetzende Einsicht in die eigene Schwäche hin. 
denen internationalen Akteure gar nicht möglich wäre, sich zurückzuziehen. Das Wechselspiel zwischen der demonstrativen Kühle, die den Geberagenturen von Regierungsseite zumeist entgegengebracht wird, mit offen zur Schau gestellter Freude, wenn neue Zusicherungen von Hilfsleistungen erreicht werden können, legt nahe, dass die Regierung tatsächlich einer ideologisch begründeten Fehleinschätzung über die internationale Position des Landes aufsitzt. Zugleich hat dieses demonstrative Unterstellen strategischer Interessenslagen eine wichtige politische Funktion. Sie eröffnet einen populistischen Rahmen für nationalistisch argumentierte Politik, der gerade angesichts des laufenden Krieges und der damit einhergehenden Kosten, die die Bevölkerung derzeit vorwiegend über die galoppierende Inflation zu bezahlen hat, für die innere Stabilität überaus wichtig ist. Außerdem liefert eine solche Unterstellung den radikalen singhalesisch-nationalistischen Kräften, sowohl in ihrer linken Strömung um die JVP als auch in ihrer buddhistischen Spielart, wie sie gegenwärtig von der JHU repräsentiert wird, ein Argumentationsmuster, mit dem sich die Rajapakse-Regierung elegant öffentlich unter Druck setzen lassen kann, ohne unter relevanten politischen Handlungszwang zu geraten. Die internationalen Akteure und die in der Phase des Friedensprozesses als mit ihnen verbündet wahrgenommene jetzige Oppositionspartei UNP, übernehmen in diesem Szenario die Rolle des Ventils, besonders aber die NGO-Szene in Colombo, die im Zuge des Friedensprozesses von den meisten bilateralen Geberagenturen als strategischer Partner im Bereich der Friedensentwicklung aufgebaut worden war.

Derartige Attacken, von Susantha Goonatilake (2006) unter dem Slogan von »Foreign Funded NGOs«, die als Instrument der neokolonialen Durchdringung fungierten, auf den Punkt gebracht, haben nicht nur den Spielraum der im friedenspolitischen Bereich tätigen zivilgesellschaftlichen Akteure stark eingeschränkt, sondern auch die Wahrnehmung der Gebergemeinschaft speziell im sich als singhalesisch verstehenden Teil der Bevölkerung nachhaltig negativ beeinflusst. Zugleich bereitet sie damit den argumentativen Boden für eine Wiederaufnahme des Krieges ${ }^{55}$ : $\gg$ Arguably, peace skeptics have been much more effective

$55 \mathrm{Ab}$ Mitte des Jahres 2006 führt die sri-lankische Armee eine Offensive im Osten des Landes durch, die in der Eroberung aller LTTE-Basen außerhalb des Vanni bis Mitte 2007 resultiert. Dies wird auch dadurch möglich, dass die abgespaltene Karuna-Fraktion, politisch als TMVP zusammengefasst, laut zahlreichen Augenzeugenberichten unmittelbar mit Armeekräften kooperiert. Die LTTE verlegt sich im Osten auf eine Guerillataktik, die sich - speziell mit den spektakulären Einsätzen der »Tamil Eelam Air Force«, die mehrere Bombardements mit Kleinflugzeugen durchführt zunehmend auch auf Colombo erstreckt. Im Zuge dieser Offensive wurden 
than the peace advocates in reaching out to societal groups « (Goodhand/Klem 2005: 85). ${ }^{56}$

So vehement diese Kritik von Betroffenen zurückgewiesen wird ${ }^{57}$, baut sie dennoch auf Problematiken auf, die nicht einfach von der Hand $\mathrm{zu}$ weisen sind und zunehmend auch von aus dem betreffenden Spektrum Kommenden kritisch diskutiert werden. Sri Lanka kann an und für sich speziell im Bereich des buddhistischen Aktivismus auf eine lange zivilgesellschaftliche Tradition zurückblicken (vgl. Wickramasinghe 2001: 76). Beispielhaft sei die von Ghandi inspirierte buddhistische Bewegung Sarvodaya erwähnt (zur Geschichte von Sarvodaya vgl. Bond 2004), die bereits seit 1983 mit dem »Sarvodaya Peace Movement« im Bereich der Friedensentwicklung aktiv ist. Allerdings hat die durch das verstärkte Engagement der Geber im zivilgesellschaftlichen Bereich bedingte Professionalisierung Erscheinungen hervorgebracht, die nicht nur von nationalistischen Kräften relativ leicht als »fremd « zu brandmarken sind, sondern auch komplexe Problemstellungen für den Zweck des Engagements selbst beinhalten: »For the Sri Lankan peace movement, this process meant a process of formalisation and the shaping of democratic structures (at least on paper), and a >monetarisation « of peace work peace work was increasingly done with money, not voluntarily, by NGOs competing on an >aid market«" (Orjuela 2004: 256).

Eine solche Praxis führt nicht nur zur Proliferation von Projekten, Aktivitäten und Trainings, deren primärer Zweck in der Zufriedenstellung der Geldgeber besteht ${ }^{58}$, sondern untergräbt jeden friedenspolitischen Aktivismus, der ohnehin auf eine nur spärlich vorhandene Traditi-

über 200.000 Menschen, vor allem in den Distrikten Batticaloa und Trincomalee, vertrieben.

56 Sowohl die JVP als auch die buddhistisch-nationalistischen Kreise bedrängten die Rajapakse-Regierung, das Waffenstillstandsabkommen mit der LTTE zum fünften Jahrestag seiner Unterzeichnung zu kündigen. Buddhistische Mönche veranstalteten sogar ein öffentliches Fasten für die Aufkündigung des Friedensprozesses. Die Regierung kam diesen Forderungen nicht nach, sondern forcierte die militärische Offensive bei einem gleichzeitigen formalen Bekenntnis zum Friedensprozess.

57 Als Beispiel sei Kumar Rupesinghes (2006b:lvi) Darstellung angeführt: »NGOs and civil society organizations working for peace are also a subject of vilification and demonization. A small but determined group of extremists have used the media and public platforms to denigrate the peace and human rights organizations in particular. Foreign funded NGOs have been a special target of attack.«

$58 \gg$ Teaching ordinary people how to solve conflicts and overcome prejudice implicitly conveyed the message that they are culpable for local insecurity and ethnic conflict, which in fact most often are problems which emanate and are sustained by actors and structures far outside of local control« (Orjuela 2004: 260). Kumar Rupesinghe beispielsweise teilt diese Kritik. 
on von zivilgesellschaftlichem Voluntarismus zurückgreifen kann: »The aid funded peace movement was stuck in these structures, relying on outside funding and having its own North-South divide, and did thus only to a limited extent manage to develop the potential for people's solidarity and voluntary civic engagement« (ebd.: 262). Ebenso wenig wie es den Gebern gelingt, »to ১buy peaceく in Sri Lanka« (Goodhand/Walton 2007: 10), ist es ihnen also möglich, einen Friedensaktivismus finanziell zu stimulieren. Stattdessen führt das Engagement zur Herausbildung einer Schicht von »Peace-Professionals« (Liyanage 2007), die sich demnach nicht durch freiwilliges Engagement, sondern durch vergleichsweise gute Ausbildung und englische Sprachkenntnisse auszeichneten und damit das prädestinierte Personalreservoir für Friedens-NGOs darstellten, die sich auf das Einfahren einer »Friedens-Rente« (ebd.) konzentrierten.

Zugleich tendiert aber auch der Konflikttransformations-Ansatz, der von den internationalen Akteuren primär im zivilgesellschaftlichen Bereich verankert wird, generell dazu, politischen Aktivismus zu untergraben. Entlang dieses Ansatzes wird nicht nur verlangt, im Sinne maximaler Chancen auf einen Friedensschluss möglichst wenig politische Position zu beziehen, gerade im Verhältnis zu den Parteien des bewaffneten Konfliktes. ${ }^{59}$ Zugleich wird postuliert, eine offene und grundsätzlich gesprächsbereite Haltung gegenüber all jenen vor allem aus dem nationalistischen Spektrum kommenden Gruppen einzunehmen, die einen militärischen Sieg gegen die LTTE einfordern. Dieses »Constructive Critical Engagement« mit so genannten »Spoilern« wird derzeit in der Gebergemeinschaft als das Um und Auf für eine erfolgreiche Wiederaufnahme von Friedensverhandlungen gesehen, was allerdings den politischen Spielraum für jede nationalistisch-kritische Initiative praktisch verunmöglicht.

Gleiches gilt für die Strategie der internationalen Akteure, im Sinne einer stärkeren Inklusivität des Friedensprozesses die politische wie organisatorische Formierung möglichst homogener ethnischer Positionen $\mathrm{zu}$ forcieren. Hatte schon das Waffenstillstandsabkommen explizit die

59 Alan Keenan (2007) kritisiert diese Vorgangsweise als »Evenhandedness«, die jeden politisch oder rechtlich, etwa an den Menschenrechten orientierten Ansatz untergraben würde. Keenan schlägt als Alternative einen »neither/nor«-Ansatz vor, der mit keiner der Konfliktparteien sympathisiert und sie gleichermaßen kritisiert. Als Beispiel für diesen Ansatz hebt er die Praxis der University Teachers of Human Rights (Jaffna), UTHR(J), hervor, die gleichermaßen kritisch mit Regierung und LTTE verfahren würden. Die Mitglieder der UTHR(J) sind von der LTTE zum Tod verurteilt und agieren im Untergrund. 
Entwaffnung aller nicht der LTTE zugehörigen tamilischen Milizen ${ }^{60}$ verlangt, was dem tamilischen Alleinvertretungsanspruch der LTTE in die Hände spielte, ist die Konsequenz aus der zentralen Kritik am Friedensprozess, dass einige wesentliche Akteure ausgeschlossen und damit politisch marginalisiert worden seien, die Formierung einer weiteren Partei: der Muslime. Über die Förderung der Bildung eines muslimischen Friedenssekretariates, des Peace Secretariat for Muslims (PSM), das von der Berghof Stiftung und Norwegen massiv vorangetrieben wurde und mittlerweile auch von USAID unterstützt wird, wird damit eine dritte ethnisch kodierte Partei festgeschrieben. ${ }^{61}$ Kritiker/innen dieses Prozesses sehen eine solche Konstruktion einer sri-lankischen muslimischen Identität als ein Projekt einer islamischen Elite, das vorwiegend zur Stärkung ihrer eigenen Position dient (vgl. etwa Ismail 1995). In jedem Fall ist es aber ein weiterer Beitrag zur entwicklungspolitischen Reproduktion ethnisierter Identität.

So stellt sich die gegenwärtige Situation der westlichen Gebergemeinschaft in Sri Lanka - gesehen im Unterschied einerseits zu Japan, das durch seinen großen finanziellen Einsatz eine vergleichsweise starke Position innehat, und zu neuen aufstrebenden asiatischen Akteuren, die nicht im DAC organisiert sind und damit auch nicht dem gängigen Bild von Entwicklungshilfe entsprechen, wie etwa China - derzeit durchwachsen dar. Einerseits hat sich die Orientierung auf Peacebuilding spätestens mit dem Beginn des jüngsten Friedensprozesses im Jahr 2001 als hauptsächlicher Ansatz etabliert, andererseits zeigen aber gerade die jüngsten Ereignisse seit der Regierungsübernahme durch Mahinda Rajapakse den äußert eingeschränkten Einfluss der internationalen Akteure auf den Verlauf des Konflikts. Dies hat zu einer neuen Skepsis geführt, die einige Geber (wie etwa Schweden) sogar dazu veranlasst, über einen Rückzug nachzudenken. In der derzeitigen Zusammensetzung forciert das GoSL einen klaren Kriegskurs, der sich nationalistisch konstruiert und damit jeder Einmischung offensiv begegnet.

60 Primär gemeint sind hier vor allem die Milizen der Eelam People's Democratic Party, EPDP, die mit der Armee zusammenarbeiten. EPDPParteichef Douglas Devananda ist derzeit auch sri-lankischer Kabinettsminister.

61 Der Leiter des PSM, M.I.M. Mohideen, begründet diesen Prozess aus der Notwendigkeit einer ethnischen Parität: »It is nothing but fair that the Muslims should also have a Muslim majority regional council to share power like the Sinhalese and Tamils of Sri Lanka« (Mohideen 2006: 333). 
Die vehementen Attacken auf die Geber und auf mit ihnen verbündete private Akteure wie INGOs oder $\mathrm{NGOs}^{62}$ durch wieder erstarkte radikale Parteien wie die $\mathrm{JVP}^{63}$ sind nur vor diesem Hintergrund zu verstehen und haben den Manövrierraum speziell für die schwerpunktmäßig im Peacebuilding-Bereich aktiven Geber schwerwiegend eingeschränkt. Diese Situation wird dadurch komplettiert, dass sich auch die LTTE, historisch gegenüber der internationalen Intervention eher aufgeschlossen, derzeit in einer »Peace Trap« (Goodhand/Walton 2007: 6) gefangen und kurzfristig keine Möglichkeit für eine Verhandlungslösung sieht.

Der historische Verlauf der internationalen entwicklungspolitischen Intervention zeigt allerdings ohnehin, dass das strategische Potenzial dieser Intervention gering ist und von einem Großteil der Akteure nie in relevantem Maße genutzt wurde. Es ist vielmehr eine Widerspiegelung internationaler Trends vorzufinden, die, nur dünn mit Analysen unterfüttert, im Sinne der vorgegebenen Effizienzkriterien - Modernisierung bis in die 1990er, Konfliktsensitivität ab den 1990ern - umgesetzt wurden. Das heißt allerdings nicht, dass keine nachhaltige Wirksamkeit dieser Interventionen festzustellen wäre. Hier zeigt sich, dass Entwicklungshilfe zumindest mittelbar, faktisch während ihrer gesamten Dauer ihrer Präsenz in Sri Lanka, auch als Hilfe zum Krieg dient. Die Selbstkritik der meisten Geber um die Jahrtausendwende hat die Vorzeichen der Intervention ohne Zweifel verschoben, schreibt aber die tendenzielle Unkalkulierbarkeit ihrer Wirkung weiter fort, was in jedem Fall garantiert, dass Entwicklungshilfe, solange die politisch relevanten Kräfte auf der Insel den Krieg wollen, auch gegen ihren Willen Mittel in diesem Krieg bleiben wird. Es ist dieser Kontext, in dem sich die Praktiken der USA und Deutschlands vollziehen, auf die ich im folgenden Abschnitt detaillierter eingehen will.

62 So wurde etwa die Repräsentanz des niederländischen NGOs ZOA, das vorwiegend in der Flüchtlingsbetreuung tätig ist, in Colombo im Jänner 2007 von JVP- und JHU-nahen Kräften physisch angegriffen, nachdem die sri-lankische Armee behauptet hatte, dass ZOA ein Feldlazarett in einem LTTE-verwalteten Camp betreiben würde. Die Polizei schritt erst ein, als der niederländische Botschafter persönlich zum Schutz der Repräsentanz am Ort des Geschehens eintraf.

$63 \mathrm{Zu}$ dieser neuen Radikalisierung vgl. Rampton/Welikala (2005). 


\section{Democracy Promotion und Peacebuilding revisited - Die entwicklungspolitischen Praxen der USA und Deutschlands}

Weder für die USA noch für Deutschland stellt Sri Lanka eine besondere außenpolitische Priorität dar. Für die USA gilt das vielleicht sogar noch mehr als für Deutschland, wenn wir in Betracht ziehen, dass sich die eingesetzten finanziellen Mittel bei beiden in der etwa gleichen Dimension bewegen, Deutschland jedoch bei den ODA-Geldern ein eindeutig höheres Volumen investiert - bis zum Stopp von Neuverpflichtungen im Jahr 2007 aus Protest gegen die Politik der RajapakseRegierung. Diese Ausgangssituation schafft grundsätzlich günstige Bedingungen für einen Vergleich der zivilen Intervention, der in Ländern, die wie etwa Kolumbien zu einer außenpolitischen Priorität der USA zählen, aufgrund der Unverhältnismäßigkeit der eingesetzten Gelder und der engen Anknüpfung der Entwicklungspolitik an den dominanten militärischen Bereich nicht so gut möglich wäre.

\section{Die Intervention der USA}

Den USA werden wie bereits erwähnt dennoch nahezu reflexartig geostrategische Interessen unterstellt, die um die Bedeutung Sri Lankas, vor allem im Hinblick auf den natürlichen Tiefseehafen von Trincomalee, für die Position der US-Marine im Indischen Ozean kreisen. Dieser könnte bei der Versorgung des abgelegenen Stützpunktes auf Diego Garcia eine besondere Rolle spielen, da Sri Lanka der Insel geographisch relativ nahe liegt (vgl. Somasundaram 2005: 139ff.). Ein solches Planspiel wird von US-Offiziellen mit dem Verweis auf die viel günstigere Situation in Singapur zurückgewiesen. Singapur kooperiert in Sicherheitsfragen eng mit den USA und stellt seinen Hafen für die USMarine zur Verfügung, daher: „Contrary to the musings of various South Asian theorists, the U.S. does not have, and has never had, any interest in the use of the harbor at Trincomalee for military purposes « (Lunstead 2006: 11). ${ }^{64}$

Die Summen, die für das Entwicklungshilfe-Programm in Sri Lanka aufgewendet wurden und werden, bestätigen diese Aussage weitestgehend (siehe Abbildung 25). ${ }^{65}$ Dennoch ist der Beginn des Engagements

64 Der hier zitierte Jeffrey Lunstead fungierte von 2003 bis 2006 als USBotschafter in Sri Lanka.

65 Das USAID-Programm in Sri Lanka ist, gemessen an seiner Mittelausstattung, im südasiatischen Vergleich das eindeutig kleinste (Bhutan und die Malediven, wo jeweils eine nur minimale Präsenz besteht, nicht einbe- 
im Jahr 1956 sehr wohl von geostrategischen Erwägungen geleitet. Der Kalte Krieg und das politisch als unzuverlässig eingestufte Indien bilden wesentliche Antriebsmomente beim Aufbau einer keinesfalls überragenden, aber doch signifikanten Präsenz im damaligen Ceylon. Die Blütezeit der US-Entwicklungshilfe in Sri Lanka beginnt mit der Regierungsübernahme von J.R. Jayawardene, dessen Liberalisierungspolitik bei gleichzeitiger Umsetzung infrastruktureller Großprojekte den Entwicklungsvisionen der USA derart entgegenkommt, dass die eingesetzten Summen (aufgerechnet auf den Wert von US\$ im Jahr 2005) die höchsten jemals investierten Beträge darstellen (vergleichsweise sogar höher als die Humanitäre Hilfe im Zuge des Tsunami).

\section{Abbildung 25: US-amerikanische ODA in Sri Lanka (in 2005-US\$)}

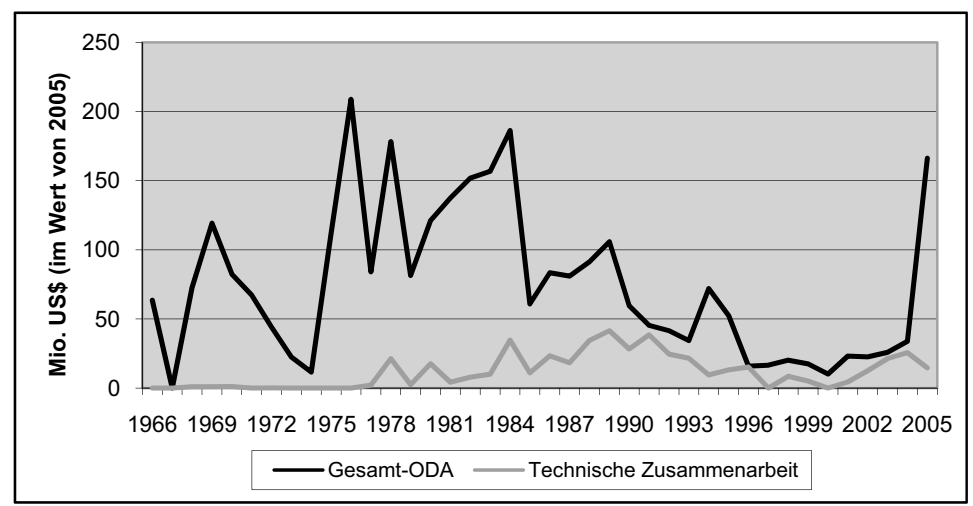

Mitte der 1980er Jahre gehen die US-Leistungen in der Folge der Zuspitzung der bewaffneten Konflikte massiv zurück, um nach Ende des Kalten Krieges überhaupt in Agonie zu verfallen. Selbst eine komplette Schließung des Programms steht zur Disposition: "At the end of the 1990s, at least some persons in USAID proposed closing the program entirely« (Lunstead 2006: 12; vgl. auch Noyahr 2006: 381; Young 2000). $\mathrm{Zu}$ einer solchen Schließung kommt es allerdings nicht, wofür neben der offiziellen Begründung, nach der Sri Lanka ein prädestiniertes Land zur Verankerung von Grundwerten der US-Außenpolitik wie Demokratie und Freihandel sei (vgl. USAID 2000c: 1), laut Jeffrey Lunstead (2006: 13) vor allem drei Faktoren verantwortlich sind: erstens die Nachwirkungen von 9/11, die die Anti-Terror-Politik der USA - die LTTE war schon seit Einführung der Listung ausländischer Terrororga-

rechnet). Das Programm in Nepal ist beispielsweise finanziell vierfach größer dimensioniert. 
nisationen im Jahr 1997 als eine solche Foreign Terrorist Organization (FTO) klassifiziert - zu einer globalen Priorität werden lassen; zweitens die gute Arbeitsgrundlage mit den UNP-geführten WickremasingheRegierungen (in der ersten Hälfte der 1990er und nach 2001); sowie drittens das persönliche Engagement des stellvertretenden Secretary of State von 2001 bis 2005, Richard Armitage ${ }^{66}$ (vgl. Samaranayake 2006: 180).

Der wahrscheinlich entscheidende Faktor für das Weiterbestehen des USAID-Programms nach den 1990ern ist der beginnende Friedensprozess, der zu einer Überarbeitung des strategischen Plans und einer daraus folgenden signifikanten Verschiebung der Interventionsschwerpunkte mündet. Waren im Strategischen Plan von 2001-2005 (vgl. USAID 2000c) noch die drei Arbeitsfelder Gesundheit, Erziehung und ökonomisches Wachstum zentral, wird im Zuge der Neuausrichtung eine Aufsplittung auf vier Schwerpunkte unternommen ${ }^{67}$ (vgl. Lunstead 2006: 18): mit Humanitärer Hilfe und ökonomischem Wachstum finden sich dabei Komponenten, die schon zuvor eine Rolle spielten, neu ist allerdings die Betonung der entwicklungspolitischen Unterstützung des Friedensprozesses und die Aufwertung des Demokratie- und GovernanceProgramms (D\&G). Dies hatte sich schon zuvor in einem ersten D\&GAssessment (MSI 2001) abgezeichnet, in dem ein Arbeitsschwerpunkt im Bereich demokratischer Reformen vorgeschlagen wurde.

Die politische Vorgangsweise der USA im Friedensprozess ist durch eine betont prinzipientreue und mit einem hohen Grad an Symbolismus verknüpfte Linie geprägt, die nicht nur jede Zusammenarbeit mit der LTTE aufgrund ihrer FTO-Klassifizierung kategorisch ausschließt, sondern auch die Einhaltung einer merklichen Distanz gegenüber dem GoSL beinhaltet, was sich im Zuge der Rajapakse-Präsidentschaft noch

66 »Armitage's involvement was so intense that officials in the State Department referred to him as >the Sri Lanka desk officer« (Lunstead 2006: 13). Die Ursache dieses Interesses ist auch für Lunstead nicht ganz erklärlich, weil weder eine nachvollziehbare persönliche Bindung von Armitage an Sri Lanka bestand, noch relevantes politisches Interesse übergeordneter Stellen feststellbar war. Eine mögliche Interpretation lautet dahingehend, dass Armitage von der Euphorie des beginnenden Friedensprozesses angesteckt worden war und er die Möglichkeit einer davon ausgehenden globalen Signalwirkung sah. In einer Rede erklärte er die Notwendigkeit des Engagements auch damit, »because it can be done« (ebd.).

67 Der modifizierte strategische Plan aus dem Jahr 2003 ist im Gegensatz zum Vorgängerplan weder über die Website noch über die zuständigen Personen bei USAID erhältlich, auch wenn er formell kein Verschlussdokument ist. Ich beziehe mich daher auf die Analyse des Plans von Jeffrey Lunstead. 
verstärkt. ${ }^{68}$ In der Umsetzung kommt es zu einer bewussten Anwendung diplomatischer, entwicklungspolitischer und auch militärischer Mittel, wobei insbesondere letztere große öffentliche Aufmerksamkeit auf sich ziehen. Laut dem zu diesem Zeitpunkt zuständigen US-Botschafter ist die militärische Unterstützung Sri Lankas ${ }^{69}$ als $»$ strategy for promoting the peace process « zu verstehen, dessen primäre Ausrichtung in einem klaren Signal an die LTTE bestünde und das daher auch mit rigiden Konditionalitäten für das GoSL versehen sei (Lunstead 2006: 18). Demgegenüber steht die Interpretation, dass diese Militärhilfe von der Regierung insbesondere in der Phase nach 2005, also nach dem Zusammenbruch der Friedensverhandlungen, als Freibrief für eine militärische Vorgangsweise gegen die LTTE verstanden wird. In jedem Fall kann festgehalten werden, dass dieser Militärhilfe augenscheinlich fast ausschließlich symbolische Bedeutung zukommt (vgl. Bastian 2005: 30). Die jährlich im Rahmen des FMF-Programms aufgewendeten Summen liegen im Bereich niedriger einstelliger Millionen-Dollar-Beträge (die IMET-Beträge bewegen sich in vergleichbarer Dimension), was sich angesichts eines sri-lankischen Wehretats, der sich nach massiven Erhöhungen in den Jahren 2006 und 2007 der 1-Mrd.-US\$-Marke annähert, verschwindend gering ausnimmt. ${ }^{70}$

68 Die politische Positionierung der USA in Sri Lanka hat sich in den letzten Jahren immer mehr derjenigen Indiens angenähert, das gegenüber der LTTE seit dem Attentat auf Rajiv Ghandi ebenfalls eine viel härtere Linie einschlägt als die meisten europäischen Akteure. Zugleich zeigt diese Positionsannäherung das sich entwickelnde strategische Bündnis zwischen den USA und Indien (vgl. Bastian 2006: 259), was auch als gegen den Einfluss Chinas gerichtet verstanden werden kann. In jedem Fall, so Bastian (ebd.), unterminierte die Vorgangsweise der USA eine einheitliche Positionierung der westlichen Gebergemeinschaft. Auch die tamilischen Medien agitieren massiv gegen dieses so wahrgenommene »Indo-US design « (vgl. Venkataramanan 2006).

69 Die militärische Unterstützung Sri Lankas erfolgt aus dem FMFProgramm, das direkte Finanz- und Logistikhilfen für befreundete Armeen vorsieht, und dem IMET-Programm, das Trainingsleistungen durch USExpert/innen und die ergänzende Ausbildung an US-Militärinstitutionen vorsieht. Die Militärkooperation läuft schon in den 1990er-Jahren (vgl. Young 2000: 2) und konzentriert sich während des Friedensprozesses vorwiegend auf den Bereich der Air Force, der technische Unterstützung speziell im Ausbau der Flugfeldanlagen und der Ausbildung gewährt wird (vgl. Noyahr 2006: 376).

70 Im gewissen Widerspruch dazu steht, dass zumindest bis zum Fiskaljahr 2007 ein Teil des Programms über Gelder aus dem Economic Support Fund finanziert wird, die ja per Definition für die Unterstützung geostrategischer Verbündeter einzusetzen sind. 
Auf politischer Ebene dominiert die bewusste Distanzierung sowohl gegenüber der LTTE als auch gegenüber dem GoSL, wenngleich die Positionierung gegenüber der LTTE naturgemäß um ein vielfaches schärfer vollzogen wird. Die Aufhebung der FTO-Klassifizierung wird im Zuge der Friedensverhandlungen zwar immer als Motivationsfaktor ins Spiel gebracht, um die LTTE in den politischen Mainstream zu holen. Dennoch wird die Einstufung zu keinem Zeitpunkt gelockert oder gar tatsächlich aufgehoben. Richard Armitage bezeichnet die Praktiken der LTTE im November 2002 im Zuge eines Geber-Treffens in Oslo sogar explizit als terroristisch, was eine wütende Reaktion des LTTEVerhandlungsführers zur Folge hat: »These unwarranted provocative comments, made by a senior US official, discrediting the Tamil freedom struggle at an international forum organised to support and encourage the parties in armed conflict to seek peace and negotiated settlement, annoyed me« (Balasingham 2004: 393). Auch zeigen die USA keine Kompromissbereitschaft, als eine LTTE-Delegation Interesse bekundet, an einem Vorbereitungstreffen zur Tokio-Geberkonferenz in Washington teilzunehmen. Die Weigerung der USA, der LTTE zugehörige Personen einreisen zu lassen, wird von dieser als Begründung zum Abbruch der Verhandlungen genutzt.

Andererseits ist nicht zu übersehen, dass sich die US-Politik gegenüber der sri-lankischen Regierung ebenfalls bewusst reserviert gestaltet. Schon im Strategischen Plan aus dem Jahr 2000 sind kaum direkte Mittelvergaben an die Zentralregierung vorgesehen (vgl. Lunstead 2006: 19), stattdessen stehen Dezentralisierung, vor allem im Sinne der Förderung von lokaler Governance, und die Finanzierung nicht-staatlicher Initiativen zur Unterstützung der Verhandlungen im Mittelpunkt. Auch auf diplomatischer Ebene kommt es zu verhältnismäßig deutlichen Äußerungen, auch wenn die US-Botschaft jene »Schrillheit« vermeiden will, die die europäischen Akteure in der Kritik der Regierung an den Tag legen. ${ }^{71}$

Trotzdem wird das GoSL immer wieder offensiv zu einem verbesserten Schutz der Menschenrechte aufgefordert, zudem wird im ersten Halbjahr 2007 erstmals der Kriegskurs der Rajapakse-Regierung explizit kritisiert. US-Botschafter Blake gibt im Februar 2007 zu Protokoll, dass die USA den Glauben der sri-lankischen Regierung an einen militärischen Sieg über die LTTE nicht teilen würden: »We respectfully disagree. ${ }^{72}$ Gleichwohl wird den USA vom sich als singhalesisch verstehen-

71 Interview mit Mark Silva, Leiter des D\&G-Programms von USAID in Colombo.

72 Siehe The Island, Colombo, 26. Februar 2007. 
den Bevölkerungsteil großes Vertrauen entgegengebracht: nach aktuellen Umfragen wollen etwa $35 \%$ dieses Bevölkerungsteils die USA als neuen Vermittler im Friedensprozess, der Norwegen ablösen sollte (Peiris 2006: 26). ${ }^{73}$

Diese relativ deutliche Positionsbeziehung der USA kann freilich nicht darüber hinwegtäuschen, dass die Bedeutung Sri Lankas für die US-Außenpolitik seit dem endgültigen Scheitern des Friedensprozesses und dem Rücktritt von Richard Armitage ${ }^{74}$ stark gesunken ist. Armitages direkter Nachfolger, der mittlerweile als Präsident zur Weltbank übergewechselte Robert Zoellick, zeigt kein vergleichbares Interesse, womit die Zuständigkeit im State Department dem Unterstaatssekretär Nicholas Burns zufällt. Burns setzt in seiner Tätigkeit allerdings andere Akzente, womit die tatsächliche diplomatische Tätigkeit in Bezug auf Sri Lanka auf die Ebene des für Süd- und Zentralasien zuständigen Assistenzstaatssekretärs Richard Boucher zurückfällt (vgl. Lunstead 2006: 33).

Die massiven Geldsummen, die in den letzten Jahren das ODABudget für Sri Lanka im Zuge der Tsunami-Hilfe aufgeblasen haben, verdecken zudem einen kontinuierlichen Rückgang der Gelder für die Entwicklungszusammenarbeit seit dem Jahr $2004 .{ }^{75}$ Diese Situation wird auch daran deutlich, dass sich das Office of Transition Initiatives, OTI, mit Ende des Jahres 2007 aus Sri Lanka zurückzieht. Das konkrete entwicklungspolitische Programm zeigt sich dennoch ambitioniert und ist von einer klaren Vision getragen, die sich im Verlauf der letzten Jahre nur unwesentlich verändert hat: »The USAID program objectives for Sri Lanka derive from a set of strategic goals that flow from a vision of Sri

73 Angeführt wird diese Umfrage von Indien, das sich $45 \%$ der sich als singhalesisch Verstehenden als Vermittler wünschen. Dies ist ein bemerkenswerter Wandel zu den 1980er Jahren, wo Indien als der wesentliche Verbündete der militanten tamilischen Unabhängigkeitsbewegung wahrgenommen wurde.

74 Armitage galt als enger Vertrauter des ehemaligen Staatssekretärs Powell und trat gemeinsam mit diesem vom Amt zurück.

75 Eine nachhaltige Änderung könnte sich dadurch ergeben, dass sich Sri Lanka anhand der gesetzten Benchmark-Kriterien für das MCA-Programm qualifiziert. Demnach wäre es für maßgebliche ODA-Leistungen der MCC empfangsberechtigt. Diese Leistungen sind allerdings noch nicht angelaufen. Der Zusammenbruch des Friedensprozesses setzt die MCC trotz ihres definiert apolitischen Ansatzes unter Argumentationszwang, da ein laufender bewaffneter Konflikt grundsätzlich nicht in das Programmschema des MCA passt. Die damit abzuwickelnden Programme seien schließlich, so Jeffrey Lunstead (2006: 21), »almost by definition not conflict sensitive«. 
Lanka as a democratic, environmentally sound, newly industrializing country« (Wickramasinghe 2001: 62). ${ }^{76}$

Obwohl sich eine starke Peacebuilding-Komponente durch die Programmatik zieht, ist es die Demokratieentwicklung, die als zentrales Ziel auszumachen ist, und nicht ein Friedensschluss im so analysierten »ethnic conflict« (USAID 2006f.). Ein solcher läge nicht im »manageable interest $«$ von USAID. ${ }^{77}$ So wird auch im entwicklungspolitischen Bereich, im markanten Unterschied zu Deutschland, aber auch zu Kanada und den meisten anderen europäischen Gebern, eine harte Linie gegenüber der LTTE eingeschlagen. Jede/r Empfänger/in eines Grants muss sich im Vorfeld vertraglich verpflichten, keine Beziehungen zur LTTE oder zu LTTE-nahen Organisationen zu haben oder solche einzugehen, andernfalls wird kein Geld zur Verfügung gestellt. Demzufolge werden auch keine USAID-Programme in den von der LTTE kontrollierten Gebieten durchgeführt. ${ }^{78}$

Der spezifisch sicherheitspolitische Charakter der US-Entwicklungszusammenarbeit entfaltet sich im aktuellen Arbeitsprogramm (vgl. ebd.), abgesehen von den mittelbaren Wirkungen der Interventionen mit der Zielsetzung eines ökonomischen Wachstums, das vor allem auf eine Stabilisierung der Livelihood in lokalen Postkonfliktsituationen abzielt $^{79}$, in den Programmelementen »Supporting the Benefits of Peace»

76 Dies wird auch vom Leiter des OTI-Programms in Colombo, Michael DeSisti, bestätigt. Wenn USAID präsent sei, so DeSisti im Interview, werde auch immer ein strategisches Ziel verfolgt. Trotz der Kleinheit des Programms und der Abwesenheit geostrategischer Interessen der USA könnte ein erfolgreicher Friedensschluss, besonders im Sinne einer demokratischen Lösung eines terroristisch geprägten Konfliktes, weit über Sri Lanka hinaus Signalwirkung haben und den von den USA geführten Kampf gegen den Terrorismus wesentlich stärken, gerade in seiner zivilen Komponente.

77 Interview mit Mark Silva, Leiter des D\&G-Programms von USAID in Colombo. Der Begriff »manageable interest « bezieht sich auf das Managementkonzept von USAID, das die Programm- und Projektzielsetzungen wesentlich an der Einschätzung der eigenen Einflussmöglichkeit festmacht. So sieht USAID in Colombo in Hinblick auf Prozesse der Demokratisierung maßgebliche Einflussmöglichkeiten, in Bezug auf den Friedensprozess jedoch nicht.

78 Es ist bemerkenswert, dass eine solche Vorgangsweise aus den FTORichtlinien nicht abzuleiten ist, da diese explizit kein Kontaktverbot mit terroristischen Organisationen vorsehen. Derartige Kontakte könnten ja prinzipiell, etwa zum Zweck von Verhandlungen, politisch notwendig werden. Es handelt sich folglich um eine Policy-Entscheidung, die entweder im State Department oder in Colombo selbst getroffen wurde.

79 »Assistance will be directed toward livelihoods and workforce development of vulnerable populations in demand-driven markets« (State Department/USAID 2007: 570). 
und »Democracy and Governance«, wobei letzteres ohnehin einem Kernbereich der internationalen Tätigkeit von USAID entspricht. Die dabei getätigten Interventionen sind, gerade im Vergleich mit dem Programm der deutschen TZ, verhältnismäßig zahlreich. Generell dominiert eine Aufteilung, nach der die Unterstützung der »Benefits of Peace« von OTI mit kurzfristig angelegten, klein dimensionierten, flexiblen und auf maximale Wirksamkeit ausgerichteten Maßnahmen organisiert wird, während die größeren, längerfristig angelegten Projekte im D\&GBereich dem D\&G-Programm von USAID obliegen. ${ }^{80}$

Abbildung 26: Programmschema von USAID in Sri Lanka

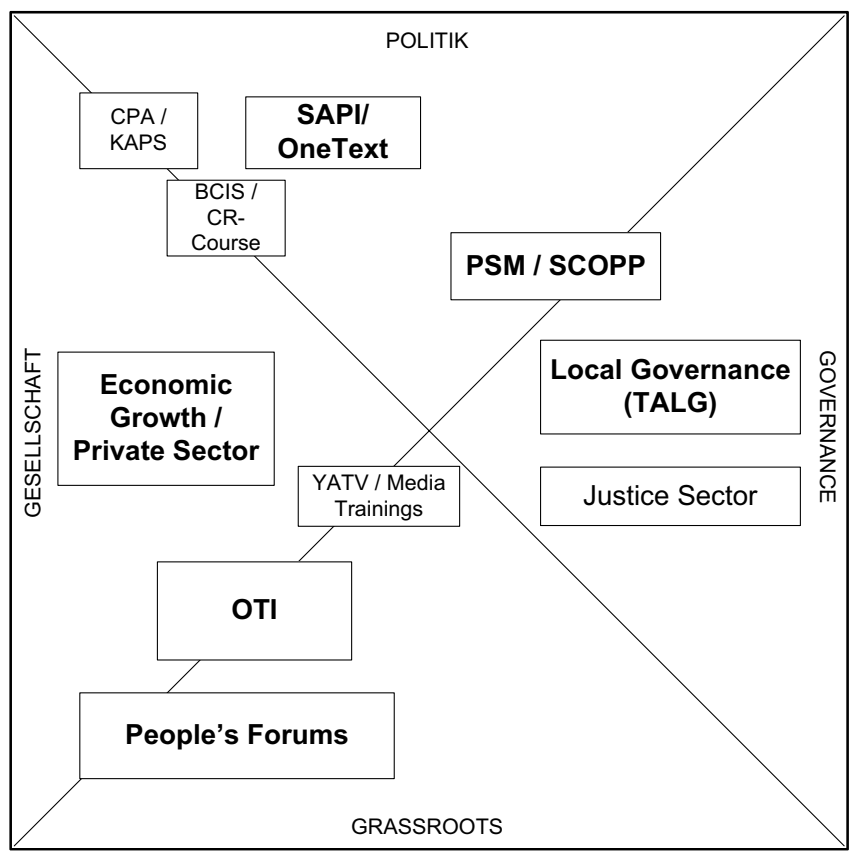

$80 \mathrm{Zu}$ dieser Aufteilung sind zwei technische Bemerkungen zu machen: zunächst ist für die Umsetzung von OTI in Sri Lanka nicht USAID direkt, sondern ein kommerzieller Kontraktor (Development Alternatives Inc., DAI) zuständig, der aber im Namen von USAID auftritt. Eine ähnliche Privatisierung ist für die nächste Phase des D\&G-Programms vorgesehen, das im Moment noch teilweise unmittelbar von USAID, teilweise über den Kontraktor AED (Academy for Educational Development) implementiert wird. Zweitens ist anzumerken, dass sich das Portfolio von USAID mit Ende des Jahres 2007 signifikant verschieben wird, was mit dem Rückzug von OTI und der Übernahme einiger Aktivitäten von OTI durch das D\&GProgramm zu tun hat. Ich beziehe mich im Folgenden auf jene Praktiken, die bis Mitte 2007 verfolgt worden sind. 
Abbildung 26 gibt einen Überblick über die laufenden Interventionen, geordnet anhand ihrer Relevanz ${ }^{81}$ und ihrem Zielsektor, wobei die angeführten kleineren Projekte ausschließlich aus dem D\&G-Programm kommen; die konkreten OTI-Projekte sind nicht einzeln ausgeführt. Die Definition der vier Zielsektoren orientiert sich an dem derzeit üblichen dreistufigen Track-Konzept: der Sektor »Politik« entspricht jener Ebene, die unter Track 1 und Track 1.5 subsumiert wird, der Sektor »Gesellschaft«, unter den ich auch die auf ökonomischer Ebene getroffenen Maßnahmen einordne, Track 2, und »Grassroots« schließlich Track 3. Zusätzlich ist es notwendig, »Governance«, die sowohl für USAID wie auch für GTZ einen Angelpunkt ihrer Interventionen ausmacht, als separaten Sektor hervorzuheben.

Es zeigt sich entlang dieser Einteilung, dass USAID in allen Sektoren schwerpunktmäßige Interventionen setzt. Dennoch ist auffällig, dass keine Komponente dem entspricht, was europäische Geber in Sri Lanka als Arbeit mit oder Aufbau der Zivilgesellschaft verstehen, und dies obwohl »Strenghten Civil Society« als eigens angeführte Programmkomponente im D\&G-Bereich existiert. Darunter zusammengefasst sind einerseits die klar auf die politische Ebene fokussierende Förderung des South Asia Peace Institute (SAPI), andererseits die »People's Forums«, die zwar einen zivilgesellschaftlichen Aspekt aufweisen, hauptsächlich jedoch auf die Forcierung des interkommunalen Austausches an den Grassroots ausgerichtet sind. Dazu kommt die Finanzierung eines Konflikttransformationskurses im Bandaranaike Centre for International Studies (BCIS), einem Postgraduate-Institut und regierungsnahem Think Tank in Colombo, sowie die Unterstützung des muslimischen Friedenssekretariates PSM, eine Maßnahme mit eindeutig politischer Stoßrichtung.

Es sind vor allem die Aktivitäten im Wirtschaftsbereich und OTI, denen die Arbeit im Sektor »Gesellschaft« schwerpunktmäßig zufällt. Mit Blick auf die eingesetzten finanziellen Mittel ist dies freilich ein tatsächlicher Schwerpunkt: OTI und die Tätigkeiten im Bereich »Economic Growth « umfassen nahezu die Hälfte des USAID-Jahresbudgets in Sri Lanka. ${ }^{82}$ Dieser finanzielle Abgleich ist allerdings nicht aussagekräf-

81 Die Relevanz bemisst sich dabei nicht nur an der Mittelausstattung, sondern an der Rolle, die das jeweilige Projekt im Gesamtprogramm spielt. Es handelt sich bei der Einordnung also nicht um eine nach objektiven Kriterien getroffene, sondern um eine Einschätzung. Die verwendeten Abkürzungen erklären sich aus dem folgenden Text.

82 Laut dem aktuellen Arbeitsprogramm (USAID 2006f.) verteilen sich die Mittel im Jahr 2007 wie folgt auf die vier strategischen Zielsetzungen: »Supporting the Benefits of Peace« (faktisch OTI) mit 2 Mio. US\$; »Eco- 
tig, weil speziell die ökonomischen Interventionen nicht nur breit gefächert sind - von Berufsausbildungsmaßnahmen über den Schwerpunkt von Energie- und Wassersektormanagement bis hin zur Förderung der Tourismusindustrie wird eine breite Palette traditioneller EZ-Maßnahmen durchgeführt -, sondern auch in Hinblick auf die politische Gesamtkonzeption des USAID-Programms eine nur marginale Rolle spielen.

Letzteres gilt auch für OTI insofern, da es als gesamtes Programm im Sinne eines mittelbaren Elements gedacht ist, das der konkreten Unterstützung von als sinnvoll angesehenen Projekten dient und damit definitionsgemäß keine unmittelbar strategischen Interventionen durchführen kann. In den fünf Jahren seines Bestehens vergibt OTI insgesamt 645 Grants (vgl. Social Impact 2007: vii), wobei der Schwerpunkt, auch hinsichtlich der eingesetzten Mittel, in der Vergabe von Förderungen im Zuge des Tsunami-Wiederaufbaus liegt. Die meisten Grants werden entweder auf Graswurzel- oder auf Zivilgesellschaftsebene vergeben, wobei hier nicht NGOs, sondern tendenziell eher traditionelle, länger bestehende Bürgerschaftsorganisationen - als ein Beispiel sei der in Sri Lanka omnipräsente Lions Club erwähnt - bei konkreten Projekten unterstützt werden. ${ }^{83}$ Das Kriterium der Sinnhaftigkeit und Umsetzbarkeit eines Projektes ist bei der Auswahl faktisch allein ausschlaggebend, weder gibt es strategische Planungsanstrengungen noch eine Anbindung an die Ausrichtung des D\&G-Programms (vgl. Social Impact 2007: 46). ${ }^{84}$

Damit liegt die Hauptverantwortung für die strategische Ausgestaltung der entwicklungspolitischen US-Intervention beim D\&G-Programm. ${ }^{85}$ Neben Kleinprogrammen, die weitgehend dem generellen Fokus von USAID entsprechen und sektorübergreifend im Sinne von Mul-

nomic Growth« 1,5 Mio. US\$; »Democracy and Governance« 3 Mio. US\$ und Humanitäre Hilfe mit 1 Mio. US\$.

83 Es ist in diesem Zusammenhang wichtig zu betonen, dass sich OTI in seiner Arbeit auf konkrete kleine Projekte konzentriert, mit dem Ziel ihrer bestmöglichen und effizienten Umsetzung. Es gibt keine längerfristige Förderung von Partnern, ebenso wenig ist Capacity-Building angestrebt.

84 Dies wird von Mike DeSisti, Leiter des OTI-Programms in Colombo, bestätigt. Die Abschlussevaluation kritisiert diesen Ansatz: »OTI did not succeed, or pay much attention to promoting advocacy or building support for >pressuring e elite decision-makers to provide the political support to a negotiated settlement. An open question is whether a >bottom-up approach to peace building could have had much impact as conditions in Sri Lanka deteriorated « (Social Impact 2007: 46). Bei der Arbeit an lokaler Governance kommt es jedoch schon zu Kooperationen zwischen OTI und D\&G, allerdings nur auf taktisch-unterstützender Ebene.

85 Einzelne Komponenten, die unter den D\&G-Bereich fallen, wurden allerdings von der OTI-Budgetlinie querfinanziert. 
tiplikatoren wirken sollen, wie etwa die Unterstützung des TV-Projektes Young Asia Television (YATV), Medientrainings oder Kooperationen im akademischen Bereich, die über das Center for Policy Alternatives (CPA) abgewickelt werden ${ }^{86}$, sind es vor allem zwei Programmkomponenten, die den Kern der strategischen Ausrichtung der Intervention bilden. Sie sind auch hinsichtlich der Rationalitäten, die in der Ausgestaltung dieser Intervention zum Tragen kommen, am aussagekräftigsten: die Förderung von lokaler Verwaltung und das Programm zur Unterstützung der Friedensentwicklung.

Von diesen beiden Komponenten ist das Capacity-Building im Bereich lokaler Verwaltungen, das dem allgegenwärtigen USAID-Schwerpunkt der Dezentralisierung entspricht, sowie die Arbeit im Justizbereich - hier wird das Justizministerium beim Aufbau so genannter »community mediation boards « unterstützt $^{87}$ - weniger überraschend. Die Tätigkeiten bestätigen den Ansatz von USAID, wonach eine dezentralisierte und gut arbeitende Verwaltung notwendige Voraussetzung für die Etablierung stabiler demokratischer Verhältnisse ist. Dies ist von Beginn an auch das Ziel des Programms, das unter dem Namen »Transparency, Accountability and Local Governance" (TALG) läuft: »The original purpose was to reduce conflict and build peace through strengthening democratic local governance and the rule of law « (Timbermann 2007: 26). Dies entspricht in der Tat genau jener Blaupause, die sich aus den strategischen Vorgaben der Schlüsseldokumente ergibt.

Als Konsequenz des Tsunami ändert sich diese Ausrichtung jedoch. Angesichts der Notwendigkeit für die 35 als Partner ausgewählten lokalen Verwaltungen, möglichst effizient auf die Katastrophe zu reagieren und im Anschluss vor allem den Wiederaufbau zu koordinieren, wird die Zielsetzung modifiziert: so geht es nicht mehr um demokratisches Capacity-Building, sondern um eine Anhebung der Kapazitäten in der Leistungserbringung, womit das Programm faktisch von einem demokratiepolitischen in einen eher technischen Bereich überführt wird. Dennoch stimmt auch diese neue Stoßrichtung mit dem überein, was als notwendige Arbeit an einer dezentralisierten Verwaltung verstanden wird, auch wenn sich die Aufgabenstellung von Bewusstseinsbildung zur Effizienzentwicklung verschiebt.

86 Finanziert werden hier etwa ein Studienaustauschprogramm und einige Studien im Rahmen der KAPS-Umfrage (Knowledge, Attitudes and Practices Survey) von CPA, die eine vertiefte Analyse der Einstellungen der Bevölkerung zum Friedensprozess ermöglichen soll.

87 Diese Programmkomponente wird von der »Asia Foundation« implementiert. 
Die zweite zentrale Komponente der politischen Arbeit von USAID ist unter dem Titel »Peace Support Program« zusammengefasst und entfaltet eine eigene, dreistufige Umsetzungslogik (vgl. im Folgenden Timbermann 2007: 7f.). Unter dem Programmbereich »build inclusive capacity for formal peace negotiations « sind jene Projekte zusammengefasst, die zentral auf die politische Ebene wirken sollen, also der Platzierung, Stärkung, Ausrichtung und, eine sri-lankische Besonderheit, auch Installierung der Akteure in der demokratischen Arena dienen. Neben dem bereits angeführten Diplomkurs in Konflikttransformation am BCIS sind es die zwei wesentlichen Interventionen im politischen Sektor, die sich hier wiederfinden.

Einerseits geht es dabei um die Installierung eines Kommunikationsmodus, dessen primäre Aufgabe es ist, ein verbindliches Forum für Expert/innen der verschiedenen politischen Kräfte in Sri Lanka zur Verfügung zu stellen, in dem politische Fragen unterhalb des offiziellen diplomatischen Levels in einer zugleich relativ verbindlichen Form diskutiert werden können. OneText, so der Name der Initiative, basiert auf einem methodischen Verfahren, das vom südafrikanischen Friedensaktivisten Hannes Siebert direkt für den sri-lankischen Kontext entwickelt wurde (vgl. Burke/Mulakala 2005: 38). Das Verfahren zielt darauf ab, dass sich die in dem Prozess beteiligten Akteure auf die Formulierung eines einheitlichen Textes einigen, der in der Folge als Unterstützung des offiziellen Verhandlungsprozesses dienen soll. Die Umsetzung erfolgt mit starker technologischer Unterstützung, wobei USAID die Einrichtung eines eigenen softwarebasierten Informations- und Distributionssystems, InfoShare, finanziert.

Ziel des Prozesses ist die Beteiligung aller maßgeblichen politischen Kräfte Sri Lankas, was mit Ausnahme der nationalistischen Parteien JVP und JHU auch gelingt. Ein gewichtiges Problem ergibt sich jedoch, als die LTTE Interesse an einer Beteiligung an OneText bekundet und schließlich im Mai 2005 erstmals dazu eingeladen wird. Obwohl USAID dieser Beteiligung grundsätzlich positiv gegenübersteht ${ }^{88}$, verunmöglicht sie zugleich, dass OneText angesichts der strikten Policy-Bestimmungen hinsichtlich der Finanzierung von Initiativen, die mit der LTTE kooperieren, weiter direkt unterstützt wird. Dies ist die Geburt von SAPI, das ursprünglich primär als vorgeschobener und LTTE-freier Finanzierungsposten von OneText eingerichtet wird (vgl. Timbermann 2007: 14).

88 USAID-Verantwortliche betonen im Gespräch, dass es ohne Zweifel notwendig sei, auch mit der LTTE, zumindest auf technischem Level, zusammenzuarbeiten. Hier vertraut USAID jedoch auf eine Art informeller Arbeitsteilung mit den europäischen Gebern. 
Dennoch erhält SAPI einen eigenen Aufgabenbereich, der sich vor allem aus verschiedenen Begleitmaßnahmen des OneText-Prozesses zusammensetzt und wesentlich auf die politische Parteienlandschaft abzielt. So ist etwa das Training des wissenschaftlichen Bereiches der fünf großen sri-lankischen Parteien, insbesondere die Kapazitätsentwicklung im Feld der Konfliktanalyse, wesentliches Ziel (vgl. USAID 2006f.).

Zudem geht es um die Anbindung dieser Parteien an die Ebene der internationalen Akteure in Sri Lanka, wo SAPI die Rolle eines Forums zufallen soll. Darüber hinaus entwickelt SAPI auch Aktivitäten auf einer Ebene, die am ehesten mit der Arbeit eines Think Tanks vergleichbar ist. So wird beispielsweise eine Artikelsammlung zur Verfassungsdebatte und dem gerade laufenden Diskussionsprozess um einen Konsens aller »südlichen« Parteien in dieser Auseinandersetzung - der so genannte APRC-Prozess mit dem Ziel eines "Southern Consensus « ${ }^{89}$ - herausgegeben (vgl. Liyanage/Sinnathamby 2007).

Interessant ist in diesem Zusammenhang, dass diese Arbeit entscheidend von Leuten geprägt wird, die aus der politisch aktiven akademischen Szene und Zivilgesellschaft stammen, also eigentlich eine Gruppe repräsentieren, die nicht zu den klassischen Partner/innen von USAID gehört. Demgemäß entwickelt sich SAPI in einer Hybridität zwischen politischer und ziviler Gesellschaft, die vielleicht ursprünglich von USAID so nicht vorgesehen war, mittlerweile aber - trotz einer überaus negativen Evaluation ${ }^{90}$ - derzeit doch angenommen wird. Trotz dieser

89 Das so genannte »All Party Representative Committee« (APRC) wird von Vertreter/innen aller Parteien gebildet, die in der »All Party Conference« (APC) von der Rajapakse-Regierung Anfang 2006 zu einer Debatte um eine Neuordnung der sri-lankischen Verfassung zur Lösung des bewaffneten Konfliktes eingeladen worden sind. Es handelt sich dabei im Wesentlichen um alle singhalesischen und muslimischen Parteien, die Parteien der Tamil/innen des Hochlandes sowie die explizit gegen die LTTE gerichteten tamilischen Parteien (vor allem die EPDP). Das in Permanenz arbeitende APRC hat zur wissenschaftlichen Stützung des Prozesses ein Expert/innengremium eingerichtet, das, nachdem keine vollständige Einigung erzielt werden konnte, Ende 2006 mit drei Berichten aufwartete. Diese Berichte wurden fast unmittelbar nach ihrer Fertigstellung bekannt, obwohl sie eigentlich nicht für die Öffentlichkeit bestimmt waren. Insbesondere der Mehrheitsreport sieht eine weitgehende Dezentralisierung des sri-lankischen Staates vor, der in Richtung einer föderalen Lösung geht, und wird dementsprechend von den meisten der sich eng an den internationalen Akteuren befindlichen Think Tanks ausführlich diskutiert und propagiert.

90 SAPI wird von einer von USAID veröffentlichten Fremdevaluation stark kritisiert. Nicht nur wird das demnach der Aufgabe nicht gewachsene, weil nicht in der notwendigen Weise vorbereitete Personal negativ hervorgehoben, generell wird die Einrichtung von SAPI als fragwürdige Idee gese- 
zivilgesellschaftlichen Schlagseite, die die OneText/SAPI-Komponente in den Jahren 2006/2007 bekommen hat, entspricht die Intervention klar den strategischen Linien von USAID, wie sie sich aus den Schlüsseldokumenten ableiten lassen. Die wesentlichen politischen Akteure, mit denen eine Arbeit als sinnvoll erachtet wird, werden zusammengefasst und in ein Wechselspiel gesetzt, dessen genauer Ausgang zwar nicht vorhersehbar, dessen Zielbestimmung aber sehr wohl definiert ist.

Ähnliches gilt auch für die zweite Intervention, die im Rahmen der Unterstützung des formellen Friedensprozesses unternommen wird: die Förderung und Stärkung der Friedenssekretariate. Neben der Unterstützung für das staatliche Friedenssekretariat SCOPP, die aber erst im Anlaufen ist, konzentriert sich die Arbeit, auch angesichts der Unmöglichkeit einer Kooperation mit dem Friedenssekretariat der LTTE, auf das muslimische PSM. USAID fungiert als eine der wichtigsten Finanzierungsinstitutionen des PSM, was auch von dessen Sekretär Mohideen (2006: 324) herausgestrichen wird, und nützt diese Kooperation auch für eine Ausweitung der eigenen Präsenz in der muslimisch dominierten Provinz Ampara.

Die Unterstützung des PSM resultiert einerseits aus der Analyse des sri-lankischen Konfliktes als »ethnischer Konflikt«, womit die Anordnung und, falls notwendig, vorherige Institutionalisierung ethnisch definierter Akteure in der politischen Arena als wesentlicher Schritt in Richtung einer Befriedung des Konfliktes und seiner Überleitung in die Bahnen des demokratischen Wettbewerbs gesehen wird. Dass damit der Prozess der ethnischen Zuordnung nicht nur reproduziert, sondern auch verstärkt wird, ist kein entscheidendes Problem, wird doch die institutionelle Verankerung einer solchen Identität als Voraussetzung der politischen Berücksichtigung von konsequenterweise ebenfalls als »ethnisch« definierten Interessenslagen gesehen. Diese Interessenslagen könnten wiederum einer gewaltsamen Entwicklung in der Ausrichtung der politischen Gruppierungen vorbauen, wie sie die tamilische Geschichte entlang dieser Lesart schmerzlich vorzeigen würde.

hen: »It is advisable to avoid creating new, non-organic organizations (such as SAPI). If, as it appears was the case with SAPI, there is no alternative to creating a new organization, then it is essential to ensure that it has top-flight leadership and there is adequate oversight « (Timbermann 2007: 4). Angesichts der Tatsache, dass der politische Raum für Initiativen wie OneText und SAPI mit dem Zusammenbruch des Friedensprozesses ohnehin schwindet, steht damit die Weiterfinanzierung im neuen D\&GProgrammschema, trotz einem grundsätzlichen Vertrauen der USAIDVerantwortlichen in die OneText-Methodik, höchstwahrscheinlich zur Disposition. 
Dass ausgerechnet das muslimische Sekretariat derart stark unterstützt wird (praktisch wurde die Einrichtung aller fünf Distriktbüros finanziert ${ }^{91}$ ), hat drei Gründe, die allesamt wieder interessante Rückschlüsse auf die Konfiguration des Gesamtprogramms liefern: Erstens ist das PSM wie gesagt das einzige der drei ethnisch definierten Friedenssekretariate, das von den USA großflächig unterstützt werden kann. Einerseits ist wie angeführt eine Arbeit mit der LTTE nicht zugelassen, womit eine Zusammenarbeit mit deren Friedenssekretariat verunmöglicht ist, andererseits ist das staatliche Friedenssekretariat SCOPP so nah bei der Regierung angesiedelt, dass eine großflächige Unterstützung auf kleinerer Ebene wird eine solche demnächst ja sehr wohl begonnen eine politische Botschaft transportieren würde, der sich die USA derzeit offenbar enthalten wollen. So kommt in dieser Entscheidung auch die grundsätzlich distanzierte Haltung zum GoSL zum Ausdruck.

Zweitens neigt die US-Entwicklungshilfe generell dazu, in ihrer Tätigkeit muslimische Zielgruppen in durchmischten konfessionellen Settings hervorzuheben. Diese Vorgangsweise entspricht einer offiziell nicht festgeschriebenen Linie, die propagandistisch die Verbundenheit der USA mit Menschen islamischen Glaubens demonstrieren und damit die Propaganda radikal antiamerikanischer islamischer Kräfte unterminieren soll.

Drittens schließlich erfolgt die Entscheidung offenbar aus einer politischen Einschätzung, die die praktische Wirksamkeit und den potenziellen Einfluss der eigenen Tätigkeit mit gegebenen politischen Notwendigkeiten abwägt und aus diesem Verhältnis die am besten geeigneten Eintrittspunkte der Arbeit bestimmt. Tatsächlich sieht USAID einen »historical lack of consensus among Muslims« als »a key contributor to the community's political marginalization $\aleph^{92}$, was sich auch im Rahmen der OneText-Arbeit am Widerspruch zwischen den beiden großen islamischen Parteien Sri Lanka Muslim Congress (SLMC) und National Unity Alliance (NUA) gezeigt hätte. Damit wird die Formierung der als objektiv gemeinsam wahrgenommenen Interessenslagen in einer einheitlichen Institution, eben dem PSM, zur wesentlichen entwicklungspolitischen Aufgabe.

Der zweite Programmbereich des »Peace Support Program«, »mobilize constituents for peace«, demonstriert schon im Namen eine im Abgleich mit den strategischen Schlüsseldokumenten überraschende Wendung: das Programm dient in seiner ursprünglichen Ausrichtung einer

91 Diese Distriktbüros befinden sich in Ampara, Batticaloa, Puttalam, Trincomalee und Galle.

92 http://www1.usaid.gov/lk/news/tos/jan06-psm.html (Zugegriffen am 1007-2007). 
möglichst breitenwirksamen Abstützung des Friedensprozesses in der Bevölkerung, weist also ein signifikantes Element jener Tendenz zur gesellschaftlichen Durchdringung auf, die eigentlich für die deutsche Entwicklungszusammenarbeit charakteristisch ist. Wenngleich sich das Programm nach dem Scheitern des Friedensprozesses in seiner Zielsetzung verschoben hat - die Idee der breitenwirksamen Rückkopplung zwischen Grassroots und politischer Ebene unter Vermittlung zivilgesellschaftlicher NGOs, der ursprüngliche Ansatz des Programms, wird in Richtung der Zielsetzung eines interethnischen Austausches verschoben -, bleibt diese grundsätzliche Tendenz dennoch erhalten.

Die Umsetzung dieser Komponente erfolgt in so genannten »People's Forums«, auf lokaler Ebene angesiedelten Gesprächs- und Aktionsgruppen, von denen derzeit insgesamt etwa 60 eingerichtet sind. Bemerkenswert ist nun, wie diese Foren in der Praxis implementiert werden. Denn obwohl die Idee originär aus der D\&G-Abteilung von USAID stammt, werden vier sehr unterschiedliche in ganz Sri Lanka aktive NGOs, allesamt wesentliche Akteure im Rahmen der professionalisierten Zivilgesellschaft, mit der Aufgabe betraut: Sarvodaya, das National Peace Council (NPC) und die zwei mit dem Namen Kumar Rupesinghe verknüpften Organisationen Foundation for Co-Existence (FCE) und National Anti-War Front.

Eine solche Auswahl ist aus mehreren Gründen bemerkenswert. Zunächst handelt es sich um sehr unterschiedliche Organisationen: Es gibt in der organisierten sri-lankischen Zivilgesellschaft, die mit Friedensarbeit befasst ist, schwerlich zwei unterschiedlichere Ansätze als das stark buddhistisch-spirituell ausgerichtete Arbeiten von Sarvodaya und den professionellen, auf den internationalen Geberdiskurs abgestimmten Ansatz von Kumar Rupesinghe. ${ }^{93}$ Tatsächlich ist es auch so, dass allen vier NGOs in der Umsetzung weitgehend freie Hand gelassen wird und diese tatsächlich auch sehr unterschiedliche Ausformungen der »People's Forums« implementieren. Erst in letzter Zeit werden die vier NGOs zu einer verstärkten Zusammenarbeit angehalten, was aber weniger als Steuerungsdrang von USAID denn als Bemühung um einen Erfahrungsaustausch und einer damit einhergehenden Effizienzsteigerung zu verstehen ist. Es ist schwierig einzuschätzen, inwieweit die Entscheidung, den

93 Kumar Rupesinghe war von 1992-1997 Generalsekretär von International Alert. In letzter Zeit ist insofern eine ideologische Annäherung von Rupesinghe an den Ansatz Sarvodayas festzustellen, als er sich zunehmend auf Ghandi bezieht und plant, in Sri Lanka ein Ghandi-Friedenszentrum einzurichten. Mehrere Interviewpartner/innen vermuteten, dass hinter dieser neuen Orientierung die Möglichkeit zur Lukrierung etwaiger indischer Fördergelder liegen könnte. 
NGOs in ihrer Arbeit diesen großen Gestaltungsspielraum zu überlassen, bewusst getroffen wurde, da ja auch noch der private US-amerikanische Kontraktor AED in der Implementierung zwischengeschaltet ist. In jedem Fall aber repräsentiert diese Praxis eine typische Vorgangsweise von USAID, die sich auf Mikrosteuerungsprozesse nicht einlässt, wie wir sie etwa bei dem vergleichbar positionierten GTZ-Projekt FLICT finden werden. ${ }^{94}$

Ebenfalls bemerkenswert ist, dass drei der vier ausgewählten NGOs (Sarvodaya enthält sich aus prinzipiellen Erwägungen weitgehend einer tagespolitischen Positionierung, die über den Grundsatz friedfertigen Handelns hinausgeht) friedenspolitische Ansätze verfolgen, die sich zwar zueinander in scharfer Konkurrenz befinden ${ }^{95}$, denen allerdings eine dem gegenwärtigen Kurs der Regierung äußerst kritisch gegenüberstehende Ausrichtung gemeinsam ist. Dies bestätigt die sich im gesamten Programm durchziehende relative Distanzierung vom srilankischen Staat, eine in als grundsätzlich freundschaftlich eingestuften Ländern unübliche Vorgangsweise, insbesondere wenn sie mit einer derart starken Einbindung der politisch aktiven Zivilgesellschaft einhergeht.

Der dritte und letzte Programmbereich des »Peace Support Program« umfasst schließlich unter dem Titel »link constituents and leadership« verschiedene kleinere, schon zuvor erwähnte Interventionen, die allerdings sehr wohl eine öffentliche Breitenwirkung anstreben, »Leadership« also nicht im Sinne eng begrenzter politischer Entscheidungsträger/innen versteht, sondern einer gesellschaftlichen Schicht, die als die gut informierten aktiven Meinungsführer/innen einer polyarchisch

94 Die Wirksamkeit der »People's Forums« wird in der jüngsten Evaluation in Frage gestellt. Obwohl es ohne Zweifel gelungen sei, die in den Foren beteiligten Personen zu begeistern und zu stärken, wird der strategische Gehalt der Intervention im Sinne der vorgegebenen programmatischen Zielsetzung angezweifelt: »The People's Forums did not achieve their original purpose of channeling people's views upwards to One Text and the national peace process. This raises a valid question about the strategic purpose of the People's Forums - especially given that there are only about 60 of them nation-wide« (Timbermann 2007: 19). Dies deutet auf eine mangelhafte konzeptionelle Vorarbeit hin, bestätigt allerdings meine Lesart der Intention des Projektes.

95 Der zentrale Unterschied lässt sich daran festmachen, dass das NPC seine Positionierung zu einer Lösung des Konfliktes sehr nahe an den ThimpuPrinzipien versteht, Tamil/innen also nicht als Minderheit im srilankischen Staat, sondern als eigene Nation definiert. FCE und die National Anti-War Front positionieren sich im Sinne eines inklusiven, supraethnisch verstandenen sri-lankischen Nationalismus. 
formierten Elitendemokratie verstanden werden können. ${ }^{96}$ Auch hier wird wiederum in der Umsetzung auf Organisationen zurückgegriffen, die wie CPA, von dem mit USAID-Geldern Umfragen, Studien und ein Ausbildungsprogramm für Journalist/innen durchgeführt werden, in der Zivilgesellschaft $\mathrm{zu}$ verorten sind oder wie das Medienunternehmen Young Asia Television (YATV) seine Ursprünge eindeutig im aktivistischen zivilgesellschaftlichen Bereich haben.

In ihren Grundzügen bestätigen diese unterschiedlichen Interventionen jene strategischen Linien, die aus den Schlüsseldokumenten der politischen und ersten Policy-Ebene herauszulesen sind. Die Zielsetzung bewegt sich im Doppelspiel der Formierung, Institutionalisierung und Platzierung von Schlüsselakteuren im bestmöglichen Rahmen, wie sie die Arbeit mit dem PSM und die SAPI/OneText-Initiative repräsentiert, bei einer gleichzeitigen Unterstützung stabilisierender Faktoren, die im Rahmen einer mit bewaffnetem Konflikt konfrontierten Form von Staatlichkeit generell in einer effizienten, maximal dezentralisierten Verwaltungsstruktur wahrgenommen werden.

Die Überraschungen, die die konkrete Praxis von USAID in Sri Lanka gegenüber den allgemeinen Vorgaben bereithält, finden sich auf zwei Ebenen, die allerdings eng miteinander verbunden sind. Zunächst erfolgt im zivilen Bereich faktisch keine nennenswerte Kooperation mit der gegenwärtigen Regierung, was impliziert, dass diese offenbar derzeit nicht als wesentlicher zu unterstützender Akteur wahrgenommen wird. Die Arbeit mit der Regierung nahestehenden Personen erfolgt ausschließlich über das Vehikel der politischen Parteien, denen die Steuerung der Prozesse des Regierens, und damit letztendlich auch der Aushandlung eines Friedens, obliegen soll. Dies würde eine noch viel stärkere gouvernementale Ausprägung des Handelns bedeuten, gäbe es nicht einen ebenso bemerkenswerten, fast krass anmutenden Kontrapunkt.

Denn der Gegenpol zu dem Ansatz auf politischer Ebene, der, wie gezeigt, auch schon unter beträchtlicher zivilgesellschaftlicher Beteiligung vollzogen wird, basiert auf einem Prinzip, das der grundlegenden Strategie von USAID auf den ersten Blick faktisch widerspricht. NGOs, und zwar solche mit prononciert politischem Charakter, werden ausgewählt, um über auf der Mikroebene gelegene Interventionen Friedenspolitik zu machen. Dies wird durch die Erwägung noch verstärkt, im nächsten Budgetjahr eventuell ein »community-based early warning system« (State Department/USAID 2007) zu unterstützen, wie es derzeit von

96 Eine solche Elitenorientierung bestätigt auch der Leiter des D\&G-Programms in Colombo, Mark Silva. 
FCE unterhalten wird. Trotz der bereits angesprochenen Einschränkung, dass die Umsetzung überaus pragmatisch und weitgehend ohne direkte Involvierung von USAID erfolgt, also mit dem noch vorzustellenden FLICT-Projekt keineswegs vergleichbar ist, wird dennoch sichtbar, dass der dominant gouvernementale Charakter des Programms durch merkliche disziplinierende Elemente ergänzt wird. Über die Ursache dieser Verschiebung lassen sich letztendlich nur Vermutungen anstellen, wenngleich eine von diesen sicher auf einer stabilen Annahme aufbauen kann: dass nämlich das Scheitern des Friedensprozesses von $2001 \mathrm{zu}$ einer nachhaltigen Erschütterung der idealtypischen Programmvorgaben der Geber geführt hat, was wiederum in einer Aufnahme neuer, tendenziell anders ausgerichteter Ansätze resultiert. Wir werden sehen, inwiefern Vergleichbares auch bei der deutschen Praxis feststellbar ist.

\section{Die Intervention Deutschlands}

Die Geschichte des deutschen Engagements in Sri Lanka ist zweigeteilt, in einer Schärfe, die bei Gebern, die derart lang (das deutsche Engagement währt bereits über vier Jahrzehnte) in einem Partnerland engagiert sind, nur selten vorzufinden ist. Der Schnittpunkt ist das Jahr 2001, als die UNP-Regierung beginnt, den Friedensprozess und die ihn begleitenden zivilen Maßnahmen zu intensivieren. Dieses Interesse wird vom BMZ zum Anlass genommen, das gesamte Länderprogramm auf den Kopf zu stellen und Sri Lanka zu einem Pilotland für eine Ausrichtung der gesamten Arbeit auf die Problemstellung »Bewaffneter Konflikt« zu machen.

Die Affinität zu UNP-Regierungen ist eine jener Kontinuitäten, die sich während der gesamten deutschen Präsenz in Sri Lanka immer wieder zeigt. Dennoch ist die Geschichte der deutschen Entwicklungshilfe in Sri Lanka mit einer Ausnahme - SLFP-Ministerpräsidentin Sirimavo Bandaranaike hatte sich 1964 im Zuge einer Auslandsreise zu zwei deutschen Staaten bekannt, was als Konsequenz der Hallstein-Doktrin zu einem einjährigen Einfrieren der entwicklungspolitischen Leistungen führte (vgl. Hein 2006: 94) - bemüht unpolitisch.

Seit den 1970er Jahren gehört Deutschland zu den größten bilateralen Gebern in Sri Lanka, was sich in der massiven Beteiligung am Mahaweli Ganga Entwicklungsprojekt beweist. Von Beginn an sind die deutschen Leistungen speziell im Bereich der finanziellen Zusammenarbeit gewichtig, wobei mit der Beschleunigung des Projektes mit der Regierungsübernahme durch die UNP unter J.R. Jayawardene die Leistungen angekurbelt werden (siehe Abbildung 30). Im Zuge des Projektes werden auch zwei Mammut-Finanzierungsleistungen getätigt, die die 
starken Ausschläge der Grafik in den Jahren 1981 und 1986 erklären: die Finanzierung der Dammstufen von Randenigala mit 400 Mio. DM und von Rantembe mit 230 Mio. DM.

\section{Abbildung 27: Deutsche ODA in Sri Lanka (in 2005-US\$)}

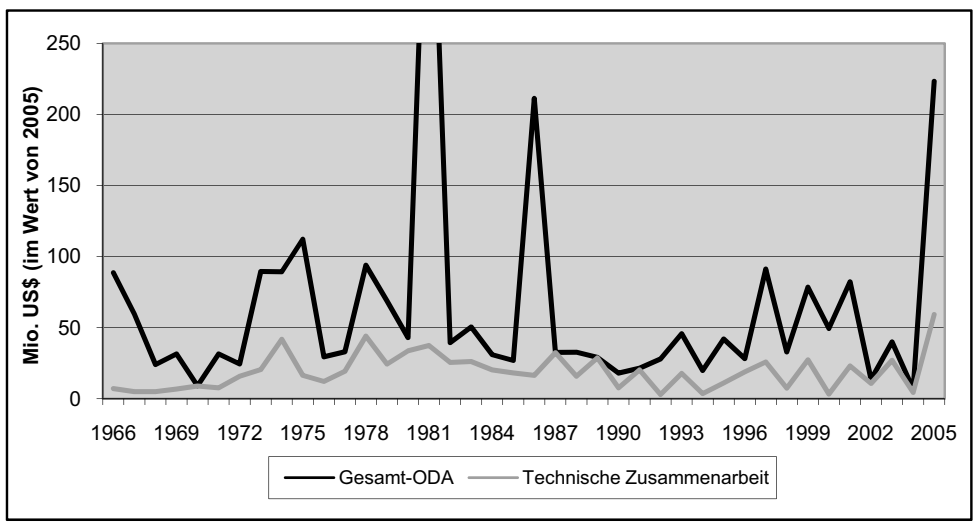

Im Zuge dieser Finanzierungen spielt es für die deutsche Entwicklungspolitik offenbar keine Rolle, dass das Mahaweli-Projekt heute als mit dem Ausbruch des Krieges in engem Zusammenhang stehend gesehen wird. Die durch das Projekt notwendig gewordenen Umsiedlungen führen in tamilischen Gebieten zu demographischen Umwälzungen, die die militanten tamilischen Gruppierungen als gezielte Binnenkolonialisierung verstehen und als eine der Begründungen bei der Aufnahme ihrer bewaffneten Kampagnen anführen. Nachdem einer der beiden Verträge für die Großfinanzierungen im Jahr 1984, also bereits nach Ausbruch der Kämpfe, geschlossen worden ist (vgl. Klingebiel 1999: 32), muss davon ausgegangen werden, dass der Zusammenhang den deutschen Verantwortlichen vor Ort entweder unbekannt bleibt - was eine beeindruckende Ignoranz gegenüber den politischen Realitäten zeigen würde oder aber für das Projekt als nicht relevant eingeschätzt wird.

Nach einem Zurückschrauben der Großfinanzierungen Ende der 1980er Jahre, als die deutschen Leistungen angesichts des JVPAufstandes auf die Weiterfinanzierung der laufenden Technischen $\mathrm{Zu}$ sammenarbeit $^{97}$ zusammenschrumpfen, kommt es Anfang der 1990er Jahre wieder $\mathrm{zu}$ einem vergleichbaren Ablauf, der heute retrospektiv

97 Wie bereits angeführt unterscheidet die deutsche Entwicklungszusammenarbeit zwischen »technischer Zusammenarbeit« (TZ), der Domäne der GTZ, und »finanzieller Zusammenarbeit« (FZ), die hauptsächlich über die Kreditanstalt für Wiederaufbau (KfW) abgewickelt wird. 
zumeist als »konfliktblind« diagnostiziert wird. Sowohl auf finanzieller wie auf technischer Ebene wird die deutsche Entwicklungshilfe zu einem der treuesten Begleiter der militärischen Offensive von Chandrika Kumaratunga, die unter dem Slogan »War for Peace« vor allem auf die Rückeroberung der Jaffna-Halbinsel von der LTTE abzielt. Dabei liefert Deutschland nicht nur wertvolle Finanzierungshilfen für vom GoSL gesteuerte Wiederaufbauprojekte, sondern ist auch selbst in den Brennpunkten präsent: so engagiert sich die GTZ, wie zuvor bereits erwähnt, stark in Rehabilitationsprojekten in Jaffna nach dessen Rückeroberung und verhilft so dem GoSL in ihm überwiegend feindlich gegenüberstehenden Gebieten zu einem wertvollen Reputationsgewinn.

Im Jahr 2001 findet schließlich, in Wechselwirkung mit der zu diesem Zeitpunkt laufenden internationalen Diskussion, die in Deutschland selbst schon stärkere Auswirkungen gezeigt und in überaus kritischen Beurteilungen der bisherigen Praxis in Sri Lanka resultiert hat (vgl. Klingebiel 1999), ein weitreichender und alle Ebenen der Zusammenarbeit betreffender Bruch statt. ${ }^{98}$ Schon gegen Ende des Jahres 2000 einigt sich das BMZ mit der damaligen SLFP-Regierung auf die Einführung eines neuen Programmschwerpunktes für die $\mathrm{GTZ}^{99}$, der unter dem Namen »Poverty Alleviation and Conflict Transformation« (PACT) nicht nur die Bearbeitung des bewaffneten Konfliktes in den Mittelpunkt rückt, sondern ihn interessanterweise auch in Zusammenhang mit der Armutsbekämpfung setzt. ${ }^{100}$

98 Einschränkend muss allerdings gesagt werden, dass die Umkrempelung des Länderprogramms nicht so durchschlagend erfolgt wie etwa in Kolumbien, wo im Zuge einer vergleichbaren Umorientierung alle laufenden Projekte gestoppt werden. In Sri Lanka werden viele bestehende Programmpunkte, wie das Wiederaufbauprogramm in Jaffna, weitergeführt, da diese, so Thania Paffenholz (2004: 30), ohnehin das Potenzial hätten, »durch das Auflegen einer >Friedensbrille< [...] kurz- oder langfristig aktiv konfliktmindernd und friedensfördernd zu wirken.« Diese Einschätzung wird von ihr allerdings nicht weiter begründet.

99 Interessanterweise bezieht sich diese Umorientierung explizit zunächst nur auf die GTZ, eine Neuausrichtung des BMZ-Programms, das alle Teile der deutschen ODA umfasst, erfolgt erst nach Scheitern des Waffenstillstandes im Oktober 2006 (vgl. Lorenz 2007: 2). Dies ist allerdings eher als symbolische Maßnahme zu verstehen, weil die praktische Umorientierung auch im Bereich der finanziellen Zusammenarbeit ebenfalls bereits mit 2001 erfolgt.

100 Es ist unklar, warum dieser Zusammenhang derart konstruiert wird. Entweder erfolgt dies vor dem Hintergrund einer generellen internationalen Tendenz, die bewaffneten Konflikt und Armut als zwei interdependente Problemfelder definiert, oder es handelt sich um die Aufrechterhaltung einer Zielbestimmung für laufende Projekte (insbesondere PIMU, siehe im Text weiter unten), die sonst nicht im Portfolio gehalten 
Dieser neue Arbeitsschwerpunkt ist nicht einfach als Ergänzung zum weitergeführten bisherigen GTZ-Programm »Dynamic market Economy and Qualified Employment« zu verstehen. Vielmehr kommt es zu einer Neuausrichtung, die, wie Abbildung 27 verdeutlicht, auch eine Veränderung der entwicklungspolitischen Arbeitsmethodik mit sich bringt: Die finanzielle Zusammenarbeit wird weitestgehend zurückgefahren und die eingesetzten Mittel auf die technische Zusammenarbeit konzentriert. So wird die »sri lankisch-deutsche Kooperation« quasi über Nacht zum »Modell für eine systematische Umsetzung einer stärker auf Friedenssicherung ausgerichteten deutschen EZ« (Paffenholz 2004: 12), zumindest in der Wahrnehmung derer, die an dieser Neuausrichtung beteiligt sind und sie auch als Modell für zukünftige Schritte der deutschen Entwicklungspolitik verstehen wollen.

Die Umsetzung der neuen Programmschiene konzentriert sich zunächst auf zwei Großprojekte. Bereits seit 1997 wird vom BMZ, damals noch im ökonomischen Fokus des Länderprogramms, die Einrichtung einer Forschungseinheit zur Untersuchung von »Poverty Impact« überlegt, was schließlich in der Einrichtung eines GTZ-Projektes mit dem Namen »Poverty Impact Monitoring Unit« (PIMU) mündet (vgl. Feyen in Gunetilleke/Jafferjee 2005: 25ff.). Im Zuge der Programmneuausrichtung wird PIMU mit einem Implementationspartner, dem »Centre for Poverty Analysis« (CEPA) ausgestattet, das wiederum, faktisch in der

hätten werden können. Auch ist denkbar, dass dies als Entgegenkommen gegenüber der internationalen Konfliktbearbeitung grundsätzlich skeptisch gegenüberstehenden SLFP gedacht ist, die zugleich die Armutsbekämpfung als wesentliches innenpolitisches Unterscheidungsmerkmal gegenüber der konservativ-liberalen UNP betont, um sich als sozialdemokratische Kraft zu positionieren. In jedem Fall wird die Konfliktanalyse in der deutschen EZ zumeist wenig exakt und kursorisch durchgeführt. Dies demonstriert etwa das Beispiel einer Evaluation über die in Sri Lanka durchgeführten ATP-Maßnahmen, die von einer der führenden Expertinnen im Bereich der Konfliktprävention durchgeführt wurde: »Die Ursachen des Konfliktes liegen in einer Kombination von Unterentwicklung und Benachteiligung überlagert von ethno-politischreligiöser Mobilisierung. Die Konzentration der politischen und ökonomischen Macht im Süden des Landes verhinderte systematisch die Entwicklung der an natürlichen Ressourcen ohnehin ärmeren Regionen im Norden und Osten des Landes« (Paffenholz 2004: 9). Während der erste Satz auf die Konstruktion eines Armutszusammenhanges hinweist, demonstriert der zweite Satz eine bemerkenswerte Unkenntnis, war doch die Erzählung einer singhalesischen Benachteiligung gegen das durch das Kolonialregime bevorzugte reiche Jaffna oder die kommerziell erfolgreichen Tamil/innen eines der wesentlichen Argumentationsmuster bei der Einführung jener diskriminierenden Verfassungsbestimmungen, die wesentlich zur Zuspitzung des Konfliktes beitrugen. 
Rolle eines GTZ-eigenen Subkontraktors, die konzeptionelle Arbeit zur Frage des Zusammenhangs von Armut und bewaffnetem Konflikt in Sri Lanka übernimmt.

Zudem schließt das BMZ mit dem GoSL einen Vertrag ab, der die Einladung der Berliner Berghof Stiftung ${ }^{101}$ als zentraler Partner der GTZ im Bereich der Konfliktbearbeitung gewährleistet. Das Berghof Foundation Sri Lanka Office nimmt im Juli 2001 seine Arbeit als Umsetzungsorganisation des Projektes »Resource Network for Conflict Studies and Transformation« (RNCST) auf, das auch von der politischen Abeilung PA4 des Schweizer Außenministeriums co-finanziert wird. Die Intervention von Berghof ist von Anfang an auf maximale Wirksamkeit ausgelegt, was sich auch daran zeigt, dass mit Norbert Ropers der damalige Geschäftsführer der Berghof Stiftung selbst den Leitungsposten in Sri Lanka antritt. Das Engagement von Berghof wird in der Folge nicht nur für die sri-lankische politisch aktive Zivilgesellschaft zu einer Umwälzung führen, sondern vor allem bedingt durch die neue Analysekapazität auch auf die Ausrichtung der gesamten deutschen Politik gegenüber Sri Lanka, von der diplomatischen Ebene bis hin zur Entwicklungshilfe, maßgeblichen Einfluss haben.

Seine derzeitige Gestalt erhält das GTZ-Programm schließlich im Jahr 2003, als mit dem Fonds »Facilitating Local Initiatives for Conflict Transformation « (FLICT) in Kooperation mit dem britischen DFID ein auf den Grassroots-Bereich fokussierter Gegenpol zum Engagement von Berghof aufgebaut wird. Damit soll ohne Zweifel auch eine Ergänzung zum stark auf Colombo ausgerichteten Engagement von Berghof geschaffen werden. Zugleich folgt die Ausrichtung der strategischen Ein-

101 Wie bereits mehrfach erwähnt, entstammt Berghof den friedenspolitischen Bemühungen zu Zeiten des Kalten Krieges und entwickelt sich im Laufe seiner Existenz zum Vehikel der Umsetzung der Konzepte von Dieter Senghaas. »[Die] als gemeinnützig anerkannte[...] Berghof Stiftung für Konfliktforschung GmbH, München [wurde] 1971 von Prof. Dr. Georg Zundel ins Leben gerufen [...]. Der Name der Stiftung erinnert an den >Berghof` bei Tübingen, das Elternhaus des Stiftungsgründers. Der Stiftungsrat der Berghof Stiftung besteht aus 9 Mitgliedern. Besonderer Wert wird auf die interdisziplinäre Zusammensetzung gelegt. Als Vorsitzender amtierte bis Dezember 2000 Prof. Dr. Dieter Senghaas. Sein Nachfolger ist Prof. Dr. Horst Fischer. Schwerpunktmäßig fördert die Berghof Stiftung anwendungsorientierte Forschung zur konstruktiven Konfliktbearbeitung sowie zu Schlüsselfragen von $>$ Naturwissenschaften und Ethik r. In Anlehnung an die Zielsetzungen der Berghof Stiftung im Bereich der Konfliktbearbeitung wurde das Berghof Forschungszentrum am 1. Juli 1993 gegründet. Es fungiert gleichzeitig als Geschäftsstelle der Stiftung« (http://www.berghof-center.org/std_page.php?LANG=d\& $\mathrm{id}=46$ \&parent $=1$, zugegriffen am 04-07-2007). 
schätzung der deutschen Entwicklungspolitik, dass eine gesellschaftliche Durchdringung mit friedenspolitischen Ansätzen und menschlicher Sicherheit Voraussetzung für eine langfristig erfolgreiche Intervention darstellt.

Schon zuvor war Sri Lanka eines der Zielgebiete im Rahmen des ATP-Programms der deutschen Bundesregierung geworden. Die gesetzten Maßnahmen, geführt unter dem Namen »Sonderprogramm für Krisenprävention und Konfliktbearbeitung«, um mit dem starken Begriff »Terrorismus« keinen der Akteure politisch zu diskreditieren (vgl. Paffenholz 2004: 14), sind dezentral angelegt und thematisch weit gestreut: Von Lehrer/innenausbildung in den LTTE-kontrollierten Gebieten über einen Schreibwettbewerb für Lehramtsanwärter/innen, mobile Berufsberatung, den Aufbau von Schulen, Austauschprogramme und Kooperationen im akademischen Bereich findet sich eine breite Palette, die nach Abschluss der einmaligen Zusatzfinanzierung zum Teil in laufende Projekte übergeführt wird. ${ }^{102}$

Generell ist die Arbeitsgrundlage mit der Wickremasinghe-Regierung in dieser Phase sehr gut, die Entwicklungsvision der UNP passt mit den Vorstellungen des BMZ zusammen (vgl. Burke/Mulakala 2005: 41), womit die Einrichtung und Umsetzung der Projekte im Zeitraum des intakten Waffenstillstandes praktisch problemlos verläuft. Ein thematischer Schwerpunkt wird auf die Vertiefung der offenbar als nicht ausreichend analysierten sri-lankischen Konfliktanalysekapazitäten gelegt. So wird in Zusammenarbeit mit der Universität Colombo, vor allem mit dem Leiter des Instituts für Politikwissenschaft, Jayadeva Uyangoda, ein umfassendes Handbuch produziert (Uyangoda 2005), das die gern als Zugabe geschätzte lokale Kompetenz sri-lankischer Wissenschaftler/innen mit dem internationalen State of the Art der Konflikttransformationstheorie und angewandten Friedensforschung übereinstimmen soll. Diese kurzfristige Theorieoffensive, die sich um das Jahr 2004 entfaltet, ist als Gegenbewegung zum sich abzeichnenden Scheitern des Friedensprozesses zu verstehen, nichtsdestotrotz bleibt ihr Einfluss eher gering.

Mit den Wahlniederlagen der UNP in den Parlaments- und Präsidentschaftswahlen und dem neuen, kriegspolitisch offensiven Kurs verschlechtern sich sowohl die Beziehungen zum GoSL als auch die konkreten Arbeitsbedingungen unmittelbar und tiefgreifend. Reagiert wird auf beiden Ebenen. Auf politischer Ebene wartet das BMZ mit einem

102 Die weitgehende Zusammenhanglosigkeit der Maßnahmen ist vermutlich auch der kurzen Vorbereitungszeit geschuldet. Die Verantwortlichen in Colombo hatten zur Erarbeitung des Umsetzungskonzeptes für die ATP-Gelder nur ein Wochenende Zeit (vgl. Paffenholz 2004: 14). 
Entscheid auf, der gerade angesichts der zu diesem Zeitpunkt schon allgemein bekannten Analysen über die Wirkungslosigkeit negativer Konditionalitäten von Entwicklungshilfe in Sri Lanka überraschend ist: Im Oktober 2006 verkündet Bundesministerin Wieczorek-Zeul, dass zur Erhöhung des Drucks auf die Konfliktparteien alle ODA-Neuzusagen an Sri Lanka bis zur Wiederaufnahme des Friedensprozesses eingefroren würden. »Es müsse endlich wieder Vernunft einkehren, damit die Bevölkerung, die durch den Tsunami dramatisch betroffen sei, wieder Mut und Hoffnung schöpfen könne«, heißt es in der Erklärung. ${ }^{103}$

Obwohl die konkreten Auswirkungen des Beschlusses, zumindest im Bereich der GTZ, zunächst gering bleiben - alle laufenden Projekte sind durch mehrjährige Budgets abgesichert, auch wurde nur ein als im Portfolio nebensächlich eingeschätztes klein dimensioniertes Projekt geschlossen - ist die öffentliche Reaktion, insbesondere von Regierungsseite, heftig und entwickelt sich für Deutschland tendenziell als Schuss nach hinten. Einerseits überzeichnen die sri-lankischen Medien die Maßnahme insofern, als sie von einem vollständigen Rückzug Deutschlands sprechen und eine solche Reaktion als neokoloniale Zwangsmaßnahme interpretieren wollen, was wiederum Klarstellungen von offizieller deutscher Seite, bis hin zur Ministerin selbst, hervorruft. Andererseits wird das Personal in Colombo, auch die Botschaft, erst kurz vor Bekanntgabe des Schrittes vor vollendete Tatsachen gestellt und damit nahezu unmittelbar in ein kommunikatives Krisenmanagement geworfen.

Zwar unterminieren diese beiden Aspekte die Effektivität der Maßnahme, angesichts der Erfahrungen selbst mit größer angelegten ODAKonditionalitäten muss allerdings ohnehin in Zweifel gezogen werden, ob sie überhaupt eine Wirksamkeit im Sinne des angestrebten »Drucks auf die Konfliktparteien« ausüben hätte können. Vielmehr ist das konkrete Resultat, dass sich der Druck auf die deutschen Repräsentanzen in Sri Lanka merklich erhöht, was die Berghof-Stiftung durch vermehrte öffentliche Anfeindungen zu spüren bekommt, die das GoSL auch auf juristischer Ebene zu kanalisieren versucht. ${ }^{104}$

Die zweite Reaktion besteht in der Einführung einer neuen Qualität von Sicherheitsmaßnahmen in das GTZ-Programm. Zu diesem Zweck

103 Pressemitteilung des BMZ vom 13.10.2006: »Bundesministerin Wieczorek-Zeul: Ohne Frieden keine Entwicklung. Deutschland erhöht den Druck auf die Konfliktparteien in Sri Lanka« (siehe http://www.bmz.de/ de/presse/pm/pm_20061013_2.html, zugegriffen am 05-07-2007).

104 So wird Berghof vor einen staatlichen NGO-Kontrollausschuss berufen, trotz der eigenen Beteuerung und entsprechender Stellungnahmen der Botschaften Deutschlands und der Schweiz, dass Berghof kein NGO, sondern Teil einer bilateralen Kooperation sei. 
wird das »GTZ Advisory Service for Conflict Transformation« (ACT) eingerichtet, dessen Aufgabe in der Bereitstellung eines »integrated approach for Conflict Transformation and Risk Management in Sri Lanka« liegt (Lorenz 2007: 1). Nachdem ein integrierter Zugang seit über fünf Jahren zur gängigen Praxis der GTZ in Sri Lanka gehört, liegt die qualitative Neuerung in der zweiten Komponente: In alle laufenden Operationen soll Risikomanagement umfassend - also von der Definition von Zielen über die konzeptionelle Umsetzung bis hin zur Implementation integriert werden. Dies hat nicht nur Konsequenzen für Auswahl und Ausbildung des Personals ${ }^{105}$, sondern legt generell einen Schwerpunkt auf Bewusstseinsarbeit. Die Auseinandersetzung mit »contingency and crisis plans« (ebd.: 4) soll Teil der alltäglichen Tätigkeiten der GTZler/innen werden.

Das gegenwärtige Programmschema wird mit diesem neuen Fokus allerdings nicht verändert, sondern nur in seiner Umsetzung modifiziert. Dementsprechend sind die Kernelemente des Programmschwerpunktes Armutsbekämpfung und Konfliktbearbeitung, die seit 2003 im Dreieck zwischen Berghof, FLICT und dem Dezentralisierungsprojekt PIP liegen $^{106}$, als einigermaßen eingespielte Struktur zu verstehen, in der es auch schon signifikante Umgestaltungen gegeben hat. Dies ermöglicht wiederum vertiefte Aussagen über die Intention und die Platzierung des Gesamtprogramms. So ist zwar heute eine strukturelle Ähnlichkeit mit dem Programm von USAID schlagend, das einen vergleichbaren, ebenfalls um einen ökonomisch-marktwirtschaftlichen Programmbereich ergänzten Aufbau zwischen Politik (SAPI/OneText), Grassroots (People's Forums) und Verwaltungsdezentralisierung und Effizienz aufweist, eine solche Aufteilung entspricht jedoch keineswegs der ursprünglichen Intention des deutschen Ansatzes.

In Abbildung 28 sind alle Projektkomponenten des PACT-Schwerpunktes aufgelistet, die nicht dem Ziel der Tsunami-Rehabilitation dienen, wiederum im Schema der vier Zielsektoren Politik, Gesellschaft, Grassroots und Governance. ${ }^{107}$ Was im Vergleich zum Aufbau des USAID-Programms sofort auffällt, ist die relevant geringere Anzahl an

105 Auf dieser Ebene zeigt diese neue Komponente bemerkenswerte Auswirkungen: So wurde für das Advisory Service ein externer britischer Sicherheitsberater angeheuert.

106 Die offiziellen Anfangsdaten der drei Projekte liegen beim RNCST im Juli 2001, bei FLICT im August 2002 und bei PIP im September 2003.

107 Insgesamt finden sich im Rahmen von PACT fünf Post-Tsunami-Projekte, die allerdings, abgesehen davon, dass sie ebenfalls »konfliktsensitiv« umgesetzt werden und das Personal damit durch dieselben Schulungs- und Trainingsmaßnahmen geht wie das der anderen Projekte, de facto nicht in die Programmstruktur von PACT eingebunden sind. 
Teilprojekten, die sich noch dazu effektiv auf die drei großen Elemente RNCST, PIP und FLICT reduzieren lässt ${ }^{108}$, die den Großteil der vorhandenen Mittel unter sich aufteilen. ${ }^{109}$ Speziell RNCST und FLICT fokussieren entlang dieses Schemas auf mehrere Zielsektoren, sind also breit angelegt, was allerdings nicht mit einer mit USAID vergleichbaren flexiblen Handhabung bei der Implementation verwechselt werden darf, wie sie etwa bei den »People's Forums« feststellbar ist. Beide Projekte arbeiten selbst tiefgreifend in die Umsetzungsebene hinein, bis hin zur Mikro-Ebene ihrer jeweiligen Arbeitsbereiche.

Abbildung 28: Programmschema der GTZ in Sri Lanka ${ }^{110}$

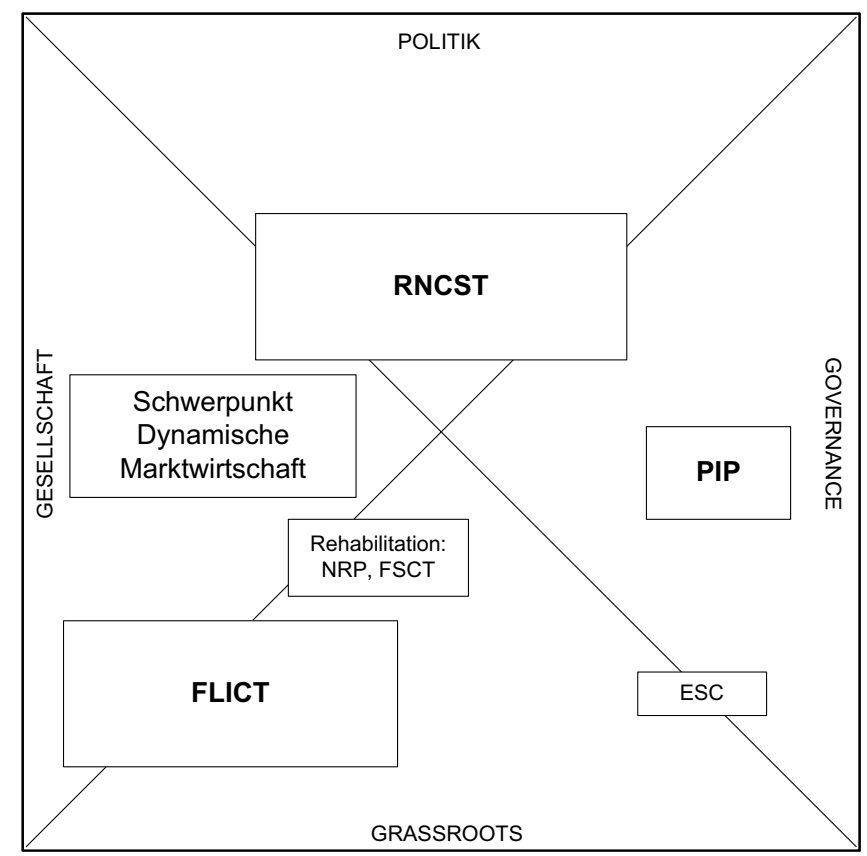

108 Bemerkenswerterweise wird bei zwei dieser Projekte, FLICT und PIP, mit DFID und der British High Commission kooperiert.

109 Es entspricht der generellen Unternehmenspolitik der GTZ, dass genaue Zahlen nicht veröffentlicht werden.

110 ESC - »Friedenserziehung «; FLICT - »Förderung lokaler Initiativen der Konflikttransformation«; FSCT - »Förderung von Ernährungssicherung und Konfliktbearbeitung in Batticaloa«; NRP - »Förderung des Wiederaufbaus im Norden Sri Lankas«; PIP - »Verwaltungsförderung in der Nordost-Provinz«; RNCST - »Netzwerk für Konfliktstudien und -transformation« (Berghof). 
Das Verwaltungsförderungsprojekt PIP ist auf den ersten Blick kein klassisches Konflikttransformationsprojekt. Der Austausch mit den beiden anderen Großprojekten, die ja ausdrücklich in diesem Bereich angesiedelt sind, ist nicht sehr weitreichend, und auch der sicherheitspolitische Charakter ist alles andere als offensichtlich. Offiziell ist das Projekt bewusst technisch gehalten, und es wirkt zunächst als eine klassische Maßnahme zur Förderung von lokaler Governance. Seinen eigentümlichen Charakter entfaltet das Projekt erst, wenn die institutionellen Partner, mit denen auf lokaler Ebene zusammengearbeitet wird, und der praktische Ablauf in Betracht gezogen werden. Daran wird schnell sichtbar, dass PIP eine relevant politische Komponente umfasst, die sich in akzentuiertem Widerspruch zur derzeitigen sri-lankischen Regierungslinie befindet, obwohl mit dem »Ministry of Nation Building and Development « der formale Counterpart auf zentralstaatlicher Ebene verortet ist. $^{111}$

Dieser besondere Gehalt des Projektes ergibt sich aus der historisch gewachsenen Komplexität der sri-lankischen Verwaltungsstruktur. Diese beruht auf zwei parallel gesetzten Säulen. Einerseits existiert das »Civil Service«, das faktisch dem ursprünglich von der Kolonialverwaltung eingerichteten Verwaltungssystem entspricht. Dieses System basiert auf den Einheiten des Distriktes und der Division, wobei die Leitungspositionen bei den Distrikten von der Zentralregierung und bei den Divisionen von den ernannten Distriktleiter/innen besetzt werden. Andererseits wurde durch die vom Indo-Sri Lanka Accord von 1987 festgeschriebene Devolution eine Parallelstruktur eingerichtet, die über Provinzen organisiert ist. Die Provinzen werden von »Provincial Councils« geleitet, die allerdings, im Gegensatz zur »Civil Service«-Struktur, nicht ernannt, sondern direkt gewählt werden. Unterhalb der Provinzen stehen die ebenfalls direkt gewählten lokalen Strukturen, die »Municipial« oder »Urban Councils«. Eine Konsequenz dieses dualen Systems ist ein permanentes Kompetenzgerangel, in dem die Zentralregierung dazu tendiert, möglichst weitgehende Kompetenzen auf die Distriktebene zu transferieren und die Council-Struktur zu unterminieren. ${ }^{112}$

111 Noch dazu obliegt die Leitung des Ministeriums direkt dem Präsidenten Mahinda Rajapakse.

112 Die Kompetenzaufteilung ist im 13. Zusatz der Verfassung, der durch den Indo-Sri Lanka Accord eingeführt wurde, in drei Listen festgelegt: in der »Provincial Council List« sind die Kompetenzen der CouncilStruktur festgelegt, in der »Reserved List« die Kompetenzen, die beim GoSL verbleiben, und in der »Concurrent List« jene Aufgabenbereiche, die gemeinsam verwaltet werden. Da die Auflistung relativ vage gehalten ist, sind zahlreiche Materien umstritten. 
PIP fokussiert nun auf das Provincial Council der Nordost-, beziehungsweise nach deren Aufspaltung im Jahr 2006 die Councils der Nord- und der Ostprovinz, mit den ihnen untergeordneten lokalen Strukturen. ${ }^{113}$ In diesem Zusammenhang ist entscheidend, dass diese Strukturen faktisch durchgängig mit Tamil/innen besetzt sind und in einer Situation eines permanenten Ausnahmezustandes agieren müssen, da ein Großteil der Nord- und Ostprovinz de facto von der sri-lankischen Armee besetzt ist. Zugleich versucht die derzeitige Regierung massiv, die Council-Struktur zu umgehen, was diese Struktur wiederum, nicht nur angesichts eines grundsätzlichen Misstrauens gegenüber Tamil/innen in der Verwaltung ${ }^{114}$, fast notwendigerweise in eine Oppositionsrolle zur Zentralregierung bringt.

Der besondere Charakter von PIP besteht darin, dass in diesem Konflikt nicht vermittelt wird, sondern speziell die sri-lankischen Projektmitarbeiter/innen, die zu einem Gutteil selbst aus den entsprechenden Provinzen stammen, diesen Widerspruch bewusst aufnehmen und ihre Arbeit eindeutig nicht nur im Sinne der Stärkung der CouncilStrukturen, sondern auch im Sinne einer Unterminierung des Zugriffes der Zentralregierung verstehen. Damit bekommt das eigentlich auf einer rein technischen Ebene angelegte Projekt einen immanent politischen Gehalt, was wiederum scharfe Reaktionen nach sich zieht. Mehrfach wurden vom PIP abgehaltene Trainings zum Ziel von Polizeiaktionen, auch das Projektbüro in Trincomalee wurde durchsucht. Mittlerweile verlegte die GTZ die Projektzentrale - zumindest vorübergehend - von Trincomalee nach Colombo ${ }^{115}$, weil die Sicherheit des Personals nicht mehr zu garantieren war.

Im Gegensatz zur Positionierung von PIP ist die Intervention der Berghof Stiftung in Sri Lanka, die im Projekt RNCST seinen formalen, von GTZ und dem Außenministerium der Schweiz gemeinsam finanzierten Rahmen findet, von Beginn an eindeutig politisch kontextualisiert. Es geht um nichts anderes als im Senghaasschen Sinne Frieden zu »machen«, und dafür bereiten die einzelnen Teilkomponenten des zivilisatorischen Hexagons die Einstiegspunkte. Es ist für Deutschland alles andere als eine Selbstverständlichkeit, ein derartiges Projekt über EZ-

113 Auch das Dezentralisierungsprojekt von USAID arbeitet mit der Council-Struktur, jedoch mit einem tatsächlich technisch gehaltenen Ansatz, den ich als im Gegensatz zu PIP nicht politisch orientiert ansehen würde. Dies ist wahrscheinlich auch der Grund dafür, dass das Dezentralisierungsprojekt von USAID nicht mit einer vergleichbaren Repression zu kämpfen hat wie PIP.

114 Interview mit Projektmitarbeitern von PIP, Colombo.

115 PIP residiert nun in einem Hotel in Colombo 06, Wellawatte, dem tamilischen Bezirk Colombos. 
Aktivitäten umzusetzen. ${ }^{116}$ Vielmehr wird hier Neuland betreten, und die Umsetzung des Projekts ist ein Beweis für den exzellenten Ruf, den die Berghof Stiftung im BMZ offenbar genießt, denn sonst wäre ihr ein derartiges Vertrauen, wie es ein solches Projekt voraussetzt, wahrscheinlich nicht entgegengebracht worden.

Die Stiftung selbst versteht ihr Engagement in Sri Lanka als Beitrag zu einem "protracted peace making« (Ropers 2003: 3, vgl. auch Ropers 2008) in einem Konflikt, den sie in ihrer auch für die gesamte deutsche Repräsentanz in Sri Lanka maßgeblichen Analyse als »protracted ethnopolitical conflict « versteht (Berghof 2006: 3). Damit setzt sie sich einerseits vom gängigen und beispielsweise auch von USAID zugrunde gelegten Analysemuster des »ethnischen Konfliktes« ab, vollzieht aber auch nicht die staatszentrierte Analyse von Jonathan Goodhand nach, wie sie etwa in den von ihm erarbeiteten geberfinanzierten »Strategic Conflict Assessments« (Goodhand 2001; Goodhand/Klem 2005) nachzulesen ist. Folglich wird Ethnizität als eine Größe verstanden, mit der, auch wenn sie vor dem Hintergrund politischer Interessen historisch und sozial konstruiert verstanden wird, praktisch umgegangen werden muss, was dem wesentlichen Grundprinzip der Berghof-Intervention, »Multipartiality« (vgl. Berghof 2006: 4), einen klaren, in einem ethnischen Kontext zu begreifenden Definitionsrahmen gibt.

Der Einstieg für Berghof, der noch vor Unterzeichnung des Waffenstillstands stattfindet, entwickelt sich zäh. Ein Versuchsballon zur Sicherheitssektorreform, bei dem es im Prinzip nur um Workshops mit Militärpersonal geht, wird angesichts starker politischer Widerstände zum Desaster und muss nach wenigen Monaten abgebrochen werden. Bis heute bildet dieser SSR-Versuch die zentrale Angriffsfläche, an der Berghof von singhalesisch-nationalistischen Kräften attackiert wird (vgl. etwa Goonatilake 2006: 121$)^{117}$ und die Stiftung zum derzeit primären Feindbild der laufenden medialen und politischen Attacken auf NGOs

116 Die Schweiz finanziert das Berghof-Projekt nicht aus EZ-Geldern, was auch nach dem seit Anfang 2007 einheitlichen Auftreten der DEZA und der PA4 des Außenministeriums in Sri Lanka einen Unterschied macht, denn die Budgetlinien bestehen nach wie vor getrennt. $\mathrm{Ob}$ die für Berghof ausgegebenen Gelder als Schweizer ODA angerechnet werden, war den Verantwortlichen in Colombo nicht bekannt, ist aber grundsätzlich nicht auszuschließen.

117 Susantha Goonatilake wiederholt regelmäßig bei öffentlichkeitswirksam organisierten Veranstaltungen die Anschuldigung, dass das deutsche NGO Berghof dem souveränen sri-lankischen Staat die Reduzierung seiner Streitkräfte befohlen hätte. Derartige Veranstaltungen werden zumeist vom »National Movement Against Terrorism« organisiert und finden breites Medienecho. 
werden lässt. Während ihrer gesamten Präsenz in Sri Lanka ist es Berghof nicht gelungen, das vom SSR-Projekt ausgehende Public RelationsDebakel in den Griff zu bekommen.

In der Folge verschiebt sich die Arbeit auf den zivilgesellschaftlichen Bereich, wo sich Berghof durch seine Analysekapazität, seinen politischen Ruf und vor allem seine enorme finanzielle Potenz schnell als einer der wesentlichen Akteure etablieren kann. Zunächst wird der Fokus auf die Heranbildung von Multiplikatoren gelegt, womit klassische Trainingstätigkeit in den Mittelpunkt rückt: »Praktisch bedeutet das erstens, Lehrmaterial für Konflikttransformation in Tamilisch und Singhalesisch zu übersetzen und auch in den ländlichen Gebieten des Landes zugänglich zu machen« (Zunzer 2003: 134). Doch dieser sehr breit gelegte Fokus entwickelt sich, gerade in Wechselwirkung zu den anlaufenden Friedensverhandlungen, schnell zu einer konzentrierten Kooperation mit der politisch aktiven Zivilgesellschaft in Colombo. Die Trainings und Workshops entfalten so den Charakter einer eindeutig politischen Intervention. Berghof konzentriert sich darauf, mit etablierten Partnern in der Zivilgesellschaft, speziell dem Centre for Policy Alternatives $(\mathrm{CPA})^{118}$, aber auch renommierten Einzelpersonen, zur Unterstützung des zu diesem Zeitpunkt bereits laufenden Friedensprozesses politische Lösungsmodelle auszuarbeiten, zu diskutieren und zu propagieren.

Konzeptionell gefasst wird diese Arbeit im so genannten »RoadMap Program«, das von Berghof in Kooperation mit CPA angestoßen und im Sinne eines kollektiven Erarbeitens von konkreten Marksteinen in einer Erweiterung des Waffenstillstandes hin zu einem Friedensvertrag und den notwendigen langfristigen Perspektiven erklärt wird: »One way of contributing to this emerging field of knowledge is to make use of the metaphor of a >Road Map $<$ and to outline in detail routes and tracks of the terrain ahead and to explore what options are available and how to assess these options « (vgl. Loganathan/Ropers 2002: 4). ${ }^{119}$ Prak-

118 Der Leiter des CPA, Paikiasothy Saravanamuttu, ist als wissenschaftlicher Experte und Vertreter der Zivilgesellschaft in die Friedensverhandlungen involviert. Dies garantiert Berghof eine enge Anbindung an die offiziellen Verhandlungen, was umso mehr dadurch gewährleistet ist, dass der Verhandlungsführer des GoSL, der damalige Minister für konstitutionelle Fragen, G.L. Peiris, als offizieller Counterpart von Berghof fungiert. Mit Peiris verbindet Berghof zu diesem Zeitpunkt ein gutes und enges Arbeitsverhältnis.

119 Die $»$ Road-Map«-Metapher ist dem zu diesem Zeitpunkt aktuellen Friedensplan der US-Administration für den Arabischen Raum entlehnt. Die so genannte »Road Map for Peace in the Middle East» war von USPräsident George W. Bush am 24. Juni 2002 angekündigt worden. Das Konzept hat Berghof also sehr rasch fasziniert. 
tisch konzentriert sich die Arbeit vor allem auf die Organisation von Diskussionen über die Analyse des sri-lankischen Konfliktes, wo es vor allem um die Dissemination des ethnopolitischen Ansatzes geht, auf die Propagierung des Föderalismus als dem zentralen Lösungskonzept (vgl. Ropers 2003: 5), sowie auf Einschätzungen des Verlaufs der Friedensverhandlungen (vgl. etwa CPA/Berghof 2003).

Der Erfolg dieser Maßnahmen zeigt sich in der engen Integration von Berghof in den inneren Zirkeln der politisch aktiven Zivilgesellschaft in Colombo, insbesondere in der Person von Norbert Ropers. ${ }^{120}$ Das hervorstechendste Symbol dieser Integration ist ein Papier mit dem Namen »The Sri Lankan Peace Process at a Crossroads«, das von Ropers gemeinsam mit vier Führungsfiguren der Zivilgesellschaft in Colombo verfasst wird (vgl. Ferdinands et al. 2004). ${ }^{121}$ Dieses Papier ist nicht nur in Hinblick auf die enge Integration von Berghof in die lokale Zivilgesellschaft bemerkenswert. Es ist auch insofern von besonderem Gehalt, als es sich um ein eindeutig politisches Dokument handelt, das klare Forderungen an verschiedene Akteursgruppen wie das GoSL, die LTTE, die Zivilgesellschaft oder die internationale Gemeinschaft formuliert und zudem unzweideutig eine Machtteilung im föderalen Sinn propagiert. Kurzum: Berghof agiert hier als NGO und wird von allen srilankischen Akteuren auch als NGO wahrgenommen, und dies obwohl Berghof in Sri Lanka formell eine vertraglich beauftragte Implementierungsagentur eines bilateral angelegten GTZ-Projektes ist, also im strikten Sinne als verlängerter Arm der GTZ agiert.

Wenn nun zugleich in Betracht gezogen wird, dass sich Berghof vor allem in seinen Stellungnahmen gegenüber den Anti-NGO-Kampagnen explizit nicht als NGO bezeichnet (vgl. Berghof 2006: 2) ${ }^{122}$, allerdings von faktisch allen zivilgesellschaftlichen Aktivist/innen, die diese nur in eingeschränktem Maße verbreiteten Stellungnahmen für gewöhnlich

120 Ein im zivilgesellschaftlichen und akademischen Bereich aktiver Interviewpartner meinte diesbezüglich sogar, dass Norbert Ropers eine von drei Personen sei, die die Zivilgesellschaft in Sri Lanka »kontrollierten«.

121 Die weiteren Autoren sind Tyrol Ferdinands, langjähriger Leiter des NPC und jetzt Leiter der politischen Consultancy INPACT, Kumar Rupesinghe, Führungsfigur von FCE und der National Anti-War Front, Paikiasothy Saravanamuttu, Leiter des CPA, und Jayadeva Uyangoda, Leiter des Departments für Politikwissenschaften an der Universität Colombo und Schlüsselperson bei der Social Scientists' Association (SSA).

122 Die genaue Formulierung in den FAQs, die Berghof als Antwort auf verschiedene Vorbehalte, Anschuldigungen und Kritiken formuliert hat, lautet: »The Berghof Foundation [...] does therefore not qualify as a nongovernmental organization« (Berghof 2006: 2). 
nicht kennen, als NGO begriffen wird ${ }^{123}$, stellt sich die Frage, inwiefern diese Vorgangsweise als politisches Kalkül gesehen und ein solches politisches Kalkül als Teil der deutschen Strategie der Friedensentwicklung in Sri Lanka verstanden werden kann. Wie dem auch sei. Die gesetzten Aktivitäten entsprechen letztlich genau dem strategischen Konzept, das sich die deutsche Entwicklungspolitik für den Umgang mit bewaffneten Konflikten zurechtgelegt hat: die Durchdringung und politische Ausrichtung der Zivilgesellschaft. So ist es nur konsequent, dass die Arbeit von Berghof, auch wenn vielleicht an Details Widersprüche auftraten, in ihrer Gesamtheit überwiegend positiv beurteilt wird.

Dennoch kommt es an genau diesem Punkt zu einer erstaunlichen Veränderung. Just am Höhepunkt von Berghofs zivilgesellschaftlichem Engagement erfolgt eine Neuausrichtung der Arbeit, die fundamentalen Charakter annimmt: der Wechsel von der Zivilgesellschaft als zentraler Zielgruppe der Intervention hin zur politischen Gesellschaft, also jene unter dem Begriff Track 1.5 zusammengefasste Gruppe von Personen aus oder nahe den politischen Parteien, denen großer und nachhaltiger Einfluss auf die Politikgestaltung nachgesagt wird. Diese Neuausrichtung, die intern als dritte Projektphase verstanden wird (vgl. Berghof 2006: 5), ist Resultat einer Evaluation der bisherigen Praxis, die gerade angesichts des zu diesem Zeitpunkt gerade endgültig scheiternden Friedensprozesses als nicht effektiv genug eingeschätzt wird. Berghof könnte demnach, gerade im Wechselspiel mit dem in den Grassroots verorteten Projekt FLICT, im Track 1.5 einen langfristig wirkungsvolleren Beitrag zu einer Wiederaufnahme der Verhandlungen leisten. ${ }^{124}$

Die gegenwärtige Interventionsstrategie von Berghof (siehe Abbildung 29) formiert sich nun als (um die Komponente »Monitoring and Assessment «, einer permanenten Begleitmaßnahme, erweitertes) Dreieck, das mit den Komponenten »Providing Multiple Perspecitves«, faktisch die Weiterführung der Propagierung von Föderalismus-Konzepten, und »Dialogue and Problem Solving" zwei Elemente aus dem RoadMap-Programm hinüberrettet, sie aber ihrer zentralen Dimension

123 Die einzigen meiner Interviewpartner/innen in Sri Lanka, die Berghof als Teil der bilateralen Kooperation und nicht als Teil der Zivilgesellschaft begriffen, kamen entweder aus der GTZ oder aus Botschaften. Alle von mir interviewten Personen aus dem zivilgesellschaftlichen Bereich, egal ob aus Sri Lanka oder aus der INGO-Szene, begriffen Berghof als INGO.

124 Es ist unklar, ob die Entscheidung von Berghof selbst oder von BMZ und PA4, den beiden Geldgebern ausgeht. Aufgrund der großen Eigenständigkeit, mit der Berghof in Sri Lanka operiert, ist die erste Variante wahrscheinlicher, ohne Abstimmung mit den Gebern allerdings ohnehin nicht vorstellbar. 
enthebt. ${ }^{125}$ Diese findet sich nun im Bereich »Conflict Resolution and Institutional Capacity Building«, der auf die Arbeit mit politischen Akteuren abzielt.

Abbildung 29: Interventionsstrategie der Berghof Foundation in Sri Lanka

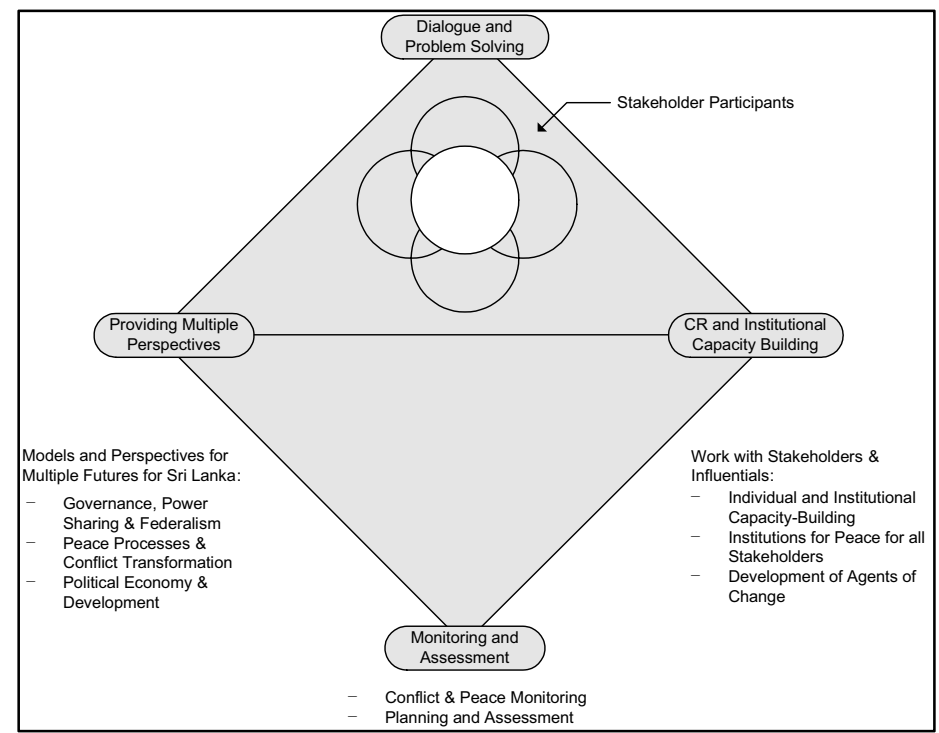

Quelle: http://www.berghof-foundation.lk/scripts/work1.htm

(Zugegriffen am 21-06-2007)

Die zu diesem Zweck identifizierten sieben Partner kommen aus sehr unterschiedlichen Zusammenhängen. Die Partnerschaften selbst haben nicht nur eine überaus ungleich ausgeprägte Tiefe, sondern eine einer-

125 Die Frage, ob Berghof hinsichtlich möglicher Lösungsmodelle eindeutig Partei beziehen sollte, ist intern umstritten, wenngleich Norbert Ropers selbst wenig Zweifel an seiner einer föderalen Lösung zugeneigten Einstellung lässt. Offiziell führt dieser interne Widerspruch, der auch mit der eigenen Positionierung gegenüber den Anfeindungen von singhalesisch-nationalistischen Kräften zu tun hat, zu Stellungnahmen, die fast schizophren klingen: »This [das Oslo-Abkommen] was the starting point for us to support all interested parties to enhance their knowledge about different power sharing arrangements including federalism. While doing that we have never propagated or proposed any specific power sharing solution. Our role has been and still is only to contribute to a better understanding of the multiplicity of models of power sharing and federalism« (Berghof 2006: 9). 
seits durch die politischen Umstände bedingte, andererseits aber auch als Pragmatismus zu verstehende unterschiedliche Entwicklung - auch wenn das formal sehr stark und mit dem Begriff »Multipartiality« vermeintlich eindeutig umrissene Konzept anderes nahe legen würde. So findet sich unter den Partnern der eigentliche Counterpart von Berghof, das Ministry of Constitutional Affairs and National Integration, zu dem seit jeher eine gute Arbeitsgrundlage besteht. Das Ministerium ist allerdings von der gegenwärtigen Regierung de facto kaltgestellt. ${ }^{126}$ Dazu kommen mehrere parteinahe Think Tanks, zu denen teilweise relativ enge Kontakte bestehen (wie SNAP, einem UNP-nahen Think Tank, das der vormaligen Präsidentin Kumaratunga nahe stehende "Council for Public Policy«, CPP, und die im tamilischen Hochland verankerte »Foundation for Community Transformation«, FCT) oder nur sehr lose Beziehungen aufrechterhalten werden können (wie etwa Kräften, die sich der gegenwärtigen Regierung zugehörig fühlen). ${ }^{127}$

Dazu kommt eine überaus enge Kooperation mit dem moslemischen Friedenssekretariat PSM (wenngleich die finanzielle Unterstützung von anderen Gebern, etwa USAID, geleistet wird) und dem »Centre for Just Peace and Democracy« (CJPD), einer tamilischen Diaspora-Organisation in der Schweiz, der ein Naheverhältnis zur LTTE nachgesagt wird. ${ }^{128}$ Mit dem CJPD wurde beispielsweise eine groß angelegte Konferenz in der Schweiz organisiert, die unter Beteiligung zahlreich eingeladener internationaler Expert/innen Perspektiven einer friedlichen Lösung nach Ende des Friedensprozesses ausloten sollte (vgl. CJPD 2006).

126 Unter dem vormaligen UNP-Minister G.L. Peiris, der Verhandlungsführer des GoSL in den Friedensgesprächen war, hatte das Ministerium einen wesentlichen Platz im Zentrum des Machtgefüges. Nach der Regierungsübernahme durch Mahinda Rajapakse und der Sistierung des Verhandlungsprozesses wurde die Leitung des Ministeriums den linken Kleinparteien überlassen - der derzeitige Minister Gunasekera gehört der Kommunistischen Partei an - und damit de facto entmachtet. Der Vollständigkeit halber sei erwähnt, dass G.L. Peiris nach einem Überlaufen von der Opposition in die Regierungskoalition mittlerweile wieder ein Ministeramt ausübt. Seine politische Rolle ist aber nicht mehr mit der von 2001-2003 zu vergleichen.

127 Zudem konnten mit keiner der relevanten singhalesisch-nationalistischen Parteien Arbeitsbeziehungen aufgebaut werden, auch wenn eine Arbeit in diesem Bereich als potenziell wichtig angesehen wird.

128 Mit der LTTE selbst wird nach Verhängung der aktuellen Emergency Regulations durch das GoSL, mit denen jeder Kontakt mit der LTTE untersagt wird, offiziell nicht mehr kooperiert. Zuvor gab es eine zeitweise enge Zusammenarbeit, etwa eine heute überaus umstrittene Informationsreise von Norbert Ropers mit führenden LTTE-Kadern nach Europa zum Zwecke der Veranschaulichung einer föderalen Verfassungsrealität. 
Entscheidend für das Verständnis der gegenwärtigen Arbeit von Berghof ist vor allem, dass es, insbesondere beim Verständnis des Capacity-Building, nicht um die Form technischer Unterstützung und Gesprächsformation geht, wie es die Kombination aus OneText und SAPI anstrebt. Berghofs Intervention strebt auch im Bereich der politischen Gesellschaft eine politische Einflussnahme an, und zwar in einem viel direkteren, unmittelbareren Sinn als es im Verhältnis von USAID zu seinen Partnern vorzufinden ist, das auf einer an Leitlinien und Prinzipien gruppierten, allgemein gehaltenen Ebene verbleibt. Berghof zielt auf direkte Transformation, auf eine möglichst weitgehende Verankerung der erarbeiteten Analysen, Konzepte und Lösungsmodelle, natürlich in einem möglichst kollektiv gestalteten und offen gehaltenen Prozess, der jedoch in seiner Intentionalität eindeutig festgelegt ist. ${ }^{129}$ Diese grundsätzliche Herangehensweise hat sich auch mit der Verschiebung von der zivilen zur politischen Gesellschaft als primäre Zielgruppe der Intervention nicht verändert.

Der Gegenpol zum Berghof-Projekt ist im GTZ-Programm das im Jahr 2003 eingerichtete Projekt »Facilitating Local Initiatives for Conflict Transformation« (FLICT). FLICT ist als Fonds organisiert, der auf Basis von an ihn herangetragenen Anträgen Förderungen vergibt. Dies klingt zunächst nach einer recht flexiblen Handhabbarkeit, doch in der realen Projektumsetzung ist das grundsätzlich offene antragsbasierte Fondsprinzip durch zwei Komponenten eingeschränkt, die sich schon im Projektnamen finden: So geht es erstens um »Local Initiatives«, was nicht nur die Verankerung an den Graswurzeln oder zumindest die Orientierung der Arbeit auf die Graswurzel-Ebene meint, sondern in erster Linie eine nur aus der spezifischen Verfasstheit der sri-lankischen Zivilgesellschaft zu verstehende Umschreibung dafür, dass die Förderung für NGOs und Initiativen außerhalb Colombos angelegt ist. Zweitens macht der Ausdruck »Facilitating [...] for Conflict Transformation« schon klar, dass es nicht in erster Linie um die Auswahl sinnvoller Projekte geht, die dann umgesetzt werden - ein Weg, den beispielsweise OTI geht -, sondern dass der Schwerpunkt der Arbeit auf CapacityBuilding und der Verankerung von Theorie und Praxis der Konflikttransformation bei den jeweiligen Partnerinitiativen ist. Der Fonds und die umgesetzten praktischen Schritte sind wenig mehr als der Rahmen,

129 Neben finanziellen Erwägungen ist es vor allem die Berghof speziell im Bereich der Konfliktanalyse nachgesagte Kompetenz, die einen solchen Einfluss ermöglicht. Die meisten der institutionellen wie persönlichen Partner Berghofs, und alle, mit denen engere Kooperationen aufgebaut werden konnten, hoben diesen Aspekt in den Interviews hervor. 
in dem ein Schulungs- und Trainingsprozess durchlaufen wird, der das eigentliche Herzstück von FLICT ausmacht.

FLICT geht auf eine gemeinsame Initiative von DFID und GTZ zurück, die die zivilgesellschaftliche Unterfütterung des Friedensprozesses stärken soll. Der Name FLICT und das Grundkonzept entsprechen zwischen den bilateralen Akteuren ausgehandelten Vorgaben, für die Ausgestaltung wird ein »FLICT design team» eingerichtet, das entlang der Zielsetzung »to enhance civil society's role in building a sustainable and just peace in Sri Lanka« (Burke/Bastian 2003: 7) ein komplexes Set aus acht Arbeitsgebieten (drei so genannte »key focus areas« und fünf »focus areas«) konzipiert. Zentral ist von Beginn an die Arbeit mit als »Minderheiten« verstandenen Gruppen, also die Zielsetzung »to build a society that includes minorities« (ebd.: 15). Spannend ist in der weiteren Ausgestaltung der Arbeitsweise in diesem ersten Konzept die Konzentration auf so genannte »Intermediaries«, also die Heranbildung von zentralen Partnerinitiativen in den acht Arbeitsgebieten. Insgesamt ist gedacht, zehn bis zwölf derartige Intermediaries anzubinden, wobei eine »reach outside Colombo« (ebd.: 33) wesentliches Auswahlmerkmal sein sollte. Interessant ist die Begründung einer auf »Intermediaries« basierenden Arbeitsweise: Die Arbeit mit kleinen Förderbeträgen würde automatisch zu einer Vervielfachung von Projekten führen und sich damit unausweichlich »donor-driven« gestalten - speziell Letzteres sollte von FLICT vermieden werden (ebd.: 30).

Schon in der ersten Umsetzungsphase entwickelt sich FLICT von einem deutsch-britischen Co-Projekt, als das es offenbar ursprünglich angedacht war, zu einem GTZ-Projekt mit britischer Co-Finanzierung. Der praktische Ablauf gestaltet sich allerdings weitgehend so, wie ihn das Projektkonzept vorsieht. Partnerinitiativen werden nach eingelangten Anträgen für eine Förderung durch den Fonds ausgewählt, in der Umsetzung ihrer geplanten Initiativen nach Bedarf begleitet, vor allem aber in ein Trainings- und Workshop-Angebot eingebunden, das die geeigneten Sichtweisen auf den Konflikt und jenes Wissen vermitteln soll, das für Konflikttransformation als maßgeblich verstanden wird. Eine aus mehreren Evaluationen bestehende Review des Projektes im Jahr 2005 bescheinigt dem Fonds denn auch einen »fresh and innovative approach« (FLICT 2005: 3), was vor allem mit der Fokussierung auf Initiativen außerhalb Colombos bedingt ist ${ }^{130}$, und eine nach wie vor bestehende Gültigkeit der Zielsetzungen (ebd.). Zugleich wird allerdings eine Reduzierung der Arbeitsschwerpunkte nahegelegt.

130 Dies bestätigt beispielsweise auch Sumanasiri Liyanage (2006: 288). 
Abbildung 30: Arbeitskonzept von FLICT

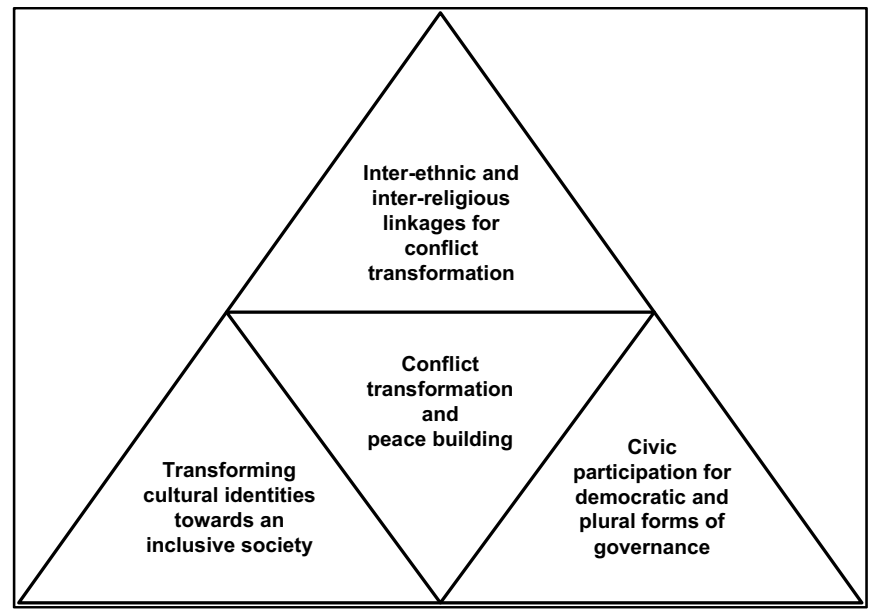

Quelle: FLICT 2006a: 15

Als Konsequenz dieser Review werden die Arbeitsbereiche reduziert (siehe Abbildung 30). Gegenwärtig beschränken sie sich auf drei ${ }^{131}$, von denen zwei die »ethnische« Konfliktdimension bearbeiten wollen, mittels Transformierung kultureller Identitäten sowie durch den Aufbau von inter-ethnischen und inter-religiösen Verbindungen, und eine das klassische strategische Zivilgesellschaftsverständnis der deutschen Entwicklungspolitik umzusetzen sucht ${ }^{132}$ : „Civic participation for democratic and plural forms of governance«. Der anleitende Grundsatz dazu ist die Idee einer starken und aktiven Bürgerschaft: »In the interest of stability,

131 Ganz im Sinne eines vollkommen durchdachten und umfassenden konzeptionellen Ansatzes entsprechen alle drei Arbeitsbereiche jeweils einer Antwort auf eine Komponente aus Galtungs Gewalttriade. So dienen die »inter-ethnic and inter-religious linkages« der Überwindung direkter Gewalt, das »transforming cultural identities« als Antwort auf kulturelle Gewalt und die »civic participation« als Gegenstrategie zu struktureller Gewalt (vgl. FLICT 2006a: 14ff.). Ein Drang zum Allumfassenden ist diesem Ansatz nicht abzusprechen.

132 Interessant ist, dass die Notwendigkeit zu einer solchen Intervention aus einer Veränderung der sri-lankischen Gesellschaft angesichts des laufenden bewaffneten Konfliktes argumentiert wird: »The continuing conflict, politicization of all facets of public life, loss of faith in public institutions and elected leaders, the rapid militarization of society as well as a deteriorating economy have placed greater demands on civil society organisations to act as monitors and activists« (FLICT 2006a: 4f.). Dies kontrastiert insofern mit der Projektgeschichte, als die betreffende Interventionsebene zu einem Zeitpunkt ausgearbeitet wird, als sich der Friedensprozess an seinem Höhepunkt befindet. 
peace and progress it is necessary to develop a strong citizenry to be more actively engaged in the governance of the country so that past mistakes are corrected and just and fair policies are implemented to address the needs of all people in a culturally diverse society « (FLICT 2006a: 5). Die Rolle der Zivilgesellschaft wird gerade in Kombination mit Governance immer zentraler, und es wird von ihr eine aktive Rolle nicht nur bei der kritischen Begleitung und aktiven Partizipation, sondern auch im »establishing $[\ldots]$ mechanisms and institutions that aim at reducing discrimination, exclusion, and exploitation« eingefordert (ebd.: 33). Dies lässt wiederum einen vielsagenden Blick auf das Selbstverständnis von FLICT zu. Denn offenbar sieht sich das Projekt nicht nur in der Lage, sondern auch in der Pflicht, jene Prozesse von »discrimination, exlusion, and exploitation « $\mathrm{zu}$ definieren und festzustellen, und die lokal verankerte Zivilgesellschaft in ihrer Kapazität so zu entwickeln, dass sie zu deren Überwindung einen effizienten und wirkungsvollen Beitrag leisten kann.

Da diese Ungerechtigkeiten nach wie vor bestehen, ist es in dieser Denklogik auch folgerichtig, dass bei der Zivilgesellschaft in diesem Bereich Defizite bestehen müssen, womit Capacity-Building eine noch viel größere Bedeutung im Rahmen der zweiten Projektphase erhält als sie es ohnehin schon hat. Zu diesem Zweck müssen nicht nur Managementkapazitäten und Organisationsfähigkeit der Partner ausgebaut werden, sondern in erster Linie ihre Fähigkeit, Konflikttransformation zu verstehen: »It has been recognised that partner organisations need to strengthen their capacities in the following areas: - conceptualising conflict transformation, - self evaluation and organisational development (linked to internal conflict transformation), - project cycle and result based management « (ebd.: 41).

In der Folge verwandelt sich FLICT von einem Fonds-basierten Förderprojekt für Kleinprojekte im Grassroots-Bereich immer mehr zu einer Trainingsinstitution für Konflikttransformations-Ansätze. Mittlerweile konzentrieren sich die Schulungsleistungen nicht mehr nur auf die Partnerinitiativen, sondern FLICT versucht, über ein Training-of-Trainers (ToT)-Programm einen regionalen »consultant pool« aufzubauen (ebd.: 45), mit dem eine noch effizientere Proliferation erreicht werden soll. ${ }^{133}$

133 Die ersten Ansätze dieses Programms sind für FLICT allerdings alles andere als ermutigend. Nachdem im Zuge dieses ToT auch eine Diplomierung erfolgt, die noch dazu von dem in Sri Lanka sehr aktiven Peace Building Department der britischen Bradford University zertifiziert ist, entwickelt sich das ToT einerseits zum Karriereprogramm und andererseits zum unvorhergesehenen Leistungstest. Über ein Drittel derjenigen, die das Programm durchlaufen, scheitern an der Prüfung, darunter eini- 
Die geförderten Projekte und Initiativen rutschen in diesem Ansatz immer stärker in die Rolle von Vehikeln zur Dissemination von Konflikttransformations-Konzepten. Dennoch bleibt der innovative Punkt von FLICT auf einer Ebene, die erst in den letzten Jahren vermehrt in den bilateralen Blickpunkt rückt: die Heranbildung einer professionalisierten Zivilgesellschaft, die den Leitbildern und den politischen Schwerpunktlegungen der Geber entspricht, außerhalb der Zentren, also die Idee der Professionalisierung der Grassroots bei einer gleichzeitigen Verankerung jener Handwerkszeuge, die für Friedensentwicklung als maßgeblich betrachtet werden.

Wie auch bei den USA findet sich im deutschen Ansatz eine Bestätigung der aus den strategischen Dokumenten herauszulesenden grundsätzlichen Ideen und Leitlinien der Intervention. Die Zivilgesellschaft ist Motor einer möglichst genau, bis hin zur Mikro-Ebene gesteuerten Ausrichtung, die entlang klarer gesellschaftlicher und politischer Orientierungspunkte vor sich gehen soll. Im Gegensatz zu den strategischen Ansatzlinien, wo Friedensentwicklung und menschliche Sicherheit noch einen lokal angepassten Gestaltungsspielraum offenlassen, hat sich die Intervention in Sri Lanka in dieser Hinsicht festgelegt: Das Programm des Eingriffes in gesellschaftliche Strukturen heißt Konflikttransformation, verstanden als Verknüpfung eines klar bestimmten politischen Programms - Föderalismus in einem als inklusiv definierten sri-lankischen Staat - mit einem spezifischen Wissenskorpus, der um eigentlich für einen relativ kleinen Kreis an Expert/innen generierte Konzepte wie Konfliktsensitivität, Konfliktanalyse, »do no harm« oder PCIA kreist. Diese Konzepte werden in die Rolle gesellschaftlicher Orientierungsmuster gepresst, die über die geförderten Aktivitäten von möglichst graswurzelnahen, aber zugleich auch maximal professionalisierten Initiativen verankert werden sollen, mit dem Idealbild eines sich entlang dieser Prinzipien entfaltenden, durch NGO-Leitbilder geprägten Zusammenlebens, das dann als friedlich identifiziert wird.

Die ursprüngliche Aufteilung, die Berghof für die Verankerung der politischen Ebene und FLICT für die Verankerung konflikttransformierender Projektpraxis bei einer außer durch räumliche Gegebenheiten (Colombo versus nicht Colombo) kaum unterscheidbaren Zielgruppe

ge, weil sie während der finalen Prüfung vollkommen identische Essays abgeben. Dieses ernüchternde Resultat sagt auch viel über das tatsächliche Verhältnis von Bedarf, Angebot und Nachfrage im Bereich der Konflikttransformation in Sri Lanka aus, auf das ich an dieser Stelle nicht weiter eingehen kann, das aber sicher nicht einer von Projekten wie FLICT diagnostizierten Situation entspricht. 
vorsieht, hat sich im Zuge der Arbeit als Konsequenz der Erfahrungen der ersten drei Arbeitsjahre und - dieser Faktor ist wichtig herauszustreichen - angesichts des Scheiterns des Friedensprozesses wesentlich verschoben. Zwar werden die generelle, stark auf disziplinierende Momente zurückgreifende Herangehensweise und die thematische Aufteilung beibehalten, es ändern sich allerdings die Zielgruppen: Während sich Berghof auf die Arbeit im viel gepriesenen Track 1.5 verlegt, die Tätigkeiten also zunehmend auf politische Akteure, vor allem im parteinahen Spektrum, verschiebt, orientiert sich FLICT immer stärker auf eine möglichst große Breitenwirkung des Programms. Die Idee der Arbeit mit Mittlerorganisationen, die sich mit der durch FLICT erworbenen Kompetenz ihren jeweiligen praktischen Schwerpunkten widmen sollen, wird dabei durch ein viel unmittelbareres Konzept von Steuerung abgelöst: das ToT-Programm dient in diesem Kontext der möglichst tiefen und flächendeckenden Dissemination einer konflikttransformativen »Projektitis«, die die ohnehin laufende enge Arbeit mit den geförderten Partnerinitiativen ergänzen und auch vertiefen soll.

Eine solche Vertiefung durch die Präsenz von FLICT-zertifizierten Trainer/innen im lokalen Setting ist eine neue Qualität disziplinarischer Durchdringung, die auf eine Tendenz hinweist, die auch bei Berghof feststellbar ist. Nachdem die Kapazitäten der vorzufindenden Zivilgesellschaft als nicht ausreichend empfunden werden, bewegen sich beide Projekte immer mehr in ein Verhaltensmuster, selbst die Rolle der Zivilgesellschaft und die ihr von den strategischen Konzepten zugedachten Funktionalitäten einzunehmen. Die sri-lankische Zivilgesellschaft wird also nicht mehr bei der Wahrnehmung der ihr zugedachten Aufgabe unterstützt, sondern sie wird substituiert und in die Doppelfunktion als Multiplikator und als lokale Legitimationsgrundlage des eigenen Handelns gedrängt.

Zugleich ist die Arbeit im Governance-Bereich überraschend gering. Es gibt so gut wie keine Kooperation mit Ebenen der Zentralverwaltung (die einzige Leistung im Sinne einer technischen Unterstützung wird, mit Ausnahme der Post-Tsunami-Maßnahmen, von der Berghof-Stiftung in ihrer Zusammenarbeit mit dem Ministry of Constitutional Affairs geleistet), und das laufende Projekt zur Stärkung der lokalen Governance hat einen klaren anti-zentralistischen Gehalt, der die Idee des starken Staates, die für die strategischen Leitdokumente prägend ist, eher unterminiert als fördert.

Diese Form der Arbeit im Governance-Bereich ähnelt stark dem USamerikanischen Zugang, und auch in den anderen Programmteilen gibt es, wenn wir die weitgehenden Unterschiede in den strategischen Konzepten in Betracht ziehen, überraschende Parallelen. Zunächst ist aber 
natürlich darauf zu verweisen, dass sich die Rationalitäten, die schon die Schlüsseldokumente prägen, in der konkreten Praxis in Sri Lanka wiederfinden, sowohl bei den USA als auch bei Deutschland. Wie letztendlich zu erwarten lässt USAID seinen Partnern einen viel größeren Spielraum, es geht in der programmgesteuerten Tätigkeit vorwiegend darum, Akteure richtig zu platzieren oder, besser formuliert, im Sinne der Entwicklung einer wettbewerbsorientierten demokratischen Perspektive in das als richtig angenommene Verhältnis zueinander zu setzen. Dass dazu Akteure, die als notwendig und zugleich nicht ausreichend institutionalisiert eingeschätzt werden, überhaupt erst geschaffen und etabliert werden (wie das Beispiel des PSM zeigt), liegt letztlich in der Logik dieses Ansatzes. Die deutsche Praxis zeigt sich demgegenüber im ebenfalls zu erwartenden disziplinarischen Gewand, das sich zuallererst im Drang zur Omnipräsenz manifestiert und die Vision einer dicht aktivistisch durchzogenen Gesellschaft als Idealbild vor sich hat.

Bei beiden Gebern ist jedoch eine Programmentwicklung sichtbar, die diese Zugänge konterkariert. Der Bruch und die Umorientierung kann zeitlich mit der Einsicht in das Scheitern des sri-lankischen Friedensprozesses festgemacht werden, der den vorhandenen überbordenden Optimismus auch innerhalb der Agenturen derart schockartig zerschlägt, dass die bisherige Ausrichtung der eigenen Praxis jeweils in ihrer Substanz in Frage gestellt wird. Infolgedessen finden wir bei beiden einen verstärkten Griff zu Ansätzen, die ursprünglich eher als Domäne des jeweils anderen gesehen werden könnten: USAID entdeckt die politisch aktive Zivilgesellschaft als Partner, sowohl in ihrer Arbeit mit Parteienvertreter/innen und den Grassroots, ein zentrales Projekt der GTZ orientiert sich weg von der Zivilgesellschaft hin zu einer Arbeit im dezidiert (partei-)politischen Bereich.

Dies zeigt eine bemerkenswerte Flexibilität im Umgang mit dem Phänomen des eigenen Scheiterns, denn auch wenn keine/r der Beteiligten dies so kommunizieren würde, ist der Zerfall des Friedensprozesses von beiden Agenturen auch in dieser Richtung verstanden worden. Obwohl beide ihren grundsätzlichen Herangehensweisen an der jeweils spezifisch rationalen Regierung im Sinne sozialer Steuerung, und als nichts anderes ist sicherheitspolitisch gesetzte Entwicklungszusammenarbeit zu verstehen, letztlich treu bleiben, zeigen sie sich bereit, andere Konzepte von Steuerung aufzugreifen und damit zu experimentieren und es ist in der Tat ein Experiment, weil für die hier beschriebenen Projekte kaum internationale Präzedenzen bestehen. Ein Weg freilich, der die Experimentierfreudigkeit so weit treiben würde, dass diese Steuerung an sich in Frage gestellt werden würde, ist auch im Angesicht des Scheiterns nicht denkbar. 



\title{
Sicherheit und Entwicklung als "White Man's Burden«? - Schlüsse und Thesen
}

\author{
»The emergence of new forms of instability has \\ reinforced the symbolic line that aid policy has \\ drawn between the pre- and post-Cold War \\ periods. Not only has conflict been rediscovered, \\ but development assistance has simultaneously \\ been granted a new lease of life as a structural \\ form of conflict prevention. Hence, despite a \\ history of failure, it has been repackaged as a \\ valuable, indeed, as an essential weapon in the \\ armoury of liberal peace."
}

(Mark Duffield)

Es stellt sich nun die Frage, welche Erkenntnisse aus der vorliegenden Untersuchung von Konzepten und Praxen jenes Komplexes, den ich als »sicherheitspolitische Entwicklungszusammenarbeit« bezeichnet habe, gewonnen werden können. In der Diskussion dieser Frage möchte ich Überinterpretationen vermeiden. Dennoch behaupte ich, und anders lieBe sich der Aufwand der Untersuchung schwerlich rechtfertigen, dass die Lesart, die ich durch meine Aufschlüsselung des empirischen Materials mit dem analytischen Rahmen als entscheidendem Raster von dessen Anordnung anzubieten in der Lage bin, Einsichten ermöglicht, die einem ersten oder vielleicht auch zweiten Blick auf die Dokumente oder die praktischen Abläufe wahrscheinlich verborgen bleiben würden.

$\mathrm{Zu}$ verstehen ist der Prozess vermutlich am besten als Erarbeitung eines spezifischen Systems von Ordnung, das zunächst durch die Form der Anordnung von Aussagen - seien sie praktisch, seien sie institutionell, seien sie diskursiv - eine genauere Einschätzung der inneren Logik 
und des strategischen Gehalts eines Dispositivs öffnet. Zugleich ermöglicht sie aber auch mittels einer Einordnung, sowohl im historischen Sinne als auch im Verhältnis zu anderen alternativen Formationen, einen Blick, der über den unmittelbaren Kontext des konkreten Untersuchungsfeldes hinausreicht.

Es gibt mittlerweile zahlreiche Untersuchungen zur Frage der Tätigkeiten bilateraler oder multilateraler Geberagenturen im Umgang mit bewaffneten Konflikten, die mit unterschiedlicher Zielsetzung, einem dementsprechend unterschiedlich gelegten Niveau und verschieden ausgerichteter Kritik die Konzepte, Abläufe und Praktiken darstellen. Sie geben einen breiten und zum Teil überaus genauen Überblick über die laufenden Maßnahmen, ihre Stärken und ihre Mängel. In diesem Zusammenhang können die von mir gewonnen Aussagen vielleicht einzig als Bestätigung einer schon vergleichsweise alten Erkenntnis entwicklungskritisch ausgelegter Untersuchungen dienen, dass die Interventionen, die Entwicklungspolitik setzt, und die Zielvorstellungen, von denen sie dabei geleitet wird, in der Tat mehr mit jenem politischen und sozialen Bezugssystem zu tun haben, in dem die Entwickler/innen selbst leben, als mit jener Realität, mit der sie in den Zielregionen ihrer Tätigkeit konfrontiert sind. Damit bestätigt sich ein ohne Zweifel vorhandener kolonialistischer Gehalt von Entwicklung (was für Sicherheit und Sicherheitspolitik im Übrigen ebenso uneingeschränkt gilt).

Dies allein wäre als Erkenntnisgewinn allerdings wenig spannend. Zwar hat eine solche Aussage (immer noch, bin ich versucht zu sagen) provokativen Gehalt und ist damit alles andere als nebensächlich, ebenso verführt sie jedoch zu Plattheiten und Vereinfachungen, denen wenig Erhellendes abzugewinnen ist. Weder ist Entwicklung die Fortsetzung des Kolonialismus mit anderen Mitteln, noch reines Mittel zur (Re-)Produktion kapitalistischer Herrschaftsverhältnisse, auch wenn sich beides in ihren vielen Verästelungen wiederfinden lässt.

Die entscheidende Frage liegt also darin, was in der Verknüpfung der Beobachtungen mit dem theoretischen Ansatz gewonnen werden kann, Entwicklung und Sicherheit als Dispositive zu interpretieren und die derart konzeptionalisierten Praktiken auf ihre Aussagen über Rationalitäten der Regierung von Menschen auszufragen. Hier denke ich, dass interessante Aspekte gewonnen werden können, die sonst hinter der relativ oberflächlichen Kritik an einer selbstverständlich eurozentristischen Praxis verborgen bleiben würden: Die Verbindung von Sicherheit und Entwicklung vollzieht sich nicht nur, sie erfüllt in der Tat einen strategischen Zweck von Herrschaft, dessen Stärke - gemeint im Sinne der wirksamen Einflechtung in das Netzwerk von Macht - nur aus dem Konkreten heraus verstanden werden kann. 
Davon ausgehend möchte ich die Resultate dieser Untersuchung in drei Punkten zusammenfassen, die sich auf die eingangs dargelegten Ausgangshypothesen beziehen, und im Anschluss daran einen Vorschlag anreißen, wie diese Resultate produktiv aufgegriffen werden könnten.

\section{(1) Sicherheit und Entwicklung verschmelzen.}

Die von mir aufgestellte Ausgangshypothese, dass sich seit Mitte der 1990er Jahre eine tendenzielle Verschmelzung von Entwicklungs- und Sicherheitspolitik abzeichnet, findet auf allen drei Ebenen, auf denen ich die Frage untersucht habe, ihre Bestätigung. Sowohl im allgemeinen theoretischen Verständnis, wie sie von Entwicklungsforschung und Konfliktanalyse repräsentiert werden, bei den strategischen Konzeptionen der führenden Geberinstitutionen wie auch in jenen Ausschnitten der Umsetzungspraxis, die ich dargelegt habe, ist diese Verschmelzung deutlich beobachten.

Die Gründe dafür sind unterschiedlich. Ich habe mich in meiner Darstellung vor allem auf jene Prozesse konzentriert, die diese Verschmelzung quasi von »oben" bedingen, habe also mehr oder minder eine »Top-Down-Analyse« angeboten. Darin sind jene Abläufe sichtbar geworden, die innerhalb der politischen Entscheidungsebenen und der zentralen entwicklungspolitischen Agenturen zum Aufgreifen der Problemstellung »Sicherheit« geführt haben. Auf dieser Ebene sind Herausforderungen wie die notwendige Neupositionierung beider Politikbereiche nach Ende des Kalten Krieges wesentlich, ebenso wie die Entdeckung von Sicherheit als logische Fortsetzung des Konzepts von Nachhaltigkeit, sowie die Einbindung von Entwicklung in sicherheitspolitische Konzepte angesichts der Aufweichung eines klassisch (neo-)realistischen Sicherheitsverständnisses.

Genauso gut könnte diese Verschmelzung jedoch auch von anderen Seiten ausgehend erzählt werden - etwa, wie eingangs erwähnt, aus der Perspektive der Betroffenen dieser Interventionen oder mit Blick auf diejenigen, die die Integration von Entwicklung und Sicherheit aus einem aktivistischen, friedens- oder entwicklungsbewegten Ansatz heraus einfordern und vorantreiben. Obwohl ich letzteren Blickwinkel an einigen Punkten in meine Darstellung miteinbezogen habe, würde eine Untersuchung, die ihren Fokus auf den zivilgesellschaftlichen, aktivistischen Bereich legen und so die Integration von Frieden und Entwicklung gleichsam von »unten« nachzeichnete, zwar vielleicht nicht unbedingt zu komplett anderen Ergebnissen führen, sicherlich aber zur Betonung von Aspekten, die in meinem Zugang nicht in gebührender Deutlichkeit hervortreten. 
Eines der wesentlichen Zeichen für die immense Integrationsfähigkeit der Kombination von Entwicklung und Sicherheit ist eben, dass es ihr über Konzepte wie »Human Security«, »Peacebuilding« oder »Democracy Promotion« gelingt, alternative und sogar oppositionelle Strömungen, die den offiziellen Projekten kritisch gegenüberstehen, wieder in den jetzt neu definierten Mainstream hereinzuholen. Dass eine solche Entwicklung nur durch Anstrengung aller Beteiligten möglich werden kann, liegt auf der Hand.

In jedem Fall beweist aber die alle Ebenen durchdringende Wirkung der Verschmelzung von Entwicklung und Sicherheit, dass dieser Prozess als eine Integration zweier bislang distinkt wirksamer Dispositive verstanden werden kann. Es ist alles andere als graue Utopie, ein neues Dispositiv von Sicherheit-Entwicklung anzunehmen, das nicht nur einen neuen, spezifischen Wissenskorpus hervorbringt, sondern auch die Praxis internationaler Interventionen entscheidend verändert hat und weiter verändern wird. Dieses neue Dispositiv bringt eine neue Form des globalen Zugriffs mit sich, die als strategische Antwort auf das letztendliche Scheitern der Sicherheits- und Entwicklungskonstruktionen des 20. Jahrhunderts an den neuen Gegebenheiten, aber auch an ihren allgemeinen Postulaten $\mathrm{zu}$ verstehen ist. Insofern wirkt dieses neue Dispositiv nicht nur als Machtverhältnis, sondern herrschaftlich, im Sinne der Reproduktion bestehender globaler Kräfteverhältnisse.

(2) Zivile Intervention vollzieht sich im Spannungsfeld zwischen disziplinarischer Kontrolle und gouvernementaler Steuerung.

Auch die zweite Ausgangshypothese, wonach sich jene Praxen, die sich bei unterschiedlichen Akteuren durch die Verschmelzung der Dispositive von Entwicklung und Sicherheit ergeben, nach neuen Rationalitäten von Regierung vollziehen und, so die dritte Annahme, akteursspezifische Besonderheiten aufweisen würden, bestätigt sich grundsätzlich. Allerdings sind hier einschränkende Bemerkungen notwendig. Denn im Unterschied zu den Formen der Interventionen, im Rahmen derer tatsächlich neuartige Schritte und Maßnahmen entwickelt werden und zur Anwendung kommen, unterscheiden sich die Rationalitäten, nach denen sich diese neuen Maßnahmen vollziehen, nicht grundlegend von jenen, die schon in der Epoche des Kolonialismus wirksam waren. ${ }^{1}$

Die Neuartigkeit beschränkt sich also, und dies ist für die historische Einordnung des Gesamtprozesses wichtig, primär auf die Konfigurationen, in denen die Rationalitäten wirken, und die neue Form des Zusammenspiels, das sie kennzeichnet. Der gouvernementale Umgang mit Ak-

1 Vgl. dazu etwa die Arbeiten von Ann Laura Stoler $(1995,2002)$ 
teuren unterscheidet sich erheblich, wenn wir das britische System der »indirect rule« betrachten oder die Stärkung politischer Akteure zu ihrem effizienten Funktionieren im Sinne eines wettbewerbsorientierten polyarchischen Systems. Die Durchdringung kolonialisierter Subjekte mit den Methoden einer militärisch gestützten Kolonialverwaltung und die Durchdringung postkolonialer Gesellschaften durch eine professionalisierte Zivilgesellschaft sind in ihrer Form und ihrem Ablauf kaum vergleichbar, zugleich sind beide eine Konsequenz aus einem Verständnis von Regierung, das hauptsächlich auf disziplinarischer Kontrolle beruht. An dieser Stelle eine Neuartigkeit zu behaupten ist nicht möglich, ohne auf die ebenso vorhandenen historischen Kontinuitäten zu verweisen.

Die Unterschiedlichkeit in der Konfiguration der Rationalitäten zeigt sich nicht nur in ihrer historischen Dimension. Der Vergleich der Strategien, Konzepte und Praktiken der beiden Akteure, die ich als Beispiele herausgegriffen habe, macht sichtbar, dass auch zeitlich parallel, in einem vergleichsweise eng umgrenzten Feld wie der sicherheitspolitischen Entwicklungszusammenarbeit, unterschiedliche Zusammensetzungen verschiedener Rationalitäten bestimmend sein können.

Zudem möchte ich vor dem Hintergrund der empirischen Untersuchungen, die ich dargelegt habe, behaupten, dass die Unterschiede, die im Denken und Handeln der USA und Deutschlands als sicherheits- wie entwicklungspolitischer Akteure sichtbar werden, nicht aus einem unterschiedlichen Arbeitsstil oder unterschiedlichen strategischen Orientierungen erklärbar sind. Gerade der Abgleich der strategischen Komponenten mit der Praxis ziviler Intervention fördert eine Widersprüchlichkeit zu Tage, die sich dennoch durch einen durchgängig und einheitlich wirksamen Charakter auszeichnet. Dieser einheitliche Charakter ist zu inkonsistent, um einfach als gut verankerte Strategie verstanden zu werden, zugleich jedoch zu einheitlich, zu konkret, um ihn - etwas einfältig - als Resultat schwer fassbarer Konzepte wie »Kultur« oder »Mentalität« (oder auch »Ideologie«) einzuordnen.

Somit macht es Sinn, von Rationalitäten zu sprechen, und wir sehen eine Zusammensetzung gouvernementaler, disziplinarischer und, in immer schwächerem Maße, souveräner Elemente sowohl in der USamerikanischen als auch in der deutschen Vorgangsweise. Dass die USA dabei eher zu einer liberalen Spielart tendieren, die gouvernementale Elemente betont, während Deutschland eher auf disziplinarische und kontrollierende Elemente im Sinne eines dichten, bürgerschaftlich geprägten Staates baut, ist nicht ohne historische Präzedenz.

Es ist dennoch eine überraschende Erkenntnis, dass sich diese Unterschiedlichkeit nicht entlang des traditionellen Widerspruches eines au- 
ßenpolitisch »idealistisch« orientierten Deutschland gegenüber den »realistisch« orientierten USA auftut, wie zunächst anzunehmen wäre. Denn alle Grundkonzepte, die bei beiden Akteuren in der momentanen Ausprägung ihres Handelns zur Anwendung gelangen, sind zutiefst idealistisch geprägt. Sowohl »Democracy Promotion« als auch »Human Security« beziehen sich, genauso wie jene Inhalte, die zumeist in Konzepten wie »Friedensentwicklung« und »Konflikttransformation« transportiert werden, auf unterstellte menschliche Bedürfnisse, die in idealtypischen Konzepten in Form gegossen und transportiert werden.

Zugespitzt ist aus den dargestellten Strategien und Vorgangsweisen ablesbar, dass sich die beiden Ansätze um zwei Schlüsselbegriffe konstruieren, die sich zur Verbindung nahezu beliebig definierbarer Idealbilder mit konkreten strategischen Konzeptionen hervorragend eignen ${ }^{2}$ : »Demokratie« und »Frieden«. Natürlich spielen beide Begriffe für jeweils beide Akteure eine wesentliche Rolle, dennoch wird die Kausalitätsfrage anders beantwortet: während »Demokratie« für die USA quasi automatisch zu »Frieden« führt, ist »Frieden« für die deutsche Herangehensweise die notwendige Voraussetzung für »Demokratie«. Während das Faktum dieser unterschiedlichen Kausalitätskonstruktion leicht aus der Empirie ablesbar ist, ist die Frage nach den Ursachen an dieser Stelle nicht zu beantworten. Selbstverständlich spielen historische Erfahrungen ebenso eine Rolle wie Fragen der politischen Mentalität, die auf Konzepte wie Prozessorientierung (Demokratie als Prozess) gegenüber $\mathrm{Zu}$ standsorientierung (Frieden als Zustand) interpretiert werden könnten.

Die von mir vorgeschlagene Lesart interpretiert die Begriffsorientierung aus den Rationalitäten von Regierung, die als jeweils dominant herausgelesen werden können. So spiegelt ein Demokratiebegriff, der mit Konzepten wie Wettbewerbsorientierung, liberaler Marktwirtschaft und polyarchisch-elitärer Ausrichtung verbunden ist, Grundelemente dessen wider, was gouvernementales Regieren definiert. Er ist, und das ist das vielleicht entscheidende Charakteristikum von Gouvernementalität, geprägt von einem Vertrauen in Prozesse der sozialen Steuerung und damit von einem Vertrauen in die eigenen Fähigkeiten, derartige Prozesse in Gang zu setzen, aber auch einem Vertrauen in die entsprechenden Fähigkeiten derjenigen, die damit in die Position der Selbststeuerung (oder, mit einem Foucaultschen Begriff, der »Techniken des Selbst«) entlassen werden.

2 Ich würde diese Begriffe daher mit Ernesto Laclau als »empty signifiers« bezeichnen: »An empty signifier is, strictly speaking, a signifier without a signified« (Laclau 1996: 36). 
Eine Orientierung auf den Friedensbegriff bedeutet tendenziell das Gegenteil. Er baut, in seiner Füllung mit dem modernen Konzept eines einheitlichen, inklusiven, aber durchaus »positiven« Friedens, auf einem Wechselspiel von Disziplin und Kontrolle auf. Ein solcher Frieden muss als Konzept gesellschaftlich durchdringend wirksam werden, und da er in seinem positiven Sinne nicht auf souveräne Überwachungsprinzipien (im Sinne eines »negativen« Friedens) reduziert werden kann, zugleich aber offenbar in der Praxis derzeit nicht funktioniert, ist er ohne solche disziplinarischen Maßnahmen, die nicht nur physisch, sondern auch auf die Bewusstseinsebene wirken müssen, nicht denkbar. Nachdem Disziplin dazu tendiert, gebrochen $\mathrm{zu}$ werden, ist die umfassende, am besten panoptische Kontrolle - wie sie ja in der Idee von »Human Security« transportiert wird - ein unabdingbarer Begleiter.

Somit baut die Arbeit mit dem Friedensbegriff in ihrem disziplinarischen Gehalt auf einem grundsätzlichen Misstrauen auf. Ein Misstrauen in jene sozialen Formationen, in die interveniert wird, weil sie ja als »unfriedlich« oder nicht in geeignetem Maße für den Frieden gerüstet verstanden werden, wie auch auf dem Misstrauen in die eigenen Fähigkeiten als intervenierender Kraft, womit die Notwendigkeit der Kontrolle und möglichst allumfassend präsenter gesellschaftlicher Partner unumgänglich wird: Dies ist der rationale Ausgangspunkt der These der produktiven Partnerschaft eines starken Staates und einer aktiven Zivilgesellschaft als Voraussetzung friedlichen Zusammenlebens.

Eine fast paradoxe Schlussfolgerung aus dieser Unterschiedlichkeit ist, dass der Zugang der USA somit als vielleicht sogar noch idealistischer verstanden werden kann als der deutsche, was allen gängigen politikwissenschaftlichen Interpretationen widerspricht. ${ }^{3}$ Trotzdem ist die Bedeutung eines solchen Schlusses zu relativieren: Es ist zweifelhaft, ob es für die von der Intervention Betroffenen einen spürbaren Unterschied macht, ob sie als zur Demokratie strebend oder zum Frieden noch nicht fähig eingestuft werden - wenngleich es zu den wenigen historischen Grundwahrheiten gehören mag, dass ein Mehr an Idealismus gewöhnlich dazu tendiert, sich in einem Mehr an kriegerischer Gewalt auszudrücken.

3 Dies gilt etwa für Robert Kagans zum Selbstläufer gewordenen Metapher, wonach »Americans are from Mars and Europeans are from Venus« (Kagan 2004: 3). Dagegen würde ich davon ausgehen, dass sowohl Amerikaner als auch Europäer solide Standbeine auf beiden Planeten haben, wenngleich sie diese Standbeine auch unterschiedlich einsetzen. 
(3) Sicherheit und Entwicklung als »White Man's Burden«?

Als ich zuvor die Unterschiede zwischen den Praxen eines gegenwärtig im Entstehen begriffenen Sicherheit-Entwicklung-Dispositivs und den Kolonialregimen hervorgehoben habe, habe ich mich in der Erwähnung von Kontinuitäten auf die gleichartigen rationalen Muster von Regierung beschränkt, die da wie dort zum Ausdruck kommen. Dies ist natürlich nur ein Teil der Gemeinsamkeiten, wie auch durch die provokative Verwendung von Rudyard Kiplings Phrase von der Bürde des weißen Mannes zum Ausdruck kommen soll. Weder Sicherheit noch Entwicklung - und schon gar nicht ihre Synthese - ist ohne ein System globaler Asymmetrien denkbar, das über simple ökonomische und militärische Herrschaftsprozesse hinausgeht.

Gerade im Kontext einer integrierten Wirksamkeit von Sicherheit und Entwicklung ist eine Asymmetrie auch in der Frage des Wissens notwendig. Ein Dispositiv kann ohne die Generierung eines entsprechenden Wissenskorpus und seine Verknüpfung mit dem Kontext von Macht, dem Macht-Wissen-Komplex, nicht in Funktion treten, was bei Dispositiven, die primär auf globaler Ebene wirken sollen, naturgemäß zu einer Frage epistemischer Qualität wird. Ein Zusammenstoß verschiedener Wissen ist nicht vermeidbar, und welches Wissen auf welchen Platz verwiesen und mit welcher Wertigkeit ausgestattet wird, wird zur Schlüsselfrage für die Effektivität oder das Scheitern eines Dispositivs.

Hier wird der Umgang mit lokalem Wissen zentral, denn es ist eine unabdingbare Notwendigkeit, eine Argumentationslogik zu konstruieren, die lokales Wissen auf jene Ebene zurückstuft, wo es nicht zum Störfaktor werden kann. Mahmood Mamdani (2001: xiv) beschreibt jenen Prozess am Feld der »area studies«, wie er ebenso im Bereich der Entwicklungs-, Konflikt- und allzu oft auch der Friedensforschung verläuft: »To the extent the enterprise of area studies was driven by a search for the latest empirical facts, it needed native informants - not native intellectuals - in the area of expertise. The result, at best, was a polite coexistence whereby local intellectuals and area study experts acknowledged one another through what had been called benign neglect in a different context.« Der in Sri Lanka feststellbare Prozess entspricht nicht diesem bestmöglichen Resultat: Ganze Programme widmen sich der Herausforderung, lokale Intellektuelle in die Rolle von Expert/innen zu pressen. Gerade im Kontext des Zusammenpralls von Entwicklungspolitik mit bewaffnetem Konflikt, im Rahmen dessen lokale Intellektuelle von den internationalen Akteuren als besonders in die Pflicht genommen verstanden werden, ist Sri Lanka alles andere als ein Einzelfall. 
Entlang des Schemas, dass diese lokalen Expert/innen zwar ein Vielfaches mehr über den lokalen und regionalen Kontext zu sagen, die internationalen Konflikt- und Entwicklungsexpert/innen jedoch nicht nur das zeitgemäße akademische Know-how, sondern einen breiten Erfahrungshorizont einzubringen hätten, wird eine produktive Symbiose behauptet, in der dennoch eine klare Richtung vorgegeben ist. Es gibt keine Capacity-Building-Workshops lokaler Intellektueller für internationale Expert/innen. Die Wissenshierarchie funktioniert umgekehrt, wobei jene lokalen Intellektuellen, deren Expertise für nützlich gehalten wird, ebenso im Ausbildungsprogramm integriert werden können wie jene, die den Wissenskorpus bereits ausreichend verinnerlicht haben. Am grundsätzlich asymmetrischen Charakter der Veranstaltung ändert das nichts: Die für die Existenz des Dispositivs unabdingbaren Formen von Wissen bleiben Teil der Intervenierenden, und ihre Verbreitung - wie schon im Kolonialismus und der »klassischen Epoche« der Entwicklungspolitik Teil des »White Man's Burden«.

Was folgt nun aus dem Gesagten? Grundsätzlich würde ich mir natürlich wünschen, dass daraus nichts folgt, wobei dieses »nichts« allerdings auch zu bedeuten hätte, dass jene Operationen, die gegenwärtig im Sinne von internationaler Sicherheit, Frieden, Demokratie und der Menschenrechte erfolgen, stoppen müssten. So will ich ein solches »nichts« in erster Linie als bewusst provokant formulierte Antwort auf jene gängige Kritik an der von mir gewählten »unkonstruktiven« Vorgangsweise verstanden wissen, die sofort geneigt ist zu fragen: Aber was ist nun zu tun angesichts des Elends, des Krieges, der Armut, der Unterentwicklung?

Ein solch moralisch durchtränkter Zugang tendiert nicht nur dazu, einfache Erklärungsmuster auf vermeintlich einfach zu durchschauende Phänomene anzuwenden und sich damit im Sinne erkennender Subjektivität in eine Position nahe der analytischen Allmacht zu versetzen. Er tendiert auch dazu, die Folgen der geforderten oder gesetzten Konsequenzen zu negieren - ganz abgesehen davon, dass er ohnehin nicht in der Lage ist, sie im Vorfeld abzuschätzen, denn dies würde wiederum jene analytische Allmacht voraussetzen, die höchstens Teil eines für gewöhnlich eurozentrisch gefärbten Idealbildes sich als politisch handhabbar gebärdender Sozialwissenschaften ist. Diesem Selbstverständnis ist das »nichts« entgegenzusetzen, ebenso wie einem dafür grundlegenden Verständnis von handlungsorientierter Wissenschaft.

»Wissenschaft gedeiht deshalb am besten fernab von Zwang und Macht. Auch Demokratien fordern von uns Wissenschaftern Wissen und Konsens - wir aber beschäftigen uns meist mit Unwissen und Widerspruch. Zum Konsens haben 
wir ein gespaltenes Verhältnis: Wir suchen ihn - und misstrauen ihm dann. Wir sind uns bewusst, dass die wissenschaftliche Wahrheit von heute schnell der Irrtum von gestern sein kann« (Schatz 2007).

Obwohl diese Bemerkungen auf die Forschung zum Klimawandel und den Zwang zum Konsens im Sinne der analytischen Bestätigung einer durch den Menschen bedingten globalen Erwärmung gemünzt sind, lassen sie sich in vollem Umfang auf Disziplinen wie Politikwissenschaft, Internationale Beziehungen, Entwicklungsforschung, Konfliktanalyse oder Friedensforschung übertragen. Mit einer wichtigen Einschränkung: Es gibt kein Wissen und damit auch keine Wissenschaft »fernab von Macht«. Jedes Wissen ist in Macht eingebunden, ebenso wie jeder Versuch, es einzufangen, darzustellen oder zu interpretieren. Damit wird aber die Frage der Positionierung von Wissenschaft, bewusst verstanden als subjektives, persönliches Unternehmen, zur Gestaltung des Verhältnisses zur Macht entscheidend.

Und hier gibt es letztendlich, naturgemäß mit allen möglichen Nuancierungen, die denkbar sind, nur zwei Pole. Entweder positioniert sich Wissenschaft im Sinne der Macht und damit der Suche nach Konsens und praktischer Anwendbarkeit, oder sie positioniert sich gegen sie und konzentriert sich auf ihr wertvollstes Gut: die Kritik. Wissenschaft, so verstanden als Prozess des Widerstandes, dem notwendigen und ständigen Begleiter von Macht, muss sich demzufolge bei der Erarbeitung von Vorschlagen und Rezepturen grundsätzlich skeptisch verhalten, will sie ihre Positionierung nicht leichtfertig aufs Spiel setzen. Entwicklung und Sicherheit sind nur zwei Beispiele für die hervorragende Integrationsfähigkeit, die Dispositive als die konkrete Operationalisierung von Macht auszeichnet.

Aber wo liegt dann die Stoßrichtung von Wissenschaft als Kritik? Wo liegt ihr Ziel? Letzteres vermag ich nicht zu sagen, und nicht nur, dass ich es nicht vermag, ich will es auch nicht. Eine Stoßrichtung zeichnet sich demnach relativ eindeutig ab, und sie orientiert sich an dem, was der herrschende Macht-Wissen-Komplex vorgibt. Natürlich bleibt sie damit in den engen epistemischen Grenzen, aber solche Grenzen werden ohnehin nicht im Elfenbeinturm gesprengt, sondern in konkreten sozialen Prozessen. Wissenschaft ist dabei notwendiger Teil, auf Seiten der Macht wie auf Seiten des Widerstandes. Wenn es stimmt, wie Foucault mutmaßt, dass Macht und Wissen in einem untrennbaren $\mathrm{Zu}$ sammenhang stehen und ohne einander nicht nur nicht funktionieren, sondern sich sogar gegenseitig hervorbringen, stimmt es auch, dass Wissenschaft eine wesentliche soziale Rolle zu spielen hat, wenn sie sich nicht krampfhaft praxisorientiert verhält. 
Hier liegt die potenzielle Bedeutung einer Haltung, die ich schon eingangs der Arbeit angeführt habe: der Haltung des Verrats. Verrat als Provokation, als Humor, aber auch als potenzielle Sprengkraft ist mehr als ein schlichtes Sich-Entziehen, ein Wegducken vor Verantwortung, wie Moralgeleitete es so gerne verstehen wollen. Es ist vielmehr ein Weg, der in der Lage ist, die Positionierung im Widerstand mit einer konkreten politischen Intervention zu verknüpfen, Kritik zu üben und sich der Konstruktivität zu enthalten. Verrat schafft einen politischen Raum, der von Dispositiven nicht zu vereinnahmen ist, weil er sich quasi natürlich auf die Suche nach den Schwachpunkten begibt, die Gegnerschaft sucht, nicht mitspielen will. Und damit wird Verrat zum Weg, der sich zwar nicht jenseits von »Zwang und Macht« bewegt, sich aber jenseits davon positioniert; und somit zur Voraussetzung wird, unter der Wissenschaft gedeihen kann. 



\section{Literatur}

Abinales, Patricio N. (1998): Images of State Power: Essays on Philippine Politics from the Margins. Quezon City: University of the Philippines Press.

Abinales, Patricio N. (2000): Making Mindanao. Cotabato and Davao in the Formation of the Philippine Nation-State. Quezon City: Ateneo de Manila University Press.

Ackermann, Alice (2003): »The Idea and Practice of Conflict Prevention. « Journal of Peace Research, 40, no. 3: 339-47.

Adam, Markus (2000): »Die Entstehung des Governance-Konzepts bei Weltbank und UN. Die EZ wird politischer.« Entwicklung und Zusammenarbeit, Jg.41/2000, no. 10: 272-74.

Addison, Tony, und S. Mansoob Murshed, Hg. (2002): »Credibility and Reputation in Peacemaking.« Journal of Peace Research, 39, no. 4: 487-501.

Agamben, Giorgio (2001): »On Security and Terror«, Frankfurter Allgemeine Zeitung 2001 (20. September) <http://www.egs.edu/faculty/ agamben/agamben-on-security-and-terror.htm $>$ (Zugegriffen am 2609-2005).

Agamben, Giorgio (2002): Homo sacer. Die souveräne Macht und das nackte Leben. Frankfurt am Main: Suhrkamp.

Agamben, Giorgio (2003): Was von Auschwitz bleibt. Das Archiv und der Zeuge. Frankfurt am Main: Suhrkamp.

Agamben, Giorgio (2004): Ausnahmezustand. Frankfurt am Main: Suhrkamp.

Albertini, Rudolf von (1985): Europäische Kolonialherrschaft 18801940. Stuttgart: Steiner.

Allen, Tim (1999): »Perceiving Contemporary Wars.« In: Tim Allen und Jean Seaton (Hg.), The Media of Conflict: War Reporting and 
Representations of Ethnic Violence. London, New York: Zed Books, $11-42$.

Allen, Tim, und Jean Seaton, Hg. (1999): The Media of Conflict: War Reporting and Representations of Ethnic Violence. London, New York: Zed Books.

Altmann, Jörn (2000): »Dezentralisierung, Demokratie und Verwaltung. $\mathrm{Zu}$ hohe Erwartungen an einen langfristigen Prozess. « Entwicklung und Zusammenarbeit, Jg.41/2000, no. 10: 275-77.

Alvares, Claude (1992): »Science.« In: Wolfgang Sachs (Hg.), The Development Dictionary. A Guide to Knowledge as Power. London, New York: Zed Books, 219-32.

Andersen, Uwe (2004): »Entwicklungspolitik/-hilfe.« In: Wichard Woyke (Hg.), Handwörterbuch Internationale Politik. Bonn: Bundeszentrale für politische Bildung, 85-95.

Anderson, Benedict (1998a [1983]): Die Erfindung der Nation. Zur Karriere eines folgenreichen Konzepts. Berlin: Ullstein.

Anderson, Benedict (1998b): »Cacique Democracy in the Philippines.« In: Benedict Anderson (Hg.), Spectre of Comparisons. Nationalism, Southeast Asia, und the World. London, New York: Verso, 192-226.

Anderson, Benedict (1998c): Spectre of Comparisons. Nationalism, Southeast Asia, und the World. London, New York: Verso.

Anderson, Lisa (2004): »Antiquated before they can ossify: States that fail before they form." Journal of International Affairs, 58, Fall 2004, no. 1: 1-16.

Anderson, Mary B (1999): Do no harm. How aid can support peace - or war. Boulder, Col., London: Lynne Rienner Publishers.

Anderson, Mary B., und Angelika Spelten (2000): Conflict Transformation. How International Assistance Can Contribute. Policy Paper der Stiftung Entwicklung und Frieden, no. 15. Bonn: Stiftung Entwicklung und Frieden.

Anderson, Mary B., und Peter J. Woodrow (1998 [1989]): Rising from the Ashes. Development Strategies in Times of Disaster. Boulder, Col., London: Lynne Rienner Publishers.

Annan, Kofi (1998): The Causes of Conflict and the Promotion of Durable Peace and Sustainable Development in Africa, Secretary General's Report to the United Nations Security Council, 16 April 1998. New York: United Nations.

Annan, Kofi (2001): Prevention of armed conflict, Report of the Secretary-General no. A/55/985 - S/2001/574. New York: United Nations. Appadurai, Arjun (2003): »Disjuncture and Difference in the Global Cultural Economy.« In: Jana Evans Braziel und Anita Mannur (Hg.), 
Theorizing Diaspora. Malden, Oxford, Melbourne, Berlin: Blackwell Publishing, 25-48.

APRC - All Party Representative Committee (2006): Report of the Sub Committee A. Colombo.

Atteslander, Peter, Hg. (1993): Kulturelle Eigenentwicklung. Frankfurt/Main, New York: Campus.

Atteslander, Peter (2003 [1968]): Methoden der empirischen Sozialforschung. Berlin, New York: De Gruyter.

Austin, Alex, Martina Fischer, und Norbert Ropers, Hg. (2004): Transforming Ethnopolitical Conflict. The Berghof Handbook. Wiesbaden: VS Verlag für Sozialwissenschaften.

Austin, Greg, Emery Brusset, Malcolm Chalmers, und Juliet Pierce, Principal Authors (2004): Evaluation of the Conflict Prevention Pools. Synthesis Report, DFID Evaluation Report no. EV 647. London: DFID.

Auswärtiges Amt (2002): Aufgaben der deutschen Außenpolitik am Beginn des 21. Jahrhunderts. Südostasien sowie Australien, Neuseeland und Pazifische Inseln. Berlin: Auswärtiges Amt.

Auswärtiges Amt (2005): Ein Jahr Aktionsplan »Zivile Krisenprävention, Konfliktlösung und Friedenskonsolidierung« der Bundesregierung. Berlin: Auswärtiges Amt.

Åkerstrøm Andersen, Niels (2003): Discursive Analytical Strategies. Understanding Foucault, Koselleck, Laclau, Luhmann. Bristol: The Policy Press.

Baechler, Günther (2004): »Conflict Transformation through State Reform.« In: Alex Austin, Martina Fischer, und Norbert Ropers (Hg.), Transforming Ethnopolitical Conflict. The Berghof Handbook. Wiesbaden: VS Verlag für Sozialwissenschaften, 273-94.

Bagchi, Amiya Kumar (2000): »The Past and the Future of the Developmental State.« Journal of World-Systems Research, VI, no. 2: 398-443.

Balasingham, Adele (2003): The Will to Freedom. An Inside View of Tamil Resistance. Mitcham: Fairmax Publishing.

Balasingham, Anton (2004): War and Peace. Armed Struggle and Peace Efforts of Liberation Tigers. Mitcham: Fairmax Publishing.

Ball, Nicole (2002): »Wiederaufbau kriegszerrütteter Gesellschaften: Welchen Beitrag können externe Akteure leisten?« In: Tobias Debiel (Hg.), Der zerbrechliche Frieden. Krisenregionen zwischen Staatsversagen, Gewalt und Entwicklung. Bonn: Dietz, 66-96.

Ball, Nicole, Principal Author (2004): The Security Sector Reform Strategy. Evaluation of the Conflict Prevention Pools, Thematic Case Study 1, DFID Evaluation Report no. EV 647. London: DFID. 
Ball, Nicole, und Tammy Halevy (1996): Making Peace Work: The Role of the International Development Community. Policy Essay, vol. 18. Washington, D.C.: Overseas Development Council.

Ballentine, Karen, und Jake Sherman, Hg. (2003): The Political Economy of Armed Conflict. Beyond Greed \& Grievance. Boulder, Col., London: Lynne Rienner Publishers.

Banton, Michael (2000): »Ethnic Conflict.« Sociology, 34, no. 3: 48198.

Banugire, Firimooni R. (1989): »Uneven and Unbalanced Development: Development Strategies and Conflict.« In: Kumar Rupesinghe (Hg.), Conflict Resolution in Uganda. Oslo: International Peace Research Institute, 207-22.

Bastian, Sunil (2005): The Economic Agenda and the Peace Process. Part of the Sri Lanka Strategic Conflict Assessment 2005. Tech. Rept. no. 5. Colombo: The Asia Foundation.

Bastian, Sunil (2006): »How Development Undermined Peace.« In: Kumar Rupesinghe (Hg.), Negotiating Peace in Sri Lanka. Efforts, Failures \& Lessons. Volume Two. Colombo: The Foundation for Co-Existence, 245-77.

Bastian, Sunil (2007): The Politics of Foreign Aid in Sri Lanka. Promoting markets and supporting peace. Colombo: International Centre for Ethnic Studies.

Baudrillard, Jean (1992): Transparenz des Bösen. Ein Essay über extreme Phänomene. Berlin: Merve Verlag.

Becker, Joachim (2004): »Konjunkturen des 〉Entwicklungsstaates «.« In: Karin Fischer, Irmi Maral-Hanak, Gerald Hödl, und Christof Parnreiter (Hg.), Entwicklung und Unterentwicklung. Eine Einführung in Probleme, Theorien und Strategien. Wien: Mandelbaum, 145-59.

Behrend, Heike (1999): Alice Lakwena \& the Holy Spirits. War in Northern Uganda 1986-1997. Oxford: James Currey Ltd.

Bellers, Jürgen, et al. (1998): Bundesdeutsche Entwicklungspolitik in der Ära Schmidt. Siegen: Universität Siegen.

Bendana, Alejandro (2006): »Peacebuilding and Neoliberalism: Will Foreign Dictated State-Building Prevail?« Journal für Entwicklungspolitik, 22, no. 3-2006: 35-54.

Benkler, Monika (2000): »Entwicklung in Sicherheit. Stand der internationalen Debatte über zivile Krisenprävention und Konfliktverarbeitung.« Akzente, 2000, no. 4: 32-33.

Berdal, Mats, und David M. Malone, Hg. (2000): Greed and Grievance. Economic Agendas in Civil War. Boulder, Col., London: Lynne Rienner Publishers. 
Berger, Mark T. (2006): »From Nation-Building to State-Building: the geopolitics of development, the nation-state system and the changing global order.« Third World Quarterly, 27, no. 1: 5-25.

Berghof Foundation for Conflict Studies - Sri Lanka Office (2006): Frequently Asked Questions. Colombo: Berghof.

Bhatia, Michael V. (2005): »Fighting words: naming terrorists, bandits, rebels and other violent actors.« Third World Quarterly, 26, no. 1: 522.

Biccum, April R. (2005): »Development and the >New८ Imperialism: a reinvention of colonial discourse in DFID promotional literature." Third World Quarterly, 26, no. 6: 1005-20.

Biekart, Kees, Claire Mainguy, Andrew Mold, und S. Mansoob Murshed, Hg. (2005): »Insecurity and Development: Regional Issues and Policies for an Interdependent World.« Special issue of European Journal of Development Research, 17, no. 3, September 2005. Routledge.

Bigdon, Christine, und Benedikt Korf (2004): »The Role of Development Aid in Conflict Transformation: Facilitating Empowerment Processes and Community Building." In: Alex Austin, Martina Fischer, und Norbert Ropers (Hg.), Transforming Ethnopolitical Conflict. The Berghof Handbook. Wiesbaden: VS Verlag für Sozialwissenschaften, 341-70.

Bilgin, Pinar, und Adam David Morton (2002): »Historicising representations of >failed states $<$ : beyond the cold-war annexation of the social science?« Third World Quarterly, 23, no. 1: 55-80.

BMZ - Bundesministerium für wirtschaftliche Zusammenarbeit und Entwicklung (1983): Fünfter Bericht zur Entwicklungspolitik der Bundesregierung, Drucksache 9/2411, Bonn: Deutscher Bundestag.

BMZ - Bundesministerium für wirtschaftliche Zusammenarbeit und Entwicklung (1985): Sechster Bericht zur Entwicklungspolitik der Bundesregierung, Drucksache 10/3028, Bonn: Deutscher Bundestag. BMZ - Bundesministerium für wirtschaftliche Zusammenarbeit und Entwicklung (1988): Siebenter Bericht zur Entwicklungspolitik der Bundesregierung, Drucksache 11/2020, Bonn: Deutscher Bundestag. $\mathrm{BMZ}$ - Bundesministerium für wirtschaftliche Zusammenarbeit und Entwicklung (1993): Neunter Bericht zur Entwicklungspolitik der Bundesregierung, Drucksache 12/4096, Bonn: Deutscher Bundestag. $\mathrm{BMZ}$ - Bundesministerium für wirtschaftliche Zusammenarbeit und Entwicklung (1995): Zehnter Bericht zur Entwicklungspolitik der Bundesregierung, Drucksache 13/3342, Bonn: Deutscher Bundestag. 
BMZ - Bundesministerium für wirtschaftliche Zusammenarbeit und Entwicklung (1997): Entwicklungszusammenarbeit und Krisenvorbeugung, BMZ-Aktuell no. 079. Bonn: BMZ.

BMZ - Bundesministerium für wirtschaftliche Zusammenarbeit und Entwicklung (1999): »Ziviler Friedensdienst - Ein neues Element der Entwicklungszusammenarbeit (Rahmenkonzept).« In: Tilman Evers (Hg.), Ziviler Friedensdienst. Fachleute für den Frieden. Idee - Erfahrungen - Ziele. Opladen: Leske + Budrich, 358-60.

BMZ - Bundesministerium für wirtschaftliche Zusammenarbeit und Entwicklung (2001a): Elfter Bericht zur Entwicklungspolitik der Bundesregierung, Drucksache 14/6496, Bonn: Deutscher Bundestag. BMZ - Bundesministerium für wirtschaftliche Zusammenarbeit und Entwicklung (2001b): Die Herausforderungen des Terrorismus Konsequenzen für die Entwicklungspolitik. Ergebnisse einer Dialogveranstaltung mit dem entwicklungs- und friedenspolitischen Umfeld des BMZ, BMZ-Spezial no. 031 / Oktober 2001. Bonn: BMZ.

BMZ - Bundesministerium für wirtschaftliche Zusammenarbeit und Entwicklung (2002a): Entwicklungspolitik als Baustein globaler Struktur- und Friedenspolitik, BMZ-Spezial no. 042 / April 2002, herausgegeben von Christina Popovic. Bonn: BMZ.

BMZ - Bundesministerium für wirtschaftliche Zusammenarbeit und Entwicklung (2002b): Good Governance, BMZ-Spezial no. 044 / Juni 2002, herausgegeben von Christina Popovic. Bonn: BMZ.

BMZ - Bundesministerium für wirtschaftliche Zusammenarbeit und

Entwicklung (2003a): Jahresbericht Entwicklungspolitik 2002. Bonn: BMZ.

BMZ - Bundesministerium für wirtschaftliche Zusammenarbeit und

Entwicklung, Referat »Entwicklungspolitische Informations- und Bildungsarbeit« (2003b): Recht - Demokratie - Frieden. Politik für Entwicklung, herausgegeben von Christian Wilmsen. Berlin, Bonn: BMZ.

BMZ - Bundesministerium für wirtschaftliche Zusammenarbeit und Entwicklung (2004a): Jahresbericht Entwicklungspolitik 2003. Bonn: BMZ.

BMZ - Bundesministerium für wirtschaftliche Zusammenarbeit und Entwicklung (2004b): On development-based and military responses to new security challenges. A BMZ discussion paper, BMZ Discourse no. 002. Bonn: BMZ.

BMZ - Bundesministerium für wirtschaftliche Zusammenarbeit und Entwicklung (2004c): Ziviler Friedensdienst, BMZ-Spezial no. 104 / Juli 2004. Bonn: BMZ. 
BMZ - Bundesministerium für wirtschaftliche Zusammenarbeit und Entwicklung (2004d): Post-Washington-Consensus - Einige Überlegungen, BMZ-Diskurs no. 03 / Mai 2004. Bonn: BMZ.

BMZ - Bundesministerium für wirtschaftliche Zusammenarbeit und Entwicklung (2004e): Menschen haben ein Recht auf Entwicklung. Entwicklungspolitischer Aktionsplan für Menschenrechte 20042007, BMZ Konzepte no. 127. Bonn: BMZ.

BMZ - Bundesministerium für wirtschaftliche Zusammenarbeit und Entwicklung (2005a): Förderung von Demokratie in der deutschen Entwicklungspolitik. Unterstützung politischer Reformprozesse und Beteiligung der Bevölkerung. Ein Positionspapier des BMZ, BMZSpezial no. 124 / Juni 2005. Bonn: BMZ.

BMZ - Bundesministerium für wirtschaftliche Zusammenarbeit und Entwicklung (2005b): Übersektorales Konzept zur Krisenprävention, Konfliktbearbeitung und Friedensförderung in der deutschen Entwicklungszusammenarbeit. Bonn: BMZ.

BMZ - Bundesministerium für wirtschaftliche Zusammenarbeit und Entwicklung (2005c): Konzept für Maßnahmen der Entwicklungsorientierten Not- und Übergangshilfe, BMZ Konzepte no. 136 / September 2005. Bonn: BMZ.

BMZ - Bundesministerium für wirtschaftliche Zusammenarbeit und Entwicklung (2006a): 40 Jahre deutsche Entwicklungspolitik. Entwicklungspolitik im Wandel. Berlin: BMZ.

BMZ - Bundesministerium für wirtschaftliche Zusammenarbeit und Entwicklung (2006b): Interministerielles Rahmenkonzept zur Unterstützung von Reformen des Sicherheitssektors in Entwicklungsund Transformationsländern. Berlin: BMZ.

BMZ - Bundesministerium für wirtschaftliche Zusammenarbeit und Entwicklung (2007): Entwicklungsorientierte Transformation bei fragiler Staatlichkeit und schlechter Regierungsführung. BMZ Konzepte no. 149 / März 2007. Berlin: BMZ.

Boeckh, Andreas (1993): »Entwicklungstheorien: Eine Rückschau.« In: Dieter Nohlen und Franz Nuscheler (Hg.), Handbuch der Dritten Welt 1: Grundprobleme, Theorien, Strategien. Bonn: Dietz, 110-30.

Boeckh, Andreas (2003): „Vergleichende Analyse peripherer Gesellschaften, oder: die Auflösung der Peripherie.« In: Dirk BergSchlosser und Ferdinand Müller-Rommel (Hg.), Vergleichende Politikwissenschaft. Opladen: Leske + Budrich, 277-96.

Bohnet, Michael (2001): »Entwicklungspolitik im 21. Jahrhundert. Potenziale und Handlungsoptionen.« Epd-Entwicklungspolitik, 2001, no. 20/21: 49-52. 
Bond, George D. (2004): Buddhism at Work. Community Development, Social Empowerment and the Sarvodaya Movement. Bloomfield, CT: Kumarian Press.

Boutros-Ghali, Boutros (1992): An Agenda for Peace. Preventive diplomacy, peacemaking and peace-keeping, Report of the SecretaryGeneral pursuant to the statement adopted by the Summit Meeting of the Security Council on 31 January 1992 no. A/47/277 - S/24111. New York: United Nations.

Bowen, John R. (1996): »The Myth of Global Ethnic Conflict.« Journal of Democracy, 7, no. 4: 3-14.

Boyce, James K. (2003): »Aid Conditionality as a Tool for Peacebuilding: Opportunities and Constraints.« In: Jennifer Milliken (Hg.), State Failure, Collapse and Reconstruction. Malden, Oxford, Melbourne, Berlin: Blackwell Publishing, 267-89.

Böge, Volker, und Tobias Debiel (2003): »Kriege und Konfliktbewältigung. « In Globale Trends 2004/2005. Fakten Analysen Prognosen, herausgegeben von Stiftung Entwicklung und Frieden, 309-27. Frankfurt am Main: Fischer.

Bøås, Morten, und Kathleen M. Jennings (2005): »Insecurity and Development: The Rhetoric of the >Failed State`.« European Journal of Development Research, 17, no. 3: 385-95.

Brahimi, Lakhdar, Chairman of the Panel on United Nations Peace Operations (2000): Report of the Panel on United Nations Peace Operations. Tech. Rept. no. A/55/305 - S/2000/809. New York: United Nations.

Brandt, Willy, Hg. (1980): Das Überleben sichern. Bericht der NordSüd-Kommission. Gemeinsame Interessen der Industrie- und Entwicklungsländer. Köln: Kiepenheuer \& Witsch.

Brigg, Morgan (2002): »Post-development, Foucault and the colonisation metaphor.« Third World Quarterly, 23, no. 3: 421-36.

Brock, Lothar (1993): »Die Dritte Welt im internationalen System. Bedrohungsvorstellungen und Konfliktpotentiale im Nord-SüdVerhältnis.« In: Dieter Nohlen und Franz Nuscheler (Hg.), Handbuch der Dritten Welt 1: Grundprobleme, Theorien, Strategien. Bonn: Dietz, 446-66.

Brock, Lothar (2001): »Nation-Building - Prelude or Belated Solution to the Failing of States?« Florence: Strategic Outreach Program of the U.S. Army War College; Office of International Programs, Purdue University.

Brock, Lothar, Uta Ruppert, Ricardo Gómez, Elisabeth Mildeberger, Felix Klauda, und Heiko Körner, Hg. (2003): »Entwicklungszusammenarbeit als Friedenssicherung - Chancen und Grenzen.« Ge- 
meinsame Ringvorlesung von Johann Wolfgang Goethe-Universität, GTZ, KfW und SID - Frankfurt Chapter, Hg. Lothar Brock, Uta Ruppert, Ricardo Gómez, Elisabeth Mildeberger, Felix Klauda, und Heiko Körner, Frankfurt am Main.

Brooks, Arthur C. (2002): »Does Civil Society Stop the Downward Spiral of Bad Government or Speed It Up?« Nonprofit and Voluntary Sector Quarterly, 31, no. 1: 140-44.

Bröckling, Ulrich, Susanne Krasmann, und Thomas Lemke, Hg. (2000): Gouvernementalität der Gegenwart. Studien zur Ökonomisierung des Sozialen. Frankfurt am Main: Suhrkamp.

Brunnett, Regina, und Stefanie Gräfe (2003): »Gouvernementalität und Anti-Terror-Gesetze. Kritische Fragen an ein analytisches Konzept.« In: Marianne Pieper und Encarnación Gutíerrez Rodríguez (Hg.), Gouvernementalität. Ein sozialwissenschaftliches Konzept in Anschluss an Foucault. Frankfurt, New York: Campus, 50-67.

Brzoska, Michael (2004): »)New Wars Discourse in Germany.« Journal of Peace Research, 41, no. 1: 107-17.

Bublitz, Hannelore, Andrea D. Bührmann, Christine Hanke, und Andrea Seier, Hg. (1999): Das Wuchern der Diskurse. Perspektiven der Diskursanalyse Foucaults. Frankfurt/Main, New York: Campus.

Bundesregierung (1979): Thesen für die Politik der Zusammenarbeit mit den Entwicklungsländern, 30. Mai 1979. Bonn: Bundesregierung.

Bundesregierung (2000): Zivile Krisenprävention, Konfliktlösung und Friedenskonsolidierung, Gesamtkonzept der Bundesregierung. Berlin: Bundesregierung.

Bundesregierung (2001): Aktionsprogramm 2015. Armut bekämpfen. Gemeinsam handeln. Der Beitrag der Bundesregierung zur weltweiten Halbierung extremer Armut. Bonn: BMZ.

Bundesregierung (2004): Zivile Krisenprävention, Konfliktlösung und Friedenskonsolidierung, Aktionsplan. Berlin: Bundesregierung.

Bundesregierung (2006): Sicherheit und Stabilität durch Krisenprävention gemeinsam stärken, 1. Bericht der Bundesregierung über die Umsetzung des Aktionsplans `Zivile Krisenprävention, Konfliktlösung und Friedenskonsolidierung<. Berichtszeitraum: Mai 2004 bis April 2006. Berlin: Bundesregierung.

Burchell, Graham (1991): »Peculiar Interests: Civil Society and Governing >The System of Natural Liberty`.« In: Graham Burchell, Colin Gordon, und Peter Miller (Hg.), The Foucault Effect. Studies in Governmentality. Chicago: The University of Chicago Press, 119-50.

Burchell, Graham (2004): »Historische Subjekte: Rassen, Nationen, Klassen. Die Grenzen liberaler Regierungsrationalität.«In: Ramón Reichert (Hg.), Governmentality Studies. Analysen liberal- 
demokratischer Gesellschaften im Anschluss an Michel Foucault. Münster: LIT Verlag, 43-61.

Burchell, Graham, Colin Gordon, und Peter Miller, Hg. (1991): The Foucault Effect. Studies in Governmentality. Chicago: The University of Chicago Press.

Burke, Adam, und Sunil Bastian (2003): FLICT. Facilitating Local Initiatives for Conflict Transformation. Concept Proposal for GTZ and DFID. Colombo: FLICT.

Burke, Adam, und Anthea Mulakala (2005): Donors and Peacebuilding. Part of the Sri Lanka Strategic Conflict Assessment 2005. Tech. Rept. no. 2. Colombo: The Asia Foundation.

Bush, Kenneth (1999): The Limits and Scope for the Use of Development Assistance Incentives and Disincentives for Influencing Conflict Situations. Case Study: Sri Lanka, Development Assistance Committee, Informal Task Force on Conflict, Peace and Development Co-Operation. Paris: OECD.

Butler, Judith (2001): Psyche der Macht. Frankfurt am Main: Suhrkamp.

Calließ, Jörg, Hg. (2003): Zivile Konfliktbearbeitung im Schatten des Terrors. Loccumer Protokolle, vol. 58/02. Rehburg-Loccum: Bertelsmann.

Campbell, David (1998 [1992]): Writing Security: United States Foreign Policy and the Politics of Identity. Minneapolis: University of Minnesota Press.

Carment, David (2003): »Assessing state failure: implications for theory and policy.« Third World Quarterly, 24, no. 3: 407-27.

Carothers, Thomas (1999): Aiding Democracy Abroad: The Learning Curve. Washington, D.C.: Carnegie Endowment for International Peace.

Carothers, Thomas (2003a): »Promoting the Rule of Law Abroad: The Problem of Knowledge.« Carnegie Endowment Working Papers, 34.

Carothers, Thomas (2003b): »Promoting Democracy and Fighting Terror.« Foreign Affairs, 82, no. 1, Jan/Feb 2003: 84-97.

Carothers, Thomas (2006): »The Backlash Against Democracy Promotion.« Foreign Affairs, 85, no. 2, Mar/Apr 2006: 55-68.

Chesterman, Simon (2005): State-Building and Human Development, Occasional Paper. Human Development Report 2005. New York: UNDP.

Chomsky, Noam (2006): Failed States. The Abuse of Power and the Assault on Democracy. New York: Metropolitan Books.

CJPD - Centre for Just Peace and Democracy, Hg. (2006): Envisioning New Trajectories for Peace in Sri Lanka. Maharagama: CJPD. 
Clapham, Christopher (2003): »The Challenge to the State in a Globalized World.« In: Jennifer Milliken (Hg.), State Failure, Collapse and Reconstruction. Malden, Oxford, Melbourne, Berlin: Blackwell Publishing, 25-44.

Clapham, Christopher (2004): »The Global-Local Politics of State Decay.» In: Robert I. Rotberg (Hg.), When States Fail. Causes and Consequences. Princeton, NJ, Oxford: Princeton University Press, 77-93.

Clements, Kevin (2004): »Towards Conflict Transformation and a Just Peace.« In: Alex Austin, Martina Fischer, und Norbert Ropers (Hg.), Transforming Ethnopolitical Conflict. The Berghof Handbook. Wiesbaden: VS Verlag für Sozialwissenschaften, 441-61.

Collier, Paul (2000): »Rebellion as a Quasi-Criminal Activity.« Journal of Conflict Resolution, 44, no. 6: 839-53.

Collier, Paul, Lani Elliott, Havard Hegre, Anke Hoeffler, Marta ReynalQuerol, und Nicholas Sambanis (2003): Breaking the Conflict Trap. Civil War and Development Policy. Washington, DC: IBRD/The World Bank and Oxford University Press.

Collier, Paul, und Anke Hoeffler (2001): Greed and Grievance in Civil War, The World Bank. New York: The World Bank.

Collier, Paul, Anke Hoeffler, und Nicholas Sambanis (2005): »The Collier-Hoeffler Model of Civil War and the Case Study Project Research Design.« In: Paul Collier und Nicholas Sambanis (Hg.), Understanding Civil War. Evidence and Analysis. Volume 1: Africa. Washington, D.C.: The World Bank, 1-33.

Collier, Paul, Anke Hoeffler, und Mans Söderbom (2004): »On the Duration of Civil War.« Journal of Peace Research, 41, no. 3: 253-73.

Collier, Paul, und Nicholas Sambanis (2002): »Understanding Civil War. A New Agenda.« Journal of Conflict Resolution, 46, no. 1: 312.

Collier, Paul, und Nicholas Sambanis, Hg. (2005): Understanding Civil War. Evidence and Analysis. Two Volumes. Washington, D.C.: The World Bank.

Commission on Human Security (2003): Human Security Now. New York: Commission on Human Security.

Conrad, Joseph (2005): The Heart of Darkness. Stilwell: Digireads.com.

Cooke, Bill (2003): »A new continuity with colonial administration: participation in development management.« Third World Quarterly, 24, no. 1: 47-61.

Cooper, Neil (2003): »State Collapse as Business: The Role of Conflict Trade and the Emerging Control Agenda.« In: Jennifer Milliken 
(Hg.), State Failure, Collapse and Reconstruction. Malden, Oxford, Melbourne, Berlin: Blackwell Publishing, 179-98.

Cooper, Robert (2004): The Breaking of Nations. Order and Chaos in the Twenty-First Century. London: Atlantic Books.

Council of the European Union (1997): Conflict Prevention and Resolution in Africa, Common Position of 2 June 1997 no. L153, 11/06/1997 P. 0001 - 0002. Brussels: European Union.

Council of the European Union (1998): The Role of Development Cooperation in Strengthening Peacebuilding, Conflict Prevention and Resolution, Council Conclusions 1998. Brussels: European Union.

Council of the European Union (2001): EU Programme for the Prevention of Violent Conflicts, adopted by the General Affairs Council and endorsed by European Council at Göteborg. Gothenburg: European Union.

CPA - Centre for Policy Alternatives (2007): War, Peace and Governance in Sri Lanka. Colombo: CPA.

CPA - Centre for Policy Alternatives, und Berghof Foundation for Conflict Studies - Sri Lanka Office (2003): Peace and Development. The Road to Tokyo.

Crawford, Gordon (2000): »Promoting Democratic Governance in the South.« European Journal of Development Research, 12, no. 1: 2357.

Crush, Jonathan, Hg. (1995): The Power of Development. London, New York: Routledge.

DAC - Development Assistance Committee (1995): Participatory Development and Good Governance, Development Co-Operation Guidelines Series. Paris: OECD.

DAC - Development Assistance Committee (2001): Helping Prevent Violent Conflict, The DAC Guidelines. Paris: OECD.

DAC - Development Assistance Committee (2003): A Development Co-operation Lens on Terrorism Prevention. Key Entry Points for Action, DAC Guidelines and Reference Series. Paris: OECD.

DAC - Development Assistance Committee (2005a): Security System Reform and Governance, DAC Guidelines and Reference Series. Paris: OECD.

DAC - Development Assistance Committee (2005b): Reporting Directives for the Creditor Reporting System - Addendum 2. Annex 5. Reporting on the Purpose of Aid. DCD/DAC(2002)21/ADD2. Paris: OECD.

DAC - Development Assistance Committee (2006a): The UNITED STATES. Development Assistance Committee (DAC) Peer Review. Paris: OECD. 
DAC - Development Assistance Committee (2006b): Whole of Government Approaches to Fragile States, DAC Guidelines and Reference Series. Paris: OECD.

DAC - Development Assistance Committee (2007): Principles for Good International Engagement in Fragile States \& Situations. Paris: OECD.

Dahl, Robert A. (1971): Polyarchy. Participation and Opposition. New Haven, London: Yale University Press.

de Abreu Fialho Gomes, Bea, Irmi Maral-Hanak, und Walter Schicho, Hg. (2006): Entwicklungszusammenarbeit. Akteure, Handlungsmuster und Interessen, Mattersburger Kreis für Entwicklungspolitik. Gesellschaft. Entwicklung. Politik, vol. 7. Wien: Mandelbaum.

de Silva, K.M. (1998): Reaping the Whirlwind. Ethnic Conflict, Ethnic Politics in Sri Lanka. New Delhi: Penguin Books.

de Silva, K.M. (2005): A History of Sri Lanka. Colombo: Vijitha Yapa Publications.

de Waal, Alex (1997): Famine Crimes. Politics \& the Disaster Relief Industry in Africa. Bloomington, Indianapolis: Indiana University Press.

Dean, Mitchell (1999): Governmentality. Power and Rule in Modern Society. London, Thousand Oaks, New Delhi: Sage Publications.

Debiel, Tobias (1995): »Von der Kriegs- zur Friedenswirtschaft. Kosten des Krieges und Sozialökonomische Bedingungen der Friedenskonsolidierung.« In: Volker Matthies (Hg.), Vom Krieg zum Frieden. Kriegsbeendigung und Friedenskonsolidierung. Bremen: Edition Temmen, 58-82.

Debiel, Tobias, Hg. (2002): Der zerbrechliche Frieden. Krisenregionen zwischen Staatsversagen, Gewalt und Entwicklung. Bonn: Dietz.

Debiel, Tobias (2004): »Wie weiter mit effektiver Krisenprävention? Der Aktionsplan der Bundesregierung im Vergleich zu den britischen Conflict Prevention Pools« Die Friedens-Warte, 79, no. 3-4, 253-98.

Debiel, Tobias, Martina Fischer, Volker Matthies, und Norbert Ropers (1999): Effektive Krisenprävention. Herausforderungen für die deutsche Außen- und Entwicklungspolitik. Policy Paper der Stiftung Entwicklung und Frieden, no. 12. Bonn: Stiftung Entwicklung und Frieden.

Debiel, Tobias, Stephan Klingebiel, Andreas Mehler, und Ulrich Schneckener (2005): Zwischen Ignorieren und Intervenieren. Strategien und Dilemmata externer Akteure in fragilen Staaten. Policy Paper der Stiftung Entwicklung und Frieden, no. 23. Bonn: Stiftung Entwicklung und Frieden. 
Debiel, Tobias, und Franz Nuscheler, Hg. (1996): Der neue Interventionismus. Humanitäre Einmischung zwischen Anspruch und Wirklichkeit. Bonn: Dietz.

Deleuze, Gilles (1991): »Was ist ein Dispositiv?« In: Francois Ewald and Bernhard Waldenfels (Hg.), Spiele der Wahrheit. Michel Foucaults Denken. Frankfurt am Main: Suhrkamp, 153-62.

Deleuze, Gilles (1992): Foucault. Frankfurt am Main: Suhrkamp.

Deleuze, Gilles, und Félix Guattari (1977): Anti-Ödipus. Kapitalismus und Schizophrenie I. Frankfurt am Main: Suhrkamp.

Deleuze, Gilles, und Félix Guattari (1997): Tausend Plateaus: Kapitalismus und Schizophrenie II. Berlin: Merve Verlag.

Dempsey, Gary T. (2002): »Old Folly in a New Disguise. Nation Building to Combat Terrorism. « Policy Analysis, 429.

Derichs, Claudia (2004): »Die Nation gestalten - Ideologische Aspekte des Nation-Building.« In: Jochen Hippler (Hg.), Nation-Building. Ein Schlüsselkonzept für friedliche Konfliktbearbeitung?. Bonn: Dietz, 69-85.

Deutsches Institut für Entwicklungspolitik (2001): Methodische Orientierung für kurze und praxisnahe Forschungsprojekte in Entwicklungsländern. Ein Leitfaden für Länderarbeitsgruppen und Gutachter, herausgegeben von Susanne Neubert. Bonn: Deutsches Institut für Entwicklungspolitik.

Development Policy Forum, Hg. (2004): »International Policy Dialogue.« In Development Policy and the Armed Forces. 14-15 September 2004. Berlin: InWEnt.

DFID - Department for International Development (2001): Making government work for poor people. Building state capability, Strategies for achieving the international development targets. London: DFID.

DFID - Department for International Development (2002a): Conducting Conflict Assessments: Guidance Notes. London: DFID.

DFID - Department for International Development (2002b): Safety, Security and Accessible Justice. Putting policy into practice. London: DFID.

DFID - Department for International Development (2002c): Understanding and Supporting Security Sector Reform. London: DFID.

DFID - Department for International Development (2004a): Non-state Justice and Security Systems, DFID Briefing no. May 2004. London: DFID.

DFID - Department for International Development (2004b): UK Policy and Strategic Priorities on Small Arms and Light Weapons 20042006. London: DFID. 
DFID - Department for International Development (2005a): Fighting poverty to build a safer world. A strategy for security and development. London: DFID.

DFID - Department for International Development (2005b): Why we need to work more effectively in fragile states. London: DFID.

DFID - Department for International Development, Foreign \& Commonwealth Office London, und Ministry of Defence (2001): The Causes of Conflict in Sub-Saharan Africa. Framework Document. London: DFID.

DFID - Department for International Development, Foreign \& Commonwealth Office London, und Ministry of Defence (2003): The Global Conflict Prevention Pool. A joint UK Government approach to reducing conflict. London: DFID.

DFID - Department for International Development, Foreign \& Commonwealth Office London, und Ministry of Defence (2004): The Africa Conflict Prevention Pool - An Information Document. A Joint UK Government Approach to Preventing and Reducing Conflict in Sub-Saharan Africa. London: DFID.

Diamond, Larry (1992): »Promoting Democracy« Foreign Policy (Washington, DC), No. 87, Summer 1992, 25-46.

Diamond, Larry (1995): Promoting Democracy in the 1990s: Actors and Instruments, Issues and Imperatives, Report to the Carnegie Commission on Preventing Deadly Conflict. Washington, D.C.: Carnegie Corporation of New York.

Diamond, Louise, und John W. McDonald (1996): Multi-Track Diplomacy: A Systems Approach to Peace. West Hartford, CT: Kumarian Press.

Dietrich, Wolfgang (1997): »Interkulturelle Kommunikation oder hybrider Diskurs? Zur schwierigen Wahrnehmung neuer und traditioneller Gemeinschaften Afrikas in aktuellen Friedens- und Entwicklungstheorien. Das Beispiel Uganda,« 4. Schlaining Workingpapers, 23. Stadtschlaining.

Dietrich, Wolfgang (1998): Periphere Integration und Frieden im Weltsystem. Ostafrika, Zentralamerika und Südostasien im Vergleich. Wien: Promedia.

Dietrich, Wolfgang (2004): »Zivilgesellschaft und Menschenwürde als Schlüsselbegriffe der Entwicklungspolitik.« In: Karin Fischer, Irmi Maral-Hanak, Gerald Hödl, und Christof Parnreiter (Hg.), Entwicklung und Unterentwicklung. Eine Einführung in Probleme, Theorien und Strategien. Wien: Mandelbaum, 257-76. 
Dietrich, Wolfgang (2006): »Kleine Archäologie des europäischen Sicherheitsdenkens« In: Alexander Siedschlag (Hg.), Jahrbuch für europäische Sicherheitspolitik. Baden-Baden: Nomos, 191-203.

Dillon, Michael (1995): »Sovereignty and Governmentality: From the Problematics of the $>$ New World Order $>$ to the Ethical Problematic of the World Order.« Alternatives, 20: 323-68.

Dillon, Michael (1996): Politics of Security. Towards a political philosophy of continental thought. London, New York: Routledge.

Dillon, Michael (2004): »The security of governance.« In: Wendy Larner und William Walters (Hg.), Global Governmentality. Governing international spaces. London: Routledge, 76-94.

Dillon, Michael, und Julian Reid (2000): »Global Governance, Liberal Peace, und Complex Emergency.« Alternatives, 25: 117-43.

Dobbins, James et al. (2003): America's Role in Nation-Building. From Germany to Iraq. Santa Monica: RAND Corporation.

Dobbins, James et al. (2005): The UN's Role in Nation-Building. From the Congo to Iraq. Santa Monica: RAND Corporation.

Donner, Jochen, und Bernhard Thibaut (1994): »Sri Lanka.« In: Dieter Nohlen und Franz Nuscheler (Hg.), Handbuch der Dritten Welt. Band 7: Südasien und Südostasien. Bonn: Dietz Verlag, 336-67.

Doornbos, Martin (2003): »State Collapse and Fresh Starts: Some Critical Reflections.« In: Jennifer Milliken (Hg.), State Failure, Collapse and Reconstruction. Malden, Oxford, Melbourne, Berlin: Blackwell Publishing, 45-62.

Dorussen, Han (2005): „Governance, Development and State Building.« European Journal of Development Research, 17, no. 3: 411-22.

Douma, P.S. (2003a): The Origins of Contemporary Conflict. A Comparison of Violence in Three World Regions. The Hague: Netherlands Institute of International Relations, Clingendael.

Douma, P.S. (2003b): The Political Economy of Internal Conflict. A Comparative Analysis of Angola, Colombia, Sierra Leone and Sri Lanka. The Hague: Netherlands Institute of International Relations, Clingendael.

Doyle, Michael W., und Nicholas Sambanis, Hg. (2006): Making War and Building Peace. United Nations Peace Operations. Princeton, NJ, Oxford: Princeton University Press.

Duffield, Mark (2000): »Globalization, Transborder Trade, und War Economies.« In: Mats Berdal und David M. Malone (Hg.), Greed and Grievance. Economic Agendas in Civil Wars. Boulder, Col., London: Lynne Rienner Publishers, 69-89. 
Duffield, Mark (2001): Global Governance and the New Wars. The Merging of Development and Security. London, New York: Zed Books.

Duffield, Mark (2002): »Reprising Durable Disorder: Network War and the Securitisation of Aid.« In: Björn Hettne und Bertil Odén (Hg.), Global Governance in the 21st Century: Alternative Perspectives on World Order. Stockholm: Almkvist \& Wiksell International, 74-105.

Duffield, Mark (2003): »Social Reconstruction and the Radicalization of Development: Aid as a Relation of Global Liberal Governance.« In: Jennifer Milliken (Hg.), State Failure, Collapse and Reconstruction. Malden, Oxford, Melbourne, Berlin: Blackwell Publishing, 291-312.

Duffield, Mark (2004): »Netzwerk-Kriege und neue sicherheitspolitische Dimensionen.« In: Sabine Kurtenbach und Peter Lock (Hg.), Kriege als (Über)Lebenswelten. Schattenglobalisierung, Kriegsökonomien und Inseln der Zivilität. Bonn: Dietz, 72-87.

Duffield, Mark (2005a): »Getting Savages to Fight Barbarians. Development, Security and the Colonial Present.« Conflict, Development and Security, 5, no. 2: 141-59.

Duffield, Mark (2005b): »Human Security: Linking Development and Security in an Age of Terror.« In: New Interfaces between Security and Development. 11th EADI General Conference - »Insecurity and Development«, Bonn.

Duffield, Mark (2006): »Racism, Migration and Development: the foundations of planetary order.« Progress in Development Studies, 6, no. 1: 68-79.

Dunaway, Wilma A (2003): »Ethnic Conflict in the Modern WorldSystem: The Dialectics of Counter-Hegemonic Resistance in an Age of Transition.« Journal of World-Systems Research, IX, no. I, Winter 2003: 3-34.

Dunne, Tim, und Nicholas J. Wheeler (2004): ") We the Peoples $<$ : Contending Discourses of Security in Human Rights Theory and Practice.« International Relations, 18, no. 1: 9-23.

EADI - European Association of Development Research and Training Institutes (2006): Insecurity and Development. Regional issues and policies for an interdependent world. Conference Report. Herausgegeben von Susanne von Itter und Jeanne Lätt. 11th EADI General Conference. Bonn: EADI, DIE.

Easterly, William (2001a): »Can Institutions Resolve Ethnic Conflict?« Economic Development and Cultural Change, 49, no. 4: 687-706.

Easterly, William (2001b): The Elusive Quest for Growth. Economist's Adventures and Misadventures in the Tropics. Cambridge, MA, London: The MIT Press. 
Easterly, William (2006): The White Man's Burden. Why the west's efforts to aid the rest have done so much ill and so little good. New York: Penguin Press.

Ebata, Michi (2003): From the Discourse to the Ground: UNDP Activities in Conflict Prevention. Bureau for Crisis Prevention and Recovery. New York: UNDP.

EC - European Commission (1996): The EU and the issue of conflicts in Africa: Peacebuilding, conflict prevention and beyond, Communication from the Commission to the Council, SEC(96) 332. Brussels: European Commission.

EC - European Commission (1999): Co-operation with ACP Countries Involved in Armed Conflicts, Communication from the Commission to the Council and the European Parliament, COM(1999) 240 final. Brussels: European Commission.

EC - European Commission (2001a): Conflict Prevention, Communication from the Commission to the Council and the European Parliament, COM(2001) 211 final. Brussels: European Commission.

EC - European Commission (2001b): The European Union's Role in Promoting Human Rights and Democratisation in Third Countries, Communication from the Commission to the Council and the European Parliament, COM(2001) 252 final. Brussels: European Commission.

EC - European Commission (2001c): Report on the Implementation of the European Initiative for Democracy and Human Rights in 2000, Commission Staff Working Document, SEC(2001) 801. Brussels: European Commission.

EC - European Commission (2003a): Governance and Development, Communication from the Commission to the Council, the European Parliament and the European Economic and Social Committee, $\operatorname{COM}(2003) 615$ final. Brussels: European Commission.

EC - European Commission (2004a): Annual Report 2004 on the European Community's Development Policy and the Implementation of External Assistance. Brussels: European Communities.

EC - European Commission (2004b): Implementation of the Commission Communication on the EU's Role in Promoting Human Rights and Democratisation in Third Countries (COM (2001) 252 final), Commission Staff Working Document, SEC(2004) 1041. Brussels: European Commission.

EC - European Commission (2005a): Annual Report 2005 on the European Community's Development Policy and the Implementation of External Assistance in 2004, Communication from the Commission 
to the Council and the European Parliament, COM(2005) 292. Brussels: European Commission.

EC - European Commission (2005b): Draft Handbook on promoting Good Governance in EC Development and Co-operation, EuropeAid Co-operation Office, J54-07/20. Brussels: European Commission.

EC - European Commission (2005c): Report on the Public Consultation on the Future of EU Development Policy, Development Policy and Sectoral Issues. Brussels: European Commission.

Ehrke, Michael (2002): »Zur politischen Ökonomie post-nationalstaatlicher Konflikte.« Internationale Politik und Gesellschaft, 2002, no. 3: 135-63.

Ehrke, Michael (2004): »Die Ökonomie innerstaatlicher Kriege - eine Kritik der Weltbank-Analysen.« In: Sabine Kurtenbach und Peter Lock (Hg.), Kriege als (Über)Lebenswelten. Schattenglobalisierung, Kriegsökonomien und Inseln der Zivilität. Bonn: Dietz, 102-21.

Eid, Uschi (1999): »Ein starkes Signal. Interview mit Staatssekretärin Dr. Uschi Eid.« Entwicklung und Zusammenarbeit, 1999, no. 1: 1113.

Eizenstat, Stuart, John Edward Porter, und Jeremy Weinstein (2005): $»$ Rebuilding Weak States.« Foreign Affairs, 84, no. 1, Jan/Feb 2005: 134-46.

Ekbladh, David (2006): »From Consensus to Crisis: The Postwar Career of Nation-Building in U.S. Foreign Relations.« In: Francis Fukuyama (Hg.), Nation-Building. Beyond Afghanistan and Iraq. Baltimore, Maryland: The Johns Hopkins University Press, 19-41.

El-Bushra, Judy, und Ibrahim M.G. Sahl (2005): Cycles of Violence. Gender Relations and Armed Conflict. Nairobi: ACORD.

Erdheim, Mario (1993): »Die gesellschaftliche Produktion von Unbewusstheit als Quelle interkultureller Konflikte.« In: Peter Atteslander (Hg.), Kulturelle Eigenentwicklung. Frankfurt/Main, New York: Campus, 86-100.

Eribon, Didier (1999 [1991]): Michel Foucault. Biographie. Frankfurt am Main: Suhrkamp.

Escobar, Arturo (1992a): »Imagining a Post-Development Era? Critical Thought, Development and Social Movements.« Social Text, 31-32: 20-56.

Escobar, Arturo (1992b): »Planning.« In: Wolfgang Sachs (Hg.), The Development Dictionary. A Guide to Knowledge as Power. London, New York: Zed Books, 132-45.

Escobar, Arturo (1995): Encountering Development. The Making and Unmaking of the Third World. Princeton, NJ: Princeton University Press. 
Escobar, Arturo (2000): »Beyond the Search for a Paradigm? PostDevelopment and beyond.« Development, 43, no. 4: 11-14.

Escobar, Arturo (2001): »Culture sits in places: reflections on globalism and subaltern strategies of localization.« Political Geography, 20: 139-74.

Escobar, Arturo (2004): »Beyond the Third World: imperial globality, global coloniality and anti-globalisation social movements.« Third World Quarterly, 25, no. 1: 207-30.

Esteva, Gustavo (1992a): »Development.« In: Wolfgang Sachs (Hg.), The Development Dictionary. A Guide to Knowledge as Power. London, New York: Zed Books, 6-25.

Esteva, Gustavo (1992b): FIESTA - jenseits von Entwicklung, Hilfe und Politik. Frankfurt am Main, Wien: Brandes und Apsel, Südwind.

Esteva, Gustavo (2001): »The Meaning and Scope of the Struggle for Autonomy.« Latin American Perspectives, 28, no. 117/2, March: $120-48$.

Esteva, Gustavo, und Madhu Suri Prakash (1998): Grassroots PostModernism. Remaking the soil of cultures. London, New York: Zed Books.

EU - European Union (2003): A Secure Europe in a Better World. European Security Strategy, approved by the European Council on 12 December 2003, herausgegeben von Javier Solana. Brussels: European Union.

Evans, Peter B., Dietrich Rueschemeyer, und Theda Skocpol, Hg. (1999 [1985]): Bringing the State Back In. Cambridge, UK: Cambridge University Press.

Evers, Tilman (1977): Bürgerliche Herrschaft in der Dritten Welt. Zur Theorie des Staates in ökonomisch unterentwickelten Gesellschaftsformationen. Köln, Frankfurt/Main: Europäische Verlagsanstalt.

Evers, Tilman, Hg. (2000): Ziviler Friedensdienst. Fachleute für den Frieden. Idee - Erfahrungen - Ziele. Opladen: Leske + Budrich.

Ewald, Francois (1991): »Eine Macht ohne Draußen.« In: Francois Ewald und Bernhard Waldenfels (Hg.), Spiele der Wahrheit. Michel Foucaults Denken. Frankfurt am Main: Suhrkamp, 163-70.

Fahrenhorst, Brigitte, Hg. (2000): Die Rolle der Entwicklungszusammenarbeit in gewalttätigen Konflikten. Dokumentation einer Fachtagung in der TU Berlin vom 3.-5.12.1999. SID-Berlin-Berichte, vol. 11. Berlin: Society for International Development.

Fahrenhorst, Brigitte, und Stefan Musto (2002): »Krisenprävention als entwicklungspolitische Querschnittaufgabe.« In: Reinhard Mutz (Hg.), Krisenprävention als politische Querschnittaufgabe. BadenBaden: Nomos Verlagsgesellschaft, 167-223. 
Falk, Richard (2002): »The Post-Westphalia Enigma.« In: Björn Hettne und Bertil Odén (Hg.), Global Governance in the 21st Century: Alternative Perspectives on World Order. Stockholm: Almkvist \& Wiksell International, 147-83.

Fanon, Frantz (1981 [1966]): Die Verdammten dieser Erde. Frankfurt am Main: Suhrkamp.

Faschingeder, Gerald (2001): Kultur und Entwicklung. Zur Relevanz soziokultureller Faktoren in hundert Jahren Entwicklungstheorie. Frankfurt am Main, Wien: Brandes \& Apsel, Südwind.

Faschingeder, Gerald, und Nikola Ornig, Hg. (2005): Globalisierung ent-wickeln. Eine Reflexion über Entwicklung, Globalisierung und Repolitisierung. Wien: Mandelbaum.

Faust, Jörg, und Dirk Messner (2004a): »Europe's New Security Strategy - Challenges for Development Policy.« Deutsches Institut für Entwicklungspolitik, Discussion Papers, Discussion Paper 3/2004.

Faust, Jörg, und Dirk Messner (2004b): »Keine Sicherheit ohne proaktive Entwicklungspolitik.« Entwicklung und Zusammenarbeit, 45, no. 11: 408-11.

Fearon, James D. (2004): „Why Do Some Civil Wars Last So Much Longer Than Others?« Journal of Peace Research, 41, no. 3: 275301.

Ferdinands, Tyrol, Kumar Rupesinghe, Palkiasothy Saravanamuttu, Jayadeva Uyangoda, und Norbert Ropers (2004): The Sri Lankan Peace Process at a Crossroads. Lessons, Opportunities and Ideas for Principled Negotiations and Conflict Transformation. Colombo: CPA, FCE, inpact, SSA, Berghof Foundation.

Ferdowsi, Mir A., Hg. (2002): Internationale Politik im 21. Jahrhundert. München: Wilhelm Fink Verlag.

Ferdowsi, Mir A., und Volker Matthies (2003a): »Kriege, Kriegsbeendigung und Friedenskonsolidierung.« In: Mir A. Ferdowsi und Volker Matthies (Hg.), Den Frieden gewinnen. Zur Konsolidierung von Friedensprozessen in Nachkriegsgesellschaften. Bonn: Dietz, 14-40.

Ferdowsi, Mir A., und Volker Matthies (2003b): »Zentrale Problemdimensionen der Friedenskonsolidierung und ihre konstruktive Bearbeitung.« In: Mir A. Ferdowsi und Volker Matthies (Hg.), Den Frieden gewinnen. Zur Konsolidierung von Friedensprozessen in Nachkriegsgesellschaften. Bonn: Dietz, 322-58.

Ferguson, James (1994 [1990]): The Anti-Politics Machine. »Development«, Depoliticization and Bureaucratic Power in Lesotho. Minneapolis, London: University of Minnesota Press.

Ferguson, James, und Akhil Gupta (2005 [2002]): »Spatializing States: Toward an Ethnography of Neoliberal Governmentality.« In: Jona- 
than Xavier Inda (Hg.), Anthropologies of Modernity. Foucault, Gouvernmentality, und Life Politics. Malden, Oxford, Carlton: Blackwell Publishing, 105-31.

Finkel, Steve E., Aníbal Pérez-Liñán, und Mitchell A. Seligson (2006): Effects of U.S. Foreign Assistance on Democracy Building: Results of a Cross-National Quantitative Study. Final Report. USAID, Vanderbilt University, University of Pittsburgh.

Finnström, Sverker (2003): Living with Bad Surroundings. War and Existential Uncertainty in Acholiland, Northern Uganda. Uppsala: Acta Universitatis Upsaliensis.

Fischer, Joschka (2005): Die Rückkehr der Geschichte. Die Welt nach dem 11. September und die Erneuerung des Westens. Köln: Kiepenheuer \& Witsch.

Fischer, Karin, Irmi Maral-Hanak, Gerald Hödl, und Christof Parnreiter, Hg. (2004): Entwicklung und Unterentwicklung. Eine Einführung in Probleme, Theorien und Strategien. Wien: Mandelbaum.

Fischer, Martina (1999): »)Krisenprävention« - Modebegriff oder friedenspolitische Notwendigkeit?« In: Österreichisches Studienzentrum für Frieden und Konfliktlösung, Schweizerische Friedensstiftung, und Institut für Konfliktlösung (Hg.), Krisenprävention: Theorie und Praxis ziviler Konfliktbearbeitung. Chur, Zürich: Verlag Rüegger, 47-76.

Fischer, Martina, und Norbert Ropers (2004): »Introduction.« In: Alex Austin, Martina Fischer, und Norbert Ropers (Hg.), Transforming Ethnopolitical Conflict. The Berghof Handbook. Wiesbaden: VS Verlag für Sozialwissenschaften, 11-22.

FLICT - Facilitating Local Initiatives for Conflict Transformation (2005): Review of FLICT. Lessons for the Future. Colombo: FLICT.

FLICT - Facilitating Local Initiatives for Conflict Transformation (2006): FLICT: Concept \& Structure. Colombo: FLICT.

Foucault, Michel (1981 [1973]): Archäologie des Wissens. Frankfurt am Main: Suhrkamp.

Foucault, Michel (1992): Was ist Kritik? Berlin: Merve Verlag.

Foucault, Michel (1994 [1976]): Überwachen und Strafen. Die Geburt des Gefängnisses. Frankfurt am Main: Suhrkamp.

Foucault, Michel (1997a [1986]): Der Gebrauch der Lüste. Sexualität und Wahrheit 2. Frankfurt am Main: Suhrkamp.

Foucault, Michel (1997b [1986]): Die Sorge um sich. Sexualität und Wahrheit 3. Frankfurt am Main: Suhrkamp.

Foucault, Michel (1999a [1977]): Der Wille zum Wissen. Sexualität und Wahrheit 1. Frankfurt am Main: Suhrkamp. 
Foucault, Michel (1999b): In Verteidigung der Gesellschaft. Frankfurt am Main: Suhrkamp.

Foucault, Michel (2000 [1991]): Die Ordnung des Diskurses. Frankfurt am Main: Fischer.

Foucault, Michel (2001 [1966]): »Michael Foucault, >Die Ordnung der Dinge «.« In: Daniel Defert und Francois Ewald (Hg.), Dits et Ecrits. Schriften. Erster Band, 1954-1969. Frankfurt am Main: Suhrkamp, 644-52.

Foucault, Michel (2002a [1973]): »Statt einer Konklusion.« In: Daniel Defert und Francois Ewald (Hg.), Dits et Ecrits. Schriften. Zweiter Band, 1970-1975. Frankfurt am Main: Suhrkamp, 519-22.

Foucault, Michel (2002b [1975]): »Gespräch über das Gefängnis; das Buch und seine Methode. «In: Daniel Defert und Francois Ewald (Hg.), Dits et Ecrits. Schriften. Zweiter Band, 1970-1975. Frankfurt am Main: Suhrkamp, 913-32.

Foucault, Michel (2002c [1971]): »Nietzsche, die Genealogie, die Historie.« In: Daniel Defert und Francois Ewald (Hg.), Dits et Ecrits. Schriften. Zweiter Band, 1970-1975. Frankfurt am Main: Suhrkamp, 166-91.

Foucault, Michel (2003a [1977]): »Die politische Funktion des Intellektuellen.« In: Daniel Defert und Francois Ewald (Hg.), Dits et Ecrits. Schriften. Dritter Band, 1976-1979. Frankfurt am Main: Suhrkamp, 145-52.

Foucault, Michel (2003b [1977]): »Das Spiel des Michel Foucault.« In: Daniel Defert und Francois Ewald (Hg.), Dits et Ecrits. Schriften. Dritter Band, 1976-1979. Frankfurt am Main: Suhrkamp, 391-429.

Foucault, Michel (2003c [1977]): »Mächte und Strategien.« In: Daniel Defert und Francois Ewald (Hg.), Dits et Ecrits. Schriften. Dritter Band, 1976-1979. Frankfurt am Main: Suhrkamp, 538-50.

Foucault, Michel (2003d [1979]): »Für eine Moral der Unbequemlichkeit.« In: Daniel Defert und Francois Ewald (Hg.), Dits et Ecrits. Schriften. Dritter Band, 1976-1979. Frankfurt am Main: Suhrkamp, 978-84.

Foucault, Michel (2003e): Die Wahrheit und die juristischen Formen.

Frankfurt am Main: Suhrkamp.

Foucault, Michel (2004a): Geschichte der Gouvernementalität I: Sicherheit, Territorium, Bevölkerung. Frankfurt am Main: Suhrkamp.

Foucault, Michel (2004b): Geschichte der Gouvernementalität II: Die

Geburt der Biopolitik. Frankfurt am Main: Suhrkamp.

Foucault, Michel (2005a [1980]): »Gespräch mit Ducio Trombadori.« In: Daniel Defert und Francois Ewald (Hg.), Dits et Ecrits. Schriften. Vierter Band, 1980-1988. Frankfurt am Main: Suhrkamp, 51-119. 
Foucault, Michel (2005b [1981]): »Omnes et singulatim«: zu einer Kritik der politischen Vernunft.« In: Daniel Defert und Francois Ewald (Hg.), Dits et Ecrits. Schriften. Vierter Band, 1980-1988. Frankfurt am Main: Suhrkamp, 165-98.

Foucault, Michel (2005c [1982]): »Subjekt und Macht.« In: Daniel Defert und Francois Ewald (Hg.), Dits et Ecrits. Schriften. Vierter Band, 1980-1988. Frankfurt am Main: Suhrkamp, 269-94.

Foucault, Michel (2005d [1984]): »Michel Foucault, ein Interview: Sex, Macht und die Politik der Identität.« In: Daniel Defert und Francois Ewald (Hg.), Dits et Ecrits. Schriften. Vierter Band, 1980-1988. Frankfurt am Main: Suhrkamp, 909-24.

Francis, Diana (2004): »Culture, Power Asymmetries and Gender in Conflict Transformation.« In: Alex Austin, Martina Fischer, und Norbert Ropers (Hg.), Transforming Ethnopolitical Conflict. The Berghof Handbook. Wiesbaden: VS Verlag für Sozialwissenschaften, 91-107.

François, Monika, und Inder Sud (2006): »Promoting Stability and Development in Fragile and Failed States.« Development Policy Review, 24, no. 2: 141-60.

Frank, André Gunder (1975 [1968]): Kapitalismus und Unterentwicklung in Lateinamerika. Frankfurt am Main, Köln: Europäische Verlagsanstalt.

Frank, André Gunder (1998): ReOrient: Global Economy in the Asian Age. Berkeley, Los Angeles, London: University of California Press.

Frank, Michael C (2004): »Kolonialismus und Diskurs: Michel Foucaults »Archäologie« in der postkolonialen Theorie.« In: Susanne Kollmann und Kathrin Schödel (Hg.), PostModerne De/Konstruktionen. Ethik, Politik und Kultur am Ende einer Epoche. Münster: LIT Verlag, 139-55.

Frerks, Georg (2006): »The Politics of Post-Tsunami Reconstruction in Wartorn Sri Lanka.« In: CJPD - Centre for Just Peace and Democracy (Hg.), Envisioning New Trajectories for Peace in Sri Lanka. Maharagama: CJPD, 75-92.

Frerks, Georg, und Bart Klem, Hg. (2005): Dealing with Diversity. Sri Lankan Discourses on Peace and Conflict. The Hague: Netherlands Institute of International Relations >Clingendaek.

Fricke, Dietmar (2002): Der Tamilen-Singhalesen-Konflikt auf Sri Lanka. Ein Handbuch. Berlin: Verlag Dr. Köster.

Fukuyama, Francis (1989): »The End of History? « The National Interest, Summer 1989: 3-18.

Fukuyama, Francis (2004): Staaten bauen. Die neue Herausforderung internationaler Politik. Berlin: Propyläen. 
Fukuyama, Francis, Hg. (2006): Nation-Building. Beyond Afghanistan and Iraq. Baltimore, Maryland: The Johns Hopkins University Press.

Fuster, Thomas (1998): Die >Good Governance` Diskussion der Jahre 1989 bis 1994. Bern, Stuttgart, Wien: Verlag Paul Haupt.

Galtung, Johan (1996): Peace by Peaceful Means. Peace and Conflict, Development and Civilization. Oslo: International Peace Research Institute.

Galtung, Johan (2000): Conflict Transformation by Peaceful Means (the Transcend Method). United Nations Disaster Management Training Programme.

Gellner, Ernest (1999): Nationalismus. Kultur und Macht. Berlin: Siedler.

Gilley, Bruce (2004): »Against the concept of ethnic conflict. Feature Review.« Third World Quarterly, 25, no. 6: 1155-66.

Glagow, Manfred (1993): »Die Nicht-Regierungsorganisationen in der internationalen Entwicklungszusammenarbeit.« In: Dieter Nohlen und Franz Nuscheler (Hg.), Handbuch der Dritten Welt 1: Grundprobleme, Theorien, Strategien. Bonn: Dietz, 304-26.

Glasius, Marlies, und Mary Kaldor (2005): »Individuals First: A Human Security Strategy for the European Union.« IPG, 2005, no. 1: 62-82.

Gleichmann, Colin, Michael Odenwald, Kees Steenken, und Adrian Wilkinson (2004): Disarmament, Demobilisation and Reintegration. A Practical Field and Classroom Guide. Frankfurt am Main: GTZ, NODEFIC, PPC, SNDC.

Gnesotto, Nicole, Hg. (2005): Die Sicherheits- und Verteidigungspolitik der EU. Die ersten fünf Jahre (1999-2004). Paris: Institut für Sicherheitsstudien der Europäischen Union.

Goetschel, Laurent (2001): »Die Europäische Union und ihre Gemeinsame Außen- und Sicherheitspolitik - eine Zivilmacht?« In: Österreichisches Studienzentrum für Frieden und Konfliktlösung (Hg.), Zivile Konfliktbearbeitung. Eine internationale Herausforderung. Münster: Agenda Verlag, 269-80.

Goodhand, Jonathan (2001): Aid, Conflict and Peace Building in Sri Lanka. London: The Conflict, Security \& Development Group.

Goodhand, Jonathan (2006): »Internationalization of the Peace Process.« In: CJPD - Centre for Just Peace and Democracy (Hg.), Envisioning New Trajectories for Peace in Sri Lanka. Maharagama: CJPD, $215-$ 24.

Goodhand, Jonathan, und Bart Klem (2005): Aid, Conflict and Peace Building in Sri Lanka 2000 - 2005. Colombo: The Asia Foundation.

Goodhand, Jonathan, und Oliver Walton (2007): »International Engagement in the Sri Lankan Peace Process.« Paper presented at the 
Seminar on the International Dimension of the Conflict in Sri Lanka. Luzern: CJPD - Centre for Just Peace and Democracy \& TRANSCEND.

Goonatilake, Susantha (2006): Recolonisation. Foreign Funded NGOs in Sri Lanka. New Delhi: Sage Publications India.

Gordon, Colin (1991): »Governmental Rationality: An Introduction.«In: Graham Burchell, Colin Gordon, und Peter Miller (Hg.), The Foucault Effect. Studies in Governmentality. Chicago: The University of Chicago Press, 1-51.

Graf, Wilfried (2001): »Konflikttransformation mit friedlichen Mitteln: Auf der Suche nach einem komplexen Paradigma ziviler Konfliktbearbeitung.« In: Österreichisches Studienzentrum für Frieden und Konfliktlösung (Hg.), Zivile Konfliktbearbeitung. Eine internationale Herausforderung. Münster: Agenda Verlag, 46-63.

Gramsci, Antonio (1991-2002): Gefängnishefte (Band 1-10). Herausgegeben von Klaus Bochmann und Wolfgang Fritz Haug. Hamburg: Argument Verlag.

Griffin, Michèle (2001): »A Stitch in Time. Making the Case for Conflict Prevention.« Security Dialogue, 32, no. 4: 481-96.

Grossmann, Georg S., und Hildegard Lingnau (2002): Vergangenheitsund Versöhnungsarbeit - wie die TZ die Aufarbeitung von gewaltsamen Konflikten unterstützen kann. Eschborn: GTZ.

GTZ - Deutsche Gesellschaft für Technische Zusammenarbeit (2003):

Friedensentwicklung und Krisenprävention in Kolumbien. Studie zur zukünftigen Gestaltung des EZ-Länderprogramms, herausgegeben von Uwe Kievelitz. Eschborn: GTZ.

GTZ - Deutsche Gesellschaft für Technische Zusammenarbeit (2004a):

Die Begriffswelt der GTZ. Eschborn: GTZ.

GTZ - Deutsche Gesellschaft für Technische Zusammenarbeit (2004b):

Education and Conflict. The role of education in the creation, prevention and resolution of societal crises - Consequences for development cooperation, herausgegeben von Klaus Seitz. Eschborn: GTZ.

GTZ - Deutsche Gesellschaft für Technische Zusammenarbeit (2004c):

Promoting good governance in post-conflict societies, Discussion Paper. Eschborn: GTZ.

GTZ - Deutsche Gesellschaft für Technische Zusammenarbeit (2005):

Sicherheit - ein Schlüssel zu nachhaltiger Entwicklung. Das Sektorvorhaben zur Reform des Sicherheitssektors (SSR) stellt sich vor. Eschborn: GTZ.

GTZ - Deutsche Gesellschaft für Technische Zusammenarbeit (2006):

Gender und Konflikte. Ein Orientierungspapier. Eschborn: GTZ. 
GTZ - Deutsche Gesellschaft für Technische Zusammenarbeit (2007a):

»Leistungsangebote Staat, Demokratie und Armutsbekämpfung« $<$ http://www.gtz.de/de/leistungsangebote/2100.htm $>$ (Zugegriffen am 11-07-2007).

GTZ - Deutsche Gesellschaft für Technische Zusammenarbeit (2007b):

Leistungsangebot des GTZ-Leistungsschwerpunkts >Demokratie, Zivilgesellschaft, Öffentliche Verwaltung«: Förderung von Good Governance. Eschborn: GTZ.

Gunetilleke, Neranjana, und Azra Jafferjee, Hg. (2005): Triangulation

Squared: Assessing Impacts of the Poverty Impact Monitoring Unit. Colombo: Centre for Poverty Analysis.

Gurr, Ted Robert (1994): »Peoples Against States: Ethnopolitical Conflict and the Changing World System.« International Studies Quarterly, 38, no. 3, September: 347-377.

Gurr, Ted Robert (2000a [1993]): Peoples versus States: Minorities at Risk in the New Century. Washington, DC: USIP.

Gurr, Ted Robert (2000b): »Ethnic Warfare on the Wane.« Foreign Affairs, 79, no. 3, May/June: 52-67.

Gutiérrez Sanín, Francisco (2003): „Criminal Rebels? A Discussion of War and Criminality from the Colombian Experience.« Crisis State Programme, Working Paper Series No. 1, vol. 27. London.

Hafner-Burton, Emilie M., und Alexander H. Montgomery (2006): »Power Positions. International Organizations, Social Networks, and Conflict.« Journal of Conflict Resolution, 50, no. 1: 3-27.

Hall, Stuart (1994): »Der Westen und der Rest: Diskurs und Macht.« In: Ulrich Mehlem, Dorothee Bohle, Joachim Gutsche, Matthias Oberg, und Dominik Schrage (Hg.), Rassismus und kulturelle Identität. Ausgewählte Schriften 2. Hamburg: Argument Verlag, 137-79.

Harbeson, John W., Donald Rothchild, und Naomi Chazan, Hg. (1994): Civil Society and the State in Africa. Boulder, Col., London: Lynne Rienner Publishers.

Hardt, Michael, und Antonio Negri (2000): Empire. Cambridge, MA, London: Harvard University Press.

Hardt, Michael, und Antonio Negri (2004): Multitude. War and Democracy in the Age of Empire. New York: Penguin Books.

Harff, Barbara, und Ted Robert Gurr (2004 [1994]): Ethnic Conflict in World Politics. Boulder, CO: Westview Press.

Harris, Simon (2005): Sri Lanka: Aid effectiveness. A scoping of development partner perceptions for DFID-SEA. Colombo: DFID.

Hasemann, Armin, Katharina Hübner-Schmid, und Anja Dargatz (2005): Konfliktanalyse zur Entwicklung von Handlungsoptionen für gesellschaftspolitische Kooperationsprogramme. Ein methodischer Leitfa- 
den. Bonn: Arbeitsgemeinschaft Entwicklungspolitische Friedensarbeit (FriEnt).

Hein, Bastian (2006): Die Westdeutschen und die Dritte Welt. Entwicklungspolitik und Entwicklungsdienste zwischen Reform und Revolte 1959-1974. München: Oldenbourg.

Heinrich Böll Stiftung, Hg. (2001): »Entwicklungspolitik als internationale Strukturpolitik.« Erstes Entwicklungspolitischen Forums der Heinrich-Böll-Stiftung, vol. 19.-20. Mai 2000. Berlin: Heinrich Böll Stiftung.

Herbst, Jeffrey (2004): »Let Them Fail: State Failure in Theory and Practice. Implications for Policy.« In: Robert I. Rotberg (Hg.), When States Fail. Causes and Consequences. Princeton, NJ, Oxford: Princeton University Press, 302-18.

Herf, Jeffrey (1986): »War, Peace, und the Intellectuals. The West German Peace Movement« International Security, 10, no. 4 (Spring, 1986): 172-200.

Hett, Julia (2005): »Provincial Reconstruction Teams in Afghanistan. Das amerikanische, britische und deutsche Modell.« ZIF - Analyse April 2005. Berlin: Zentrum für Internationale Friedenseinsätze.

Hettne, Björn, und Bertil Odén, Hg. (2002): Global Governance in the 21st Century: Alternative Perspectives on World Order. Stockholm: Almkvist \& Wiksell International.

Hippler, Jochen (2003): »USA und Europa: unterschiedliche Sicherheitspolitiken.« In: Stiftung Entwicklung und Frieden (Hg.), Globale Trends 2004/2005. Fakten Analysen Prognosen. Frankfurt am Main: Fischer, 293-307.

Hippler, Jochen, Hg. (2004a): Nation-Building. Ein Schlüsselkonzept für friedliche Konfliktbearbeitung? Bonn: Dietz.

Hippler, Jochen (2004b): »Nationalstaaten aus der Retorte? NationBuilding zwischen militärischer Intervention, Krisenprävention und Entwicklungspolitik.« In: Jochen Hippler (Hg.), Nation-Building. Ein Schlüsselkonzept für friedliche Konfliktbearbeitung? Bonn: Dietz, 245-70.

Hobbes, Thomas (1996 [1651]): Leviathan. Oxford, New York: Oxford University Press.

Hobsbawm, Eric J. (1972): Die Banditen. Frankfurt am Main: Suhrkamp.

Hobsbawm, Eric J. (1973): Revolutionaries. London: Weidenfeld and Nicolson.

Hobsbawm, Eric J. (1979): Sozialrebellen. Archaische Sozialbewegungen im 19. und 20. Jahrhundert. Gießen: Focus Verlag. 
Hobsbawm, Eric J. (1992): Nations and Nationalism since 1780. Programme, Myth, Reality. Cambridge, UK: Cambridge University Press.

Hobsbawm, Eric J. (1998): Das Zeitalter der Extreme. Weltgeschichte des 20. Jahrhunderts. München: Dtv.

Hoebink, Paul (2006): »European Donors and >Good Governance〈: Condition or Goal?« European Journal of Development Research, 18, no. 1: 131-61.

Hoffman, Mark (2004): »Peace and Conflict Impact Assessment Methodology.« In: Alex Austin, Martina Fischer, und Norbert Ropers (Hg.), Transforming Ethnopolitical Conflict. The Berghof Handbook. Wiesbaden: VS Verlag für Sozialwissenschaften, 171-91.

Holert, Tom, und Mark Terkessidis (2003): Entsichert. Krieg als Massenkultur im 21. Jahrhundert. Köln: Kiepenheuer \& Witsch.

Holm, Hans-Henrik (1998): »The Responsibility That Will Not Go Away. Weak States in the International System. « Paper presented at the Failed States and International Security: Causes, Prospects, und Consequences, Purdue University, West Lafayette.

Holm, Hans-Henrik (2001): »Writing the Rules for Disaggregated World Order. Policy towards failed states as an example.« Paper presented at the Failed States IV: Structures, Cases and Policies. Florence: Strategic Outreach Program of the U.S. Army War College; Office of International Programs, Purdue University.

Holm, Hans-Henrik (2002): »Failing Failed States: Who Forgets the Forgotten?« Security Dialogue, 33, no. 4: 457-71.

Holmberg, Björn, Inger Buxton, und Olle Kvist (2003): Reflections on Development Co-operation and Violent Conflict, Department for Cooperation with NGOs, Humanitarian Assistance and Conflict Management, Sida. Stockholm: Sida.

Holsti, Kalevi J (1996): The State, War, und the State of War. Cambridge, UK: Cambridge University Press.

Holtz, Uwe (2006): »Abschied von der Gießkanne. Stationen aus 50 Jahren deutscher Entwicklungspolitik« eins / Entwicklungspolitik Information Nord-Süd, 23-24/2006: 32-36.

Hoogvelt, Ankie (1997): Globalization and the Postcolonial World. The New Political Economy of Development. Baltimore, Maryland: The Johns Hopkins University Press.

Hook, Steven W (1997): Mission Without A Mandate: U.S. Foreign Aid After The Cold War. Case 378. Washington, DC: Institute for the Study of Diplomacy, Georgetown University.

Hopp, Ulrike, und Adolf Kloke-Lesch (2004): »Nation-Building versus Nationenbildung - Eine entwicklungspolitische Perspektive.« In: Jo- 
chen Hippler (Hg.), Nation-Building. Ein Schlüsselkonzept für friedliche Konfliktbearbeitung? Bonn: Dietz, 195-214.

Horowitz, Donald (2000 [1985]): Ethnic Groups in Conflict. Berkeley, Los Angeles: University of California Press.

Howarth, David (2000): Discourse. Concepts in the Social Science. Buckingham, Philadelphia: Open University Press.

Howarth, David, und Yannis Stavrakakis (2000): »Introducing discourse theory and political analysis.« In: David Howarth, Aletta J. Norval, und Yannis Stavrakakis (Hg.), Discourse theory and political analysis. Identities, hegemonies and social change. Manchester, New York: Manchester University Press, 1-23.

Hödl, Gerald (2004): Österreich und die Dritte Welt. Außen- und Entwicklungspolitik der Zweiten Republik bis zum EU-Beitritt 1995. Wien: Promedia.

Human Security Centre (2005): The Human Security Report 2005: War and Peace in the 21st Century. New York: Oxford University Press.

Huntington, Samuel P (1991): The Third Wave: Democratization in the Late Twentieth Century. Norman, OK: University of Oklahoma Press.

Huntington, Samuel P. (1996a [1968]): Political Order in Changing Societies. New Haven, London: Yale University Press.

Huntington, Samuel P. (1996b [1993]): »The Clash of Civilizations?« In: Samuel P. Huntington (Hg.), The Clash of Civilizations? The Debate. New York: Foreign Affairs, 1-25.

Huntington, Samuel P. (1996c [1993]): »If Not Civilizations, What? Paradigms of the Post-Cold War World.« In: Samuel P. Huntington (Hg.), The Clash of Civilizations? The Debate. New York: Foreign Affairs, 56-67.

Huntington, Samuel P. (2003 [1996]): The Clash of Civilizations and the Remaking of World Order. New York: Simon \& Schuster.

ICG - International Crisis Group (2006): Sri Lanka: The Failure of the

Peace Process, Asia Report no. 124. Colombo, Brussels: ICG.

Ignatieff, Michael (1993): Blood and Belonging: Journeys into the New

Nationalism. New London: BBC Books.

Ignatieff, Michael (1997): The Warrior's Honor. Ethnic War and the

Modern Conscience. New York: Henry Holt and Company.

Ignatieff, Michael (2000): Die Zivilisierung des Krieges. Ethnische Konflikte, Menschenrechte, Medien. Hamburg: Rotbuch Verlag.

Illich, Ivan (2005 [1971]): »Development as Planned Poverty.« In: Majid Rahnema und Victoria Bawtree (Hg.), The Post-Development Reader. London, New York: Zed Books, 94-101. 
Imbusch, Peter (1999): »Moderne und postmoderne Perspektiven der Gewalt.« In: Sighard Neckel und Michael Schwab-Trapp (Hg.), Ordnungen der Gewalt. Beiträge zu einer politischen Soziologie der Gewalt und des Krieges. Opladen: Leske + Budrich, 147-60.

Inda, Jonathan Xavier, Hg. (2005): Anthropologies of Modernity. Foucault, Governmentality, und Life Politics. Malden, Oxford, Carlton: Blackwell Publishing.

International Alert und Saferworld (2005): Developing an EU Strategy to Address Fragile States: Priorities for the UK Presidency of the EU in 2005. London: International Alert, Saferworld.

International Alert und Woman Waging Peace (2004): Inclusive Security, Sustainable Peace: A Toolkit for Advocacy and Action. London, Washington, DC: International Alert, Woman Waging Peace.

Ismail, Qadri (1995): »Unmooring Identity. The Antinomies of Elite Muslim Self-Representation in Modern Sri Lanka.« In: Pradeep Jeganathan und Qadri Ismail (Hg.), Unmaking the Nation. The Politics of Identity and History in Modern Sri Lanka. Colombo: Social Scientists' Association, 55-105.

Ismail, Qadri (2005): Abiding by Sri Lanka. On Peace, Place, und Postcoloniality. Minneapolis, London: University of Minnesota Press.

Jäger, Siegfried (2001): »Diskurs und Wissen. Theoretische und methodische Aspekte einer Kritischen Diskurs- und Dispositivanalyse.« In: Reiner Keller, Andreas Hirseland, Werner Schneider, und Willy Viehöver (Hg.), Handbuch Sozialwissenschaftliche Diskursanalyse. Band 1: Theorien und Methoden. Opladen: Leske + Budrich, 81112.

Jean, Francois, und Jean-Christophe Rufin, Hg. (1999): Ökonomie der Bürgerkriege. Hamburg: Hamburger Edition.

Jeganathan, Pradeep, und Qadri Ismail, Hg. (1995): Unmaking the Nation. The Politics of Identity and History in Modern Sri Lanka. Colombo: Social Scientists' Association.

Joxe, Alain (2004): »Der Wandel des Krieges im Zeitalter der Globalisierung. Europäische Multipolarität versus US-amerikanische Hegemonie.« In: Sabine Kurtenbach und Peter Lock (Hg.), Kriege als (Über)Lebenswelten. Schattenglobalisierung, Kriegsökonomien und Inseln der Zivilität. Bonn: Dietz, 62-71.

Kagan, Robert (2004): Of Paradise and Power. America and Europe in the New World Order. New York: Vintage.

Kagan, Robert (2008): The Return of History and the End of Dreams. New York: Alfred A. Knopf.

Kaldor, Mary (2001 [1999]): New and Old Wars. Organized Violence in a Global Era. Stanford, Cal.: Stanford University Press. 
Kaldor, Mary (2002): Civil Society and Accountability, Occasional Paper. Background paper for HDR 2002. New York: UNDP.

Kaldor, Mary (2003): Global Civil Society. An Answer to War. Cambridge: Polity Press.

Kaltefleiter, Viola (1995): Die Entwicklungshilfe der Europäischen Union: Rechtfertigung, Effizienz und politische Ökonomie staatlicher Entwicklungshilfe. Heidelberg: Physica-Verlag.

Kant, Immanuel (1996 [1795]): Zum ewigen Frieden. Stuttgart: Philipp Reclam Jun.

Kaplan, Robert D. (1994): Balkan Ghosts: A Journey Through History. New York: Vintage.

Kaplan, Robert D. (2001): The Coming Anarchy. Shattering the Dreams of the Post Cold War. New York: Random House.

Kapoor, Ilan (2002): „Capitalism, culture, agency: dependency versus postcolonial theory.« Third World Quarterly, 23, no. 4: 647-64.

Keen, David (1999): »»Who's it Between?« „Ethnic War^ and Rational Violence.« In: Tim Allen und Jean Seaton (Hg.), The Media of Conflict: War Reporting and Representations of Ethnic Violence. London, New York: Zed Books, 81-101.

Keenan, Alan (2007): »The Trouble with Evenhandedness: On the Politics of Human Rights and Peace Advocacy in Sri Lanka.« In: Michel Feher (Hg.), Nongovernmental Politics. New York: Zone Books, 88117.

Keethaponcalan, S.I. (2005): »The Ethnic Conflict in Sri Lanka and Dynamics of Third-Party Activities.« In: Jayadeva Uyangoda (Hg.), Conflict, Conflict Resolution \& Peace Building: An Introduction to Theories and Practices. Colombo: GTZ Sri Lanka, IMCAP, University of Colombo, 204-24.

Kelegama, Saman (2006): »Transforming Conflict with an Economic Dividend: The Sri Lankan Experience.« In: Kumar Rupesinghe (Hg.), Negotiating Peace in Sri Lanka. Efforts, Failures \& Lessons. Volume Two. Colombo: The Foundation for Co-Existence, 205-43.

Keller, Reiner (2004): Diskursforschung. Eine Einführung für SozialwissenschaftlerInnen. Wiesbaden: VS Verlag für Sozialwissenschaften.

Kemp, Walter A. (2004): »The Business of Ethnic Conflict.« Security Dialogue, 35, no. 1: 43-59.

Khittel, Stefan, und Jan Pospisil (2006): Europäische Entwicklungspolitik als Alternative zum >Global War on Terrorism?^Zur Entwicklungszusammenarbeit der EU in Krisenregionen. Wien: ÖFSE. 
Kiely, Ray (1999): »The Last Refuge of the Noble Savage? A Critical Assessment of Post-Development Theory.« European Journal of Development Research, 11, no. 1: 30-55.

Kievelitz, Uwe (2003): »Strategic Conflict Assessments in German Development Cooperation: Approaches, Experiences, Impacts.« In: Uwe Kievelitz und Roman Poeschke (Hg.), Friedens- und Konfliktarbeit in Forschung und EZ-Praxis und der Beitrag der Entwicklungsethnologie. Saarbrücken: Verlag für Entwicklungspolitik, 16578.

Kievelitz, Uwe, Gabriele Kruk, und Norbert Frieters (2003): Joint Utstein Study of Peacebuilding. National Report on Germany. Commissioned by the Evaluation Division of the German Federal Ministry for Economic Cooperation and Development (BMZ). Eschborn: GTZ.

Kievelitz, Uwe, und Roman Poeschke, Hg. (2003): Friedens- und Konfliktarbeit in Forschung und EZ-Praxis und der Beitrag der Entwicklungsethnologie. Entwicklungsethnologie, vol. Heft 1+2, 2003. Saarbrücken: Verlag für Entwicklungspolitik.

Kievelitz, Uwe, und Eva Schrottshammer (2005): Dezentralisierung und Konflikte. Handreichung, Sektorberatungsvorhaben Krisenprävention und Konfliktbearbeitung. Eschborn: GTZ.

Klem, Bart (2002): Sharing Studies on Development and Conflict in Sri Lanka. Synthesis of Eight Studies, Netherlands Institute of International Relations - Clingendael. The Hague: Clingendael.

Klemp, Ludgera (2006): »Difficult cooperation with fragile states. « Development and Cooperation, 33, no. 5: 208-09.

Klingebiel, Stephan (1999): Impact of Development Cooperation in Conflict Situations. Cross-section Report on Evaluations of German Development Cooperation in Six Countries, German Development Institute, GDI. Berlin: Deutsches Institut für Entwicklungspolitik.

Klingebiel, Stephan (2001): Ansatzpunkte für eine krisenpräventive und konfliktsensible Entwicklungszusammenarbeit, Deutsches Institut für Entwicklungspolitik DIE no. Analysen und Stellungnahmen 4/2001. Bonn: Deutsches Institut für Entwicklungspolitik.

Klingebiel, Stephan (2002): Strukturelle Stabilität: Ansatzpunkte für die Entwicklungszusammenarbeit, Beitrag zur VAD-Konferenz 2002. Bonn: Deutsches Institut für Entwicklungspolitik.

Klingebiel, Stephan, Hg. (2006): New interfaces between security and development: changing concepts and approaches. Studies / Deutsches Institut für Entwicklungspolitik, vol. 13. Bonn: Deutsches Institut für Entwicklungspolitik. 
Klingebiel, Stephan, und Katja Roehder (2004): Entwicklungspolitischmilitärische Schnittstellen. Neue Herausforderungen in Krisen und Post-Konflikt-Situationen, Deutsches Institut für Entwicklungspolitik DIE no. Berichte und Gutachten 3/2004. Bonn: Deutsches Institut für Entwicklungspolitik.

Kloke-Lesch, Adolf, und Hans-Peter Baur (2000): »Friedensentwicklung und Krisenprävention als Strategieelemente der Entwicklungspolitik.« In: Tilman Evers (Hg.), Ziviler Friedensdienst. Fachleute für den Frieden. Idee - Erfahrungen - Ziele. Opladen: Leske + Budrich, 189-98.

Knuchel, Lars (2004): Ohne Entwicklung keine Sicherheit - keine Entwicklung ohne Sicherheit: Anmerkungen zu aktuellen Tendenzen in der EU-Außenpolitik, Direktion für Entwicklung und Zusammenarbeit DEZA no. ep@brief 04/04. Bern: Direktion für Entwicklung und Zusammenarbeit, DEZA.

Korf, Benedikt (2006): »Cargo Cult Science, Armchair Empiricism and the Idea of Violent Conflict.« Third World Quarterly, 27, no. 3: 45976.

Korff, Rüdiger (1997): »Der Stellenwert der Entwicklungspolitik in der Bundesrepublik Deutschland.« Universität Bielefeld, Fakultät für Soziologie, Working Paper no. 273. Bielefeld: Universität Bielefeld.

Kramer, Paul A. (2006): The Blood of Government. Race, Empire, the United Sates \& the Philippines. Chapel Hill: The University of North Carolina Press.

Kothari, Uma, und Martin Minogue, Hg. (2002): Development Theory and Practice. Critical Perspectives. Houndmills, Basingstoke, Hampshire: Palgrave.

Krasner, Stephen D., und Carlos Pascual (2005): "Addressing State Failure.« Foreign Affairs, 84, no. 4, Jul/Aug 2005: 153-163.

Krause, Joachim (2003): »Multilaterale Ordnung oder Hegemonie? Zur transatlantischen Debatte über die weltpolitische Neuordnung. « Aus Politik und Zeitgeschichte, 2003, no. B31-32, 28. Juli: 6-14.

Kreidler, Corinna (1995): »Schwerter zu Pflugscharen - Zur Demobilisierung und Reintegration ehemaliger Kombattantinnen und Kombattanten.« In: Volker Matthies (Hg.), Vom Krieg zum Frieden. Kriegsbeendigung und Friedenskonsolidierung. Bremen: Edition Temmen, 83-109.

Krummenacher, Heinz (2001): »Early Warning: Eine Momentaufnahme in Theorie und Praxis.« In: Österreichisches Studienzentrum für Frieden und Konfliktlösung (Hg.), Zivile Konfliktbearbeitung. Eine internationale Herausforderung. Münster: Agenda Verlag, 77-88. 
Krummenacher, Heinz, Günther Baechler, und Susanne Schmeidl (1999): »Beitrag der Frühwarnung zur Krisenprävention. Möglichkeiten und Grenzen in Theorie und Praxis.« In: Österreichisches Studienzentrum für Frieden und Konfliktlösung, Schweizerische Friedensstiftung, und Institut für Konfliktlösung (Hg.), Krisenprävention: Theorie und Praxis ziviler Konfliktbearbeitung. Chur, Zürich: Verlag Rüegger, 77-97.

Kurtenbach, Sabine (2004): »Nachhaltige Strategien zum Umgang externer Akteure mit Gewalt.« In: Sabine Kurtenbach und Peter Lock (Hg.), Kriege als (Über)Lebenswelten. Schattenglobalisierung, Kriegsökonomien und Inseln der Zivilität. Bonn: Dietz, 306-20.

Kurtenbach, Sabine, und Peter Lock, Hg. (2004): Kriege als (Über)Lebenswelten. Schattenglobalisierung, Kriegsökonomien und Inseln der Zivilität. Bonn: Dietz.

Kurtenbach, Sabine, und Andreas Mehler, Hg. (2002): Die Vielfalt von Gewaltkonflikten. Analysen aus regionalwissenschaftlicher Perspektive. Schriften des Deutschen Übersee-Instituts Hamburg. Hamburg: DÜI.

Laclau, Ernesto (1996): Emancipation(s). London: Verso.

Laclau, Ernesto, und Chantal Mouffe. 2001 [1985]. Hegemony and Socialist Strategy. Towards a Radical Democratic Politics. London, New York: Verso.

Lancaster, Carol (2000a): Transforming Foreign Aid: United States Assistance in the $21^{\text {st }}$ Century. Washington, DC: Institute for International Economics.

Lancaster, Carol (2000b): »Redesigning Foreign Aid.« Foreign Affairs, 79, no. 5, Sept/Oct 2000: 74-88.

Lancaster, Carol, und Ann van Dusen (2005): Organizing U.S. Foreign Aid: Confronting the Challenges of the Twenty-first Century. Washington, DC: The Brookings Institution.

Larner, Wendy, und William Walters, Hg. (2004): Global Governmentality. Governing international spaces. London: Routledge.

Latto, Benedict (2002): »Governance and Conflict Management: implications for donor intervention.« Crisis State Programme, Working Paper Series No. 1, vol. 9. London.

Lederach, John Paul (1997): Building Peace: Sustainable Reconciliation in Divided Societies. Washington, DC: USIP.

Lederach, John Paul (2003): The Little Book of Conflict Transformation. Clear articulation of the guiding principles by a pioneer in the field. Intercourse, PA: Good Books.

Lederer, William J., und Eugene Burdick (1999 [1958]): The Ugly American. New York, London: W.W. Norton\&Company. 
Lemke, Thomas (1997): Eine Kritik der politischen Vernunft. Hamburg: Argument Verlag.

Lemke, Thomas (1999): »Antwort auf eine Frage: Ist Foucaults >Geschichte der Wahrheit» eine wahre Geschichte? « In: Hannelore Bublitz, Andrea D. Bührmann, Christine Hanke, und Andrea Seier (Hg.), Das Wuchern der Diskurse. Perspektiven der Diskursanalyse Foucaults. Frankfurt/Main, New York: Campus, 177-93.

Lemke, Thomas (2004): »Governance, Gouvernementalität und die Dezentrierung der Ökonomie.« In: Ramón Reichert (Hg.), Governmentality Studies. Analysen liberal-demokratischer Gesellschaften im Anschluss an Michel Foucault. Münster: LIT Verlag, 63-73.

Lemke, Thomas, Susanne Krasmann, und Ulrich Bröckling (2000): »Gouvernementalität, Neoliberalismus und Selbsttechnologien. Eine Einleitung.« In: Ulrich Bröckling, Susanne Krasmann, und Thomas Lemke (Hg.), Gouvernementalität der Gegenwart. Studien zur Ökonomisierung des Sozialen. Frankfurt am Main: Suhrkamp, 7-40.

Leonhardt, Manuela (2000): Conflict Impact Assessment of EU Development Co-operation with ACP Countries. A Review of Literature and Practice. London: International Alert, Saferworld.

Lilly, Damian, Robin Luckham, und Michael von Tangen Page (2002): A Goal Orientated Approach to Governance and Security Sector Reform. London: International Alert.

Link, Werner (2002): »Hegemonie und Gleichgewicht der Macht.« In: Mir A. Ferdowsi (Hg.), Internationale Politik im 21. Jahrhundert. München: Wilhelm Fink Verlag, 33-52.

Lischer, Sarah Kenyon (2005): Dangerous Sanctuaries. Refugee Camps, Civil War, und the Dilemmas of Humanitarian Aid. Ithaca, London: Cornell University Press.

Lister, Sarah (2003): »NGO Legitimacy. Technical Issue or Social Construct?« Critique of Anthropology, 23, no. 2: 175-92.

Liyanage, Sumanasiri (2005): »Right of Self-Determination in Diverse Societies: The Case of Sri Lanka.« In: Jayadeva Uyangoda (Hg.), Conflict, Conflict Resolution \& Peace Building: An Introduction to Theories and Practices. Colombo: GTZ Sri Lanka, IMCAP, University of Colombo, 120-48.

Liyanage, Sumanasiri (2006): »Civil Society and the Peace Process.« In:

Kumar Rupesinghe (Hg.), Negotiating Peace in Sri Lanka. Efforts, Failures \& Lessons. Volume Two. Colombo: The Foundation for Co-Existence, 279-302.

Liyanage, Sumanasiri (2007): »The Rise of 〉Peace〈 Professionals in Sri Lanka: Rent-Seeking in the Peace Sector.« Daily News (Colombo) 16/March, Financial Times Supplement: B3. 
Liyanage, Sumanasiri, und M. Sinnathamby, Hg. (2007): A Glimmer of Hope. A New Phase in Constitutional Reforms in Sri Lanka. Colombo: South Asian Peace Institute.

Loganathan, Ketheshwaran, und Norbert Ropers (2002): Conceptualizing a Road Map for Peace in Sri Lanka. Colombo: Berghof.

Loges, Bastian, und Ulrich Menzel (2004): »Staatszerfall und humanitäre Intervention.« Entwicklung und Zusammenarbeit, 45, no. 4: 14851.

Lorenz, Kathrin (2005): »Conflict and Conflict Analysis in Sri Lanka.« In: Jayadeva Uyangoda (Hg.), Conflict, Conflict Resolution \& Peace Building: An Introduction to Theories and Practices. Colombo: GTZ Sri Lanka, IMCAP, University of Colombo, 190-201.

Lorenz, Kathrin (2007): Good Development Practice in Conflict Environments and Fragile States: Recent Experiences from GTZ Sri Lanka. Colombo: GTZ.

Lund, Michael, Andreas Mehler, Luc van de Goor, und Céline Moyroud, Principal Contributors (2001): Conflict Prevention and Peacebuilding: A Practical Guide. CD-Rom. Berlin: Stiftung Wissenschaft und Politik, Conflict Prevention Network (SWP-CPN).

Lunstead, Jeffrey (2006): The United States' Role in Sri Lanka's Peace Process 2002-2006. Colombo: The Asia Foundation.

Mair, Stefan (2002): Die Globalisierung privater Gewalt. Kriegsherren, Rebellen, Terroristen und organisierte Kriminalität, SWP-Studie. Berlin: Stiftung Wissenschaft und Politik.

Mamdani, Mahmood (1996): Citizen and Subject. Contemporary Africa and the Legacy of Late Colonialism. London: James Currey Ltd.

Mamdani, Mahmood (2001): When Victims Become Killers. Colonialism, Nativism, und the Genocide in Rwanda. Princeton, Oxford: Princeton University Press.

Management Systems International (2006): Democracy and Governance: Sri Lanka Assessment. Colombo: USAID.

Maral-Hanak, Irmi (2004): »Feministische Entwicklungstheorien.« In: Karin Fischer, Irmi Maral-Hanak, Gerald Hödl, und Christof Parnreiter (Hg.), Entwicklung und Unterentwicklung. Eine Einführung in Probleme, Theorien und Strategien. Wien: Mandelbaum, 179-97.

Marasinghe, Lakshman (2007): The Evolution of Constitutional Governance in Sri Lanka. Colombo: Vijitha Yapa Publications.

Marriage, Zoë (2005): »Shame and Assistance - Does it Help?« Civil Wars, 7, no. 4: 357-76.

Marriage, Zoë (2006a): Not Breaking the Rules, Not Playing the Game. London: Hurst \& Company. 
Marriage, Zoë (2006b): »The Comfort of Denial: External Assistance in Southern Sudan.« Development and Change, 37, no. 3: 479-500.

Matthies, Volker (1993): »Kriege in der Dritten Welt.« In: Dieter Nohlen und Franz Nuscheler (Hg.), Handbuch der Dritten Welt 1: Grundprobleme, Theorien, Strategien. Bonn: Dietz, 359-73.

Matthies, Volker, Hg. (1995a): Vom Krieg zum Frieden. Kriegsbeendigung und Friedenskonsolidierung. Bremen: Edition Temmen.

Matthies, Volker (1995b): »Der Transformationsprozess vom Krieg zum Frieden - ein vernachlässigtes Forschungsfeld.« In: Volker Matthies (Hg.), Vom Krieg zum Frieden. Kriegsbeendigung und Friedenskonsolidierung. Bremen: Edition Temmen, 8-38.

Matthies, Volker (1997): »Zwischen Kriegsbeendigung und Friedenskonsolidierung.« In: Dieter Senghaas (Hg.), Frieden machen. Frankfurt am Main: Suhrkamp, 527-59.

Matthies, Volker (2002): »Krisenprävention und Friedenskonsolidierung.« In: Mir A. Ferdowsi (Hg.), Internationale Politik im 21. Jahrhundert. München: Wilhelm Fink Verlag, 123-46.

Maull, Hanns W. (1990): »Germany and Japan: The New Civilian Powers. « Foreign Affairs, 69, no. 5, Winter 1990/1991: 91-106.

Maull, Hanns W. (1997): »Zivilmacht Deutschland. Vierzehn Thesen für eine neue deutsche Außenpolitik.« In: Dieter Senghaas (Hg.), Frieden machen. Frankfurt am Main: Suhrkamp, 527-59.

Maull, Hanns W. (1999): »Die deutsche Außenpolitik am Ende der Ära Kohl.« In: Erich Reiter (Hg.), Jahrbuch für Internationale Sicherheitspolitik 1999. Hamburg: Verlag E.S. Mittler \& Sohn, 172-84.

Maull, Hanns W. (2004): "»Normalisierung` oder Auszehrung? Deutsche Außenpolitik im Wandel.« Aus Politik und Zeitgeschichte, 2004, no. B11: 17-23.

Maull, Hanns W. (2007): »Deutschland als Zivilmacht.« In: Siegmar Schmidt, Gunther Hellmann, und Reinhard Wolf (Hg.), Handbuch zur deutschen Außenpolitik. Opladen: VS Verlag für Sozialwissenschaften, 73-84.

Mbaine, Adolf E., Hg. (2006): Media in Situations of Conflict. Roles, Challenges and Responsibility. Kampala: Fountain Publishers Ltd.

Mbembe, Achille (2001): On the Postcolony. Berkeley, Los Angeles, London: University of California Press.

Mehler, Andreas, und Claude Ribaux (2000): Krisenprävention und Konfliktbearbeitung in der Technischen Zusammenarbeit. Ein Überblick zur nationalen und internationalen Diskussion. Wiesbaden: Universum Verlagsanstalt.

Menon, Nivedita (2004): Recovering Subversion. Feminist Politics Beyond the Law. Champaign, Il: University of Illinois Press. 
Menzel, Ulrich (1988): Auswege aus der Abhängigkeit. Die entwicklungspolitische Aktualität Europas. Frankfurt am Main: Suhrkamp.

Menzel, Ulrich (1992): Das Ende der Dritten Welt und das Scheitern der großen Theorie. Frankfurt am Main: Suhrkamp.

Menzel, Ulrich (1993): »40 Jahre Entwicklungsstrategie $=40$ Jahre Wachstumsstrategie.« In: Dieter Nohlen und Franz Nuscheler (Hg.), Handbuch der Dritten Welt 1: Grundprobleme, Theorien, Strategien. Bonn: Dietz, 131-55.

Menzel, Ulrich, und Dieter Senghaas (1986): Europas Entwicklung und die Dritte Welt. Eine Bestandsaufnahme. Frankfurt am Main: Suhrkamp.

Meyer, Éric (2003): Sri Lanka: Biography of an Island. Between Local \& Global. Negombo: Viator Publications.

Mies, Maria (2003): »Über die Notwendigkeit, Europa zu entkolonisieren.« In: Claudia von Werlhof, Veronika Bennholdt-Thomsen, und Nicholas Faraclas (Hg.), Subsistenz und Widerstand. Alternativen zur Globalisierung. Wien: Promedia, 19-40.

Migdal, Joel S. (1988): Strong Societies and Weak States. State-Society Relations and State Capabilities in the Third World. Princeton: Princeton University Press.

Migdal, Joel S. (2001): State in Society. Studying how states and societies transform and constitute one another. Cambridge, UK: Cambridge University Press.

Miller, Gale, und Kathryn J. Fox (2004): »Building bridges: the possibility of analytical dialogue between ethnography, conversation analysis and Foucault.« In: David Silverman (Hg.), Qualitative Research. Theory, Method and Practice. London, Thousand Oaks, New Delhi: Sage Publications, 35-55.

Milliken, Jennifer, Hg. (2003): State Failure, Collapse and Reconstruction. Malden, Oxford, Melbourne, Berlin: Blackwell Publishing.

Milliken, Jennifer, und Keith Krause (2003): »State Failure, State Collapse and State Reconstruction: Concepts, Lessons and Strategies.« In: Jennifer Milliken (Hg.), State Failure, Collapse and Reconstruction. Malden, Oxford, Melbourne, Berlin: Blackwell Publishing, 121.

Ministry of Defence (2005): Africa Conflict Prevention Pool. The UK Sub-Saharan Strategy for Conflict Prevention. London: Ministry of Defence.

Mohideen, M.I.M. (2006): »Sri Lankan Peace Process and the Muslim Question.« In: Kumar Rupesinghe (Hg.), Negotiating Peace in Sri Lanka. Efforts, Failures \& Lessons. Volume Two. Colombo: The Foundation for Co-Existence, 314-37. 
Moore, David (1996): »Reading Americans on Democracy in Africa: From the CIA to >Good Governance«.« European Journal of Development Research, 8, no. 1: 123-49.

Moore, Jonathan, Hg. (1998): Hard Choices. Moral Dilemmas in Humanitarian Intervention. Lanham, MD., Oxford: Rowman \& Littlefield Publishers.

Moreno Torres, Magüi, und Michael Anderson (2004): Fragile States: Defining Difficult Environments for Poverty Reduction, PRDE Working Paper no. 1. London: DFID.

Mosse, David (2005): Cultivating Development. An Ethnography of Aid Policy and Practice. London, Ann Arbor: Pluto Press.

MSI - Management Systems International (2001): Sri Lanka Democracy and Governance Assessment. Colombo: USAID.

MSI - Management Systems International (2006): Democracy and Governance: Sri Lanka Assessment. Colombo: USAID.

Mueller, John (2000): »The Banality of 〉Ethnic Warı.« International Security, 25, no. 1: 42-70.

Murshed, S. Mansoob (2002): »Conflict, Civil War and Underdevelopment: An Introduction.« Journal of Peace Research, 39, no. 4: 38793.

Musto, Stefan (2000): »Wer Gewalt sät, erntet EZ? Einige Überlegungen zur Genese, Kasernierung und Prävention kollektiver Gewalt.« In: Brigitte Fahrenhorst (Hg.), Die Rolle der Entwicklungszusammenarbeit in gewalttätigen Konflikten. Berlin: Society for International Development, 21-31.

Müller, Barbara (2001): »Konfliktursachen in der Konfliktbearbeitung Ein Problemaufriss zum Verhältnis von Konfliktursachen zu Arbeitsfeldern der zivilen Konfliktbearbeitung.« In: Österreichisches Studienzentrum für Frieden und Konfliktlösung (Hg.), Zivile Konfliktbearbeitung. Eine internationale Herausforderung. Münster: Agenda Verlag, 27-45.

Münkler, Herfried (2002): Die neuen Kriege. Reinbek bei Hamburg: Rowohlt Verlag (Lizenzausgabe für die Bundeszentrale für politische Bildung).

Nandy, Ashis (1992): »State.« In: Wolfgang Sachs (Hg.), The Development Dictionary. A Guide to Knowledge as Power. London, New York: Zed Books, 264-74.

Narayan Swamy, M.R. (20023): Tigers of Lanka. From Boys to Guerrillas. New Delhi: Konark Publishers.

Neckel, Sighard, und Michael Schwab-Trapp, Hg. (1999): Ordnungen der Gewalt. Beiträge zu einer politischen Soziologie der Gewalt und des Krieges. Opladen: Leske + Budrich. 
Nederveen Pieterse, Jan (1998): »My Paradigm or Yours? Alternative Development, Post-Development, Reflexive Development.« Development and Change, 29, no. 2: 343-73.

Newman, Edward (2004): »The \New Wars` Debate: A Historical Perspective Is NeedHg.« Security Dialogue, 35, no. 2: 173-89.

Nilsson, Anders (2005): Reintegrating Ex-Combatants in Post-Conflict Societies. Stockholm: Sida.

Nohlen, Dieter, und Franz Nuscheler, Hg. (1993): Handbuch der Dritten Welt. Bonn: Dietz.

Nowels, Larry, und Connie Veillette (2006): Restructuring U.S. Foreign Aid: The Role of the Director of Foreign Assistance. CRS Report for Congress. Washington, DC: Congressional Research Service.

Noyahr, Keith (2006): »The Role of the International Community.« In: Kumar Rupesinghe (Hg.), Negotiating Peace in Sri Lanka. Efforts, Failures \& Lessons. Volume Two. Colombo: The Foundation for Co-Existence, 355-404.

Nöst, Barbara (2003): »Den Staat links liegen lassen?« In: Bea de Abreu Fialho Gomes, Irmi Hanak, und Walter Schicho (Hg.), Die Praxis der Entwicklungszusammenarbeit. Akteure, Interessen und Handlungsmuster. Wien: Mandelbaum, 77-88.

Nöst, Barbara (2006): »Entwicklungszusammenarbeit mit oder gegen den Staat - Partnerschaften mit leistungsschwachen und fragilen Staaten.« In: Bea de Abreu Fialho Gomes, Irmi Maral-Hanak, und Walter Schicho (Hg.), Entwicklungszusammenarbeit. Akteure, Handlungsmuster und Interessen. Wien: Mandelbaum, 89-102.

Nuscheler, Franz (1993): »Menschenrechte und Entwicklung - Recht auf Entwicklung.« In: Dieter Nohlen und Franz Nuscheler (Hg.), Handbuch der Dritten Welt 1: Grundprobleme, Theorien, Strategien. Bonn: Dietz, 269-86.

Nuscheler, Franz (1996): Lern- und Arbeitsbuch Entwicklungspolitik. Bonn: Dietz.

Nuscheler, Franz, Hg. (2000): Entwicklung und Frieden im Zeichen der Globalisierung. Bonn: Bundeszentrale für politische Bildung.

Nuscheler, Franz (2002): »Überforderte Entwicklungspolitik. Veränderungen nach dem 11. September.« Internationale Politik, 2002, no. Nr. 11, November.

Nuscheler, Franz (2006): »Deutsche Entwicklungspolitik. Interessensgeleitete Kontinuitäten und Veränderungen.« In: Bea de Abreu Fialho Gomes, Irmi Maral-Hanak, und Walter Schicho (Hg.), Entwicklungszusammenarbeit. Akteure, Handlungsmuster und Interessen. Wien: Mandelbaum, 189-205. 
Nye, Jr., Joseph S. (1999): »Redefining the National Interest.« Foreign Affairs, 78, no. 4, Jul/Aug 1999: 22-35.

Ofstad, Arve (2002): »Countries in Violent Conflict and Aid Strategies: The Case of Sri Lanka.« World Development, 30, no. 2: 165-80.

Orjuela, Camilla (2004): Civil Society in Civil War. Peace Work and Identity Politics in Sri Lanka. Göteborg: Department of Peace and Development Research, Göteborg University.

Orr, Robert C., Hg. (2004): Winning the Peace. An American Strategy for Post-Conflict Reconstruction. Washington, D.C.: The CSIS Press.

Osborne, Thomas (2004): »Techniken und Subjekte: Von den >Governmentality Studies $<$ zu den 〉Studies of Governmentality<.« In: Ramón Reichert (Hg.), Governmentality Studies. Analysen liberaldemokratischer Gesellschaften im Anschluss an Michel Foucault. Münster: LIT Verlag, 33-42.

OSCE - Organization for Security and Co-Operation in Europe (1997): The Relationship Between Large-Scale Forced Migration and the Electoral Process. Vienna: OSCE's Refugee Election Steering Group.

Ottaway, Marina (2002): »Nation-Building.» Foreign Policy, 132, Sept/Oct 2002: 16-24.

Ottaway, Marina (2003): »Rebuilding State Institutions in Collapsed States.« In: Jennifer Milliken (Hg.), State Failure, Collapse and Reconstruction. Malden, Oxford, Melbourne, Berlin: Blackwell Publishing, 245-66.

Österreichisches Studienzentrum für Frieden und Konfliktlösung, Hg. (2001): Zivile Konfliktbearbeitung. Eine internationale Herausforderung. Münster: Agenda Verlag.

Österreichisches Studienzentrum für Frieden und Konfliktlösung, Schweizerische Friedensstiftung, und Institut für Konfliktlösung, Hg. (1999): Krisenprävention: Theorie und Praxis ziviler Konfliktbearbeitung. Chur, Zürich: Verlag Rüegger.

Paffenholz, Thania (1995): »Vermittlung - Kriegsbeendigung und Konfliktregelung durch friedliche Einmischung.« In: Volker Matthies (Hg.), Vom Krieg zum Frieden. Kriegsbeendigung und Friedenskonsolidierung. Bremen: Edition Temmen, 39-56.

Paffenholz, Thania (2001): »Ansätze ziviler Konfliktbearbeitung.» In: Österreichisches Studienzentrum für Frieden und Konfliktlösung (Hg.), Zivile Konfliktbearbeitung. Eine internationale Herausforderung. Münster: Agenda Verlag, 15-26.

Paffenholz, Thania (2002): Stärkung von Friedensallianzen. Eschborn: GTZ. 
Paffenholz, Thania (2004): Erfahrungen Aus dem ATP Sri Lanka. Eschborn: GTZ.

Paffenholz, Thania (2006): »Peacebuilding: A Task for Development Cooperation.« Journal für Entwicklungspolitik, 22, no. 3-2006: 6-34.

Paffenholz, Thania, und Dunja Brede (2004): Lernerfahrungen aus dem ATP. Möglichkeiten und Grenzen der Technischen Zusammenarbeit in der Krisenprävention und Friedensentwicklung im Kontext terrorgefährdeter Länder. Eschborn: GTZ.

Paffenholz, Thania, und Luc Reychler (2007): Aid for Peace. A Guide to Planning and Evaluation for Conflict Zones. Baden-Baden: Nomos.

Pakenham, Thomas (1992): The Scramble for Africa 1876-1912. London: Abacus.

Paris, Roland (2001): »Human Security. Paradigm Shift or Hot Air?« International Security, 26, no. 2: 87-102.

Paris, Roland (2004): At War's End. Building Peace After Civil Conflict. Cambridge: Cambridge University Press.

Pearson, Frederic S. (2001): »Dimensions of Conflict Resolution in Ethnopolitical Disputes.« Journal of Peace Research, 38, no. 3: 275-87.

Pei, Minxin et al. (2006): »Building Nations. The American Experience.« In: Francis Fukuyama (Hg.), Nation-Building. Beyond Afghanistan and Iraq. Baltimore, Maryland: The Johns Hopkins University Press, 64-85.

Peiris, Pradeep (2006): »The International Community: Friend or Foe?« Polity, 30, no. 4: 24-26.

Perry, Richard Warren, und Bill Maurer, Hg. (2003): Globalization under Construction. Governmentality, Law, und Identity. Minneapolis, London: University of Minnesota Press.

Peters, Dirk (2001): »The debate about a new German foreign policy after unification.« In: Volker Rittberger (Hg.), German foreign policy since unification. Theories and case studies. Manchester: Manchester University Press, 11-33.

Pfaff-Czarnecka, Joanna (2004): »Demokratisierung und NationBuilding in >geteilten Gesellschaften «.« In: Jochen Hippler (Hg.), Nation-Building. Ein Schlüsselkonzept für friedliche Konfliktbearbeitung? Bonn: Dietz, 49-68.

Pherson, Randolph (2001): »Developing a More Effective Conflict Prevention Capacity in an Increasingly Unstable World.« Paper presented at the The Role of Foreign Assistance in Conflict Prevention. Washington, D.C.: U.S. Agency for International Development; The Woodrow Wilson International Center for Scholars. 
Philipson, Liz, und Yuvi Thangarajah (2005): The Politics of the NorthEast. Part of the Sri Lanka Strategic Conflict Assessment 2005. Tech. Rept. no. 4. Colombo: The Asia Foundation.

Picciotto, Robert, Funmi Olonisakin, und Michael Clarke (2005): Global Development and Human Security: Towards a Policy Agenda. A policy review commissioned by the Ministry for Foreign Affairs, Sweden. Global Development Studies, vol. 3. Stockholm: EGDI Secretariat, Ministry for Foreign Affairs, Sweden.

Pieper, Marianne, und Encarnación Gutíerrez Rodríguez, Hg. (2003): Gouvernementalität. Ein sozialwissenschaftliches Konzept in Anschluss an Foucault. Frankfurt am Main, New York: Campus.

Pirani, Cenan, und Ahlan Kadirgamar (2006): »Internationalisation of Sri Lanka's Peace Process and Governance. A Review of Strategic Conflict Assessments. « Economic and Political Weekly, 1789-95.

Piza Lopez, Eugenia, und Susan Schmeidl (2002): Gender and Conflict Early Warning: A Framework for Action. London: International Alert, Swiss Peace Foundation.

Plattform Zivile Konfliktbearbeitung (2003): Frieden braucht Gesellschaft! Gesellschaftliche Ansätze in der Zivilen Konfliktbearbeitung. Wahlenau: Initiativkreis Plattform Zivile Konfliktbearbeitung e.V.

Polanyi, Karl (2001 [1944]): The Great Transformation. The Political and Economic Origins of Our Time. Boston, MA: Beacon Press.

Pospisil, Jan (2004): »Das Intellektuellen-Partei-Dilemma der CPPNPA, Philippinen.« Südostasien Working Papers, Kommission für Sozialanthropologie an der Österreichischen Akademie der Wissenschaften, 4.

Pospisil, Jan (2005): »On the Edge of Escalation. How US Development Policy Deals with the Political Economy of War in Mindanao.« Kasarinlan. Philippine Journal of Third World Studies, 20, no. 2: 10024.

Pospisil, Jan (2006a): Development, Security and Research, KEF Fact Sheet no. 1/06. Wien: KEF.

Pospisil, Jan (2006b): »Joseph Kony, der ICC und die Suche nach Frieden in Acholiland.« In >Agency vs. Structure? «: Personalisierung in den Internationalen Beziehungen. Panel 1: Personalisierung im Krieg conducted at the 3-Länder-Tagung ÖGPW-SVPW-DVPW: Politik und Persönlichkeit, Wien.

Pospisil, Jan, und Stefan Khittel (2005a): »EU Development Policy as Alternative to the »Global War on Terrorism? « An examination of security-related development programmes in two crisis regions.« In European Development Cooperation to 2010. 11th EADI General 
Conference - »Insecurity and Development.« Bonn: European Association of Development Research and Training Institutes.

Pospisil, Jan, und Stefan Khittel (2005b): »Front-Runners or Appendices? Assessing the Potential of Small States in Security-Related Development Cooperation." Paper presented at the CEPSA Annual Conference 2005. Vienna: Central European Political Science Association.

Radelet, Steven (2003): »Bush and Foreign Aid.« Foreign Affairs, 82, no. 5, Sept/Oct 2003: 104-17.

Rahnema, Majid (1992): »Participation.« In: Wolfgang Sachs (Hg.), The Development Dictionary. A Guide to Knowledge as Power. London, New York: Zed Books, 116-31.

Rahnema, Majid, und Victoria Bawtree, Hg. (2005 [1997]): The PostDevelopment Reader. London, New York: Zed Books.

Rainford, Charan (2007): »Sri Lanka: Relapse Into Conflict. Limits of Donor Interventions.« Development »In Perspective« (Madrid), Case Studies 08, no. May 2007.

Rajasingham Semanayake, Darini (2006): »The Political Economy of Aid, Conflict, und Peace Building in Sri Lanka.« Polity, 30, no. 5\&6: 7-13.

Raman, B., N. Sathiya Moorthy, und Kalpana Chittaranjan, Hg. (2006): Sri Lanka: Peace Without Process. Colombo: Vijitha Yapa Publications.

Rampton, David, und Asanga Welikala (2005): The Politics of the South. Part of the Sri Lanka Strategic Conflict Assessment 2005. Tech. Rept. no. 3. Colombo: The Asia Foundation.

Ravlo, Hilde, Nils Petter Gleditsch, und Han Dorussen (2003): »Colonial War and the Democratic Peace.« Journal of Conflict Resolution, 47, no. 4: 520-48.

Regan, Patrick M. (2000): »Substituting Policies during U.S. Interventions in Internal Conflicts. A Little of This, a Little of That.« Journal of Conflict Resolution, 44, no. 1: 90-106.

Reichert, Ramón, Hg. (2004): Governmentality Studies. Analysen liberal-demokratischer Gesellschaften im Anschluss an Michel Foucault. Münster: LIT Verlag.

Reid, Julian (2005): »The Biopolitics of the War on Terror: a critique of the >return of imperialism< thesis in international relations. « Third World Quarterly, 26, no. 2: 237-52.

Reimann, Cordula (2001a): »Engendering the Field of Conflict Management: Why Gender Does Not Matter! Thoughts from a Theoretical Perspective.« Peace Studies Papers, Department of Peace Stu- 
dies, University of Bradford, Fourth Series, no. Working Paper 2: $19-45$.

Reimann, Cordula (2001b): Towards gender mainstreaming in crisis prevention and conflict management. Guidelines for the German Technical Co-operation. Eschborn: GTZ.

Reinfeldt, Sebastian, und Richard Schwarz (2004): »»Naissance de la biopolitique.< Liberalismus und Biopolitik.« In: Ramón Reichert (Hg.), Governmentality Studies. Analysen liberal-demokratischer Gesellschaften im Anschluss an Michel Foucault. Münster: LIT Verlag, 101-11.

Reinhardt, Dieter (1999): »Staaten und Krisenprävention. Die Aussenpolitik der skandinavischen Regierungen.« In: Österreichisches Studienzentrum für Frieden und Konfliktlösung, Schweizerische Friedensstiftung, und Institut für Konfliktlösung (Hg.), Krisenprävention: Theorie und Praxis ziviler Konfliktbearbeitung. Chur, Zürich: Verlag Rüegger, 335-52.

Reinhardt, Dieter (2001): »Bilaterale zivile Konfliktbearbeitung von Staaten in Westeuropa.« In: Österreichisches Studienzentrum für Frieden und Konfliktlösung (Hg.), Zivile Konfliktbearbeitung. Eine internationale Herausforderung. Münster: Agenda Verlag, 281-302.

Reinhardt, Klaus (2005): »Konflikt oder Zusammenarbeit zwischen Entwicklungs- und Sicherheitspolitik.« In: Conflict or convergence between security and development policy. 11th EADI General Conference - »Insecurity and Development.« Bonn: European Association of Development Research and Training Institutes.

Reis, Elisa P. (2004): »The Lasting Marriage Between Nation and State Despite Globalization.« International Political Science Review, 25, no. 3: 251-57.

Reno, William (2003): »The Politics of Insurgency in Collapsing States.« In: Jennifer Milliken (Hg.), State Failure, Collapse and Reconstruction. Malden, Oxford, Melbourne, Berlin: Blackwell Publishing, 83-103.

Reychler, Luc, und Thania Paffenholz, Hg. (2001): Peacebuilding: A Field Guide. Boulder, Col., London: Lynne Rienner Publishers.

Richards, Paul, Hg. (2005): No Peace, No War. An Anthropology of Contemporary Armed Conflict. London: James Currey Ltd.

Richmond, Oliver P. (2004): »The Globalization of Responses to Conflict and the Peacebuilding Consensus." Cooperation and Conflict: Journal of the Nordic International Studies Association, 39, no. 2: 129-50. 
Ricigliano, Robert (2003): »Networks of Effective Action: Implementing an Integrated Approach to Peacebuilding." Security Dialogue, 34, no. 4: 445-62.

Ricigliano, Robert, Hg. (2005): Choosing to engage: armed groups and peace processes. Accord issue, vol. 16. London: Accord.

Risse, Thomas (2004): »Kontinuität durch Wandel: Eine `neueく deutsche Außenpolitik?.« Aus Politik und Zeitgeschichte, 2004, no. B11: 2431.

Robinson, Clive (2006): »Whose security? Integration and integrity in EU policies for security and development.« In: Stephan Klingebiel (Hg.), New interfaces between security and development: changing concepts and approaches. Bonn: Deutsches Institut für Entwicklungspolitik, 69-92.

Robinson, William I. (1996): Promoting Polyarchy. Globalization, US Intervention, und Hegemony. Cambridge, UK: Cambridge University Press.

Rokkan, Stein (2000): Staat, Nation und Demokratie in Europa. Frankfurt am Main: Suhrkamp.

Ropers, Norbert (1994): »Ethno-soziale Konflikte und ihre Bearbeitung durch Drittpartei-Interventionen gesellschaftlicher Träger.« In: Österreichisches Studienzentrum für Frieden und Konfliktlösung and Schweizerische Friedensstiftung (Hg.), Krieg und gewaltfreie Konfliktlösung. Friedensbericht 1994. Chur, Zürich: Verlag Rüegger, 213-30.

Ropers, Norbert (1995): Friedliche Einmischung. Strukturen, Prozesse und Strategien zur konstruktiven Bearbeitung ethnopolitischer Konflikte. Berghof-Report Nr. 1. Berlin: Berghof Forschungszentrum für konstruktive Konfliktbearbeitung.

Ropers, Norbert (2002): Friedensentwicklung, Krisenprävention und Konfliktbearbeitung. Technische Zusammenarbeit im Kontext von Krisen, Konflikten und Katastrophen. Eschborn: GTZ.

Ropers, Norbert (2003): The Art of Protracted Peace Making. Lecture on the Occasion of the Opening Ceremony of the Foundation for CoExistence Colombo. Colombo.

Ropers, Norbert (2008): Systemic Conflict Transformation: Reflections on the Conflict and Peace Process in Sri Lanka. Berghof-Handbook Dialogue Series No. 6. Berlin: Berghof Research Center for Constructive Conflict Management.

Rostow, Walt W. (1991 ${ }^{3}$ [1960]): The Stages of Economic Growth: A Non-Communist Manifesto. Cambridge: Cambridge University Press. 
Rotberg, Robert I., Hg. (1999): Creating Peace in Sri Lanka. Civil War and Reconciliation. Washington, DC: Brookings Institution Press.

Rotberg, Robert I. (2002): »The New Nature of Nation-State Failure.« The Washington Quarterly, 25, no. 3: 85-96.

Rotberg, Robert I., Hg. (2003): State Failure and State Weakness in a Time of Terror. Cambridge, MA, Washington, DC: World Peace Foundation, Brookings Institution Press.

Rotberg, Robert I., Hg. (2004): When States Fail. Causes and Consequences. Princeton, NJ, Oxford: Princeton University Press.

Rufin, Jean-Christophe (1999): »Kriegswirtschaft in internen Konflikten.« In: Francois Jean und Jean-Christophe Rufin (Hg.), Ökonomie der Bürgerkriege. Hamburg: Hamburger Edition, 15-46.

Ruloff, Dieter (2004 [1985]): Wie Kriege beginnen. Ursachen und Formen. München: Verlag C.H. Beck.

Runge, Peter (1999): »Entwicklungszusammenarbeit und humanitäre Hilfe im Spannungsfeld von Krisenprävention und Konfliktverlängerung.« In: Österreichisches Studienzentrum für Frieden und Konfliktlösung, Schweizerische Friedensstiftung, und Institut für Konfliktlösung (Hg.), Krisenprävention: Theorie und Praxis ziviler Konfliktbearbeitung. Chur, Zürich: Verlag Rüegger, 137-52.

Rupesinghe, Kumar, Hg. (1989): Conflict Resolution in Uganda. Peace Research Monograph, vol. 16. Oslo: International Peace Research Institute.

Rupesinghe, Kumar, Hg. (2006a): Negotiating Peace in Sri Lanka. Efforts, Failures \& Lessons. Volume One. Colombo: The Foundation for Co-Existence.

Rupesinghe, Kumar, Hg. (2006b): Negotiating Peace in Sri Lanka. Efforts, Failures \& Lessons. Volume Two. Colombo: The Foundation for Co-Existence.

Rynning, Sten (2001): »Providing Relief or Promoting Democracy? The European Union and Crisis Management.« Security Dialogue, 32, no. 1: 87-101.

Sachs, Wolfgang, Hg. (1992): The Development Dictionary. A Guide to Knowledge as Power. London, New York: Zed Books.

Said, Edward W. (1983): The World, the Text, und the Critic. Cambridge: Harvard University Press.

Said, Edward W. (1993): Culture and Imperialism. London: Vintage.

Said, Edward W. (1995 [1978]): Orientalism. Western Concepts of the Orient. London: Penguin Books.

Saikal, Amin (2000): »Dimensions of state disruption and international responses.« Third World Quarterly, 21, no. 1: 39-49. 
Samaranayake, Gamini (2006): »Of Phases and Paces.« In: B. Raman, N. Sathiya Moorthy, und Kalpana Chittaranjan (Hg.), Sri Lanka: Peace Without Process. Colombo: Vijitha Yapa Publications, 16395.

Santiso, Carlos (2001): »International Co-operation for Democracy and Good Governance: Moving Towards a Second Generation?« European Journal of Development Research, 13, no. 1: 154-80.

Saull, Richard (2005): »Locating the Global South in the Theorisation of the Cold War: capitalist development, social revolution and geopolitical conflict.« Third World Quarterly, 26, no. 2: 253-80.

Schade, Jeanette (2004): »Zwischen Projektitis und Gegenmachtbildung - NGOs in Prozessen des Nation-Building.« In: Jochen Hippler (Hg.), Nation-Building. Ein Schlüsselkonzept für friedliche Konfliktbearbeitung? Bonn: Dietz, 178-94.

Schatz, Gottfried (2007): »Erdfieber: Das Unbehagen der Wissenschaft bei der Klimadebatte.« Neue Zürcher Zeitung (Zürich) 23. Juli, Nr. $168,19$.

Schmidt, Hajo (1994): »Pazifizierung - Zivilisierung - Demokratisierung. Philosophische Reflexionen über die Grundlagen europäischer Friedenspolitik.« In: Österreichisches Studienzentrum für Frieden und Konfliktlösung (Hg.), Europa, Zukunft eines Kontinents: Friedenspolitik oder Rückfall in die Barbarei? Münster: Agenda Verlag, 61-78.

Schneckener, Ulrich (2005a): »Frieden Machen: Peacebuilding und peacebuilder « Die Friedens-Warte, 80, no. 1-2, 17-39.

Schneckener, Ulrich (2005b): Die Zivilmacht Europa und die präwestfälische Herausforderung. Marburg: Zentrum für Konfliktforschung der Philipps-Universität Marburg.

Scott, David (1995): »Dehistoricising History.« In: Pradeep Jeganathan und Qadri Ismail (Hg.), Unmaking the Nation. The Politics of Identity and History in Modern Sri Lanka. Colombo: Social Scientists' Association, 10-24.

Scott, David (1999): Refashioning Futures. Criticism After Postcoloniality. Princeton, NJ: Princeton University Press.

Scott, David (2005 [1999]): »Colonial Governmentality.« In: Jonathan Xavier Inda (Hg.), Anthropologies of Modernity. Foucault, Governmentality, und Life Politics. Malden, Oxford, Carlton: Blackwell Publishing, 23-49.

Scott, James C. (1998): Seeing Like a State. How Certain Schemes to Improve the Human Condition Have FailHg. New Haven, London: Yale University Press. 
Seaton, Jim (1999): »The New 〉Ethnic « Wars and the Media.« In: Tim Allen and Jean Seaton (Hg.), The Media of Conflict: War Reporting and Representations of Ethnic Violence. London, New York: Zed Books, 43-63.

Senghaas, Dieter (1982): Von Europa lernen. Entwicklungsgeschichtliche Betrachtung. Frankfurt am Main: Suhrkamp.

Senghaas, Dieter (1992): Friedensprojekt Europa. Frankfurt am Main: Suhrkamp.

Senghaas, Dieter (1993): »Therapeutische Konfliktintervention in Europa.« In: Peter Atteslander (Hg.), Kulturelle Eigenentwicklung. Frankfurt/Main, New York: Campus, 65-85.

Senghaas, Dieter (1994): Wohin driftet die Welt? Über die Zukunft friedlicher Koexistenz. Frankfurt am Main: Suhrkamp.

Senghaas, Dieter (1995): »Frieden als Zivilisierungsprojekt.« In: Dieter Senghaas (Hg.), Den Frieden denken. Si vis pacem, para pacem. Frankfurt am Main: Suhrkamp, 196-223.

Senghaas, Dieter, Hg. (1997a): Frieden machen. Frankfurt am Main: Suhrkamp.

Senghaas, Dieter (1997b): »Frieden - Ein mehrfaches Komplexprogramm.« In: Dieter Senghaas (Hg.), Frieden machen. Frankfurt am Main: Suhrkamp, 560-76.

Shapiro, Isaac, und Nancy Birdsall (2002): How Does The Proposed Level Of Foreign Economic Aid Under The Bush Budget Compare with Historical Level? And What Would Be The Effects Of Bush's New >Millennium Challenge Account $\prec$ ? Washington, DC: Center for Global Development, Center on Budget and Policy Priorities.

Singer, Mona (2005): Geteilte Wahrheit. Feministische Epistemologie, Wissenssoziologie und Cultural Studies. Wien: Löcker.

Smith, Anthony D. (1987): The Ethnic Origins of Nations. Oxford, Malden: Blackwell Publishers.

Smith, Anthony D. (1998): Nationalism and Modernism. London, New York: Routledge.

Smith, Chris (1999): „South Asia’s Enduring War.« In: Robert I. Rotberg (Hg.), Creating Peace in Sri Lanka. Civil War and Reconciliation. Washington, DC: Brookings Institution Press, 17-40.

Smith, Dan (2004a): Towards a Strategic Framework for Peacebuilding: Getting Their Act Together. Overview report of the Joint Utstein Study of Peacebuilding. Oslo: Royal Norwegian Ministry of Foreign Affairs.

Smith, Dan (2004b): »Trends and Causes of Armed Conflict.« In: Alex Austin, Martina Fischer, und Norbert Ropers (Hg.), Transforming 
Ethnopolitical Conflict. The Berghof Handbook. Wiesbaden: VS Verlag für Sozialwissenschaften, 111-27.

Social Impact, Inc. (2007): Final Evaluation: The Sri Lanka Transition Initiative (2003-2007). Colombo: USAID.

Solheim, Erik (2006): »Interview with Erik Solheim, Minister of Development [Norway] and Facilitator of the Sri Lankan Peace Process.« In: Kumar Rupesinghe (Hg.), Negotiating Peace in Sri Lanka. Efforts, Failures \& Lessons. Volume Two. Colombo: The Foundation for Co-Existence, 339-54.

Somasundaram, Ramash (2005): Strategic Significance of Sri Lanka. Pannipitiya: Stamford Lake.

Söderberg, Mimmi, und Thomas Ohlson (2003): Democratisation and Armed Conflicts in Weak States, Sida. Stockholm: Sida.

Sørensen, Georg (2001): »War and State-Making. Why Doesn't It Work in the Third World? « Security Dialogue, 32, no. 3: 341-54.

Spelten, Angelika (1999): »Präventive Maßnahmen in der Entwicklungszusammenarbeit. Indikatorenkatalog zur Bestimmung des Einsatzzeitpunktes.« In: Österreichisches Studienzentrum für Frieden und Konfliktlösung, Schweizerische Friedensstiftung, und Institut für Konfliktlösung (Hg.), Krisenprävention: Theorie und Praxis ziviler Konfliktbearbeitung. Chur, Zürich: Verlag Rüegger, 121-36.

Spelten, Angelika (2000): »Wie erkennt man Krisenpotential? Entwurf eines Indikatorenkatalogs.« Entwicklung und Zusammenarbeit, 2000, no. 3: 70-72.

Spelten, Angelika (2001): »Entwicklungszusammenarbeit als Instrument der Krisenprävention und des nachhaltigen Wiederaufbaus.« In: Österreichisches Studienzentrum für Frieden und Konfliktlösung (Hg.), Zivile Konfliktbearbeitung. Eine internationale Herausforderung. Münster: Agenda Verlag, 110-25.

Spelten, Angelika (2004): »Stabilisierung durch >Friedensökonomie?«.» In: Sabine Kurtenbach und Peter Lock (Hg.), Kriege als (Über) Lebenswelten. Schattenglobalisierung, Kriegsökonomien und Inseln der Zivilität. Bonn: Dietz, 274-85.

Spivak, Gayatri Chakravorty (1988): »Can the Subaltern Speak?« In: Cary Nelson und Lawrence Grossberg (Hg.), Marxism and the Interpretation of Culture. Urbana, Chicago: University of Illinois Press, 271-313.

Spranger, Carl-Dieter (1996): »Krisenprävention: eine Aufgabe der Entwicklungspolitik« Internationale Politik, 1996, no. 4, 1-7.

Stepanova, Ekaterina (2003): Anti-terrorism and Peacebuilding During and After Conflict. Stockholm: Sipri. 
Stokke, Kristian (2006): »Building the Tamil Eelam State: Emerging State Institutions and Forms of Governance in LTTE-Controlled Areas in Sri Lanka.« Third World Quarterly, 27, no. 6: 1021-40.

Stokke, Olav (1997): »Violent Conflict Prevention and Development Co-operation: Coherent or Conflicting Perspectives?« Forum for Development Studies, 1997, no. 2: 195-250.

Stoler, Ann Laura (1995): Race and the Education of Desire. Foucault's History of Sexuality and the Colonial Order of Things. Durham, London: Duke University Press.

Stoler, Ann Laura (2002): Carnal Knowledge and Imperial Power. Race and the Intimate in Colonial Rule. Berkeley, Los Angeles, London: University of California Press.

Storey, Andy (2000): »Post-Development Theory: Romanticism and Pontius Pilate politics.« Development, 43, no. 4: 40-46.

Strategic Foresight Group (2006): Cost of Conflict in Sri Lanka. Mumbai: Strategic Foresight Group.

Study Group on Europe's Security Capabilities (2004): A Human Security Doctrine for Europe, Presented to EU High Representative for Common Foreign and Security Policy Javier Solana. Barcelona.

Sutton, Francis X. (2006): »Nation-Building in the Heyday of the Classic Development Ideology. Ford Foundation Experience in the 1950s and 1960s« In: Francis Fukuyama (Hg.), Nation-Building. Beyond Afghanistan and Iraq. Baltimore, Maryland: The Johns Hopkins University Press, 42-63.

Taake, Hans-Helmut (2002): »Der Nährboden für den Terrorismus: Was tun? Überlegungen des Deutschen Instituts für Entwicklungspolitik.« Entwicklung und Zusammenarbeit, 43, no. 1: 11-12.

Talentino, Andrea Kathryn (2002): »Intervention as Nation-Building: Illusion or Possibility? « Security Dialogue, 33, no. 1: 27-43.

Terry, Fiona (2002): Condemned to Repeat? The Paradox of Humanitarian Action. Ithaca, London: Cornell University Press.

Tetzlaff, Rainer (2004): »Globalisierung und Nation-Building - kein Widerspruch.« In: Jochen Hippler (Hg.), Nation-Building. Ein Schlüsselkonzept für friedliche Konfliktbearbeitung? Bonn: Dietz, 31-48.

The Congress of the United States, Congressional Budget Office (1997):

The Role of Foreign Aid in Development. Washington, D.C.: CBO.

The White House (1997): Managing Complex Contingency Operations.

Presidential Decision Directive, PDD/NSC 56, Washington, D.C.: The White House.

The White House (2002): The National Security Strategy of the United States of America. Washington, D.C.: The White House. 
The White House (2006): The National Security Strategy of the United States of America. Washington, D.C.: The White House.

Tilly, Charles (1985): »War Making and State Making as Organized Crime.« In: Peter B. Evans, Dietrich Rueschemeyer, und Theda Skocpol (Hg.), Bringing the State Back In. Cambridge, UK: Cambridge University Press, 169-87.

Tilly, Charles (1992): Coercion, Capital, und European States, AD 9901992. Cambridge, MA, Oxford, UK: Blackwell Publishing.

Timbermann, David G. (2007): Evaluation of the AED Peace Support Program (Phase III) and the TAF-ICMA Transparent and Accountable Local Governance (TALG) Program, Prepared for USAID Sri Lanka. Colombo: USAID.

Tiruchelvam, Neelan (1999): »Devolution and the Elusive Quest for Peace.« In: Robert I. Rotberg (Hg.), Creating Peace in Sri Lanka. Civil War and Reconciliation. Washington, DC: Brookings Institution Press, 189-201.

Travis, Rick (1998): »The Promotion of Democracy at the End of the Twentieth Century: A New Polestar for American Foreign Policy?« In: James M. Scott (Hg.), After the End: Making U.S. Foreign Policy in the Post-Cold War World. Durham, NC: Duke University Press, 251-76.

Trotha, Trutz von (1999): »Formen des Krieges. Zur Typologie kriegerischer Aktionsmacht.« In: Sighard Neckel und Michael SchwabTrapp (Hg.), Ordnungen der Gewalt. Beiträge zu einer politischen Soziologie der Gewalt und des Krieges. Opladen: Leske + Budrich, 71-96.

Tuminez, Astrid S. (2005): Ancestral Domain in Comparative Perspective, United States Institute of Peace Special Report no. 151. Washington, D.C.: USIP.

UNDP - United Nations Development Programme (1994): Human Development Report 1994. New dimensions of human security. New York, Oxford: Oxford University Press.

UNDP - United Nations Development Programme (2002): Human Development Report 2002. Deepening democracy in a fragmented world. New York, Oxford: Oxford University Press.

UNDP - United Nations Development Programme (2003a): Human Development Report 2003. Millennium Development Goals: A compact among nations to end human poverty. New York, Oxford: Oxford University Press.

UNDP - United Nations Development Programme (2005): Human Development Report 2005. International cooperation at a crossroads. 
Aid, trade and security in an unequal world. New York, Oxford: Oxford University Press.

United Nations Security Council (2000): On women and peace and security, Resolution 1325 (2000) Adopted by the Security Council at its 4213th meeting, on 31 October 2000 no. S/RES/1325 (2000). New York: United Nations.

USAID - U.S. Agency for International Development (1994): Strategies for Sustainable Development, USAID Office of the Administrator, herausgegeben von Brian J. Atwood. Washington, D.C.: USAID.

USAID - U.S. Agency for International Development (1997): Strategic Plan [1997]. Washington, D.C.: USAID.

USAID - U.S. Agency for International Development, Center for Democracy and Governance (1998a): Civil-Military Relations: USAID's Role, Technical Paper Series. Washington, D.C.: USAID.

USAID - U.S. Agency for International Development, Center for Democracy and Governance (1998b): Democracy and Governance: A Conceptual Framework, Technical Paper Series. Washington, D.C.: USAID.

USAID - U.S. Agency for International Development (1998c): USAID/Sri Lanka, Revised Country Program Strategy, 1998-2000. Colombo: USAID.

USAID - U.S. Agency for International Development (2000a): USAID Strategic Plan 1997 (revised 2000). Washington, D.C.: USAID.

USAID - U.S. Agency for International Development, Center for Democracy and Governance (2000b): Conducting a DG Assessment: A Framework for Strategy Development, Technical Publication Series. Washington, D.C.: USAID.

USAID - U.S. Agency for International Development (2000c): USAID/Sri Lanka: Country Program Strategy 2001-2005. Colombo: USAID.

USAID - U.S. Agency for International Development (2001a): Elections and Conflict: An Issues Paper, herausgegeben von Michele Schimpp und Aud Frances McKernan. Washington, D.C.: USAID.

USAID - U.S. Agency for International Development, Office of Transition Initiatives (2001b): Guide to Program Options in Conflict-Prone Settings. Washington, D.C.: USAID.

USAID - U.S. Agency for International Development (2002): Foreign Aid in the National Interest. Promoting Freedom, Security, und Opportunity, herausgegeben von Susan Merrill. Washington, D.C.: USAID.

USAID - U.S. Agency for International Development, Office of Private and Voluntary Cooperation (2003): Operating in Conflict. Current 
Practices in the Development Community, herausgegeben von Cailtin Davitt. Washington, D.C.: USAID.

USAID - U.S. Agency for International Development, Office of Democracy and Governance (2004a): Civil Society Groups and Political Parties. Supporting Constructive Relationships, Occasional Paper Series. Washington, D.C.: USAID.

USAID - U.S. Agency for International Development, Bureau for Policy and Program Coordination (2004b): U.S. Foreign Aid: Meeting the Challenges of the Twenty-first Century, White Paper. Washington, D.C.: USAID.

USAID - U.S. Agency for International Development, Bureau for Policy and Program Coordination (2005a): Conflict Mitigation and Management Policy. Washington, D.C.: USAID.

USAID - U.S. Agency for International Development (2005b): Conducting a Conflict Assessment. A Framework for Strategy and Program Development. Washington, D.C.: USAID.

USAID - U.S. Agency for International Development (2005c): Fragile States Strategy. Washington, D.C.: USAID.

USAID - U.S. Agency for International Development (2005d): At Freedom's Frontiers. A Democracy and Governance Strategic Framework. Washington, D.C.: USAID.

USAID - U.S. Agency for International Development (2005e): U.S. Overseas Loans and Grants. Obligations and Loan Authorizations. July 1, 1945 - September 30, 2003, Greenbook. Washington, D.C.: USAID.

USAID - U.S. Agency for International Development (2005f): Budget Justification to the Congress. Fiscal Year $2006<$ http://www.usaid. gov/policy/budget/cbj2006/> (Zugegriffen am 06-11-2005).

USAID - U.S. Agency for International Development (2005g): USAID/Sri Lanka Annual Report, FY 2005. Colombo: USAID.

USAID - U.S. Agency for International Development (2005h): Performance Management Plan, USAID/Sri Lanka, FY 2003-FY 2007. Colombo: USAID.

USAID - U.S. Agency for International Development, Office of Conflict Management and Mitigation (2005i): Land and Conflict. A Toolkit and Intervention. Washington, D.C.: USAID.

USAID - U.S. Agency for International Development, Office of Conflict Management and Mitigation (2005j): Minerals and Conflict. A Toolkit for Intervention. Washington, D.C.: USAID.

USAID - U.S. Agency for International Development, Office of Conflict Management and Mitigation (2005k): Youth and Conflict. A Toolkit for Intervention. Washington, D.C.: USAID. 
USAID - U.S. Agency for International Development, Office of Conflict Management and Mitigation (20051): Livelihoods and Conflict. A Toolkit for Intervention. Washington, D.C.: USAID.

USAID - U.S. Agency for International Development, Office of Conflict Management and Mitigation (2005m): Forest and Conflict. A Toolkit for Intervention. Washington, D.C.: USAID.

USAID - U.S. Agency for International Development (2006a): »USAID History« < http://www.usaid.gov/about_usaid/usaidhist.html > Washington, D.C. (Zugegriffen am 11-08-2006).

USAID - U.S. Agency for International Development (2006b): Policy Framework for Bilateral Foreign Aid. Implementing Transformational Diplomacy Through Development. Washington, D.C.: USAID.

USAID - U.S. Agency for International Development, DCHA/DG (2006c): User's Guide to DG Programming. Washington, D.C.: USAID.

USAID - U.S. Agency for International Development (2006d): USAID/Sri Lanka, Operational Plan, FY 2006. Colombo: USAID.

USAID - U.S. Agency for International Development (2006e): USAID/Sri Lanka: Economic Growth \& Conflict Assessment. Colombo: USAID.

USAID - U.S. Agency for International Development (2006f): Budget Justification to the Congress. Fiscal Year $2007<$ http://www.usaid. gov/policy/budget/cbj2007/> (Zugegriffen am 05-09-2006).

USAID - U.S. Agency for International Development, Office of Conflict Management and Mitigation (2007): Women \& Conflict. An Introductory Guide For Programming. Washington, D.C.: USAID.

USAID - U.S. Agency for International Development and Woodrow Wilson International Center for Scholars (2001): »The Role of Foreign Assistance in Conflict Prevention.« Conference Report. Washington, D.C.: USAID.

U.S. Department of State, und USAID - U.S. Agency for International Development (2003): Strategic Plan, Fiscal Years 2004-2009. Security, Democracy, Prosperity. Washington, D.C.: Department of State/USAID Publication.

U.S. Department of State, und USAID - U.S. Agency for International Development (2007a): Strategic Plan, Fiscal Years 2007-2012. Transformational Diplomacy. Washington, D.C.: Department of State/USAID Publication.

U.S. Department of State, und USAID - U.S. Agency for International Development (2007b): Budget Justification to the Congress. Fiscal 
Year $2008<$ http://www.usaid.gov/policy/budget/cbj2008/> (Zugegriffen am 06-06-2007).

Uyangoda, Jayadeva (1999): »A Political Culture of Conflict.» In:

Robert I. Rotberg (Hg.), Creating Peace in Sri Lanka. Civil War and Reconciliation. Washington, DC: Brookings Institution Press, 15768.

Uyangoda, Jayadeva, Hg. (2005): Conflict, Conflict Resolution \& Peace Building. Colombo: Department of Political Science and Public Policy and IMCAP, University of Colombo.

Uyangoda, Jayadeva, und Morina Perera, Hg. (2003): Sri Lanka’s Peace Process 2002: Critical Perspectives. Colombo: Social Scientists' Association.

Van Creveld, Martin L. (1998 [1991]): Die Zukunft des Krieges. München: Gerling Akademie Verlag.

Van Creveld, Martin L. (1999): Aufstieg und Untergang des Staates. München: Gerling Akademie Verlag.

Van Edig, Helmut (2004): »Nation-Building: Eine Strategie für regionale Stabilisierung und Konfliktprävention.« In: Jochen Hippler (Hg.), Nation-Building. Ein Schlüsselkonzept für friedliche Konfliktbearbeitung? Bonn: Dietz, 215-32.

Vaux, Tony (2001): The Selfish Altruist. Relief Work in Famine and War. London, Sterling, VA: Earthscan.

Venkataramanan, K. (2006): »Dilemmas of External Actors.« In: B. Raman, N. Sathiya Moorthy, und Kalpana Chittaranjan (Hg.), Sri Lanka: Peace Without Process. Colombo: Vijitha Yapa Publications, 197-225.

Veyne, Paul (1992 [1978]): Foucault: Die Revolutionierung der Geschichte. Frankfurt am Main: Suhrkamp.

Volkan, Vamik (1997): Bloodlines: From Ethnic Pride to Ethnic Terrorism. Boulder, CO: Westview Press.

Walby, Sylvia (2003): »The Myth of the Nation-State: Theorizing Society and Polities in a Global Era.« Sociology, 37, no. 3: 529-46.

Wallerstein, Immanuel (1986): Das moderne Weltsystem: Kapitalistische Landwirtschaft und die Entstehung der europäischen Weltwirtschaft im 16. Jahrhundert. Frankfurt am Main: Syndikat.

Wallerstein, Immanuel (1990a): »Die Konstruktion von Völkern: Rassismus, Nationalismus, Ethnizität.« In Rasse, Klasse, Nation. Ambivalente Identitäten, herausgegeben von Etienne Balibar und Immanuel Wallerstein, 87-106. Hamburg: Argument Verlag.

Wallerstein, Immanuel (1990b): »Soziale Konflikte in den unabhängigen Staaten Schwarzafrikas.« In: Etienne Balibar und Immanuel Wal- 
lerstein (Hg.), Rasse, Klasse, Nation. Ambivalente Identitäten. Hamburg: Argument Verlag, 227-46.

Wallerstein, Immanuel (1999): The End of the World as we know it. Social Science for the Twenty-First Century. Minneapolis, London: University of Minnesota Press.

Wallerstein, Immanuel (2004): World-System Analysis. An Introduction. Durham, London: Duke University Press.

Walter, Barbara F. (1999): »Designing Transitions from Civil War. Demobilization, Democratization, und Commitments to Peace.« International Security, 24, no. 1: 127-55.

Wanie, Renate, und Hans Hartmann (2000): „Schöner intervenieren. Gewaltfreie Intervention - ein verkappter Friedenskolonialismus?« In: Tilman Evers (Hg.), Ziviler Friedensdienst. Fachleute für den Frieden. Idee - Erfahrungen - Ziele. Opladen: Leske + Budrich, 8693.

Weilenmann, Markus (1999): »Konfliktregelungsverfahren am Kreuzpunkt zwischen Mediation und Recht. Plädoyer für einen gesellschaftlich integrativen Ansatz der Konfliktbearbeitung.« In: Österreichisches Studienzentrum für Frieden und Konfliktlösung, Schweizerische Friedensstiftung, und Institut für Konfliktlösung (Hg.), Krisenprävention: Theorie und Praxis ziviler Konfliktbearbeitung. Chur, Zürich: Verlag Rüegger, 99-117.

Weinstein, Jeremy M., John Edward Porter, und Stuart E. Eizenstat, Commission on Weak States and US National Security (2004): On the Brink. Weak States and US National Security. Washington, D.C.: Center for Global Development.

Werlhof, Claudia von (1991): Was haben die Hühner mit dem Dollar zu tun? Frauen und Ökonomie. München: Frauenoffensive.

Werther-Pietsch, Ursula (2001): Strategie für Entwicklung auf Grundlage der Menschenrechte und Demokratisierung. Europäische Union: Entwicklungs- und Außenpolitik. Cotonou-Abkommen. ÖFSE Forum, vol. 15. Wien: ÖFSE.

Wickramasinghe, Nira (2001): Civil Society in Sri Lanka: New Circles of Power. New Delhi, Thousand Oaks, London: Sage Publications.

Wickramasinghe, Somasari, und Nilmal Wickramasinghe (2005): S.D. and the Family Roots in Sri Lankan Politics. Rajagiriya: Rasika Printers.

Wieczorek-Zeul, Heidemarie (1999a): »Entwicklungspolitik als Friedenspolitik. Interview mit Ministerin Heidemarie Wieczorek-Zeul.« Entwicklung und Zusammenarbeit, 1999, no. 1: 8-10. 
Wieczorek-Zeul, Heidemarie (1999b): »Security Aspects of Development Cooperation.« Development and Cooperation, 1999, no. 6: 912.

Wieczorek-Zeul, Heidemarie (2000): »Entwicklungspolitik ist Friedenspolitik. Neue Herausforderungen für Frieden und Entwicklung auf dem Weg ins 21. Jahrhundert.« In: Franz Nuscheler (Hg.), Entwicklung und Frieden im Zeichen der Globalisierung. Bonn: Bundeszentrale für politische Bildung, 131-43.

Wieczorek-Zeul, Heidemarie (2002): »Entwicklungspolitik nach dem 11. September. Ein umfassender friedens- und sicherheitspolitischer Ansatz.« Entwicklung und Zusammenarbeit, 43, no. 1: 8-10.

Wieczorek-Zeul, Heidemarie (2004): »»Es darf keine Vermischung der Finanzen geben.< Interview mit Entwicklungsministerin Heidemarie Wieczorek-Zeul.« Entwicklung und Zusammenarbeit, 45, no. 4: 14447.

Williams, Glyn (1999): French Discourse Analysis. The method of poststructuralism. London, New York: Routledge.

Wimmer, Andreas (1997): »Who owns the state? Understanding ethnic conflict in post-colonial societies.« Nations and Nationalism, 3, no. 4: 631-65.

Wittkopf, Eugene R., Charles W. Kegley, Jr., und James M. Scott (2003): American Foreign Policy: Pattern and Process. 6th Hg. Belmont, CA: Wadsworth.

Wodak, Ruth, und Michael Meyer, Hg. (2002): Methods of Critical Discourse Analysis. London, Thousand Oaks, New Delhi: Sage Publications.

Wolf, Eric R. (1997 [1982]): Europe and the People Without History. Berkeley, Los Angeles, London: University of California Press.

Wood, Bernard (2003 [2001]): Development Dimensions of Conflict Prevention and Peacebuilding. An independent study prepared for the Bureau for Crisis Prevention \& Recovery, UNDP. New York: UNDP.

World Bank (1983): World Development Report 1983. World Economic Recession and Prospects for Recovery; Management in Development; World Development Indicators. Washington, D.C.: The World Bank.

World Bank (1989): Sub-Saharan Africa: From Crisis to Sustainable Growth. A Long-Term Perspective Study. Washington, D.C.: The World Bank.

World Bank (1997): World Development Report 1997. The State in a Changing World. Oxford, New York: Oxford University Press. 
World Bank (2003): Nachhaltige Entwicklung in einer dynamischen Welt. Institutionen, Wachstum und Lebensqualität verbessern. Bonn: UNO-Verlag.

World Bank (2004a): Funktionierende Dienstleistungen für arme Menschen. Bonn: UNO-Verlag.

World Bank (2004b): Sri Lanka: Development Policy Review. Colombo: The World Bank Colombo Office.

World Bank (2005a): Ein besseres Investitionsklima für Jeden. Bonn: UNO-Verlag.

World Bank (2005b): Global Development Finance 2005: Mobilizing Finance and Managing Vulnerability. Washington, D.C.: The World Bank.

Yack, Bernard (2001): »Nationalism and the Modern Doctrine of Popular Sovereignty.« Political Theory, 29, no. 4: 517-36.

Young, Miriam (2000): »Sri Lanka’s Long War.« Foreign Policy in Focus, 5 , no. 35 .

Youngs, Richard (2003): »European approaches to democracy assistance: learning the right lessons?« Third World Quarterly, 24, no. 1: 127-38.

Zanger, Sabine C. (2000): „Good Governance and European Aid. The Impact of Political Conditionality. « European Union Politics, 1, no. 3: 293-317.

Zdunnek, Gabriele (2003): »Gender Mainstreaming in der Konfliktanalyse und -bearbeitung." In: Uwe Kievelitz und Roman Poeschke (Hg.), Friedens- und Konfliktarbeit in Forschung und EZ-Praxis und der Beitrag der Entwicklungsethnologie. Saarbrücken: Verlag für Entwicklungspolitik, 157-64.

Ziai, Aram (2003a): »Foucault in der Entwicklungstheorie.« Peripherie, 23, no. 92: 406-29.

Ziai, Aram (2003b): »Governance und Gouvernementalität.« Nord-Süd aktuell, XVII, no. 3: 411-22.

Ziai, Aram (2004a): Entwicklung als Ideologie? Entwicklungsparadigma und die Post-Development-Kritik. Ein Beitrag zur Analyse des Entwicklungsdiskurses. Hamburg: DÜI.

Ziai, Aram (2004b): »The ambivalence of post-development: between reactionary populism and radical democracy.« Third World Quarterly, 25, no. 6: 1045-60.

Ziai, Aram (2004c): »Zur Ordnung und Transformation des Entwicklungsdiskurses.« In: Susanne Kollmann und Kathrin Schödel (Hg.), PostModerne De/Konstruktionen. Ethik, Politik und Kultur am Ende einer Epoche. Münster: LIT Verlag, 157-69. 
Ziai, Aram (2006): Zwischen Global Governance und Post-Development. Entwicklungspolitik aus diskursanalytischer Perspektive. Münster: Verlag Westfälisches Dampfboot.

Zunzer, Wolfram (2003): »Zivile Konfliktbearbeitung in Sri Lanka und die Besonderheiten Des Einsatzes Terroristischer Mittel Durch die Liberation Tigers of Tamil Eelam (LTTE).« In: Jörg Calließ (Hg.), Zivile Konfliktbearbeitung Im Schatten Des Terrors. RehburgLoccum: Bertelsmann, 131-41. 



\section{Tabellen und Grafiken}

Tabelle 1: Aufwendungen ausgewählter Geber für

DAC-Codes 150/152 166

Tabelle 2: Bilaterale US-Hilfe nach Regionen in Prozentanteilen 196

Abbildung 1: »Branding« der post-politischen Konfliktanalyse 116

Abbildung 2: Kriegsformen im historischen Verlauf nach

AKUF-Kriegstypen

Abbildung 3: Die »Civilizations« nach Huntington

Abbildung 4: Das zivilisatorische Hexagon von Senghaas

Abbildung 5: ODA der größten bilateralen Geber in Anteilen an

Gesamt-ODA

Abbildung 6: Der institutionelle Apparat des Dispositivs

Entwicklung

Abbildung 7: US-Demokratieförderung in den 1990ern nach Regionen

Abbildung 8: US-Demokratieförderung im Verhältnis zur

Gesamt-ODA in den 1990ern

Abbildung 9: US-Demokratieförderung im Verhältnis zu den

Gesamtmilitärausgaben der USA in den 1990ern

Abbildung 10: Aufteilung der US-amerikanischen

Demokratieförderung nach Institutionen im Jahr 1998

Abbildung 11: ODA-Aufwendungen der USA

Abbildung 12: ODA-Aufwendungen der USA

im Verhältnis zum BIP

Abbildung 13: Institutionelle Verankerung des Demokratie- und

Konfliktbereiches in USAID 
Abbildung 14: Schlüsseldokumente der US-Entwicklungspolitik im Konfliktkontext

Abbildung 15: Historische Entwicklung der deutschen

ODA-Leistungen

Abbildung 16: Deutsche ODA-Aufwendungen für DAC-Code 150245

Abbildung 17: Deutscher Anteil an den Gesamtaufwendungen der DAC-Mitgliedsstaaten für Code 150

Abbildung 18: Friedensaktivistin in BMZ-Publikation

Abbildung 19: Schlüsseldokumente der deutschen

Entwicklungspolitik im Konfliktkontext

Abbildung 20: Institutionelle Verflechtung der zivilen

Friedensentwicklung in Deutschland

Abbildung 21: Schema des deutschen Konzepts zur Herstellung verlässlicher staatlicher Strukturen

Abbildung 22: Zivilisatorisches Hexagon der staatlichen deutschen Friedensentwicklung

Abbildung 23: Politische Karte von Sri Lanka

Abbildung 24: ODA in Sri Lanka

Abbildung 25: US-amerikanische ODA in Sri Lanka

Abbildung 26: Programmschema von USAID in Sri Lanka

Abbildung 28: Programmschema der GTZ in Sri Lanka

Abbildung 29: Interventionsstrategie der Berghof Foundation

in Sri Lanka

Abbildung 30: Arbeitskonzept von FLICT

Anmerkung zu den Statistiken: Wenn nicht anders angegeben, wurden für die quantitativen Statistiken die von der OECD veröffentlichten Zahlen des Development Assistance Committee verwendet. Die Daten sind über die Website der OECD abrufbar: http://www.oecd.org/dac/stats/ idsonline. 


\section{Global Studies}
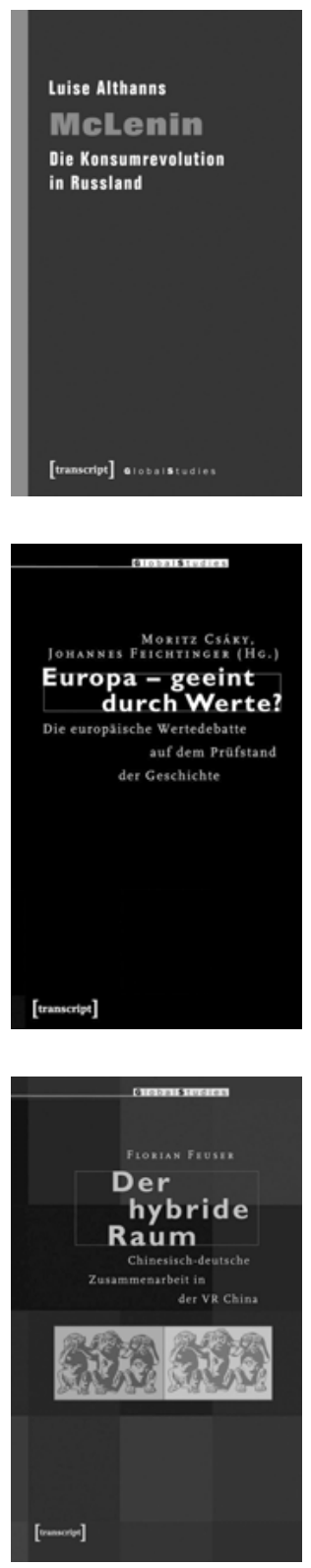

Luise Althanns

\section{McLenin}

Die Konsumrevolution in Russland

Oktober 2009, ca. 294 Seiten, kart., ca. 29,80€, ISBN 978-3-8376-I254-7

MORITZ CSÁKY, JOHANNES FEICHTINGER (HG.)

Europa - geeint durch Werte?

Die europäische Wertedebatte auf dem Prüfstand der Geschichte

2007, 2I8 Seiten, kart., 24,80€, ISBN 978-3-89942-785-I

\section{FLORIAN FEUSER}

Der hybride Raum

Chinesisch-deutsche Zusammenarbeit in der VR China

2006, 344 Seiten, kart., 29,80€, ISBN 978-3-89942-58I-9 


\section{Global Studies}

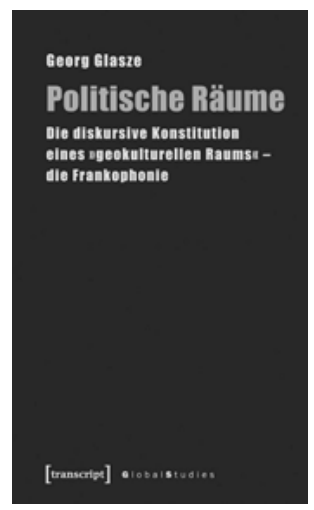

Georg Glasze

Politische Räume

Die diskursive Konstitution

eines »geokulturellen Raums«die Frankophonie

August 2009, ca. 256 Seiten, kart., ca. 27,80€, ISBN 978-3-8376-I232-5

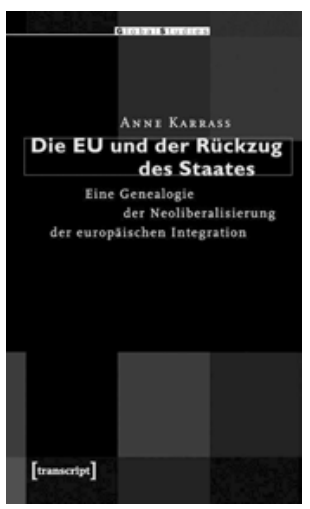

ANNE KARRASS

Die EU und der Rückzug des Staates Eine Genealogie der Neoliberalisierung der europäischen Integration

Januar 2009, 280 Seiten, kart., 28,80€, ISBN 978-3-8376-1067-3

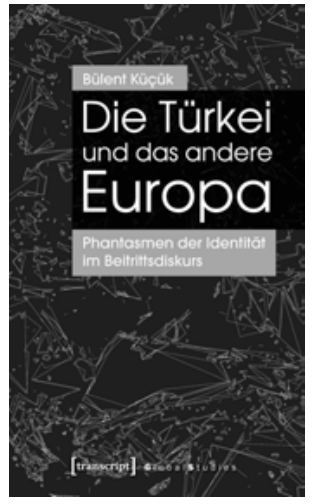

\section{BÜLENT KüÇÜK}

Die Türkei und das andere Europa

Phantasmen der Identität im

Beitrittsdiskurs

2008, 236 Seiten, kart., zahlr. Abb., 25,80€, ISBN 978-3-8376-IOI2-3 


\section{Global Studies}

Christian Berndt,

Johannes GlüCKLER (HG.)

Denkanstöße zu einer anderen

Geographie der Ökonomie

2006, I72 Seiten, kart., I7,80€,

ISBN 978-3-89942-454-6

Heiner Depner

Transnationale Direkt-

investitionen und kulturelle

Unterschiede

Lieferanten und Joint Ventures

deutscher Automobilzulieferer

in China

2006, 240 Seiten, kart., $25,80 €$,

ISBN 978-3-89942-567-3

\section{UlRich HeInZE}

Hautkontakt der Schriftsysteme

Japan im Zeichen der

Globalisierung: Geldflüsse

und Werbetexte

2006, 208 Seiten, kart., $25,80 €$,

ISBN 978-3-89942-513-0

\section{WiebKe Keim}

\section{Vermessene Disziplin}

Zum konterhegemonialen

Potential afrikanischer und

lateinamerikanischer Soziologien 2008, 564 Seiten, kart., $35,80 €$, ISBN 978-3-89942-838-4

Christian Kellermann

Die Organisation

des Washington Consensus

Der Internationale

Währungsfonds und

seine Rolle in der

internationalen

Finanzarchitektur

2006, 326 Seiten, kart., $28,80 €$,

ISBN 978-3-89942-553-6
Lutz Leisering, Petra Buhr,

Ute Traiser-DioP

Soziale Grundsicherung

in der Weltgesellschaft

Monetäre Mindestsicherungs-

systeme in den Ländern des

Südens und des Nordens.

Weltweiter Survey und

theoretische Verortung

2006, 342 Seiten, kart., 29,80€,

ISBN 978-3-89942-460-7

Ivo Mossig

Netzwerke der Kulturökonomie

Lokale Knoten und globale

Verflechtungen der Film- und

Fernsehindustrie in Deutschland und den USA

2006, 228 Seiten, kart., $26,80 €$, ISBN 978-3-89942-523-9

Matthias OtTen

Interkulturelles Handeln in der globalisierten Hochschulbildung Eine kultursoziologische Studie 2006, 318 Seiten, kart., 28,80€, ISBN 978-3-89942-434-8

Helen Schwenken

Rechtlos, aber nicht ohne Stimme

Politische Mobilisierungen um irreguläre Migration in die Europäische Union 2006, 374 Seiten, kart., 29,80€, ISBN 978-3-89942-516-I

Shingo Shimada, Christian TAGsold

Alternde Gesellschaften im Vergleich

Solidarität und Pflege in

Deutschland und Japan

2006, I78 Seiten, kart., I8,80€, ISBN 978-3-89942-476-8 
